\title{
Stoffübergang in Druckblasensäulen mit organischen Flüssigkeiten
}

\author{
Von der Fakultät für Lebenswissenschaften \\ der Technischen Universität Carolo-Wilhelmina \\ zu Braunschweig
}

zur Erlangung des Grades eines

Doktors der Naturwissenschaften (Dr. rer. nat.)

genehmigte

Dissertation

von

Uwe Jordan

aus Potsdam Babelsberg 
1. Referent

2. Referent

eingereicht am:

mündliche Prüfung (Disputation) am:

Druckjahr:
Prof. Dr. Adrian Schumpe

Prof. Dr. Peter Jomo Walla

09.05 .2016

09.09.2016

2016 


\section{Vorveröffentlichungen der Dissertation}

Teilergebnisse aus dieser Arbeit wurden mit Genehmigung der Fakultät für

Lebenswissenschaften, vertreten durch den Mentor der Arbeit, in folgenden Beiträgen vorab veröffentlicht:

\section{Publikationen}

\section{U. JORDAN, K. TERASAKA, A. SCHUMPE,}

Mass transfer in high-pressure bubble columns with organic liquids.

Preprints of the 5th German-Japanese Symposium Bubble Columns, Dresden 28.-30.

Mai 2000, S. 259-264, TU Bergakademie, Freiberg.

U. JORDAN, K. TERASAKA, G. KUNDU, A. SCHUMPE,

Stoffübergang in Druckblasensäulen mit organischen Flüssigkeiten.

Chem. Ing. Tech. 73 (2001) 8, 982-985.

U. JORDAN, A. SCHUMPE,

The gas density effect on mass transfer in bubble columns with organic liquids.

Chem. Eng. Sci. 56 (2001) 21/22, 6267-6272.

U. JoRdAn, K. TERASAKA, G. KUndU, A. SCHUMPE,

Mass transfer in high-pressure bubble columns with organic liquids.

Chem. Eng. Technol. 25 (2002) 3, 262-265.

U. Jordan, A. SAXENA, A. SCHUMPE,

Dynamic gas disengagement in a high-pressure bubble column.

Canad. J. Chem. Eng. 81 (2003) 491-498.

S. NedeltChev, U. JoRdan, A. SCHUMPe,

A new correction factor for theoretical prediction of mass transfer coefficients in bubble columns.

J. Chem. Eng. Japan $\underline{39}$ (2006) 12, 1237-1242.

S. NedelTCHEV, U. JoRdAn, A. SCHUMPE,

Correction of the penetration theory applied to predict mass transfer coefficients in a bubble column with organic liquids.

Chem. Eng. Technol. 29 (2006) 9, 1113-1117.

S. NedeltCheV, U. JoRdAn, A. SCHUMPe,

Identification of flow regime transitions in a bubble column based on chaos analysis of both liquid concentration and gas holdup fluctuations.

Preprints of the $7^{\text {th }}$ German/Japanese Symposium Bubble Columns, Goslar, 20.-23. Mai 2006, S. 144-149. 
S. Nedeltchev, U. Jordan, O. LoREnZ, A. SchumPe,

Identification of various transition velocities in a bubble column based on Kolmogorov entropy.

Chem. Eng. Technol. 30 (2007) 4, 534-539.

\section{S. NeDELTCHEV, U. JoRdAN, A. SCHUMPE}

Correction of the penetration theory based on mass transfer data from bubble columns operated in the homogeneous regime under high pressure.

Chem. Eng. Sci. 62 (2007) 22, 6263-6273.

S. Nedeltchev, U. Jordan, A. Schumpe,

Semi-theoretical prediction of volumetric mass transfer coefficients in bubble columns with organic liquids at ambient and elevated temperatures.

Canad. J. Chem. Eng. 8 8 (2010) 4, 523-532.

\section{Tagungsbeiträge:}

\section{U. JORDAN, K. TERASAKA, A. SCHUMPE,}

Mass transfer in high-pressure bubble columns with organic liquids.

5th German-Japanese Symposium Bubble Columns, Dresden 28.-30. Mai 2000.

\section{U. JORDAN, G. KUNDU, A. SCHUMPE,}

The gas density effect on mass transfer in bubble columns with organic liquids.

$5^{\text {th }}$ Int. Conf. on Gas-Liquid and Gas-Liquid-Solid Reactor Engineering $/ 6^{\text {th }}$ World Congress of Chemical Engineering, Melbourne, 23.-27. September 2001; Proceedings of the 6th World Congress of Chemical Engineering (on CD-ROM) ISBN 0734022018.

\section{U. JORDAN, A. SAXENA, A. SCHUMPE,}

Dynamic gas disengagement in a high-pressure bubble column.

$6^{\text {th }}$ International Conference on Gas-Liquid and Gas-Liquid-Solid Reactor Engineering, Vancouver, Canada, 17.-20. August 2003.

\section{S. NedeltCHEV, U. JoRdAN, A. SCHUMPE,}

Identification of flow regime boundaries based on chaos analysis of mass transfer data obtained in a bubble column operated with nitrogen-gasoline system.

$7^{\text {th }}$ International Conference on Gas/Liquid and Gas/Liquid/Solid Reactor Engineering, Strasbourg, Frankreich, 21.-24. August 2005.

\section{S. NeDELTCHEV, U. JORDAN, A. SCHUMPE,}

Identification of flow regime transitions in a bubble column based on chaos analysis of both liquid concentration and gas holdup fluctuations.

$7^{\text {th }}$ German/Japanese Symposium Bubble Columns, Goslar, 20.-23. Mai 2006.

\section{S. NedelTCHEV, U. JoRdAN, A. SCHUMPE,}

Correction of the penetration theory applied to predict $k_{L} a$ in a bubble column with organic liquids.

$7^{\text {th }}$ German/Japanese Symposium Bubble Columns, Goslar, 20.-23. Mai 2006. 
S. NedeltCHEV, U. JoRdAn, O. LORENZ, A. SCHUMPE,

A new approach for the prediction of gas holdup in bubble columns operated under homogeneous regime and various pressures.

Poster, $8^{\text {th }}$ International Conference on Gas/Liquid and Gas/Liquid/Solid Reactor Design (GLS8), Neu Delhi, Indien, 16.-19. Dezember 2007.

S. NedeltCheV, U. Jordan, A. Schumpe,

Semi-theoretical prediction of mass transfer coefficients in a bubble column operated at elevated temperatures.

$9^{\text {th }}$ International Conference on Gas/Liquid and Gas/Liquid/Solid Reactor Engineering (GLS9), Montreal, Kanada, 23.-27. August 2009.

U. JORDAN, S. NEDELTCHEV 2 A. SCHUMPE,

Mass transfer in bubble columns with organic liquids.

$10^{\text {th }}$ International Conference on Gas/Liquid and Gas/Liquid/Solid Reactor Engineering (GLS10), Braga, Portugal, 26.-29. Juni 2011. 



\section{Danksagung}

Nach Fertigstellung dieser Arbeit möchte ich mich bei Professor Schumpe bedanken. Er hat mich nicht nur bei der praktischen Durchführung der Arbeit betreut, sondern er hat viel Geduld bewiesen und mich nach nun über 15 Jahren ermuntert, den schriftlichen Teil abzuschließen und so den letzten Schritt zur Promotion zu gehen. 



\section{Inhalt}

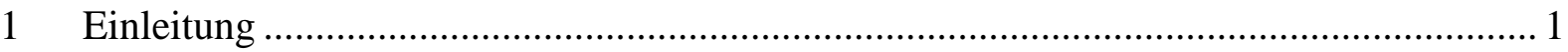

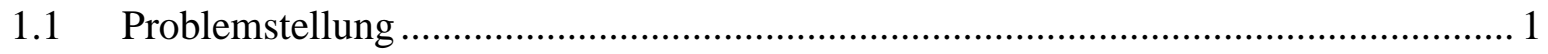

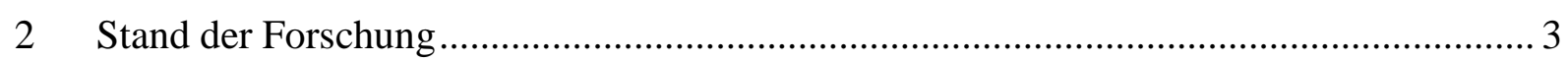

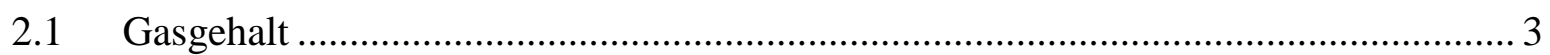

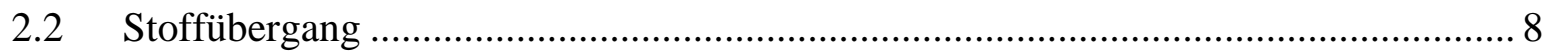

2.3 Stoffübergang und Blasenaufstiegsgeschwindigkeit ............................................ 11

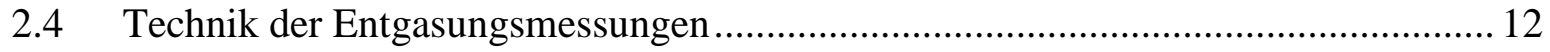

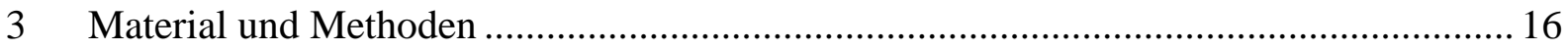

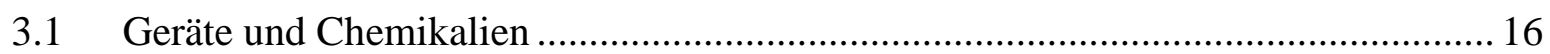

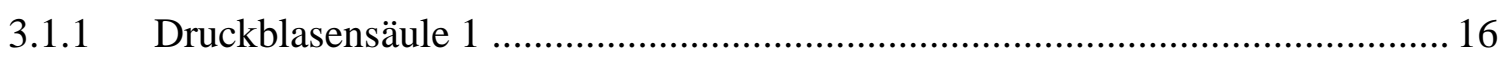

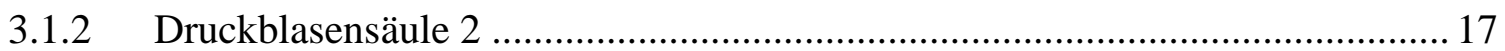

3.1.3 Massendurchflussregler (Mass-Flow-Controller; MFC) ................................... 19

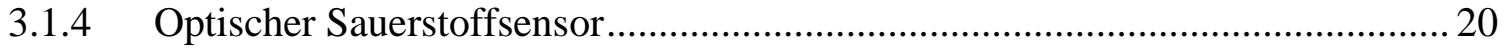

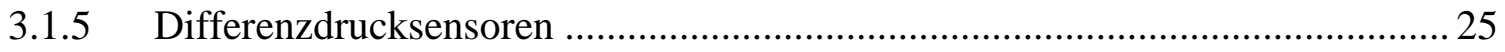

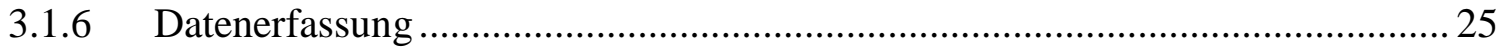

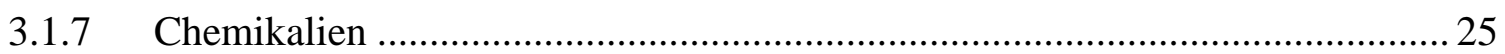

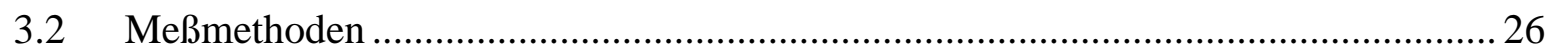

3.2.1 Entgasungsmessungen (Dynamic Gas Disengagement; DGD) ......................... 26

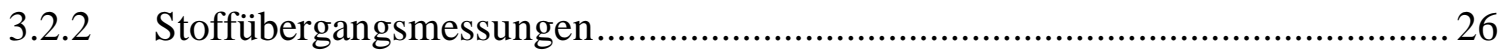

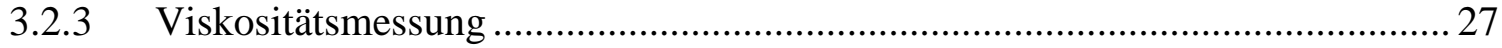

3.2.4 Messung der Oberflächenspannung …......................................................... 27

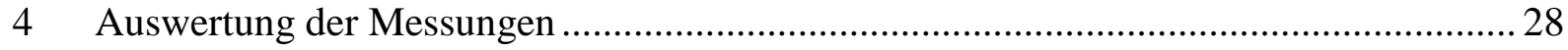

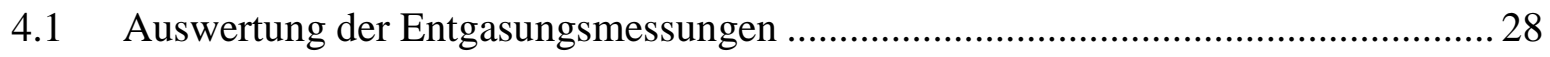

4.1.1 Entgasungsmessungen mit der Dispersionshöhe............................................2 28

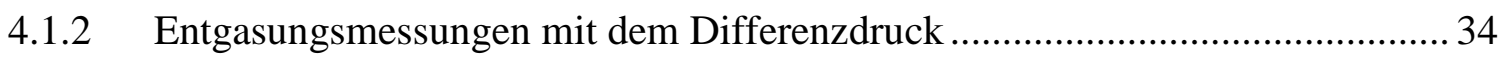

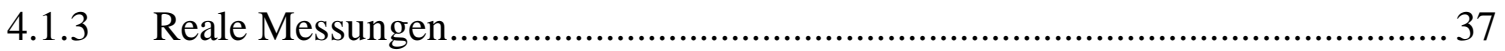

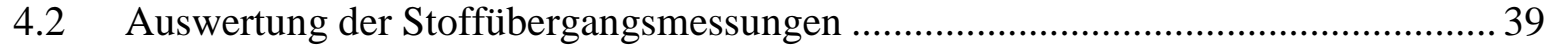

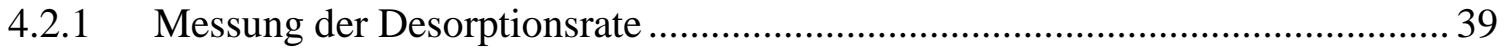

4.2.2 Gasphasekonzentration während der Desorption ........................................... 43

4.2.3 Berechnung der Gasphasekonzentration des Sauerstoffes im homogenen

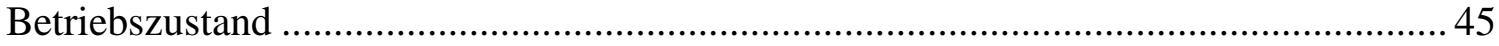

4.2.4 Berechnung der Gasphasekonzentration des Sauerstoffs im heterogenen

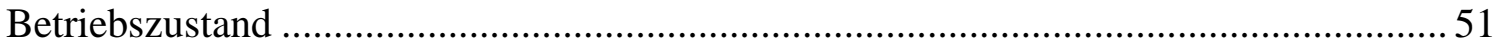

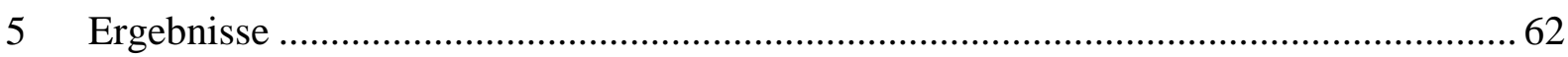

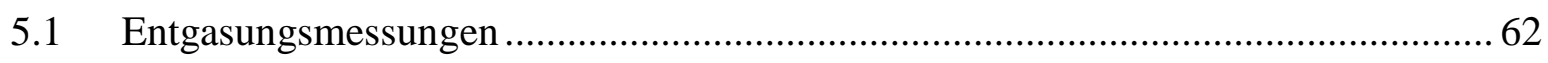

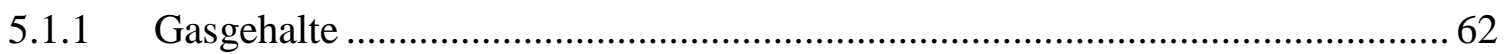

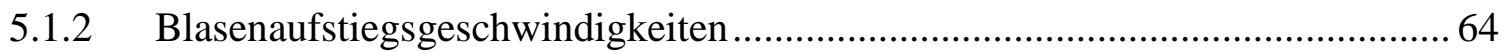


5.1.3 Einfluss der Viskosität auf den Gasgehalt.................................................... 66

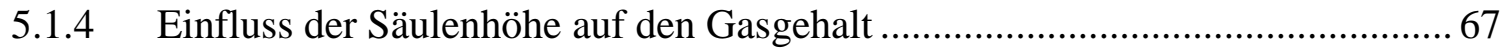

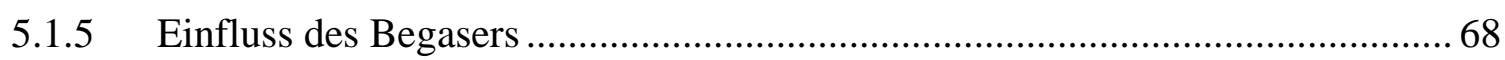

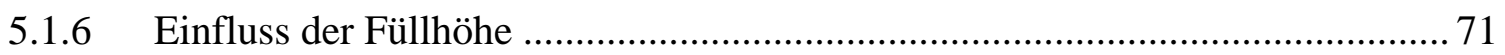

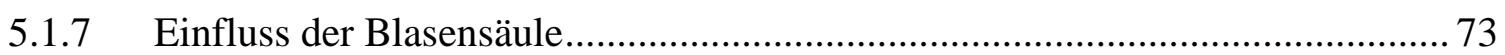

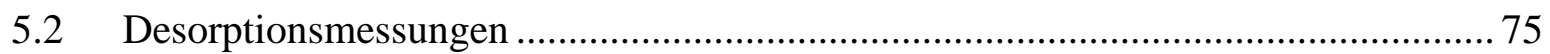

5.2.1 Einfluss von Gasdichte und Gasleerrohrgeschwindigkeit................................ 75

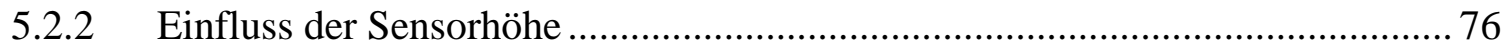

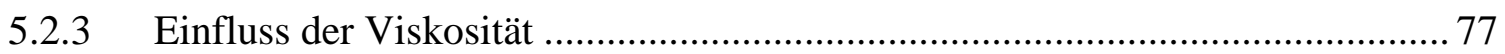

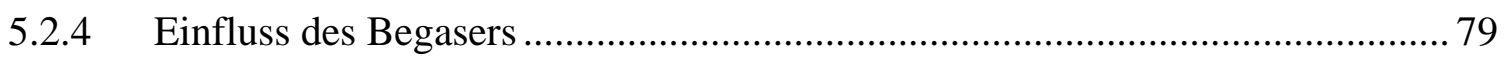

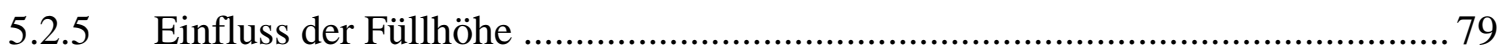

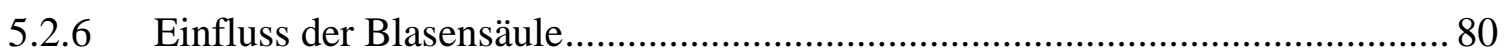

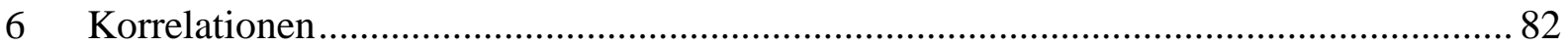

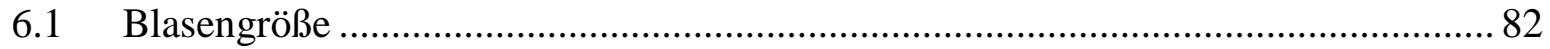

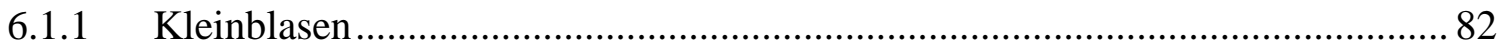

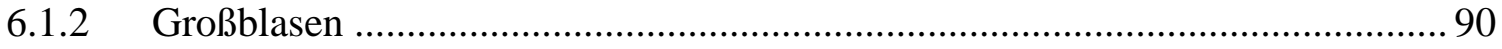

6.2 Übergang vom homogenen zum heterogenen Blasenregime ................................. 93

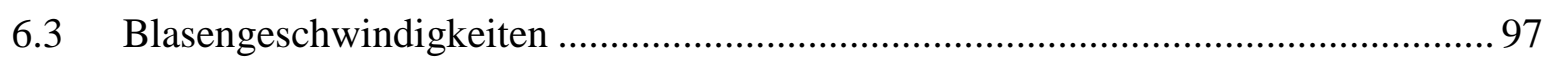

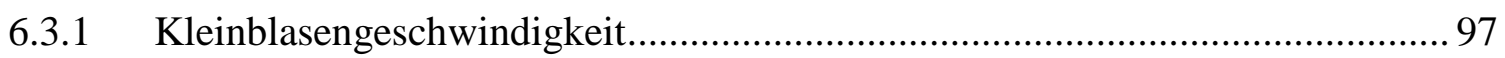

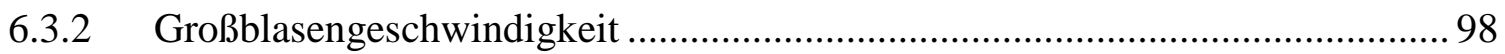

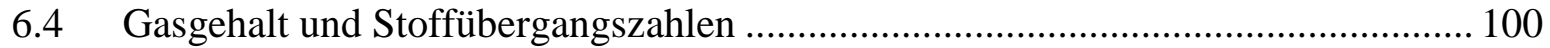

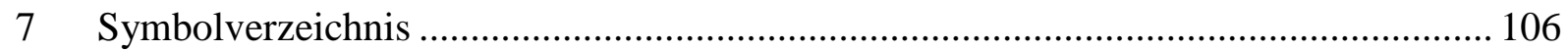

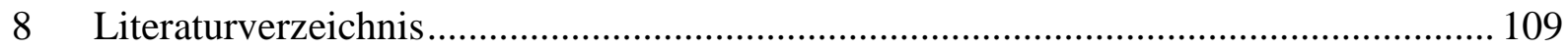

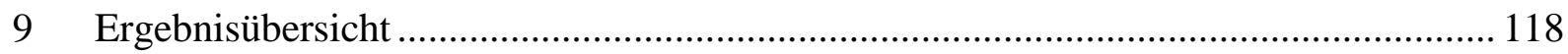




\section{Einleitung}

\subsection{Problemstellung}

Blasensäulen sind technische Reaktionsapparate, in denen ein Gas in Form von Blasen mit einer Flüssigkeit in Kontakt gebracht wird. Dabei können Substanzen aus einer Phase in die andere übergehen. Dies ist zum Beispiel der Fall, wenn sich Gase in der Flüssigkeit lösen und dort zur Reaktion kommen, oder wenn leichtflüchtige Bestandteile der Flüssigkeit in die Gasphase übergehen und mit dem Gasstrom ausgetragen werden. Die Flüssigkeit kann zusätzlich inerte, katalytisch aktive oder reaktive Feststoffpartikel enthalten, die durch die Mischwirkung der Gasphase in Suspension gehalten werden. Man spricht dann von einer Suspensionsblasensäule.

Die einfachste Form einer Blasensäule ist ein lotrecht gestelltes Rohr, das mit einer Flüssigkeit oder Suspension gefüllt ist. Am unteren Ende des Rohres befindet sich ein Gasverteiler. Das Gas steigt in Form von Blasen durch die Flüssigkeit auf und entweicht durch die Oberfläche der Flüssigkeit. Die Flüssigkeit kann dabei stationär in der Blasensäule verweilen oder kontinuierlich durch die Blasensäule geleitet werden. Dies kann von unten nach oben im Gleichstrom oder von oben nach unten im Gegenstrom zur Gasphase erfolgen. Einige industriell angewendete Prozesse sind in Tabelle 1-1 aufgeführt.

Tabelle 1-1: Beispiele chemischer Prozesse in Blasensäulen ("Ullmann's encyclopaedia of industrial chemistry", Fifth edition, Volume B4)

\begin{tabular}{|l|l|l|}
\hline Prozesse & Druck & Temperatur \\
\hline Oxidation von Ethylen zu Acetaldehyd & 3 bar & $400 \mathrm{~K}$ \\
\hline Oxidation von Cumol zu Phenol & $5-10$ bar & $360-400 \mathrm{~K}$ \\
\hline Epoxidierung von Propen & $9-12$ bar & $320-350 \mathrm{~K}$ \\
\hline Chlorierung von Ethylen & $4-5$ bar & $360 \mathrm{~K}$ \\
\hline Oxidation von Toluol zu Benzoesäure & 2 bar & $140^{\circ} \mathrm{C}$ \\
\hline Sulfonierung von Alkylbenzolen & & $40-50^{\circ} \mathrm{C}$ \\
\hline Vinylierung höherer Carbonsäuren & & $200-230^{\circ} \mathrm{C}$ \\
\hline Oxidation von Montanwachsen & $1-5$ bar & $100-125^{\circ} \mathrm{C}$ \\
\hline
\end{tabular}

Je nach Anwendung und Größe der Blasensäule können Einbauten zur Anwendung kommen, die die Rückvermischung und Verteilung der Phasen beeinflussen.

In dieser Arbeit wird eine einfache Blasensäule ohne suspendierte Feststoffe, ohne Einbauten und ohne externe Flüssigkeitszirkulation verwendet.

Trotz des einfachen Aufbaus der Blasensäule liegen komplexe hydrodynamische Bedingungen vor. Die Zielgrößen Gasgehalt $\varepsilon_{\mathrm{G}}$, Stoffübergangszahl $\mathrm{k}_{\mathrm{L}} \mathrm{a}$ und spezifische Phasengrenzfläche a hängen von einer Reihe einstellbarer und nicht einstellbarer Größen ab. $\mathrm{Zu}$ den einstellbaren Größen gehören die Gasleerrohrgeschwindigkeit $\mathrm{u}_{\mathrm{G}}$ und der Druck $\mathrm{p}_{\mathrm{G}}$. $\mathrm{Zu}$ den bedingt einstellbaren Größen gehören die Dimensionen der Blasensäule, insbesondere der Blasensäulendurchmesser und die Art des Begasers. Diese Werte sind durch das experimentelle Setup vorgegeben. $\mathrm{Zu}$ den nicht einstellbaren Größen gehören die physikalischen Eigenschaften der eingesetzten Gase und Flüssigkeiten. Dazu zählen 
Viskosität, Dichte der Flüssigkeit, Oberflächenspannung und Dampfdruck der Flüssigkeit sowie die Molmasse des Gases.

Insbesondere der Einfluss der Gasdichte auf den Gasgehalt wurde lange Zeit unterschätzt. Die experimentellen Ergebnisse bei kleinen Gasdichten zeigen keinen signifikanten Einfluss. Erst seit den siebziger Jahren des 20. Jahrhunderts wurden verstärkt Experimente bei hohen Drücken durchgeführt. Dabei zeigte sich ein stark ansteigender Gasgehalt mit steigender Gasdichte (Stegeman ET. AL. 1996; KoJima ET. AL. 1997; LetZEL ET. AL. 1998; LetZEL ET. AL. 1999; OYEVAAR ET. AL. 1991; KRISHNAET. AL. 1991).

Während die Messung des Gasgehaltes relativ einfach ist, wirft die Messung der Stoffübergangszahl $\mathrm{k}_{\mathrm{L}}$ a einige Schwierigkeiten auf. Es wurden verschiedene Meßmethoden entwickelt. Sie lassen sich in physikalische und chemische Techniken einteilen (MARQUEZ ET. AL. 1994).

Tabelle 1-2: Chemische Absorptionstechniken zur Messung der Stoffübergangszahlen $\mathrm{k}_{\mathrm{L}} \mathrm{a}$

\begin{tabular}{|l|l|l|}
\hline Methode & Reaktion & Flüssige Phase \\
\hline Sulfitoxidationsmethode & $\mathrm{SO}_{3}^{2-}+0,5 \mathrm{O}_{2} \stackrel{\text { Kat }}{\rightarrow} \mathrm{SO}_{4}^{2-}$ & wässrige Lösung \\
\hline Hydrazinoxidation & $\mathrm{N}_{2} \mathrm{H}_{4}+\mathrm{O}_{2} \stackrel{\mathrm{Me}^{2+}}{\rightarrow} \mathrm{N}_{2}+2 \mathrm{H}_{2} \mathrm{O}$ & wässrige Lösung \\
\hline
\end{tabular}

Bei den chemischen Absorptionstechniken wird die absorbierte gasförmige Komponente durch eine Reaktion in der flüssigen Phase ständig verbraucht. Diese Techniken sind zum größten Teil nur für wässrige Lösungen geeignet (FLEISCHER ET. AL. 1995; SPRINGMANN ET. AL. 1991; MARQUEZ ET. AL. 1994).

Die physikalischen Absorptionstechniken bzw. Desorptionstechniken lassen sich wiederum in zwei Gruppen einteilen. Die stationären Methoden nutzen externe Desorber, um absorbierte Gase aus der recyklierten Flüssigkeit zu entfernen. Bei den dynamischen Methoden wird die absorbierte Komponente in der flüssigen Phase bis zum Gleichgewicht akkumuliert.

Bei den Stoffübergangszahlen zeigt sich ein ähnlicher Gasdichteeinfluss wie bei den Gasgehaltsmessungen. Der Stoffübergang nimmt mit steigender Gasdichte bei sonst gleichen Bedingungen zu (KOJIMA ET. AL. 1997; LETZEL ET. AL. 1999; ÖZTÜRK ET. AL. 1987).

Nach neueren Forschungsarbeiten gibt es bei höheren Gasleerohrgeschwindigkeiten, im heterogenen Strömungsregime, zwischen dem Gasgehalt und den Stoffübergangzahlen einen engen Zusammenhang. Der Quotient $k_{L} a / \varepsilon_{G}$ ist bei gegebenem Stoffsystem eine Konstante (VANDU, KRISHNA I; 2004; VANDU, KRISHNA II; 2004).

Die Datenbasis der Gasgehalte und besonders der Stoffübergangszahlen in organischen Flüssigkeiten ist zurzeit noch relativ klein. Besonders unter erhöhtem Druck wurden wenige Arbeiten durchgeführt. Im Rahmen dieser Arbeit sollen folgende Punkte untersucht werden:

- Einfluss der Gasdichte und der Gasleerrohrgeschwindigkeit auf den Gasgehalt und dessen Struktur

- Einfluss der Gasdichte und der Gasleerrohrgeschwindigkeit auf den Stofftransport

- Einfluss der Flüssigkeitseigenschaften auf Gasgehalt und Stofftransport 


\section{Stand der Forschung}

\subsection{Gasgehalt}

Der Gasgehalt ist eine leicht zu messende und sehr wichtige Größe für die Auslegung von Blasensäulenreaktoren. Der Gasgehalt bewirkt eine Expansion der Flüssigkeitssäule beim Batchbetrieb und eine Verringerung der mittleren Dichte der Dispersion. Anhand dieser beiden Effekte lässt sich der Gasgehalt bestimmen.

$$
\varepsilon_{\mathrm{G}}=\frac{\mathrm{V}_{\mathrm{G}}}{\mathrm{V}_{\mathrm{G}}+\mathrm{V}_{\mathrm{L}}}
$$

Gleichung 2-1: Definition Gasgehalt

Der Gasgehalt lässt sich aus dem Füllstand berechnen, indem man den Füllstand während der Begasung $\mathrm{H}_{0}$ und die Höhe der Flüssigkeitssäule ohne Begasung $\mathrm{H}_{\mathrm{L}}$ bestimmt:

$$
\varepsilon_{\mathrm{G}}=\frac{\mathrm{H}_{0}-\mathrm{H}_{\mathrm{L}}}{\mathrm{H}_{0}}
$$

Gleichung 2-2: Berechnung des Gasgehaltes aus der Füllstandsmessung

Eine elegantere Methode ist die Messung des hydrostatischen Druckes des Reaktionsgemisches über eine feste Höhendifferenz. Aus dem Differenzdruck während der Begasung $\Delta \mathrm{p}_{0}$ und dem Differenzdruck ohne Begasung $\Delta \mathrm{p}_{\mathrm{E}}$ lässt sich der Gasgehalt ebenfalls einfach berechnen:

$$
\varepsilon_{\mathrm{G}}=\frac{\Delta \mathrm{p}_{\mathrm{E}}-\Delta \mathrm{p}_{0}}{\Delta \mathrm{p}_{\mathrm{E}}}
$$

Gleichung 2-3: Berechnung des Gasgehaltes aus der Differenzdruckmessung

Für den Gesamtgasgehalt existiert in der Literatur eine Reihe von empirischen Korrelationen (siehe Tabelle 2-1). In den ersten Jahren wurde der Einfluss der Gasdichte auf den Gasgehalt ignoriert (Hughmark 1967; AKITA, YoshidA 1974; HikITA, KIKUWA 1974; GESTRICH, RÄHSE 1975; BACH, PILHOFER 1978). Entweder wurde die Gasdichte bei den Experimenten gar nicht variiert oder der abgedeckte Dichtebereich war zu klein, um einen Gasdichteeffekt zu beobachten.

In späteren Veröffentlichungen ist die Gasdichte als Einflussfaktor in die Korrelationen aufgenommen worden (MERSMANN 1978; HIKITA 1980; HAMMER 1984; IDOGAWA 1985; REILlY 1986; IDOGAWA ET. AL. 1987; BEKISH ET AL 2006). Bemerkenswert sind hier die Gleichungen von IDOGAWA, bei denen der Druck (als Ausdruck der Gasdichte) nicht die Basis eines Exponenten bildet, sondern selbst im Exponenten steht.

Im letzten Jahrzehnt ging man dazu über, den Gasgehalt in Großblasen und Kleinblasen einzuteilen und die verschiedenen Blasenklassen und Strömungsregime getrennt zu behandeln (KRISHNA ET. AL. 1991; LETZEL ET. AL. 1999; LEMOINE ET AL 2008). 
Tabelle 2-1: Korrelationen des Gasgehaltes

\begin{tabular}{|c|c|c|}
\hline Autor & Korrelation & Erläuterungen \\
\hline $\begin{array}{l}1967 \\
\text { HUGHMARK }\end{array}$ & $\varepsilon_{\mathrm{G}}=\left[2+\frac{0,35}{\mathrm{u}_{\mathrm{G}}} \cdot\left(\frac{\rho_{\mathrm{L}} \cdot \sigma}{72}\right)^{\frac{1}{3}}\right]^{-1}$ & $\begin{array}{l}\mathrm{u}_{\mathrm{G}} \text { in }[\mathrm{m} / \mathrm{s}] \\
\rho_{\mathrm{L}} \text { in }\left[\mathrm{kg} / \mathrm{m}^{3}\right] \\
\sigma \text { in }[\mathrm{N} / \mathrm{m}]\end{array}$ \\
\hline $\begin{array}{l}1974 \\
\text { AKITA; } \\
\text { YOSHIDA }\end{array}$ & $\frac{\varepsilon_{\mathrm{G}}}{\left(1-\varepsilon_{\mathrm{G}}\right)^{4}} \cdot \mathrm{c}=\mathrm{Bo}^{\frac{1}{8}} \cdot \mathrm{Ga}^{\frac{1}{12}} \cdot \mathrm{Fr}$ & $\begin{array}{l}\mathrm{d}=\mathrm{d}_{\mathrm{BS}} \\
\text { (Blasensäulendurchmesser) } \\
\mathrm{c}=0,2 \text { für reine Flüssigkeiten } \\
\mathrm{c}=0,25 \text { für } \\
\text { Elektrolytlösungen }\end{array}$ \\
\hline $\begin{array}{l}1974 \\
\text { HIKITA; } \\
\text { KIKUWA }\end{array}$ & $\varepsilon_{\mathrm{G}}=0,505 \cdot \mathrm{u}_{\mathrm{G}}^{0,47} \cdot\left(\frac{0,072}{\sigma}\right)^{\frac{2}{3}} \cdot\left(\frac{0,001}{\eta_{\mathrm{L}}}\right)^{0,05}$ & $\begin{array}{l}\mathrm{u}_{\mathrm{G}} \text { in }[\mathrm{m} / \mathrm{s}] \\
\sigma \text { in }[\mathrm{N} / \mathrm{m}] \\
\eta_{\mathrm{L}} \text { in }[\mathrm{Pa} \cdot \mathrm{s}]\end{array}$ \\
\hline $\begin{array}{l}1975 \\
\text { GESTRICH; } \\
\text { RÄHSE }\end{array}$ & $\begin{array}{l}\varepsilon_{\mathrm{G}}=0,89 \cdot\left(\frac{\mathrm{h}_{\mathrm{L}}}{\mathrm{d}_{\mathrm{BS}}}\right)^{0,035 \cdot(-15,7+\mathrm{lg}(\mathrm{K}))} \cdot\left(\frac{\mathrm{d}_{\mathrm{B}}}{\mathrm{d}_{\mathrm{BS}}}\right)^{0,3} \\
\cdot\left(\frac{\mathrm{u}_{\mathrm{G}}^{2}}{\mathrm{~d}_{\mathrm{B}} \cdot \mathrm{g}}\right)^{0,025 \cdot(2,6+\mathrm{lg}(\mathrm{K}))} \cdot \mathrm{K}^{0,047}-0,05 \\
\mathrm{~K}=\frac{\rho_{\mathrm{L}} \cdot \sigma^{3}}{\mathrm{~g} \cdot \eta_{\mathrm{L}}^{4}}\end{array}$ & $\mathrm{~d}_{\mathrm{B}}=0,003 \mathrm{~m}$ \\
\hline $\begin{array}{l}1976 \\
\text { KUMAR }\end{array}$ & $\begin{array}{l}\varepsilon_{\mathrm{G}}=0,728 \cdot \mathrm{U}-0,485 \cdot \mathrm{U}^{2}+0,0975 \mathrm{U}^{3} \\
\mathrm{U}=\mathrm{u}_{\mathrm{G}} \cdot\left(\frac{\rho_{\mathrm{L}}^{2}}{\sigma \cdot \Delta \rho \cdot \mathrm{g}}\right)^{\frac{1}{4}}=(\mathrm{We} \cdot \mathrm{Fr})^{\frac{1}{4}}\end{array}$ & \\
\hline $\begin{array}{l}1978 \\
\text { BACH; } \\
\text { PILHOFER }\end{array}$ & $\frac{\varepsilon_{\mathrm{G}}}{1-\varepsilon_{\mathrm{G}}}=0,0115 \cdot\left(\frac{\mathrm{u}_{\mathrm{G}}^{3} \cdot \rho_{\mathrm{L}}^{2}}{\eta_{\mathrm{L}} \cdot \mathrm{g} \cdot \Delta \rho}\right)^{0,23}=0,0115 \cdot(\mathrm{Re} \cdot \mathrm{Fr})^{0,23}$ & \\
\hline $\begin{array}{l}1978 \\
\text { MERSMANN }\end{array}$ & $\begin{array}{l}\frac{\varepsilon_{\mathrm{G}}}{\left(1-\varepsilon_{\mathrm{G}}\right)^{4}}= \\
0,14 \cdot \mathrm{u}_{\mathrm{G}} \cdot\left(\frac{\rho_{\mathrm{L}}^{2}}{\sigma \cdot \Delta \rho \cdot \mathrm{g}}\right)^{\frac{1}{4}} \cdot\left(\frac{\rho_{\mathrm{L}}^{2} \cdot \sigma^{3}}{\eta_{\mathrm{L}}^{4} \cdot \Delta \rho \cdot \mathrm{g}}\right)^{\frac{1}{24}} \cdot\left(\frac{\rho_{\mathrm{L}}}{\rho_{\mathrm{G}}}\right)^{\frac{1}{36}} \cdot\left(\frac{\rho_{\mathrm{L}}}{\Delta \rho}\right)^{\frac{1}{3}} \\
=0,14 \cdot(\mathrm{We} \cdot \mathrm{Fr})^{\frac{1}{4}} \cdot\left(\frac{1}{\mathrm{Mo}}\right)^{\frac{1}{24}} \cdot\left(\frac{\rho_{\mathrm{L}}}{\rho_{\mathrm{G}}}\right)^{\frac{1}{36}} \cdot\left(\frac{\rho_{\mathrm{L}}}{\Delta \rho}\right)^{\frac{1}{3}}\end{array}$ & \\
\hline
\end{tabular}




\begin{tabular}{|c|c|c|}
\hline $\begin{array}{l}1980 \\
\text { HIKITA }\end{array}$ & $\varepsilon_{\mathrm{G}}=0,672 \cdot\left(\frac{1}{\mathrm{Fl}}\right)^{0,578} \cdot \mathrm{Mo}^{-0,131} \cdot\left(\frac{\rho_{\mathrm{G}}}{\rho_{\mathrm{L}}}\right)^{0,062} \cdot\left(\frac{\eta_{\mathrm{G}}}{\eta_{\mathrm{L}}}\right)^{0,107}$ & \\
\hline $\begin{array}{l}1984 \\
\text { HAMMER }\end{array}$ & $\frac{\varepsilon_{\mathrm{G}}}{1-\varepsilon_{\mathrm{G}}}=0,672 \cdot\left(\frac{1}{\mathrm{Fl}}\right)^{0,87} \cdot \mathrm{Mo}^{-0,27} \cdot\left(\frac{\rho_{\mathrm{G}}}{\rho_{\mathrm{L}}}\right)^{0,17}$ & \\
\hline $\begin{array}{l}1985 \\
\text { IDOGAWA }\end{array}$ & $\frac{\varepsilon_{\mathrm{G}}}{1-\varepsilon_{\mathrm{G}}}=1,44 \cdot \mathrm{u}_{\mathrm{G}}^{0,58} \cdot \rho_{\mathrm{G}}^{0,12} \cdot \sigma^{-0,16 \cdot \exp (-\mathrm{p})}$ & $\begin{array}{l}\sigma \text { in }[\mathrm{mN} / \mathrm{m}] \\
\mathrm{p} \text { in }[\mathrm{MPa}]\end{array}$ \\
\hline $\begin{array}{l}1986 \\
\text { REILLY }\end{array}$ & $\varepsilon_{\mathrm{G}}=296 \cdot \mathrm{u}_{\mathrm{G}}^{0,44} \cdot \rho_{\mathrm{L}}^{-0,98} \cdot \sigma^{-0,16} \cdot \rho_{\mathrm{G}}^{0,19}+0,009$ & \\
\hline $\begin{array}{l}1987 \\
\text { IDOGAWA }\end{array}$ & $\frac{\varepsilon_{\mathrm{G}}}{1-\varepsilon_{\mathrm{G}}}=0,059 \cdot \mathrm{u}_{\mathrm{G}}^{0,8} \cdot \rho_{\mathrm{G}}^{0,17} \cdot\left(\frac{\sigma}{72}\right)^{-0,22 \cdot \exp (-\mathrm{p})}$ & $\begin{array}{l}\sigma \text { in }[\mathrm{mN} / \mathrm{m}] \\
\mathrm{p} \text { in }[\mathrm{MPa}] \\
\mathrm{u}_{\mathrm{G}} \text { in }[\mathrm{cm} / \mathrm{s}]\end{array}$ \\
\hline $\begin{array}{l}1991 \\
\text { KRISHNA }\end{array}$ & $\begin{array}{l}\text { homogenes Regime: } \\
\varepsilon_{\mathrm{G}}=4 \cdot \mathrm{u}_{\mathrm{G}} \\
\text { heterogenes Regime: } \\
\varepsilon_{\mathrm{G}}=\varepsilon_{\mathrm{G}, \mathrm{Klein}}+\varepsilon_{\mathrm{G}, \mathrm{Gro}}=4 \cdot \mathrm{u}_{\mathrm{G}, \text { trans }}+\mathrm{A} \cdot\left(\mathrm{u}_{\mathrm{G}}-\mathrm{u}_{\mathrm{G}, \text { trans }}\right)^{\mathrm{n}}\end{array}$ & $\begin{array}{l}A=1 \\
n=0,8 \\
u_{G, \text { trans }}=f\left(\rho_{G}\right) \text { (experimentell } \\
\text { ermittelt) }\end{array}$ \\
\hline $\begin{array}{l}1992 \\
\text { WILKINSON }\end{array}$ & $\begin{array}{l}\text { homogenes Regime: } \\
\varepsilon_{\mathrm{G}}=\frac{\mathrm{u}_{\mathrm{G}}}{\mathrm{v}_{\mathrm{B}, \text { Klein }}} \\
\text { heterogenes Regime: } \\
\varepsilon_{\mathrm{G}}=\frac{\mathrm{u}_{\mathrm{G}, \text { trans }}}{\mathrm{v}_{\mathrm{B}, \text { Klein }}}+\frac{\mathrm{u}_{\mathrm{G}}-\mathrm{u}_{\mathrm{G}, \text { trans }}}{\mathrm{v}_{\mathrm{B}, \mathrm{Gro \beta}}} \\
\frac{\mathrm{v}_{\mathrm{B}, \text { Klein }} \cdot \eta_{\mathrm{L}}}{\sigma}=2,25 \cdot \mathrm{Mo}^{0,273} \cdot\left(\frac{\rho_{\mathrm{L}}}{\rho_{\mathrm{G}}}\right)^{0,03} \\
\frac{\mathrm{v}_{\mathrm{B}, \text { Groß }} \cdot \eta_{\mathrm{L}}}{\sigma}=\frac{\mathrm{v}_{\mathrm{B}, \text { Klein }} \cdot \mu_{\mathrm{L}}}{\sigma}+ \\
2,4 \cdot\left(\frac{\left(\mathrm{u}_{\mathrm{G}}-\mathrm{u}_{\mathrm{G}, \text { trans }}\right) \cdot \eta_{\mathrm{L}}}{\sigma}\right) \mathrm{Mo}^{0,077} \cdot\left(\frac{\rho_{\mathrm{L}}}{\rho_{\mathrm{G}}}\right)^{0,077} \\
\frac{\mathrm{u}_{\mathrm{G}, \text { trans }}}{\mathrm{v}_{\mathrm{B}, \text { Klein }}}=\varepsilon_{\mathrm{G}, \text { trans }}=0,5 \cdot \mathrm{e}^{\left(-193 \cdot \mathrm{g}_{\mathrm{G}}^{-0,61} \cdot \eta_{\mathrm{L}}^{0,5} \sigma^{0,11}\right)}\end{array}$ & $\begin{array}{l}\rho_{\mathrm{G}} \text { in }\left[\mathrm{kg} / \mathrm{m}^{3}\right] \\
\eta_{\mathrm{L}} \text { in }[\mathrm{Pa} \cdot \mathrm{s}] \\
\sigma \text { in }[\mathrm{N} / \mathrm{m}]\end{array}$ \\
\hline
\end{tabular}




\begin{tabular}{|c|c|c|}
\hline \begin{tabular}{|l}
1999 \\
LETZEL
\end{tabular} & $\begin{array}{l}\text { heterogenes Regime: } \\
\varepsilon_{\mathrm{G}}=\varepsilon_{\mathrm{G}, \mathrm{Gro \beta}}+\left(1-\varepsilon_{\mathrm{G}, \mathrm{Gro \beta}}\right) \cdot \varepsilon_{\mathrm{df}} \\
\varepsilon_{\mathrm{G}, \mathrm{Gro \beta}}=0,268 \cdot \frac{1}{\mathrm{~d}_{\mathrm{BS}}^{0,18}} \cdot \frac{\left(\mathrm{u}_{\mathrm{G}}-\mathrm{u}_{\mathrm{G}, \mathrm{df}}\right)^{\frac{4}{5}}}{\left(\mathrm{u}_{\mathrm{G}}-\mathrm{u}_{\mathrm{G}, \mathrm{df}}\right)^{0,22}} \cdot\left(\frac{\rho_{\mathrm{G}}}{\rho_{\mathrm{atm}}}\right)^{0,5} \\
\mathrm{u}_{\mathrm{G}, \mathrm{df}}=\mathrm{v}_{\mathrm{B}, \text { Klein }} \cdot \varepsilon_{\mathrm{G}, \text { trans }} \cdot\left(1-\varepsilon_{\mathrm{G}, \text { trans }}\right) \\
\varepsilon_{\mathrm{G}, \text { trans }}=0,59 \cdot \mathrm{B}^{1,5} \cdot \sqrt{\frac{\rho_{\mathrm{G}}^{0,96}}{\rho_{\mathrm{L}}} \cdot \sigma^{0,12}} \\
\mathrm{v}_{\mathrm{B}, \text { Klein }}=\frac{1}{2,84} \cdot \frac{1}{\rho_{\mathrm{G}}^{0,04}} \cdot \sigma^{0,12}\end{array}$ & $\begin{array}{l}\text { subscript: } \\
\text { df: „dense phase hold-up“ } \\
\varepsilon_{\mathrm{df}}=\varepsilon_{\mathrm{G}, \text { trans }} \\
\text { B: dimensionslose } \\
\text { Konstante } \\
\text { B } \sim 4\end{array}$ \\
\hline \begin{tabular}{|l|}
2006 \\
BEKISH
\end{tabular} & $\begin{array}{l}\varepsilon_{\mathrm{G}}=4,49 \cdot 10^{-3} \cdot\left(\frac{\rho_{\mathrm{L}}^{0,415} \cdot \rho_{\mathrm{G}}^{0,177}}{\eta_{\mathrm{L}}^{0,174} \sigma_{\mathrm{L}}^{0,27}}\right) \cdot \mathrm{u}_{\mathrm{G}}^{0,553} . \\
\left(\frac{\mathrm{p}}{\mathrm{p}-\mathrm{p}_{\mathrm{L}}^{\mathrm{vap}}}\right)^{0,203} \cdot\left(\frac{\mathrm{d}_{\mathrm{BS}}}{\mathrm{d}_{\mathrm{BS}}-1}\right)^{-0,117} \cdot \\
\Gamma^{0,053} \cdot \mathrm{e}^{\left[-2,231 \cdot \mathrm{c}_{\mathrm{v}}-0,157 \cdot \rho_{\mathrm{P}} \cdot \mathrm{d}_{\mathrm{P}}-0,242 \cdot \mathrm{x}_{\mathrm{W}}\right]}\end{array}$ & $\begin{array}{l}\Gamma: \text { Einfluss des Begasers } \\
\text { e-Term: Einfluss von } \\
\text { suspendierten Feststoffen }\end{array}$ \\
\hline $\begin{array}{l}2008 \\
\text { LEMOINE }\end{array}$ & $\begin{array}{l}\varepsilon_{\mathrm{G}}-\text { siehe BEKISH } 2006 \\
\varepsilon_{\mathrm{G}-\text { large }}=\varepsilon_{\mathrm{G}}^{0,84} \cdot\left(1-3,04 \cdot 10^{-6} \cdot \frac{\rho_{\mathrm{L}}^{0,97}}{\eta_{\mathrm{L}}^{0,16}} \cdot \varepsilon^{\left(4,50 \cdot \mathrm{X}_{\mathrm{W}}-4,49 \cdot \mathrm{C}_{\mathrm{V}}\right)}\right. \\
\varepsilon_{\mathrm{G}-\text { small }}=\varepsilon_{\mathrm{G}}-\varepsilon_{\mathrm{G}-\text { larg e }}\end{array}$ & \\
\hline
\end{tabular}




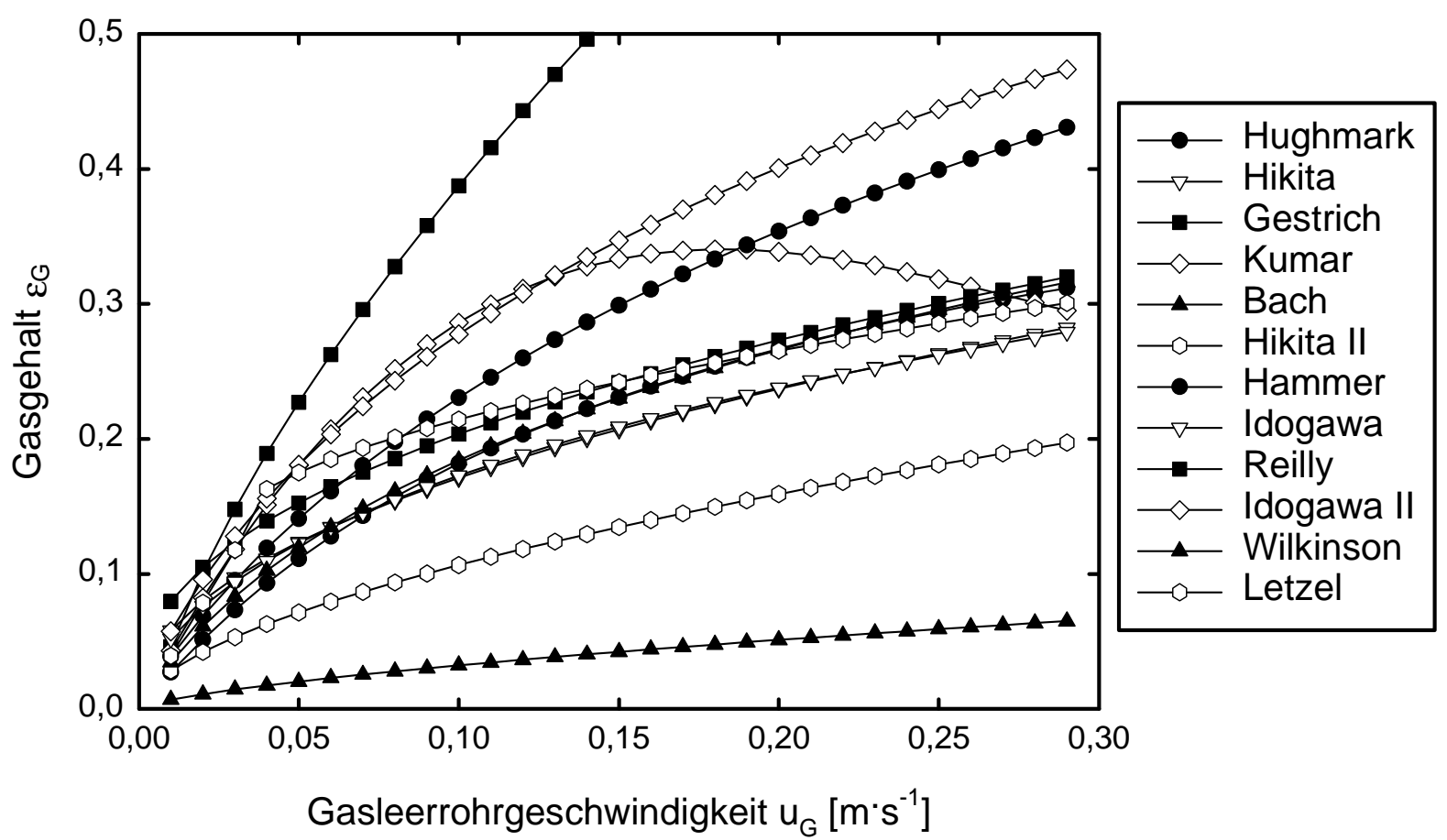

Abbildung 2-1: Berechnete Gasgehalte für Wasser bei $20^{\circ} \mathrm{C}$ und 1 bar $\mathrm{N}_{2}$ als Funktion der Gasleerrohrgeschwindigkeit; die Ergebnisse für Akita \& Yoshida und Mersmann fehlen, da die Gleichungen nicht nach $\varepsilon_{\mathrm{G}}$ lösbar sind

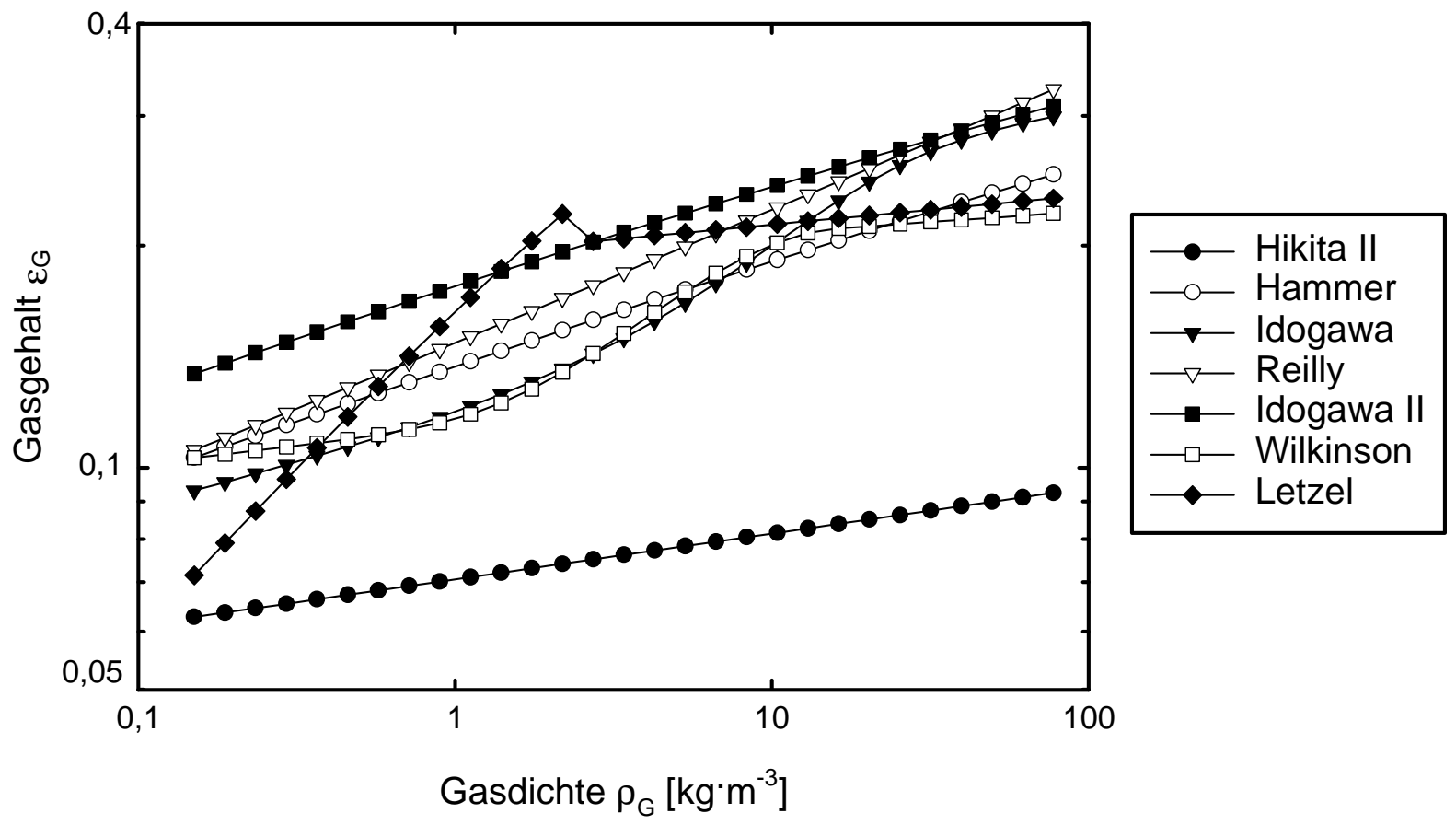

Abbildung 2-2: Berechnete Gasgehalte für Wasser bei $20^{\circ} \mathrm{C}$ und $\mathrm{u}_{\mathrm{G}}=5 \mathrm{~cm} / \mathrm{s}$ als Funktion der Gasdichte; es werden nur die Kurven mit signifikanter Gasdichteabhängigkeit gezeigt; die Auftragung ist doppeltlogarithmisch 
Der generelle Trend der Korrelationen ist ein steigender Gasgehalt mit zunehmender Gasleerrohrgeschwindigkeit. Die verschiedenen Korrelationen liefern für das gleiche System, hier Wasser mit Stickstoff bei Zimmertemperatur und Normaldruck, stark abweichende Ergebnisse. In der Regel sind Korrelationen nur auf die Systeme anwendbar, aus denen sie hervorgegangen sind. Der Gültigkeitsbereich ist bei einigen Gleichungen überschritten. Bemerkenswert sind hier die Gleichungen mit dem Term $\varepsilon_{\mathrm{G}} /\left(1-\varepsilon_{\mathrm{G}}\right)^{4}$. Diese Gleichungen lassen sich analytisch nicht nach dem Gasgehalt auflösen. Zur Ermittlung des Gasgehaltes müssen iterative Verfahren verwendet werden.

Nur ein Teil der Korrelationen zeigt eine deutliche Gasdichteabhängigkeit des Gasgehaltes. In Abbildung 2-2 sind diese Gleichungen aufgetragen. Der generelle Trend ist eine Zunahme des Gasgehaltes mit der Gasdichte. Aber auch hier weichen die Ergebnisse zum Teil stark voneinander ab. Bemerkenswert ist die Tatsache, dass ein einfacher Potenzansatz zur Beschreibung der Gasdichteabhängigkeit einigen Autoren nicht ausreichend erschien. Andernfalls müssten sich in der doppeltlogarithmischen Auftragung Geraden ergeben.

Des Weiteren sind einige der Korrelationen Größengleichungen. Die eingeführten Konstanten sind einheitenbehaftet. Dies lässt den Schluss $\mathrm{zu}$, dass diese Korrelationen rein phänomenologisch erstellt wurden.

\subsection{Stoffübergang}

Der Stoffübergang in einer Blasensäule ist wesentlich schwieriger zu messen, als der Gasgehalt. Dazu muss der Stoffstrom durch die Phasengrenzfläche ermittelt werden und gleichzeitig die Triebkraft des Stoffüberganges, die Konzentrationsdifferenz, bekannt sein. Im Allgemeinen wird davon ausgegangen, dass der Hauptwiderstand des Stoffüberganges in der flüssigkeitsseitigen Grenzschicht liegt. Der gasseitige Widerstand und der Durchtrittswiderstand durch die Phasengrenzfläche werden vernachlässigt.

$$
\frac{\mathrm{dn}}{\mathrm{dt}}=\mathrm{k}_{\mathrm{L}} \cdot \mathrm{A} \cdot\left(\mathrm{c}_{\mathrm{L}}^{*}-\mathrm{c}_{\mathrm{L}}\right)=\mathrm{k}_{\mathrm{L}} \mathrm{a} \cdot \mathrm{V} \cdot\left(\mathrm{c}_{\mathrm{L}}^{*}-\mathrm{c}_{\mathrm{L}}\right)
$$

Gleichung 2-4: Stoffstrom durch die Phasengrenzfläche

In Tabelle 2-2 sind verschiedene Korrelationen für den Stoffübergangszahlen $\mathrm{k}_{\mathrm{L}} \mathrm{a}$ zusammengetragen. Es handelt sich dabei in den meisten Fällen um mehr oder weniger komplizierte Potenzansätze. Hervorzuheben ist der Ansatz von LETZEL: Der $\mathrm{k}_{\mathrm{L}} \mathrm{a}-$ Wert ist proportional dem Gasgehalt. Die Proportionalitätskonstante ist vom Stoffsystem abhängig (VANDU, KRISHNA I; 2004; VANDU, KRISHNA II; 2004). 
Tabelle 2-2: Korrelationen des kLa-Wertes

\begin{tabular}{|c|c|c|}
\hline Autor & Korrelation & Erläuterung \\
\hline $\begin{array}{l}1987 \\
\text { ÖZTÜRK }\end{array}$ & $\mathrm{Sh}=0,62 \cdot \mathrm{Sc}^{0,5} \cdot \mathrm{Bo}^{0,33} \cdot \mathrm{Ga}^{0,29} \cdot \mathrm{Fr}^{0,68} \cdot\left(\frac{\rho_{\mathrm{G}}}{\rho_{\mathrm{L}}}\right)^{0,04}$ & $\mathrm{~d}=\mathrm{d}_{\mathrm{B}}=0,003 \mathrm{~m}$ \\
\hline $\begin{array}{l}1994 \\
\text { WILKINSON }\end{array}$ & $\mathrm{Sh}=\mathrm{k}_{\mathrm{M}} \cdot \mathrm{Sc}^{0,5} \cdot \mathrm{Bo}^{\frac{4}{7}} \cdot \mathrm{Ga}^{\frac{2}{7}} \cdot \varepsilon_{\mathrm{G}}^{1,18}$ & $\begin{array}{l}\mathrm{k}_{\mathrm{M}}: \text { dimensionslose } \\
\text { Konstante } \\
\mathrm{k}_{\mathrm{M}}=2,6 \text { für } 0,8 \mathrm{M} \mathrm{Na}_{2} \mathrm{SO}_{4}- \\
\text { Lösung } \\
\varepsilon_{\mathrm{G}}: \text { experimenteller } \\
\quad \text { Gasgehalt; Korrelation } \\
\text { siehe Tabelle 2-1 }\end{array}$ \\
\hline $\begin{array}{l}1996 \\
\text { DUDUKOVI } \\
\text { C }\end{array}$ & $\mathrm{Sh}=0,0318 \cdot \mathrm{Re}^{0,790} \cdot \mathrm{Sc}^{0,5}$ & $\begin{array}{l}\text { REYNOLDS-Zahl nicht } \\
\text { definiert }\end{array}$ \\
\hline $\begin{array}{l}1997 \\
\text { KOJIMA }\end{array}$ & $\begin{array}{l}\mathrm{k}_{\mathrm{L}} \mathrm{a}=\mathrm{C} \cdot \varepsilon_{\mathrm{G}}^{\mathrm{D}} \cdot \mathrm{We}^{\mathrm{E}} \cdot\left(\frac{\mathrm{p}}{\mathrm{p}_{0}}\right)^{\mathrm{F}} \\
\mathrm{We}=\frac{\mathrm{v}^{2} \cdot \mathrm{d} \cdot \rho}{\sigma}=\frac{\rho_{\mathrm{L}} \cdot \dot{\mathrm{V}}_{\mathrm{G}}^{2}}{\mathrm{~d}_{0}^{3} \cdot \sigma}\end{array}$ & $\begin{array}{l}\mathrm{C} ; \mathrm{D} ; \mathrm{E} ; \mathrm{F}: \\
\text { flüssigkeitsspezifische } \\
\text { Konstanten } \\
\mathrm{d}_{0}: \text { Durchmesser } \\
\text { Begaseröffnung } \\
\text { Sauerstoffverbrauch in } \\
\text { Enzymlösungen }\end{array}$ \\
\hline $\begin{array}{l}1999 \\
\text { LETZEL }\end{array}$ & $\mathrm{k}_{\mathrm{L}} \mathrm{a}=0,5 \cdot \varepsilon_{\mathrm{G}}$ & $\varepsilon_{\mathrm{G}}:$ siehe Tabelle 2-1 \\
\hline $\begin{array}{l}2008 \\
\text { LEMOINE }\end{array}$ & $\begin{array}{l}\mathrm{d}_{\mathrm{S}}=37,19 \cdot \frac{\eta_{\mathrm{L}}^{0,08} \cdot \sigma_{\mathrm{L}}^{1,22} \cdot \rho_{\mathrm{G}}^{0,02} \cdot \mathrm{T}^{1,66}}{\rho_{\mathrm{L}}^{1,52} \cdot \mathrm{M}_{\mathrm{W}-\mathrm{Gas}}^{0,12}} \cdot \mathrm{u}_{\mathrm{G}}^{0,14} \cdot \\
\left(\frac{\mathrm{d}_{\mathrm{BS}}}{\mathrm{d}_{\mathrm{BS}}+1}\right)^{0,30} \cdot\left(1-\varepsilon_{\mathrm{G}}\right)^{1,56} \cdot \Gamma^{-0,02} \cdot \varepsilon^{\left(-2,29 \cdot \mathrm{X}_{\mathrm{w}}+2,81 \cdot \mathrm{C}_{\mathrm{v}}+\right.} \\
\mathrm{k}_{\mathrm{L}} \mathrm{a}=6,14 \cdot 10^{4} \cdot \frac{\rho_{\mathrm{L}}^{0,26} \eta_{\mathrm{L}}^{0,12} \cdot \varepsilon_{\mathrm{G}}^{1,21} \cdot \mathrm{D}_{\mathrm{L}}^{0,5}}{\sigma_{\mathrm{L}}^{0,52} \cdot \rho_{\mathrm{G}}^{0,06} \cdot \mathrm{u}_{\mathrm{G}}^{0,12} \cdot \mathrm{d}_{\mathrm{S}}^{0,05} \cdot \mathrm{T}^{0,68}} \cdot \Gamma \\
\left(\frac{\mathrm{d}_{\mathrm{BS}}}{\mathrm{d}_{\mathrm{BS}}-1}\right)^{0,40}\end{array}$ & $\begin{array}{l}\varepsilon_{\mathrm{G}}: \text { siehe Tabelle } 2-1 \\
\Gamma: \text { Begasereinfluss }\end{array}$ \\
\hline
\end{tabular}


In Abbildung 2-3 sind die $\mathrm{k}_{\mathrm{L}} \mathrm{a}$-Werte als Funktion der Gasleerrohrgeschwindigkeit $\mathrm{u}_{\mathrm{G}}$ aufgetragen. Die Kurve für die Gleichung von Dudukovic fehlt, da die Geschwindigkeit v in der REYNOLDS-Zahl nicht näher definiert wurde und so keine Berechnung möglich ist. Die Korrelation von KoJIMA ergibt bei hohen Gasleerrohrgeschwindigkeiten extrem große $\mathrm{k}_{\mathrm{L}} \mathrm{a}-$ Werte. Der Grund dafür ist wahrscheinlich, dass die zugrunde liegenden Daten mit Enzymlösungen gemessen wurden, die Sauerstoff verbrauchen. Diese Systeme haben eine komplexe Kinetik. Der Verbrauch des Sauerstoffes wird dabei nicht nur vom Stoffübergang limitiert, sondern kann durch unbekannte Effekte beeinflusst werden. Die verbleibenden drei Kurven von ÖZTÜRK, WILKINSON und LETZEL zeigen einen ziemlich einheitlichen Verlauf.

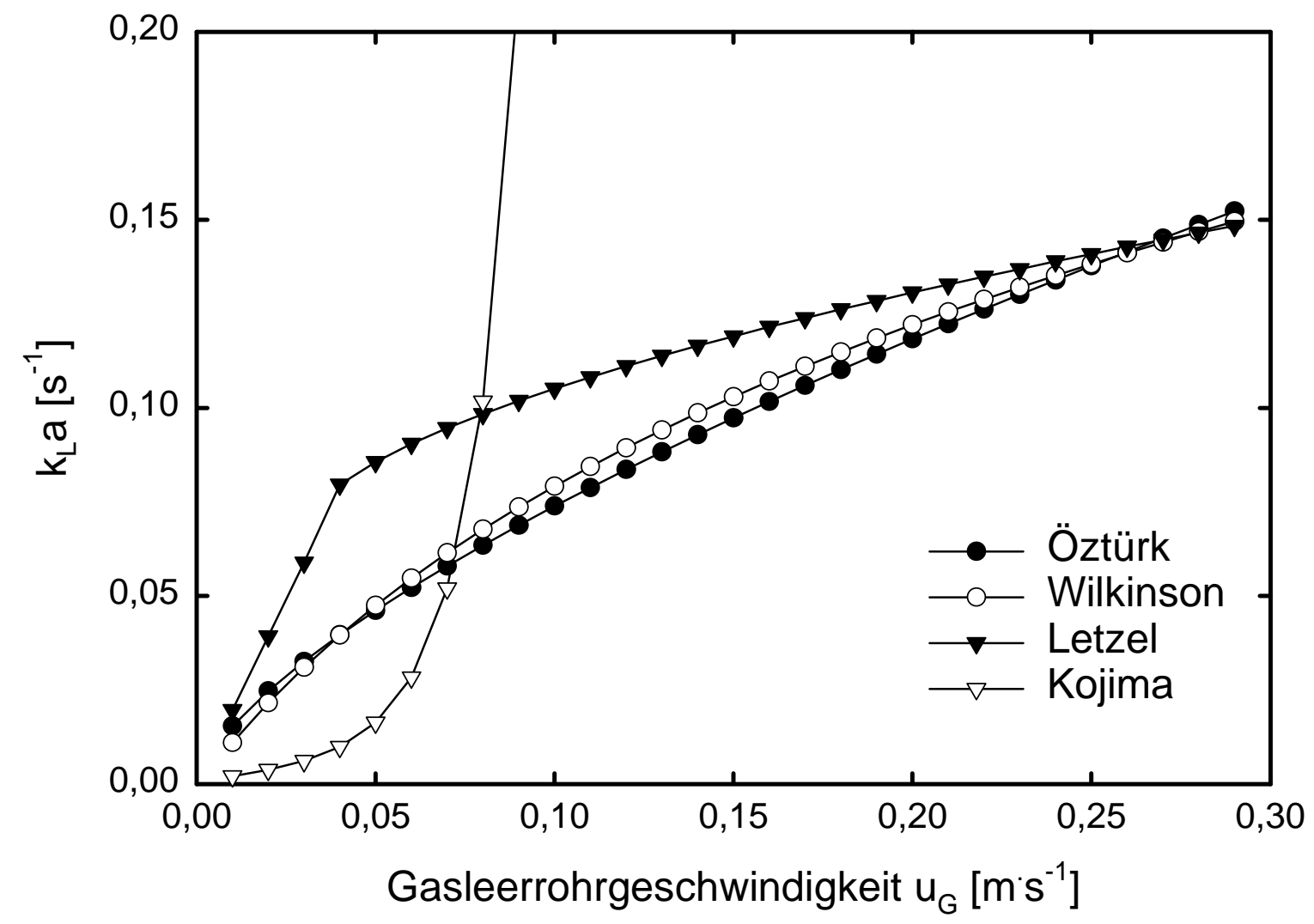

Abbildung 2-3: Berechnete $\mathrm{k}_{\mathrm{L}} \mathrm{a}-$ Werte für Wasser bei $20^{\circ} \mathrm{C}$ und 1 bar $\mathrm{N}_{2}$ als Funktion der Gasleerrohrgeschwindigkeit 


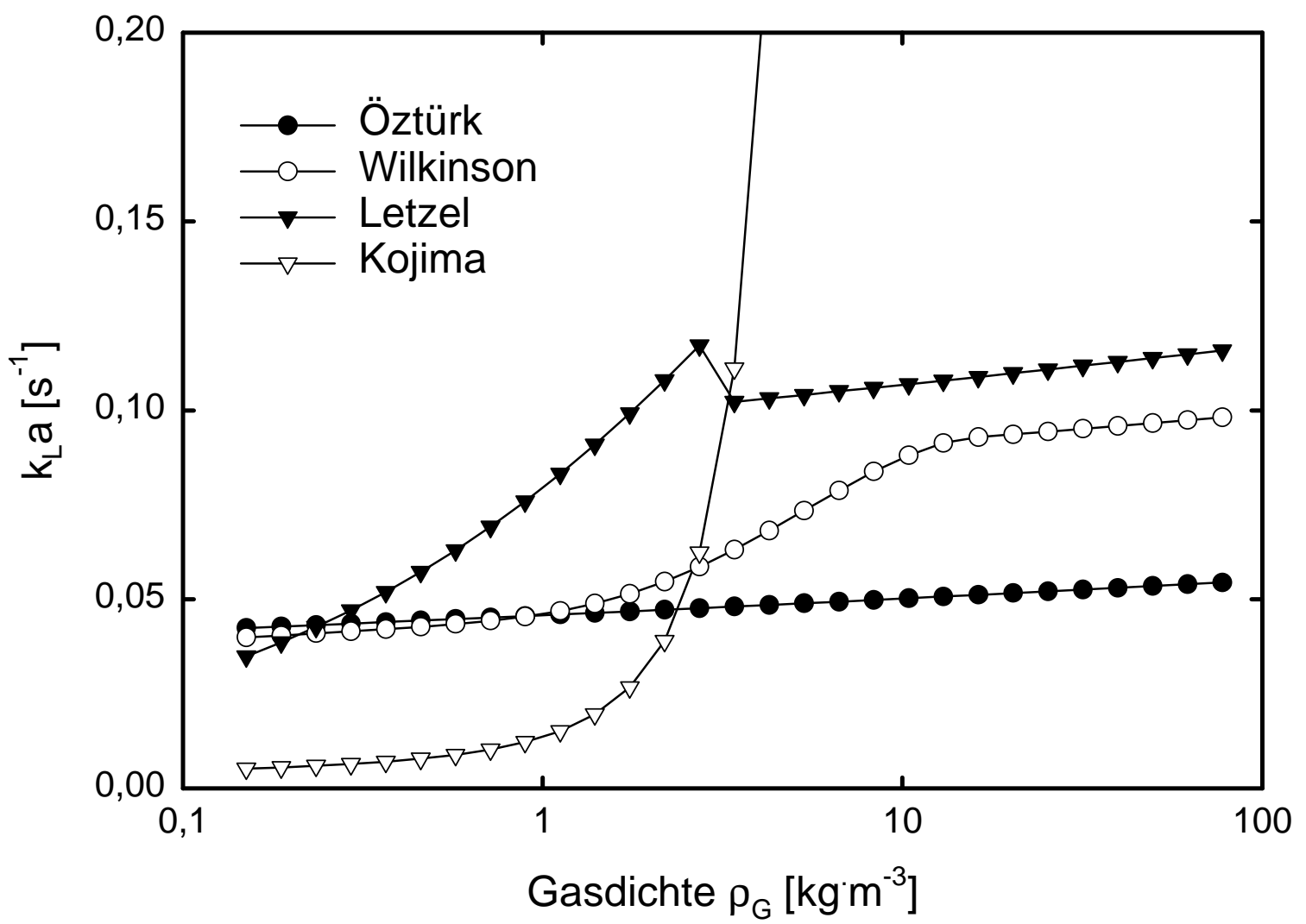

Abbildung 2-4: Berechnete $\mathrm{k}_{\mathrm{L}} \mathrm{a}-$ Werte für Wasser bei $20^{\circ} \mathrm{C}$ und $\mathrm{u}_{\mathrm{G}}=5 \mathrm{~cm} / \mathrm{s}$ als Funktion der Gasdichte; halblogarithmische Auftragung

Die Gasdichteabhängigkeit des $\mathrm{k}_{\mathrm{L}} \mathrm{a}$-Wertes hingegen zeigt größere Unterschiede zwischen den Korrelationen. Die Gleichung von KoJIMA kann aufgrund ihrer speziellen Datenbasis ignoriert werden. Die Kurve von ÖZTÜRK zeigt bei der gewählten Gasleerrohrgeschwindigkeit nur eine geringe Gasdichteabhängigkeit. Die Kurven von WILKINSON und LETZEL zeigen stärkere Gasdichteabhängigkeiten. Die Gasdichteabhängigkeit ändert sich wiederum mit der Gasdichte: bei niedrigen Gasdichten ist die Abhängigkeit stärker. In diesem Bereich befindet sich die Blasensäule im heterogenen Betriebszustand. Mit steigender Gasdichte nimmt der Anteil der Kleinbasen zu und der Anteil der Großblasen nimmt ab. Da Kleinblasen eine höhere spezifische Oberfläche haben, nimmt der Stoffübergang mit steigendem Kleinblasenanteil zu. Bei hohen Gasdichten sind keine Großblasen mehr vorhanden. Die Blasensäule befindet sich im homogenen Betriebszustand. Da sich die Gasstruktur bei hohen Gasdichten nicht mehr wesentlich ändert, erhält man in diesem Bereich eine geringe Gasdichteabhängigkeit des Stoffübergangs.

Trotz dieser Gemeinsamkeiten zeigen die Kurven von WILKINSON und LETZEL unterschiedliche Verläufe.

\subsection{Stoffübergang und Blasenaufstiegsgeschwindigkeit}

Die volumenbezogene Stoffübergangszahl $\mathrm{k}_{\mathrm{L}} \mathrm{a}$ setzt sich zusammen aus der spezifischen Phasengrenzfläche a und dem flüssigkeitsseitigen Stoffübergangskoeffizienten $\mathrm{k}_{\mathrm{L}}$. Die 
spezifische Phasengrenzfläche ergibt sich aus dem Gasgehalt und aus dem mittleren Blasendurchmesser, dem SAUTER-Durchmesser $\mathrm{d}_{\mathrm{S}}$ :

$\mathrm{a}=\frac{6 \cdot \varepsilon_{\mathrm{G}}}{\mathrm{d}_{\mathrm{s}}}$

Gleichung 2-5: Spezifische Phasengrenzfläche

Für eine größere Phasengrenzfläche muss also der Gasgehalt erhöht und/oder der SAUTERDurchmesser der Blasen verringert werden.

Für den $\mathrm{k}_{\mathrm{L}}$-Wert findet man bei COCKX 1995 folgende Korrelation:

$\frac{\mathrm{k}_{\mathrm{L}}}{\mathrm{v}_{\text {slip }}} \cdot \mathrm{Sc}^{0,5}=$ const $\approx 0,1 \pm 0,02$

Gleichung 2-6: Korrelation des $\mathrm{k}_{\mathrm{L}}$-Wertes

Der $\mathrm{k}_{\mathrm{L}}$-Wert steigt mit dem Diffusionskoeffizienten der untersuchten Substanz in der Flüssigkeit und sinkt mit der Viskosität der Flüssigkeit. Gleichzeitig hat die Relativgeschwindigkeit $v_{\text {slip }}$ zwischen Flüssigkeit und Gas noch einen Einfluss auf den Stoffübergang: mit steigender Geschwindigkeit nimmt der Stoffübergang zu.

\subsection{Technik der Entgasungsmessungen}

Eine Technik zur Charakterisierung des Gasgehaltes ist die dynamische Entgasungsmessung (Dynamic Gas Disengagement; DGD). Dabei wird an einer Blasensäule im stationären Betrieb die Gaszufuhr sehr schnell abgeschaltet. Die in diesem Moment in der Flüssigkeit enthaltenen Blasen entweichen bei unterschiedlicher Größe unterschiedlich schnell. Der Entgasungsvorgang kann über die Messung der Dispersionshöhe oder über die Messung des hydrostatischen Druckes verfolgt werden. Aus den so gewonnenen Messkurven kann auf den Anteil der unterschiedlichen Blasenklassen am Gasgehalt geschlossen werden.

Allerdings herrscht in der Literatur noch keine Einigkeit über die theoretische Betrachtung des Entgasungsvorganges. Daraus folgen dann auch unterschiedliche, z. T. widersprüchliche Ansätze in der Auswertung der Entgasungsmessungen.

Zum besseren Verständnis wird im Folgenden nur mit zwei Blasenklassen gearbeitet: Großblasen und Kleinblasen. Folgende Beschreibungen des Entgasungsvorganges sind in der Literatur zu finden (SRIRAM, MANN 1977; PATEl ET AL 1989; DALY ET AL 1992; SCHUMPE, GRUND 1986; ELLENBERGER, KRISHNA 1994):

Unabhängiges Entgasen (SriraM, MANN 1977; DALY ET AL 1992; PATEL ET AL 1989)

Beide Blasenklassen werden getrennt behandelt. Jeder Blasenklasse wird entsprechend ihrem Anteil am Gasgehalt ein Anteil am Flüssigkeitsvolumen zugewiesen. Während der Entgasung hinterlässt jede Blasenklasse ihren Anteil blasenfreier Flüssigkeit.

Diese Betrachtung führt zu einer relativ einfachen Berechnung der Blasenklassengehalte. Allerdings werden folgende Dinge nicht beachtet: 
Da die verschiedenen Blasenklassen unterschiedlich schnell aufsteigen, müssen die Flüssigkeitsspiegel in den jeweiligen Flüssigkeitsvolumina unterschiedlich schnell sinken. Dies ist jedoch nicht möglich.

Das freiwerdende Volumen der entweichenden Großblasen wird in diesem Ansatz von der Flüssigkeit ausgefüllt. Tatsächlich steigen die Großblasen in einem Kleinblasen-FlüssigkeitsGemisch auf, das das frei werdende Volumen ausfüllt. Dadurch ist der Kleinblasengehalt während der zweiten Entgasungsphase größer als während des stationären Betriebes. Der Kleinblasengehalt kann nicht unabhängig vom Großblasengehalt betrachtet werden.

Durch diesen Ansatz wird der Kleinblasengehalt zu groß eingeschätzt.

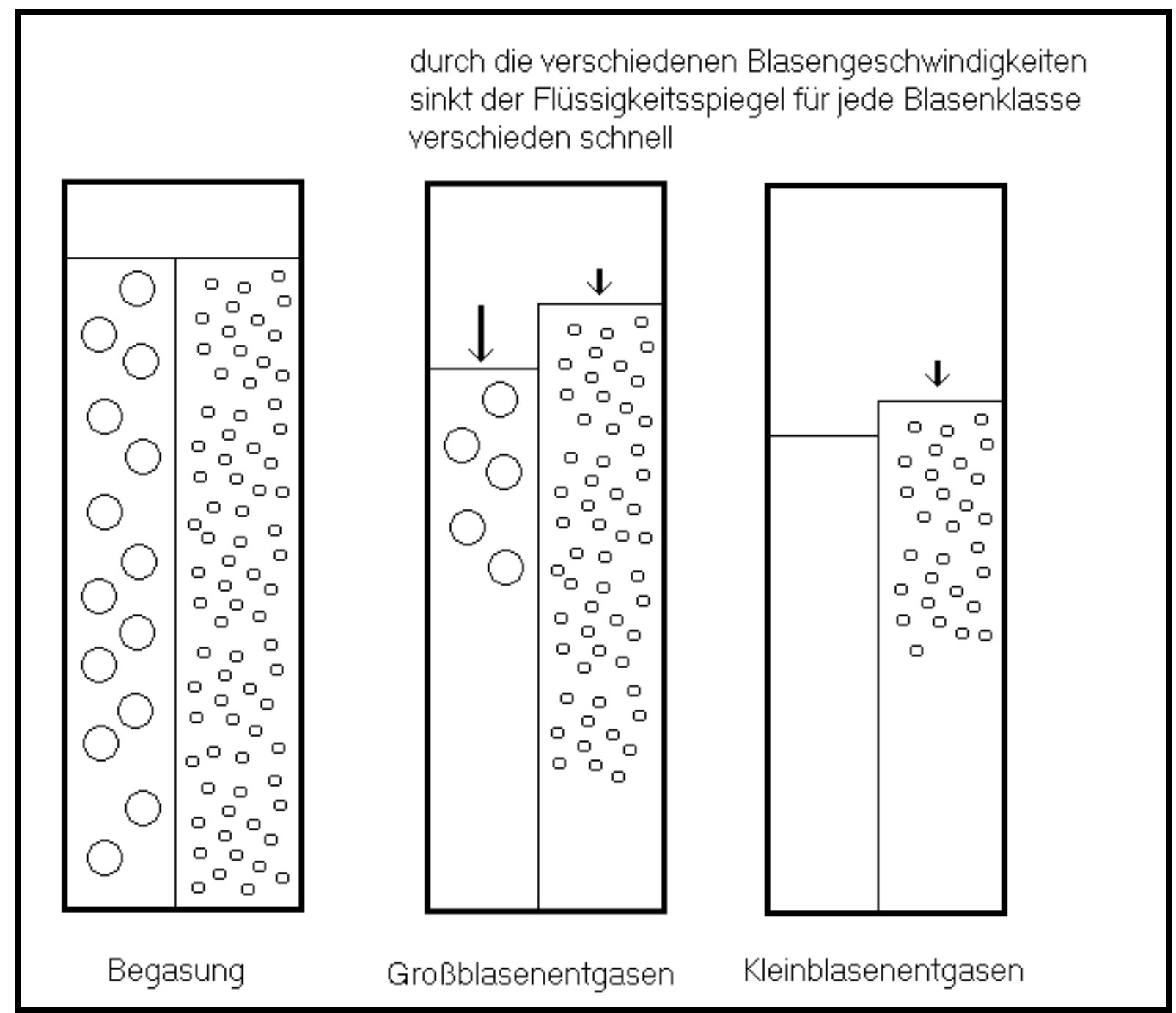

Abbildung 2-5: Schematische Darstellung des unabhängigen Entgasens; das unterschiedlich schnelle Absinken der Flüssigkeitsspiegel wurde in der zitierten Literatur nicht beachtet

\section{Sequentielles Entgasen (Ellenberger, Krishna 1994; PATEl Et Al 1989)}

In diesem Fall wird das Entgasen der Blasenklassen nicht räumlich, sondern zeitlich getrennt. Zunächst steigen die schnellen Großblasen in einem Kleinblasen-Flüssigkeits-Gemisch auf. Haben alle Großblasen die Flüssigkeit verlassen, setzt das Entgasen der langsamen Kleinblasen ein. 
In diesem Ansatz wird die Wechselwirkung zwischen Großblasen und Kleinblasen beachtet. Dennoch ist die Annahme, dass die Kleinblasen erst nach dem vollständigen Entweichen der Großblasen aufsteigen, falsch:

In diesem Ansatz verharren die Kleinblasen während der ersten Entgasungphase an Ort und Stelle. In der Realität bewegt sich die hintere Kleinblasenfront schon während der ersten Entgasungsphase nach oben. Gleichzeitig verlassen die ersten Kleinblasen die Flüssigkeit.

Durch diesen Ansatz wird der Kleinblasengehalt zu klein eingeschätzt.

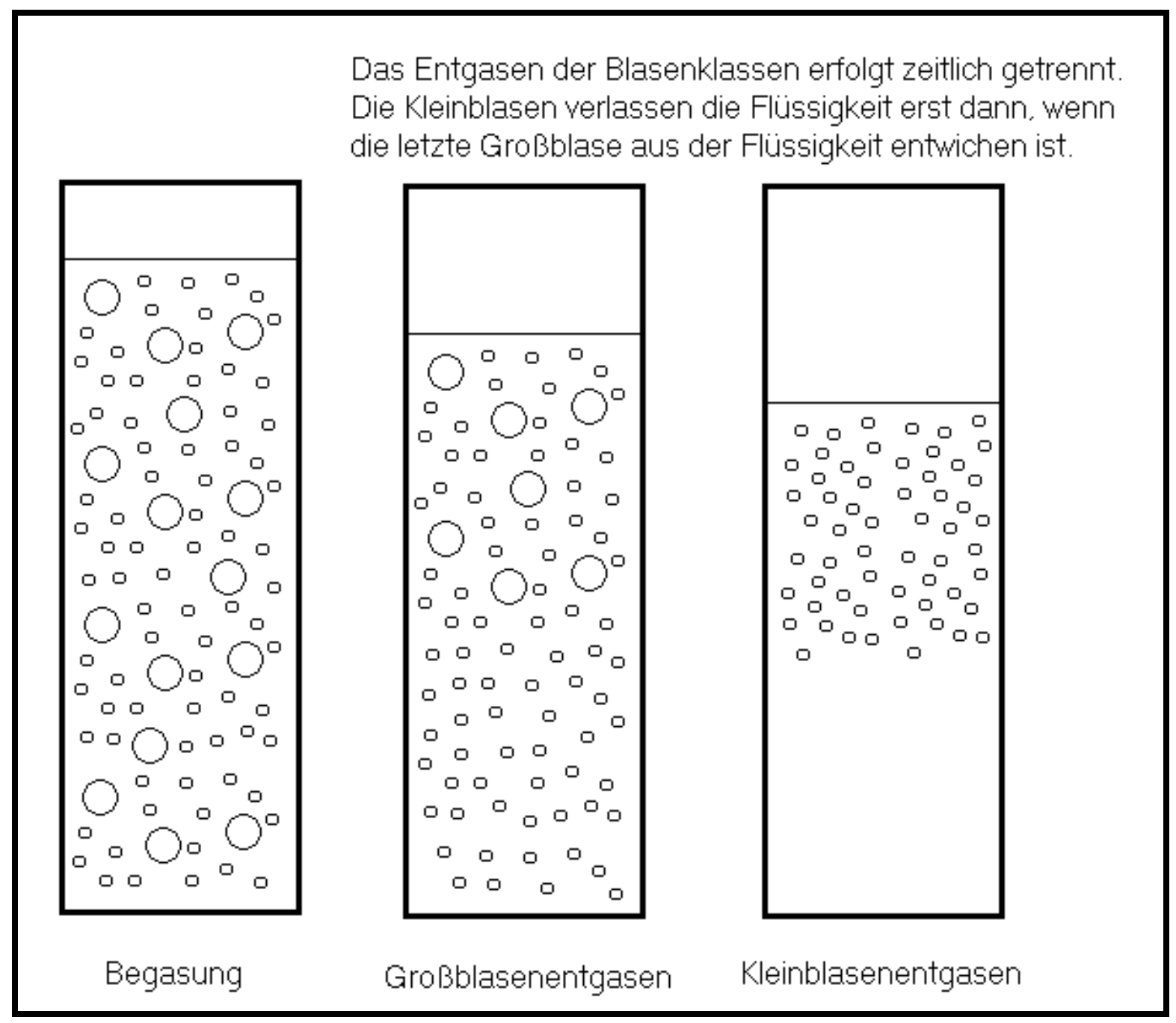

Abbildung 2-6: Schematische Darstellung des sequentiellen Entgasens

\section{Konstante Relativgeschwindigkeit ( SCHUMPE, GRUND 1986; DEWES 1996)}

Bei diesem Ansatz wird vorausgesetzt, dass die Relativgeschwindigkeit zwischen Blase und umgebender Flüssigkeit konstant ist. In diesem Fall ist es nötig, die Geschwindigkeit der abströmenden Flüssigkeit in die Rechnungen einzubeziehen, da dadurch die aufsteigenden Blasen abgebremst werden. Dadurch wird die Berechnung kompliziert, aber die Schwächen der ersten beiden Ansätze werden vermieden. Darum soll mit diesem Ansatz weitergearbeitet werden.

Es gibt verschiedene Arbeiten, in denen mit diesem Ansatz Entgasungsmessungen ausgewertet wurden. Leider sind die verwendeten Formeln widersprüchlich und zum Teil 
fehlerhaft. Darum soll das mathematische Handwerkszeug zur Auswertung der Entgasungsmessungen neu entwickelt und mit den zitierten Arbeiten verglichen werden.

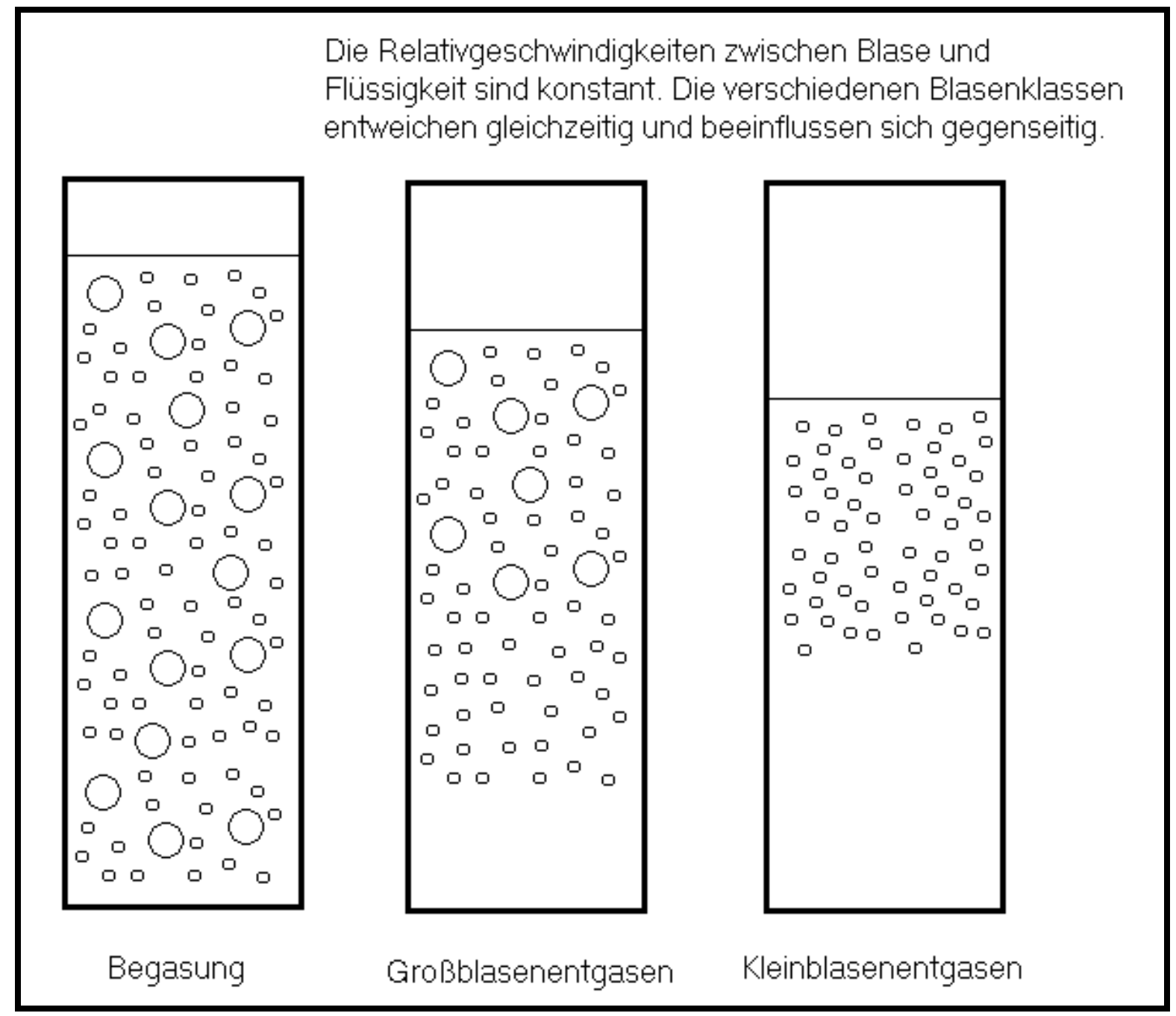

Abbildung 2-7: Schematische Darstellung des Entgasens mit konstanter Relativgeschwindigkeit 


\section{Material und Methoden}

Im Rahmen dieser Arbeit wurden zwei verschiedene Druckblasensäulen, verschiedene Flüssigkeiten und Gase verwendet. Stoffübergang, Gasgehalt und Gasstruktur wurden untersucht.

\subsection{Geräte und Chemikalien}

\subsubsection{Druckblasensäule 1}

Bei der Blasensäule 1 handelt es sich um einen modifizierten Bioreaktor der Firma Bioengineering (Wald, Schweiz). (siehe Abbildung 3-1).

Der Reaktorkörper hat im unteren zylindrischen Teil einen Innendurchmesser von $115 \mathrm{~mm}$ und eine Höhe von 1000 mm. Der Kopfteil erweitert sich konisch auf einen Durchmesser von $200 \mathrm{~mm}$ und hat eine Höhe von $240 \mathrm{~mm}$. Die Gesamthöhe beträgt $1370 \mathrm{~mm}$ und das Gesamtvolumen beträgt 201.

Die Gasversorgung erfolgt mit Stickstoff und Druckluft aus Druckgasflaschen bzw. aus dem hauseigenen Druckluftnetz. Der Gasstrom wird mit zwei Massendurchflussreglern eingestellt. Der kleinere Massendurchflussregler hat einen Regelbereich von 0 - $50 \mathrm{Nl} / \mathrm{min}$ (Normliter pro Minute; siehe Abschnitt 3.1.3), der größere Massendurchflussregler von $0-250 \mathrm{Nl} / \mathrm{min}$. Das Gas gelangt durch eine Lochplatte $(7 \times 1 \mathrm{~mm})$ in den Blasensäulenreaktor. Im Kopfteil der Blasensäule befinden sich der Gasauslass und der Drucksensor. Der Kopfdruck der Blasensäule wird durch ein an der Abluftleitung befindliches Stellventil auf $+/-0,01$ bar genau geregelt.

Gaseinlass und Gasauslass können durch elektromagnetische Ventile geschlossen werden.

Die Temperierung erfolgt über einen Doppelmantel um den unteren zylindrischen Teil der Blasensäule. Die Temperatur lässt sich auf +/- 0,1 K genau einstellen.

Der Reaktor hat eine Reihe von Anschlussstutzen auf einer Höhe von 65 mm und $900 \mathrm{~mm}$ über dem Begaser. Der Sauerstoffsensor wurde an einem der unteren Stutzen platziert. Die Differenzdrucksensoren wurden am unteren und am oberen Stutzenring platziert.

Die Datenerfassung erfolgt über einen Analog-Digital-Wandler mit einem PC. 


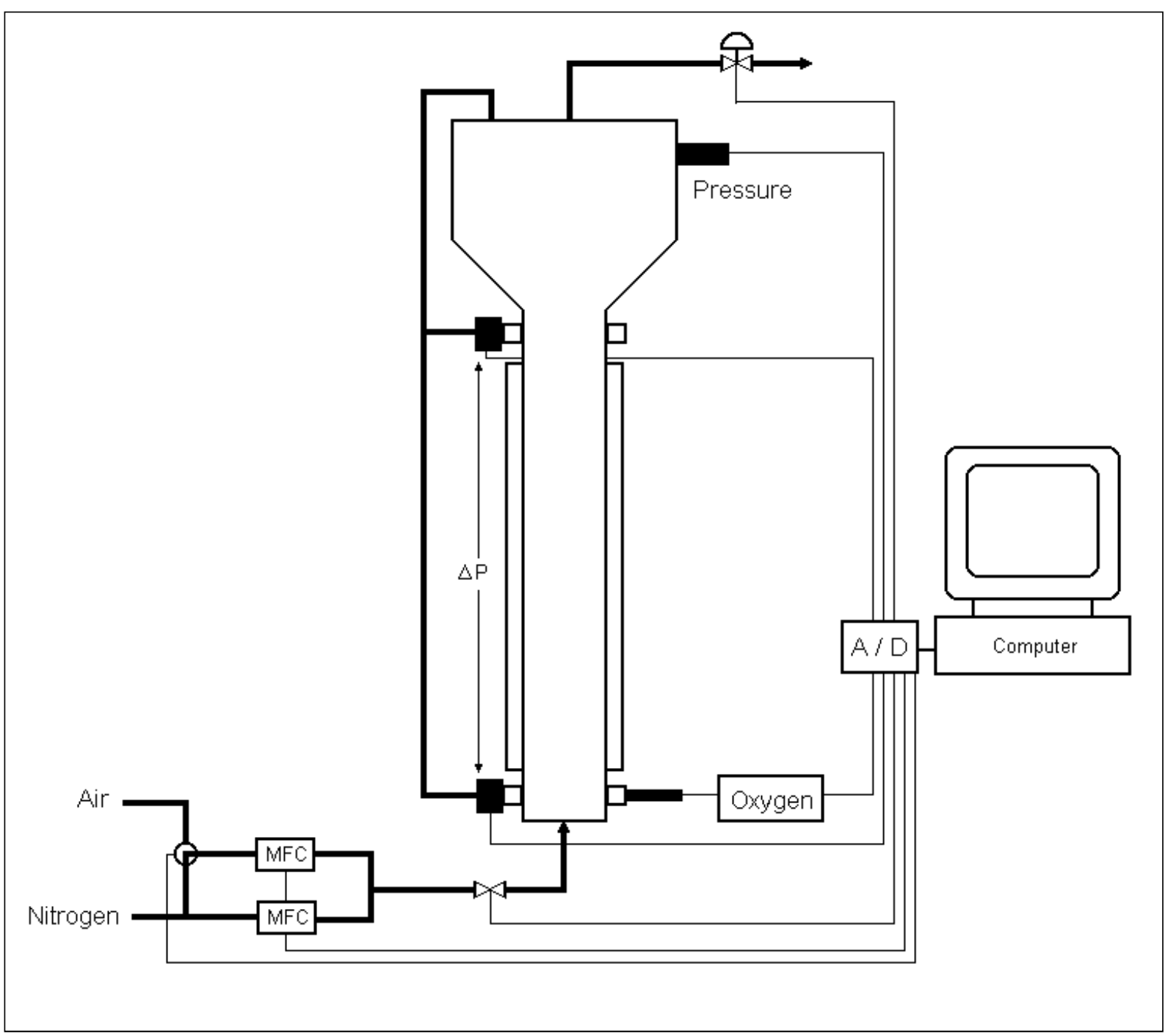

Abbildung 3-1: Schematischer Aufbau der Druckblasensäule 1

\subsubsection{Druckblasensäule 2}

Die Blasensäule wurde von der Firma Ernst Haage Apparatebau GmbH \& Co KG (Deutschland; Mühlheim a. d. Ruhr) hergestellt. Der Reaktorkörper besteht aus Edelstahl und ist ein Zylinder von $102 \mathrm{~mm}$ Innendurchmesser und $2400 \mathrm{~mm}$ Höhe. Auf den Höhen $0 \mathrm{~mm}$; $650 \mathrm{~mm} ; 1200 \mathrm{~mm} ; 1800 \mathrm{~mm}$ und im Deckel der Blasensäule gibt es jeweils mehrere Anschlussstutzen. Auf $900 \mathrm{~mm}$ und $2100 \mathrm{~mm}$ Höhe sind je ein Paar gegenüberliegende Fenster zur Beobachtung der Flüssigkeit vorhanden. Die Thermostatisierung erfolgt über einen Doppelmantel mit einem Kühlthermostat F 33 der Firma Julabo im unteren Teil der Säule. Der Sauerstoffsensor wurde auf einer Höhe von $650 \mathrm{~mm}$ über dem Begaser platziert. Die vier Differenzdrucksensoren befinden sich auf $0 \mathrm{~mm} ; 650 \mathrm{~mm} ; 1200 \mathrm{~mm}$ und $1800 \mathrm{~mm}$ über dem Begaser.

Die Gasversorgung erfolgt aus Gasdruckflaschen mit Stickstoff oder Helium bzw. aus der hauseigenen Stickstoffleitung. Der Gasstrom wird durch drei Massendurchflussregler mit den Regelbereichen $0-50 \mathrm{Nl} / \mathrm{min} ; 0-500 \mathrm{Nl} / \mathrm{min}$ und $0-5000 \mathrm{Nl} / \mathrm{min}$ geregelt.

Der Gasstrom kann wahlweise direkt in die Blasensäule geleitet werden oder in einem Sättiger (Hersteller: Kempe Apparate- und Maschinenbau; Deutschland; Schwaigern) auf die Betriebstemperatur gebracht und mit Lösemitteldampf gesättigt werden. Im Normalfall wurde mit Sättiger gearbeitet, da so Lösemittelverluste in der Blasensäule vermieden werden. 
Nach dem Sättiger kann bei Bedarf mit Hilfe eines vierten Massendurchflussreglers $\left(\begin{array}{ll}0 & -5\end{array}\right.$ $\mathrm{Nl} / \mathrm{min}$ ) Sauerstoff in den Gasstrom dosiert werden. Des Gasstrom gelangt durch einen austauschbaren Gasverteiler in die Blasensäule (Tabelle 3-1: verwendete Begaser)

Tabelle 3-1: verwendete Begaser der Druckblasensäule 2

\begin{tabular}{|c|c|c|}
\hline Begaser & Lochanzahl & Lochgröße \\
\hline Sinterplatte & $? ?$ & ca. $100 \mu \mathrm{m}$ \\
\hline Einzelloch $1 \times 4,3$ & 1 & $4,3 \mathrm{~mm}$ \\
\hline Einzelloch $1 \times 1$ & 1 & $1 \mathrm{~mm}$ \\
\hline Lochplatte 19x1 & 19 & $1 \mathrm{~mm}$ \\
\hline
\end{tabular}

Die Druckregelung erfolgt über zwei Stellventile am Gasauslass der Blasensäule. Der Gaseinlass und der Gasauslass können für die Entgasungsmessungen mit je einem elektropneumatischen Ventil schnell geschlossen werden. Die Messdatenerfassung erfolgt über einen Analog-Digital-Wandler mit dem PC.

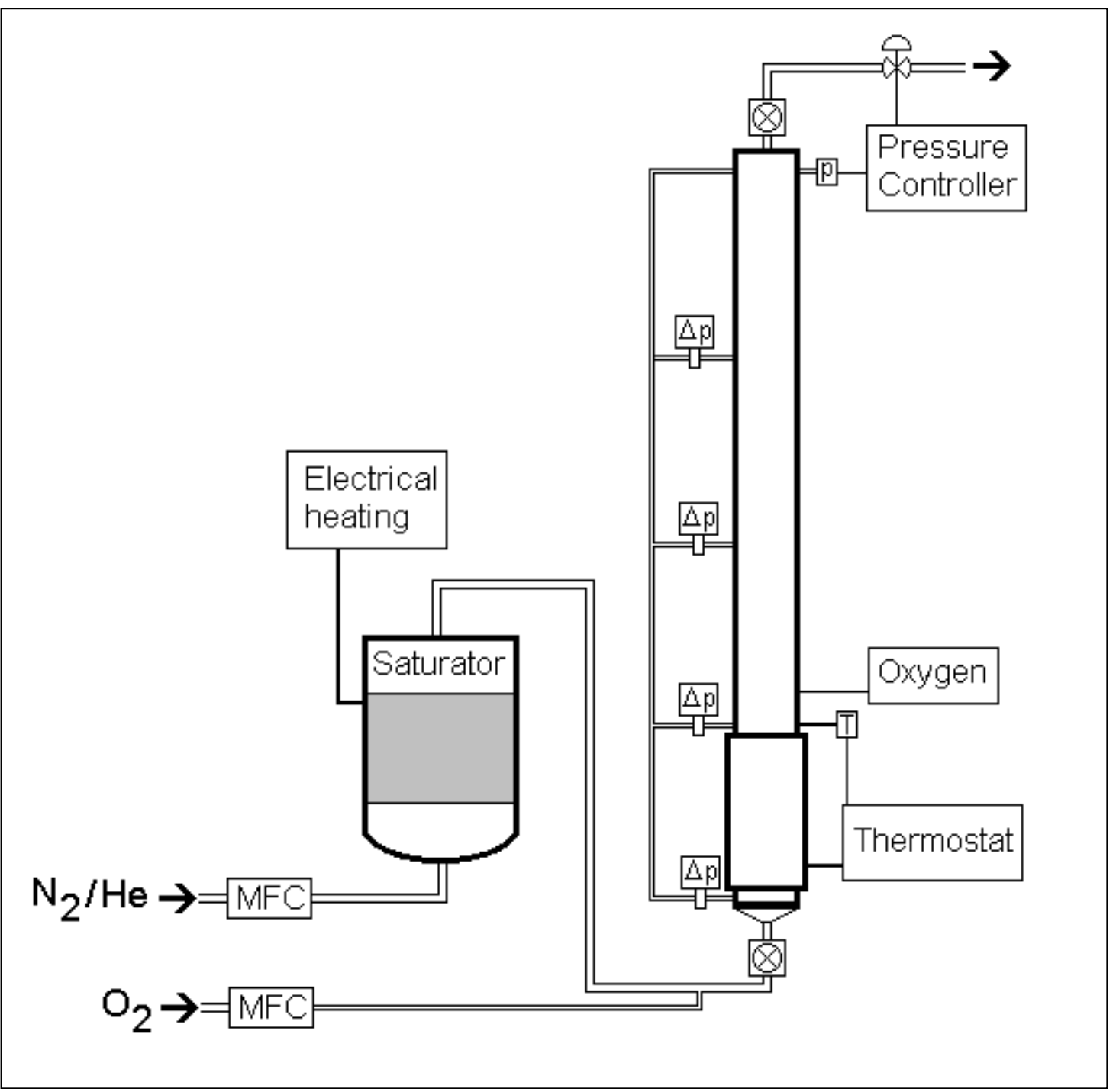

Abbildung 3-2: Schematischer Aufbau der Druckblasensäule 2 


\subsubsection{Massendurchflussregler (Mass-Flow-Controller; MFC)}

Die verwendeten Massendurchflussregler wurden von Bronkhorst Hi-Tec (Ruurlo, Niederlande) hergestellt. Der Massendurchfluss wird thermisch gemessen. Dazu wird der Gasstrom in den Massendurchflussreglern zunächst laminarisiert. Dann wird ein definierter Teilstrom durch den eigentlichen Messsensor geleitet. Der Hauptstrom wird durch ein Laminar-Durchfluss-Element geleitet. Dabei bleibt das Verhältnis Hauptstrom zu Nebenstrom unabhängig vom Gesamtstrom konstant.

Der Messsensor besteht aus zwei Temperatursensoren und einer Heizung. Die Heizung gibt eine konstante Heizleistung an den Nebenstrom ab. Mit den Temperatursensoren wird die dadurch erreichte Temperaturdifferenz vor und nach der Heizung gemessen. Es ergibt sich folgende Wärmebilanz:

$\mathrm{P}_{\mathrm{Q}}=\Delta \mathrm{T} \cdot \mathrm{c}_{\mathrm{P}} \cdot \dot{\mathrm{m}}_{\mathrm{G}}$

Gleichung 3-1: Heizleistung des Massendurchflusssensors

Aus der Gleichung lässt sich erkennen, dass für jede Gasart eine eigene Kalibrierung nötig ist. Der Grund sind die unterschiedlichen Wärmekapazitäten der Gase. Wird auf Neukalibrierungen verzichtet, müssen für verschiedene Gase die vom Hersteller angegebenen Korrekturfaktoren verwendet werden.

Die gemessenen Massenströme werden auf die Einheit $\mathrm{Nl} / \mathrm{min}$ (Normliter pro Minute) umgerechnet. Ein Normliter ist die Gasmenge, die bei $101325 \mathrm{~Pa}$ und 273,15 K das Volumen von 11 einnimmt.

Die Genauigkeit der eingestellten Gasströme berechnet sich laut Hersteller:

$\Delta \dot{\mathrm{V}}=0,005 \cdot \dot{\mathrm{V}}+0,001 \cdot \dot{\mathrm{V}}_{\max }$

Gleichung 3-2: Regelgenauigkeit der Massendurchflussregler

Der relative Fehler nimmt bei kleinen Volumenströmen stark zu. (siehe Abbildung 3-3) Die Massendurchflussregler sollten deshalb nicht unterhalb von 5\% ihres Maximalwertes betrieben werden. 


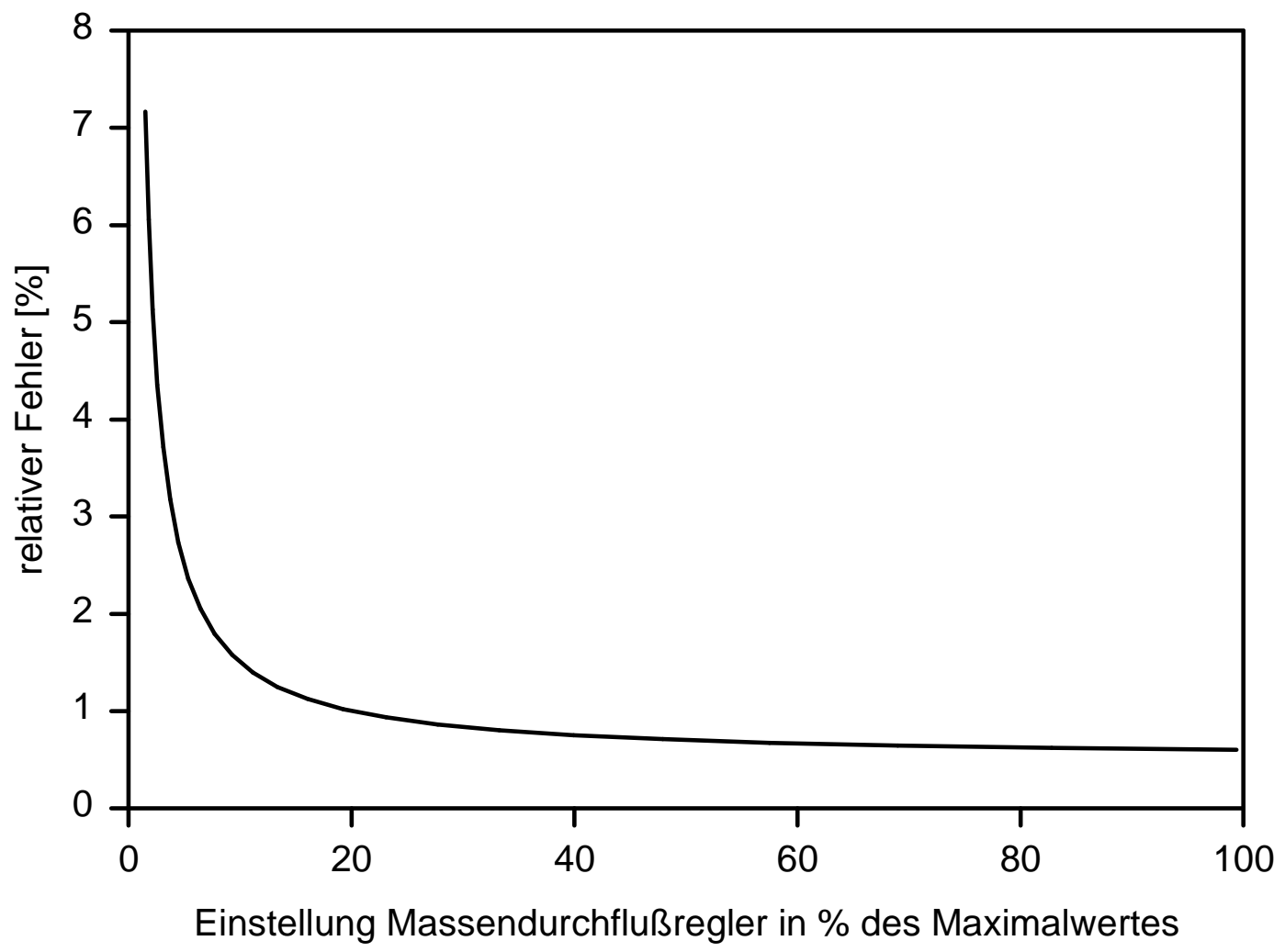

Abbildung 3-3: Regelabweichung der verwendeten Massendurchflussregler

\subsubsection{Optischer Sauerstoffsensor}

Der Sauerstoffsensor, ein mikroprozessorgesteuerter optischer portabler Sauerstoffsensor (MOPS), wurde von der Firma „COMTE - moderne Analysensysteme“ (Hannover; Deutschland) hergestellt.

Er nutzt die Fluoreszenzlöschung bestimmter Fluorophore (fluoreszierende Farbstoffe) durch molekularen Sauerstoff. Das Fluorophor wird durch kurzwelliges blaues Licht $(\lambda=470 \mathrm{~nm})$ angeregt. Das Licht gelangt durch eine Glasfaser $\mathrm{zu}$ dem in einer Silikonmatrix immobilisierten Fluorophor. Die dadurch ausgelöste Fluoreszenz im langwelligen roten Bereich $(\lambda=580 \mathrm{~nm})$ gelangt über die Glasfaser zurück in den Sauerstoffsensor und wird über einen Strahlteiler in einen Photomultiplier geleitet. Die dort gemessene Lichtintensität ist ein Maß für die Sauerstoffkonzentration. Den mathematischen Zusammenhang zwischen Fluoreszenzintensität und Sauerstoffkonzentration stellt die STERN-VOLLMER-Gleichung her.

$\frac{\mathrm{I}_{0}}{\mathrm{I}}-1=\mathrm{K}_{\mathrm{SV}} \cdot \mathrm{c}_{\mathrm{O}_{2}}$

Gleichung 3-3: STERN-VOLLMER-Gleichung

Um die Signalverzögerung durch die Diffusion des Sauerstoffes durch die Silikonmatrix zu umgehen, wurde das Fluorophor direkt in der Flüssigkeit gelöst. Da das originale Fluorophor nur in polaren organischen Flüssigkeiten löslich ist, mussten für Wasser und unpolare organische Flüssigkeiten andere, weniger geeignete Farbstoffe gewählt werden (siehe Tabelle 3-2 und Abbildung 3-4). 
Tabelle 3-2: verwendete Fluoreszenzfarbstoffe

\begin{tabular}{|c|c|c|}
\hline Fluorophor & Flüssigkeit & Konz. $\left[\mathrm{kg}^{-3} \mathrm{~m}^{-3}\right]$ \\
\hline $\begin{array}{l}\text { Tris-(2,2'bipyridyl)-Ruthenium(II)chlorid } \\
\text { (RuBPY) }\end{array}$ & Wasser & 0,1 \\
\hline $\begin{array}{l}\text { Tris-(4,7diphenyl, 1,10-phenanthrolin)- } \\
\text { Ruthenium(II)chlorid * } \\
\text { (RuDPP) }\end{array}$ & $\begin{array}{c}\text { Ethanol, } \\
\text { 1-Butanol } \\
\text { Ethylenglycol }\end{array}$ & 0,02 \\
\hline $\begin{array}{c}2,3,7,8,12,13,17,18 \text {-Octaethyl-21H,23H-porphin } \\
\text { (OEP) }\end{array}$ & $\begin{array}{l}\text { Toluol } \\
\text { Decalin }\end{array}$ & 0,1 \\
\hline
\end{tabular}

* Originalfluorophor

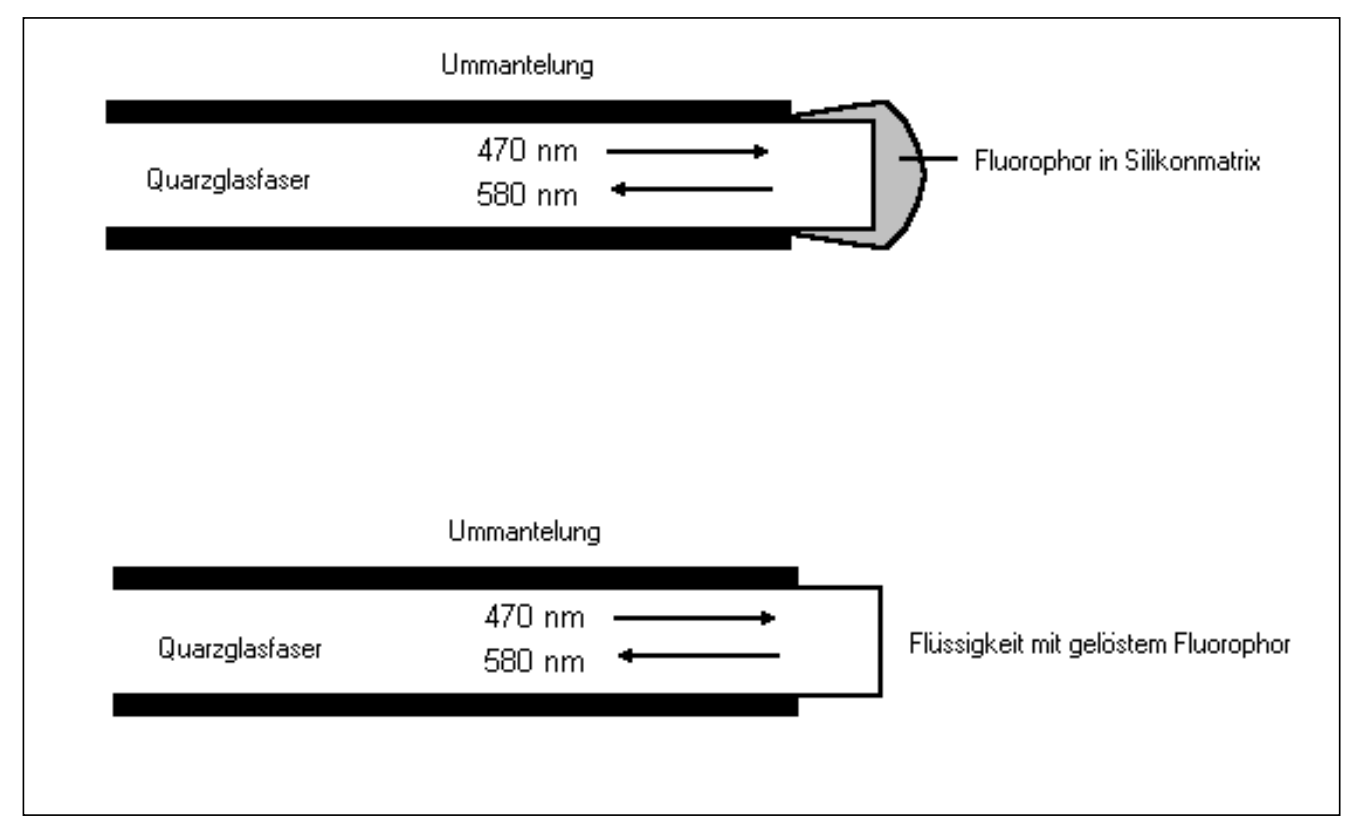

Abbildung 3-4: Optode mit immobilisiertem und gelöstem Fluorophor

Da für die Messungen nicht das vom Hersteller gelieferte immobilisierte Fluorophor genutzt wurde, konnte die im Messgerät gespeicherte Kalibrierung für die Messungen nicht genutzt werden. Es mussten eigene Kalibrierungen erstellt werden. Dazu wurde das Messgerät so modifiziert, dass die Spannung des Photomultipliers ausgelesen werden konnte.

Die Fluoreszenzintensitäten $\mathrm{I}$ und $\mathrm{I}_{0}$ werden nach den Angaben des Herstellers aus der Spannung des Photomultipliers berechnet

$$
\begin{aligned}
& \mathrm{I}_{0}=\frac{\mathrm{U}_{0}-\mathrm{U}_{100}}{\text { konst. }} \\
& \mathrm{I}=\mathrm{U}-\mathrm{U}_{0}+\mathrm{I}_{0}
\end{aligned}
$$

Also ergibt sich für die Konzentration des Sauerstoffes in der Lösung:

$$
\mathrm{c}_{\mathrm{O}_{2}}=\frac{1}{\mathrm{~K}_{\mathrm{SV}}} \cdot\left(\frac{\frac{\mathrm{U}_{0}-\mathrm{U}_{100}}{\text { konst. }}}{\mathrm{U}-\mathrm{U}_{0}+\frac{\mathrm{U}_{0}-\mathrm{U}_{100}}{\text { konst. }}}-1\right)
$$

Gleichung 3-4: modifizierte STERN-VOLLMER-Gleichung 
Während der Messung wird die Spannung U aufgezeichnet und in die Konzentration $\mathrm{c}_{\mathrm{O} 2}$ umgerechnet. Die Werte für $\mathrm{U}_{0}, \mathrm{U}_{100}$, die Konstante „konst.“ und die STERN-VollmerKonstante $\mathrm{K}_{\mathrm{SV}}$ werden vor der Messung mit Hilfe einer Kalibrierreihe ermittelt. Dazu wird die Flüssigkeit mit Stickstoff-Sauerstoff-Gemischen verschiedener Zusammensetzung begast (0 mbar; 10,6 mbar; 21,3 mbar; 53,2 mbar; 106,4 mbar und 212,8 mbar Sauerstoffpartialdruck; dies entspricht $0 \%, 5 \%, 10 \%, 25 \%, 50 \%$ und $100 \%$ der Sauerstoffkonzentration der Luft) und die Spannung des Photomultipliers gemessen.

Die Werte für $\mathrm{U}_{0}$ und $\mathrm{U}_{100}$ entsprechen den Werten für $0 \%$ bzw. 100\% Sauerstoffsättigung. Die Werte für konst. und $\mathrm{K}_{\mathrm{SV}}$ werden mit der Methode der kleinsten Fehlerquadrate angepasst.

Beispiele für die Kalibrierungen für die drei Fluorophore sind in Tabelle 3-3 aufgelistet.

Tabelle 3-3: Beispiele für die Kalibrierung der verschiedenen Fluorophore

\begin{tabular}{|c|c|c|c|c|}
\hline Fluorophor & $\mathrm{U}_{0}[\mathrm{~V}]$ & $\mathrm{U}_{100}[\mathrm{~V}]$ & $\mathrm{K}_{\mathrm{SV}}$ (willkürliche Einheit) & konst. \\
\hline RuDPP & 9,02 & 4,48 & 0,1484 & 0,937 \\
\hline RuBPY & 9,23 & 8,13 & $6,17 \cdot 10^{-3}$ & 0,382 \\
\hline OEP & 8,87 & 8,25 & $3,20 \cdot 10^{-3}$ & 0,244 \\
\hline
\end{tabular}

Die Genauigkeit der Sauerstoffmessung ist durch die Messabweichung des Photomultipliers vorgegeben. Dis Spannung des Photomultipliers kann mit einer Genauigkeit von $\Delta U=+/$ 0,01 V ausgelesen werden. Der absolute Fehler der gemessenen Sauerstoffkonzentration liegt damit in der Regel unter $\Delta \mathrm{c}_{\mathrm{O} 2}<+/-2 \%$ Luftsättigung (siehe Abbildung 3-5).

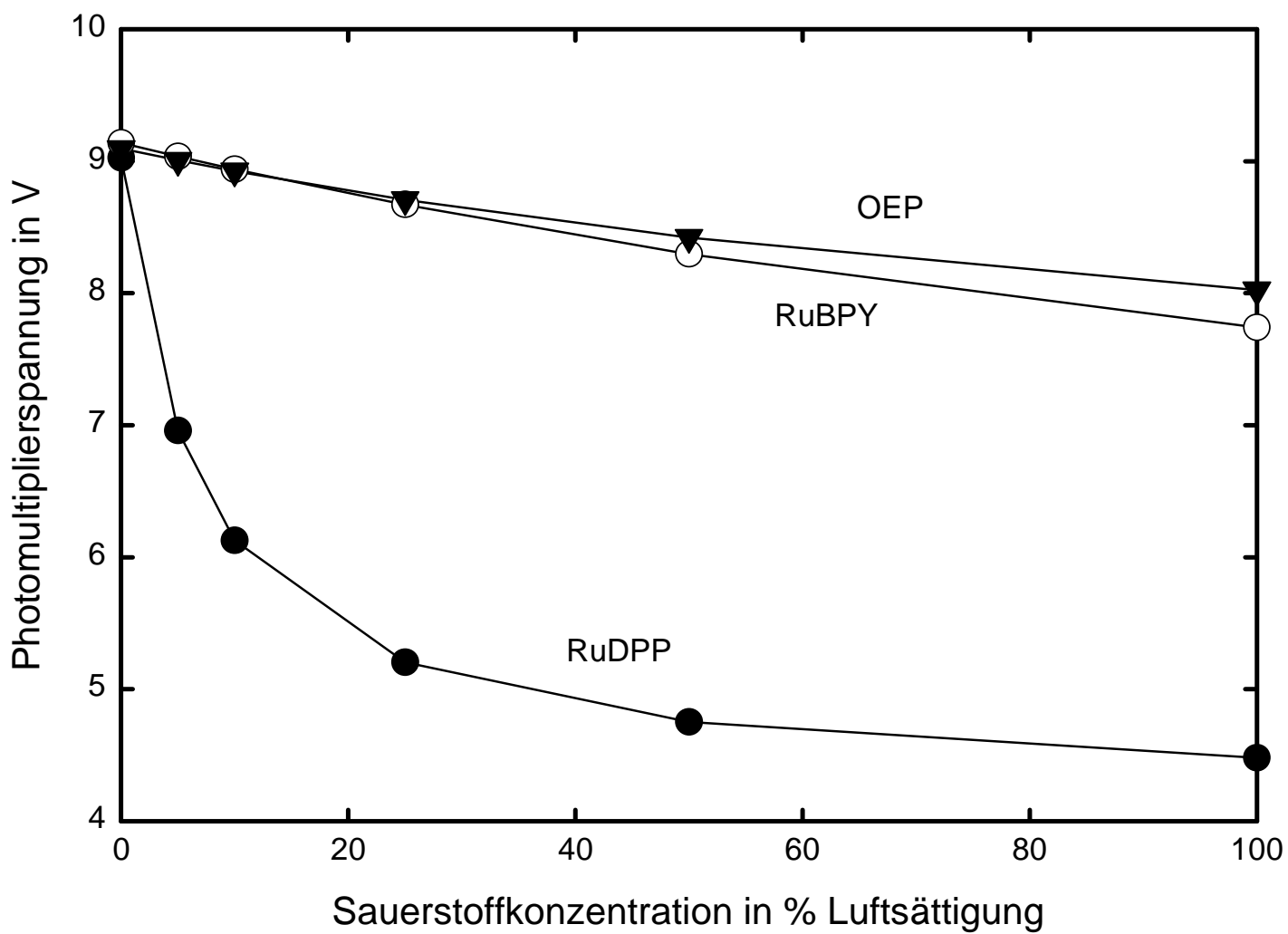




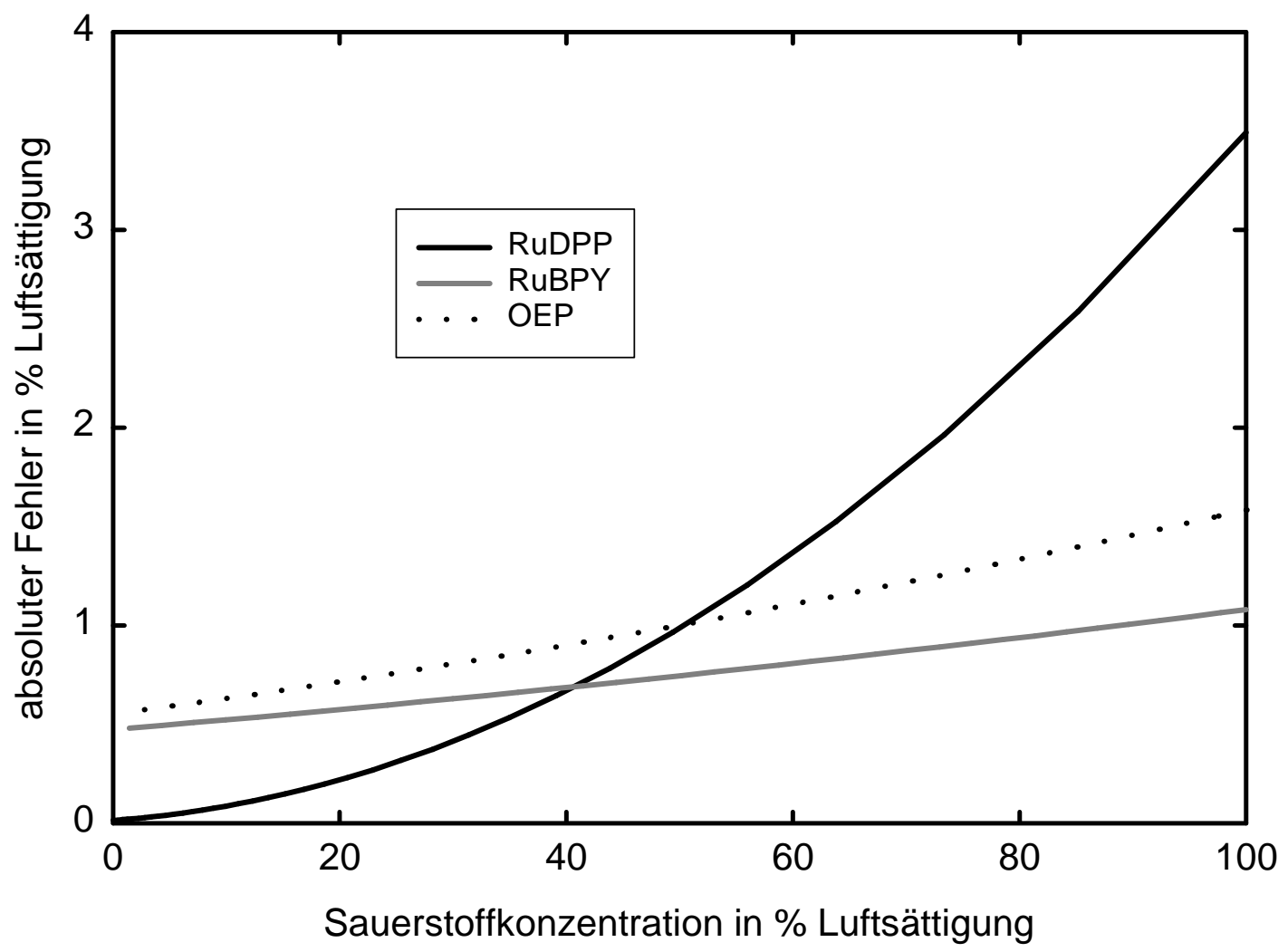

Abbildung 3-5: Durch die Messgenauigkeit des Photomultipliers bedingter absoluter Fehler der Sauerstoffmessung

Die Meßmethoden beruht auf der Fluoreszenzlöschung der Farbstoffe durch Übertragung der Anregungsenergie auf das Sauerstoffmolekül. Daneben ist auch eine thermische Löschung der angeregten Moleküle durch Stöße mit der umgebenden Flüssigkeit möglich. Dieser Vorgang ist temperaturabhängig und nimmt mit steigender Temperatur zu. In Abbildung 3-6 ist die Abhängigkeit der Photomultiplierspannung von der Temperatur bei verschiedenen Sauerstoffkonzentrationen dargestellt. Der daraus resultierende absolute Fehler der Sauerstoffkonzentration ist in Abbildung 3-7 dargestellt. Je kleiner die Temperaturschwankungen, desto kleiner fällt auch dieser Fehler aus. Die Temperatur in der Blasensäule konnte während der Desorptionsmessungen auf $\Delta \mathrm{T}=+/-0,1 \mathrm{~K}$ konstant gehalten werden. Der absolute Fehler ist also kleiner als $\Delta \mathrm{c}_{\mathrm{O} 2}=+/-0,4 \%$ Luftsättigung. 


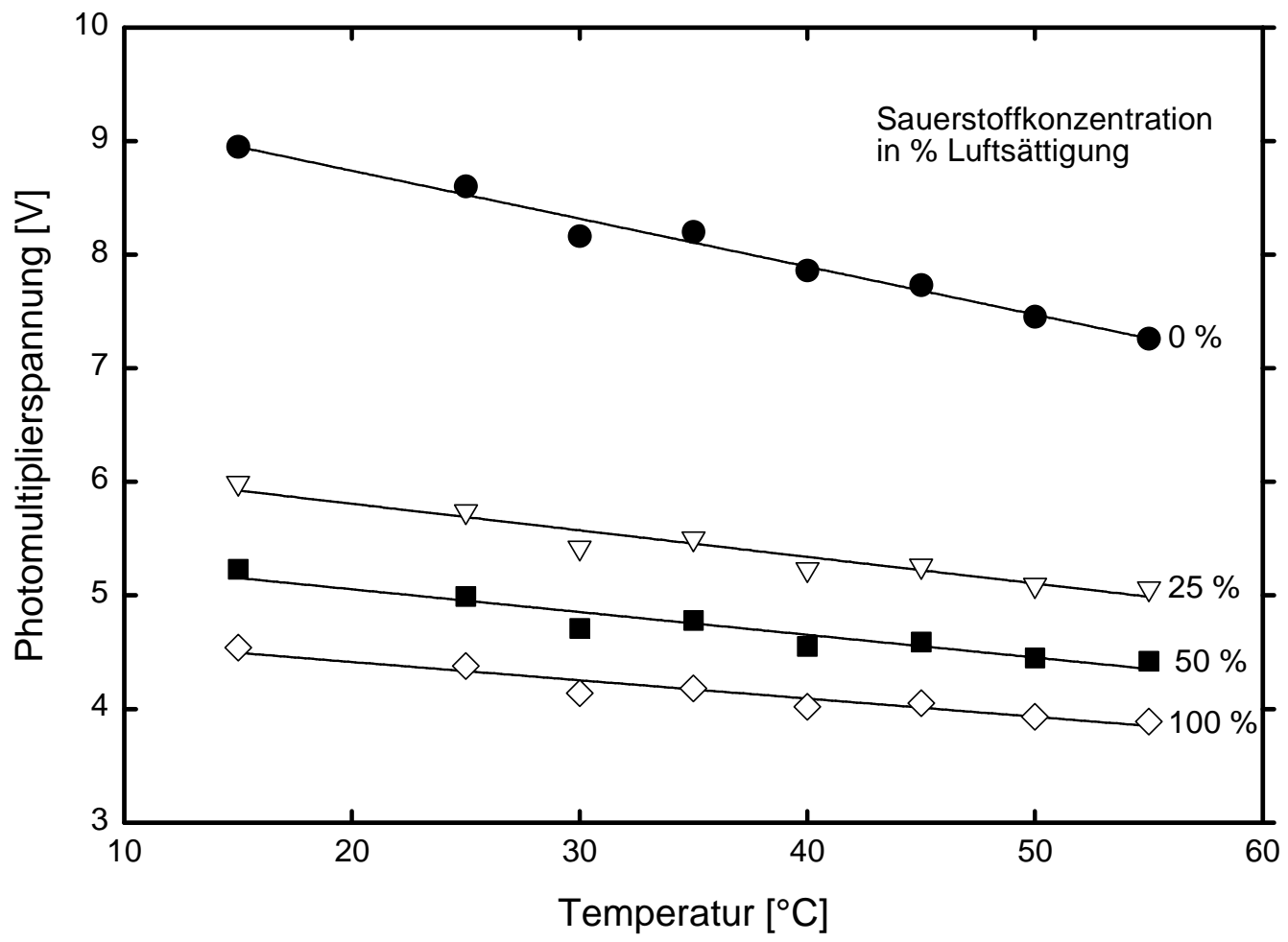

Abbildung 3-6: Einfluss der Temperatur auf die Fluoreszenzintensität von RuDPP in Ethanol

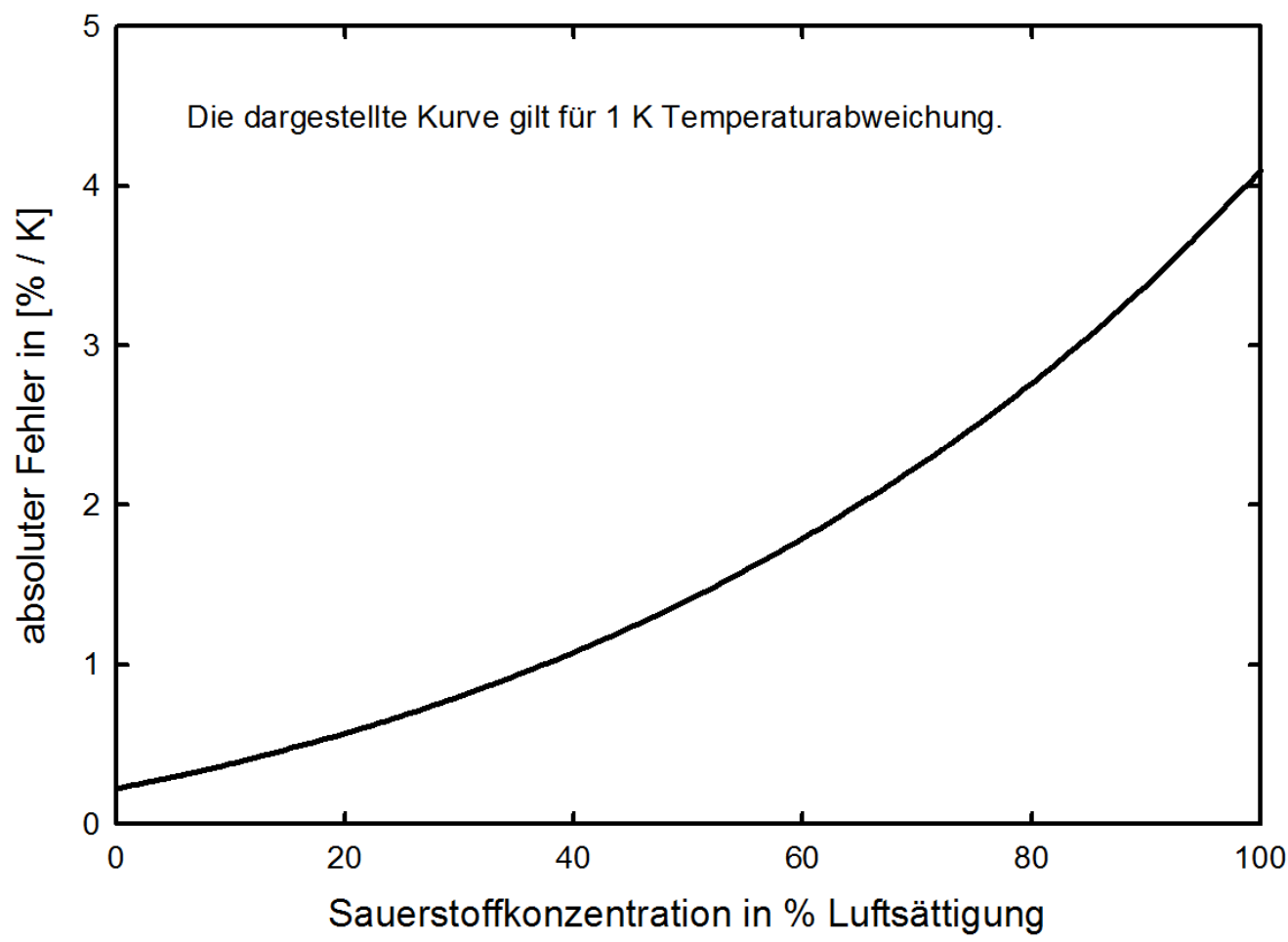

Abbildung 3-7: Durch Temperaturabweichung bedingter Fehler der gemessenen Sauerstoffkonzentration; gleiche Bedingungen wie in Abbildung 3-6 


\subsubsection{Differenzdrucksensoren}

An der Blasensäule I sind Differenzdrucksensoren der Firma BURSTER (Gernsbach; Deutschland) montiert. Der Messbereich ist $p=0-+/-200$ mbar. Der Messfehler beträgt nach Herstellerangaben $0,5 \%$. Dies entspricht einem absoluten Fehler von $\Delta p=+/-1$ mbar.

An der Blasensäule II sind Differenzdrucksensoren der Firma LABOM Mess- und Regeltechnik GmbH (Hude; Deutschland) montiert. Zwei Sensoren haben einen Messbereich $p=0-1000$ mbar; zwei weitere Sensoren haben einen Messbereich vom $p=0-2500$ mbar. Der Messfehler beträgt nach Herstellerangaben 0,2\%. Dies entspricht einem absoluten Fehler von $\Delta \mathrm{p}=+/-2$ mbar bzw. $\Delta \mathrm{p}=+/-5$ mbar.

\subsubsection{Datenerfassung}

Die Datenerfassung erfolgt über die Analog-Digital-Wandler-Karte DAS-1701 ST-DA der Firma Keithley Instruments Inc. (Taunton; USA) mit einem PC und der graphischen Programmiersoftware LabView 4.0 der Firma National Instruments (Austin; Texas; USA).

\subsubsection{Chemikalien}

- Toluol, zur Synthese; Gehalt $>99 \%$

- Leitungswasser

- Ethanol, technisch: Gehalt ca. $96 \%$

- 1-Butanol zur Synthese; Gehalt > 99\%

- Ethylenglycol zur Synthese; Gehalt $>99 \%$

- Decalin zur Synthese, Isomerengemisch, Isomerengehalt $>99 \%$

- Tris-(4,7diphenyl,1,10-phenanthrolin)-Ruthenium(II)chlorid, (RuDPP); Hersteller: COMTE (Hannover; Deutschland)

- Tris-(2,2’bipyridyl)-Ruthenium(II)chlorid, (RuBPY); Fluka Nr.: 93307

- 2,3,7,8,12,13,17,18-Octaethyl-21H,23H-porphin, (OEP); Fluka Nr: 74714

- Stickstoff; technisch, Qualität: 2.3

- Helium; Qualität: 4.5

- Druckluft; technisch, trocken

- Sauerstoff; Qualität: 5.0 


\subsection{Meßmethoden}

\subsubsection{Entgasungsmessungen (Dynamic Gas Disengagement; DGD)}

Die Entgasungsmessungen liefern Informationen über den Gasgehalt und die Gasstruktur. Es werden die Zeit, der Schaltzustand der elektropneumatischen Ventile, die Daten der Differenzdrucksensoren und der Gesamtdruck aufgezeichnet. Die Datenerfassung erfolgt zehnmal in der Sekunde.

Für die Messung wird die Blasensäule in den gewünschten stationären Zustand gebracht. Die Datenerfassung wird gestartet. Nach einem ausreichenden Vorlauf von mind. $10 \mathrm{~s}$ werden die Ventile an Gaseinlass und Gasauslass gleichzeitig geschlossen. Die Gasblasen entweichen aus der Flüssigkeit. Nach Erreichen des gasfreien Zustandes wird noch ein ausreichender Nachlauf aufgezeichnet.

\begin{tabular}{|ccccccc|}
\hline Temperatur & Druck & Volumenstrom & Lösemittel & Gas & Begaser & \\
20 & 5 & 471,3 & 1-Butanol & N2 & $19 \times 1 \mathrm{~mm}$ & \\
Zeit & Abschalt & Sensor IV & Sensor III & Sensor II & Sensor I & Druck \\
0,436 & 0 & 10,449 & 37,808 & 67,265 & 100,333 & 5,031 \\
0,563 & 0 & 11,274 & 38,878 & 67,624 & 102,372 & 5,033 \\
0,66 & 0 & 9,336 & 38,661 & 68,773 & 102,631 & 5,034 \\
0,76 & 0 & 8,51 & 39,6 & 67,373 & 102,53 & 5,036 \\
0,866 & 0 & 9,551 & 36,608 & 67,804 & 102,559 & 5,035 \\
0,963 & 0 & 8,582 & 37,504 & 69,492 & 102,516 & 5,035 \\
1,059 & 0 & 10,09 & 38,935 & 69,851 & 102,631 & 5,035 \\
1,179 & 0 & 9,084 & 41,003 & 68,522 & 103,004 & 5,034 \\
1,276 & 0 & 10,305 & 38,834 & 69,923 & 102,789 & 5,034 \\
$:$ & $:$ & $:$ & $:$ & $:$ & $:$ & $:$ \\
\hline
\end{tabular}

Abbildung 3-8: Beispiel für die Messdaten einer Entgasungsmessung

\subsubsection{Stoffübergangsmessungen}

$\mathrm{Zu}$ Beginn jeder Messreihe ist eine Kalibrierung des MOPS nötig.

Für die Stoffübergangsmessungen werden die Photomultiplierspannung des MOPS, die Zeit, der Druck und der Gesamtgasgehalt aufgezeichnet. Die Datenerfassung erfolgt je nach System mit einem Zeitabstand von $0,5 \mathrm{~s}$ bis $5 \mathrm{~s}$.

Für die Messung wird die Blasensäule in den gewünschten stationären Zustand gebracht. Dann wird Sauerstoff in den Gasstrom dosiert. Der Inertgasstrom wird dabei so reduziert, dass sich der Gesamtgasstrom nicht ändert. Da der Massendurchflussregler zur Dosierung des Sauerstoffs maximal $5 \mathrm{Nl} / \mathrm{min} \mathrm{O}_{2}$ liefert, sind die maximal erreichbaren Sauerstoffkonzentrationen in der Flüssigkeit begrenzt (siehe Tabelle 3-4).

Tabelle 3-4: maximal erreichbare Sauerstoffkonzentration in der Flüssigkeit

\begin{tabular}{|c|c|c|c|c|c|}
\hline $\mathrm{u}_{\mathrm{G}}[\mathrm{cm} / \mathrm{s}]$ & 1 & 2 & 5 & 10 & 20 \\
\hline $\begin{array}{c}\text { erreichbare Sauerstoffkonzentration in } \\
\text { \% Luftsättigung }\end{array}$ & $>100 \%$ & $>100 \%$ & $\sim 100 \%$ & $53 \%$ & $26 \%$ \\
\hline
\end{tabular}

Ist die gewünschte Sauerstoffkonzentration erreicht, wird der Sauerstoffstrom abgeschaltet, der Inertgasstrom im gleichen Maße wieder erhöht und die Messdatenerfassung gestartet. 
Die Dauer der Messung sollte im Interesse der Auswertung möglichst lang sein. Als Faustregel für die Mindestdauer der Messung gilt entweder die vierfache Halbwertszeit des Sauerstoffs in der Flüssigkeit oder Messung bis zu einem Zehntel der Ausgangskonzentration.

$$
\mathrm{t}_{0,5}=\frac{\ln (2)}{\mathrm{k}_{\mathrm{L}} \mathrm{a}^{\prime}}
$$

\begin{tabular}{|cccc|}
\hline Konst. & SVK & U0 & U100 \\
0,933 & 7,207 & 8,61 & 4,422 \\
& & & \\
Flüssigkeit:1-Butanol mit Helium & & \\
Temperatur:20,00 C & & & \\
Volumenstrom:133,701/min & & Druck & Gasgehalt \\
Spannung MOPS & Zeit & 1,975 & 0,213 \\
5,132 & 0,201 & 1,982 & 0,181 \\
5,136 & 0,801 & 1,989 & 0,206 \\
5,117 & 1,4 & 1,992 & 0,227 \\
5,128 & 2 & 1,995 & 0,224 \\
5,125 & 2,6 & 1,996 & 0,205 \\
5,154 & 3,199 & 1,992 & 0,215 \\
5,185 & 3,801 & 1,991 & 0,195 \\
5,232 & 4,399 & $:$ & $:$ \\
$:$ & $:$ & & \\
\hline
\end{tabular}

Abbildung 3-9: Beispiel für die Messdaten einer Desorptionsmessung

\subsubsection{Viskositätsmessung}

Die Viskositäten der Flüssigkeiten wurden mit einem Kapillar-Viskosimeter gemessen. Die Messung erfolgte mit dem automatischen Viskositäts-Messsystem AVS/G der Firma SchottGeräte (Deutschland; Hofheim).

\subsubsection{Messung der Oberflächenspannung}

Die Oberflächenspannung der Flüssigkeiten wurde mit der Ring-Methode in dem ProcessorTensiometer K12 der Firma Krüss gemessen. 


\section{Auswertung der Messungen}

\subsection{Auswertung der Entgasungsmessungen}

Es gibt zwei Möglichkeiten, den Entgasungsvorgang zu verfolgen. Zum einen durch Messung der Dispersionshöhe, z.B. durch eine Videokamera oder einen Schwimmer. Zum anderen durch Messung des hydrostatischen Druckes, z. B. durch Differenzdrucksensoren.

Zunächst wird die Messung der Dispersionshöhe besprochen, da sie in der Literatur weiter verbreitet ist. Danach wird die Messung des hydrostatischen Druckes diskutiert, da sie bei den experimentellen Arbeiten zur Anwendung kommt.

Die hier dargestellten Gedanken und Ergebnisse wurden bereits publiziert (JORDAN ET.AL. 2003). Da sie im Rahmen dieser Arbeit entstanden sind, sollen sie noch einmal dargelegt werden.

\subsubsection{Entgasungsmessungen mit der Dispersionshöhe}

In Abbildung 4-1 sind die Vorgänge in der Blasensäule während der Entgasungsmessung skizziert. In Abbildung 4-2 ist die dazugehörige (idealisierte) Messkurve aufgetragen. Zum Zeitpunkt $\mathrm{t}=0$ befindet sich die Blasensäule im kontinuierlichen Betrieb. Die Dispersionshöhe hat ihren maximalen Wert von $\mathrm{H}_{0}$. Nach dem Abschalten der Gaszufuhr entweichen die Blasen aus der Flüssigkeit, ohne dass neue Blasen vom Begaser in die Flüssigkeit eingebracht werden. Dadurch sinkt die Dispersionshöhe. Zum Zeitpunkt $\mathrm{t}_{2}$ hat die letzte Großblase die Flüssigkeit verlassen. In diesem Moment beträgt die Dispersionshöhe $\mathrm{H}_{1}$. Nun entweichen nur noch die langsamen Kleinblasen. Die Dispersionshöhe sinkt jetzt langsamer. Zum Zeitpunkt $\mathrm{t}_{4}$ hat auch die letzte Kleinblase die Flüssigkeit verlassen. Die Dispersionshöhe entspricht nun der Höhe der reinen Flüssigkeit $\mathrm{H}_{\mathrm{L}}$. Die Entgasung ist abgeschlossen. 

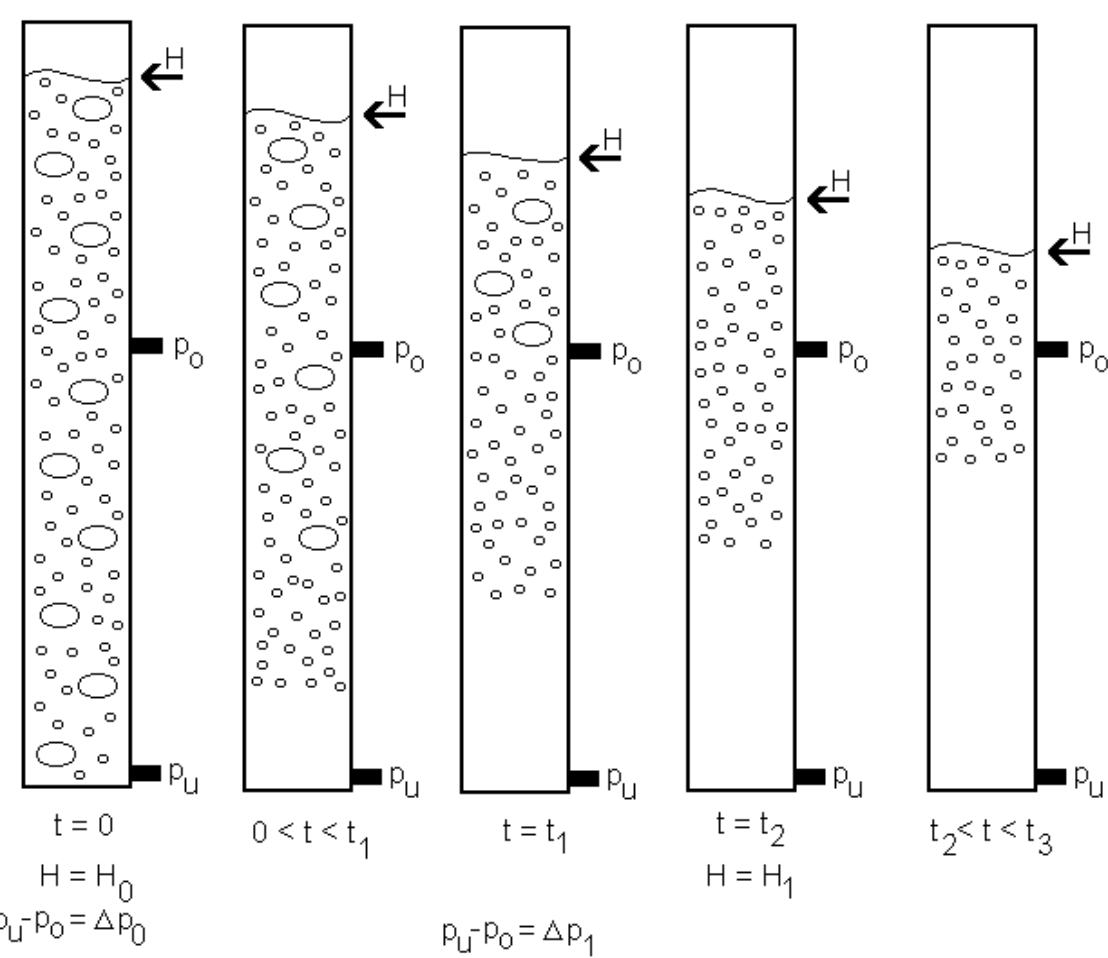
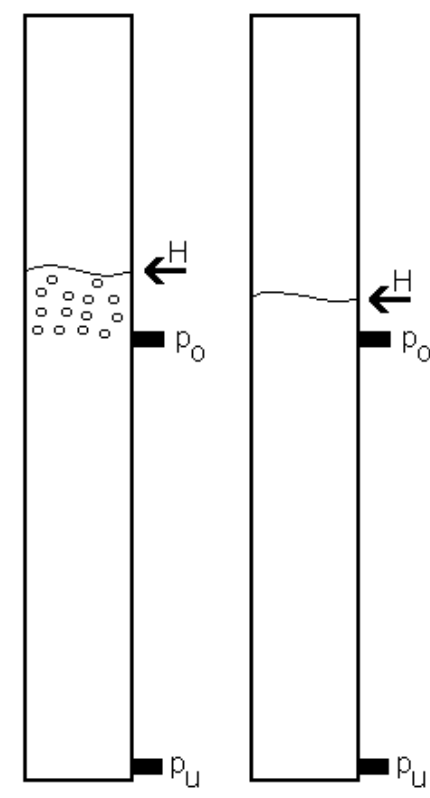

$\mathrm{t}=\mathrm{t}_{4}$ $\mathrm{H}=\mathrm{H}_{\mathrm{L}}$

Abbildung 4-1: Schema Entgasungsmessung

In Abbildung 4-2 sind noch einige zusätzliche Geraden eingetragen. Die Höhe $\mathrm{H}_{2}$ entspricht dem Schnittpunkt des verlängerten zweiten Geradenabschnittes mit der Senkrechten über dem Nullpunkt. Die Geschwindigkeiten $\mathrm{v}_{\mathrm{B}, \mathrm{gro}}$ und $\mathrm{v}_{\mathrm{B}, \mathrm{klein}}$ entsprechen den scheinbaren beobachteten Geschwindigkeiten der unteren Blasenfronten der jeweiligen Blasenklasse.

Der Gesamtgasgehalt ergibt sich aus der Messung:

$\varepsilon_{\mathrm{G}}=\frac{\mathrm{H}_{0}-\mathrm{H}_{\mathrm{L}}}{\mathrm{H}_{0}}$

Gleichung 4-1: Gasgehalt aus der Messung der Dispersionshöhe

Die Summe des Gasgehaltes der einzelnen Blasenklassen entspricht dem Gesamtgasgehalt:

$\varepsilon_{\mathrm{G}}=\varepsilon_{\mathrm{G}, \mathrm{Gro \beta}}+\varepsilon_{\mathrm{G}, \text { Klein }}$

Gleichung 4-2: Summe der Blasenklassengehalte

Ein Problem der Entgasungsmessungen ist, dass während des Großblasenentgasens auch Kleinblasen die Flüssigkeit verlassen. Die Abnahme der Dispersionshöhe von $\mathrm{H}_{0}$ auf $\mathrm{H}_{1}$ ist also auf das Entweichen der Großblasen und eines Teils der Kleinblasen zurückzuführen. Beim darauffolgenden Kleinblasenentgasen ist nur noch ein Teil der ursprünglich bei $t_{0}$ enthaltenen Kleinblasen beteiligt. Die Frage ist, wie viele Kleinblasen haben die Flüssigkeit parallel mit den Großblasen verlassen? 


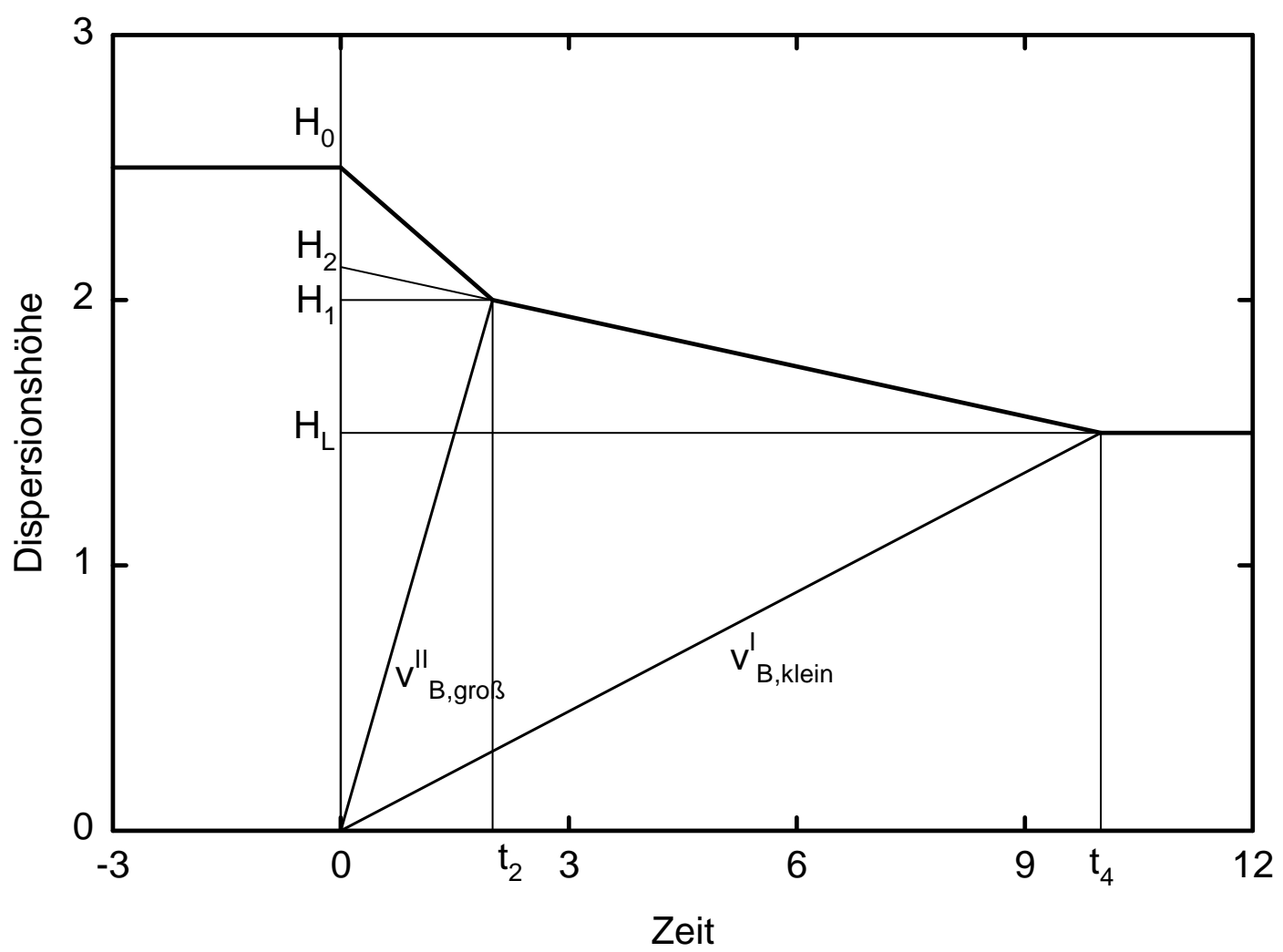

Abbildung 4-2: Schema Entgasungskurve bei Messung der Dispersionshöhe

Zur Lösung des Problems wird postuliert, dass die Relativgeschwindigkeit zwischen Blase und Flüssigkeit konstant ist. Dabei spielt es keine Rolle, ob sich die betrachtete Blase im homogenen oder heterogenen Bereich aufhält. Als zweite Voraussetzung wird angenommen, dass der Volumenstrom der hinab strömenden Flüssigkeit gleich dem Volumenstrom der aufsteigenden Gasblasen ist.

Die beobachtete (scheinbare) Großblasengeschwindigkeit $\mathrm{v}_{\mathrm{B}, \mathrm{II} \text { (I)ß }}$ erhält man aus der schematischen Entgasungskurve folgendermaßen:

$\mathrm{v}_{\mathrm{B}, \mathrm{Gro \beta}}^{\mathrm{II}}=\frac{\mathrm{H}_{1}}{\mathrm{t}_{2}}$

Gleichung 4-3: Beobachtete Großblasengeschwindigkeit

Die scheinbare Kleinblasengeschwindigkeit $\mathrm{v}_{\text {B,Klein }}^{\mathrm{I}}$ erhält man aus:

$\mathrm{v}_{\mathrm{B}, \text { Klein }}^{\mathrm{I}}=\frac{\mathrm{H}_{\mathrm{L}}}{\mathrm{t}_{4}}$

Gleichung 4-4: Beobachtete Kleinblasengeschwindigkeit im homogenen Bereich 


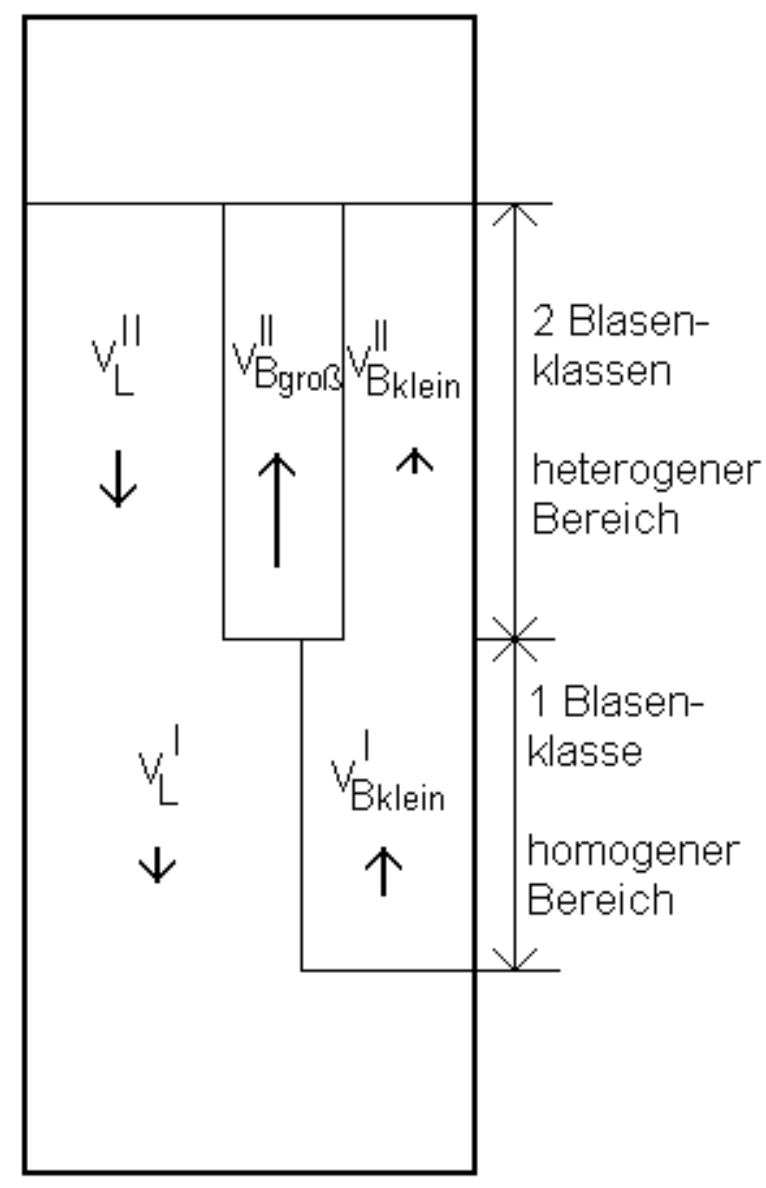

Abbildung 4-3: Schematische Darstellung der heterogenen Entgasung

Diese Geschwindigkeiten sind nur scheinbare Geschwindigkeiten, da die abströmende Flüssigkeit die ursprüngliche Aufwärtsbewegung der Blasen abbremst. Für die Berechnung der Relativgeschwindigkeiten müssen noch die jeweiligen Flüssigkeitsgeschwindigkeiten bekannt sein. Die Flüssigkeitsgeschwindigkeiten $\mathrm{v}_{\mathrm{L}}^{\mathrm{II}}$ und $\mathrm{v}_{\mathrm{L}}^{\mathrm{I}}$ ergeben sich aus der Sinkgeschwindigkeit des Flüssigkeitsspiegels:

$\mathrm{v}_{\mathrm{L}}^{\mathrm{I}}=\frac{\mathrm{H}_{1}-\mathrm{H}_{\mathrm{L}}}{\mathrm{t}_{4}-\mathrm{t}_{2}}=\frac{\mathrm{H}_{2}-\mathrm{H}_{\mathrm{L}}}{\mathrm{t}_{4}}$

Gleichung 4-5: Flüssigkeitsgeschwindigkeit im homogenen Bereich

$\mathrm{v}_{\mathrm{L}}^{\mathrm{II}}=\frac{\mathrm{H}_{0}-\mathrm{H}_{1}}{\mathrm{t}_{2}}$

Gleichung 4-6: Flüssigkeitsgeschwindigkeit im heterogenen Bereich

Aus der Phase des Kleinblasenentgasens kann nun die Relativgeschwindigkeit der Kleinblasen zur Flüssigkeit bestimmt werden: 


$$
\begin{aligned}
& \mathrm{v}_{\mathrm{B}, \text { Klein }}=\mathrm{v}_{\mathrm{B}, \text { Klein }}^{\mathrm{I}}+\mathrm{v}_{\mathrm{L}}^{\mathrm{I}} \\
& \mathrm{v}_{\mathrm{B}, \text { Klein }}=\frac{\mathrm{H}_{2}}{\mathrm{t}_{4}}
\end{aligned}
$$

Gleichung 4-7: Kleinblasengeschwindigkeit aus der Entgasungsmessung

$\mathrm{Zu}$ diesem Ergebnis gelangt man auch, wenn eine Volumenstrombilanz gemacht wird. Der nach oben strömende Gasvolumenstrom entspricht dem nach unten strömenden Flüssigkeitsvolumenstrom:

$$
\begin{aligned}
\dot{\mathrm{V}}_{\mathrm{L}} & =\dot{\mathrm{V}}_{\mathrm{G}} \\
\mathrm{v}_{\mathrm{L}}^{\mathrm{I}} \cdot\left(1-\varepsilon_{\mathrm{df}}\right) & =\mathrm{v}_{\mathrm{B}, \text { Klein }}^{\mathrm{I}} \cdot \varepsilon_{\mathrm{df}} \\
\mathrm{v}_{\mathrm{B}, \text { Klein }} & =\mathrm{v}_{\mathrm{B}, \text { Klein }}^{\mathrm{I}}+\mathrm{v}_{\mathrm{L}}^{\mathrm{I}} \\
\mathrm{v}_{\mathrm{B}, \text { Klein }} & =\mathrm{v}_{\mathrm{B}, \text { Klein }}^{\mathrm{I}} \cdot\left(1+\frac{\varepsilon_{\mathrm{df}}}{1-\varepsilon_{\mathrm{df}}}\right) \\
\mathrm{v}_{\mathrm{B}, \text { Klein }} & =\mathrm{v}_{\mathrm{B}, \text { Klein }}^{\mathrm{I}} \cdot \frac{\mathrm{H}_{2}}{\mathrm{H}_{\mathrm{L}}} \\
\mathrm{v}_{\mathrm{B}, \text { Klein }} & =\frac{\mathrm{H}_{2}}{\mathrm{t}_{4}}
\end{aligned}
$$

Gleichung 4-8: Kleinblasengeschwindigkeit aus der Entgasungsmessung über die Volumenstrombilanz hergeleitet

Der Term $\varepsilon_{\mathrm{df}}$ ist der „dense phase hold-up“ und entspricht dem Kleinblasengehalt im homogenen Bereich, in dem keine Großblasen vorliegen. Dieser Begriff stammt aus der Charakterisierung von fluidisierten Festbetten (ELLENBERGER 1994). Der „,dense phase holdup“ ist größer als der Kleinblasengehalt im heterogenen Bereich.

$\varepsilon_{\mathrm{df}}=\frac{\mathrm{H}_{2}-\mathrm{H}_{\mathrm{L}}}{\mathrm{H}_{2}}>\varepsilon_{\mathrm{G}, \text { Klein }}$

Gleichung 4-9: dense phase holdup

Die Relativgeschwindigkeit der Großblasen kann ebenfalls als Summe der Flüssigkeitsgeschwindigkeit und der scheinbaren Großblasengeschwindigkeit berechnet werden:

$$
\mathrm{v}_{\mathrm{B}, \mathrm{Gro \beta}}=\mathrm{v}_{\mathrm{B}, \mathrm{Gro \beta}}^{\mathrm{II}}+\mathrm{v}_{\mathrm{L}}^{\mathrm{II}}=\frac{\mathrm{H}_{0}}{\mathrm{t}_{2}}
$$

Gleichung 4-10: Großblasengeschwindigkeit aus der Entgasungsmessung

Zur Ermittlung der Blasenklassengehalte wird nun eine Volumenstrombilanz für die heterogene Entgasung erstellt. 


$$
\begin{aligned}
\dot{\mathrm{V}}_{\mathrm{L}} & =\dot{\mathrm{V}}_{\mathrm{G}} \\
\mathrm{v}_{\mathrm{L}}^{\mathrm{II}} \cdot\left(1-\varepsilon_{\mathrm{G}}\right) & =\mathrm{v}_{\mathrm{B}, \mathrm{Gro \beta}} \cdot \varepsilon_{\mathrm{G}, \mathrm{Gro \beta}}+\left(\mathrm{v}_{\mathrm{B}, \text { Klein }}-\mathrm{v}_{\mathrm{L}}^{\mathrm{II}}\right) \cdot \varepsilon_{\mathrm{G}, \text { Klein }}
\end{aligned}
$$

Gleichung 4-11: Volumenstrombilanz für heterogene Blasenströmung

Diese Gleichung wird nach $\varepsilon_{\mathrm{G}, \mathrm{Gro}}$ umgestellt und aufgelöst. So erhält man den Großblasengehalt:

$$
\begin{aligned}
\mathrm{v}_{\mathrm{L}}^{\mathrm{II}} \cdot\left(1-\varepsilon_{\mathrm{G}}\right) & =\mathrm{v}_{\mathrm{B}, \mathrm{Gro \beta}}^{\mathrm{II}} \cdot \varepsilon_{\mathrm{G}, \mathrm{Gro \beta}}+\left(\mathrm{v}_{\mathrm{B}, \text { Klein }}-\mathrm{v}_{\mathrm{L}}^{\mathrm{II}}\right) \cdot \varepsilon_{\mathrm{G}, \text { Klein }} \\
\mathrm{v}_{\mathrm{L}}^{\mathrm{II}} \cdot\left(1-\varepsilon_{\mathrm{G}}\right) & =\mathrm{v}_{\mathrm{B}, \mathrm{Gro} \beta}^{\mathrm{II}} \cdot \varepsilon_{\mathrm{G}, \mathrm{Gro \beta}}+\left(\mathrm{v}_{\mathrm{B}, \text { Klein }}-\mathrm{v}_{\mathrm{L}}^{\mathrm{II}}\right) \cdot\left(\varepsilon_{\mathrm{G}}-\varepsilon_{\mathrm{G}, \mathrm{Gro \beta}}\right) \\
\varepsilon_{\mathrm{G}, \mathrm{Gro \beta}} & =\frac{\mathrm{v}_{\mathrm{L}}^{\mathrm{II}} \cdot\left(1-\varepsilon_{\mathrm{G}}\right)-\varepsilon_{\mathrm{G}} \cdot\left(\mathrm{v}_{\mathrm{B}, \text { Klein }}-\mathrm{v}_{\mathrm{L}}^{\mathrm{II}}\right)}{\mathrm{v}_{\mathrm{B}, \mathrm{Gro \beta}}^{\mathrm{II}}-\mathrm{v}_{\mathrm{B}, \text { Klein }}+\mathrm{v}_{\mathrm{L}}^{\mathrm{II}}} \\
\varepsilon_{\mathrm{G}, \mathrm{Gro \beta}}= & \frac{\frac{\mathrm{H}_{0}-\mathrm{H}_{1}}{\mathrm{t}_{2}}-\frac{\mathrm{H}_{0}-\mathrm{H}_{\mathrm{L}}}{\mathrm{H}_{0}} \cdot \frac{\mathrm{H}_{2}}{\mathrm{t}_{4}}}{\frac{\mathrm{H}_{0}}{\mathrm{t}_{2}}-\frac{\mathrm{H}_{2}}{\mathrm{t}_{4}}}
\end{aligned}
$$

Gleichung 4-12: Großblasengehalt aus Volumenstrombilanz

Analog kann für den Kleinblasengehalt vorgegangen werden.

$$
\begin{aligned}
v_{\mathrm{L}}^{\mathrm{II}} \cdot\left(1-\varepsilon_{\mathrm{G}}\right)= & \mathrm{v}_{\mathrm{B}, \mathrm{Gro \beta}}^{\mathrm{II}} \cdot \varepsilon_{\mathrm{G}, \text { Groß }}+\left(\mathrm{v}_{\mathrm{B}, \text { Klein }}-\mathrm{v}_{\mathrm{L}}^{\mathrm{II}}\right) \cdot \varepsilon_{\mathrm{G}, \text { Klein }} \\
\mathrm{v}_{\mathrm{L}}^{\mathrm{II}} \cdot\left(1-\varepsilon_{\mathrm{G}}\right)= & \mathrm{v}_{\mathrm{B}, \mathrm{Gro \beta}}^{\mathrm{II}} \cdot\left(1-\varepsilon_{\mathrm{G}, \text { Klein }}\right)+\left(\mathrm{v}_{\mathrm{B}, \text { Klein }}-\mathrm{v}_{\mathrm{L}}^{\mathrm{II}}\right) \cdot \varepsilon_{\mathrm{G}, \text { Klein }} \\
\varepsilon_{\mathrm{G}, \text { Klein }}= & \frac{\mathrm{v}_{\mathrm{L}}^{\mathrm{II}} \cdot\left(1-\varepsilon_{\mathrm{G}}\right)-\mathrm{v}_{\mathrm{B}, \mathrm{Gro \beta}}^{\mathrm{II}} \cdot \varepsilon_{\mathrm{G}}}{\mathrm{v}_{\mathrm{B}, \text { Klein }}-\mathrm{v}_{\mathrm{L}}^{\mathrm{II}}-\mathrm{v}_{\mathrm{B}, \mathrm{Gro \beta}}^{\mathrm{II}}} \\
\varepsilon_{\mathrm{G}, \text { Klein }}= & \frac{\frac{\mathrm{H}_{1}-\mathrm{H}_{\mathrm{L}}}{\mathrm{t}_{2}}}{\frac{\mathrm{H}_{0}}{\mathrm{t}_{2}}-\frac{\mathrm{H}_{2}}{\mathrm{t}_{4}}}
\end{aligned}
$$

Gleichung 4-13: Kleinblasengehalt aus Volumenstrombilanz

Die experimentellen Schwierigkeiten bei der Auswertung der Entgasungsmessungen sind die starken Fluktuationen während des Großblasenentgasens. Im Normalfall lässt sich der erste Teil der Entgasung nicht durch eine Gerade annähern. Es lässt sich aber zeigen, dass die Flüssigkeitsgeschwindigkeit zum Beginn der Entgasung der Gasleerrohrgeschwindigkeit $\mathrm{u}_{\mathrm{G}}$ entspricht: 


$$
\begin{aligned}
& \mathrm{u}_{\mathrm{G}}=\mathrm{v}_{\mathrm{B}, \mathrm{Gro \beta}} \cdot \varepsilon_{\mathrm{G}, \mathrm{Gro \beta}}+\mathrm{v}_{\mathrm{B}, \text { Klein }} \cdot \varepsilon_{\mathrm{G}, \text { Klein }} \\
& \mathrm{u}_{\mathrm{G}}=\frac{\mathrm{H}_{0}}{\mathrm{t}_{2}} \cdot \frac{\frac{\mathrm{H}_{0}-\mathrm{H}_{1}}{\mathrm{t}_{2}}-\frac{\mathrm{H}_{0}-\mathrm{H}_{\mathrm{L}}}{\mathrm{H}_{0}} \cdot \frac{\mathrm{H}_{2}}{\mathrm{t}_{4}}}{\frac{\mathrm{H}_{0}}{\mathrm{t}_{2}}-\frac{\mathrm{H}_{2}}{\mathrm{t}_{4}}}+\frac{\mathrm{H}_{2}}{\mathrm{t}_{4}} \cdot \frac{\frac{\mathrm{H}_{1}-\mathrm{H}_{\mathrm{L}}}{\frac{\mathrm{t}_{2}}{\mathrm{H}_{0}}-\frac{\mathrm{H}_{2}}{\mathrm{t}_{4}}}}{\mathrm{u}_{\mathrm{G}}}=\frac{\mathrm{H}_{0}-\mathrm{H}_{1}}{\mathrm{t}_{2}} \\
& \mathrm{u}_{\mathrm{G}}=\mathrm{v}_{\mathrm{L}}^{\mathrm{II}}
\end{aligned}
$$

Gleichung 4-14: Flüssigkeitsgeschwindigkeit als Funktion der Gasleerrohrgeschwindigkeit

Die Flüssigkeitsgeschwindigkeit $\mathrm{v}_{\mathrm{L}}^{\mathrm{II}}$ entspricht der Gasleerrohrgeschwindigkeit $\mathrm{u}_{\mathrm{G}}$. In diesem Punkt ist der Ansatz von SCHUMPE \& GRUND (1986) korrekt. Im Appendix 1 wird ausführlich gezeigt, dass die von SCHUMPE \& GRUND gefundene Lösung mit der hier beschriebenen Lösung übereinstimmt.

\subsubsection{Entgasungsmessungen mit dem Differenzdruck}

Eine elegantere Methode der Entgasungsmessung ist die Verfolgung des hydrostatischen Druckes zwischen zwei Messstellen. Diese Methode wurde in der Promotion von DewES (1996) angewendet. In Abbildung 4-4 ist eine schematisierte Messkurve dargestellt. Die Differenzdrücke sind die Druckunterschiede zwischen den Sensoren $\mathrm{p}_{u}$ und $\mathrm{p}_{\mathrm{o}}$ in Abbildung 4-1. Bis zum Zeitpunkt $t_{0}$ wird kontinuierlich begast. Der Differenzdruck bleibt bis zu diesem Zeitpunkt konstant bei $\Delta \mathrm{p}_{0}$. Bei $\mathrm{t}_{0}$ wird die Gaszufuhr gestoppt. Die Gasblasen entweichen aus dem Messvolumen. Bis zum Zeitpunkt $t_{1}$ erfolgt ein steiler Druckanstieg bis auf den Differenzdruck $\Delta \mathrm{p}_{1}$. In dieser Zeit entweichen die schnellen Großblasen. Danach erfolgt bis zum Zeitpunkt $\mathrm{t}_{3}$ ein langsamer Druckanstieg bis auf den Differenzdruck $\Delta \mathrm{p}_{\mathrm{E}}$. In dieser Zeit entweichen die langsamen Kleinblasen. Ab dem Zeitpunkt $t_{3}$ ist das Messvolumen blasenfrei. Der Differenzdruck $\Delta \mathrm{p}_{\mathrm{E}}$ entspricht dem hydrostatischen Druck der reinen Flüssigkeit. 


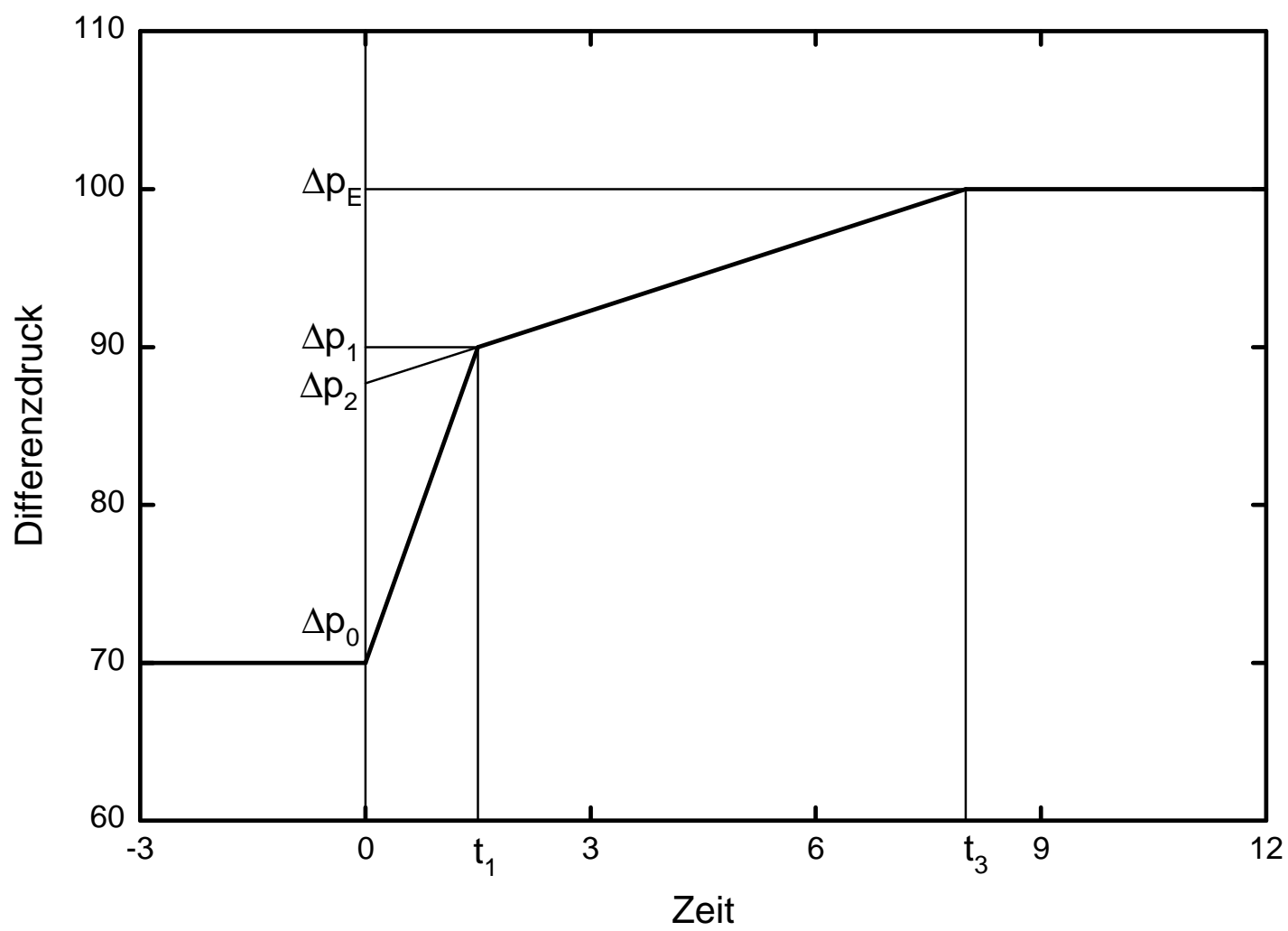

Abbildung 4-4: Schema Entgasungskurve bei Messung des hydrostatischen Druckes

Der Gesamtgasgehalt $\varepsilon_{\mathrm{G}}$ berechnet sich wie in Kapitel 2 beschrieben.

$\varepsilon_{\mathrm{G}}=\frac{\Delta \mathrm{p}_{\mathrm{E}}-\Delta \mathrm{p}_{0}}{\Delta \mathrm{p}_{\mathrm{E}}}$

Gleichung 4-15: Gesamtgasgehalt aus der Differenzdruckmessung

Entsprechend erhält man den „Dense Phase Hold-up“ für das Kleinblasenentgasen aus $\Delta \mathrm{p}_{\mathrm{E}}$ und $\Delta \mathrm{p}_{2}$ :

$\varepsilon_{\mathrm{df}}=\frac{\Delta \mathrm{p}_{\mathrm{E}}-\Delta \mathrm{p}_{2}}{\Delta \mathrm{p}_{\mathrm{E}}}$

Gleichung 4-16: „Dense Phase Hold-up“ aus der Differenzdruckmessung

Die scheinbaren Blasengeschwindigkeiten ermittelt man analog Gleichung 4-3 und Gleichung 4-4 aus den Aufstiegszeiten $\mathrm{t}_{1}$ und $\mathrm{t}_{3}$ und dem Abstand der Drucksensoren $\Delta \mathrm{h}$ :

$\mathrm{v}_{\mathrm{B}, \text { Klein }}^{\mathrm{I}}=\frac{\Delta \mathrm{h}}{\mathrm{t}_{3}}$

Gleichung 4-17: Beobachtete Kleinblasengeschwindigkeit aus Differenzdruckmessung 
$\mathrm{v}_{\mathrm{B}, \mathrm{Gro \beta}}^{\mathrm{II}}=\frac{\Delta \mathrm{h}}{\mathrm{t}_{1}}$

Gleichung 4-18: Beobachtete Großblasengeschwindigkeit aus Differenzdruckmessung

Die Flüssigkeitsgeschwindigkeiten für beide Entgasungsphasen erhält man aus der Steilheit der Druckanstiege. Der Druckanstieg im Messvolumen wird durch das Hineinströmen der Flüssigkeit verursacht. Dabei muss beachtet werden, dass der Flüssigkeit nicht der gesamte Blasensäulenquerschnitt zur Verfügung steht:

$$
\begin{aligned}
& \frac{\Delta \mathrm{p}_{\mathrm{E}}-\Delta \mathrm{p}_{2}}{\mathrm{t}_{3}}=\mathrm{v}_{\mathrm{L}}^{\mathrm{I}} \cdot\left(1-\varepsilon_{\mathrm{df}}\right) \cdot \rho_{\mathrm{L}} \cdot \mathrm{g} \\
& \mathrm{v}_{\mathrm{L}}^{\mathrm{I}}=\frac{\Delta \mathrm{p}_{\mathrm{E}}-\Delta \mathrm{p}_{2}}{\mathrm{t}_{3} \cdot\left(1-\varepsilon_{\mathrm{df}}\right)} \cdot \frac{\Delta \mathrm{h}}{\Delta \mathrm{p}_{\mathrm{E}}}
\end{aligned}
$$

Gleichung 4-19: Flüssigkeitsgeschwindigkeit in homogener Entgasungsphase aus

Differenzdruckmessung

$$
\begin{aligned}
& \frac{\Delta \mathrm{p}_{1}-\Delta \mathrm{p}_{0}}{\mathrm{t}_{1}}=\mathrm{v}_{\mathrm{L}}^{\mathrm{II}} \cdot\left(1-\varepsilon_{\mathrm{G}}\right) \cdot \rho_{\mathrm{L}} \cdot \mathrm{g} \\
& \mathrm{v}_{\mathrm{L}}^{\mathrm{II}}=\frac{\Delta \mathrm{p}_{1}-\Delta \mathrm{p}_{0}}{\mathrm{t}_{1} \cdot\left(1-\varepsilon_{\mathrm{G}}\right)} \cdot \frac{\Delta \mathrm{h}}{\Delta \mathrm{p}_{\mathrm{E}}}
\end{aligned}
$$

Gleichung 4-20: Flüssigkeitsgeschwindigkeit in heterogener Entgasungsphase aus

\section{Differenzdruckmessung}

Die Relativgeschwindigkeiten der Blasen, die den Aufstiegsgeschwindigkeiten während der Begasung entsprechen, berechnen sich analog Gleichung 4-7 und Gleichung 4-10. Die Gleichung lassen sich dabei vereinfachen:

$$
\begin{aligned}
\mathrm{v}_{\mathrm{B}, \text { Klein }} & =\mathrm{v}_{\mathrm{B}, \text { Klein }}^{\mathrm{I}}+\mathrm{v}_{\mathrm{L}}^{\mathrm{I}} \\
& =\frac{\Delta \mathrm{h}}{\mathrm{t}_{3}}+\frac{\Delta \mathrm{p}_{\mathrm{E}}-\Delta \mathrm{p}_{2}}{\mathrm{t}_{3} \cdot\left(1-\varepsilon_{\mathrm{df}}\right)} \cdot \frac{\Delta \mathrm{h}}{\Delta \mathrm{p}_{\mathrm{E}}} \\
& =\frac{\Delta \mathrm{h}}{\mathrm{t}_{3}} \cdot \frac{\Delta \mathrm{p}_{\mathrm{E}}}{\Delta \mathrm{p}_{2}}
\end{aligned}
$$

Gleichung 4-21: Kleinblasengeschwindigkeit aus Differenzdruckmessung

$$
\begin{aligned}
\mathrm{v}_{\mathrm{B}, \text { Groß }} & =\mathrm{v}_{\mathrm{B}, \mathrm{Gro \beta}}^{\mathrm{II}}+\mathrm{v}_{\mathrm{L}}^{\mathrm{II}} \\
& =\frac{\Delta \mathrm{h}}{\mathrm{t}_{1}}+\frac{\Delta \mathrm{p}_{1}-\Delta \mathrm{p}_{0}}{\mathrm{t}_{1} \cdot\left(1-\varepsilon_{\mathrm{G}}\right)} \cdot \frac{\Delta \mathrm{h}}{\Delta \mathrm{p}_{\mathrm{E}}} \\
& =\frac{\Delta \mathrm{h}}{\mathrm{t}_{1}} \cdot \frac{\Delta \mathrm{p}_{1}}{\Delta \mathrm{p}_{0}}
\end{aligned}
$$

Gleichung 4-22: Großblasengeschwindigkeit aus Differenzdruckmessung

Die Gehalte der einzelnen Blasenklassen werden wiederum aus Gleichung 4-11, der Volumenstrombilanz für heterogene Blasenströmung, hergeleitet. Es ergeben sich umfangreiche Gleichungen, die sich aber gut vereinfachen lassen: 
$\varepsilon_{\mathrm{G}, \mathrm{Gro \beta}}=\frac{\mathrm{v}_{\mathrm{L}}^{\mathrm{II}} \cdot\left(1-\varepsilon_{\mathrm{G}}\right)-\varepsilon_{\mathrm{G}} \cdot\left(\mathrm{v}_{\mathrm{B}, \text { Klein }}-\mathrm{v}_{\mathrm{L}}^{\mathrm{II}}\right)}{\mathrm{v}_{\mathrm{B}, \mathrm{Gro \beta}}^{\mathrm{II}}-\mathrm{v}_{\mathrm{B}, \text { Klein }}+\mathrm{v}_{\mathrm{L}}^{\mathrm{II}}}$

$\varepsilon_{\mathrm{G}, \mathrm{Gro \beta}}=\frac{\frac{\Delta \mathrm{h}}{\mathrm{t}_{1}} \cdot \frac{\Delta \mathrm{p}_{1}-\Delta \mathrm{p}_{0}}{\Delta \mathrm{p}_{0}}-\frac{\Delta \mathrm{h}}{\mathrm{t}_{3}} \cdot \frac{\Delta \mathrm{p}_{\mathrm{E}}-\Delta \mathrm{p}_{0}}{\Delta \mathrm{p}_{2}}}{\frac{\Delta \mathrm{h}}{\mathrm{t}_{1}} \cdot \frac{\Delta \mathrm{p}_{1}}{\Delta \mathrm{p}_{0}}-\frac{\Delta \mathrm{h}}{\mathrm{t}_{3}} \cdot \frac{\Delta \mathrm{p}_{\mathrm{E}}}{\Delta \mathrm{p}_{2}}}$

Gleichung 4-23: Großblasengehalt aus Differenzdruckmessungen

$\varepsilon_{\mathrm{G}, \text { Klein }}=\frac{\mathrm{v}_{\mathrm{L}}^{\mathrm{II}} \cdot\left(1-\varepsilon_{\mathrm{G}}\right)-\mathrm{v}_{\mathrm{B}, \mathrm{Gro} \beta}^{\mathrm{II}} \cdot \varepsilon_{\mathrm{G}}}{\mathrm{v}_{\mathrm{B}, \text { Klein }}-\mathrm{v}_{\mathrm{L}}^{\mathrm{II}}-\mathrm{v}_{\mathrm{B}, \mathrm{Gro} \beta}^{\mathrm{II}}}$

$\varepsilon_{\mathrm{G}, \text { Klein }}=\frac{\frac{\Delta \mathrm{h}}{\mathrm{t}_{1}} \cdot \frac{\Delta \mathrm{p}_{\mathrm{E}}-\Delta \mathrm{p}_{1}}{\Delta \mathrm{p}_{\mathrm{E}}}}{\frac{\Delta \mathrm{h}}{\mathrm{t}_{1}} \cdot \frac{\Delta \mathrm{p}_{1}}{\Delta \mathrm{p}_{0}}-\frac{\Delta \mathrm{h}}{\mathrm{t}_{3}} \cdot \frac{\Delta \mathrm{p}_{\mathrm{E}}}{\Delta \mathrm{p}_{2}}}$

Gleichung 4-24: Kleinblasengehalt aus Differenzdruckmessungen

Wie bei der Messung der Dispersionshöhe kann die erste steile Steigung der Entgasungskurve aus der eingestellten Gasleerrohrgeschwindigkeit hergeleitet werden. Die Flüssigkeitsgeschwindigkeit $v_{L}{ }_{L}$ ist gleich der Gasleerrohrgeschwindigkeit $u_{G}$. Die Gleichung 4-20 braucht nur umgestellt zu werden:

$$
\frac{\Delta \mathrm{p}_{1}-\Delta \mathrm{p}_{0}}{\mathrm{t}_{1}}=\mathrm{u}_{\mathrm{G}} \cdot\left(1-\varepsilon_{\mathrm{G}}\right) \cdot \frac{\Delta \mathrm{p}_{\mathrm{E}}}{\Delta \mathrm{h}}
$$

Gleichung 4-25: Anfangssteigung der Entgasungskurve

\subsubsection{Reale Messungen}

Die mathematische Behandlung der Entgasungsmessungen wurde in Kapitel 4.1.2 ausführlich behandelt. An dieser Stelle soll nur noch die Anpassung der theoretischen Messkurve an die gemessenen Daten beschrieben werden.

In Abbildung 4-5 und Abbildung 4-6 sind zwei typische Entgasungsmessungen dargestellt. Die Abbildung 4-5 zeigt die Entgasung des homogenen Betriebszustandes. Im stationären Betrieb bleibt der gemessene Differenzdruck konstant, es ist nur ein leichtes Rauschen zu erkennen. Nach dem Abschalten des Gasstromes erfolgt ein linearer Druckanstieg bis zum Differenzdruck der gasfreien Flüssigkeit.

Die Abbildung 4-6 zeigt eine Entgasungsmessung aus dem heterogenen Betriebszustand heraus. Im stationären Betrieb ist der gemessene Differenzdruck im Mittel konstant, durch die Großblasen wird jedoch ein starkes Rauschen verursacht. Nach dem Abschalten erfolgt ein schneller Druckanstieg. In dieser Zeit entweichen die Großblasen aus dem Messvolumen. Dieser Druckanstieg ist ebenfalls von einem starken Rauschen überlagert. Danach erfolgt ein langsamerer Druckanstieg bis zum Differenzdruck der gasfreien Flüssigkeit. Dieser Abschnitt ist weitgehend rauschfrei. Die Anfangssteigung der Entgasungskurve kann aus der 
eingestellten Leerrohrgeschwindigkeit und dem Gesamtgasgehalt ermittelt werden (siehe Gleichung 4-25).

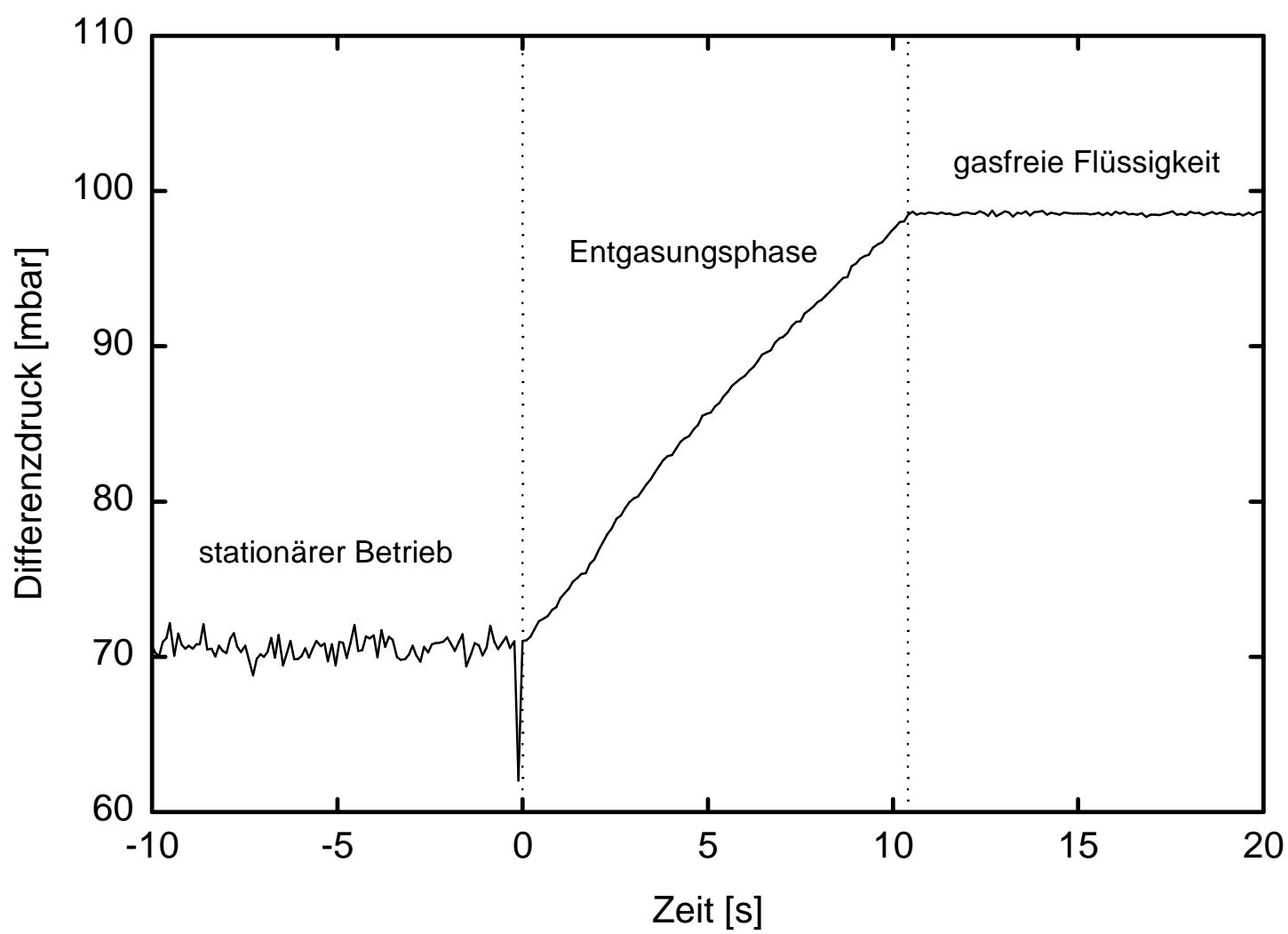

Abbildung 4-5: Typische Entgasungskurve im homogenen Strömungsregime

Im homogenen Fall ist der Druckanstieg in der Entgasungsphase durch die Randbedingungen vorgegeben. Die theoretische Kurve kann mit der gemessenen Kurve verglichen werden. Bei einer zu starken Abweichung des berechneten und des gemessenen Druckanstieges muss ein Fehler in der Messung oder Auswertung vorliegen.

Im heterogenen Fall ist der Druckanstieg während des Großblasenentgasens durch Gleichung 4-25 vorgegeben. So kann das starke Rauschen während des Großblasenentgasens umgangen werden. Der Druckanstieg während des Kleinblasenentgasens wird mit der Methode der kleinsten Fehlerquadrate angepasst. 


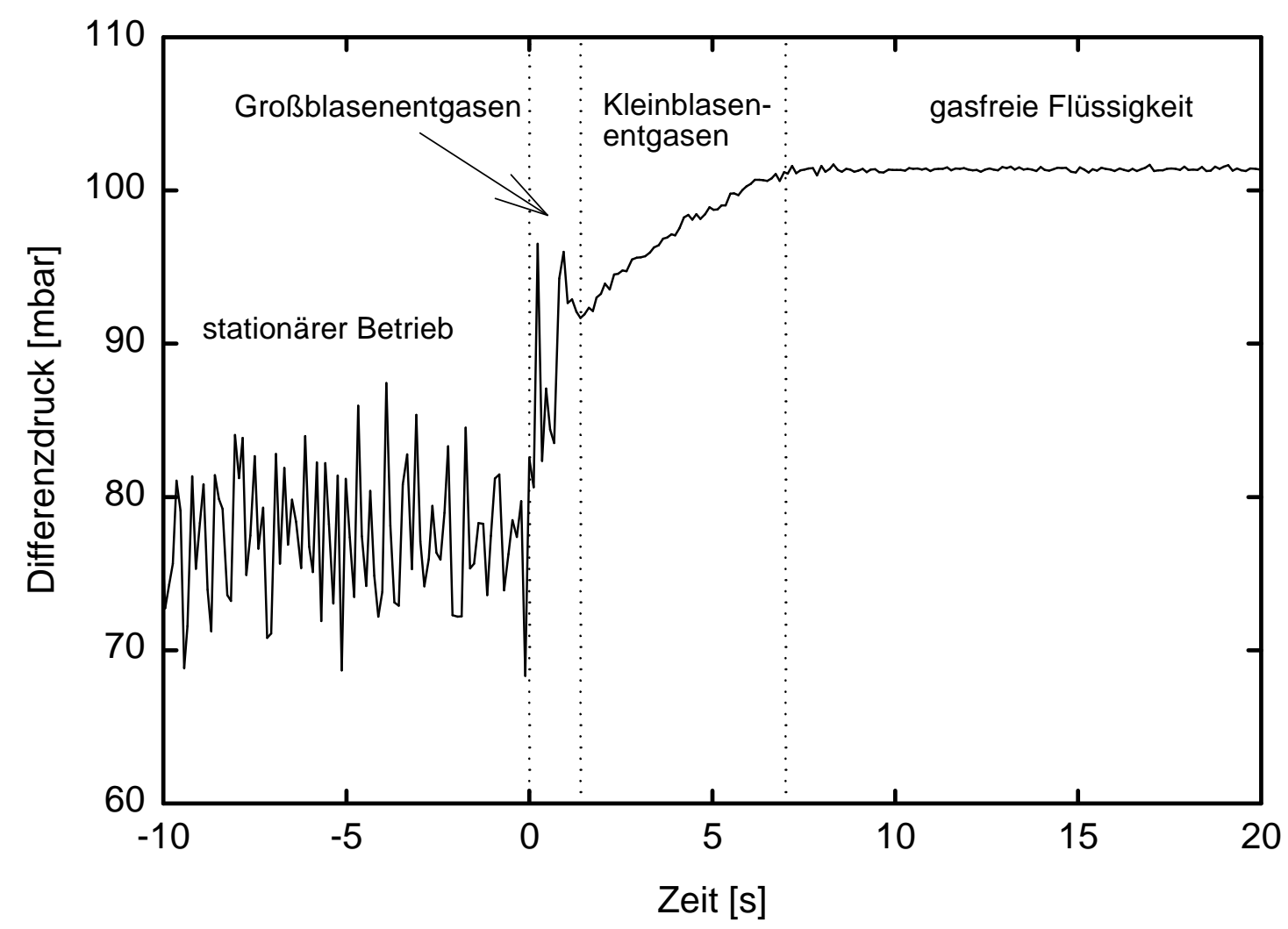

Abbildung 4-6: Typische Entgasungskurve im heterogenen Strömungsregime

\subsection{Auswertung der Stoffübergangsmessungen}

\subsubsection{Messung der Desorptionsrate}

Für die Stoffübergangsmessungen wurde in der Flüssigkeit gelöster Sauerstoff mit einem Inertgasstrom (Helium oder Stickstoff) ausgetrieben. Es handelt sich um physikalische Desorption. Es wird die Sauerstoffkonzentration in der Flüssigkeit gemessen. Für den Stoffstrom durch die Phasengrenzfläche gilt:

$\frac{d n_{O_{2}}}{d t}=k_{L} \cdot A \cdot\left(c_{O_{2}, L}^{*}-c_{O_{2}, L}\right)$

Gleichung 4-26: Stoffmengenstrom durch die Phasengrenzfläche A

Es wird davon ausgegangen, dass der gesamte Stofftransportwiderstand auf der Flüssigkeitsseite liegt. Die Konzentration $\mathrm{c}_{\mathrm{O}_{2}, \mathrm{~L}}^{*}$ ist die Sauerstoffkonzentration in der Flüssigkeit direkt an der Phasengrenzfläche und steht mit der Gasphasekonzentration im Gleichgewicht. Die Phasengrenzfläche A wird durch den Term $a \cdot \frac{V_{L}}{\left(1-\varepsilon_{G}\right)}$ ersetzt. Eine Division durch $\mathrm{V}_{\mathrm{L}}$ überführt den Stoffmengenstrom in die Konzentrationsänderung. 


$$
\begin{aligned}
\frac{d n_{O_{2}}}{d t} & =k_{L} a \cdot \frac{V_{L}}{\left(1-\varepsilon_{G}\right)} \cdot\left(c_{O_{2}, L}^{*}-c_{O_{2}, L}\right) \\
\frac{d c_{O_{2}, L}}{d t} & =\frac{k_{L} a}{\left(1-\varepsilon_{G}\right)} \cdot\left(L_{O_{2}} \cdot c_{O_{2}, G}-c_{O_{2}, L}\right)
\end{aligned}
$$

Gleichung 4-27: Sauerstoffabnahme durch physikalische Desorption

In der Gleichung 4-27 taucht der Gasgehalt $\varepsilon_{\mathrm{G}}$ auf. Zur einfacheren Behandlung wird folgende Ersetzung vorgenommen:

$$
\mathrm{k}_{\mathrm{L}} \mathrm{a}^{\prime}=\frac{\mathrm{k}_{\mathrm{L}} \mathrm{a}}{\left(1-\varepsilon_{\mathrm{G}}\right)}
$$

Gleichung 4-28: Flüssigkeitsbezogene Stoffübergangszahl

Der $\mathrm{k}_{\mathrm{L}} \mathrm{a}^{\prime}-$ Wert ist die flüssigkeitsbezogene Stoffübergangszahl.

Da während der Desorption mit sauerstofffreiem Inertgas begast wird, wird in erster Näherung angenommen, dass die Gasphasekonzentration des Sauerstoffs $c_{O 2, G}=0$ ist. Die Variablen in Gleichung 4-27 können nun getrennt und unbestimmt integriert werden:

$$
\ln \left(c_{O_{2}, L}\right)=-k_{L} a^{\prime} \cdot t+\text { const }
$$

Gleichung 4-29: Bestimmung von $\mathrm{k}_{\mathrm{L}^{\prime}} \mathrm{a}^{\prime}$ aus der Messung

Der gesuchte $\mathrm{k}_{\mathrm{L}} \mathrm{a}^{\prime}$-Wert lässt sich aus der Messung leicht ermitteln, indem man den Logarithmus der Konzentration über der Zeit aufträgt. Es ergibt sich eine Gerade, die negative Steigung der Gerade entspricht dem $\mathrm{k}_{\mathrm{L}} \mathrm{a}^{\prime}$-Wert. (Abbildung 4-8).

Bei den Messungen kommt es manchmal vor, dass die Glasfaser von einer Blase berührt wird. In diesem Fall kann der MOPS keine Fluoreszenzintensität feststellen und es erscheint ein Ausreißer zu hohen Sauerstoffkonzentrationen. Solche Werte werden vor der eigentlichen Auswertung eliminiert. 


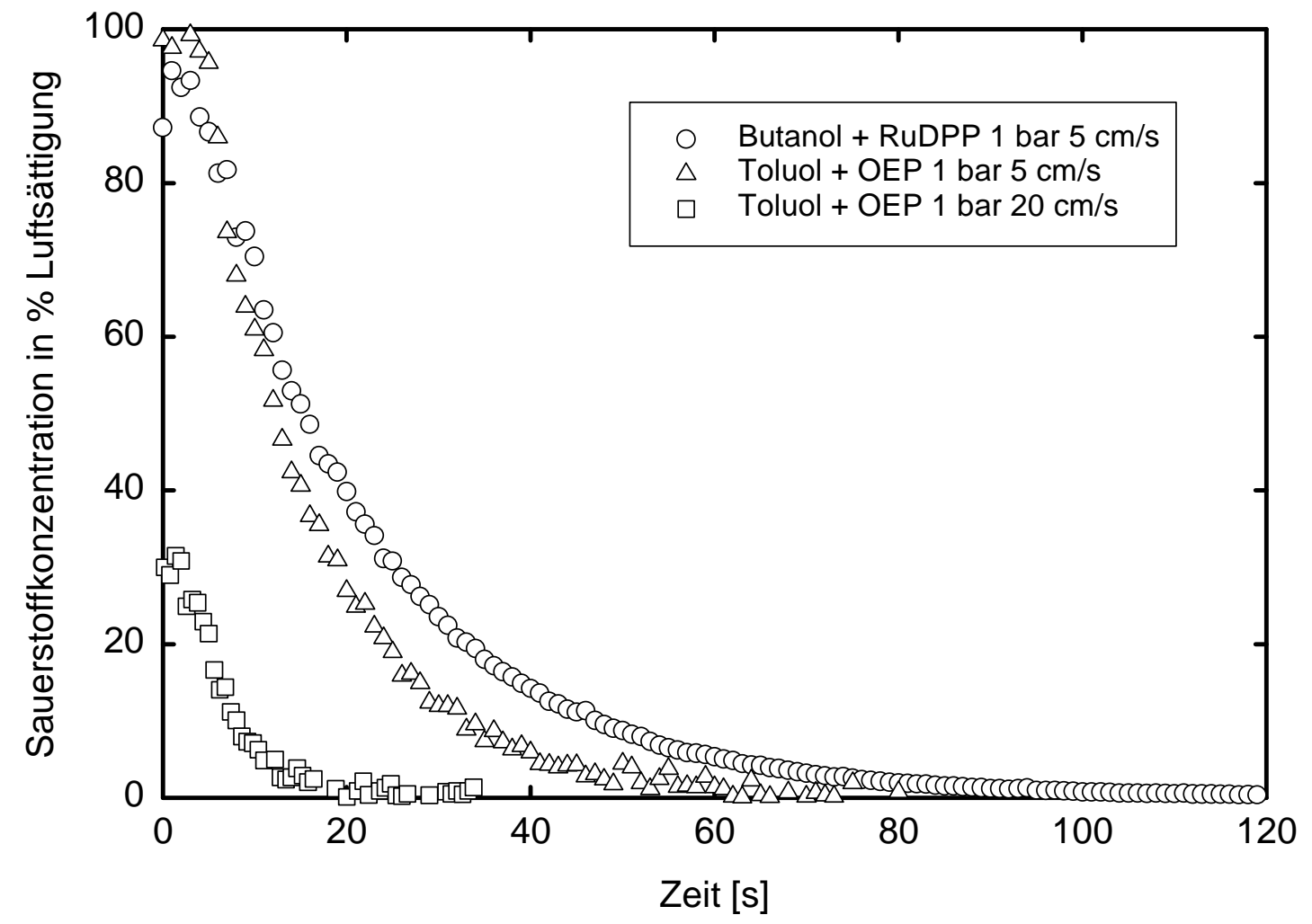

Abbildung 4-7: Drei Beispiele für die Desorptionsmessungen

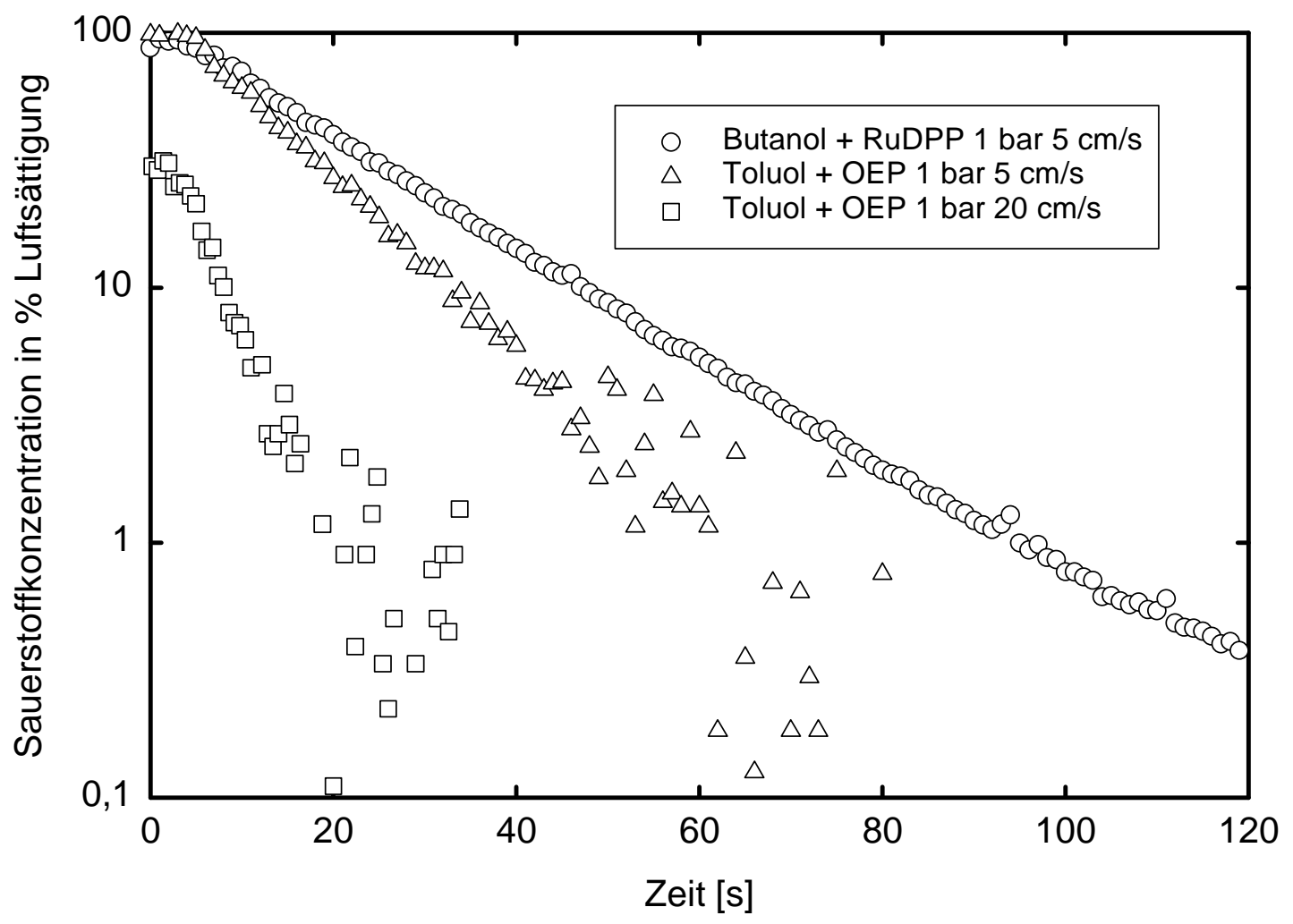

Abbildung 4-8: Drei Beispiele für Desorptionsmessungen in halblogarithmischer Auftragung 
Die logarithmische Auftragung ist jedoch nur bei relativ hohen Sauerstoffkonzentrationen für die Ermittlung des $\mathrm{k}_{\mathrm{L}} \mathrm{a}^{\prime}$-Wertes nutzbar. Bei kleinen Sauerstoffkonzentrationen nimmt der relative Messfehler überproportional zu. Bei sehr großen $\mathrm{k}_{\mathrm{L}} \mathrm{a}^{\prime}-$ Werten nimmt die Konzentration sehr schnell ab und fällt bereits nach kurzer Messzeit unter die Grenze, die mit Hilfe der logarithmischen Auftragung noch nutzbar ist (siehe Abbildung 4-8 und Abbildung 4-9). Gerade bei hohen Gasleerrohrgeschwindigkeiten wird die maximal mögliche Startkonzentration von $\mathrm{c}_{\mathrm{O} 2}=100 \%$ nicht erreicht, so dass sich das nutzbare Teilstück der Messung für die Auswertung nochmals verkürzt. Es ist zu erkennen, dass diese Probleme vor allem bei den Fluorophoren RuBPY (für Wasser) und OEP (für unpolare organische Flüssigkeiten) auftreten. Das Fluorophor RuDPP ist gerade bei sehr kleinen Sauerstoffkonzentrationen sehr sensitiv, so dass die typische Trichterform der Fehlerabweichung erst bei sehr kleinen Sauerstoffkonzentrationen auftritt. (Siehe Abbildung 4-9)

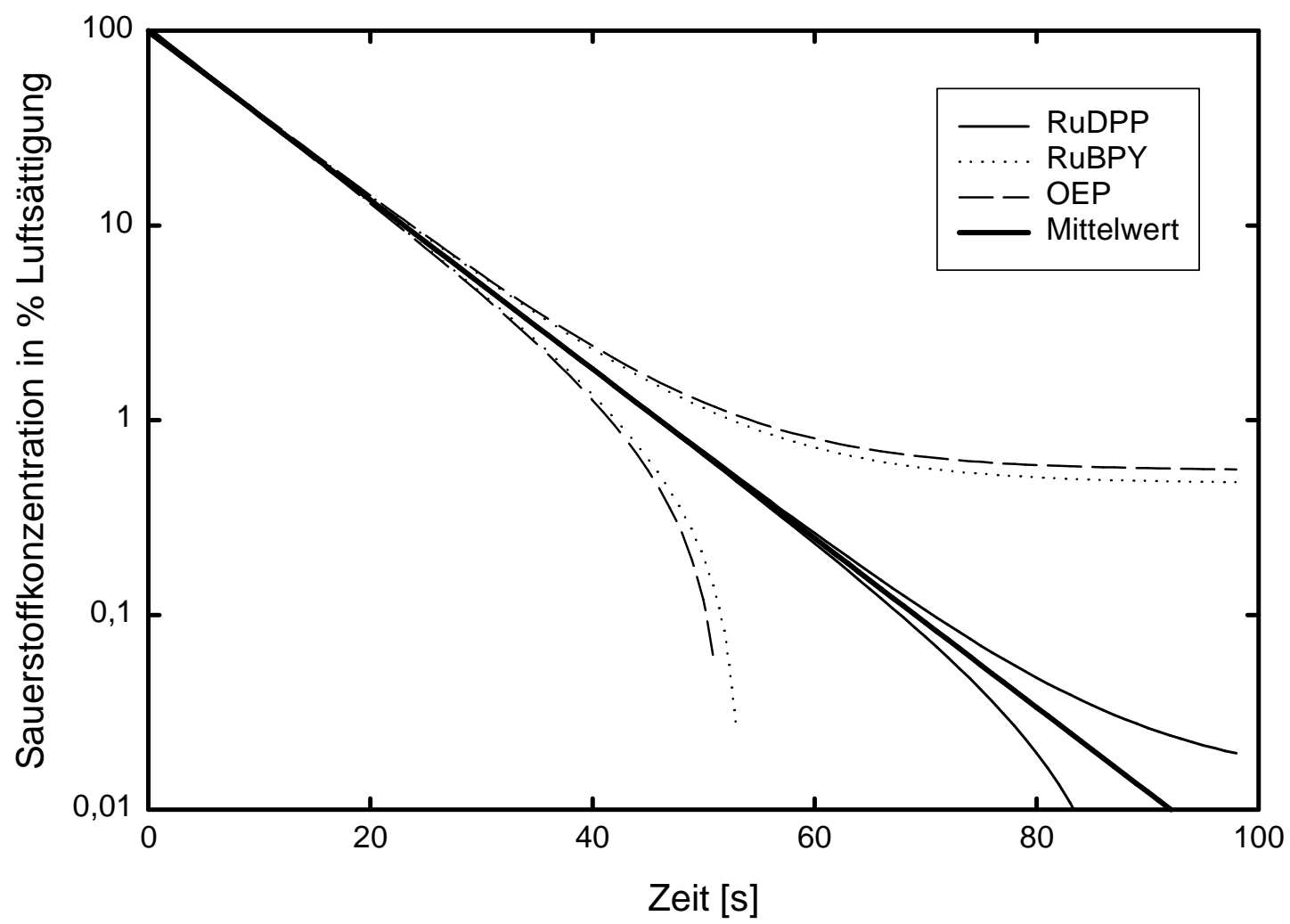

Abbildung 4-9: Theoretische Fehlerbreite der Sauerstoffkonzentration in halblogarithmischer Auftragung

Statt der linearen Regression der logarithmierten Messdaten wurde folgende Gleichung nach der Methode der kleinsten Fehlerquadrate an die Messdaten angepasst:

$\mathrm{c}_{\mathrm{O}_{2}}=\mathrm{c}_{\mathrm{O}_{2} \text {,start }} \cdot \exp \left(-\mathrm{k}_{\mathrm{L}} \mathrm{a}^{\prime} \cdot \mathrm{t}\right)+\mathrm{c}_{\mathrm{O}_{2} \text {, delta }}$

Gleichung 4-30: Sauerstoffkonzentration während der Desorptionsmessung inklusive Nullpunktsdrift

Der Wert für $\mathrm{c}_{\mathrm{O} 2 \text {,start }}$ ist die Sauerstoffkonzentration zum Zeitpunkt $\mathrm{t}=0$. Der Wert $\mathrm{c}_{\mathrm{O} 2 \text {,delta }}$ steht für die Nullpunktsdrift. Die Nullpunktsdrift kann durch Undichtigkeiten der Sauerstoffinjektion und durch interne Schwankungen des MOPS verursacht werden. Diese 
Werte und der $\mathrm{k}_{\mathrm{L}} \mathrm{a}^{\prime}$-Wert werden optimiert. Dies hat den Vorteil, dass die Messdaten in ihrer vollen Länge genutzt werden können (siehe Abbildung 4-10) und die Nullpunktsdrift gut erfasst werden kann. Gleichzeitig wird der Fehler durch die Anfangsverzögerung minimiert, da die ausgewertete Messzeit verlängert wird.

Die Anfangsverzögerung ist die Zeitspanne, die dadurch entsteht, dass nach der Umschaltung das sauerstofffreie Gas durch den Begaser und die Flüssigkeitssäule bis zum Sensor gelangen muss, bevor eine Abnahme des Sauerstoffgehaltes gemessen wird. (In Abbildung 5-19 wird gezeigt, dass die Sensorhöhe keinen Einfluss auf die ermittelte Stoffübergangszahl hat.)

Die Messungen wurden bis zu neun Mal wiederholt, um durch Mittelwertbildung den Fehler weiter zu minimieren.

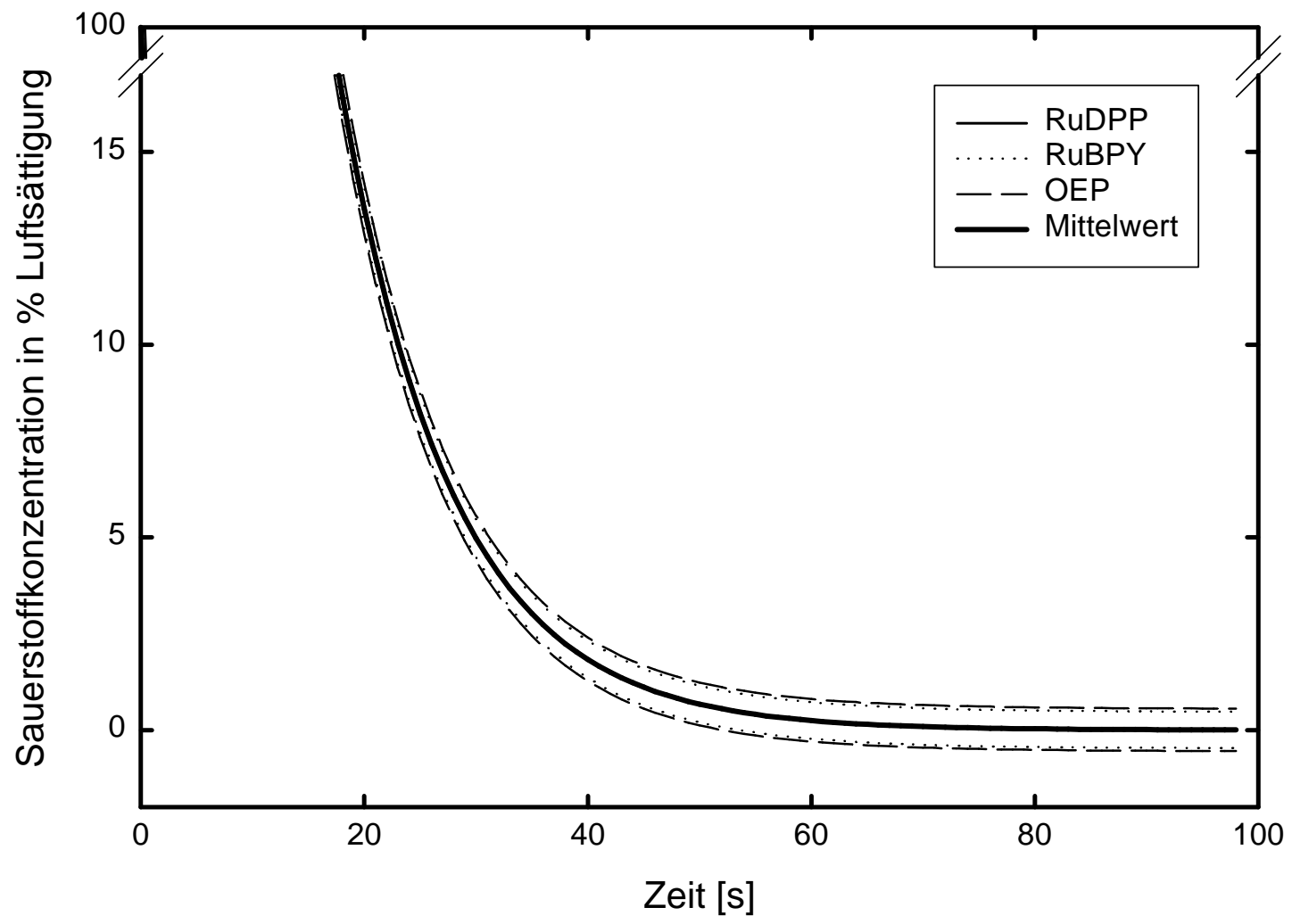

Abbildung 4-10: Theoretische Fehlerbreite der Sauerstoffkonzentration in linearer Auftragung

\subsubsection{Gasphasekonzentration während der Desorption}

Die bisherigen Berechnungen wurden unter der Voraussetzung gemacht, dass die Gasphasekonzentration gegenüber der Flüssigphasekonzentration vernachlässigbar klein ist. Diese Annahme soll nun überprüft werden.

Die aus der Flüssigkeit ausgetragene Sauerstoffmenge ist durch die Messungen bekannt:

$$
\begin{aligned}
& \frac{d n_{O_{2}}}{d t}=V_{L} \cdot \frac{d c_{O_{2}}}{d t} \\
& \frac{d n_{O_{2}}}{d t}=-V_{L} \cdot c_{L, O_{2}} \cdot k_{L} a^{\prime}
\end{aligned}
$$

Gleichung 4-31: Aus der Flüssigkeit ausgetragener Sauerstoffstrom 
Genau diese Menge Sauerstoff muss durch den Gasstrom aus dem Dispersionsvolumen heraus transportiert werden:

$\frac{d n_{O_{2}}}{d t}=-\dot{V}_{G} \cdot c_{G, O_{2}}$

Gleichung 4-32: mit dem Gasstrom transportierter Sauerstoffstrom

Beide Stoffströme müssen gleich groß sein:

$\mathrm{V}_{\mathrm{L}} \cdot \mathrm{c}_{\mathrm{L}, \mathrm{O}_{2}} \cdot \mathrm{k}_{\mathrm{L}} \mathrm{a}^{\prime}=\dot{\mathrm{V}}_{\mathrm{G}} \cdot \mathrm{c}_{\mathrm{G}, \mathrm{O}_{2}}$

Gleichung 4-33: Sauerstoffbilanz

Für die Gasphasekonzentration am Gasauslass kann in erster Näherung also gesagt werden:

$\mathrm{c}_{\mathrm{G}, \mathrm{O}_{2}}=\frac{\mathrm{V}_{\mathrm{L}} \cdot \mathrm{c}_{\mathrm{L}, \mathrm{O}_{2}} \cdot \mathrm{k}_{\mathrm{L}} \mathrm{a}^{\prime}}{\dot{\mathrm{V}}_{\mathrm{G}}}$

Gleichung 4-34: Gasphasekonzentration während der Desorption am Gasauslass

Um abschätzen zu können, ob diese Gasphasekonzentration vernachlässigbar klein oder für den Stoffübergang relevant ist, wird sie mit der Gleichgewichtskonzentration der Flüssigphase verglichen. Die Gleichgewichtskonzentration lässt sich mit dem OSTWALD-Koeffizienten berechnen:

$\mathrm{c}_{\mathrm{G}, \max }=\frac{\mathrm{c}_{\mathrm{L}, \mathrm{O}_{2}}}{\mathrm{~L}_{\mathrm{O}_{2}}}$

Gleichung 4-35: Gleichgewichtskonzentration der Gasphase

Hat die Gasphase diese Konzentration erreicht, so ist sie gesättigt. Das Verhältnis der Gasphasekonzentration zur maximalen Konzentration berechnet sich wie folgt:

$\frac{\mathrm{c}_{\mathrm{G}, \mathrm{O}_{2}}}{\mathrm{c}_{\mathrm{G}, \max }}=\frac{\mathrm{V}_{\mathrm{L}} \cdot \mathrm{L}_{\mathrm{O}_{2}} \cdot \mathrm{k}_{\mathrm{L}} \mathrm{a}^{\prime}}{\dot{\mathrm{V}}_{\mathrm{G}}}$

Gleichung 4-36: Sättigungsgrad der Gasphase während der Desorption

Ist dieser Sättigungsgrad gleich eins, ist die Gasphase praktisch im Gleichgewicht mit der Flüssigphase. Der Sättigungsgrad sollte möglichst nahe bei Null liegen. In Tabelle 4-1 sind die Sättigungsgrade für verschiedene Versuchsbedingungen aufgelistet. Für Wasser und Ethylenglycol sind diese Sättigungsgrade klein. Für die anderen Flüssigkeiten sind die Gasphasekonzentrationen so groß, dass sie nicht mehr vernachlässigt werden können. Die aufsteigenden Gasblasen werden so stark mit Sauerstoff angereichert, dass die Triebkraft für den Stoffübergang, die Konzentrationsdifferenz, signifikant kleiner wird. Dies bedeutet, dass der wahre $\mathrm{k}_{\mathrm{L}} \mathrm{a}^{\prime}$-Wert größer als der gemessene ist. Der gemessene Wert wird darum in der weiteren Arbeit als $\mathrm{k}_{\mathrm{L}}{ }{ }^{*}$-Wert, als Desorptionsrate bezeichnet. 
Tabelle 4-1: Abschätzung des Sättigungsgrades nach Gleichung 4-36 für verschiedene Versuchsbedingungen

\begin{tabular}{|c|c|c|c|c|c|c|}
\hline Flüssigkeit & $\begin{array}{c}\text { Druck } \\
{[\text { bar }]}\end{array}$ & $\begin{array}{c}\mathrm{u}_{\mathrm{G}} \\
{[\mathrm{cm} / \mathrm{s}]}\end{array}$ & $\mathrm{L}_{\mathrm{O} 2}$ & $\begin{array}{c}\mathrm{V}_{\mathrm{L}} \\
{[1]}\end{array}$ & $\begin{array}{c}\mathrm{k}_{\mathrm{L}} \mathrm{a}^{\prime} \\
{[1 / \mathrm{s}]}\end{array}$ & $\begin{array}{c}\mathrm{c}_{\mathrm{G}_{\mathrm{O}}} \\
\mathrm{c}_{\mathrm{G}, \max }\end{array}$ \\
\hline 1-Butanol & 1 & 0,98 & 0,2 & 10,6 & 0,0129 & 0,341 \\
\hline 1-Butanol & 1 & 4,90 & 0,2 & 10,6 & 0,0557 & 0,293 \\
\hline 1-Butanol & 1 & 19,8 & 0,2 & 10,6 & 0,1751 & 0,231 \\
\hline 1-Butanol & 40 & 1,03 & 0,2 & 10,6 & 0,0169 & 0,427 \\
\hline 1-Butanol & 40 & 5,15 & 0,2 & 10,6 & 0,1127 & 0,569 \\
\hline Decalin & 1 & 0,98 & 0,22 & 10,6 & 0,0161 & 0,471 \\
\hline Decalin & 1 & 4,90 & 0,22 & 10,6 & 0,0637 & 0,371 \\
\hline Decalin & 40 & 1,03 & 0,22 & 10,6 & 0,0192 & 0,533 \\
\hline Decalin & 40 & 5,15 & 0,22 & 10,6 & 0,1158 & 0,643 \\
\hline Ethanol & 1 & 1,03 & 0,23 & 10,6 & 0,0212 & 0,613 \\
\hline Ethanol & 1 & 5,19 & 0,23 & 10,6 & 0,0799 & 0,460 \\
\hline Ethanol & 1 & 20,8 & 0,23 & 10,6 & 0,2275 & 0,328 \\
\hline Ethanol & 40 & 1,03 & 0,23 & 10,6 & 0,0323 & 0,936 \\
\hline Ethanol & 40 & 5,16 & 0,23 & 10,6 & 0,1114 & 0,646 \\
\hline Ethylenglycol & 1 & 0,96 & 0,031 & 10,6 & 0,0017 & 0,007 \\
\hline Ethylenglycol & 1 & 4,83 & 0,031 & 10,6 & 0,0075 & 0,006 \\
\hline Ethylenglycol & 1 & 19,3 & 0,031 & 10,6 & 0,0216 & 0,004 \\
\hline Ethylenglycol & 40 & 1,03 & 0,031 & 10,6 & 0,0042 & 0,016 \\
\hline Ethylenglycol & 40 & 5,15 & 0,031 & 10,6 & 0,0225 & 0,018 \\
\hline Wasser & 1 & 0,99 & 0,033 & 10,6 & 0,0079 & 0,034 \\
\hline Wasser & 1 & 4,97 & 0,033 & 10,6 & 0,0345 & 0,030 \\
\hline Wasser & 1 & 19,8 & 0,033 & 10,6 & 0,1071 & 0,023 \\
\hline Wasser & 40 & 1,03 & 0,033 & 10,6 & 0,0172 & 0,072 \\
\hline Wasser & 40 & 5,15 & 0,033 & 10,6 & 0,09 & 0,075 \\
\hline Toluol & 1 & 1,00 & 0,22 & 10,6 & 0,0229 & 0,654 \\
\hline Toluol & 1 & 5,03 & 0,22 & 10,6 & 0,0776 & 0,441 \\
\hline Toluol & 1 & 20,1 & 0,22 & 10,6 & 0,2022 & 0,288 \\
\hline Toluol & 40 & 1,03 & 0,22 & 10,6 & 0,0235 & 0,652 \\
\hline Toluol & 40 & 5,15 & 0,22 & 10,6 & 0,1258 & 0,698 \\
\hline
\end{tabular}

Die Gasphasekonzentration an Sauerstoff ist signifikant erhöht. Zur Berechnung des $\mathrm{k}_{\mathrm{L}} \mathrm{a}^{\prime}-$ Wertes ist die Kenntnis der Gasphasekonzentration notwendig. Die Gasphasekonzentration an Sauerstoff kann über eine Sauerstoffbilanz ermittelt werden. Dies soll in den folgenden Abschnitten durchgeführt werden.

\subsubsection{Berechnung der Gasphasekonzentration des Sauerstoffes im homogenen Betriebszustand}

Zur Berechnung der Gasphasekonzentration wird vorausgesetzt, dass die Blasen kugelförmig sind und ihre Aufstiegsgeschwindigkeit und ihr Durchmesser während des gesamten Aufstieges konstant sind.

Die Flüssigkeit sei ideal durchmischt, d.h. die Flüssigkeit habe an jedem Punkt die gleiche Sauerstoffkonzentration, die sich folgendermaßen berechnen lässt: 
$\mathrm{c}_{\mathrm{L}, \mathrm{O}_{2}}=\mathrm{c}_{\mathrm{L}, \mathrm{O}_{2}, 0} \cdot \exp \left(-\mathrm{k}_{\mathrm{L}} \mathrm{a}^{*} \cdot \mathrm{t}\right)$

Gleichung 4-37: Flüssigphasekonzentration als Funktion der Zeit

Die Blase hat die Startkonzentration $\mathrm{c}_{\mathrm{L}, \mathrm{O}_{2}}=0$ und nimmt während ihres Aufstieges Sauerstoff auf. Der in die Blase diffundierende Stoffstrom berechnet sich:

$$
\frac{\mathrm{dn}_{\mathrm{O}_{2}}}{\mathrm{dt}}=\mathrm{k}_{\mathrm{L}} \cdot \mathrm{A}_{\mathrm{B}} \cdot\left(\mathrm{c}_{\mathrm{L}, \mathrm{O}_{2}}-\mathrm{c}_{\mathrm{B}, \mathrm{O}_{2}} \cdot \mathrm{L}_{\mathrm{O}_{2}}\right)=\mathrm{k}_{\mathrm{L}} \cdot \pi \cdot \mathrm{d}_{\mathrm{B}}^{2} \cdot\left(\mathrm{c}_{\mathrm{L}, \mathrm{O}_{2}}-\mathrm{c}_{\mathrm{B}, \mathrm{O}_{2}} \cdot \mathrm{L}_{\mathrm{O}_{2}}\right)
$$

Gleichung 4-38: Sauerstoffstrom in die Gasblase

Die Konzentrationsänderung in der Blase ergibt sich demnach:

$$
\frac{d c_{B, O_{2}}}{d t}=k_{L} \cdot \frac{A_{B}}{V_{B}} \cdot\left(c_{L, O_{2}}-c_{B, O_{2}} \cdot L_{O_{2}}\right)=k_{L} \cdot \frac{6}{d_{B}} \cdot\left(c_{L, O_{2}}-c_{B, O_{2}} \cdot L_{O_{2}}\right)
$$

Gleichung 4-39: Konzentrationsänderung Sauerstoff in der Blase

Der Blasendurchmesser $d_{B}$ wird durch den SAUTER-Durchmesser $d_{S}$ ersetzt:

$$
\mathrm{d}_{\mathrm{S}}=\frac{6 \cdot \varepsilon_{\mathrm{G}}}{\mathrm{a}}
$$

Gleichung 4-40: SAUTER-Durchmesser

$$
\frac{\mathrm{dc}_{\mathrm{B}, \mathrm{O}_{2}}}{\mathrm{dt}}=\frac{\mathrm{k}_{\mathrm{L}} \mathrm{a}}{\varepsilon_{\mathrm{G}}} \cdot\left(\mathrm{c}_{\mathrm{L}, \mathrm{O}_{2}}-\mathrm{c}_{\mathrm{B}, \mathrm{O}_{2}} \cdot \mathrm{L}_{\mathrm{O}_{2}}\right)
$$

Gleichung 4-41: Konzentrationsänderung Sauerstoff in der Blase

Und die Flüssigphasekonzentration $\mathrm{c}_{\mathrm{L}, \mathrm{O} 2}$ wird durch Gleichung 4-37 ersetzt:

$$
\frac{\mathrm{dc}_{\mathrm{B}, \mathrm{O}_{2}}}{\mathrm{dt}}=\frac{\mathrm{k}_{\mathrm{L}} \mathrm{a}}{\varepsilon_{\mathrm{G}}} \cdot\left(\mathrm{c}_{\mathrm{L}, \mathrm{O}_{2}, 0} \cdot \exp \left(-\mathrm{k}_{\mathrm{L}} \mathrm{a}^{*} \cdot \mathrm{t}\right)-\mathrm{c}_{\mathrm{B}, \mathrm{O}_{2}} \cdot \mathrm{L}_{\mathrm{O}_{2}}\right)
$$

Gleichung 4-42: Konzentrationsänderung Sauerstoff in der Blase

Mit dieser Gleichung lässt sich der zeitliche Konzentrationsverlauf in einer Blase berechnen (siehe Abbildung 4-11). Die Blase stellt kurz nach ihrer Entstehung eine Sauerstoffsenke dar und reichert sich mit Sauerstoff an. Im dargestellten Beispiel befinden sich Gasblase und Flüssigkeit nach ca. $7 \mathrm{~s}$ im Gleichgewicht. Die Sauerstoffkonzentration in der Gasblase erreicht in diesem Punkt ihr Maximum. Da die Flüssigphasekonzentration weiterhin abnimmt, wird die Gasblase nun zur Sauerstoffquelle und gibt Sauerstoff in die Flüssigkeit ab. Dies zeigt, dass die Gasphasekonzentration am Gasauslass auch höher als die aktuelle Gleichgewichtskonzentration sein kann. Weiterhin wird klar, dass der gemessene $\mathrm{k}_{\mathrm{L}} \mathrm{a}^{*}$-Wert mit zunehmender Verweilzeit der Blasen bei sonst gleichen Bedingungen kleiner wird. 


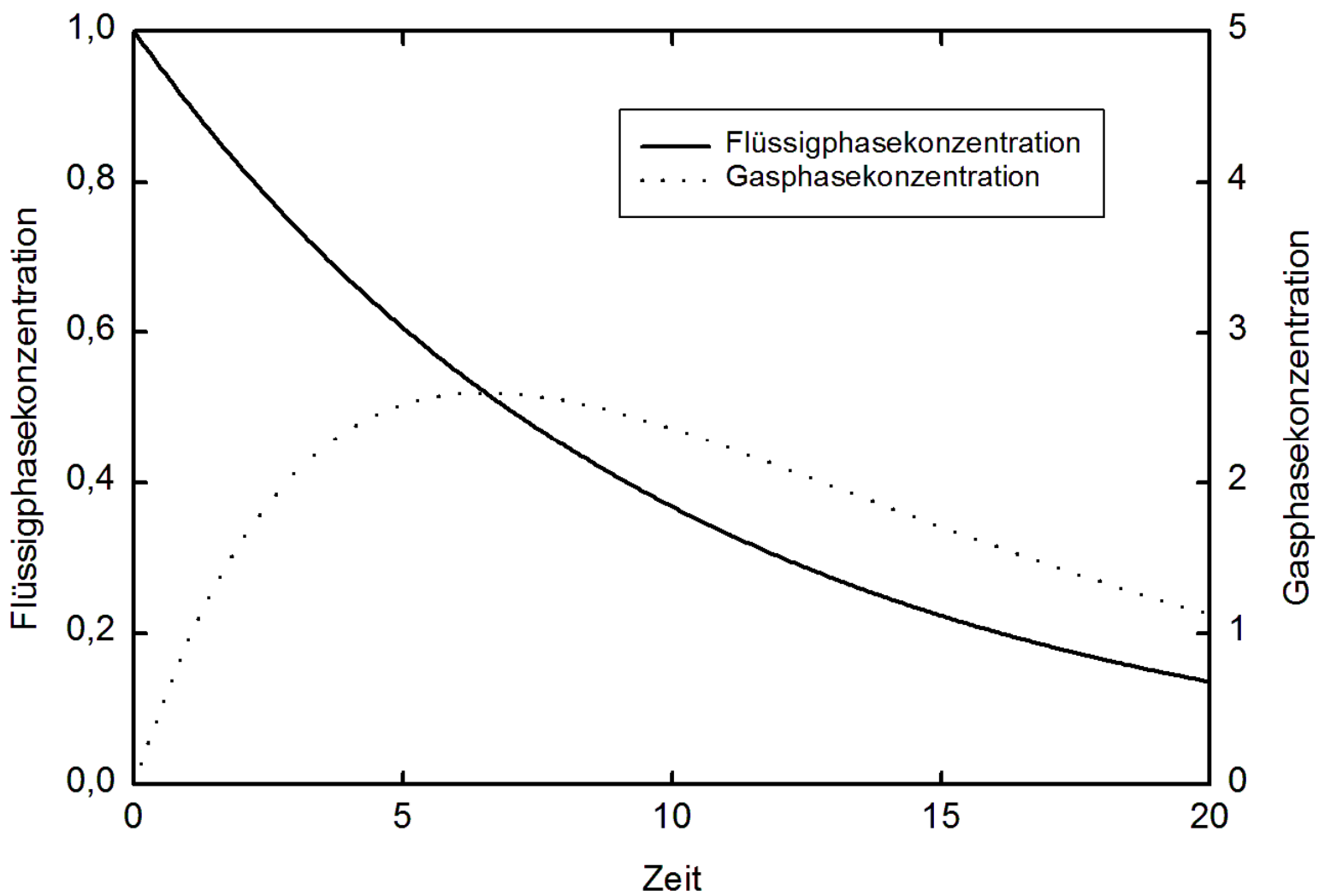

Abbildung 4-11: Zeitlicher Verlauf der Sauerstoffkonzentration einer Gasblase während einer Desorptionsmessung, $\mathrm{k}_{\mathrm{L}} \mathrm{a}^{*}=0,1 \mathrm{~s}^{-1}, \mathrm{~L}_{\mathrm{O} 2}=0,2, \mathrm{~d}_{\mathrm{B}}=3 \mathrm{~mm}$

Zur genauen Berechnung von $\mathrm{k}_{\mathrm{L}}$ a aus $\mathrm{k}_{\mathrm{L}} \mathrm{a}^{*}$ muss Gleichung 4-42 integriert werden. Je nach Randbedingungen ergeben sich zwei Lösungen:

Fall 1: $\mathrm{k}_{\mathrm{L}} \mathrm{a}^{*}-\mathrm{k}_{\mathrm{L}} \mathrm{a} \frac{\mathrm{L}_{\mathrm{O}_{2}}}{\varepsilon_{\mathrm{G}}} \neq 0$

$\mathrm{c}_{\mathrm{B}, \mathrm{O}_{2}}=\frac{\mathrm{k}_{\mathrm{L}} \mathrm{a} \cdot \mathrm{c}_{\mathrm{L}, \mathrm{O}_{2}, 0}}{\varepsilon_{\mathrm{G}} \cdot\left(\mathrm{k}_{\mathrm{L}} \mathrm{a}^{*}-\mathrm{k}_{\mathrm{L}} \mathrm{a} \frac{\mathrm{L}_{\mathrm{O}_{2}}}{\varepsilon_{\mathrm{G}}}\right)} \cdot\left(\exp \left(-\frac{\mathrm{k}_{\mathrm{L}} \mathrm{a} \cdot \mathrm{L}_{\mathrm{O}_{2}}}{\varepsilon_{\mathrm{G}}} \cdot \mathrm{t}\right)-\exp \left(-\mathrm{k}_{\mathrm{L}} \mathrm{a}^{*} \cdot \mathrm{t}\right)\right)$

Fall 2: $\mathrm{k}_{\mathrm{L}} \mathrm{a}^{*}-\mathrm{k}_{\mathrm{L}} \mathrm{a} \frac{\mathrm{L}_{\mathrm{O}_{2}}}{\varepsilon_{\mathrm{G}}}=0$

$\mathrm{c}_{\mathrm{B}, \mathrm{O}_{2}}=\frac{\mathrm{k}_{\mathrm{L}} \mathrm{a} \cdot \mathrm{c}_{\mathrm{L}, \mathrm{O}_{2}, 0}}{\varepsilon_{\mathrm{G}}} \cdot \exp \left(-\mathrm{k}_{\mathrm{L}} \mathrm{a}^{*} \cdot \mathrm{t}\right) \cdot \mathrm{t}$

Gleichung 4-43: $\mathrm{c}_{\mathrm{B}, \mathrm{O} 2}$ als Funktion der Verweilzeit $\mathrm{t}$

Der Wert $c_{\mathrm{L}, \mathrm{O} 2,0}$ ist die Flüssigphasekonzentration zum Startzeitpunkt $\mathrm{t}=0$ der entsprechenden Blase am Begaser.

Für die Berechnung der Gasphasekonzentration ist aber nicht der zeitliche Verlauf einer Gasblase notwendig, sondern die mittlere Konzentration aller vorliegenden Gasblasen. Da die Gasblasen zu unterschiedlichen Zeitpunkten starten, liegt für jede Gasblase eine andere Startkonzentration $\mathrm{C}_{\mathrm{L}, \mathrm{O} 2,0}$ in der Flüssigkeit vor. Diese Startkonzentration lässt sich ebenfalls 
mit dem $\mathrm{k}_{\mathrm{L}} \mathrm{a}^{*}$-Wert nach Gleichung 4-37 berechnen. Als Zeit wird hierbei eine negative Zeit eingesetzt. Diese Zeit entspricht der bisherigen Lebensdauer der Blase. Die Flüssigphasekonzentration zum Startzeitpunkt der Blase wird also aus der gegenwärtigen Flüssigkeitskonzentration zurückgerechnet. Die bisherige Lebensdauer der Blase lässt sich leicht aus der Blasengeschwindigkeit $\mathrm{v}_{\mathrm{B}}$ und der erreichten Steighöhe h ermitteln:

$\mathrm{t}=\frac{\mathrm{h}}{\mathrm{v}_{\mathrm{B}}}$

Gleichung 4-44: Lebensdauer einer Blase

Einsetzen der Startkonzentrationen und Verweilzeiten:

Fall 1: $\mathrm{k}_{\mathrm{L}} \mathrm{a}^{*}-\mathrm{k}_{\mathrm{L}} \mathrm{a} \frac{\mathrm{L}_{\mathrm{O}_{2}}}{\varepsilon_{\mathrm{G}}} \neq 0$

$\mathrm{c}_{\mathrm{B}, \mathrm{O}_{2}}=\frac{\mathrm{k}_{\mathrm{L}} \mathrm{a} \cdot \mathrm{c}_{\mathrm{L}, \mathrm{O}_{2}} \cdot \exp \left(\mathrm{k}_{\mathrm{L}} \mathrm{a}^{*} \cdot \mathrm{t}\right)}{\varepsilon_{\mathrm{G}} \cdot\left(\mathrm{k}_{\mathrm{L}} \mathrm{a}^{*}-\mathrm{k}_{\mathrm{L}} \mathrm{a} \frac{\mathrm{L}_{\mathrm{O}_{2}}}{\varepsilon_{\mathrm{G}}}\right)} \cdot\left(\exp \left(-\frac{\mathrm{k}_{\mathrm{L}} \mathrm{a} \cdot \mathrm{L}_{\mathrm{O}_{2}}}{\varepsilon_{\mathrm{G}}} \cdot \mathrm{t}\right)-\exp \left(-\mathrm{k}_{\mathrm{L}} \mathrm{a}^{*} \cdot \mathrm{t}\right)\right)$

$\mathrm{c}_{\mathrm{B}, \mathrm{O}_{2}}=\frac{\mathrm{k}_{\mathrm{L}} \mathrm{a} \cdot \mathrm{c}_{\mathrm{L}, \mathrm{O}_{2}}}{\varepsilon_{\mathrm{G}} \cdot\left(\mathrm{k}_{\mathrm{L}} \mathrm{a}^{*}-\mathrm{k}_{\mathrm{L}} \mathrm{a} \frac{\mathrm{L}_{\mathrm{O}_{2}}}{\varepsilon_{\mathrm{G}}}\right)} \cdot\left(\exp \left(\left(\mathrm{k}_{\mathrm{L}} \mathrm{a}^{*}-\frac{\mathrm{k}_{\mathrm{L}} \mathrm{a} \cdot \mathrm{L}_{\mathrm{O}_{2}}}{\varepsilon_{\mathrm{G}}}\right) \cdot \mathrm{t}\right)-1\right)$

Fall 2: $\mathrm{k}_{\mathrm{L}} \mathrm{a}^{*}-\mathrm{k}_{\mathrm{L}} \mathrm{a} \frac{\mathrm{L}_{\mathrm{O}_{2}}}{\varepsilon_{\mathrm{G}}}=0$

$c_{B, O_{2}}=\frac{k_{L} a \cdot c_{L, O_{2}}}{\varepsilon_{G}} \cdot t=\frac{k_{L} a \cdot c_{L, O_{2}}}{\varepsilon_{G}} \cdot \frac{h_{B}}{v_{B}}$

Gleichung 4-45: $\mathrm{c}_{\mathrm{B}, \mathrm{O} 2}$ als Funktion der Aufstiegshöhe

Die Zeit $\mathrm{t}$ entspricht hier der bisherigen Verweilzeit der Blase in der Flüssigkeit. Die Zeit ergibt sich als Quotient aus der Höhe $h_{B}$ der Blase über dem Begaser und der Aufstiegsgeschwindigkeit der Blase $\mathrm{v}_{\mathrm{B}}$. Die Flüssigphasekonzentration $\mathrm{c}_{\mathrm{L}, \mathrm{O} 2}$ entspricht der aktuell gemessenen Konzentration.

In der Abbildung 4-12 ist die so berechnete Gasphasekonzentration im Vergleich zur Flüssigphasekonzentration dargestellt. Die Steighöhe ist in Sekunden angegeben. Auch hier ist zu erkennen, dass Blasen mit langer Verweilzeit eine Sauerstoffquelle darstellen und nicht zur Desorption beitragen.

Um die mittlere Gasphasekonzentration zu erhalten, muss über alle im Dispersionsvolumen enthaltenen Gasblasen gemittelt werden. 


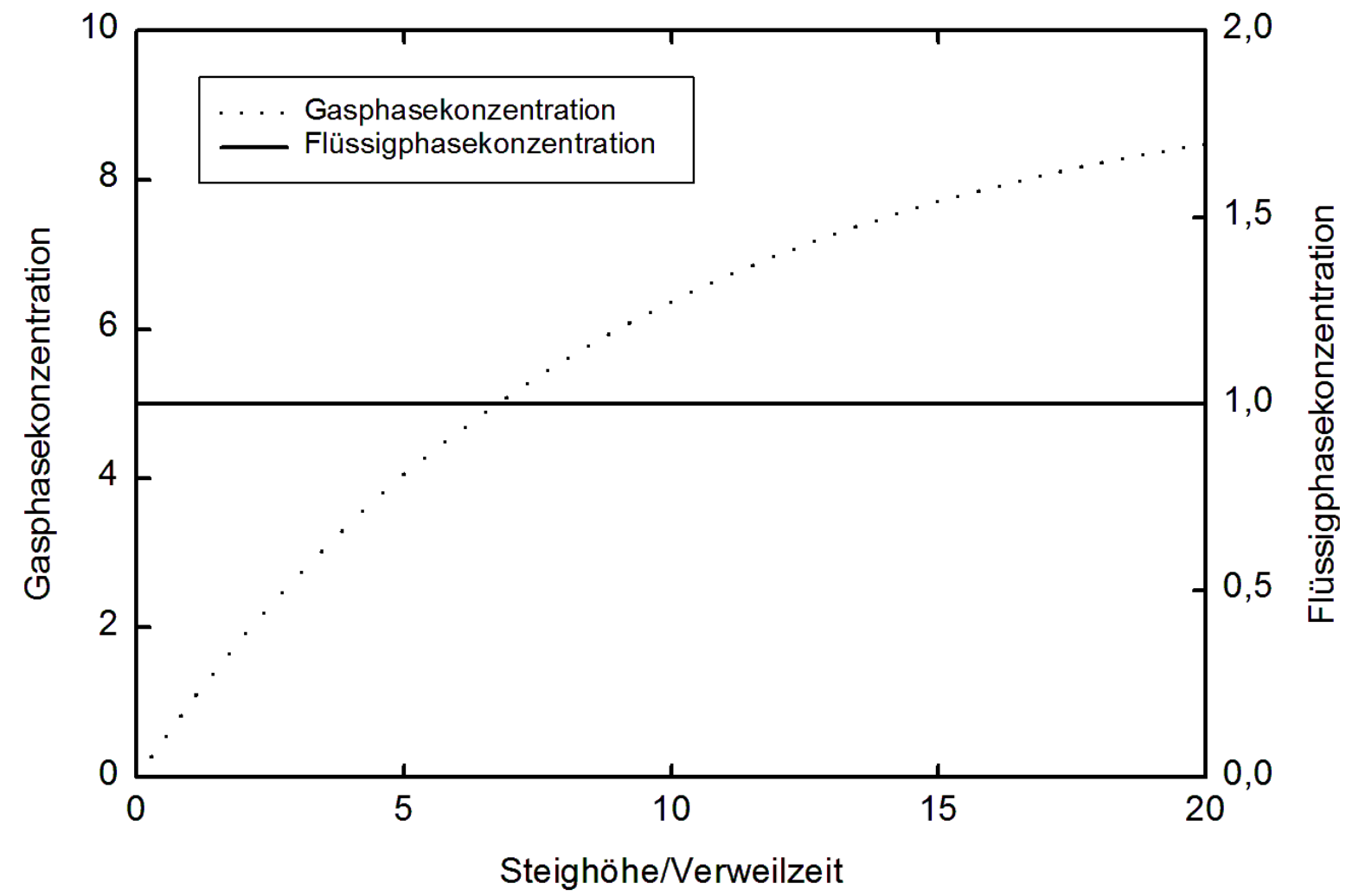

Abbildung 4-12: Axialer Verlauf der Gasphasekonzentration; die Flüssigphase wird als vollständig durchmischt angenommen, es wird eine konstante Aufstiegsgeschwindigkeit angenommen; gleiche Bedingungen wie in Abbildung 4-11

Es ergeben sich für die verschiedenen Randbedingungen wieder zwei Lösungen:

Fall 1: $\mathrm{k}_{\mathrm{L}} \mathrm{a}^{*}-\mathrm{k}_{\mathrm{L}} \mathrm{a} \frac{\mathrm{L}_{\mathrm{O}_{2}}}{\varepsilon_{\mathrm{G}}} \neq 0$

$$
\begin{aligned}
& \int_{0}^{t_{V}} c_{B} \cdot d t=\int_{0}^{t_{V}} \frac{k_{L} a \cdot c_{L, O_{2}}}{\varepsilon_{G} \cdot\left(k_{L} a^{*}-k_{L} a \frac{L_{O_{2}}}{\varepsilon_{G}}\right)} \cdot\left(\exp \left(\left(k_{L} a^{*}-\frac{k_{L} a \cdot L_{O_{2}}}{\varepsilon_{G}}\right) \cdot t\right)-1\right) \cdot d t \\
& \int_{0}^{t_{v}} c_{B} \cdot d t=\frac{k_{L} a \cdot c_{L, O_{2}}}{\varepsilon_{G} \cdot\left(k_{L} a^{*}-k_{L} a \frac{L_{O_{2}}}{\varepsilon_{G}}\right)} \cdot\left(\frac{\exp \left(\left(k_{L} a^{*}-\frac{k_{L} a \cdot L_{O_{2}}}{\varepsilon_{G}}\right) \cdot t_{V}\right)-1}{\left(k_{L} a^{*}-k_{L} a \frac{L_{O_{2}}}{\varepsilon_{G}}\right)}-t_{v}\right) \\
& \bar{c}_{\mathrm{G}, \mathrm{O}_{2}}=\frac{\mathrm{k}_{\mathrm{L}} \mathrm{a} \cdot \mathrm{c}_{\mathrm{L}, \mathrm{O}_{2}}}{\mathrm{t}_{\mathrm{V}} \cdot \varepsilon_{\mathrm{G}} \cdot\left(\mathrm{k}_{\mathrm{L}} \mathrm{a}^{*}-\mathrm{k}_{\mathrm{L}} \mathrm{a} \frac{\mathrm{L}_{\mathrm{O}_{2}}}{\varepsilon_{\mathrm{G}}}\right)} \cdot\left(\frac{\exp \left(\left(\mathrm{k}_{\mathrm{L}} \mathrm{a}^{*}-\frac{\mathrm{k}_{\mathrm{L}} \mathrm{a} \cdot \mathrm{L}_{\mathrm{O}_{2}}}{\varepsilon_{\mathrm{G}}}\right) \cdot \mathrm{t}_{\mathrm{V}}\right)-1}{\left(\mathrm{k}_{\mathrm{L}} \mathrm{a}^{*}-\mathrm{k}_{\mathrm{L}} \mathrm{a} \frac{\mathrm{L}_{\mathrm{O}_{2}}}{\varepsilon_{\mathrm{G}}}\right)}-\mathrm{t}_{\mathrm{V}}\right)
\end{aligned}
$$


Fall 2: $\mathrm{k}_{\mathrm{L}} \mathrm{a}^{*}-\mathrm{k}_{\mathrm{L}} \mathrm{a} \frac{\mathrm{L}_{\mathrm{O}_{2}}}{\varepsilon_{\mathrm{G}}}=0$

$\int_{0}^{t_{V}} c_{B} \cdot d t=\int_{0}^{t_{V}} \frac{k_{L} a \cdot c_{L, O_{2}}}{\varepsilon_{G}} \cdot t \cdot d t$

$\int_{0}^{t_{V}} c_{B} \cdot d t=\frac{k_{L} a \cdot c_{L, O_{2}}}{\varepsilon_{G}} \cdot \frac{t_{V}^{2}}{2}$

$\overline{\mathrm{c}}_{\mathrm{G}, \mathrm{O}_{2}}=\frac{\mathrm{k}_{\mathrm{L}} \mathrm{a} \cdot \mathrm{c}_{\mathrm{L}, \mathrm{O}_{2}}}{\varepsilon_{\mathrm{G}}} \cdot \frac{\mathrm{t}_{\mathrm{V}}}{2}$

Gleichung 4-46: Mittlere Gasphasekonzentration aus $\mathrm{k}_{\mathrm{L}} \mathrm{a}^{*}$

Der Wert $\bar{c}_{\mathrm{G}, \mathrm{O}_{2}}$ ist die mittlere Gasphasekonzentration über die gesamte Dispersionshöhe. Die Zeit $t_{v}$ ist die Verweilzeit des Gases im Dispersionsvolumen. Sie ergibt sich aus der Flüssigkeitshöhe $\mathrm{h}_{\mathrm{L}}$, dem Gasgehalt $\varepsilon_{\mathrm{G}}$ und der Leerrohrgeschwindigkeit $\mathrm{u}_{\mathrm{G}}$ :

$\mathrm{t}_{\mathrm{V}}=\frac{\mathrm{h}_{\mathrm{L}} \cdot \varepsilon_{\mathrm{G}}}{\left(1-\varepsilon_{\mathrm{G}}\right) \cdot \mathrm{u}_{\mathrm{G}}}$

Gleichung 4-47: Verweilzeit der Blasen im Dispersionsvolumen

Diese Gasphasekonzentration kann man nutzen, um die Triebkraft des Stoffübergangs zu berechnen.

$\frac{\mathrm{dc}_{\mathrm{L}, \mathrm{O}_{2}}}{\mathrm{dt}}=\mathrm{k}_{\mathrm{L}} \mathrm{a}^{*} \cdot \mathrm{c}_{\mathrm{L}, \mathrm{O}_{2}}=\frac{\mathrm{k}_{\mathrm{L}} \mathrm{a}}{\left(1-\varepsilon_{\mathrm{G}}\right)} \cdot\left(\mathrm{c}_{\mathrm{L}, \mathrm{O}_{2}}-\mathrm{L}_{\mathrm{O}_{2}} \cdot \overline{\mathrm{c}}_{\mathrm{G}, \mathrm{O}_{2}}\right)$

Gleichung 4-48: Änderung der Flüssigphasekonzentration des Sauerstoffes

Das Einsetzen von $\overline{\mathrm{c}}_{\mathrm{G}, \mathrm{O}_{2}}$ liefert je nach Randbedingung wieder zwei Lösungen:

Fall 1: $\mathrm{k}_{\mathrm{L}} \mathrm{a}^{*}-\mathrm{k}_{\mathrm{L}} \mathrm{a} \frac{\mathrm{L}_{\mathrm{O}_{2}}}{\varepsilon_{\mathrm{G}}} \neq 0$

$$
\mathrm{k}_{\mathrm{L}} \mathrm{a}=\frac{\mathrm{k}_{\mathrm{L}} \mathrm{a}}{1-\mathrm{L}_{\mathrm{O}_{2}} \cdot \frac{\mathrm{k}_{\mathrm{L}} \mathrm{a}^{*} \cdot\left(1-\varepsilon_{\mathrm{G}}\right)}{\mathrm{t}_{\mathrm{V}} \cdot \varepsilon_{\mathrm{G}} \cdot\left(\mathrm{k}_{\mathrm{L}} \mathrm{a}^{*}-\mathrm{k}_{\mathrm{L}} \mathrm{a} \frac{\mathrm{L}_{\mathrm{O}_{2}}}{\varepsilon_{\mathrm{G}}}\right)} \cdot\left(\frac{\exp \left(\left(\mathrm{k}_{\mathrm{L}} \mathrm{a}^{*}-\mathrm{k}_{\mathrm{L}} \mathrm{a} \frac{\mathrm{L}_{\mathrm{O}_{2}}}{\varepsilon_{\mathrm{G}}}\right) \cdot \mathrm{t}_{\mathrm{V}}\right)-1}{\left(\mathrm{k}_{\mathrm{L}} \mathrm{a}^{*}-\mathrm{k}_{\mathrm{L}} \mathrm{a} \frac{\mathrm{L}_{\mathrm{O}_{2}}}{\varepsilon_{\mathrm{G}}}\right)}-\mathrm{t}_{\mathrm{V}}\right)}
$$

Fall 2: $\mathrm{k}_{\mathrm{L}} \mathrm{a}^{*}-\mathrm{k}_{\mathrm{L}} \mathrm{a} \frac{\mathrm{L}_{\mathrm{O}_{2}}}{\varepsilon_{\mathrm{G}}}=0$ 


$$
\mathrm{k}_{\mathrm{L}} \mathrm{a}=\frac{\mathrm{k}_{\mathrm{L}} \mathrm{a}^{*} \cdot\left(1-\varepsilon_{\mathrm{G}}\right)}{1-\mathrm{L}_{\mathrm{O}_{2}} \cdot \frac{\mathrm{k}_{\mathrm{L}} \mathrm{a}}{\varepsilon_{\mathrm{G}}} \cdot \frac{\mathrm{t}_{\mathrm{V}}}{2}}
$$

Gleichung 4-49: Berechnung der Stoffübergangszahl $\mathrm{k}_{\mathrm{L}} \mathrm{a}$ aus der Desorptionsrate $\mathrm{k}_{\mathrm{L}} \mathrm{a}^{*}$

Bei diesem Schritt kürzt sich $\mathrm{c}_{\mathrm{L}, \mathrm{O} 2}$ aus der Gleichung heraus. Die so erfolgte Korrektur ist also unabhängig von der eigentlichen Sauerstoffkonzentration. Dies kann man sich dadurch veranschaulichen, dass nicht nur die Flüssigphasekonzentration während der Desorption exponentiell abnimmt, sondern auch die mittlere Gasphasekonzentration einer Exponentialkurve folgt. Da Exponentialkurven selbstähnlich sind, d.h. dass sie bei unterschiedlichen Vergrößerungen stets die gleiche Gestalt haben, ist das Verhältnis zwischen Gasphasekonzentration und Flüssigphasekonzentration konstant. Der Fall 1 der Gleichung 4-49 kann nicht nach $\mathrm{k}_{\mathrm{L}}$ a aufgelöst werden. Es ist aber möglich, durch eine Iteration zu einer stabilen Lösung zu kommen. Als Startwert für $\mathrm{k}_{\mathrm{L}} \mathrm{a}$ wird $\mathrm{k}_{\mathrm{L}} \mathrm{a}^{*}$ eingesetzt. Nach spätestens 20 Iterationsschritten gelangt man zu einem stabilen Endwert.

Diese Berechnungen gelten nur für homogene Strömungsregimes, bei denen alle Blasen weitgehend die gleiche Größe und Geschwindigkeit haben. Im heterogenen Regime ist es notwendig, die Blasenklassen separat $\mathrm{zu}$ behandeln, da unterschiedlich große Blasen unterschiedliche Oberflächen-Volumen-Verhältnisse haben und unterschiedliche schnell aufsteigen.

\subsubsection{Berechnung der Gasphasekonzentration des Sauerstoffs im heterogenen Betriebszustand}

Es wird von zwei Blasenklassen ausgegangen. Jeder Blasenklasse kann ein mittlerer Durchmesser $\mathrm{d}_{\mathrm{B}, \text { Klein }}$ und $\mathrm{d}_{\mathrm{B}, \mathrm{Groß}}$ zugeordnet werden. Weiterhin sollen die Verweilzeiten $\mathrm{t}_{\mathrm{V}, \text { Klein }}$ und $t_{\mathrm{V}, \mathrm{Gro}}$ und die Blasenaufstiegsgeschwindigkeiten $\mathrm{v}_{\mathrm{B}, \mathrm{Klein}}$ und $\mathrm{v}_{\mathrm{B}, \mathrm{Gro}}$ bekannt sein.

Zunächst wird der Stoffstrom an Sauerstoff, der aus der Flüssigkeit in das Gas übergeht, auf die Großblasen und Kleinblasen aufgeteilt.

$$
\frac{\mathrm{dn}_{\mathrm{O}_{2}}}{\mathrm{dt}}=\left(\frac{\mathrm{dn}_{\mathrm{O}_{2}}}{\mathrm{dt}}\right)_{\text {Klein }}+\left(\frac{\mathrm{dn}_{\mathrm{O}_{2}}}{\mathrm{dt}}\right)_{\mathrm{Gro \beta}}
$$

Gleichung 4-50: Aufteilung des Sauerstoffstromes auf Großblasen und Kleinblasen

Das Verhältnis beider Stoffströme zueinander ergibt sich aus verschiedenen Parametern, die aus der Gleichung 4-37 für den Stoffstrom durch die Phasengrenzfläche ersichtlich sind. Der $\mathrm{k}_{\mathrm{L}}$-Wert ändert sich mit der Aufstiegsgeschwindigkeit $\mathrm{v}_{\mathrm{B}}$ der jeweiligen Blasenklasse [COCKX 1995]: 
$\frac{\mathrm{k}_{\mathrm{L}}}{\mathrm{v}_{\mathrm{B}}} \cdot \mathrm{Sc}^{0,5}=$ const.

$\mathrm{Sc}=\frac{\mu_{\mathrm{L}}}{\rho_{\mathrm{L}} \cdot \mathrm{D}_{\mathrm{L}}}$

$\frac{\mathrm{k}_{\mathrm{L}}}{\mathrm{v}_{\mathrm{B}}}=$ const.

Gleichung 4-51: Abhängigkeit des $\mathrm{k}_{\mathrm{L}}$-Wertes von der Blasengeschwindigkeit $\mathrm{v}_{\mathrm{B}}$

Die SCHMIDT-Zahl Sc enthält nur stoffspezifische Werte, ist also ebenfalls eine Konstante. Die Blasenaufstiegsgeschwindigkeiten $\mathrm{v}_{\mathrm{B}}$ sind als bekannt vorausgesetzt. Dabei handelt es sich um die Slip-Geschwindigkeit, also die Relativgeschwindigkeit zwischen Gas und Flüssigkeit und nicht um die Schwarmgeschwindigkeit, die aus den Entgasungsmessungen ermittelt wurde. Die Slip-Geschwindigkeiten lassen sich aus den Blasendurchmessern nach MENDELSON und MANERI berechnen.

Die Phasengrenzfläche $A_{L, G}$ für jede Blasenklasse ist abhängig von Gasgehalt $\varepsilon_{G}$ und mittlerem Blasendurchmesser $\mathrm{d}_{\mathrm{B}}$ :

$$
\begin{aligned}
& \mathrm{a}=\frac{\mathrm{A}_{\mathrm{L}, \mathrm{G}}}{\mathrm{V}}=\frac{6 \cdot \varepsilon_{\mathrm{G}}}{\mathrm{d}_{\mathrm{B}}} \\
& \mathrm{A}_{\mathrm{L}, \mathrm{G}}=\frac{6 \cdot \varepsilon_{\mathrm{G}} \cdot \mathrm{V}}{\mathrm{d}_{\mathrm{B}}}
\end{aligned}
$$

Gleichung 4-52: Phasengrenzfläche einer Blasenklasse

Der Gasgehalt $\varepsilon_{\mathrm{G}}$ für jede Blasenklasse ist aus den Entgasungsmessungen bekannt. Das Volumen V entspricht dem Dispersionsvolumen und ist für alle Blasenklassen gleich. Der Blasendurchmesser $\mathrm{d}_{\mathrm{B}}$ wird als bekannt vorausgesetzt.

Weiterhin ändert sich der Stoffstrom, wenn sich die Gasphasekonzentration $\mathrm{c}_{\mathrm{G}, \mathrm{O} 2}$ ändert.

Das Verhältnis der Stoffströme in die Großblasen und in die Kleinblasen berechnet sich also:

$$
\frac{\left(\frac{\mathrm{dn}_{\mathrm{O}_{2}}}{\mathrm{dt}}\right)_{\text {Klein }}}{\left(\frac{\mathrm{dn}_{\mathrm{O}_{2}}}{\mathrm{dt}}\right)_{\mathrm{Gro \beta}}}=\frac{\mathrm{v}_{\mathrm{B}, \text { Klein }} \cdot \varepsilon_{\mathrm{G}, \text { Klein }} \cdot\left(\mathrm{c}_{\mathrm{L}}-\mathrm{L}_{\mathrm{O}_{2}} \cdot \overline{\mathrm{c}}_{\mathrm{G}, \text { Klein }}\right)}{\mathrm{v}_{\mathrm{B}, \text { Groß }} \cdot \varepsilon_{\mathrm{G}, \text { Groß }} \cdot\left(\mathrm{c}_{\mathrm{L}}-\mathrm{L}_{\mathrm{O}_{2}} \cdot \overline{\mathrm{c}}_{\mathrm{G}, \text { Gro }}\right)} \cdot \frac{\mathrm{d}_{\mathrm{S}, \text { Groß }}}{\mathrm{d}_{\mathrm{S}, \text { Klein }}}
$$

Gleichung 4-53: Verhältnis der Stoffströme für Großblasen und Kleinblasen im heterogenen Regime

Verknüpft man nun Gleichung 4-50 und Gleichung 4-53 so erhält man für die Stoffmengenströme: 


$$
\begin{aligned}
& \left(\frac{\mathrm{dn}_{\mathrm{O}_{2}}}{\mathrm{dt}}\right)_{\text {Klein }}=\frac{\left(\frac{\mathrm{dn}_{\mathrm{O}_{2}}}{\mathrm{dt}}\right)}{1+\frac{\mathrm{v}_{\mathrm{B}, \text { Groß }} \cdot \varepsilon_{\mathrm{G}, \text { Groß }} \cdot\left(\mathrm{c}_{\mathrm{L}}-\mathrm{L}_{\mathrm{O}_{2}} \cdot \overline{\mathrm{c}}_{\mathrm{G}, \text { Groß }}\right)}{\mathrm{v}_{\mathrm{B}, \text { Klein }} \cdot \varepsilon_{\mathrm{G}, \text { Klein }} \cdot\left(\mathrm{c}_{\mathrm{L}}-\mathrm{L}_{\mathrm{O}_{2}} \cdot \overline{\mathrm{c}}_{\mathrm{G}, \text { Klein }}\right)} \cdot \frac{\mathrm{d}_{\mathrm{S}, \text { Klein }}}{\mathrm{d}_{\mathrm{S}, \mathrm{Gro} \beta}}} \\
& \left(\frac{\mathrm{dn}_{\mathrm{O}_{2}}}{\mathrm{dt}}\right)_{\text {Groß }}=\frac{\left(\frac{\mathrm{dn}_{\mathrm{O}_{2}}}{\mathrm{dt}}\right)}{1+\frac{\mathrm{v}_{\mathrm{B}, \text { Klein }} \cdot \varepsilon_{\mathrm{G}, \text { Klein }} \cdot\left(\mathrm{c}_{\mathrm{L}}-\mathrm{L}_{\mathrm{O}_{2}} \cdot \overline{\mathrm{c}}_{\mathrm{G}, \text { Klein }}\right)}{\mathrm{v}_{\mathrm{B}, \text { Groß }} \cdot \varepsilon_{\mathrm{G}, \text { Groß }} \cdot\left(\mathrm{c}_{\mathrm{L}}-\mathrm{L}_{\mathrm{O}_{2}} \cdot \overline{\mathrm{c}}_{\mathrm{G}, \text { Groß }}\right)} \cdot \frac{\mathrm{d}_{\mathrm{S}, \text { Groß }}}{\mathrm{d}_{\mathrm{S}, \text { Klein }}}}
\end{aligned}
$$

Gleichung 4-54: Blasenklassenbezogene Stoffmengenströme

Diese Stoffmengenströme werden nun mit Gleichung 4-38 gleichgesetzt. Die Phasengrenzfläche A wird durch den Term $a \cdot \frac{V_{L}}{\left(1-\varepsilon_{G}\right)}$ ersetzt Man erhält für jede Blasenklasse:

$$
\begin{aligned}
& \left(\frac{\mathrm{dn}_{\mathrm{O}_{2}}}{\mathrm{dt}}\right)_{\text {Klein }}=\frac{\mathrm{V}_{\mathrm{L}}}{1-\varepsilon_{\mathrm{G}}} \cdot \mathrm{k}_{\mathrm{L}} \mathrm{a}_{\text {Klein }} \cdot\left(\mathrm{c}_{\mathrm{L}, \mathrm{O}_{2}}-\mathrm{L}_{\mathrm{O}_{2}} \cdot \overline{\mathrm{c}}_{\mathrm{G}, \text { Klein }}\right) \\
& =\frac{\mathrm{V}_{\mathrm{L}} \cdot \mathrm{k}_{\mathrm{L}} \mathrm{a}^{*} \cdot \mathrm{c}_{\mathrm{L}, \mathrm{O}_{2}}}{1+\frac{\mathrm{v}_{\mathrm{B}, \text { Groß }} \cdot \varepsilon_{\mathrm{G}, \text { Groß }} \cdot\left(\mathrm{c}_{\mathrm{L}, \mathrm{O}_{2}}-\mathrm{L}_{\mathrm{O}_{2}} \cdot \overline{\mathrm{c}}_{\mathrm{G}, \text { Groß }}\right)}{\mathrm{v}_{\mathrm{B}, \text { Klein }} \cdot \varepsilon_{\mathrm{G}, \text { Klein }} \cdot\left(\mathrm{c}_{\mathrm{L}, \mathrm{O}_{2}}-\mathrm{L}_{\mathrm{O}_{2}} \cdot \overline{\mathrm{c}}_{\mathrm{G}, \text { Klein }}\right)} \cdot \frac{\mathrm{d}_{\mathrm{B}, \text { Klein }}}{\mathrm{d}_{\mathrm{B}, \text { Groß }}}} \\
& \left(\frac{\mathrm{dn}_{\mathrm{O}_{2}}}{\mathrm{dt}}\right)_{\mathrm{Gro \beta}}=\frac{\mathrm{V}_{\mathrm{L}}}{1-\varepsilon_{\mathrm{G}}} \cdot \mathrm{k}_{\mathrm{L}} \mathrm{a}_{\mathrm{Gro \beta}} \cdot\left(\mathrm{c}_{\mathrm{L}, \mathrm{O}_{2}}-\mathrm{L}_{\mathrm{O}_{2}} \cdot \overline{\mathrm{c}}_{\mathrm{G}, \mathrm{GrO \beta}}\right) \\
& =\frac{\mathrm{V}_{\mathrm{L}} \cdot \mathrm{k}_{\mathrm{L}} \mathrm{a}^{*} \cdot \mathrm{c}_{\mathrm{L}, \mathrm{O}_{2}}}{1+\frac{\mathrm{v}_{\mathrm{B}, \text { Klein }} \cdot \varepsilon_{\mathrm{G}, \text { Klein }} \cdot\left(\mathrm{c}_{\mathrm{L}, \mathrm{O}_{2}}-\mathrm{L}_{\mathrm{O}_{2}} \cdot \overline{\mathrm{c}}_{\mathrm{G}, \text { Klein }}\right)}{\mathrm{v}_{\mathrm{B}, \text { Gro }} \cdot \varepsilon_{\mathrm{G}, \text { Gro }} \cdot\left(\mathrm{c}_{\mathrm{L}, \mathrm{O}_{2}}-\mathrm{L}_{\mathrm{O}_{2}} \cdot \overline{\mathrm{c}}_{\mathrm{G}, \text { Groß }}\right)} \cdot \frac{\mathrm{d}_{\mathrm{B}, \text { Groß }}}{\mathrm{d}_{\mathrm{B}, \text { Klein }}}}
\end{aligned}
$$

Gleichung 4-55: Stoffmengenströme der Blasenklassen

Diese Gleichungen werden nach $\mathrm{k}_{\mathrm{L}} \mathrm{a}_{\mathrm{Klein}}$ bzw. $\mathrm{k}_{\mathrm{L}} \mathrm{a}_{\mathrm{Groß}}$ aufgelöst:

$$
\begin{gathered}
\mathrm{k}_{\mathrm{L}} \mathrm{a}_{\text {Klein }}=\frac{\left(1-\varepsilon_{\mathrm{G}}\right) \cdot \mathrm{k}_{\mathrm{L}} \mathrm{a}^{*} \cdot \mathrm{c}_{\mathrm{L}, \mathrm{O}_{2}}}{\left(\mathrm{c}_{\mathrm{L}, \mathrm{O}_{2}}-\mathrm{L}_{\mathrm{O}_{2}} \cdot \bar{c}_{\mathrm{G}, \text { Klein }}\right)+\frac{\mathrm{v}_{\mathrm{B}, \mathrm{Gro \beta}} \cdot \varepsilon_{\mathrm{G}, \mathrm{Gro \beta}} \cdot\left(\mathrm{c}_{\mathrm{L}, \mathrm{O}_{2}}-\mathrm{L}_{\mathrm{O}_{2}} \cdot \overline{\mathrm{c}}_{\mathrm{G}, \mathrm{Gro \beta}}\right)}{\mathrm{v}_{\mathrm{B}, \text { Klein }} \cdot \varepsilon_{\mathrm{G}, \text { Klein }}} \cdot \frac{\mathrm{d}_{\mathrm{B}, \text { Klein }}}{\mathrm{d}_{\mathrm{B}, \mathrm{Gro \beta}}}} \\
\mathrm{k}_{\mathrm{L}} \mathrm{a}_{\text {Groß }}=\frac{\left(1-\varepsilon_{\mathrm{G}}\right) \cdot \mathrm{k}_{\mathrm{L}} \mathrm{a}^{*} \cdot \mathrm{c}_{\mathrm{L}, \mathrm{O}_{2}}}{\left(\mathrm{c}_{\mathrm{L}, \mathrm{O}_{2}}-\mathrm{L}_{\mathrm{O}_{2}} \cdot \overline{\mathrm{c}}_{\mathrm{G}, \mathrm{Gro \beta}}\right)+\frac{\mathrm{v}_{\mathrm{B}, \text { Klein }} \cdot \varepsilon_{\mathrm{G}, \text { Klein }} \cdot\left(\mathrm{c}_{\mathrm{L}, \mathrm{O}_{2}}-\mathrm{L}_{\mathrm{O}_{2}} \cdot \overline{\mathrm{c}}_{\mathrm{G}, \text { Klein }}\right)}{\mathrm{v}_{\mathrm{B}, \mathrm{Gro \beta}} \cdot \varepsilon_{\mathrm{G}, \text { Groß }}} \cdot \frac{\mathrm{d}_{\mathrm{B}, \text { Groß }}}{\mathrm{d}_{\mathrm{B}, \text { Klein }}}}
\end{gathered}
$$

Gleichung 4-56: Blasenklassenbezogene Stoffübergangszahlen

Die globale Stoffübergangszahl ist die Summe dieser beiden einzelnen Stoffübergangszahlen. 
$\mathrm{k}_{\mathrm{L}} \mathrm{a}=\mathrm{k}_{\mathrm{L}} \mathrm{a}_{\text {Klein }}+\mathrm{k}_{\mathrm{L}} \mathrm{a}_{\text {Groß }}$

Gleichung 4-57: $\mathrm{k}_{\mathrm{L}}$ a als Summe der blasenklassenbezogene Stoffübergangszahl

Für die mittleren Gasphasekonzentrationen $\bar{c}_{\mathrm{G}, \text { Groß }}$ und $\bar{c}_{\mathrm{G}, \text { Klein }}$ wird Gleichung 4-58 eingesetzt, die sich für die einzelnen Blasenklassen aus Gleichung 4-46 ergibt. Dabei kürzt sich die Flüssigphasekonzentration $\mathrm{c}_{\mathrm{L}, \mathrm{O} 2}$ heraus. Das Ergebnis ist also unabhängig von der Sauerstoffkonzentration. Durch Iteration von Gleichung 4-58 und Gleichung 4-56 erhält man nach spätestens 30 Iterationen konstante Werte.

Für die Berechnung ist die Kenntnis der Blasengeschwindigkeiten und Blasendurchmesser nötig. Für die Blasendurchmesser wurden bereits Berechnungsvorschriften im Kapitel „Korrelationen“ vorgestellt. Die Slip-Geschwindigkeiten ergeben sich aus den Blasendurchmessern nach MENDELSON und MANERI. Die Verweilzeiten erhält man aus den scheinbaren Aufstiegsgeschwindigkeiten der jeweiligen Blasenklasse.

Fall 1: $\mathrm{k}_{\mathrm{L}} \mathrm{a}^{*}-\mathrm{k}_{\mathrm{L}} \mathrm{a} \frac{\mathrm{L}_{\mathrm{O}_{2}}}{\varepsilon_{\mathrm{G}}} \neq 0$

$$
\begin{aligned}
& \bar{c}_{\mathrm{G}, \mathrm{Gro} \beta}=\frac{\mathrm{k}_{\mathrm{L}} \mathrm{a}_{\mathrm{Gro} \beta} \cdot \mathrm{c}_{\mathrm{L}, \mathrm{O}_{2}}}{\mathrm{t}_{\mathrm{V}, \mathrm{Gro} \beta} \cdot \varepsilon_{\mathrm{G}, \mathrm{Gro \beta}} \cdot\left(\mathrm{k}_{\mathrm{L}} \mathrm{a}^{*}-\mathrm{k}_{\mathrm{L}} \mathrm{a}_{\mathrm{Gro \beta}} \frac{\mathrm{L}_{\mathrm{O}_{2}}}{\varepsilon_{\mathrm{G}, \mathrm{Gro} \beta}}\right)} \cdot\left(\frac{\exp \left(\left(\mathrm{k}_{\mathrm{L}} \mathrm{a}^{*}-\frac{\mathrm{k}_{\mathrm{L}} \mathrm{a}_{\mathrm{Gro} \beta} \cdot \mathrm{L}_{\mathrm{O}_{2}}}{\varepsilon_{\mathrm{G}, \mathrm{Gro} \beta}}\right) \cdot \mathrm{t}_{\mathrm{V}, \mathrm{Gro \beta}}\right)-1}{\left(\mathrm{k}_{\mathrm{L}} \mathrm{a}^{*}-\frac{\mathrm{k}_{\mathrm{L}} \mathrm{a}_{\mathrm{Gro \beta}} \cdot \mathrm{L}_{\mathrm{O}_{2}}}{\varepsilon_{\mathrm{G}, \mathrm{Gro} \beta}}\right)}-\mathrm{t}_{\mathrm{V}, \text { Groß }}\right) \\
& \bar{c}_{\mathrm{G}, \text { Klein }}=\frac{\mathrm{k}_{\mathrm{L}} \mathrm{a}_{\text {Klein }} \cdot \mathrm{c}_{\mathrm{L}, \mathrm{O}_{2}}}{\mathrm{t}_{\mathrm{V}, \text { Klein }} \cdot \varepsilon_{\mathrm{G}, \text { Klein }} \cdot\left(\mathrm{k}_{\mathrm{L}} \mathrm{a}^{*}-\mathrm{k}_{\mathrm{L}} \mathrm{a}_{\text {Klein }} \frac{\mathrm{L}_{\mathrm{O}_{2}}}{\varepsilon_{\mathrm{G}, \text { Klein }}}\right)} \cdot\left(\frac{\exp \left(\left(\mathrm{k}_{\mathrm{L}} \mathrm{a}^{*}-\mathrm{k}_{\mathrm{L}} \mathrm{a}_{\text {Klein }} \frac{\mathrm{L}_{\mathrm{O}_{2}}}{\varepsilon_{\mathrm{G}, \text { Klein }}}\right) \cdot \mathrm{t}_{\mathrm{V}, \text { Klein }}\right)-1}{\left(\mathrm{k}_{\mathrm{L}} \mathrm{a}^{*}-\mathrm{k}_{\mathrm{L}} \mathrm{a}_{\text {Klein }} \frac{\mathrm{L}_{\mathrm{O}_{2}}}{\varepsilon_{\mathrm{G}, \text { Klein }}}\right)}-\mathrm{t}_{\mathrm{V}, \text { Klein }}\right)
\end{aligned}
$$

Fall 2: $\mathrm{k}_{\mathrm{L}} \mathrm{a}^{*}-\mathrm{k}_{\mathrm{L}} \mathrm{a} \frac{\mathrm{L}_{\mathrm{O}_{2}}}{\varepsilon_{\mathrm{G}}}=0$

$$
\begin{aligned}
& \overline{\mathrm{c}}_{\mathrm{G}, \mathrm{Gro \beta}}=\frac{\mathrm{k}_{\mathrm{L}} \mathrm{a}_{\mathrm{Gro \beta}} \cdot \mathrm{c}_{\mathrm{L}, \mathrm{O}_{2}}}{\varepsilon_{\mathrm{G}, \text { Groß }}} \cdot \frac{\mathrm{t}_{\mathrm{v}, \text { Groß }}}{2} \\
& \overline{\mathrm{c}}_{\mathrm{G}, \text { Klein }}=\frac{\mathrm{k}_{\mathrm{L}} \mathrm{a}_{\text {Klein }} \cdot \mathrm{c}_{\mathrm{L}, \mathrm{O}_{2}}}{\varepsilon_{\mathrm{G}, \text { Klein }}} \cdot \frac{\mathrm{t}_{\mathrm{v}, \text { Klein }}}{2}
\end{aligned}
$$

Gleichung 4-58: Mittlere Gasphasekonzentrationen der Blasenklassen im heterogenen Betriebszustand

Mit diesen Gleichungen können nun Messungen miteinander verglichen werden, bei denen mit unterschiedlichen Füllhöhen gearbeitet wurde. Im Kapitel „Ergebnisse“ sind die Ergebnisse von Desorptionsmessungen mit unterschiedlichen Füllhöhen dargestellt.

Zur Ermittlung des $\mathrm{k}_{\mathrm{L}} \mathrm{a}-\mathrm{Wertes}$ wird unter Vernachlässigung der Gasphasekonzentration die Desorptionsrate $\mathrm{k}_{\mathrm{L}}{ }{ }^{*}$ mit dem Gasgehalt verknüpft. Die Desorptionsrate $\mathrm{k}_{\mathrm{L}}{ }^{*}{ }^{*}$ entspricht in diesem Fall dem flüssigkeitsbezogenen Stoffübergangszahl $\mathrm{k}_{\mathrm{L}} \mathrm{a}^{\prime}$ : 
$\mathrm{k}_{\mathrm{L}} \mathrm{a}=\mathrm{k}_{\mathrm{L}} \mathrm{a}^{*} \cdot\left(1-\varepsilon_{\mathrm{G}}\right)$

Gleichung 4-59: „Klassische“ Berechnung der Stoffübergangszahl aus der Desorptionsrate

Die so berechneten Stoffübergangszahlen sind stets kleiner als die Desorptionsraten $\mathrm{k}_{\mathrm{L}} \mathrm{a}^{*}$.

In Abbildung 4-13 sind die gemessenen $\mathrm{k}_{\mathrm{L}} \mathrm{a}^{*}$-Werte und die daraus ermittelten Stoffübergangszahlen für eine ausgewählte Messreihe vergleichend aufgetragen.

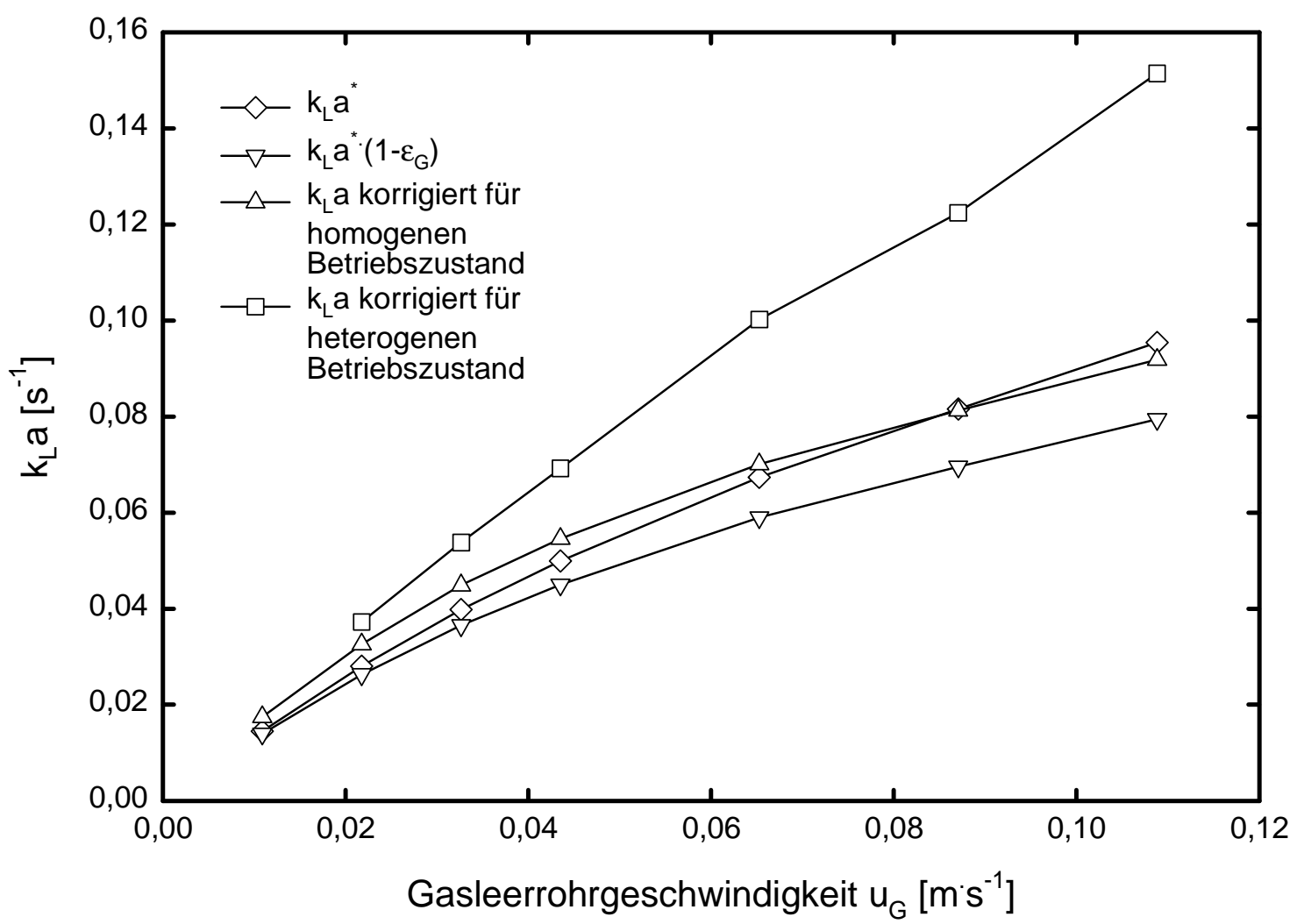

Abbildung 4-13: Vergleich der verschiedenen Stoffübergangswerte für 1-Butanol bei 1 bar $\mathrm{N}_{2}$ und $20{ }^{\circ} \mathrm{C}$ und $135 \mathrm{~cm}$ Füllstand

Die nach Gleichung 4-59 berechnete Stoffübergangszahl ist erwartungsgemäß die am niedrigsten liegende Kurve. Es wird davon ausgegangen, dass die Gasphase praktisch keinen Sauerstoff enthält. Die Konzentrationsdifferenz als treibende Kraft des Stoffüberganges zwischen den Phasen ist maximal. Der so berechnete $\mathrm{k}_{\mathrm{L}} \mathrm{a}-$ Wert liegt beim theoretischen Minimum für die jeweilige Messung.

Die $\mathrm{k}_{\mathrm{L}} \mathrm{a}-$ Werte, die sich aus der Korrektur für den homogenen Betriebszustand ergeben, sind höher. In diesem Fall wurde angenommen, dass sich die Gasphase beim Aufstieg signifikant mit Sauerstoff anreichert, so dass die Konzentrationsdifferenz zwischen den Phasen entsprechend kleiner wird. Aus den gleichen Desorptionsraten ergeben sich dadurch höhere $\mathrm{k}_{\mathrm{L}} \mathrm{a}-$ Werte. Die Unterschiede der Stoffübergangszahlen liegen beim angeführten Beispiel zwischen 10 und $20 \%$.

Berechnet man die Korrektur des $\mathrm{k}_{\mathrm{L}} \mathrm{a}-\mathrm{Wertes}$ für den heterogenen Betriebszustand, so erhält man noch höhere Werte für die Stoffübergangszahl. Die Ergebnisse betragen teilweise das Doppelte der „klassisch“ ermittelten $\mathrm{k}_{\mathrm{L}} \mathrm{a}$-Werte.

In Abbildung 4-14 sind die ,klassisch“ ermittelten $\mathrm{k}_{\mathrm{L}} \mathrm{a}$-Werte für zwei verschiedene Füllhöhen mit denen mit der Korrektur für den homogenen Fall gewonnenen $\mathrm{k}_{\mathrm{L}} \mathrm{a}-\mathrm{Werte}$ verglichen. Die homogen korrigierten Werte liegen höher, als die klassisch ermittelten $\mathrm{k}_{\mathrm{L}} \mathrm{a}-$ Werte. Bei niedrigen Gasgeschwindigkeiten $\mathrm{u}_{\mathrm{G}}<0,04 \mathrm{~m}^{-1}$ liegen die nach der neuen 
Methode ermittelten Kurven genau übereinander. Die Korrekturmethode ist also in der Lage, den Einfluss der Füllhöhe auf den Stoffübergang bei homogener Blasenströmung genau zu beschreiben.

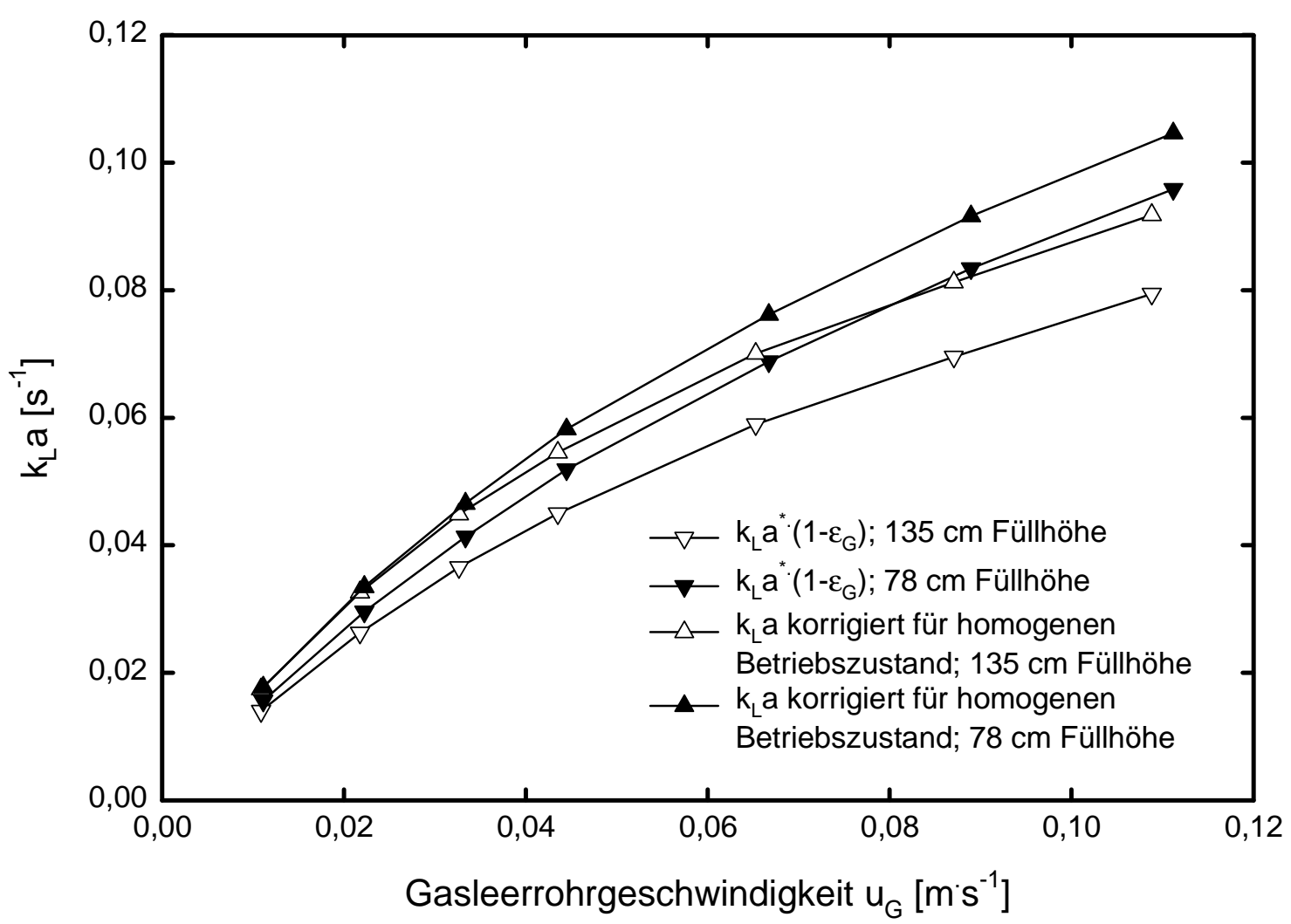

Abbildung 4-14: Vergleich der herkömmlichen Methode zur Berechnung von $\mathrm{k}_{\mathrm{L}}$ a mit der Korrektur für den homogenen Betriebszustand für verschiedenen Füllhöhen in 1Butanol

In Abbildung 4-15 sind die korrigierten $\mathrm{k}_{\mathrm{L}} \mathrm{a}$-Werte für den homogenen und heterogenen Betriebszustand aufgetragen. Die Kurven für den heterogenen Betriebszustand verlaufen zwar parallel, liegen aber nicht wie erwartet übereinander. Für diese Abweichung gibt es zwei mögliche Ursachen:

Zum Ersten sind für die Korrektur im heterogenen Betriebszustand die Kenntnis über die Blasendurchmesser, die Slipgeschwindigkeiten, die Verweilzeiten und die Anteile der Blasenklassen am Gesamtgasgehalt nötig. Die Blasenklassengehalte und die Verweilzeiten sind aus den Entgasungsmessungen zugänglich. Diese Werte sind relativ genau bekannt. Die Blasendurchmesser und die Slipgeschwindigkeiten hingegen können nur geschätzt werden. Durch die Nutzung dieser acht fehlerbehafteten Größen ergibt sich natürlich eine größere Fehlerbreite bei den berechneten Stoffübergangszahlen.

Zum zweiten ist der Anteil der Begaserzone bei niedrigem Füllstand am gesamten Dispersionsvolumen höher. In der Begaserzone liegt in der Regel ein niedrigerer Gasgehalt vor (siehe Kapitel „Ergebnisse“). Ein geänderter Gesamtgasgehalt hat Einfluss auf die Stoffübergangszahl. Außerdem benötigen die Großblasen einige Zeit, um sich aus den Kleinblasen zusammenzuschließen. Bei niedrigen Füllhöhen kann es also vorkommen, dass die Großblasen ihre endgültige Größe nicht erreichen und somit einen anderen Anteil an der Phasengrenzfläche haben. 
Die Korrektur für den heterogenen Betriebszustand ist durch die Vielzahl der Einflussgrößen sehr fehleranfällig. Für die Korrektur des homogenen Betriebszustandes ist nur die Kenntnis des Gasgehaltes und der Füllhöhe notwendig. Sie ist darum weniger fehleranfällig.

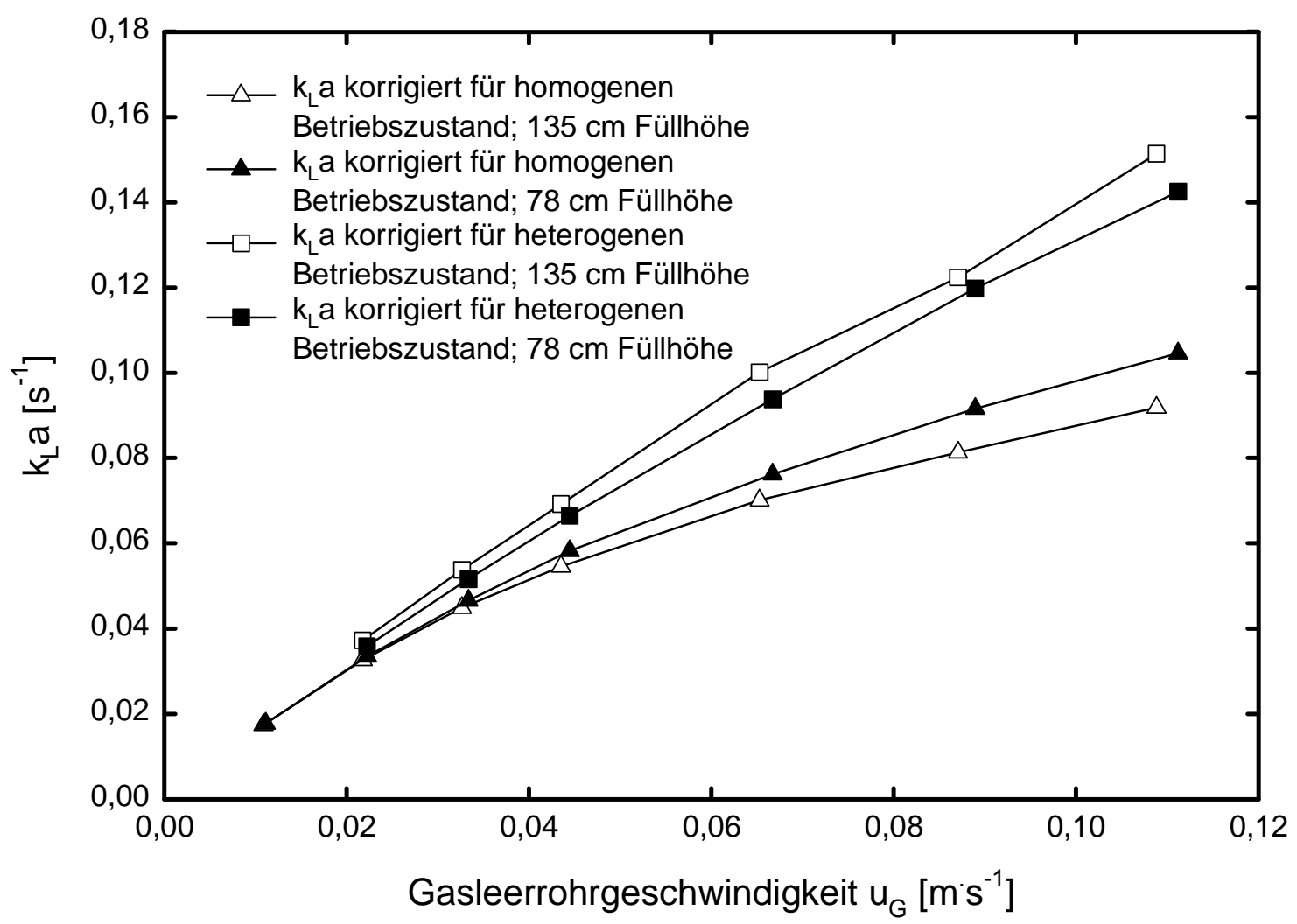

Abbildung 4-15: Vergleich der Korrektur für den homogenen und heterogenen Betriebszustand mit zwei verschiedenen Füllhöhen von 1-Butanol 


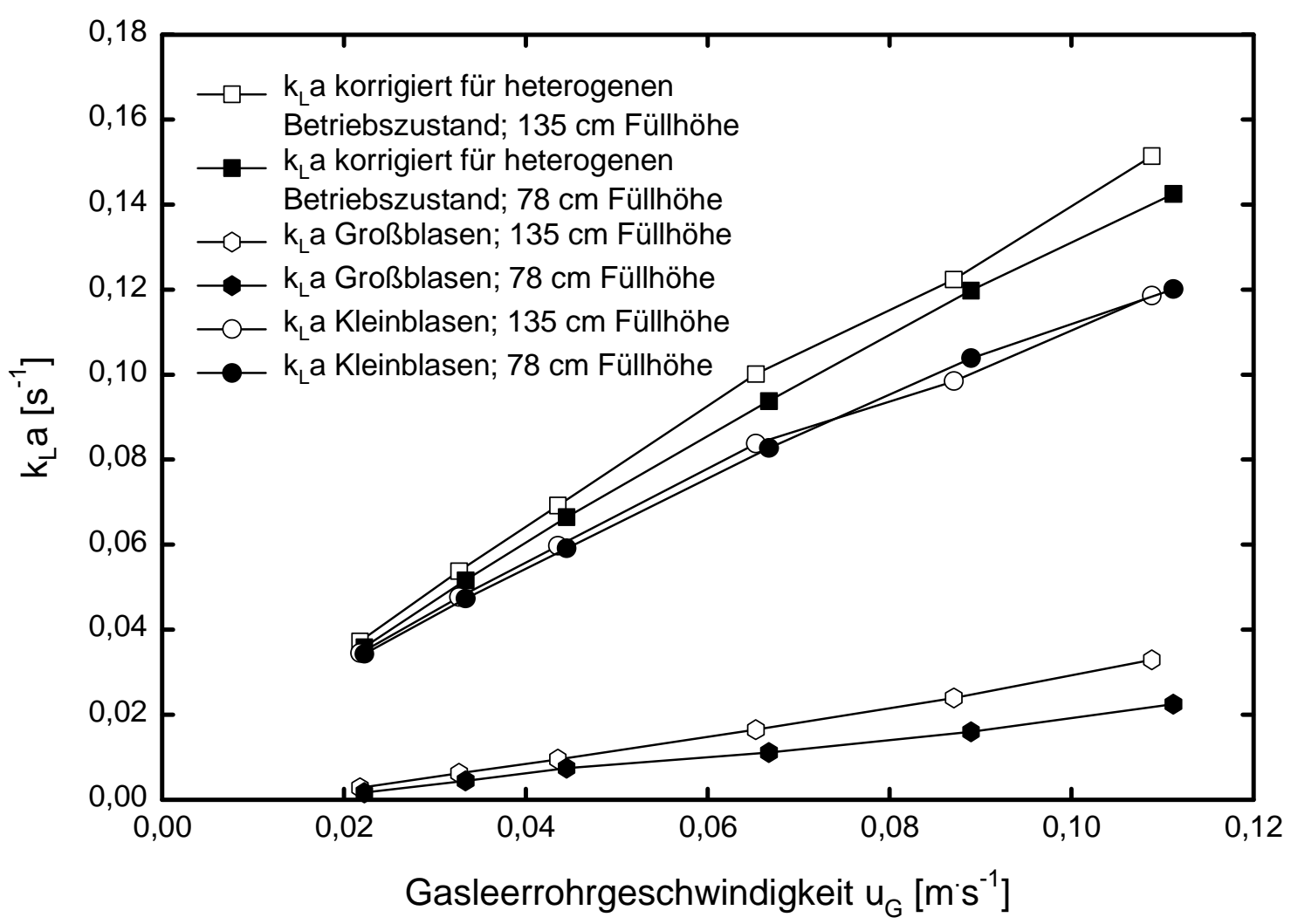

Abbildung 4-16: Aufteilung des Stoffstromes auf die einzelnen Blasenklassen in 1-Butanol

In Abbildung 4-16 sind die Stoffübergangszahlen für den heterogenen Fall für die beiden Blasenklassen getrennt aufgetragen. Für die Berechnung unterscheidet man zwei Gasströme, die durch die Flüssigkeit aufsteigen:

Die Kleinblasen haben ein hohes Oberfläche-Volumen-Verhältnis. Sie steigen langsam auf und sie stellen in der Regel den kleineren Teil des Gesamtgasstromes dar. Durch ihre große Oberfläche tragen die Kleinblasen den Hauptteil des Stoffüberganges.

Die Großblasen haben ein niedriges Oberfläche-Volumen-Verhältnis. Sie steigen schnell auf und stellen den Hauptteil des Gesamtgasstromes dar. Durch ihre kleine Oberfläche und ihre kurze Verweilzeit in der Flüssigkeit nehmen sie nur sehr wenig Sauerstoff auf und tragen nur zum geringen Teil zum Stoffübergang bei.

Dies zeigt, dass Großblasen für einen effektiven Stoffaustausch in der Blasensäule kontraproduktiv sind: Sie erzeugen nur wenig Phasengrenzfläche und transportieren einen großen Teil des Gases schnell durch die Flüssigkeit. Dieses Gas nimmt dabei kaum am Stoffaustausch teil.

Führt man die Korrekturen für den homogenen und heterogenen Betriebszustand für die gemessenen $\mathrm{k}_{\mathrm{L}} \mathrm{a}^{\prime}$-Werte durch, so zeigt sich dass die heterogen korrigierten Stoffübergangszahlen oft sehr große Werte annehmen bzw. sich erst gar nicht berechnen lassen. (siehe Abbildung 4-17) Diese Werte sind offensichtlich falsch. Der Grund für diesen Fehler liegt in der Annahme, dass die einzelnen Blasenklassen unabhängig voneinander aufsteigen und kein Stoffaustausch zwischen den Blasenklassen stattfindet. 


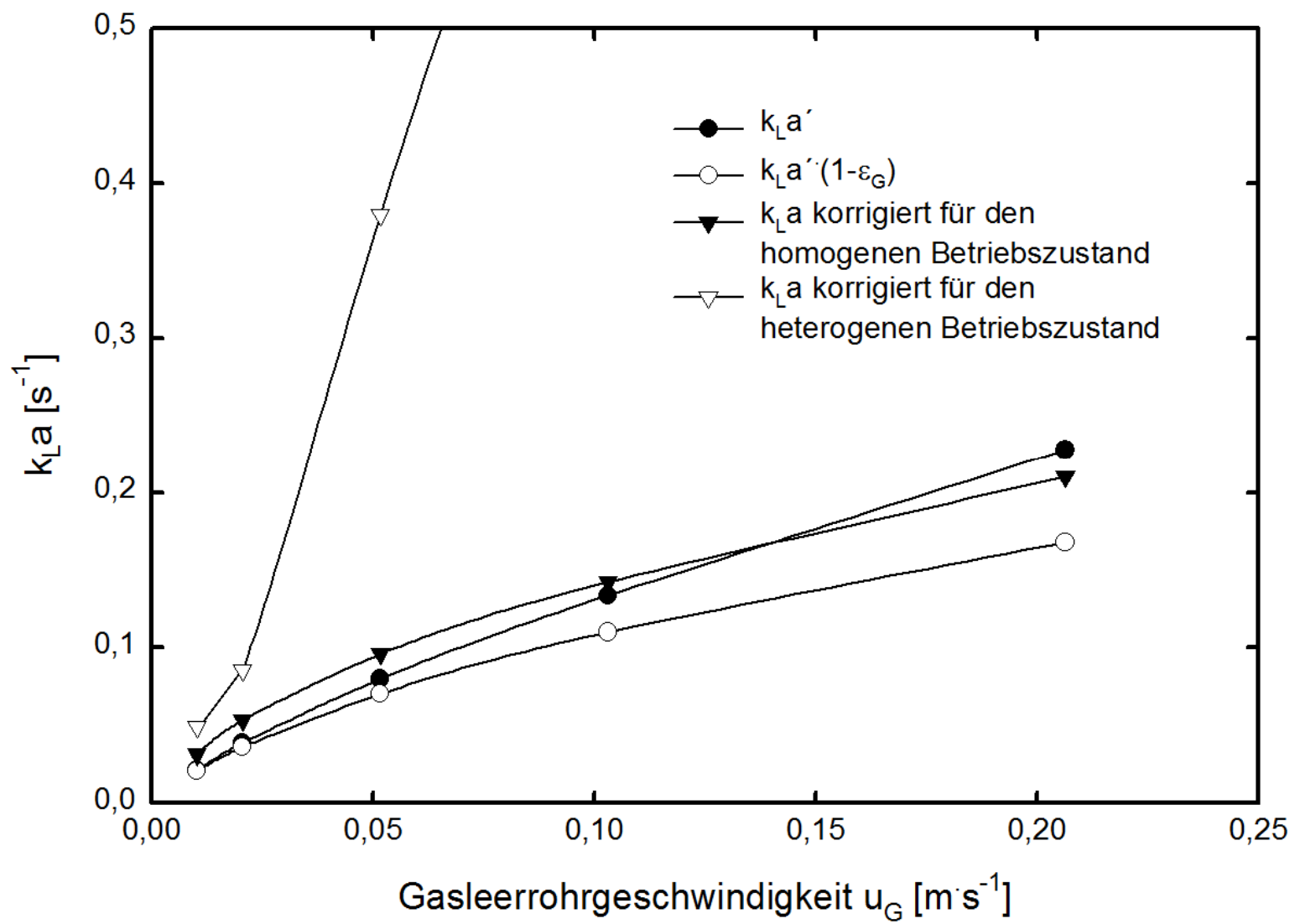

Abbildung 4-17: Die verschiedenen Stoffübergangszahlen in Ethanol bei 1 bar $\mathrm{N}_{2}$ und $20^{\circ} \mathrm{C}$

Realistischer ist dagegen die Annahme, dass die Großblasen bei ihrem Aufstieg mit Kleinblasen koaleszieren, wodurch sich die Sauerstoffkonzentration in der Großblase erhöht. Hat die Großblasen ihre Maximalgröße erreicht, werden von ihr ständig kleinere Blasen abgespalten. Diese abgespaltenen Blasen haben eine geringere Sauerstoffkonzentration, als die umgebenden Kleinblasen. Dadurch wird die mittlere Sauerstoffkonzentration der Kleinblasen verringert.

Dieser Stoffstrom zwischen den Blasenklassen wird von dem Modell zur Korrektur des $\mathrm{k}_{\mathrm{L}} \mathrm{a}-$ Wertes im heterogenen Betriebszustand nicht erfasst. Tatsächlich entspricht die Korrektur im homogenen Betriebszustand einer vollständigen Vermischung der beiden Blasenklassen. Es empfiehlt sich also, die einfachere Variante der Korrektur zu verwenden. Allerdings gibt es auch bei diesen Ergebnissen Probleme: Die Konzentration der Gasphase beim Verlassen des Dispersionsvolumens liegt zum Teil über der Gleichgewichtskonzentration der Flüssigkeit. Die Gasblasen sind in diesen Fällen keine Sauerstoffsenken, sondern Sauerstoffquellen (siehe Diskussion zu Abbildung 4-11). In diesem Bereich sind die Ergebnisse der Korrektur sehr sensibel gegen kleine Abweichungen der Eingangsdaten, wie z. B. des Füllstandes, des Gasgehaltes und der gemessenen Desorptionsrate $\mathrm{k}_{\mathrm{L}} \mathrm{a}^{*}$.

Zur Abschätzung der Wirksamkeit und Genauigkeit der $\mathrm{k}_{\mathrm{L}} \mathrm{a}-$ Korrektur kann die STANTONZahl herangezogen werden:

$\mathrm{St}=\frac{\mathrm{k}_{\mathrm{L}} \mathrm{a} \cdot \mathrm{V}_{\text {Disp }}}{\dot{\mathrm{V}}_{\mathrm{G}}}$

Gleichung 4-60: STANTON-Zahl 
Der $\mathrm{k}_{\mathrm{L}} \mathrm{a}$-Wert ist ein $\mathrm{Ma}$ für die Konzentrationsabnahme in der Flüssigphase. Er ist umgekehrt proportional der Halbwertszeit des Sauerstoffes in der flüssigen Phase während der

Desorption. Der Term $V_{\text {Disp }} / \dot{V}_{G}$ entspricht der mittleren Verweilzeit $t_{V}$ der Gasblasen im der Flüssigkeit. Eine niedrige STANTON-Zahl bedeutet eine geringe Belastung der Blasen mit Sauerstoff. Die Sauerstoffkonzentration der entweichenden Blasen ist noch weit von der Sättigungskonzentration entfernt. Eine hohe STANTON-Zahl bedeutet, dass die Gasphase viel Sauerstoff aufnimmt. Die entweichenden Gasblasen sind mit Sauerstoff gesättigt.

Allerdings reicht die STANTON-Zahl zur Abschätzung nicht aus. Ein weiterer Parameter ist das Kapazitätsverhältnis $\mathrm{K}_{\mathrm{L} / \mathrm{G}}$ der Gas-Flüssig-Dispersion:

$$
\mathrm{K}_{\mathrm{L} / \mathrm{G}}=\frac{\mathrm{L}_{\mathrm{O}_{2}} \cdot\left(1-\varepsilon_{\mathrm{G}}\right)}{\varepsilon_{\mathrm{G}}}
$$

Gleichung 4-61: Kapazitätsverhältnis der fluiden Phasen

Das Kapazitätsverhältnis vergleicht die Sauerstoffaufnahmekapazität der Flüssigphase mit der Gasphase. Ein Kapazitätsverhältnis von $\mathrm{K}_{\mathrm{L} / \mathrm{G}}=1$ bedeutet, dass im Gleichgewicht in Gasphase und Flüssigphase die gleiche Sauerstoffmenge $\mathrm{n}_{\mathrm{O} 2}$ vorhanden ist. Ein niedriges Kapazitätsverhältnis besagt, dass die Gasphase viel Sauerstoff aus der Flüssigphase aufnehmen kann. In diesem Fall wird die Gasphase die Sättigungskonzentration nicht erreichen. Ein hohes Kapazitätsverhältnis steht für ein geringes Aufnahmevermögen der Gasphase. In diesem Fall wird der Gasstrom gesättigt aus der Flüssigkeit entweichen.

Verknüpft man beide Kennzahlen miteinander, so erhält man einen zuverlässigen Indikator für die Einschätzung der Messung:

$$
\mathrm{St}^{*}=\mathrm{St} \cdot\left(\mathrm{K}_{\mathrm{L} / \mathrm{G}}+1\right)
$$

Gleichung 4-62: modifizierte STANTON-Zahl

Dieser Indikator ist eine modifizierte STANTON-Zahl St ${ }^{*}$. In Abbildung 4-18 ist die relative Gasaustrittskonzentration nach Fehler! Verweisquelle konnte nicht gefunden-werden.-als Funktion der modifizierten STANTON-Zahl aufgetragen. Die relative Gasaustrittskonzentration ergibt sich aus der gemessenen Sauerstoffkonzentration in der Flüssigkeit und der sich aus der Bilanz ergebenden Sauerstoffkonzentration in der Gasphase beim Verlassen der Flüssigkeit nach Gleichung 4-45. Dabei wird für $\mathrm{h}_{\mathrm{B}}$ die Dispersionshöhe eingesetzt.

Im Diagramm ergibt sich ein enger Zusammenhang: 


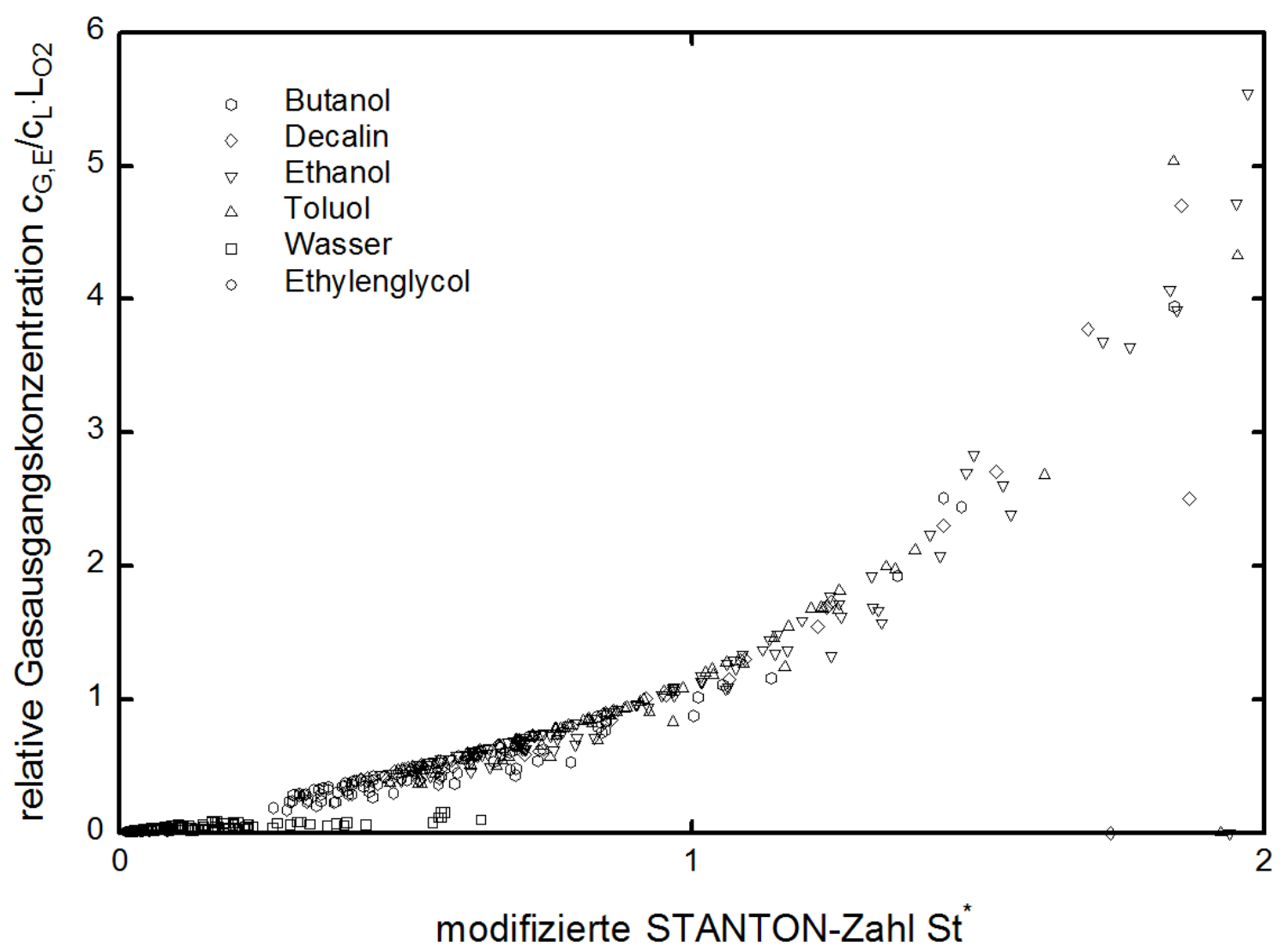

Abbildung 4-18: Die relative Gasaustrittskonzentration als eine Funktion der modifizierten STANTON-Zahl

Wird die modifizierte STANTON-Zahl St ${ }^{*}>1$, dann ist die relative Gasausgangskonzentration ebenfalls größer als 1. Der Gasstrom ist beim Verlassen der Flüssigkeit gesättigt. Ab einer STANTON-Zahl von $\mathrm{St}^{*}>1,1$ wird die iterative Korrektur unzuverlässig. Unterhalb von $\mathrm{St}^{*}<$ 0,2 ist die Gasphase so weit ungesättigt, dass eine iterative Korrektur unnötig ist. 


\section{Ergebnisse}

\subsection{Entgasungsmessungen}

Die Entgasungsmessungen liefern Informationen über den Gesamtgasgehalt, den Gasgehalt der einzelnen Blasenklassen sowie über die Aufstiegsgeschwindigkeit der Blasen.

\subsubsection{Gasgehalte}

Die Gesamtgasgehalte nehmen mit steigender Gasleerrohrgeschwindigkeit $\mathrm{u}_{\mathrm{G}}$ und steigender Gasdichte $\rho_{\mathrm{G}} \mathrm{zu}$ (siehe Abbildung 5-1). Dies entspricht den Erwartungen, die sich aus vielen früheren Veröffentlichungen ergeben. In halblogarithmischer Auftragung ergibt sich keine Gerade, sondern eine nach oben gebogene Kurve. Die Abhängigkeit des Gasgehaltes von der Gasdichte lässt sich demnach nicht problemlos mit einem einfachen Potenzansatz beschreiben. Ähnliches gilt auch für die Abhängigkeit des Gasgehalts von der Leerrohrgeschwindigkeit: Der Einfluss der Gasleerrohrgeschwindigkeit nimmt mit steigender Gasgeschwindigkeit ab.

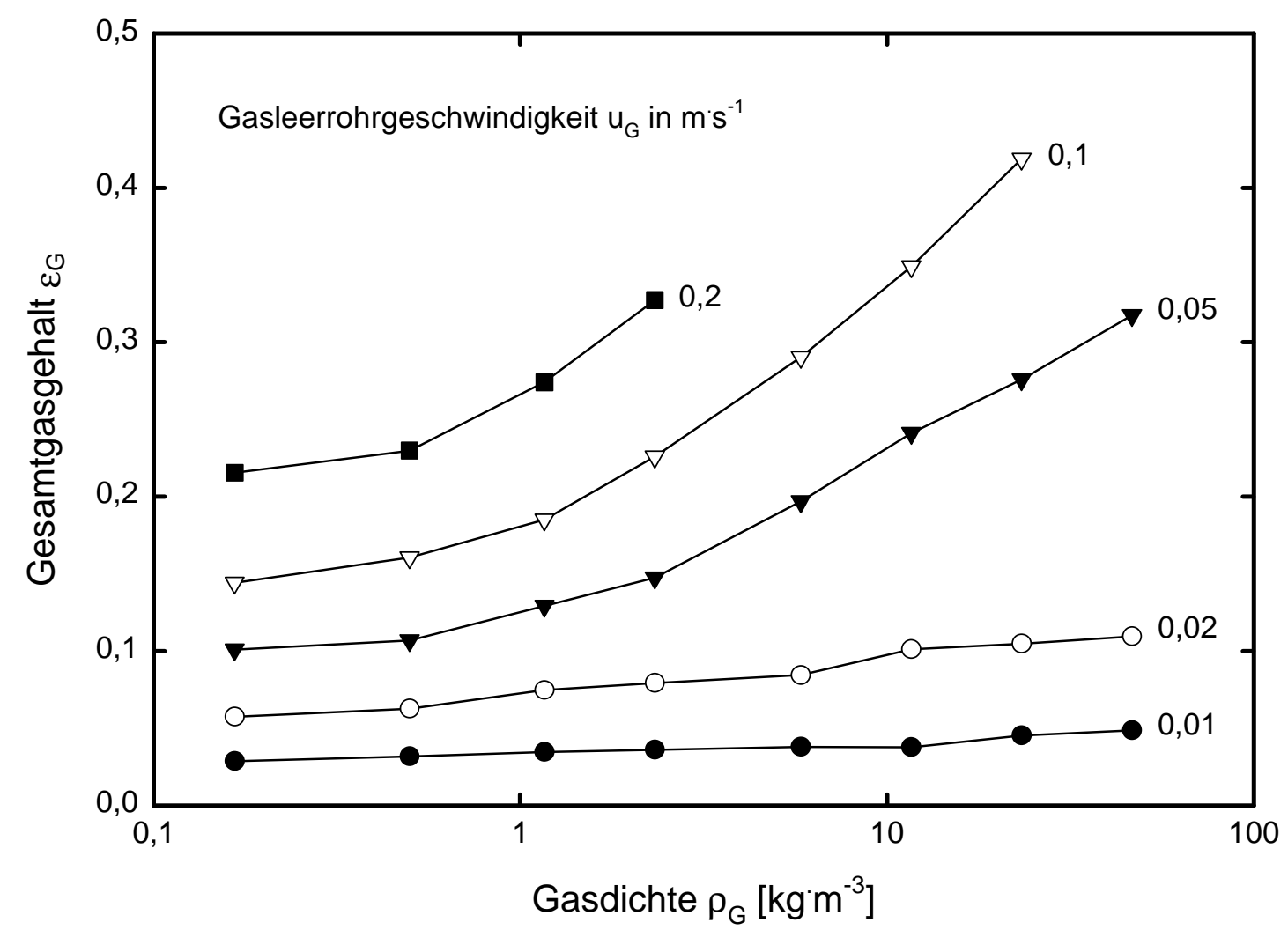

Abbildung 5-1: Gasgehalte in Toluol 


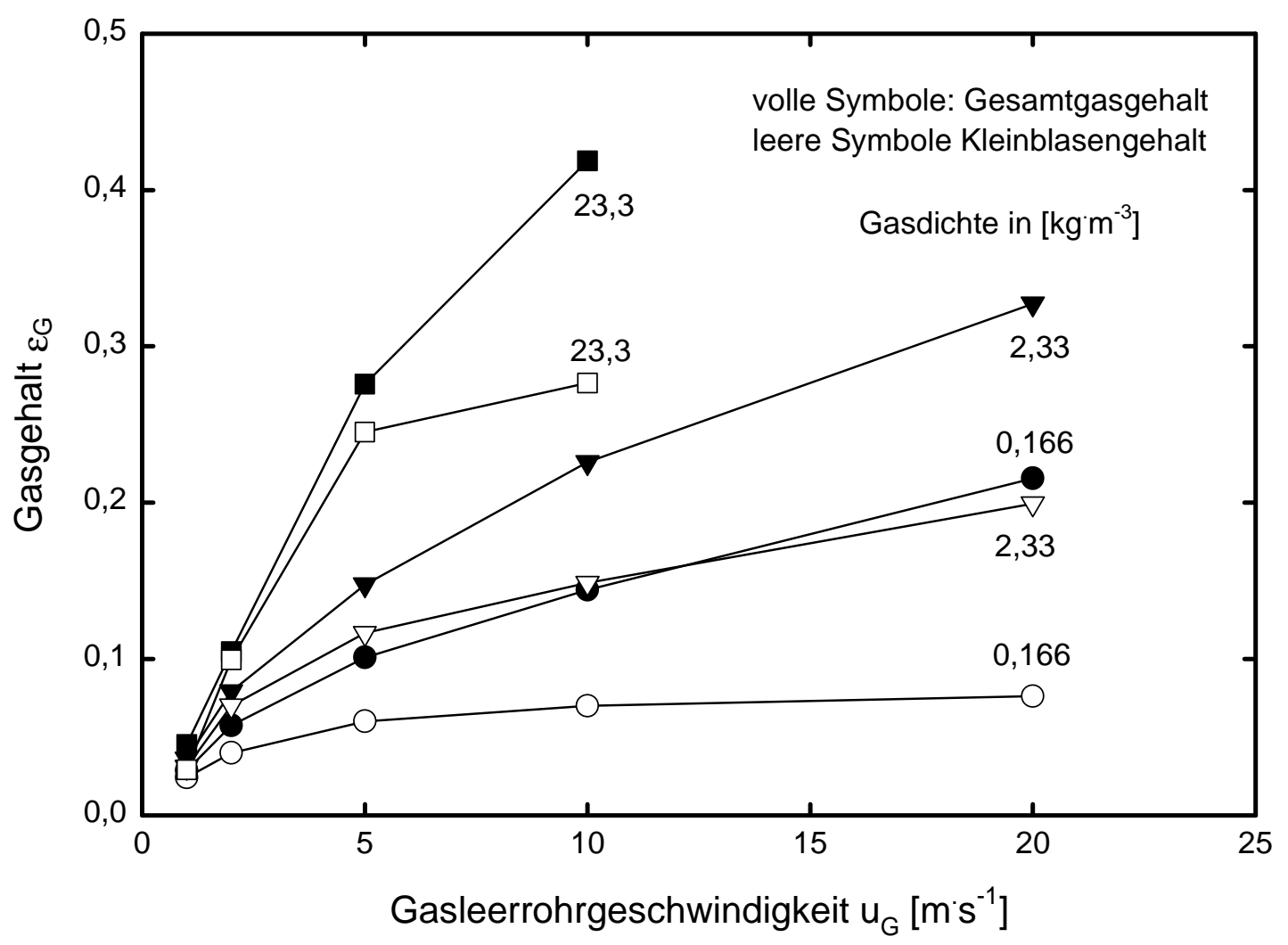

Abbildung 5-2: Gasstruktur in Toluol; der Abstand zwischen Gesamtgasgehalt und Kleinblasengehalt entspricht dem Großblasengehalt

Die globale Größe Gasgehalt lässt sich in der Regel in Großblasengehalt und Kleinblasengehalt aufteilen. In Abbildung 5-2 ist der Kleinblasengehalt mit dem Gesamtgasgehalt für drei verschiedene Gasdichten aufgetragen. Bei sehr kleinen Gasleerrohrgeschwindigkeiten wird der gesamte Gasgehalt durch Kleinblasen repräsentiert. Erst $\mathrm{ab}$ einer bestimmten Grenzgasgeschwindigkeit $\mathrm{u}_{\mathrm{G}, \mathrm{t}}$ (,transition gas velocity“ Übergangsgasgeschwindigkeit: Übergang zwischen homogenem und heterogenem Strömungsregime), treten Großblasen auf. Die Grenzgasgeschwindigkeit steigt mit zunehmender Gasdichte an. In der Abbildung ist gut zu erkennen, dass bei einer Gasdichte von $\rho_{\mathrm{G}}=0,166 \mathrm{~kg} \mathrm{~m}^{-3}$ schon bei $\mathrm{u}_{\mathrm{G}}=2 \mathrm{~cm}^{-1}$ ein deutlicher Anteil Großblasen vorhanden ist, während bei $\rho_{\mathrm{G}}=23,3 \mathrm{~kg} \mathrm{~m}^{-3}$ noch bei $\mathrm{u}_{\mathrm{G}}=5 \mathrm{~cm}^{-1}$ fast nur Kleinblasen vorliegen. Dabei ist bemerkenswert, dass der Kleinblasengehalt mit der Gasgeschwindigkeit steigt, auch wenn die Grenzgasgeschwindigkeit schon überschritten ist.

In Abbildung 5-3 ist der Großblasengehalt in Abhängigkeit von der Gasdichte aufgetragen. Die Gasdichte hat keinen starken Einfluss auf den Großblasengehalt. Der Großblasengehalt scheint mit steigender Gasdichte sogar leicht abzunehmen. Dies bedeutet, dass der ansteigende Gasgehalt in Abbildung 5-1 allein auf den zunehmenden Kleinblasengehalt zurückzuführen ist. 


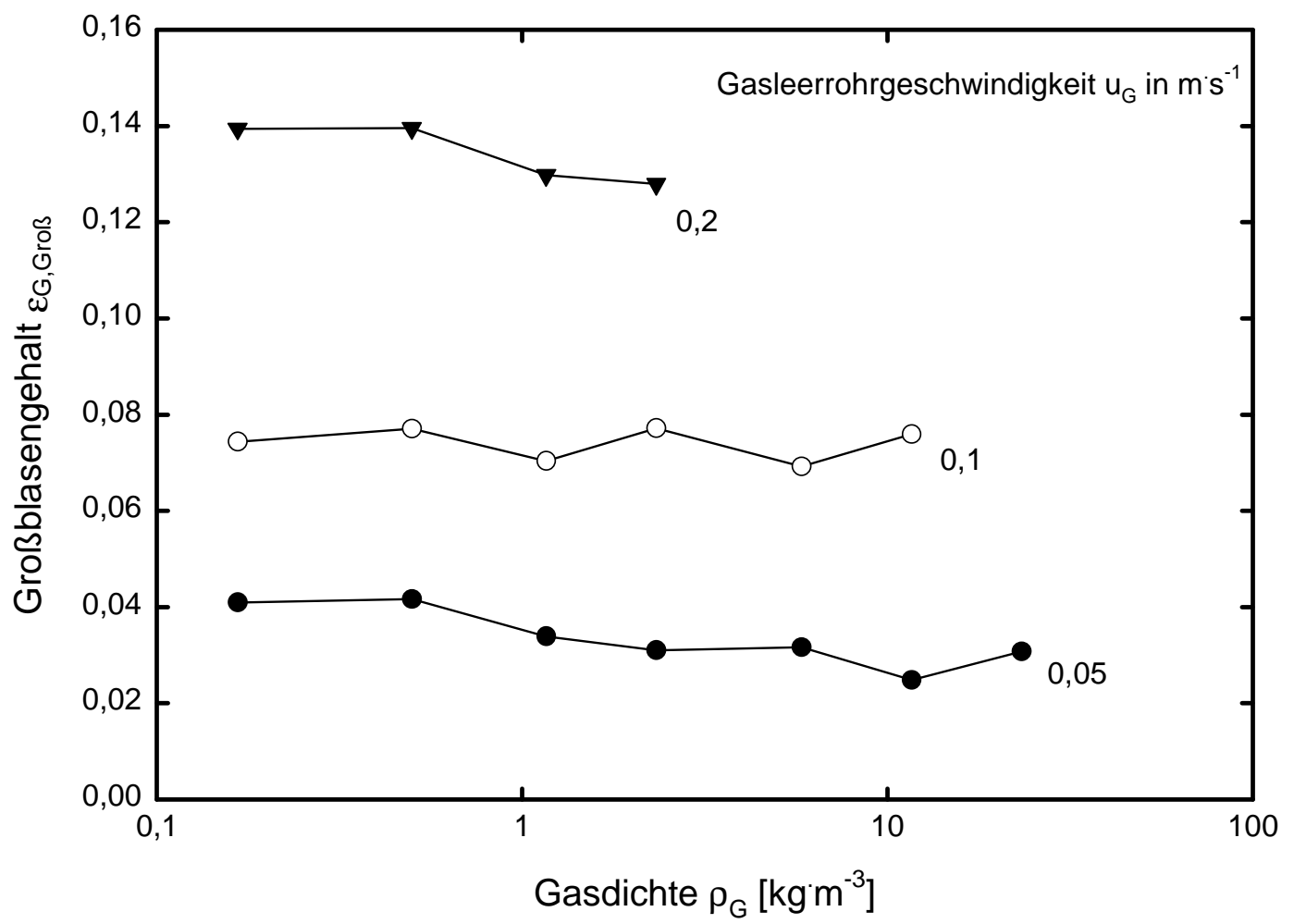

Abbildung 5-3: Großblasengehalt in Toluol

\subsubsection{Blasenaufstiegsgeschwindigkeiten}

Ebenfalls ermittelt wurden die Blasenaufstiegsgeschwindigkeiten $\mathrm{v}_{\mathrm{B}}$. Die gemessenen Kleinblasengeschwindigkeiten haben alle die gleiche Größenordnung (siehe Abbildung 5-4) und liegen bei $\mathrm{v}_{\mathrm{B}} \sim 0,2 \mathrm{~m}^{-1}$. Es ist aber zu sehen, dass die Kleinblasengeschwindigkeit mit steigender Gasdichte und steigender Gasleerrohrgeschwindigkeit abnimmt. Dies ist ein Indiz dafür, dass der Kleinblasendurchmesser sich mit diesen Parametern ebenfalls ändert. Es kann also nicht von einer konstanten Kleinblasengröße ausgegangen werden. Ebenfalls ist zu bedenken, dass es sich hier nicht um die Aufstiegsgeschwindigkeiten von Einzelblasen

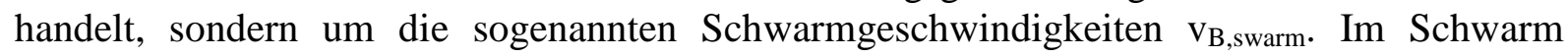
steigen Kleinblasen in der Regel langsamer auf, als Einzelblasen. Die Einzelblasengeschwindigkeit wird höher sein als die gemessenen Werte.

In Abbildung 5-5 sind die gemessenen Großblasengeschwindigkeiten aufgetragen. Zunächst ist $\mathrm{zu}$ sehen, dass die Großblasengeschwindigkeit mit steigender Leerrohrgeschwindigkeit zunimmt. Dies könnte bedeuten, dass die Großblasen mit steigender Gasgeschwindigkeit größer werden und dementsprechend schneller aufsteigen.

Die Gasdichteabhängigkeit der Großblasengeschwindigkeit lässt sich grob in zwei Abschnitte einteilen: Bei kleinen Gasdichten für $\rho_{\mathrm{G}}<2 \mathrm{~kg}^{-3}$ gibt es keinen Zusammenhang. Die Großblasen steigen bei verschiedenen Gasdichten gleich schnell auf. Bei hohen Gasdichten nimmt die Großblasengeschwindigkeit mit steigender Gasdichte ab. Dies lässt auf einen abnehmenden Durchmesser der Großblasen schließen. Extrapoliert man diesen Effekt auf noch höhere Gasdichten, so erreichen die Großblasen die Größe der Kleinblasen und sind von ihnen nicht mehr zu unterscheiden. Ab einer gewissen Gasdichte arbeitet die Blasensäule demnach nicht mehr im heterogenen Regime. 


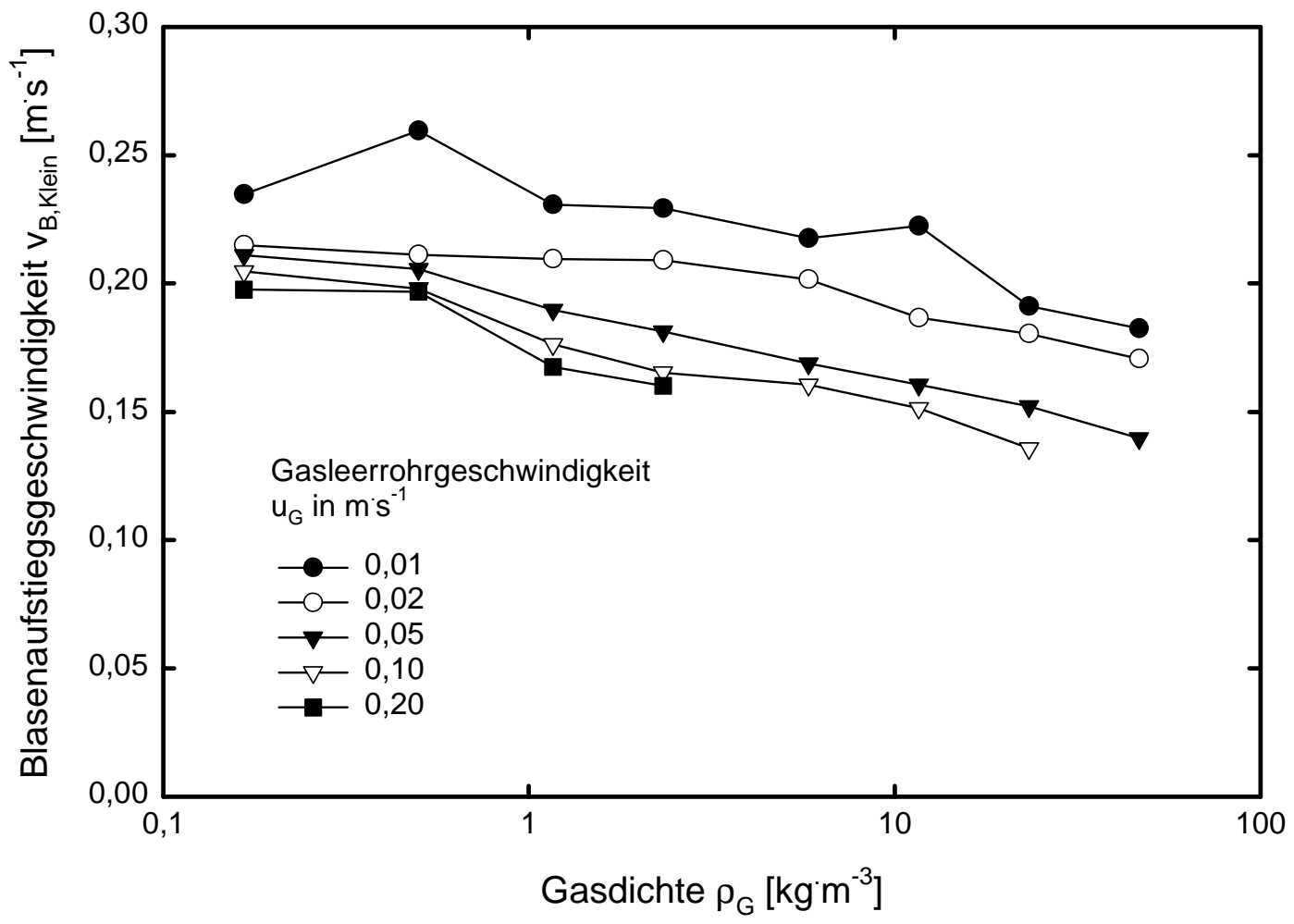

Abbildung 5-4: Kleinblasengeschwindigkeit in Toluol

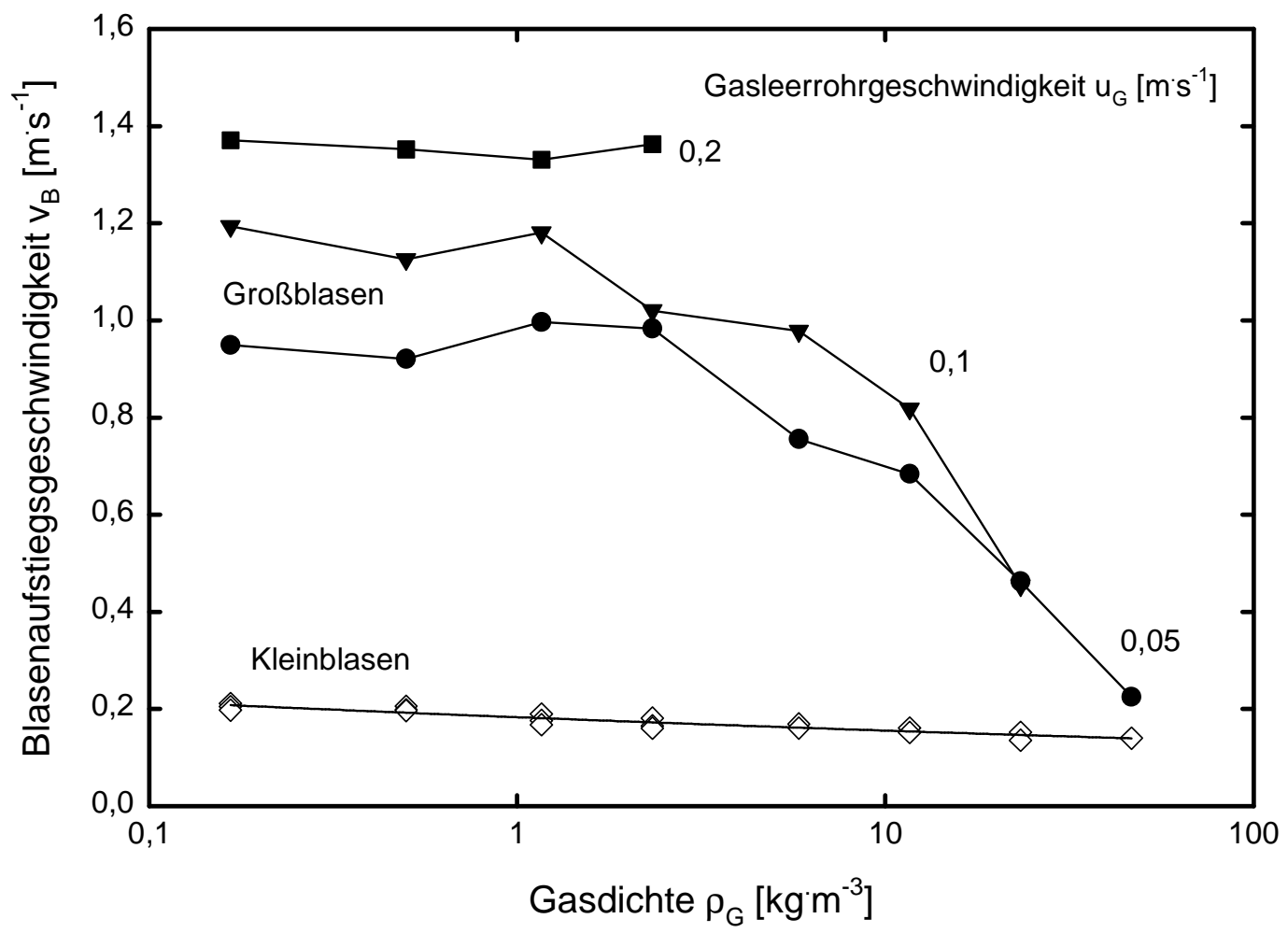

Abbildung 5-5: Großblasengeschwindigkeit in Toluol; zum Vergleich ist die Kleinblasengeschwindigkeit ebenfalls eingetragen 


\subsubsection{Einfluss der Viskosität auf den Gasgehalt}

Ethylenglycol zeigt eine starke Abhängigkeit seiner Viskosität von der Temperatur. Durch Änderung der Temperatur lässt sich die Viskosität variieren, ohne andere Parameter wesentlich zu beeinflussen (siehe Tabelle 5-1)

Tabelle 5-1: Änderung wichtiger Stoffparameter im System Ethylenglycol-Stickstoff bei Temperaturänderung

\begin{tabular}{|c|c|c|c|c|c|}
\hline Temperatur $\left[{ }^{\circ} \mathrm{C}\right]$ & $\eta_{\mathrm{L}}[\mathrm{Pa} \cdot \mathrm{s}]$ & $\eta_{\mathrm{G}}[\mathrm{Pa} \cdot \mathrm{s}]$ & $\rho_{\mathrm{L}}\left[\mathrm{kg}^{-3}{ }^{-3}\right]$ & $\rho_{\mathrm{G}}\left[\mathrm{kg} \cdot \mathrm{m}^{-3}\right]$ & $\sigma\left[\mathrm{kg} \cdot \mathrm{s}^{-2}\right]$ \\
\hline 10 & 0,0315 & $170 \cdot 10^{-06}$ & 1119 & 1,206 & 0,0487 \\
\hline 20 & 0,0205 & $175 \cdot 10^{-06}$ & 1112 & 1,165 & 0,048 \\
\hline 30 & 0,0136 & $179 \cdot 10^{-06}$ & 1105 & 1,126 & 0,0473 \\
\hline 50 & 0,0068 & $188 \cdot 10^{-06}$ & 1091 & 1,056 & 0,0458 \\
\hline 70 & 0,0039 & $196 \cdot 10^{-06}$ & 1077 & 0,995 & 0,0441 \\
\hline 80 & 0,0031 & $200 \cdot 10^{-06}$ & 1071 & 0,967 & 0,0432 \\
\hline
\end{tabular}

Das Ergebnis der Messungen ist in Abbildung 5-6 aufgetragen. Der Gasgehalt nimmt mit steigender Temperatur leicht zu. Dies entspricht den Erwartungen, da Flüssigkeiten mit hohen Viskositäten koaleszenzfördernd wirken. Eine stärkere Koaleszenz bedeutet einen höheren Großblasenanteil. Die Großblasen haben eine höhere Aufstiegsgeschwindigkeit, was zu einem geringeren Gasgehalt führt.

Allerdings kann solch eine geringe Abnahme des Gasgehaltes seine Ursache auch in der Änderung der anderen Stoffparameter, wie z. B. der Oberflächenspannung haben. Die Viskosität hat jedenfalls keinen signifikanten Einfluss auf den Gasgehalt.

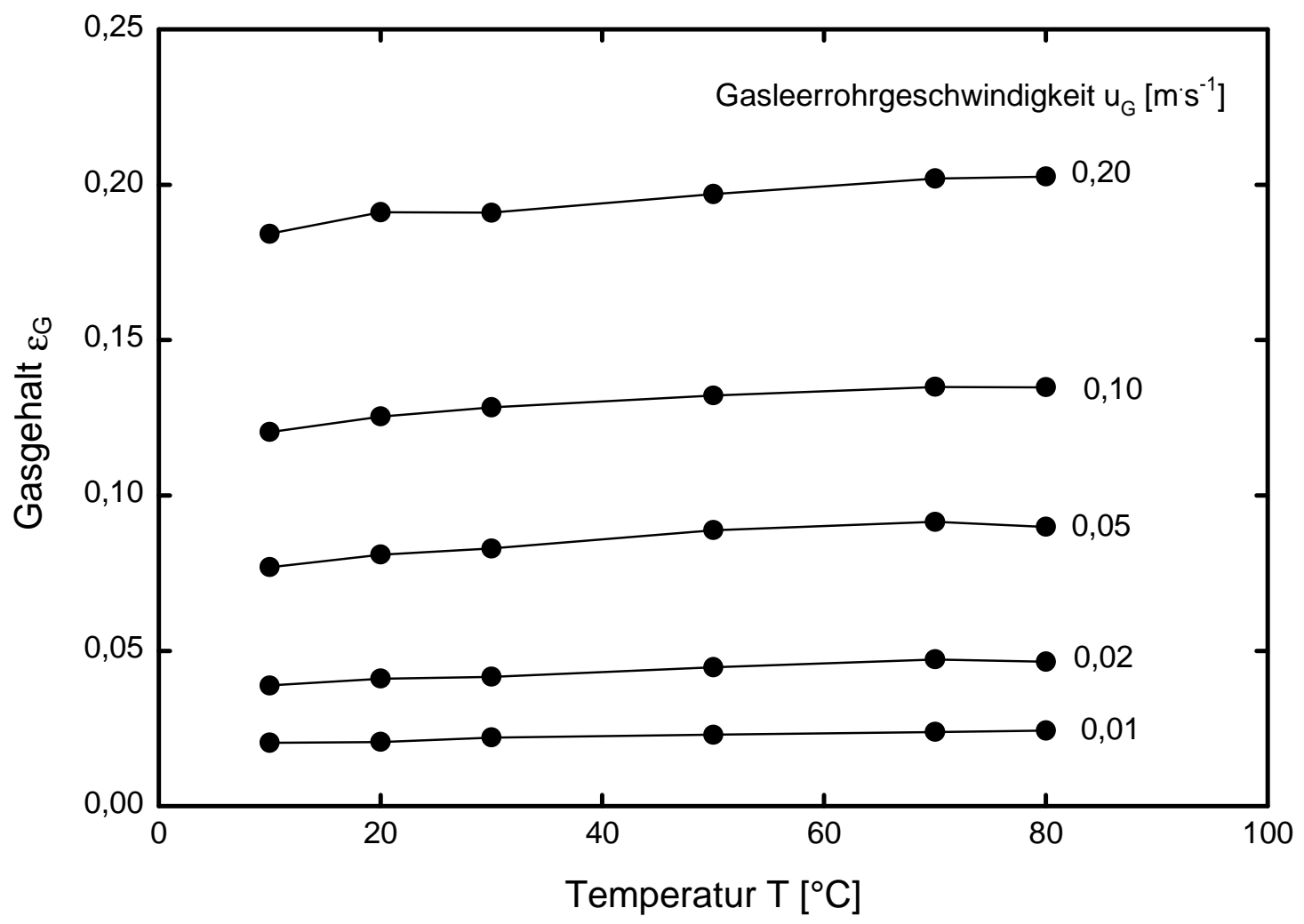

Abbildung 5-6: Einfluss der Temperatur auf den Gasgehalt in Ethylenglycol 


\subsubsection{Einfluss der Säulenhöhe auf den Gasgehalt}

In einer Blasensäule werden in der Regel drei Abschnitte unterschieden: Die Begaserzone, die Entgasungszone und die eigentliche Aufstiegszone.

In der Begaserzone verteilt sich das Gas in der Flüssigkeit. Hier erfolgt die eigentliche Blasenbildung. Die Blasen steigen durch die Aufstiegszone nach oben und verlassen die Flüssigkeit in der Entgasungszone. Die Entgasungszone ist eine mehr oder weniger stark ausgeprägte Schaumschicht mit geringem Flüssigkeitsanteil.

Der Gasgehalt in der Begaserzone weicht in der Regel von dem Gasgehalt in der restlichen Blasensäule ab.

In Abbildung 5-7 sind die Gasgehalte in Toluol in verschiedenen Höhen über dem Begaser vergleichend aufgetragen. Die Begaserzone zeigt stets einen geringeren Gasgehalt, als der Rest der Blasensäule. Wählt man als Begaser eine Sinterplatte (siehe Abbildung 5-8), dann kann die Begaserzone bei niedrigen bis mittleren Gasgeschwindigkeiten auch höhere Gasgehalte als der Rest der Blasensäule aufweisen. Bei hohen Gasgeschwindigkeiten kehrt sich das Verhältnis wieder um und die Begaserzone zeigt niedrigere Gasgehalte.

Der Gasgehalt der Begaserzone unterliegt offensichtlich zahlreichen Einflüssen, wie z.B. Begaserart, Gasdichte und Gasleerrohrgeschwindigkeit.

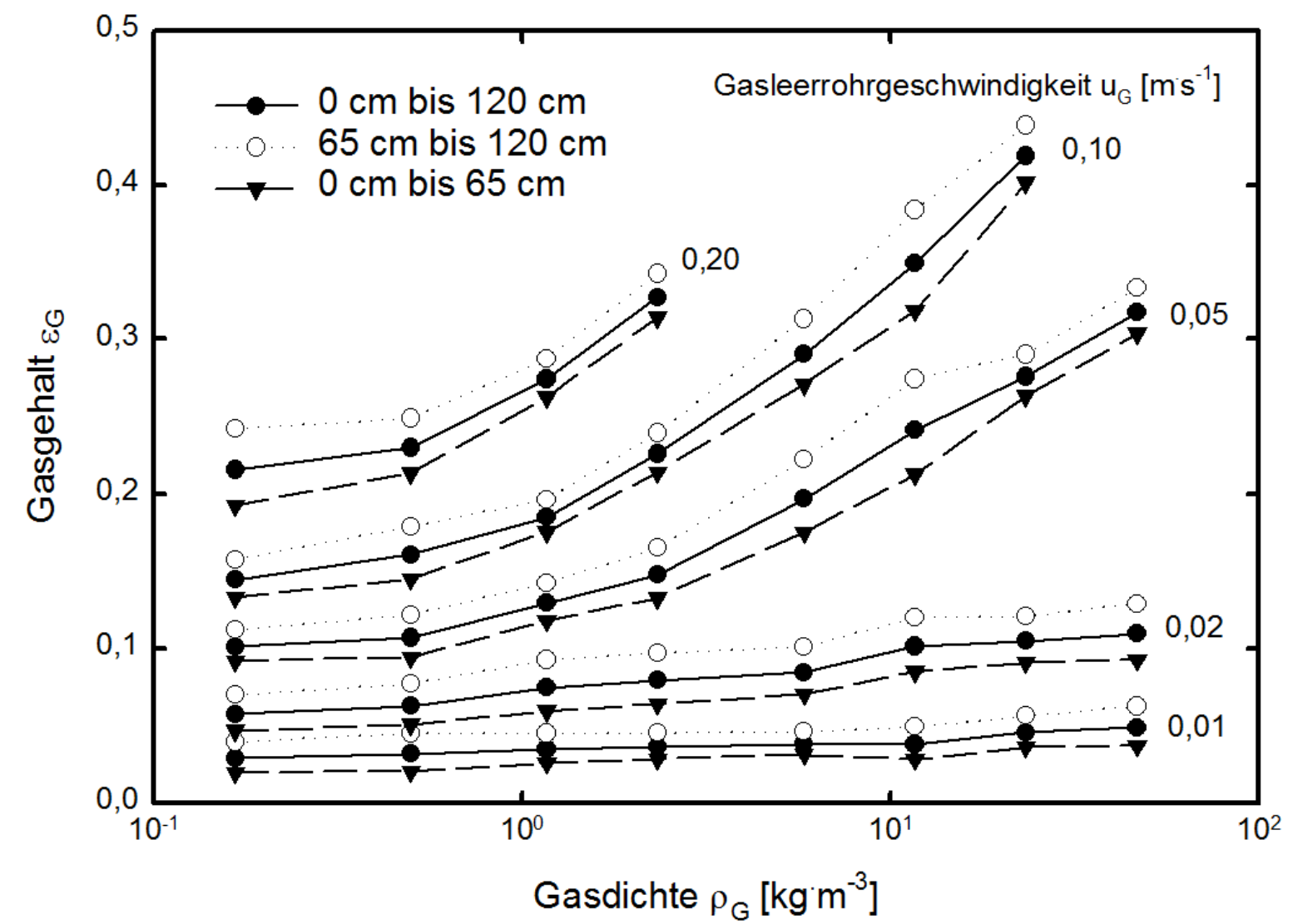

Abbildung 5-7: Gasgehalte in Toluol abhängig von der Höhe über dem Begaser (Lochplatte 19 x $1 \mathrm{~mm})$ 


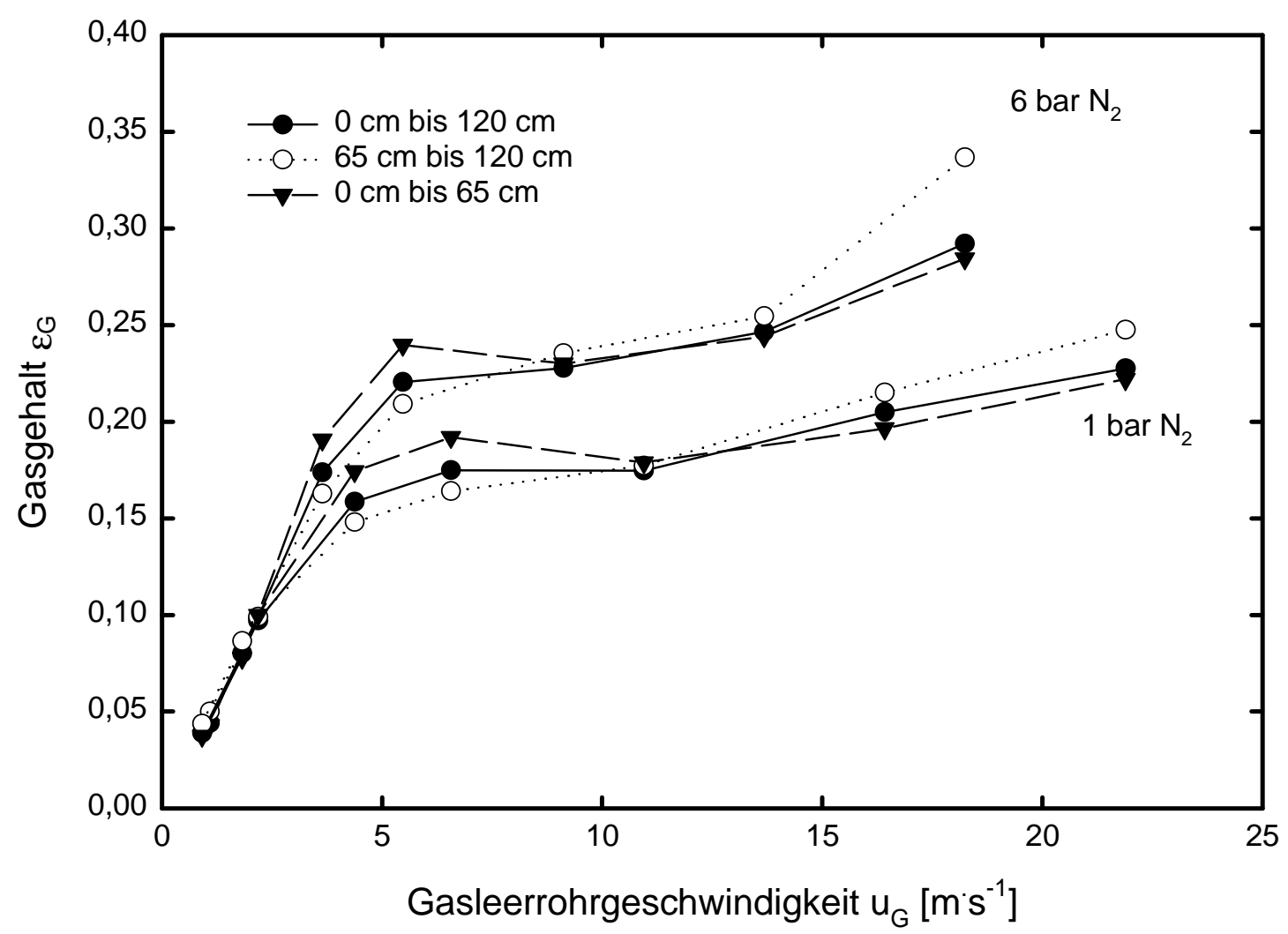

Abbildung 5-8: Gasgehalte in Wasser abhängig von der Höhe über dem Begaser (Sinterplatte)

\subsubsection{Einfluss des Begasers}

Die Art des Begasers beeinflusst die Größe der entstehenden Blasen und damit auch die Aufstiegsgeschwindigkeit, die Gasgehaltsstruktur und letztendlich den Gasgehalt in der Blasensäule. In Abbildung 5-9 sind für das System Wasser - Stickstoff die Gasgehalte für zwei verschiedene Begasertypen aufgetragen. Die Sinterplatte erzeugt erwartungsgemäß höhere Gasgehalte als der Einzellochbegaser. Der Unterschied ist bei mittleren Gasleerrohrgeschwindigkeiten um $u_{G}=5 \mathrm{~cm} / \mathrm{s}$ am größten. Der Gasgehalt für die Sinterplatte ist fast doppelt so groß wie der beim Einzellochbegaser. Bei sehr hohen Gasleerrohrgeschwindigkeiten von $\mathrm{u}_{\mathrm{G}}>20 \mathrm{~cm} / \mathrm{s}$ jedoch verschwindet dieser Unterschied. 


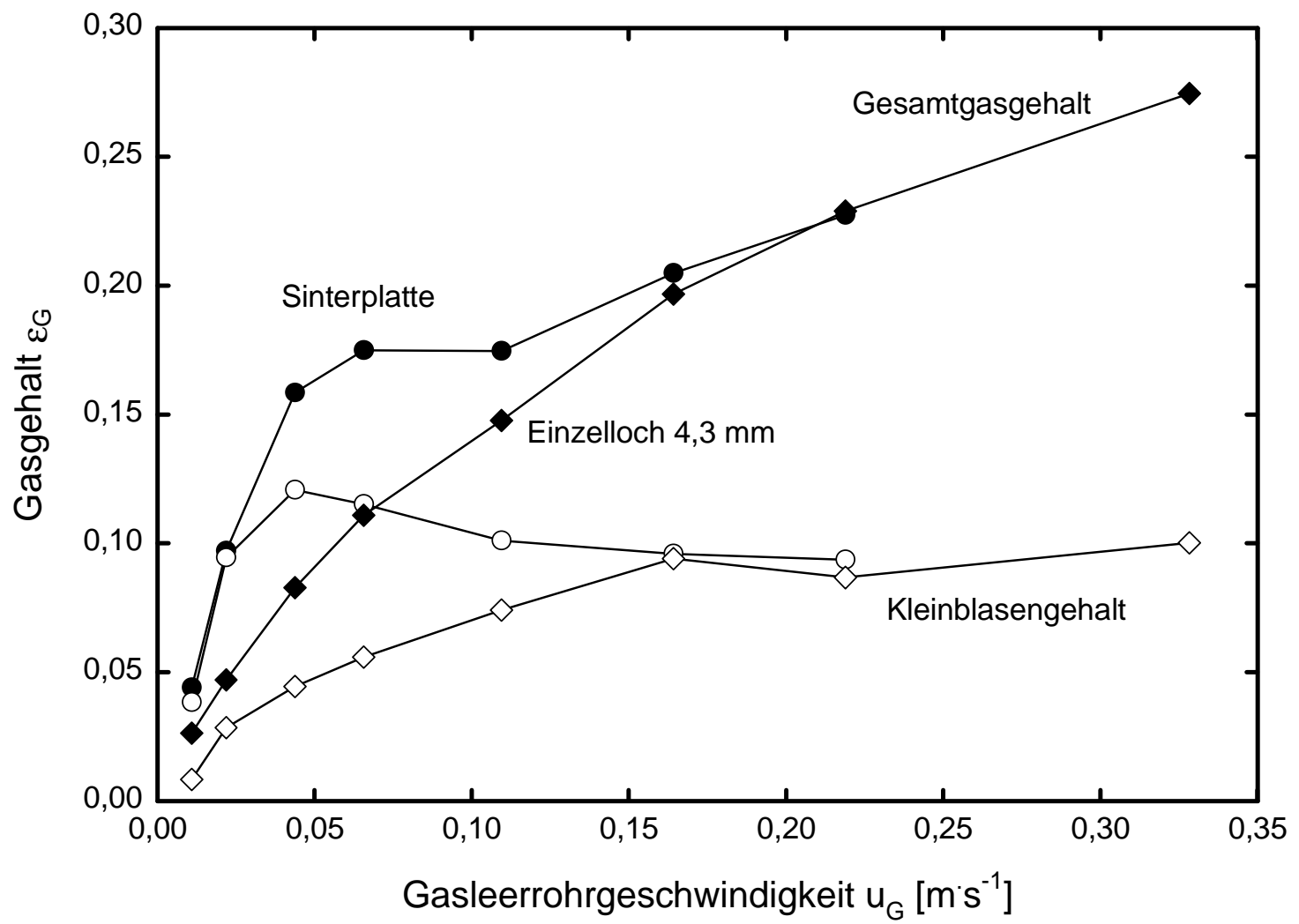

Abbildung 5-9: Gasgehalt und Gasstruktur in Wasser bei $20^{\circ} \mathrm{C}$ und 1 bar $\mathrm{N}_{2}$ mit verschiedenen Begasern

In der Gasstruktur ergeben sich ebenfalls Unterschiede. Die Sinterplatte erzeugt bei mittleren Gasgeschwindigkeiten einen wesentlich höheren Kleinblasengehalt als der Einzellochbegaser. Der Großblasengehalt hingegen unterscheidet sich kaum. Die Erhöhung des Gesamtgasgehaltes ist also auf die Erhöhung des Kleinblasengehaltes zurückzuführen. Bei hohen Gasgeschwindigkeiten verschwinden diese Unterschiede wieder.

Betreibt man die Blasensäule bei höheren Gasdichten (siehe Abbildung 5-10), so sieht man, dass der Einzellochbegaser durchaus auch höhere Gasgehalte als die Sinterplatte erzeugen kann. Bei kleinen Gasgeschwindigkeiten erhält man mit der Sinterplatte höhere Gasgehalte. Arbeitet man jedoch bei hohen Gasgeschwindigkeiten $\mathrm{u}_{\mathrm{G}}>10 \mathrm{~cm} / \mathrm{s}$, so erzeugt der Einzellochbegaser die höheren Gasgehalte. Auch in diesem Fall ist der Kleinblasenanteil für die Unterschiede verantwortlich. Der Großblasengehalt zeigt unabhängig vom Begaser den gleichen Verlauf.

Zwischen Sinterplatte und Einzellochbegaser gibt es offensichtlich große Unterschiede. Andere Begaser zeigen trotz unterschiedlichem Aufbau sehr ähnliche Ergebnisse in Gasgehalt und Gasgehaltsstruktur (siehe Abbildung 5-11 und Abbildung 5-12). Es wurden eine Lochplatte mit 19 Öffnungen mit $1 \mathrm{~mm}$ Durchmesser und ein Einzellochbegaser mit $1 \mathrm{~mm}$ Öffnungsdurchmesser verwendet. Der Einzellochbegaser zeigt leicht höhere Gasgehalte als die Lochplatte. Die Unterschiede sind allerdings sehr klein. 


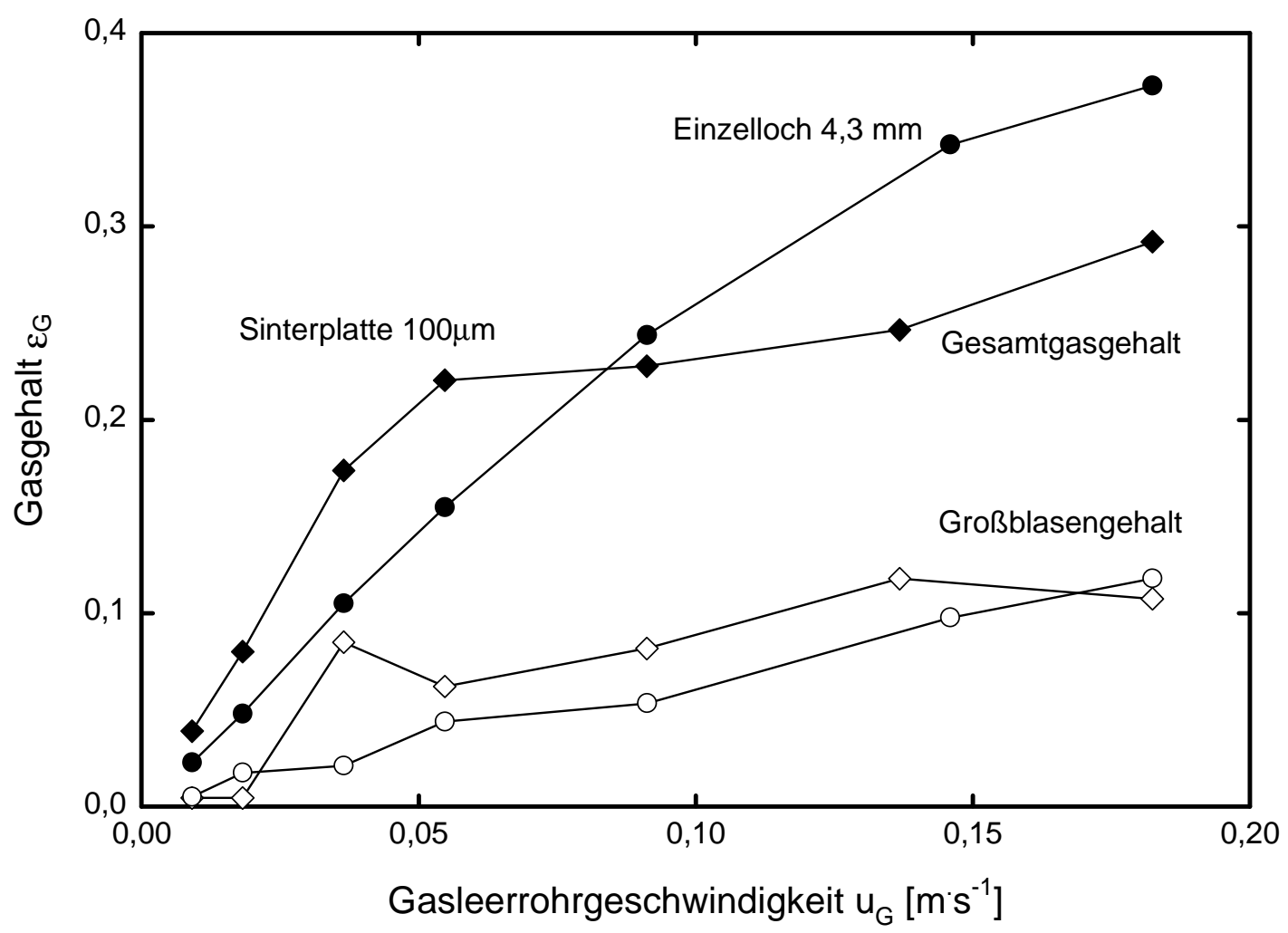

Abbildung 5-10: Gasgehalt und Gasstruktur in Wasser bei $20^{\circ} \mathrm{C}$ und 6 bar $\mathrm{N}_{2}$ mit verschiedenen Begasern

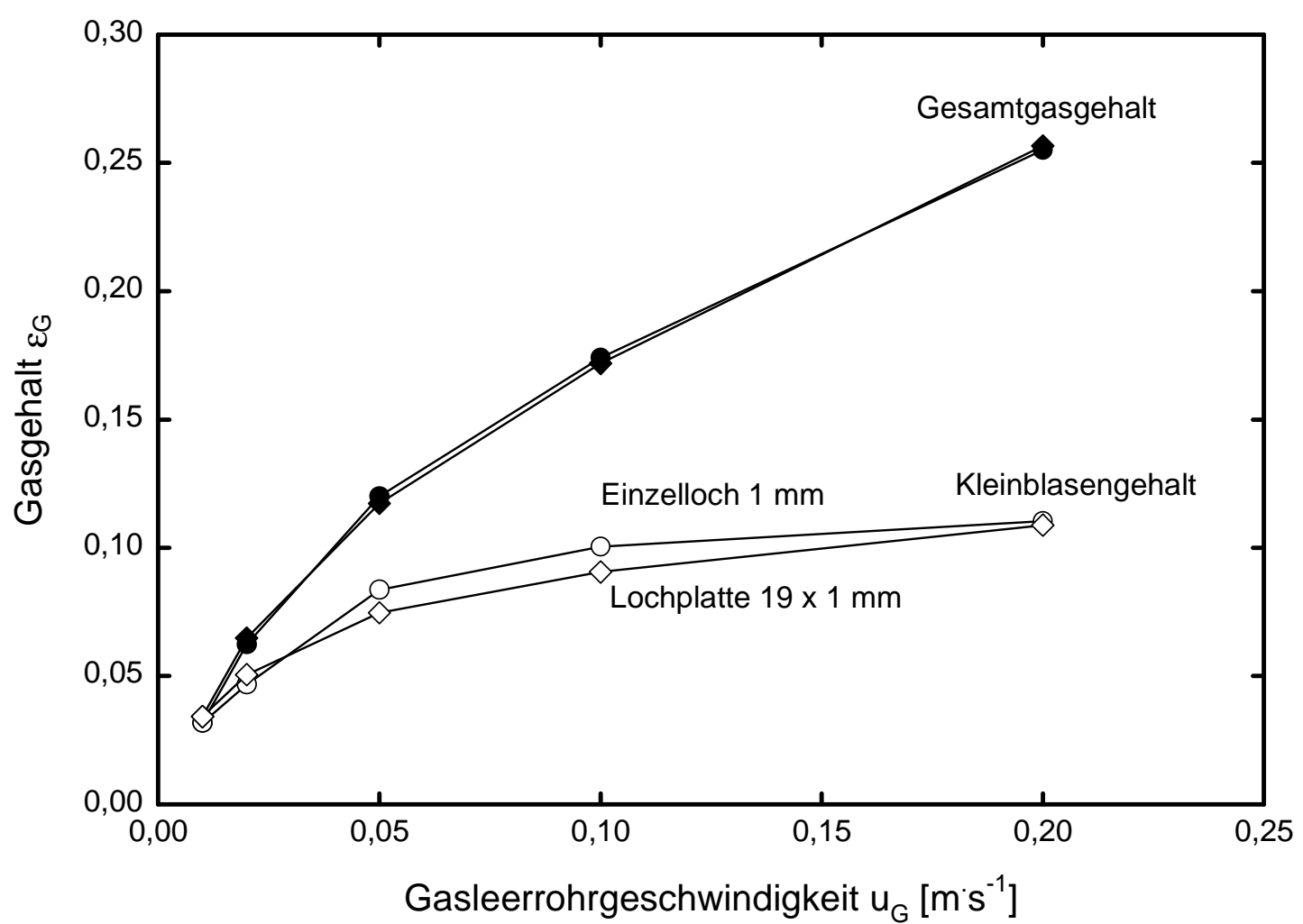

Abbildung 5-11: Gasgehalt und Gasstruktur in Ethanol bei $20^{\circ} \mathrm{C}$ und 1 bar $\mathrm{N}_{2}$ mit verschiedenen Begasern 


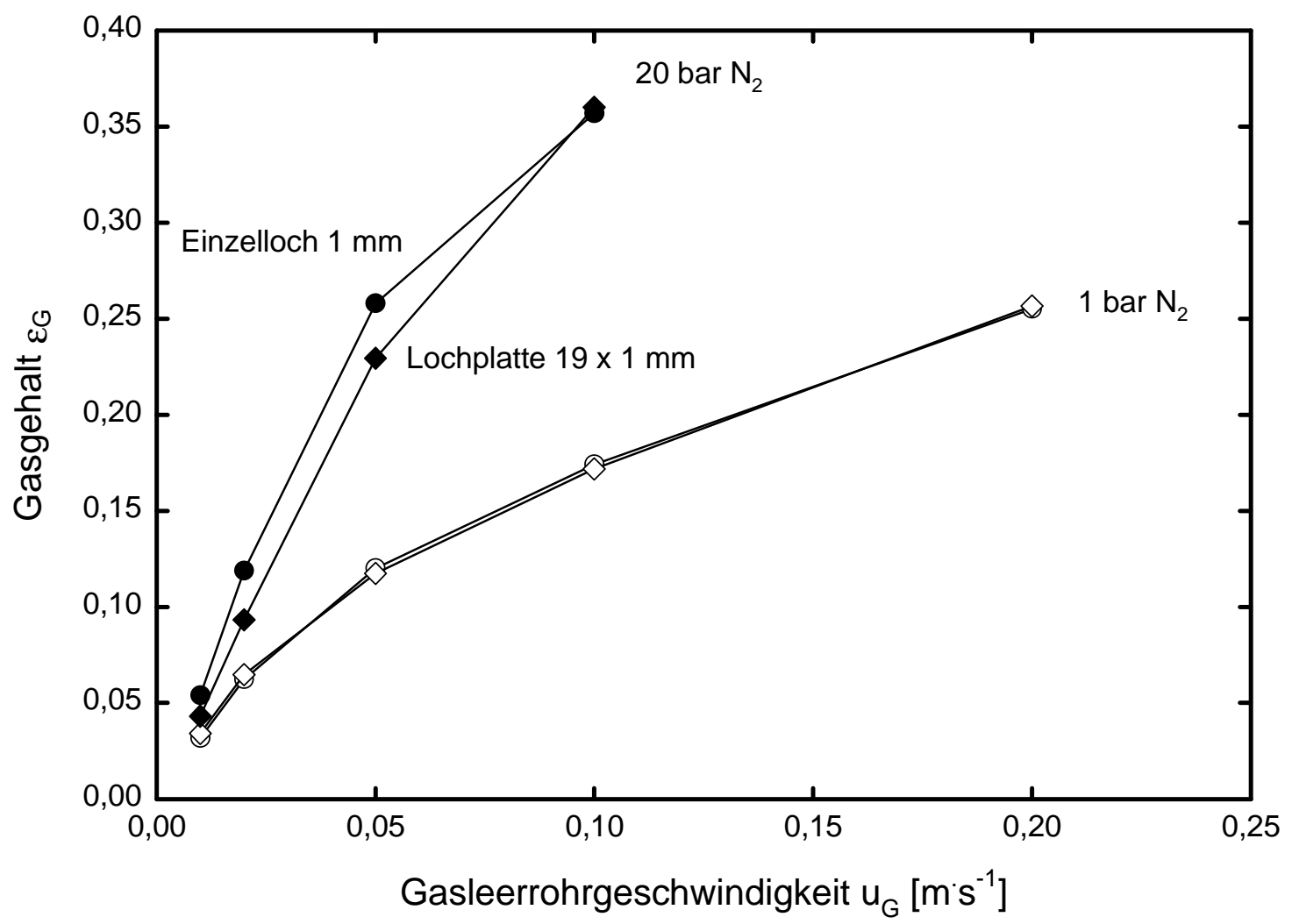

Abbildung 5-12: Gasgehalt in Ethanol bei $20^{\circ} \mathrm{C}$ und bei verschiedenen Drücken und Begasern

\subsubsection{Einfluss der Füllhöhe}

Die Flüssigkeit in der Blasensäule erzeugt einen hydrostatischen Druck, der den mittleren Druck im Dispersionsvolumen erhöht. Dadurch wird das Gas komprimiert und der Gasgehalt verringert. Dieser Effekt ist leicht zu berechnen und wird mit steigendem Kopfdruck der Blasensäule geringer.

Außerdem nimmt mit sinkender Füllhöhe der Anteil der Begaserzone am gesamten Dispersionsvolumen zu und ändert so den Gasgehalt in Abhängigkeit von der Füllhöhe.

In Abbildung 5-13 sind die Gasgehalte in Wasser bei verschiedenen Füllhöhen unter sonst gleichen Bedingungen aufgetragen. In dem gemessenen Bereich sind die Gasgehalte für beide Füllhöhen annähernd gleich. In diesem Fall ist kein Einfluss der Füllhöhe auf den Gasgehalt festzustellen.

In Abbildung 5-14 sind die Gasgehalte für 1-Butanol bei verschiedenen Füllhöhen dargestellt. Da bei einer Füllhöhe von $78 \mathrm{~cm}$ nicht mehr der Differenzdrucksensor auf $120 \mathrm{~cm}$ Höhe genutzt werden kann, musste auf den Sensor auf $65 \mathrm{~cm}$ Höhe zurückgegriffen werden. Für den niedrigeren Füllstand ergeben sich niedrigere Gasgehalte. Dies entspricht den Ergebnissen aus Abschnitt 5.1.4, nach denen in der Begaserzone kleinere Gasgehalte zu erwarten sind. Allerdings nimmt die Abweichung mit steigender Gasgeschwindigkeit stärker $\mathrm{zu}$ als erwartet. Offensichtlich spielt die Füllhöhe eine Rolle bei der Ausbildung der Begaserzone. Bei sinkendem Füllstand ist die Begaserzone stärker ausgeprägt. 


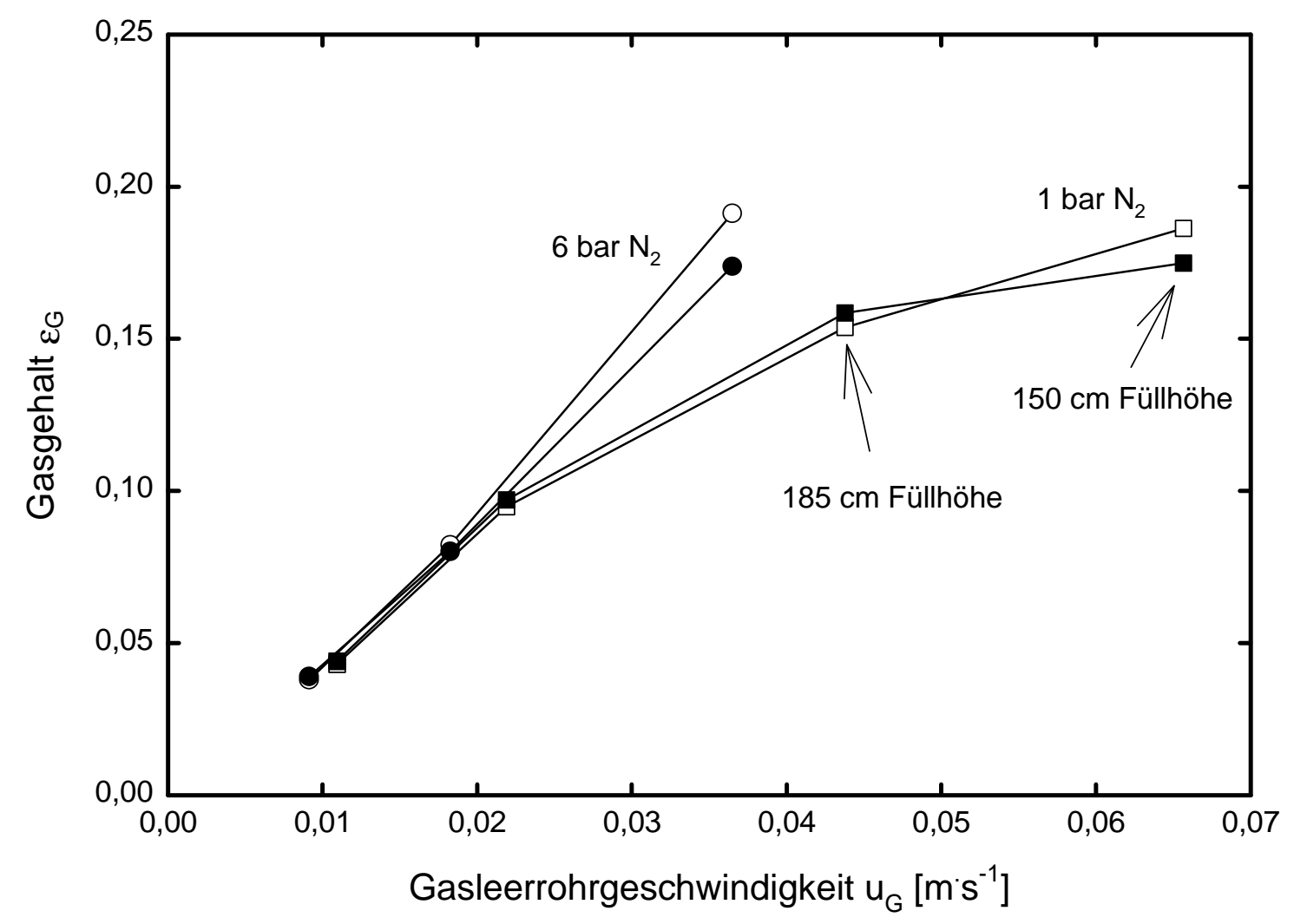

Abbildung 5-13: Gasgehalt in Wasser bei verschiedenen Füllhöhen $\left(20^{\circ} \mathrm{C}\right.$; Sinterplatte)

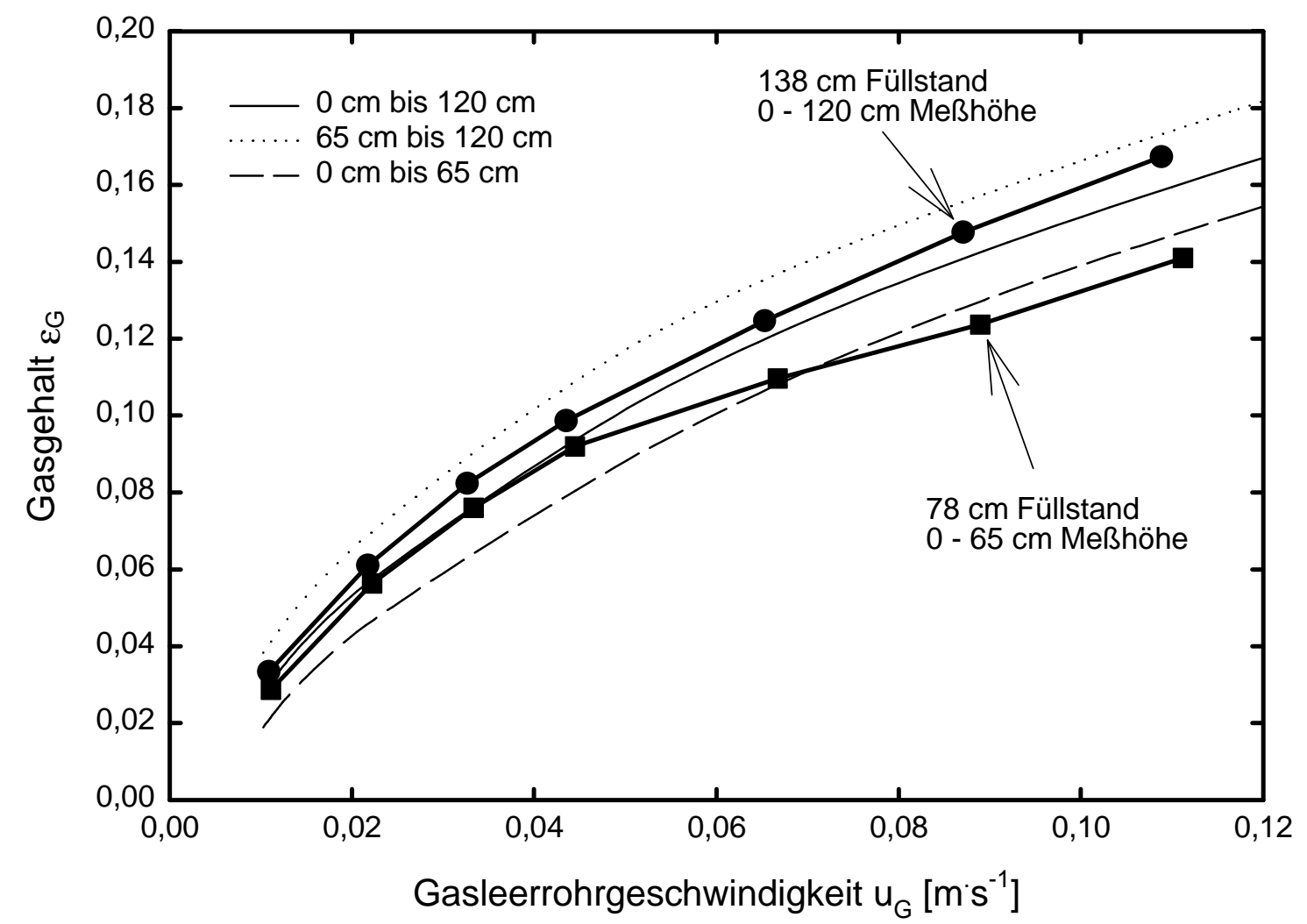

Abbildung 5-14: Gasgehalte in 1-Butanol bei verschiedenen Füllhöhen $\left(20^{\circ} \mathrm{C} ; 1\right.$ bar $\mathrm{N}_{2}$; $19 \mathrm{x} 1 \mathrm{~mm}$ ); zum Vergleich sind die Gasgehalte aus Kapitel 5.1.4 eingetragen 


\subsubsection{Einfluss der Blasensäule}

Es wurden Versuche mit zwei verschiedenen Blasensäulenreaktoren durchgeführt. Die Blasensäulen unterscheiden sich in ihrem Durchmesser, in ihrer Höhe und in ihrer Form. Außerdem kommen unterschiedliche Begaser zum Einsatz.

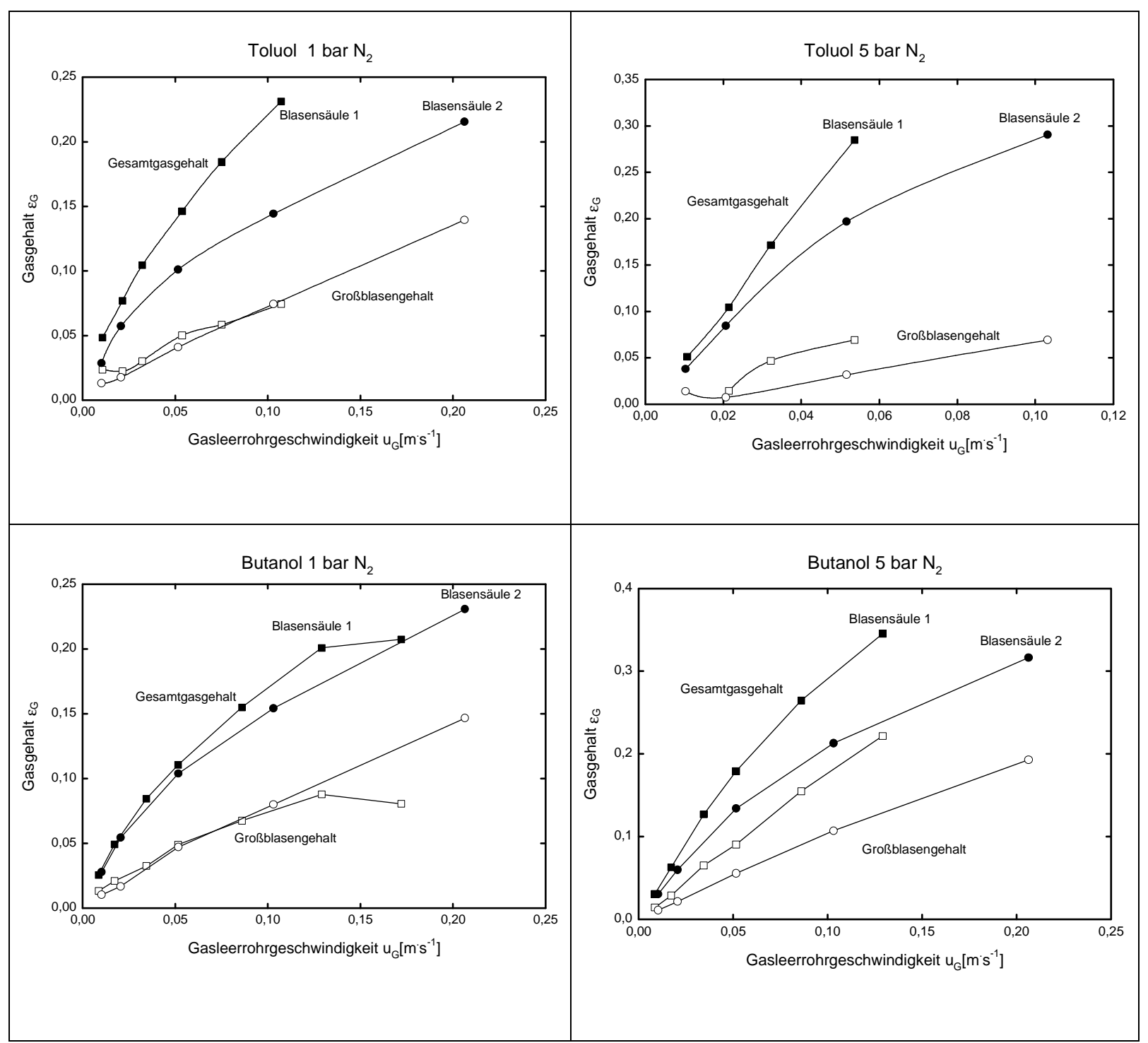

Abbildung 5-15: Vergleich von Gasgehalt und Gasstruktur in organischen Flüssigkeiten in zwei verschiedenen Blasensäulen

In Abbildung 5-15 sind die Gesamtgasgehalte und Großblasengehalte für Butanol und Toluol bei 1 bar und 5 bar Stickstoffdruck für die beiden verwendeten Blasensäulen vergleichend aufgetragen. Es ist zu sehen, dass die Blasensäule 1 in allen Fällen den größeren Gasgehalt aufweist. Dies ist ungewöhnlich, da bei Blasensäulen mit größerem Durchmesser im Allgemeinen ein kleinerer Gasgehalt entsteht.

Betrachtet man die Großblasengehalte, so sieht man, dass der Unterschied im Gasgehalt bei 1 bar Druck durch unterschiedlichen Kleinblasengehalt verursacht wird. Bei 5 bar Druck hingegen sind die unterschiedlichen Großblasengehalte für die Differenz in den Gasgehalten verantwortlich. 
Die Unterschiede im Großblasengehalt lassen sich durch die unterschiedlichen Aufstiegsgeschwindigkeiten erklären (siehe Abbildung 5-16 und Abbildung 5-17). Bei erhöhtem Druck sind die Großblasengeschwindigkeiten in der Blasensäule 2 höher als die der Blasensäule 1. Bei niedrigem Druck sind die Unterschiede kleiner bzw. kehren sich bei Butanol und hohen Gasgeschwindigkeiten um.

Die Unterschiede in den Großblasengeschwindigkeiten sind vermutlich bedingt durch die Bildungsgeschwindigkeiten der Großblasen. Die am Begaser entstandenen Kleinblasen müssen zu Großblasen koaleszieren. Zur Bildung einer Großblase mit dem Zehnfachen des Kleinblasendurchmessers sind tausend Kleinblasen erforderlich. Bei niedrigen Messhöhen haben die Großblasen das Messvolumen bereits verlassen, bevor sie ihre endgültige Größe erreicht haben. Das Messvolumen endet bei der Blasensäule 1 bereits $91 \mathrm{~cm}$ über dem Begaser und bei Blasensäule 2 bei $120 \mathrm{~cm}$ über dem Begaser. Bei diesen Messhöhen bleibt den Großblasen lediglich ein Zeitraum von $1-2 \mathrm{~s}$ bevor sie das Messvolumen verlassen. $\mathrm{Ob}$ diese Zeit ausreicht, um zur endgültigen Größe zu wachsen, ist fraglich. Gleichzeitig ist die Messgenauigkeit der Großblasengeschwindigkeit bei einer zeitlichen Auflösung von 0,1 s bei hohen Geschwindigkeiten nicht sehr hoch.

Die Kleinblasengeschwindigkeiten unterscheiden sich in beiden Blasensäulen kaum voneinander.

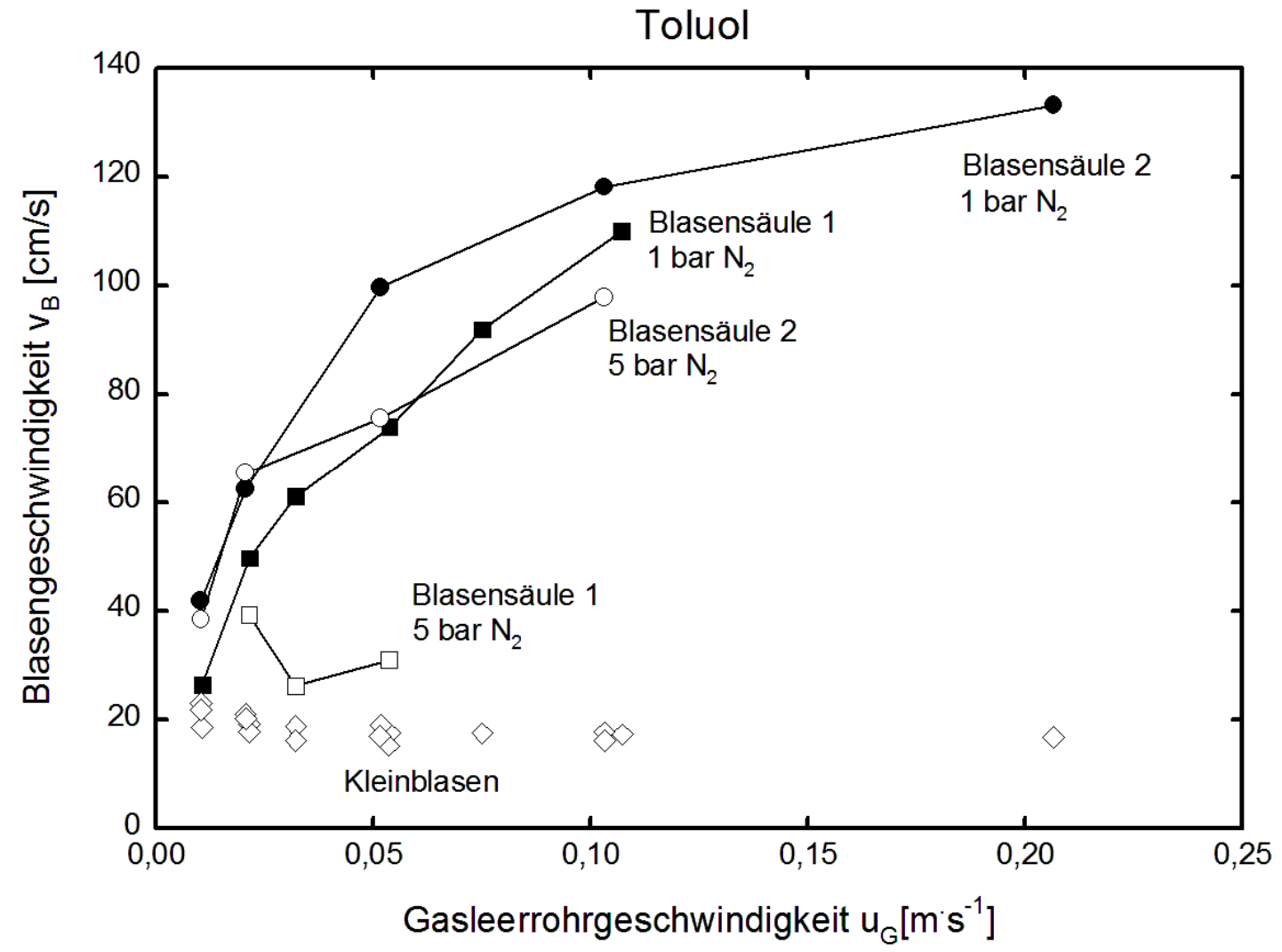

Abbildung 5-16: Blasengeschwindigkeiten in Toluol 


\section{Butanol}

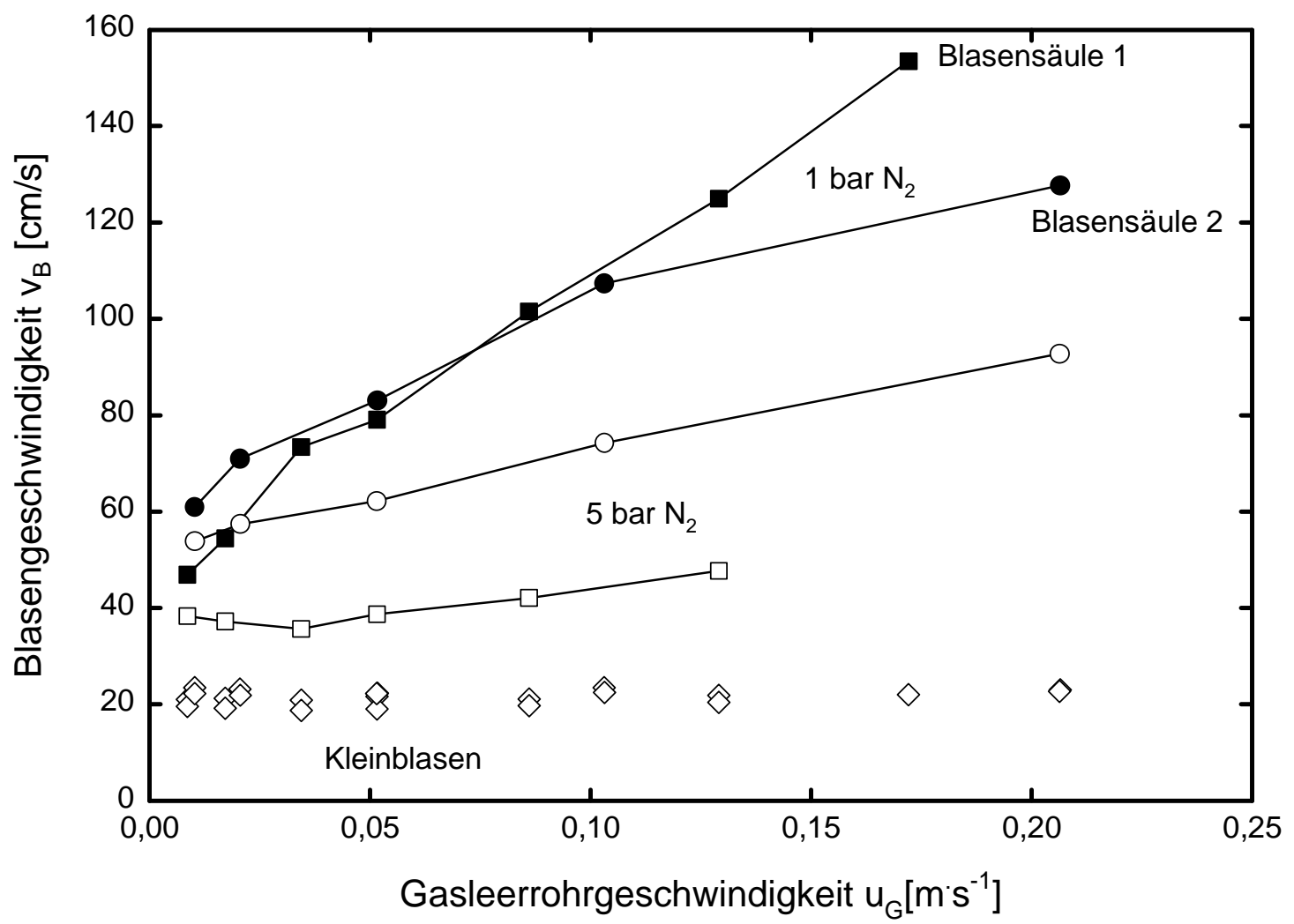

Abbildung 5-17: Blasengeschwindigkeiten in 1-Butanol

\subsection{Desorptionsmessungen}

\subsubsection{Einfluss von Gasdichte und Gasleerrohrgeschwindigkeit}

In Abbildung 5-18 sind die gemessenen Desorptionsraten $\mathrm{k}_{\mathrm{L}} \mathrm{a}^{*}$ für 1-Butanol aufgetragen. Der Stoffübergang steigt mit zunehmender Gasdichte und zunehmender Gasleerrohrgeschwindigkeit. Dies entspricht den Erwartungen, die sich aus früheren Arbeiten ergeben. Allerdings erlaubt der $\mathrm{k}_{\mathrm{L}}{ }{ }^{*}$-Wert noch keine genauen Aussagen über den tatsächlichen $\mathrm{k}_{\mathrm{L}} \mathrm{a}-$ Wert, da noch eine recht komplizierte Korrektur vorgenommen werden muss. Dazu müssen für den heterogenen Betriebszustand die Blasendurchmesser bekannt sein. Vor der eigentlichen Interpretation der Ergebnisse sind noch einige Rechnungen nötig. 


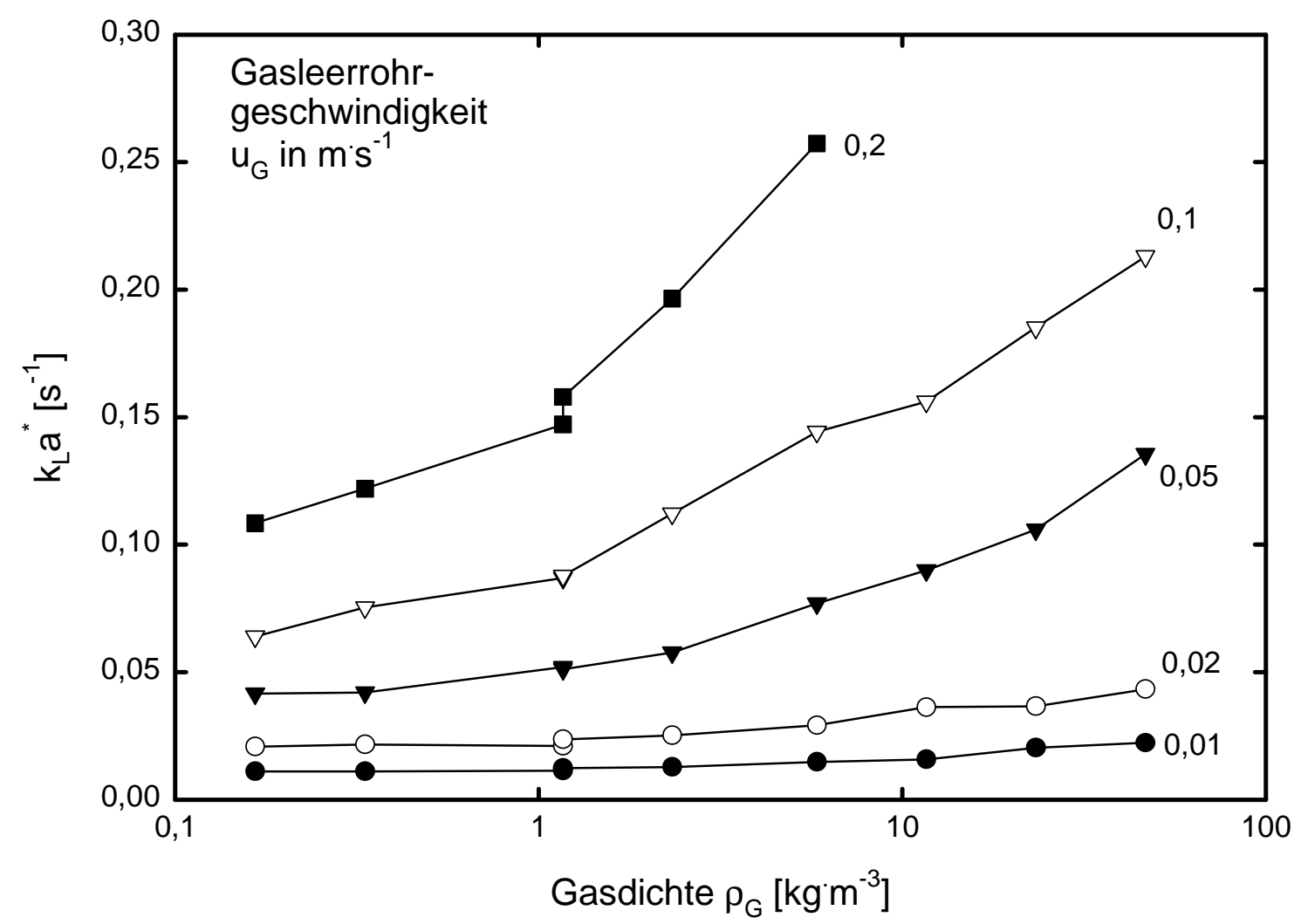

Abbildung 5-18: Desorptionsrate $\mathrm{k}_{\mathrm{L}} \mathrm{a}^{*}$ in 1-Butanol

\subsubsection{Einfluss der Sensorhöhe}

Der Sauerstoffsensor wurde im Normalfall $65 \mathrm{~cm}$ über dem Begaser positioniert. Zur Überprüfung, ob die Sensorposition das Ergebnis beeinflusst, wurden Messungen mit dem Sauerstoffsensor $120 \mathrm{~cm}$ über dem Begaser unter sonst gleichen Bedingungen gemacht. In Abbildung 5-19 sind die Ergebnisse aufgetragen. Die Sensorposition beeinflusst das Ergebnis nicht. Die Durchmischung der flüssigen Phase ist also gut genug, um keine axialen Konzentrationsgradienten entstehen zu lassen. 


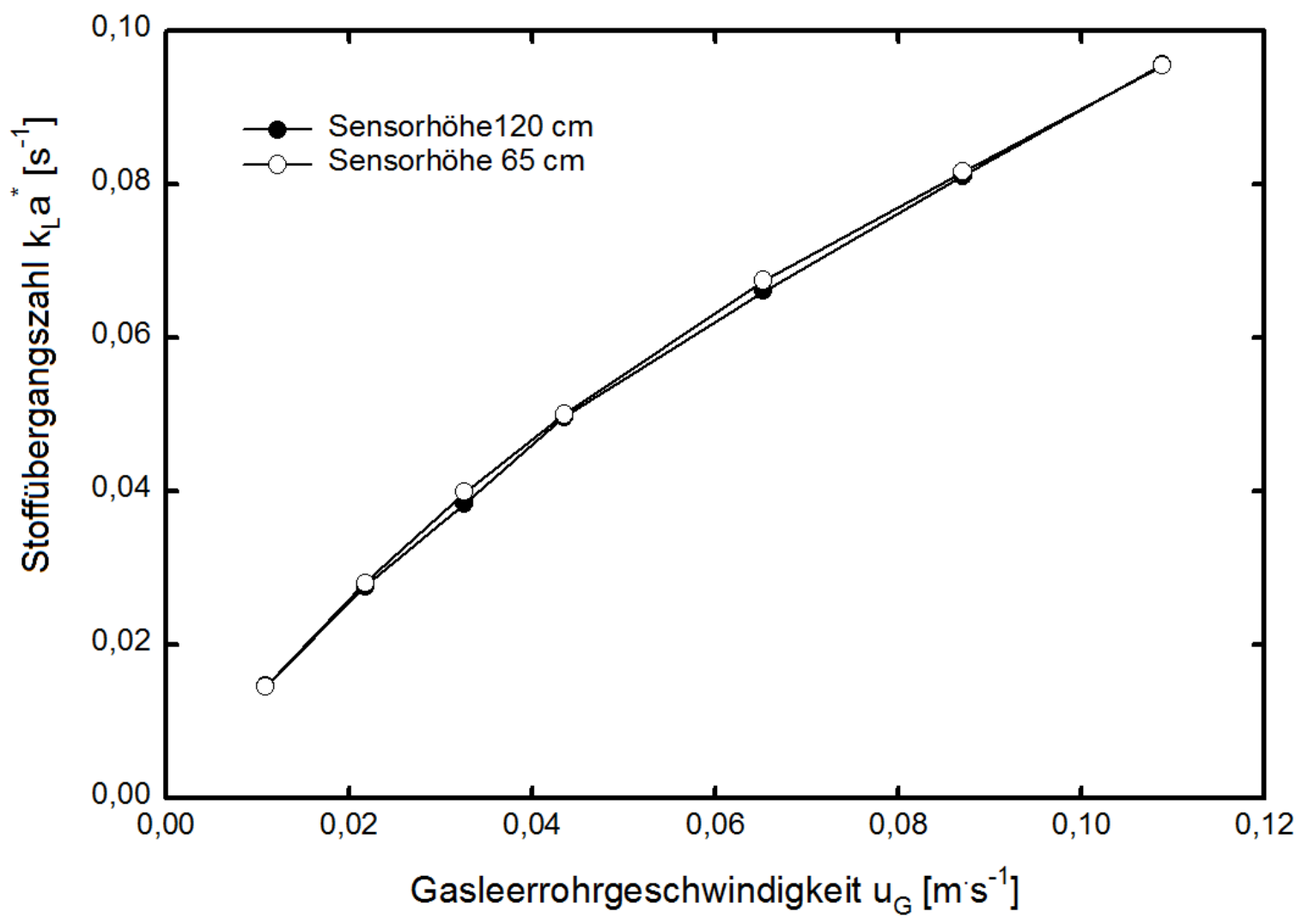

Abbildung 5-19: Einfluss der Sensorhöhe auf die gemessenen Stoffübergangszahlen in 1Butanol $\left(1\right.$ bar $\left.\mathrm{N}_{2} ; 20^{\circ} \mathrm{C} ; 19 \times 1 \mathrm{~mm}\right)$

\subsubsection{Einfluss der Viskosität}

Der geschwindigkeitsbestimmende Schritt beim Stoffübergang ist die Diffusion durch die flüssigkeitsseitige laminare Grenzschicht. Die Diffusionsgeschwindigkeit nimmt mit steigender Viskosität der Flüssigkeit ab.

Zur Änderung der Viskosität wurde Ethylenglycol bei verschiedenen Temperaturen eingesetzt. Die Ergebnisse sind in Abbildung 5-20 dargestellt. Die Desorptionsrate nimmt mit steigender Temperatur stark zu. In Tabelle 5-1 sind die entsprechenden Stoffparameter für die verschiedenen Temperaturen aufgelistet. Für die Viskosität ergibt sich folgender Zusammenhang:

$\mathrm{k}_{\mathrm{L}} \mathrm{a}^{*} \propto \mu_{\mathrm{L}}^{\mathrm{x}}$

Wobei der Exponent $\mathrm{x}$ je nach Gasleerrohrgeschwindigkeit zwischen $\mathrm{x}=-0,83$ und $\mathrm{x}=-0,91$ liegt. Die Desorptionsrate ist nahezu umgekehrt proportional zur Viskosität.

In Abbildung 5-21 sind für verschiedene organische Flüssigkeiten die Stoffübergangszahlen als Funktion der Viskosität aufgetragen. Bei hohen Viskositäten nimmt die Stoffübergangszahl mit steigender Viskosität ab. Bei niedrigen Viskositäten schwächt sich dieser Einfluss ab und scheint sich unterhalb einer gewissen Schwelle sogar umzukehren. Allerdings wird hier der Einfluss anderer Stoffparameter, wie der Oberflächenspannung, ignoriert.

Im Gegensatz zum Gasgehalt hängt der Stoffübergang stark von der Viskosität ab. 


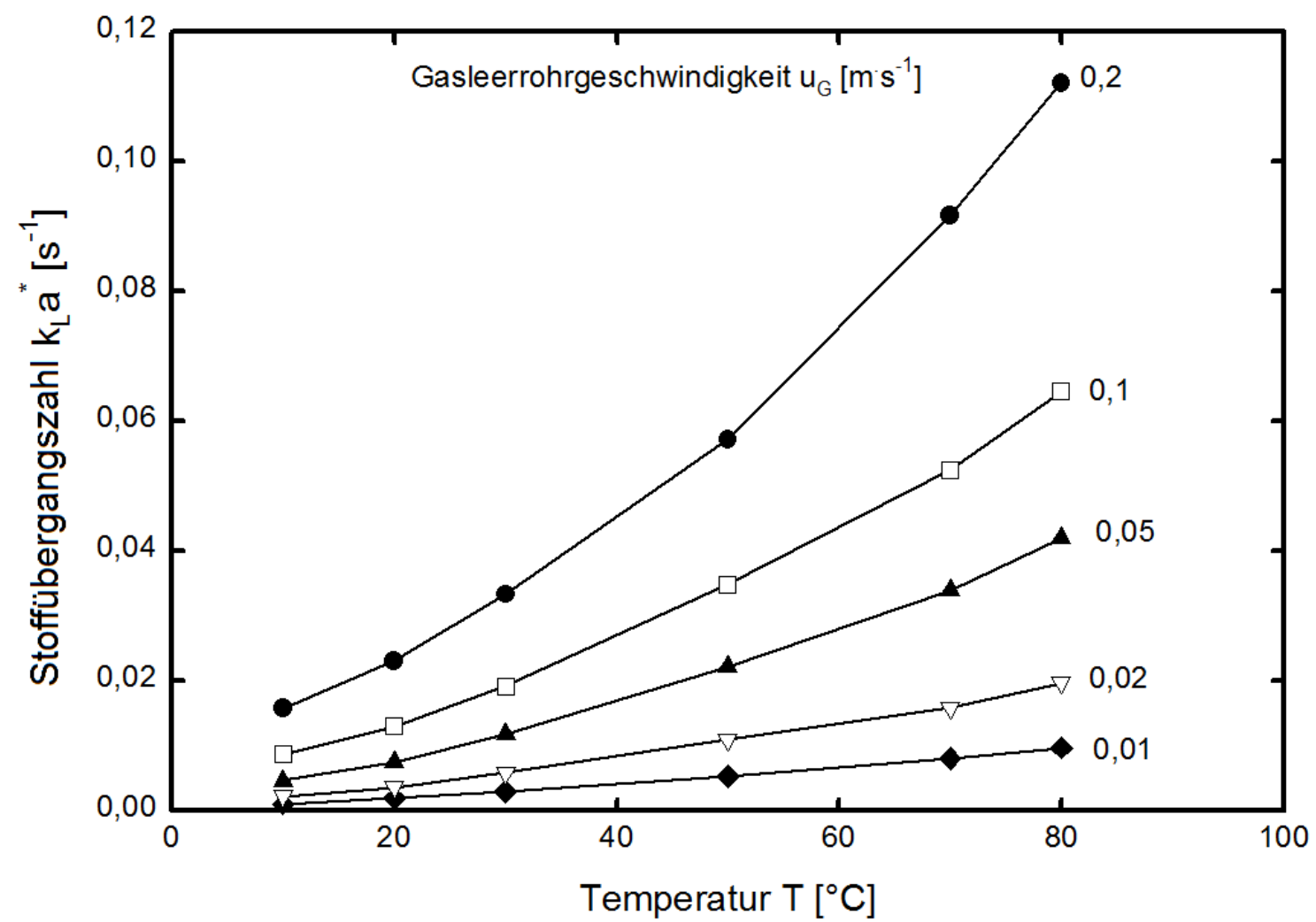

Abbildung 5-20: Änderung der Stoffübergangszahl in Ethylenglycol mit der Temperatur bei 1 bar $\mathrm{N}_{2}$

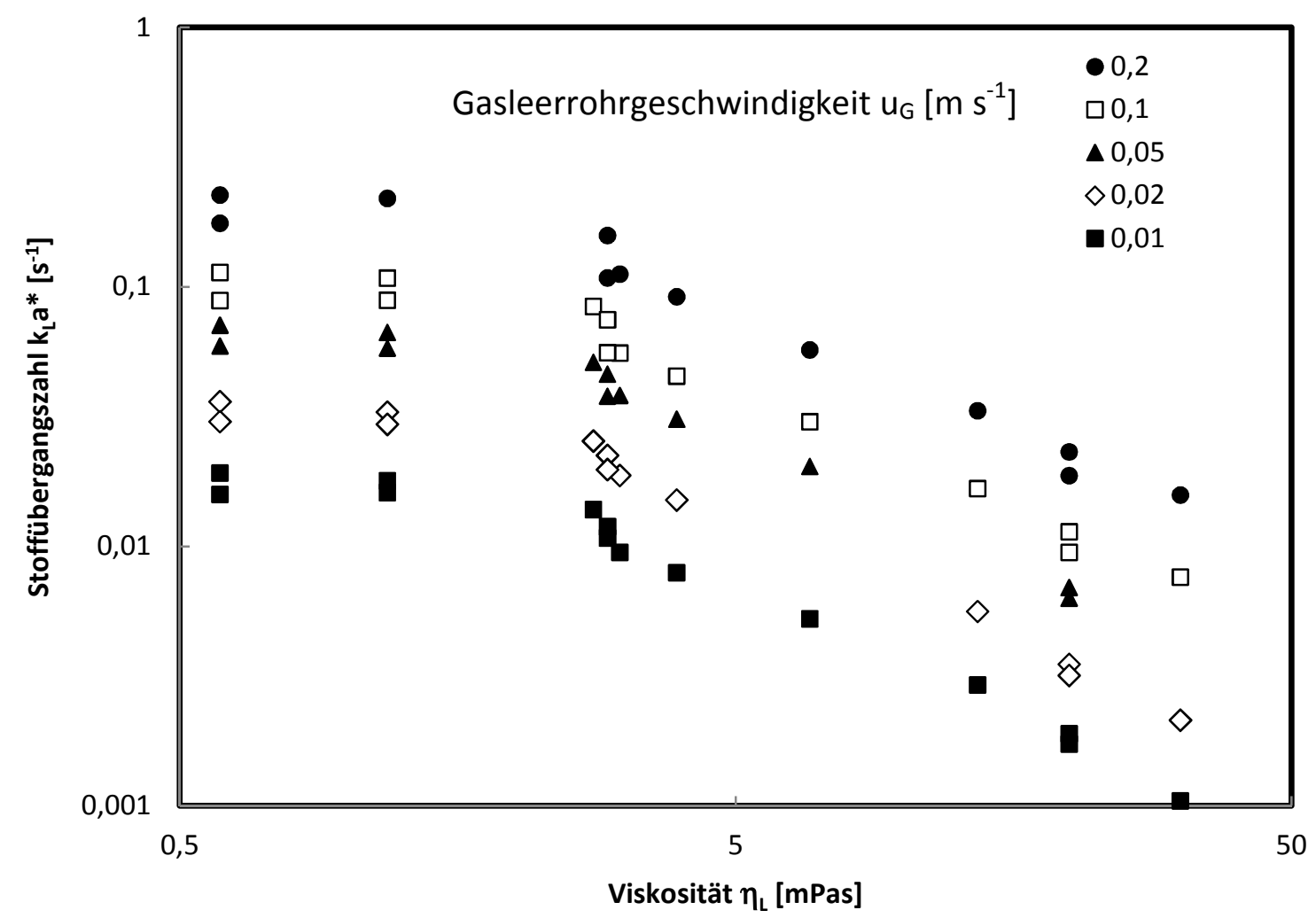

Abbildung 5-21: Änderung der Stoffübergangszahl mit der Viskosität der Flüssigkeit (Toluol, Ethanol, Decalin, 1-Butanol und Ethylenglycol); Einzellochbegaser 4,3 mm 


\subsubsection{Einfluss des Begasers}

Die Art des Begasers beeinflusst die Größe der Primärblasen. Kleinere Blasen erzeugen bei gleichen Gasgehalten größere spezifische Phasengrenzflächen a. Ein Gasverteiler, der kleinere Blasen erzeugt, sollte demnach auch höhere Stoffübergangszahlen erreichen. In Abbildung 5-22 sind die Messungen in Ethanol dargestellt. Die Begaser zeigen einen schwachen Trend: in der Reihenfolge 1 x 4,3 mm $<19$ x $1 \mathrm{~mm}<1$ x $1 \mathrm{~mm}$ nimmt der Stoffübergang leicht zu. Der Effekt ist jedoch schwach und liegt innerhalb der Fehlerbreite der Stoffübergangsmessungen. Die Zunahme des Stoffüberganges ist ähnlich gering wie die Zunahme des Gasgehaltes (siehe Abbildung 5-12) mit Änderung des Begasers.

Der Gasverteiler hat in der hier verwendeten Anordnung keinen signifikanten Einfluss.

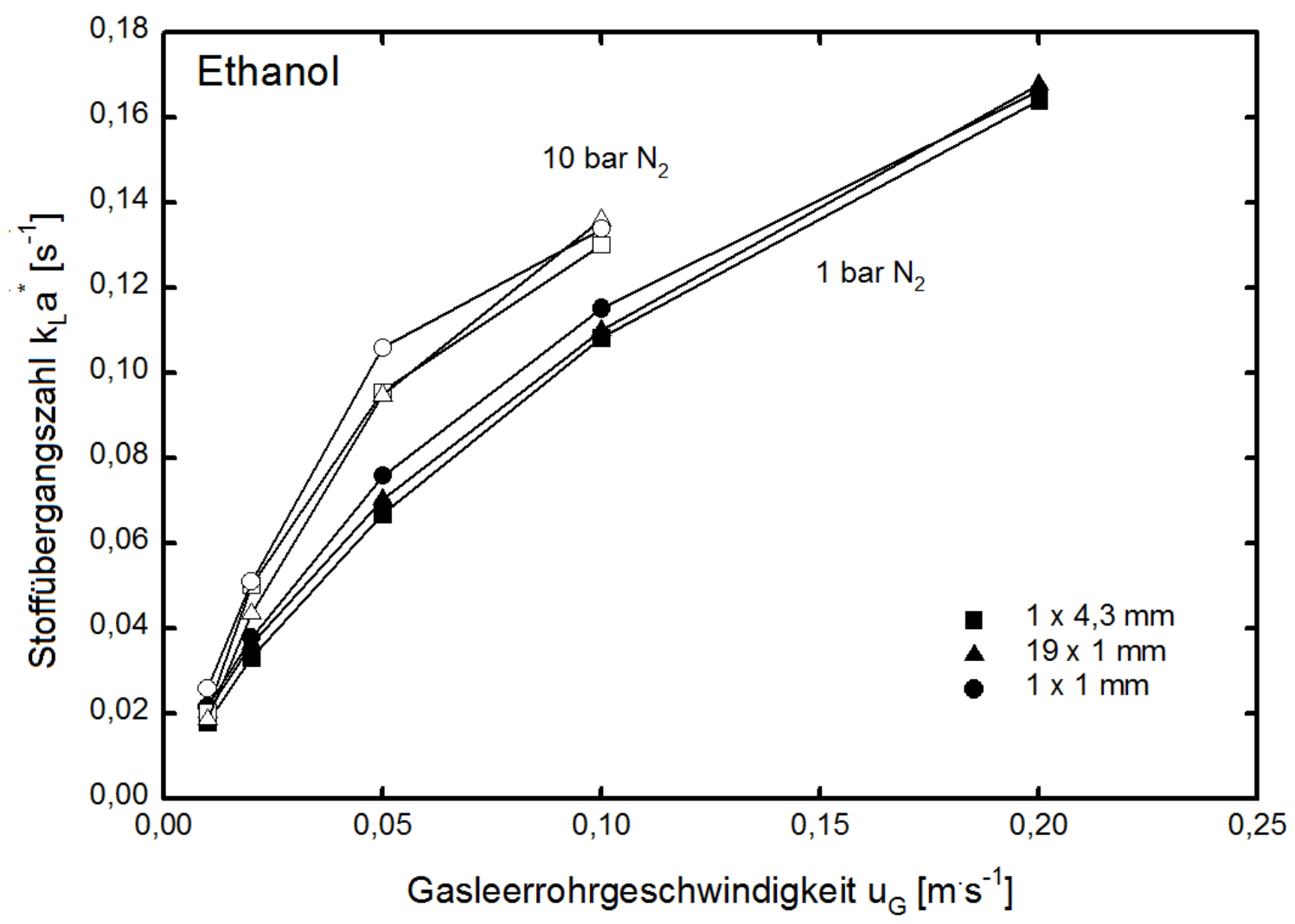

Abbildung 5-22: Vergleich der Stoffübergangszahlen in Ethanol mit verschiedenen Begasern

\subsubsection{Einfluss der Füllhöhe}

Unterschiedliche Füllhöhen der Blasensäule entsprechen unterschiedlichen Flüssigkeitsvolumina. Die Sauerstoffmenge, die die flüssige Phase aufnehmen und abgeben kann ist proportional zu ihrem Volumen. Steigt die Flüssigkeitsmenge an, so ist die Sauerstoffmenge die der Gasstrom aus dem Dispersionsvolumen transportieren muss um die gleiche Desorptionsrate zu erreichen, dementsprechend größer. Gesetzt den Fall, dass sich die aufsteigenden Gasblasen schnell mit Sauerstoff sättigen, nimmt die Desorptionsrate mit steigender Füllhöhe ab. In Abbildung 5-23 sind die Ergebnisse der entsprechenden Versuche dargestellt. Die Stoffübergangszahl nimmt mit steigender Füllhöhe erwartungsgemäß ab.

Dies ist umso bemerkenswerter, da der Gasgehalt in der gleichen Richtung zunimmt (siehe Abbildung 5-14). Dies bedeutet, dass der Gasstrom schon bei den relativ geringen Füllhöhen von $135 \mathrm{~cm}$ stark mit Sauerstoff gesättigt ist, wenn er das Dispersionsvolumen verlässt. 


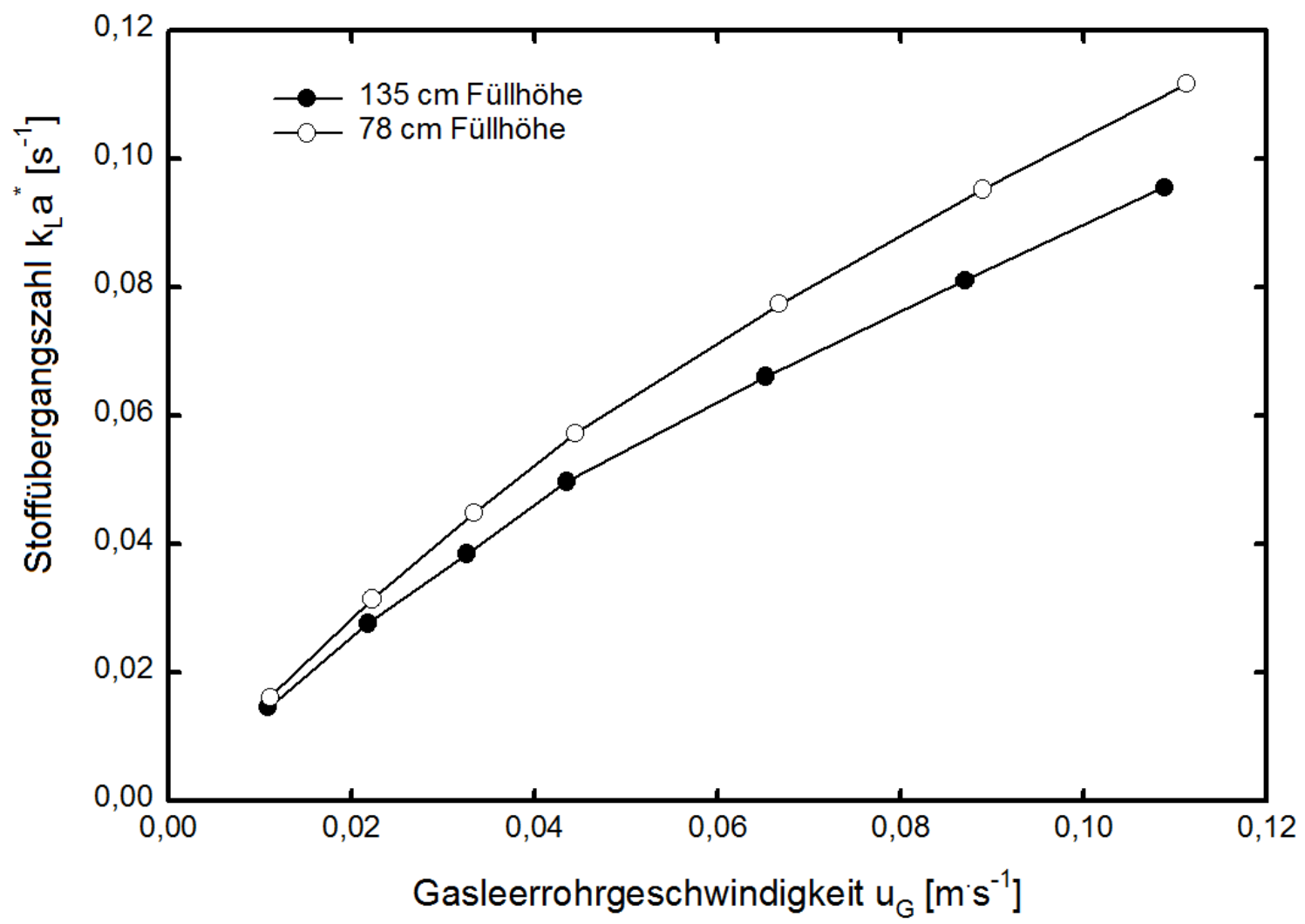

Abbildung 5-23: Einfluss der Füllhöhe auf die gemessenen Stoffübergangszahlen in 1Butanol $\left(1\right.$ bar $\left.\mathrm{N}_{2} ; 20^{\circ} \mathrm{C} ; 19 x 1 \mathrm{~mm}\right)$

\subsubsection{Einfluss der Blasensäule}

Die Desorptionsmessungen wurden in verschiedenen Blasensäulen durchgeführt. Die Ergebnisse der Messungen sind in Abbildung 5-24 und Abbildung 5-25 aufgetragen. Die Stoffübergangszahl nimmt mit steigendem Druck und steigender Leerrohrgeschwindigkeit wie erwartet zu. Die Blasensäule 1 zeigt in allen Fällen eine höhere Desorptionsrate als die Blasensäule 2. Dieser Trend geht konform mit dem höheren Gasgehalt in Blasensäule 1. (siehe Kapitel 5.1.7). Ein höherer Gasgehalt ermöglicht auch immer einen besseren Stoffübergang, da die spezifische Phasengrenzfläche a mit dem Gasgehalt zunimmt.

Ein zweiter Grund für den Unterschied kann auch die unterschiedliche Füllhöhe der Blasensäulen sein. Die Blasensäule 1 wurde $h_{L}=95 \mathrm{~cm}$ mit Flüssigkeit gefüllt, die Blasensäule 2 wies mindestens einen Füllstand von $h_{L}=130 \mathrm{~cm}$ auf. In Kapitel 5.2.5 wurde bereits gezeigt, dass ein niedrigerer Füllstand höhere Desorptionsraten verursacht. Die Blasensäulen wurden weiterhin mit verschiedenen Gasverteilern betrieben. Blasensäule 1 war mit einer Lochplatte mit 7 x $1 \mathrm{~mm}$ Löchern versehen. Blasensäule 2 hatte eine Lochplatte mit 19 x 1 mm Löchern. 


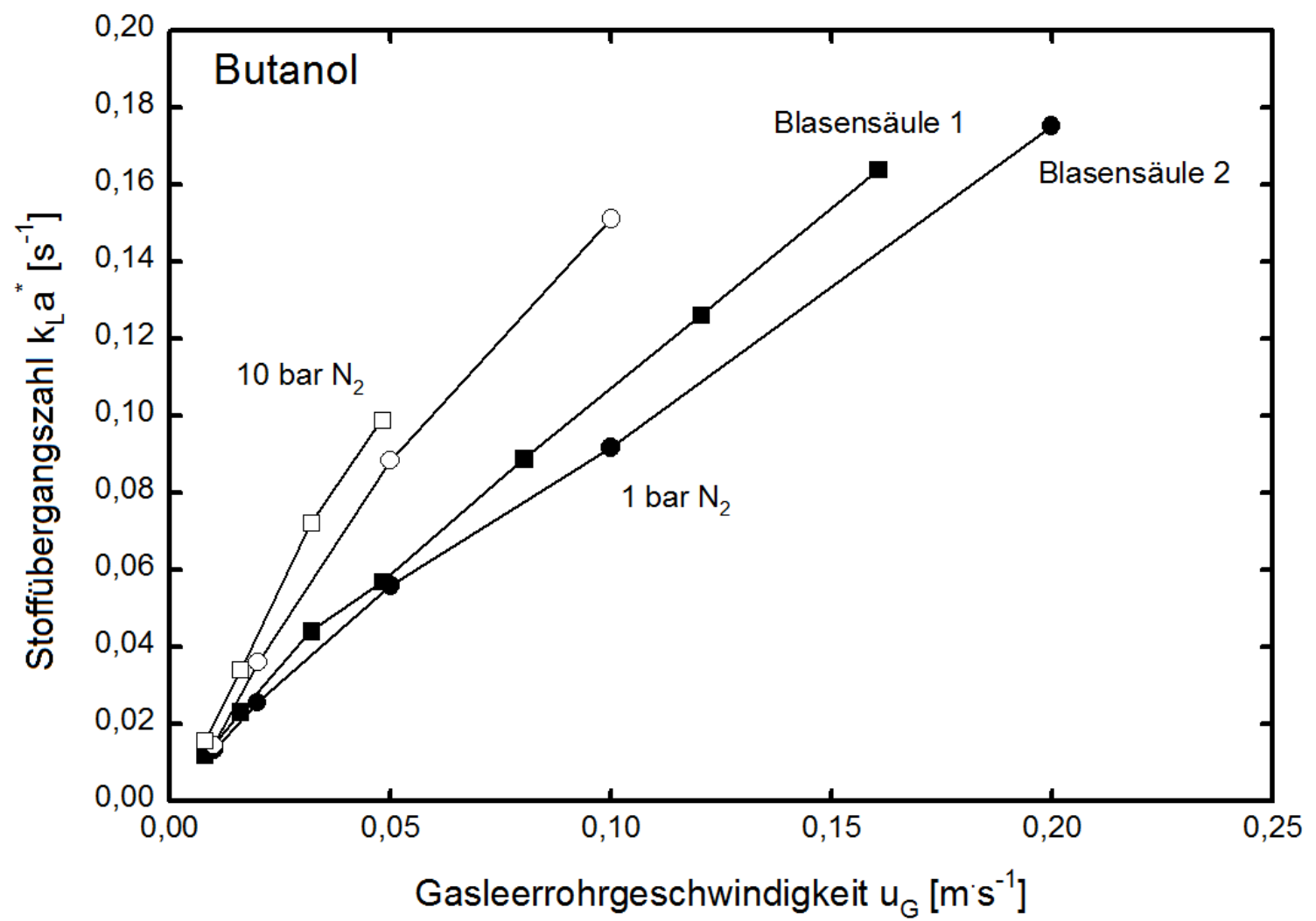

Abbildung 5-24: Vergleich des Stoffüberganges in Butanol in den verwendeten Blasensäulen

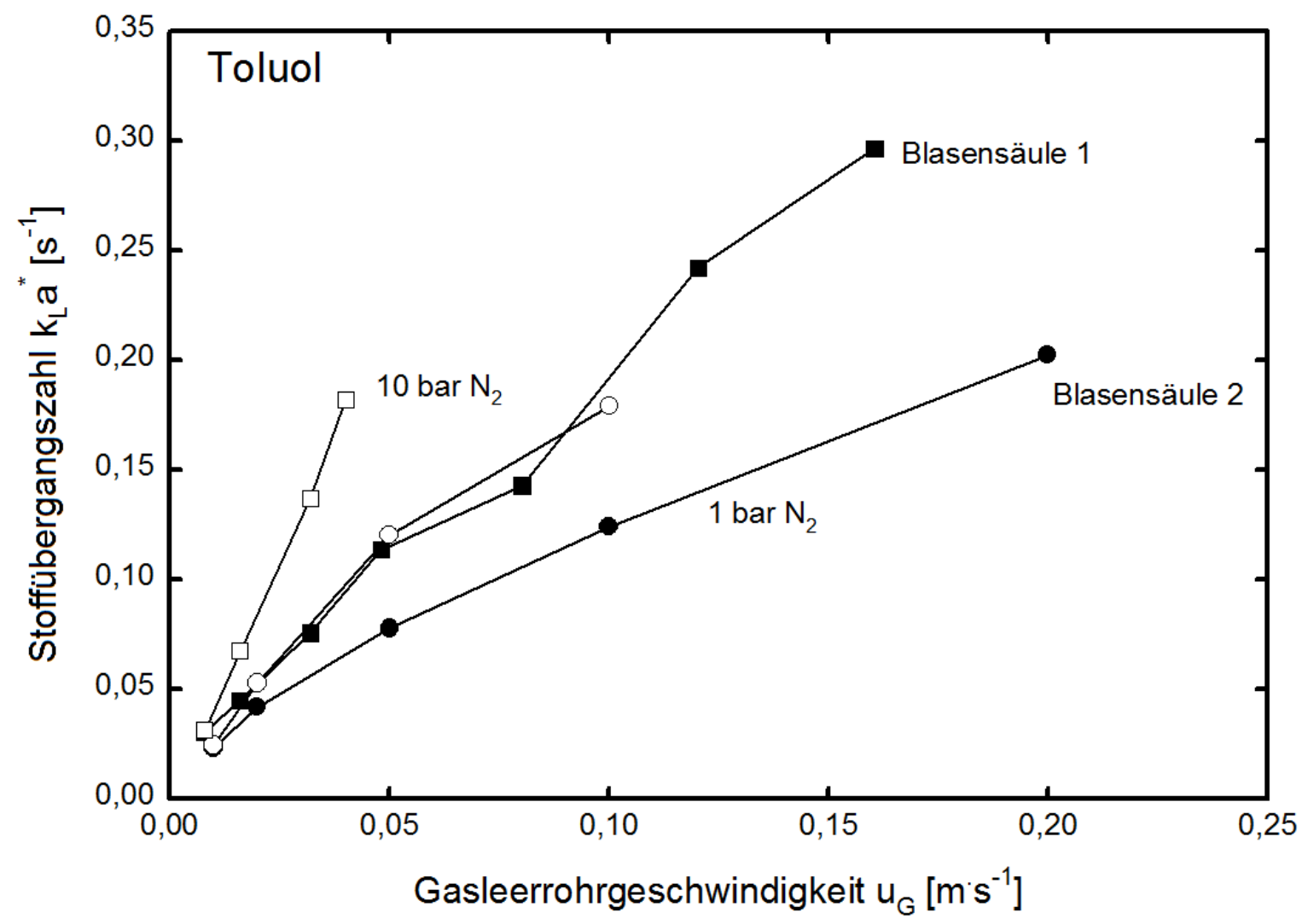

Abbildung 5-25: Vergleich des Stoffüberganges in Toluol in den verwendeten Blasensäulen 


\section{Korrelationen}

\subsection{Blasengröße}

\subsubsection{Kleinblasen}

\section{Zusammenhang zwischen Blasengröße und Aufstiegsgeschwindigkeit}

Die Blasengrößen werden nicht direkt gemessen. Es werden die Aufstiegsgeschwindigkeiten der Kleinblasen gemessen. Daraus lässt sich dann auf die Blasengröße schließen. Für den Zusammenhang zwischen Blasengröße und Blasengeschwindigkeit gibt es in der Literatur etliche Korrelationen (siehe Tabelle 6-1). Diese Korrelationen haben unterschiedliche Gültigkeitsbereiche.

Die Korrelationen 1 und 2 betrachten die Blase als starres Objekt, das von der Flüssigkeit umströmt wird. Der Strömungswiderstand berechnet sich ähnlich wie bei einem festen Körper. Dabei gilt das bekannte STOKES'sche Gesetz für den rein laminaren Fall.

Die Korrelationen 3 und 4 beruhen auf der Wellentheorie. Nach der Wellentheorie ist die Geschwindigkeit einer Welle abhängig von ihrer Wellenlänge. Bei kleinen Wellenlängen nimmt die Geschwindigkeit mit steigender Wellenlänge $a b$, durchläuft ein Minimum und nimmt dann mit wachsender Wellenlänge wieder zu. In diesen Modellen entspricht der Blasendurchmesser der Wellenlänge. Für ausreichend große Blasen werden mit diesem Ansatz gute Ergebnisse erzielt.

Die Korrelationen 5 und 6 decken den gesamten Größenbereich der Blasen ab. Sie sind dementsprechend komplizierter in ihrer mathematischen Formulierung. Für Korrelation 5 werden drei Parameter benötigt, die Aussagen über die Qualität der verwendeten Flüssigkeit enthalten. Diese Parameter sind empirisch ermittelt und lassen sich nicht aus den physikalischen Stoffdaten herleiten. Die Korrelation 6 ist rekursiv aufgebaut und kann nur iterativ gelöst werden.

In Abbildung 6-1 sind die so berechneten Aufstiegsgeschwindigkeiten in ihren Gültigkeitsbereichen aufgetragen. Man sieht, dass die Korrelationen 3 bis 6 im Bereich großer Blasen $\left(d_{B}>2 \mathrm{~mm}\right)$ sehr ähnliche Ergebnisse liefern. Es ist also möglich, in diesem Bereich eine der einfachen Korrelationen zu verwenden.

In Abbildung 6-2 ist der Bereich der kleinen Blasendurchmesser vergrößert abgebildet. Hier zeigen sich die wesentlichen Unterschiede zwischen den einzelnen Korrelationen. Im Bereich des STOKES'schen Gesetzes liegen Nummer 5 und 6 noch dicht beieinander. Beim Übergang zum Bereich der Wellentheorie jedoch zeigt Nummer 5 einen weichen, geschwungenen Übergang, während bei Nummer 6 ein scharfer Knick zu sehen ist. Die Differenz zwischen beiden Kurven ist im Bereich dieses Knicks z. T. recht erheblich.

Die aufgeführten Korrelationen liefern bei Blasendurchmessern von $\mathrm{d}_{\mathrm{B}}<0,5 \mathrm{~mm}$ und $\mathrm{d}_{\mathrm{B}}>2$ $\mathrm{mm}$ einheitliche Ergebnisse. Für diese Bereiche kann der Zusammenhang zwischen Blasendurchmesser und Blasengeschwindigkeit berechnet werden. Dabei spielen die Blasen mit $d_{B}<0,5 \mathrm{~mm}$ keine Rolle, denn Blasengeschwindigkeiten von $\mathrm{v}_{\mathrm{B}}<5 \mathrm{~cm} / \mathrm{s}$ wurden nicht beobachtet.

Für die künftigen Berechnungen wird die Korrelation 4 von MENDELSON und MANERI verwendet. Diese Gleichung enthält als Parameter die Gasdichte und die Eigenschaften der Flüssigkeit. Im Gegensatz zu Korrelation 5 und 6 ist sie mathematisch einfach zu handhaben und wird auch in anderen Publikationen häufig zitiert. 
Tabelle 6-1: verschiedene Korrelationen für die Berechnung der Aufstiegsgeschwindigkeit aus dem Blasendurchmesser

\begin{tabular}{|c|c|c|c|}
\hline & Autoren & Formel & Gültigkeitsbereich \\
\hline 1 & STOKES & $d_{B}=\sqrt{\frac{18 \cdot \mu_{L} \cdot v_{B}}{g \cdot\left(\rho_{L}-\rho_{G}\right)}}$ & $\begin{array}{l}\text { laminare Strömung } \\
\operatorname{Re}<2\end{array}$ \\
\hline 2 & \begin{tabular}{|l|} 
PEEBLES und \\
GARBER \\
$(1953)$ \\
\end{tabular} & $\mathrm{d}_{\mathrm{B}}=4,76 \cdot\left(\frac{\mu_{\mathrm{L}}}{\rho_{\mathrm{L}}}\right)^{0,41} \cdot \frac{\mathrm{v}_{\mathrm{B}}^{0,78}}{\mathrm{~g}^{0,59}}$ & $2 \leq \operatorname{Re} \leq 4,02 \cdot\left(\frac{\mathrm{g} \cdot \mu_{\mathrm{L}}^{4}}{\rho_{\mathrm{L}} \cdot \sigma_{\mathrm{L}}^{3}}\right)^{-0,214}$ \\
\hline 3 & ClifT & $\mathrm{v}_{\mathrm{B}}=\sqrt{\frac{2,14 \cdot \sigma_{\mathrm{L}}}{\rho_{\mathrm{L}} \cdot \mathrm{d}_{\mathrm{B}}}+0,505 \cdot \mathrm{g} \cdot \mathrm{d}_{\mathrm{B}}}$ & $\mathrm{dB}>1,3 \mathrm{~mm}$ \\
\hline 4 & $\begin{array}{l}\text { MENDELSON } \\
\text {;MANERI } \\
\text { (MANERI } \\
1995)\end{array}$ & $\mathrm{v}_{\mathrm{B}}=\sqrt{\frac{2 \cdot \mathrm{c} \cdot \sigma_{\mathrm{L}}}{\rho_{\mathrm{L}} \cdot \mathrm{d}_{\mathrm{B}}}+\frac{\rho_{\mathrm{L}}-\rho_{\mathrm{G}}}{\rho_{\mathrm{L}}} \cdot \frac{\mathrm{g} \cdot \mathrm{d}_{\mathrm{B}}}{2}}$ & $\begin{array}{l}\mathrm{c}=1,2 \text { für reine Flüssigkeiten } \\
\mathrm{c}=1,4 \text { für Flüssigkeitsgemische } \\
\mathrm{d}_{\mathrm{B}}>1,4 \mathrm{~mm}\end{array}$ \\
\hline 5 & $\begin{array}{l}\text { FAN; } \\
\text { TSUCHIYA } \\
\text { (LIN 1998) }\end{array}$ & 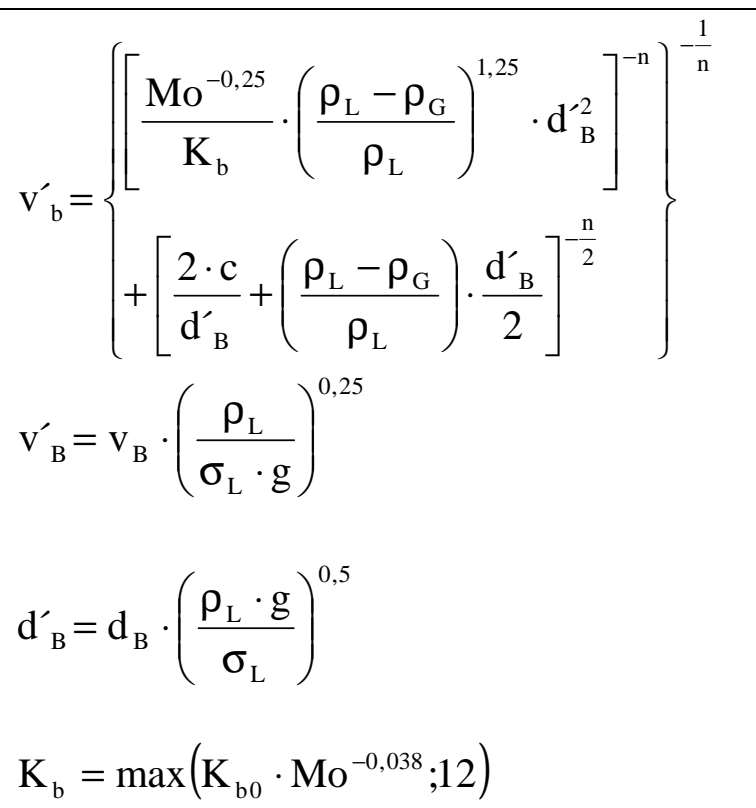 & $\begin{array}{l}\mathrm{c}=1,2 \text { für reine Flüssigkeiten } \\
\mathrm{c}=1,4 \text { für Flüssigkeits- } \\
\text { gemische } \\
\mathrm{n}=0,8 \text { für verunreinigte } \\
\text { Flüssigkeiten } \\
\mathrm{n}=1,6 \text { für reine Flüssigkeiten } \\
\mathrm{K}_{\mathrm{b} 0}=14,7 \text { für wässrige } \\
\text { Lösungen } \\
\mathrm{K}_{\mathrm{b} 0}=10,2 \text { für organische } \\
\text { Flüssigkeiten / Gemische }\end{array}$ \\
\hline 6 & $\begin{array}{l}\text { TOMIYAMA } \\
\text { (LIN 1998) }\end{array}$ & $\begin{array}{l}c_{\mathrm{W}}=\frac{4 \cdot g \cdot\left(\rho_{\mathrm{L}}-\rho_{\mathrm{G}}\right) \cdot \mathrm{d}_{\mathrm{B}}}{3 \cdot \rho_{\mathrm{L}} \cdot \mathrm{v}_{\mathrm{B}}^{2}} \\
\mathrm{c}_{\mathrm{W}}=\max \left\{\begin{array}{l}\min \left(\frac{16}{\operatorname{Re}} \cdot\left(1+0,15 \cdot \mathrm{Re}^{0,687}\right) ; \frac{48}{\mathrm{Re}}\right) \\
; \frac{8}{3} \cdot \frac{\mathrm{Eo}}{\mathrm{Eo}+4}\end{array}\right\}\end{array}$ & \\
\hline
\end{tabular}




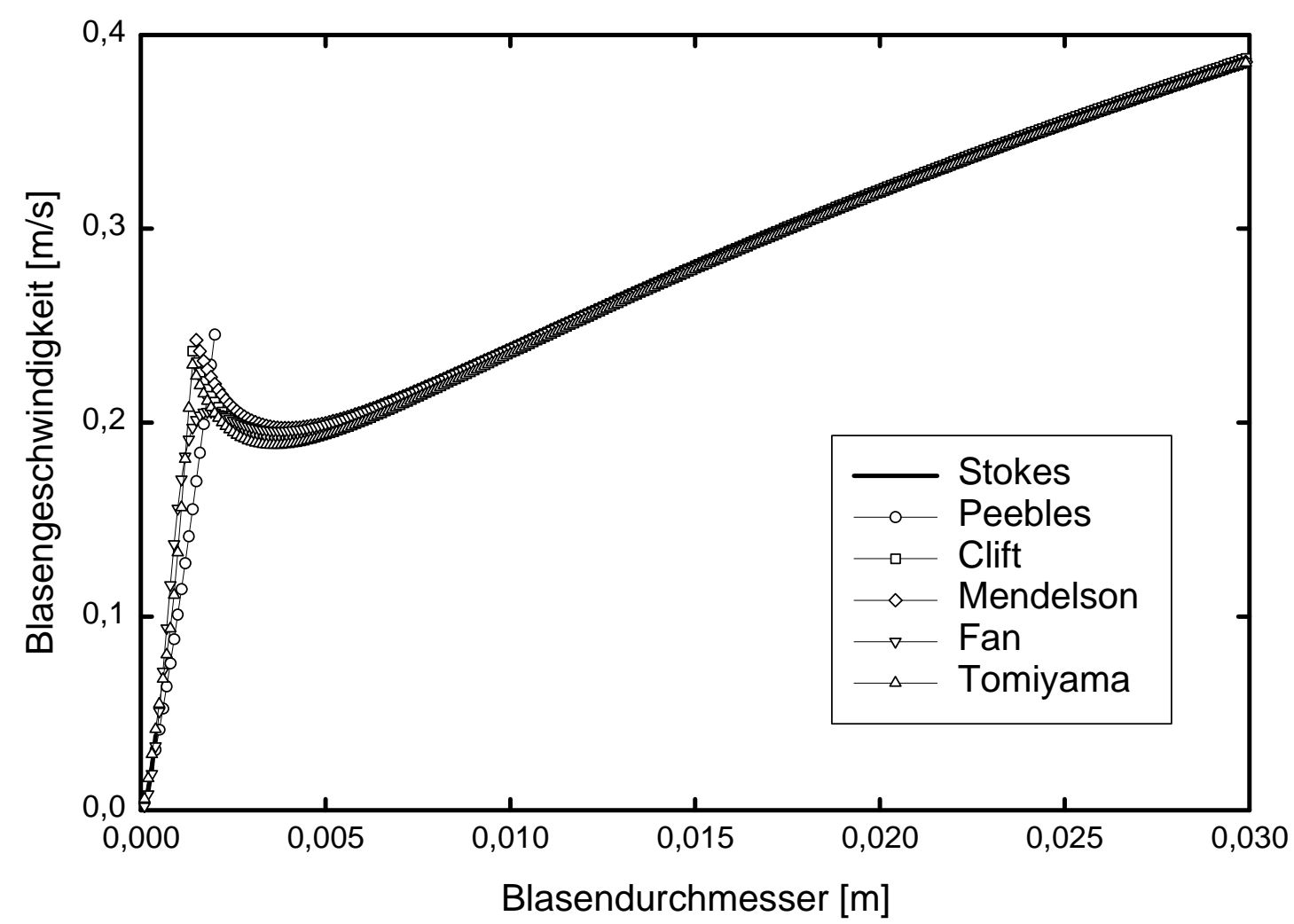

Abbildung 6-1: Aus Tabelle 6-1 errechnete Blasenaufstiegsgeschwindigkeiten für Decalin bei 1 bar $\mathrm{N}_{2}$

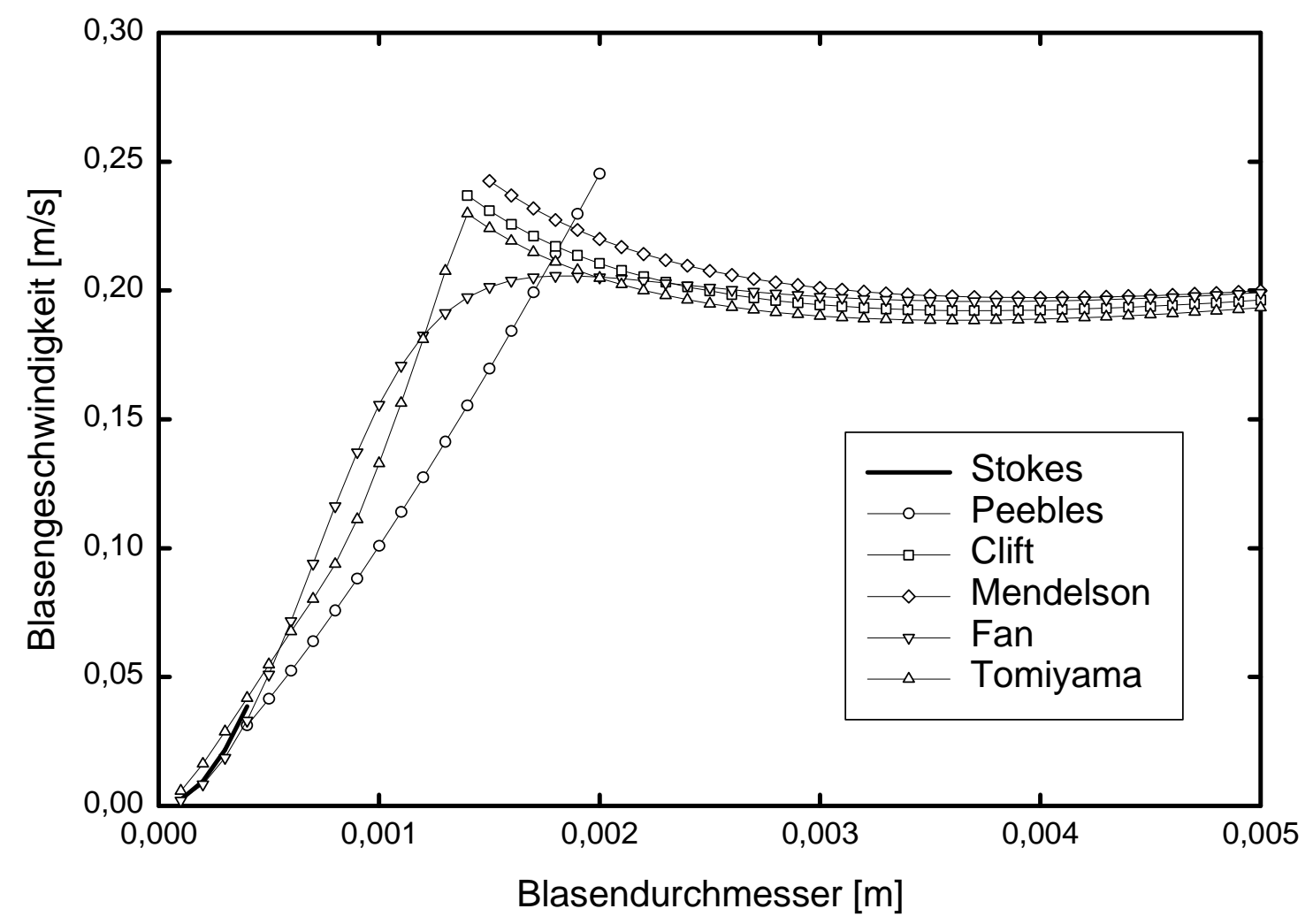

Abbildung 6-2: Wie Abbildung 6-1 mit verändertem Maßstab 


\section{Kleinblasengeschwindigkeit im Blasenschwarm}

Die nach MENDELSON und MANERI berechneten Blasengeschwindigkeiten gelten für Einzelblasen, die in freier Flüssigkeit aufsteigen. In einer Blasensäule bewegen sich die Blasen im Kollektiv und beeinflussen sich gegenseitig. Das hat zur Folge, dass sich die Geschwindigkeit der Blasen verringert.

Für die Schwarmgeschwindigkeit gilt folgender Ansatz:

$$
\mathrm{v}_{\text {sedim ent }}=\mathrm{v}_{0} \cdot\left(1-\varepsilon_{\mathrm{G}}\right)^{\mathrm{Z}}
$$

Gleichung 6-1: Schwarmgeschwindigkeit nach RICHARDSON-ZAKI

Der RichaRdSON-ZAKI-Exponent $Z$ liegt je nach untersuchtem System zwischen $2 \leq Z \leq 5$. Dabei gelten die hohen Werte für laminar umströmte Partikel, die niedrigen Werte für turbulent umströmte Partikel. Für eine durchschnittliche Kleinblase mit $d_{B}=3 \mathrm{~mm}$ und $v_{B}=$ $20 \mathrm{~cm} / \mathrm{s}$ gilt der turbulente Fall. Der Exponent ist also $\mathrm{Z}=2$.

Die experimentellen Daten für diese Schwarmgeschwindigkeiten stammen aus Messungen der Sedimentationsgeschwindigkeiten verschiedener Partikel. Dabei wurde stets die beobachtete Geschwindigkeit, aber nicht die Relativgeschwindigkeit zwischen Partikel und Flüssigkeit gemessen. Die durch das Absinken der Partikel verursachte Gegenströmung wurde nicht erfasst. Darum entspricht die so erhaltene Schwarmgeschwindigkeit der scheinbaren Blasengeschwindigkeit bei den Entgasungsmessungen:

$$
\mathrm{v}_{\text {schein }}=\mathrm{v}_{0} \cdot\left(1-\varepsilon_{\mathrm{G}}\right)^{\mathrm{Z}}
$$

Im Abschnitt „Auswertung“ wurde die Gegenströmung der Flüssigkeit bereits rechnerisch erfasst. Vereinfacht man diese Gleichung, so erhält man:

$$
\mathrm{v}_{\mathrm{B}}=\frac{\mathrm{h}}{\mathrm{t}_{\mathrm{E}}}+\frac{\Delta \mathrm{p}_{\mathrm{E}}-\Delta \mathrm{p}_{0}}{\mathrm{~g} \cdot \Delta \rho \cdot \mathrm{t}_{\mathrm{E}}} \cdot \frac{1}{\left(1-\varepsilon_{\mathrm{G}}\right)}=\frac{\mathrm{h}}{\mathrm{t}_{\mathrm{E}}} \cdot\left(\frac{1}{1-\varepsilon_{\mathrm{G}}}\right)
$$

Dies entspricht:

$$
\mathrm{v}_{\text {schein }}=\frac{\mathrm{h}}{\mathrm{t}_{\mathrm{E}}}=\mathrm{v}_{\text {swarm }} \cdot\left(1-\varepsilon_{\mathrm{G}}\right)
$$

Für die Berechnung der ,wahren“ Schwarmgeschwindigkeit $\mathrm{v}_{\text {swarm }}$ aus der freien Geschwindigkeit $\mathrm{v}_{0}$ muss also der RICHARDSON-ZAKI-Exponent $\mathrm{Z}$ um 1 verringert werden. So erhält man:

$$
\mathrm{v}_{\text {swarm }}=\mathrm{v}_{0} \cdot\left(1-\varepsilon_{\mathrm{G}}\right)^{\mathrm{Z}-1}
$$

Für $\mathrm{Z}=2$ wird der Exponent $(\mathrm{Z}-1)=1$. Umgekehrt kann so aus der gemessenen Schwarmgeschwindigkeit durch Umrechnen mit dem Gasgehalt die freie Aufstiegsgeschwindigkeit $\mathrm{v}_{0}$ ermittelt werden.

Anmerkung: Wird die Kleinblasengeschwindigkeit im heterogenen System behandelt, so wird für den Gasgehalt $\varepsilon_{\mathrm{G}}$ der Kleinblasengehalt $\varepsilon_{\mathrm{G}, \text { Klein }}$ eingesetzt. 


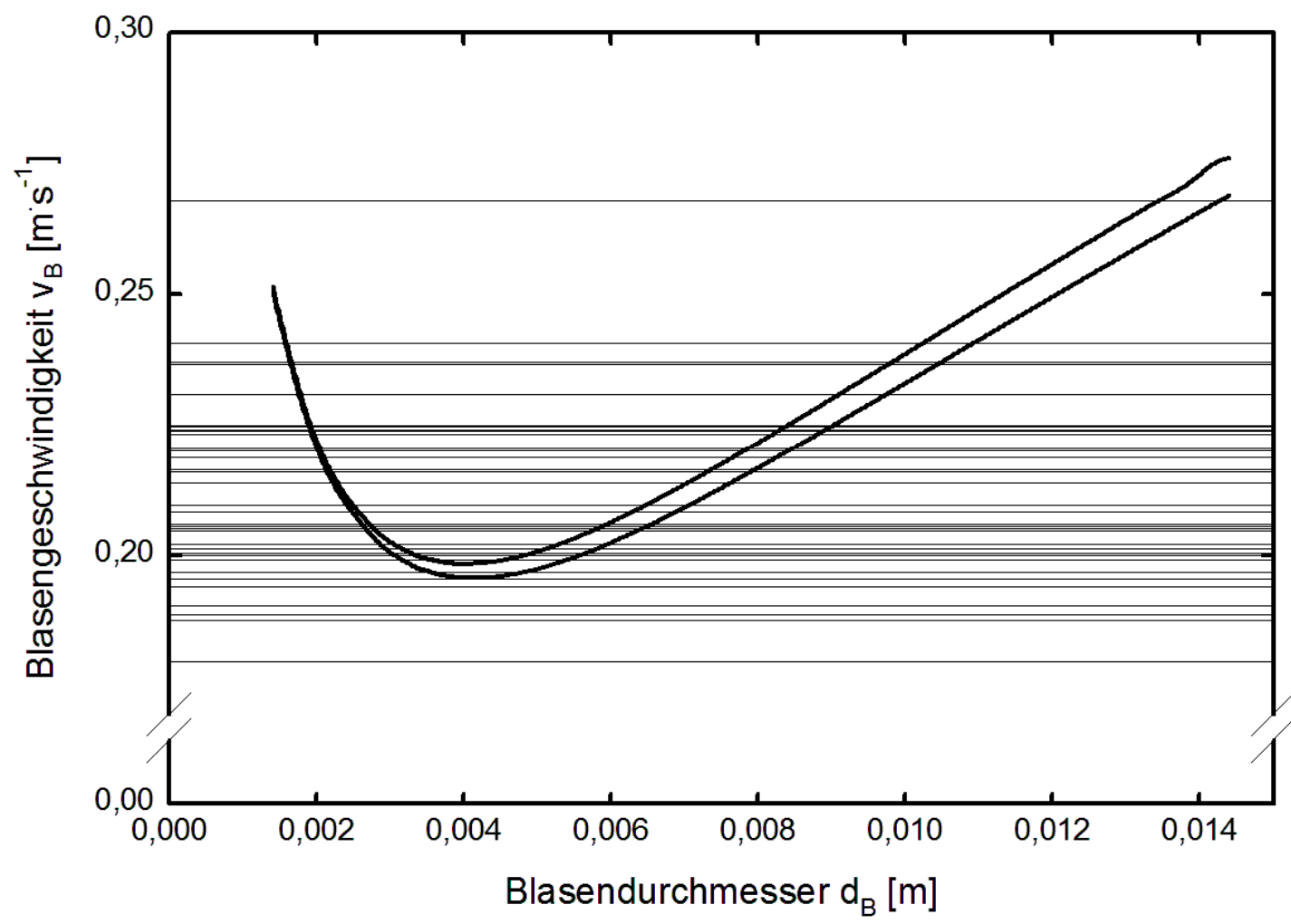

Abbildung 6-3:Die dünnen Linien sind die freien Kleinblasengeschwindigkeiten in Toluol bei verschiedenen Gasgeschwindigkeiten und Gasdichten. Zum Vergleich sind nach MENDELSON und MANERI berechneten Blasengeschwindigkeiten für die höchste und niedrigste Gasdichte schwarz eingetragen.

Die so berechneten freien Aufstiegsgeschwindigkeiten sind in Abbildung 6-3 mit den Geschwindigkeiten nach MENDELSON und MANERI zum Vergleich aufgetragen. Einige Werte liegen unter dem Minimum der theoretischen Kurven. Dies ist vermutlich auf Messungenauigkeiten zurückzuführen. Die meisten Werte liegen im „erlaubten“ Bereich. Für diese Blasengeschwindigkeiten sind zwei verschiedene Blasengrößen als Lösung möglich. Es ist kein eindeutiger Rückschluss von der Blasengeschwindigkeit auf die Blasengröße möglich. Zudem bedingt bereits ein kleiner Fehler in der Bestimmung der Blasengeschwindigkeit einen großen Fehler bei der Berechnung der Blasengröße. Der Rückschluss von der Aufstiegsgeschwindigkeit auf die Blasengröße ist sehr fehlersensitiv. Die so bestimmten Blasengrößen können nur als grobe Abschätzung dienen. Es wird ein anderer Zugang zur Blasengröße benötigt.

\section{Korrelationen für die Blasengröße}

Die Kleinblasen entstehen zunächst am Begaser. Dabei wird zwischen Blasenbegasen und Strahlbegasen unterschieden. Beim Blasenbegasen bildet das in die Flüssigkeit strömende Gas eine Blase, die sich durch ihre Auftriebskraft von der Begaseröffnung löst. Beim Strahlbegasen strömt das Gas so schnell durch die Begaseröffnung, dass sich ein Gasstrahl bildet, der sich erst in einiger Entfernung von der Begaseröffnung in die einzelnen Blasen aufteilt. Die Begaser der Blasensäule wurden so ausgelegt, dass beim normalen Betrieb keine Flüssigkeit durch den Begaser regnet. Dies bedingt, dass in den meisten Fällen Strahlbegasen vorliegt. Lediglich bei sehr kleinen Gasdichten und Gasleerrohrgeschwindigkeiten liegt Blasenbegasen vor. 
Für die Blasengröße am Begaser gibt es zahlreiche Veröffentlichungen (IDOGEWA e.a. 1987; Tsuge e.a. 1997; Tsuge e.a. 1981; Kling 1962; MiYAHIRA e.a. 1997, GAdDIS 1986). In Tabelle 6-2 sind einige der daraus entstandenen Korrelationen aufgelistet. Dabei sind die Korrelationen 1, 2 und 3 einfache Korrelationen, bei denen die Gasgeschwindigkeit $\mathrm{v}_{\mathrm{G}, \mathrm{O}}$ durch den Begaser keiner Rolle spielt. Die dabei zu Grunde liegenden Versuche erfolgten bei Gasgeschwindigkeiten, bei denen die Ablösung der Gasblasen durch die Auftriebskraft erfolgt.

Tabelle 6-2: Korrelationen zur Abschätzung der Blasengröße am Begaser

\begin{tabular}{|c|c|c|c|}
\hline $\mathrm{Nr}$. & Autor & Korrelation & Randbedingungen \\
\hline 1 & MIYAHARA 1997 & $d_{B}=2,9 \cdot\left(\frac{d_{O} \cdot \sigma}{\rho_{L} \cdot g}\right)^{1 / 3}$ & $\frac{\mathrm{We}}{\mathrm{Fr}^{0,5}} \leq 1$ \\
\hline 2 & $\begin{array}{l}\text { IDOGAWA } 1987 \\
\text { (I) }\end{array}$ & $\mathrm{d}_{\mathrm{B}}=3,91 \cdot \rho_{\mathrm{G}}^{-0,07} \cdot\left(\frac{\sigma}{\sigma_{\mathrm{H}_{2} \mathrm{O}}}\right)^{0,22 \cdot \exp \left(-\mathrm{p}_{\mathrm{i}}\right)}$ & $\begin{array}{l}\rho_{\mathrm{G}}=0,084-120,8 \mathrm{~kg}^{-3} \\
\mathrm{u}_{\mathrm{G}}=0,5-5 \mathrm{~cm} \cdot \mathrm{s}^{-1} \\
\sigma=0,0226-0,0721 \mathrm{~N} \cdot \mathrm{m}^{-1} \\
\mathrm{~d}_{\mathrm{O}}=1 \mathrm{~mm}\end{array}$ \\
\hline 3 & SARRAFI 1999 & $d_{B}=\left(\frac{6 \cdot d_{O} \cdot \sigma}{g \cdot \Delta \rho}\right)^{1 / 3}$ & Einzelblasenentstehung \\
\hline 4 & $\begin{array}{l}\text { GADDIS; } \\
\text { VOGELPOHL } \\
1986\end{array}$ & $\begin{array}{l}\mathrm{d}_{\mathrm{B}}^{3}=\mathrm{S}+\frac{\mathrm{L}}{\mathrm{d}_{\mathrm{B}}}+\frac{\mathrm{T}}{\mathrm{d}_{\mathrm{B}}^{2}} \\
\mathrm{~S}=\frac{6 \cdot \mathrm{d}_{\mathrm{O}} \cdot \sigma}{\Delta \rho \cdot \mathrm{g}} \cdot\left(1-\frac{\mathrm{We}_{\mathrm{G}}}{4}\right) \\
\mathrm{L}=\frac{81 \cdot \eta_{\mathrm{L}} \cdot \dot{\mathrm{V}}}{\pi \cdot \Delta \rho \cdot \mathrm{g}} \\
\mathrm{T}=\left(\frac{135}{4 \cdot \pi^{2}}+\frac{27 \cdot \rho_{\mathrm{G}}}{\pi^{2} \cdot \rho_{\mathrm{L}}}\right) \cdot \frac{\rho_{\mathrm{L}} \cdot \dot{\mathrm{V}}^{2}}{\Delta \rho \cdot \mathrm{g}} \\
\mathrm{We}_{\mathrm{G}}=\frac{\mathrm{d}_{\mathrm{O}} \cdot \rho_{\mathrm{G}} \cdot \mathrm{v}_{\mathrm{G}, \mathrm{O}}^{2}}{\sigma}\end{array}$ & $\begin{array}{l}\mathrm{We}_{\mathrm{G}} \leq 4 \cdot\left(1-\frac{\mathrm{d}_{\mathrm{O}}}{\mathrm{d}_{\mathrm{B}}}\right) \\
\mathrm{d}_{\mathrm{O}} \leq \sqrt{\frac{6 \cdot \sigma}{\rho_{\mathrm{L}} \cdot \mathrm{g}}} \\
\text { vereinfachte Form: } \\
\mathrm{d}_{\mathrm{B}}=\left[\mathrm{S}^{4 / 3}+\mathrm{L}+\mathrm{T}^{4 / 5}\right]^{1 / 4}\end{array}$ \\
\hline 5 & $\begin{array}{l}\text { IDOGAWA } 1987 \\
\text { (II) }\end{array}$ & $\begin{array}{l}\mathrm{d}_{\mathrm{B}}=0,02 \cdot \mathrm{We}^{-0,02} \cdot \operatorname{Re}_{\mathrm{G}}^{-0,16} \\
\operatorname{Re}_{\mathrm{G}}=\frac{\mathrm{v}_{\mathrm{O}} \cdot \mathrm{d}_{\mathrm{O}} \cdot \rho_{\mathrm{G}}}{\mu_{\mathrm{G}}} \\
\mathrm{We}=\frac{\mathrm{v}_{\mathrm{O}}^{2} \cdot \mathrm{d}_{\mathrm{O}} \cdot \rho_{\mathrm{L}}}{\sigma}\end{array}$ & $\begin{array}{l}40 \mathrm{~cm} \text { Höhe über dem } \\
\text { Begaser }\end{array}$ \\
\hline
\end{tabular}

Die Korrelation von GADDIS setzt sich aus drei Einzeltermen zusammen. Der Term S erfasst das Zusammenspiel zwischen Oberflächenspannung und Auftriebskraft. Dieser Term ist in ähnlicher Form auch in Korrelation 1 und 3 vorhanden. Die Auftriebskraft muss groß genug sein, um die Oberflächenenergie aufzubringen, die zum „Verschließen“ der fehlenden Oberfläche zwischen Begaseröffnung und Blase nötig ist. Bei kleinen Begaseröffnungen ist wenig Energie nötig, es entstehen kleine Blasen, bei großen Öffnungen entstehen größere Blasen. Dabei darf die Begaseröffnung eine bestimmte Größe nicht übersteigen (siehe Tabelle 6-2: Randbedingungen). Der Term S spielt nur bei sehr kleinen Gasgeschwindigkeiten eine Rolle. 
Der zweite Term L erfasst den Widerstand, den die Flüssigkeit dem Blasenaufstieg in der Anfangsphase entgegensetzt. Je höher die Viskosität der Flüssigkeit, desto langsamer löst sich die Blase vom Begaser und desto mehr Gas nimmt die Blase in sich auf, bevor sie sich vom Begaser löst. Dieser Effekt spielt bei viskosen Flüssigkeiten und höheren Gasströmen eine Rolle. Er liegt in der Größenordnung des Terms S.

Der dritte Term $\mathrm{T}$ erfasst den mechanischen Impuls, den das Gas durch seine Bewegung durch die Begaseröffnung in die Blase hineinträgt. Diese Geschwindigkeit muss durch die Oberfläche der Blase abgefangen werden. Dadurch verformt sich die Blase. Die eingetragene Energie wird in Oberflächenenergie umgewandelt, die Blase wächst. Schon bei moderaten Gasgeschwindigkeiten ist der Term T der bestimmende Term der Korrelation. Mit steigender Gasgeschwindigkeit und steigender Gasdichte nimmt die Blasengröße zu.

Die Korrelation von GADDIS gilt jedoch nur für das Blasenbegasen. Die meisten Versuche fanden im Bereich des Strahlbegasens statt. Für diesen Bereich gibt es nur wenig Literatur. Die Korrelation 5 ist eine empirisch ermittelte Gleichung. Der Blasendurchmesser nimmt mit steigender Gasdichte und Gasgeschwindigkeit ab.

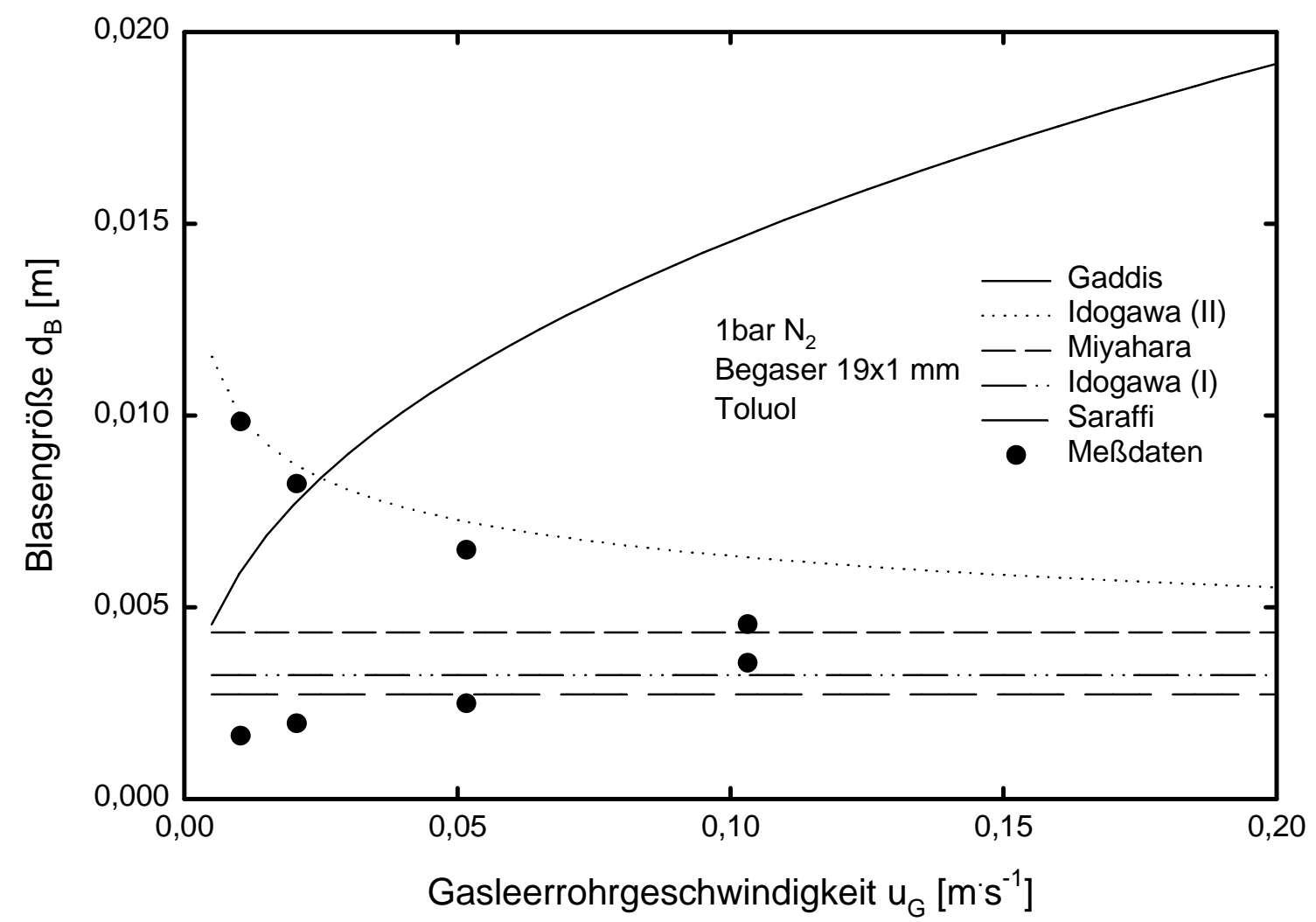

Abbildung 6-4: Berechnete Blasendurchmesser für Toluol bei $20^{\circ} \mathrm{C}$ und 1 bar $\mathrm{N}_{2}$ nach den Korrelationen aus Tabelle 6-2 als Funktion der Gasgeschwindigkeit 


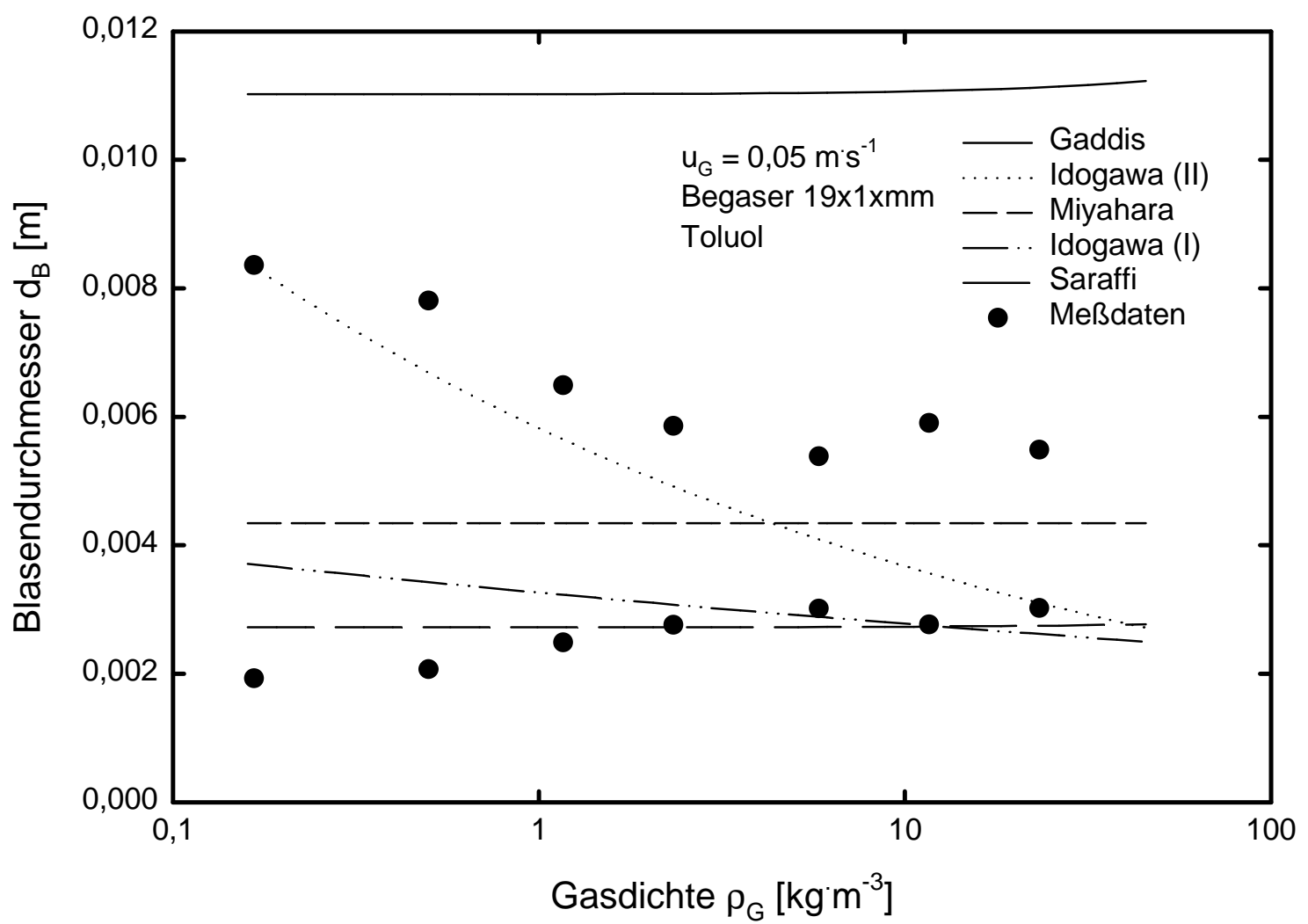

Abbildung 6-5: Berechnete Blasendurchmesser für Toluol bei $20^{\circ} \mathrm{C}$ nach den Korrelationen aus Tabelle 6-2 als Funktion der Gasdichte

In Abbildung 6-4 und Abbildung 6-5 sind die Verläufe der Korrelationen als Funktion der Gasgeschwindigkeit bzw. Gasdichte eingetragen. Zum Vergleich sind die entsprechenden Werte für die Blasengröße aus Abbildung 6-3 als schwarze Punkte eingetragen. Da es für jede Blasengeschwindigkeit zwei mögliche Blasengrößen gibt, sind die Messwerte paarweise vorhanden. Der obere Punkt entspricht dem größeren Blasendurchmesser. Einige Blasengeschwindigkeiten liegen im ,verbotenen“ Bereich. Diese Werte wurden nicht mit aufgenommen.

Die Korrelation von GADDIS liefert zu hohe Werte für den Blasendurchmesser. Dies ist nicht verwunderlich, da die meisten Messwerte ohnehin außerhalb des Gültigkeitsbereiches liegen. Die Gleichungen von MIYAHARA und SARAFFI zeigen keine sichtbare Abhängigkeit des Blasendurchmessers, weder von der Gasdichte, noch von der Blasengeschwindigkeit. Bei den Messwerten ist dagegen eine eindeutige Abhängigkeit von diesen Einflussgrößen zu erkennen. Diese Korrelationen können als nicht zutreffend verworfen werden.

Die Korrelation Nummer 4 von IDOGAwA beschreibt die Tendenz für die größeren der Messwerte ziemlich gut: Die Blasengröße nimmt mit steigender Gasgeschwindigkeit und Gasdichte ab. Für die kleinere Lösung der Messwerte dagegen bietet sich keine der betrachteten Korrelationen an.

Zur Abschätzung der Blasengröße der Kleinblasen kann die Gleichung von IDOGAWA herangezogen werden. 


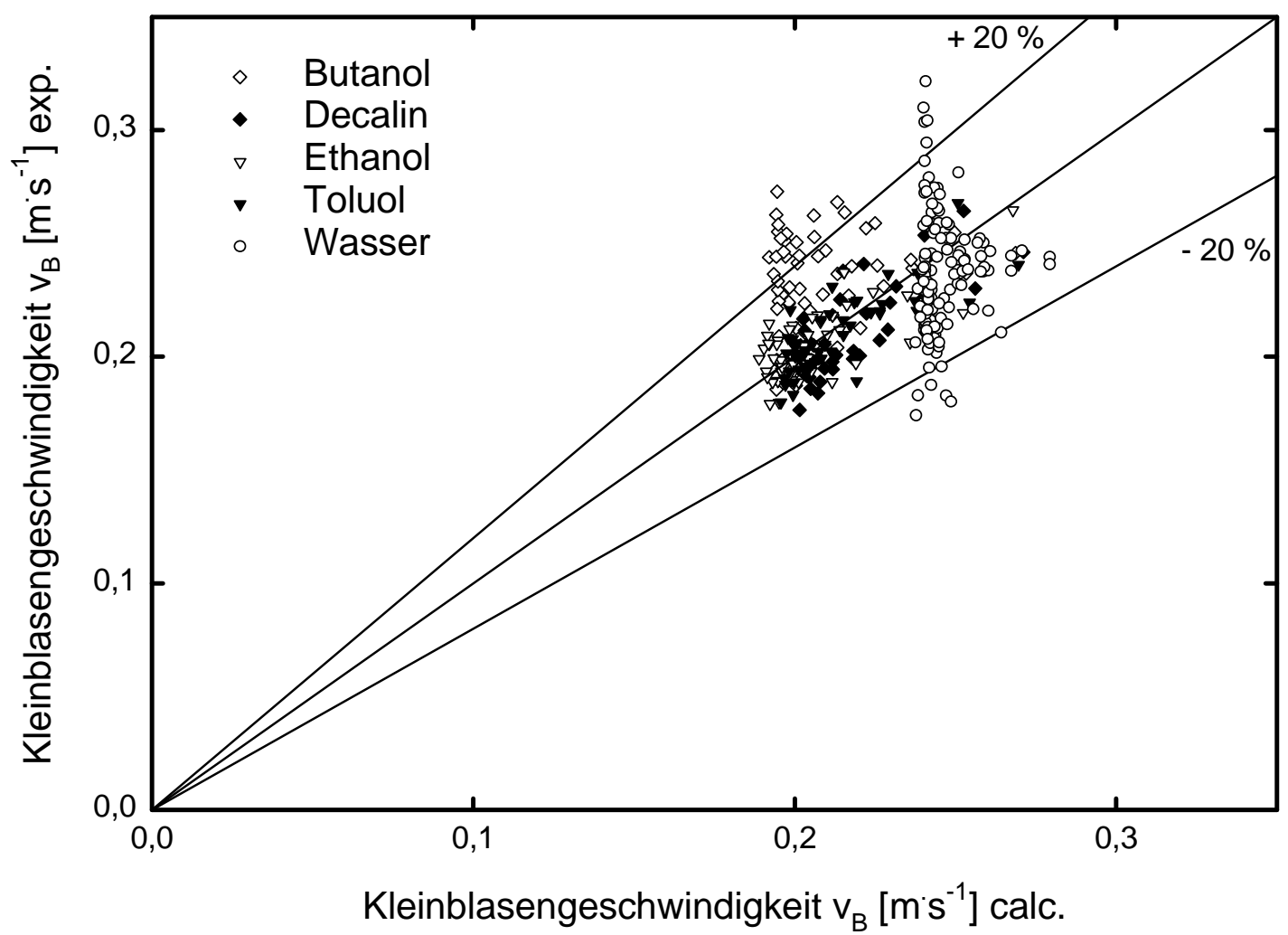

Abbildung 6-6: Paritätsplot der berechneten und der experimentell ermittelten

Kleinblasengeschwindigkeiten

In Abbildung 6-6 sind die so berechneten Kleinblasengeschwindigkeiten mit den gemessenen Kleinblasengeschwindigkeiten als Paritätsplot aufgetragen. Die Übereinstimmung ist für die Flüssigkeiten Ethanol, Toluol und Decalin sehr gut. Für Butanol werden etwas zu niedrige Werte berechnet. Bei den Werten für Wasser gibt es einige Ausreißer zu höheren und niedrigeren Geschwindigkeiten.

Die Abweichungen bei 1-Butanol lassen sich mit der Viskosität der Flüssigkeit erklären. Bei viskosen Flüssigkeiten gibt es keine scharf abgrenzbaren Blasenklassen, sondern eine breite Blasengrößenverteilung. In den Entgasungsmessungen erhält man keine scharfen Knicke in der Druckkurve sondern eher weiche Übergänge. Dadurch lassen sich die Blasenklassen nicht scharf voneinander trennen und die Bestimmung der Blasengeschwindigkeiten und Gasgehalte der Blasenklassen wird ungenauer. Aus diesem Grund gibt es keine solchen Werte für Ethylenglycol.

Die Kleinblasengeschwindigkeiten lassen sich gut mit den berechneten Primärblasengrößen vorhersagen. Es spricht nichts dagegen, die Kleinblasengröße gleich der berechneten Primärblasengröße zu setzen.

\subsubsection{Großblasen}

Blasen sind nur bis zu einer bestimmten Größe stabil. Wird diese Maximalgröße überschritten, dann zerfällt die Blase. In jüngeren Veröffentlichungen wurde häufig die Kelvin-HelmholtZ-Instabilität als Grund für den Blasenzerfall angeführt. (LETZEL 1998; WILKINSON 1990; Dissertation WILKINSON 1991) Dabei wird das Wachstum von Wellen an der Gas-Flüssig-Grenzfläche betrachtet. Kleine Störungen der glatten Grenzfläche schaukeln 
sich unter bestimmten Bedingungen $\mathrm{zu}$ großen Wellen auf, die die Blase letztendlich zerstören. Der Wachstumsfaktor $\omega$ einer Störung ergibt sich:

$\omega^{2}=\frac{\rho_{\mathrm{L}} \cdot \rho_{\mathrm{G}} \cdot \mathrm{v}_{\text {slip }}^{2} \cdot \mathrm{K}^{2}}{\left(\rho_{\mathrm{L}}+\rho_{\mathrm{G}}\right)^{2}}-\frac{\sigma \cdot \mathrm{K}^{3}-\mathrm{g} \cdot\left(\rho_{\mathrm{L}}-\rho_{\mathrm{G}}\right) \cdot \mathrm{K}}{\rho_{\mathrm{L}}+\rho_{\mathrm{G}}}$

Gleichung 6-2: Kelvin-Helmoltz-Instabilität

Dabei ist K die Wellenzahl

$\mathrm{K}=\frac{2 \cdot \pi}{\lambda}$

Ist die rechte Seite der Gleichung 6-2 negativ, dann wird eine auftretende Störung gedämpft und die Blase ist stabil. Ist die rechte Seite jedoch positiv, dann wächst die Störung, auftretende Wellen werden mit der Zeit größer. Die maximale stabile Wellenlänge ergibt sich $\mathrm{zu}$ :

$$
\lambda_{\text {max }, \text { stabil }}=\frac{2 \cdot \pi \cdot \sqrt{\frac{\sigma}{g \cdot \Delta \rho}}}{\frac{\rho_{\mathrm{L}}}{\rho_{\mathrm{L}}+\rho_{\mathrm{G}}} \cdot \frac{\rho_{\mathrm{G}} \cdot \mathrm{v}_{\text {slip }}^{2}}{2 \cdot \sqrt{\sigma \cdot g \cdot \Delta \rho}}+\sqrt{1+\frac{\left(\rho_{\mathrm{L}} \cdot \rho_{\mathrm{G}} \cdot \mathrm{v}_{\text {slip }}^{2}\right)^{2}}{4 \cdot \sigma \cdot g \cdot \Delta \rho \cdot\left(\rho_{\mathrm{L}}+\rho_{\mathrm{G}}\right)^{2}}}}
$$

Gleichung 6-3: Maximale stabile Wellenlänge nach Kelvin-Helmholtz

Die größte Instabilität ergibt sich bei etwas größerer Wellenlänge $\lambda$ :

$$
\lambda_{\text {instabil }}=\frac{2 \cdot \pi \cdot \sqrt{\frac{3 \cdot \sigma}{\mathrm{g} \cdot \Delta \rho}}}{\frac{\rho_{\mathrm{L}}}{\rho_{\mathrm{L}}+\rho_{\mathrm{G}}} \cdot \frac{\rho_{\mathrm{G}} \cdot \mathrm{v}_{\text {slip }}^{2}}{2 \cdot \sqrt{3 \cdot \sigma \cdot g \cdot \Delta \rho}}+\sqrt{1+\frac{\left(\rho_{\mathrm{L}} \cdot \rho_{\mathrm{G}} \cdot \mathrm{v}_{\text {slip }}^{2}\right)^{2}}{12 \cdot \sigma \cdot g \cdot \Delta \rho \cdot\left(\rho_{\mathrm{L}}+\rho_{\mathrm{G}}\right)^{2}}}}
$$

Gleichung 6-4: Instabilste Wellenlänge nach Kelvin-Helmholtz

Die zerfallende Blase muss auf jeden Fall größer sein, als die sie zerstörende Welle. Ist die Blase kleiner als $\lambda_{\text {max,stabil }}$, dann ist die Blase auf jeden Fall stabil. Mit steigender Gasdichte nehmen $\lambda_{\text {max,stabil }}$ und $\lambda_{\text {instabil }}$ ab. Die maximale stabile Blasengröße wird mit steigender Gasdichte erwartungsgemäß kleiner. Allerdings ist nicht klar, in welchem Verhältnis Blasengröße und Wellenlänge zueinander stehen müssen, damit die Blase tatsächlich zerfällt. Weiterhin ist die Relativgeschwindigkeit $\mathrm{v}_{\text {slip }}$ zwischen Gas und Flüssigkeit nicht bekannt. Die Aufstiegsgeschwindigkeit der Blase $\mathrm{v}_{\mathrm{B}}$ stellt hier nur einen Maximalwert dar. In großen Blasen (oder Tropfen) gibt es eine innere Zirkulation. Das Gas strömt in der Mitte der Blase nach oben und an den seitlichen Wänden nach unten (siehe Abbildung 6-7 und CLIFT „Bubbles, Drops and Particles“). Dadurch wird $\mathrm{v}_{\text {slip }}$ wesentlich verringert und der Wachstumsfaktor $\omega$ wird negativ: die auftretenden Störungen werden gedämpft.

Die Kelvin-Helmholtz-Theorie ist also kein praktikabler Ansatz zur Abschätzung der Blasengröße. 


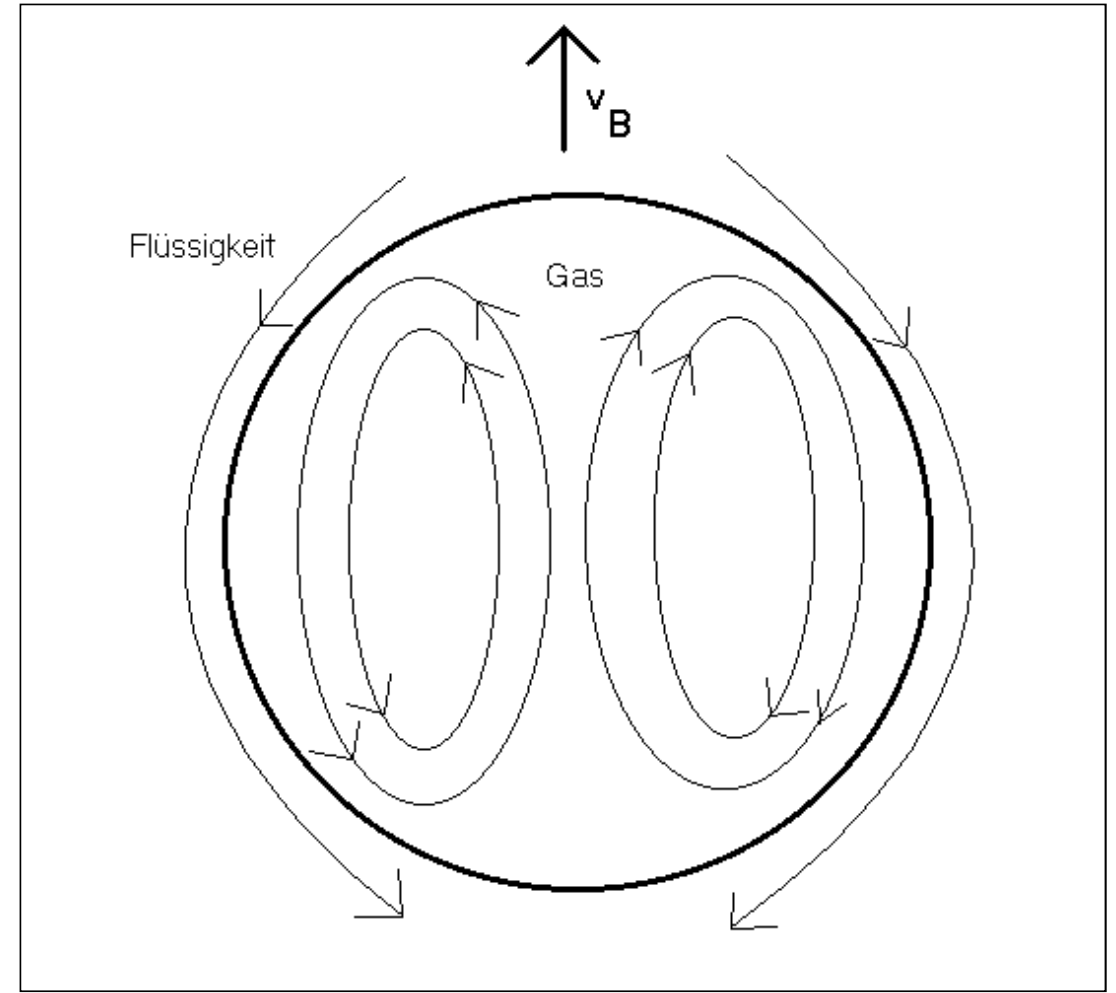

Abbildung 6-7: innere Zirkulation einer Blase

Ein Maß für die Stabilität einer Blase ist die WEBER-Zahl:

$W e=\frac{v_{B}^{2} \cdot d_{B} \cdot \rho_{G}}{\sigma}$

Gleichung 6-5: Weber-Zahl

Sie vergleicht die Trägheitskraft des Gases mit der Oberflächenspannung. Ist die Masse des Gases groß genug, um die Oberflächenspannung zu überwinden, dann zerfällt die Blase. Die kritische WEBER-Zahl wird meistens mit $\mathrm{We}=1$ angegeben.

Die Geschwindigkeit $\mathrm{v}_{\mathrm{B}}$ berechnet sich aus dem Blasendurchmesser $\mathrm{d}_{\mathrm{B}}$ nach MENDELSON und MANERI:

$v_{B}=\sqrt{\frac{2 \cdot c \cdot \sigma_{L}}{\rho_{L} \cdot d_{B}}+\frac{\rho_{L}-\rho_{G}}{\rho_{L}} \cdot \frac{g \cdot d_{B}}{2}}$

Gleichung 6-6: Blasengeschwindigkeit nach Mendelson Maneri

Durch Einsetzen von Gleichung 6-6 in Gleichung 6-5 und Umstellen der Gleichung nach dem Blasendurchmesser kann man durch einsetzen der kritischen WEBER-Zahl einen maximalen Blasendurchmesser ermitteln. Für den maximalen Blasendurchmesser ergibt sich:

$d_{B, \max }=\sqrt{\frac{W e_{c} \cdot \rho_{L} \cdot 2 \cdot \sigma}{\left(\rho_{L}-\rho_{G}\right) \cdot \rho_{G} \cdot g}-\frac{4 \cdot c \cdot \sigma}{\left(\rho_{L}-\rho_{G}\right) \cdot g}}$

Gleichung 6-7: Maximaler Großblasendurchmesser

Bei kleinen Gasdichten kann der maximale Blasendurchmesser dabei größer als der Blasensäulendurchmesser werden. Dies ist nicht möglich: darum wird die Blasengröße auf den halben Blasensäulendurchmesser begrenzt. Trägt man nun die Blasengröße über der 
Gasdichte auf, dann erhält man eine Kurve in zwei Abschnitten (siehe Abbildung 6-8): Bei Gasdichten $\rho_{\mathrm{G}}<2 \mathrm{kgm}^{-3}$ beträgt die Blasengröße konstant ihr Maximum des halben Blasensäulendurchmessers. Liegt die Gasdichte $\rho_{\mathrm{G}}>2 \mathrm{kgm}^{-3}$ nimmt die Blasengröße mit steigender Gasdichte ab. Der Exponent der Gasdichte ist in diesem Fall -0,5. Berechnet man daraus die Blasenaufstiegsgeschwindigkeit, so erhält man eine ähnliche Kurve, wie in Abschnitt „Ergebnisse“ für die Großblasengeschwindigkeit. Allerdings sind die berechneten Großblasengeschwindigkeiten mit maximal $\mathrm{v}_{\mathrm{B}}=0,5 \mathrm{~ms}^{-1}$ wesentlich kleiner als die gemessenen Geschwindigkeiten mit bis $\mathrm{zu} \mathrm{v}_{\mathrm{B}}=1,4 \mathrm{~m}^{-1} \mathrm{~s}^{-1}$.

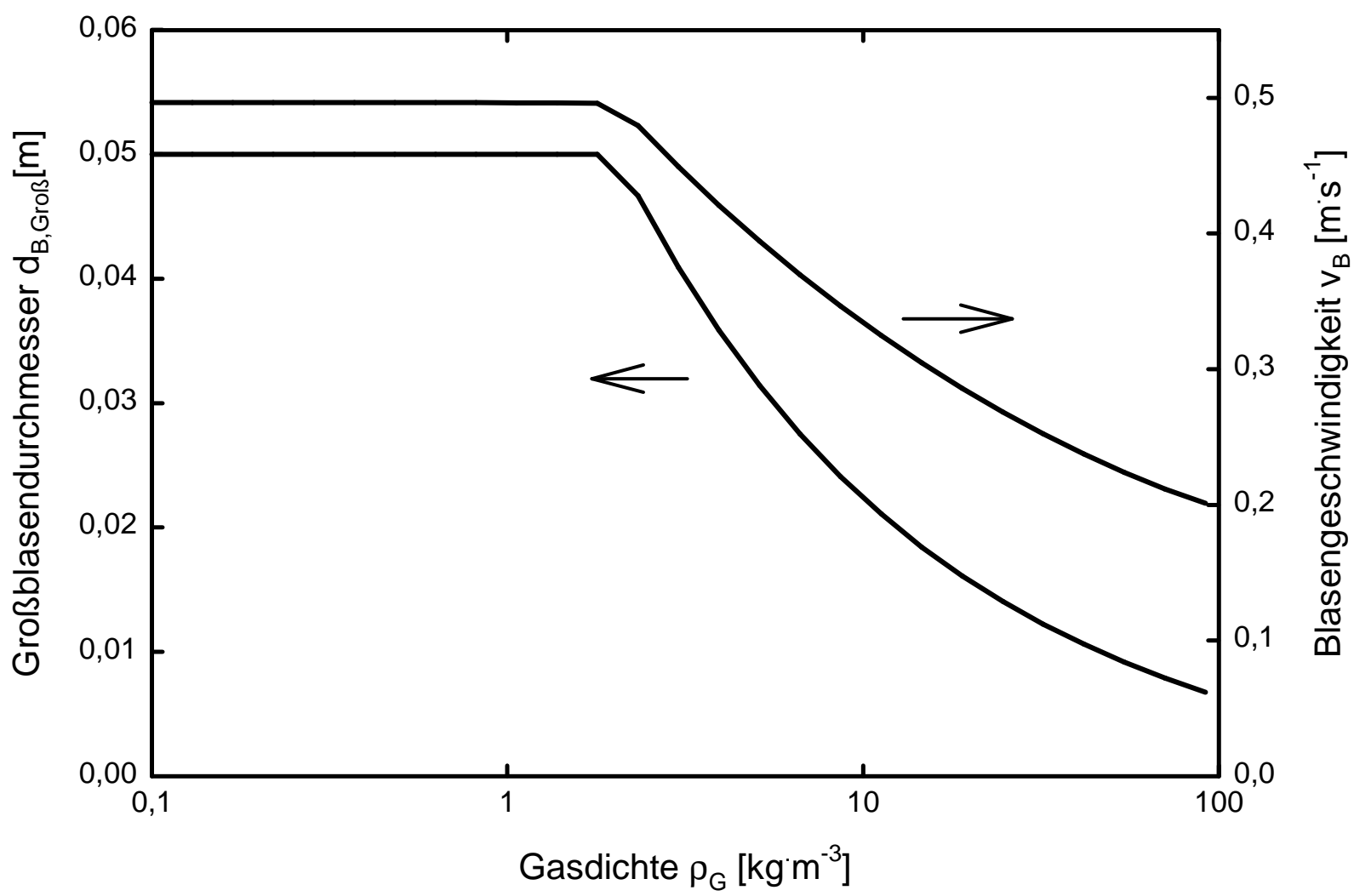

Abbildung 6-8: Maximaler Großblasendurchmesser und Großblasengeschwindigkeit als Funktion der Gasdichte

\section{2 Übergang vom homogenen zum heterogenen Blasenregime}

Steigert man bei sonst gleichen Betriebsbedingungen die Gasleerohrgeschwindigkeit $\mathrm{u}_{\mathrm{G}}$, so wird irgendwann ein Punkt erreicht, an dem sich ein Teil der Kleinblasen zu Großblasen vereinigt und sehr schnell aus dem Dispersionsvolumen entweicht. Diese Übergangsgasgeschwindigkeit $\mathrm{u}_{\mathrm{G}, \mathrm{t}}(\mathrm{t}=$ transition) ist abhängig von den Stoffeigenschaften Oberflächenspannung und Viskosität und wächst mit zunehmender Gasdichte.

Eine gute Veröffentlichung zu diesem Thema stammt von REILLY et. al. (1994). Eine Reihe organischer Flüssigkeiten und Wasser wurden genutzt, um den Gasgehalt bei verschiedenen Gasdichten zu ermitteln. Für den Gasgehalt am Übergangspunkt wurde folgende Beziehung gefunden: 
$\varepsilon_{\mathrm{G}, \mathrm{t}}=0,59 \cdot \mathrm{B}^{1,5} \cdot \sqrt{\frac{\rho_{\mathrm{G}}^{0,96} \cdot \sigma^{0,12}}{\rho_{\mathrm{L}}}}$

Gleichung 6-8: Übergangsgasgehalt nach REILLY

Die Konstante B lag im Mittel bei $\mathrm{B}=3,9$. Es ist aber anzunehmen, dass sich für andere Versuchsanlagen andere Werte für B ergeben.

Der Gasgehalt im Übergangspunkt $\varepsilon_{\mathrm{G}, \mathrm{t}}$ entspricht dem Kleinblasengehalt im heterogenen Regime. So kann die Gleichung 6-8 anhand der eigenen Daten überprüft werden. In Abbildung 6-9 sind die Werte für Toluol im heterogenen Regime aufgetragen. Der neue Wert für die Konstante $\mathrm{B}$ wäre $\mathrm{B}=3,33$. Allerdings ist keine gute Linearität gegeben. Die Gleichung 6-8 von REILLY stellt für unsere Messungen nur eine grobe Näherung dar.

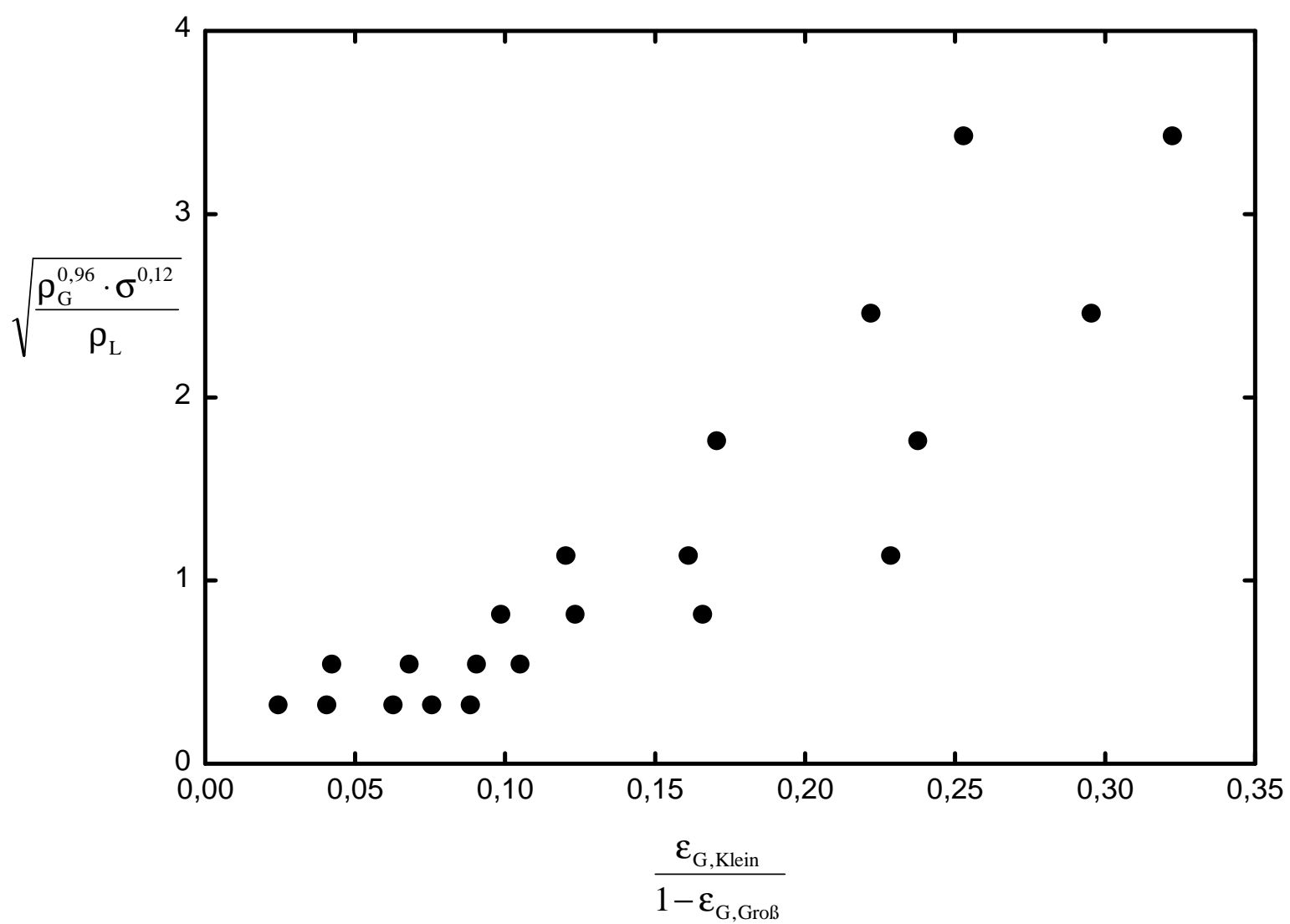

Abbildung 6-9: Auftragung von Gleichung 6-8 über den Kleinblasengehalt im heterogenen Regime (Toluol)

Ein anderes interessantes Bild ergibt sich, wenn man den Kleinblasenstrom über der Kleinblasengröße aufträgt. (siehe Abbildung 6-10) Die Daten für den heterogenen Betriebszustand zeigen einen engen Zusammenhang zwischen der Blasengröße und dem maximalen Kleinblasenstrom. Dabei zeigen die Gasdichte und die Leerrohrgeschwindigkeit keinen Einfluss. Je größer die Kleinblasen, desto geringer ist der maximale Kleinblasenstrom. Die Blasengröße ist der limitierende Faktor für die Leerrohrgeschwindigkeit.

In der Promotion von WILKINSON findet sich ein interessanter Ansatz zur Erklärung dieses Phänomens. 


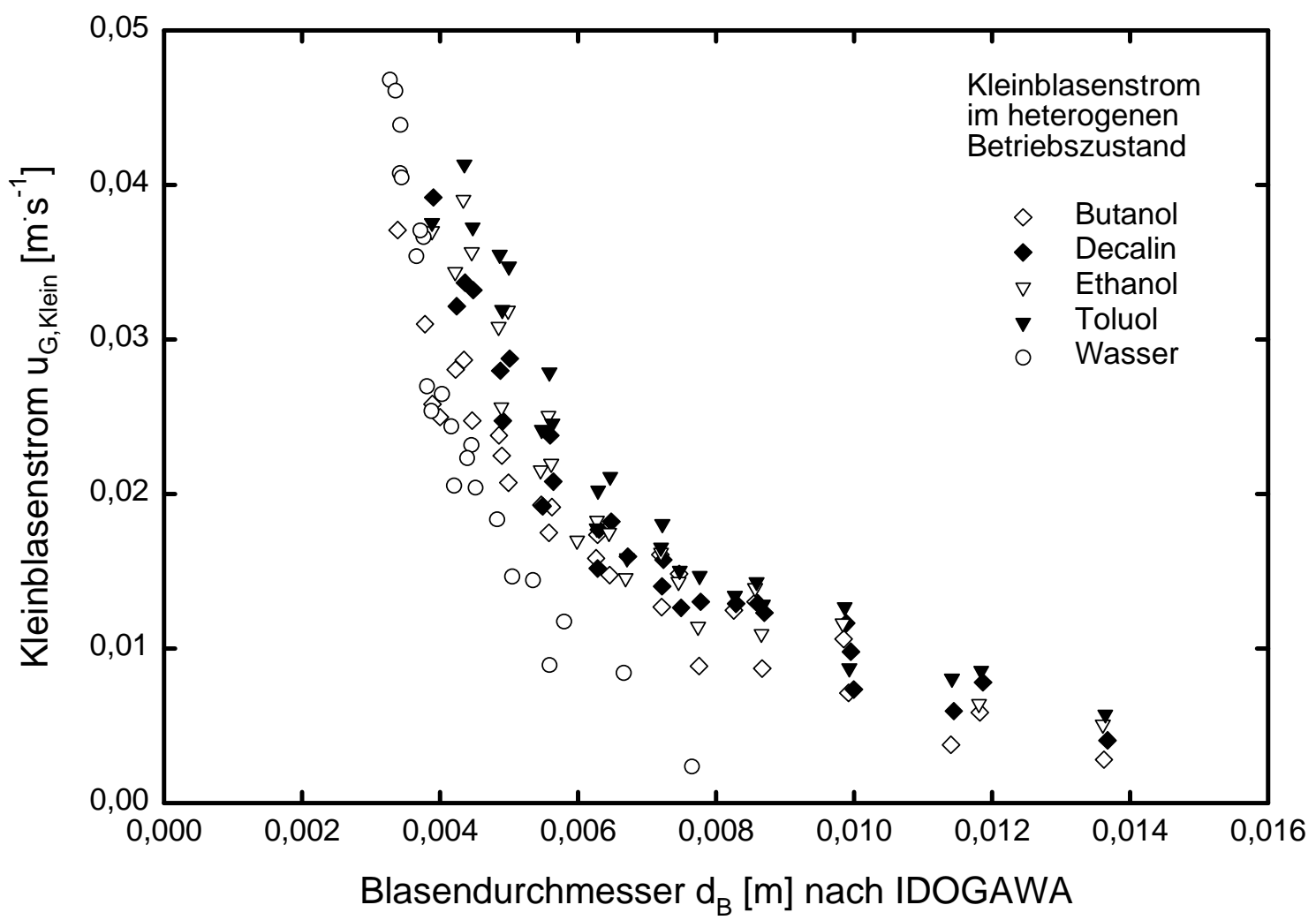

Abbildung 6-10: Kleinblasenstrom als Funktion des Kleinblasendurchmesser im heterogenen Betriebszustand

Eine Blase ist stabil, solange die lokale Schubspannung $\tau$ durch Mikroturbulenzen kleiner ist, als die Oberflächenspannung der Blasen.

$\tau>\frac{\sigma}{\mathrm{d}_{\mathrm{B}}}$

Gleichung 6-9: Schubspannung

Der Grenzfall lässt sich durch die kritische WEBER-Zahl beschreiben:

$\mathrm{We}_{\mathrm{krit}}=\frac{\tau \cdot \mathrm{d}_{\mathrm{B}, \max }}{\sigma}$

Gleichung 6-10: Kritische Weber-Zahl für die Schubspannung auf Kleinblasen

Die Schubspannung kann auch durch die Geschwindigkeit der Mikroturbulenzen beschrieben werden:

$\tau=\frac{\rho_{\mathrm{L}}}{2} \cdot \mathrm{v}_{\text {turb }}^{2}$

Die Geschwindigkeit der Mikroturbulenzen $\mathrm{v}_{\text {turb }}$ hängt von der in die Flüssigkeit eingetragenen Energie und der Größe der Turbulenzen ab. Die eingetragene Energie entspricht der potenziellen Energie, die die Blasen während ihres Aufstieges freisetzen. Diese Energie wird vollständig durch die innere Reibung der Flüssigkeit in Wärme umgewandelt. 
$\mathrm{v}_{\text {turb }}^{2}=2 \cdot\left(\frac{\mathrm{d}_{\text {turb }}}{\rho_{\mathrm{L}}} \cdot \frac{\mathrm{P}}{\mathrm{V}_{\mathrm{L}}}\right)^{2 / 3}=2 \cdot\left(\frac{\mathrm{d}_{\text {turb }}}{\rho_{\mathrm{L}}} \cdot \mathrm{u}_{\mathrm{G}, \text { Klein }} \cdot \mathrm{g} \cdot \Delta \rho\right)^{2 / 3}$

Gleichung 6-11: Turbulenzgeschwindigkeiten

WILKINSON ging davon aus, dass die Mikroturbulenzen den Zerfall von Großblasen verursachen. Dies konnte jedoch nicht bestätigt werden. Die Großblasenstabilität lässt sich mit der gasbezogenen WEBER-Zahl We $\mathrm{G}_{\mathrm{G}}$ wesentlich besser abschätzen.

Dennoch haben ausreichend schnelle Mikroturbulenzen einen Einfluss auf die Blasen. Die Blasen werden jedoch nicht zerstört, sondern lediglich aus ihrer Bahn abgelenkt und ins Innere des Wirbels gezogen. Gelangen zwei oder mehr Kleinblasen in den Bereich eines ausreichend starken Mikrowirbels, so werden alle Blasen in das Zentrum des Wirbels gesogen. Dort kommt es dann zur Koaleszenz der Blasen und zur Bildung einer Großblase.

Die kritische WEBER-Zahl We krit liegt je nach Veröffentlichung zwischen 0,6 bis 4,7.

Die Größe der Mikroturbulenz $d_{\text {turb }}$ ist nicht bekannt. Sie muss jedoch in der Größenordnung der Blasen liegen. Eine geringere Wirbelgröße hat nicht genügend Energie, mehr als eine Blase aufzusaugen. Ein größerer Wirbel transportiert die Blase im Ganzen weiter, er hat nicht genügend Schergefälle, um die Blase zu deformieren. Mit Gleichung 6-12 lässt sich die kritische WEBER-Zahl aus den Messdaten ermitteln.

$\mathrm{We}_{\text {krit }}=\frac{\mathrm{d}_{\mathrm{B}, \max }}{\sigma} \cdot \rho_{\mathrm{L}} \cdot\left(\frac{\mathrm{d}_{\mathrm{B}, \max }}{\rho_{\mathrm{L}}} \cdot \mathrm{u}_{\mathrm{G}, \text { Klein }} \cdot \mathrm{g} \cdot \Delta \rho\right)^{2 / 3}$

Gleichung 6-12: Kritische Weber-Zahl für die Koaleszenz von Kleinblasen

Dabei wird für die Blasengröße $\mathrm{d}_{\mathrm{B}, \max }$ die nach IDOGAWA berechnete Blasengröße eingesetzt. Die kritische WEBER-Zahl lässt sich nur für den heterogenen Betriebszustand bestimmen, da ansonsten der Kleinblasenstrom $\mathrm{u}_{\mathrm{G}, \mathrm{Klein}}$ noch nicht sein Maximum erreicht hat.

Für die durchgeführten Versuche ergeben sich WEBER-Zahlen in dem Bereich von We $=0,6-$ 3,5. Die so ermittelte WEBER-Zahl ist also keine Konstante, sondern wiederum abhängig von Gasdichte, Gasgeschwindigkeit und den anderen Einflussgrößen.

Kritisch ist die Gleichsetzung der Turbulenzgröße mit dem Blasendurchmesser $\mathrm{d}_{\text {turb }}=\mathrm{d}_{\mathrm{B} \text {, max }}$. Die Größe der Mikroturbulenzen hängt nicht allein von der Größe der Kleinblasen ab, sondern auch von der eingetragenen Energie und den spezifischen Stoffeigenschaften der Flüssigkeit.

Wandelt man Gleichung 6-12 leicht ab, so erhält man eine brauchbare Abschätzung des maximalen Kleinblasenstromes:

Konst. $=\mathrm{d}_{\mathrm{B}, \max } \cdot \rho_{\mathrm{L}} \cdot\left(\mathrm{u}_{\mathrm{G}, \text { Klein }} \cdot \mathrm{g} \cdot \frac{\Delta \rho}{\rho_{\mathrm{L}}}\right)^{2 / 3}$

Gleichung 6-13: Abgewandelte Form von Gleichung 6-12

Die Oberflächenspannung $\sigma$ und die Turbulenzgröße $d_{\text {turb }}$ verschwinden aus der Gleichung und man erhält für alle Flüssigkeiten einen konstanten Wert.

In Abbildung 6-11 sind die Ergebnisse über dem Kleinblasenstrom $\mathrm{u}_{\mathrm{G}, \mathrm{Klein}}$ aufgetragen. Der Mittelwert aller Messungen ist Konst. $=1,57$. Dabei zeigen die einzelnen Flüssigkeiten Abweichungen von diesem Mittelwert (siehe Tabelle 6-3). 


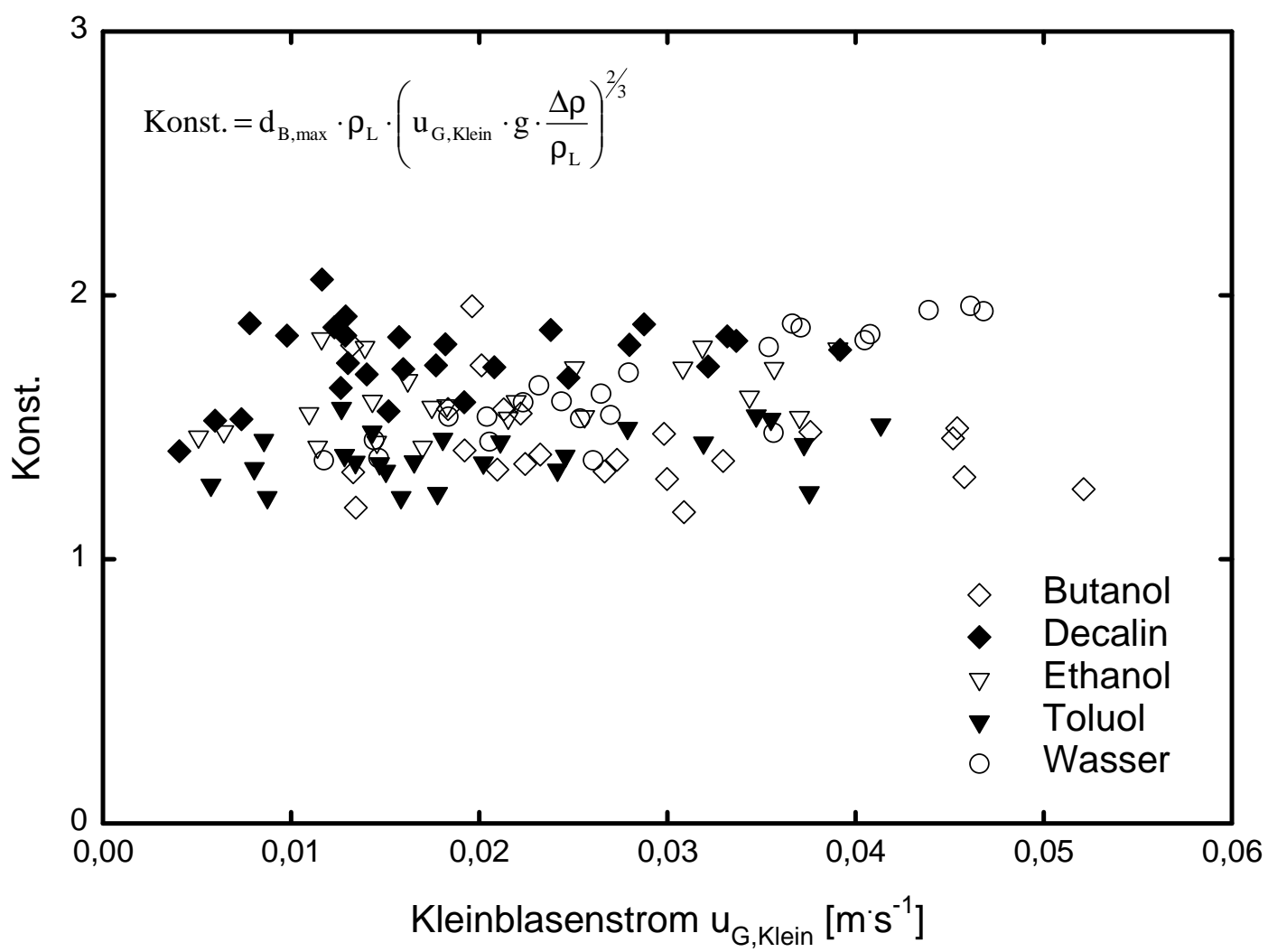

Abbildung 6-11: Auftragung der Ergebnisse von Gleichung 6-13 über dem Kleinblasenstrom

Tabelle 6-3: Mittlere Werte für den Term Konst aus Gleichung 6-13

\begin{tabular}{|c|c|c|c|c|c|c|}
\hline Flüssigkeit & Butanol & Decalin & Ethanol & Toluol & Wasser & Mittelwert \\
\hline Konstante & 1,45 & 1,76 & 1,61 & 1,40 & 1,65 & 1,57 \\
\hline
\end{tabular}

Es ist nun möglich, die Grenzgasgeschwindigkeit $\mathrm{u}_{\mathrm{G}, \mathrm{t}}$ für den Übergang zwischen homogenem und heterogenem Betriebszustand abzuschätzen. Dabei handelt es sich um einen empirisch gefundenen Zusammenhang. Eine bessere Abschätzung der Größe der Mikroturbulenzen $d_{\text {turb }}$ könnte dieses Modell wesentlich verbessern.

\subsection{Blasengeschwindigkeiten}

Die Blasengeschwindigkeiten hängen von zwei Faktoren ab. Die Blasengröße determiniert die Aufstiegsgeschwindigkeit einer Einzelblase in der freien Flüssigkeit. Die Berechnung erfolgt mit der Gleichung nach MENDELSON und MANERI. Gleichzeitig wird die Blase in ihrem Aufstieg durch benachbarte Blasen und die Reaktorwand behindert und sie kann durch herauf strömende Flüssigkeit beschleunigt werden.

\subsubsection{Kleinblasengeschwindigkeit}

Die Kleinblasengeschwindigkeit wurde im Kapitel 6.1.1 ausführlich behandelt. 


\subsubsection{Großblasengeschwindigkeit}

Der Durchmesser der Großblasen $\mathrm{d}_{\mathrm{B}, \mathrm{Gro}}$ berechnet sich nach Gleichung 6-7. Die Blasenaufstiegsgeschwindigkeit in der freien Flüssigkeit wird nach MENDELSON und MANERI ermittelt. In einer Blasensäule wird der Aufstieg einer großen Blase durch Wandeffekte gehemmt. Diese Hemmung ist schon bei einem Blasendurchmesser von $1 / 10$ des Blasensäulendurchmessers signifikant und wird mit zunehmenden Blasendurchmesser schnell größer. Für mittlere Blasengrößen findet sich bei CLIFT („Bubbles, Drops and Particles“) folgende Beziehung:

$\frac{\mathrm{v}_{\mathrm{B}}}{\mathrm{v}_{\mathrm{B}, \mathrm{frei}}}=\left[1-\left(\frac{\mathrm{d}_{\mathrm{B}}}{\mathrm{d}_{\mathrm{BS}}}\right)^{2}\right]^{3 / 2}$

Gleichung 6-14: Blasenaufstiegshemmung für mittlere Blasen

Diese Gleichung gilt für Blasen mit Bo $<40 ; \mathrm{Re}>200$ und $\mathrm{d}_{\mathrm{B}} / \mathrm{d}_{\mathrm{BS}}<0,6$.

Für große Blasen wurde eine andere Beziehung gefunden (LETZEL ET AL 1999):

$\frac{\mathrm{v}_{\mathrm{B}}}{\mathrm{v}_{\mathrm{B}, \mathrm{frei}}}=1,13 \cdot \mathrm{e}^{-\frac{\mathrm{d}_{\mathrm{B}}}{\mathrm{d}_{\mathrm{BS}}}}$

Gleichung 6-15: Blasenaufstiegshemmung für große Blasen

Diese Gleichung gilt für Bo $>40$ und für $0,125<\mathrm{d}_{\mathrm{B}} / \mathrm{d}_{\mathrm{BS}}<0,6$. Für $\mathrm{d}_{\mathrm{B}} / \mathrm{d}_{\mathrm{BS}}<0,125$ können die Wandeffekte vernachlässigt werden; die Aufstiegsgeschwindigkeit entspricht der freien Aufstiegsgeschwindigkeit. 


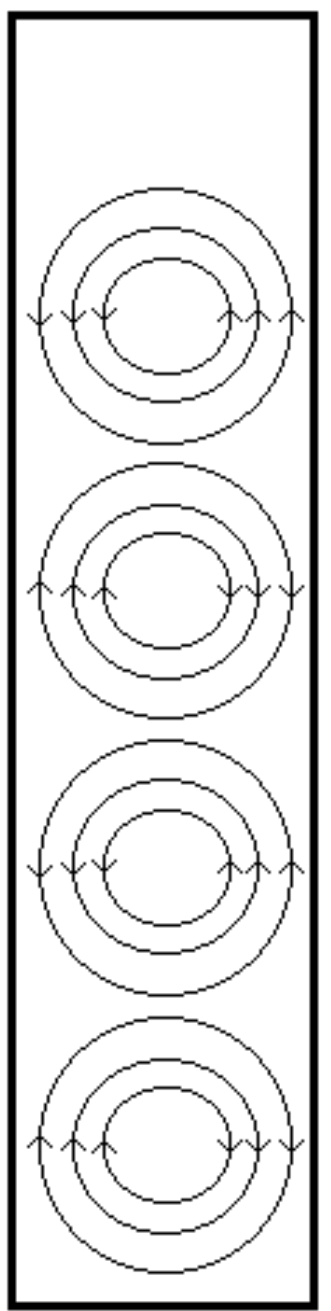

Abbildung 6-12: Makroturbulenzen in einer Blasensäule

Die Großblasengeschwindigkeit dürfte in diesem Fall $\mathrm{v}_{\mathrm{B}}=0,4 \mathrm{~ms}^{-1}$ nicht überschreiten, tatsächlich werden aber wesentlich höhere Geschwindigkeiten beobachtet. Da die Relativgeschwindigkeit der Blasen zur Flüssigkeit nicht wachsen kann, muss die Flüssigkeit die Blasen in ihrer Bewegung mitschleppen. Die Turbulenzgeschwindigkeit der Flüssigkeit kann mit empirischen Gleichungen beschrieben werden. In der Literatur trifft man oft auf die Korrelation von ZEHNER (1986) (KRISHNA 1999; GROEN 1996; VIAL 2001)

$v_{\text {turb }}=0,737 \cdot\left(u_{G} \cdot g \cdot d_{B S}\right)^{1 / 3}$

Addiert man die so ermittelte Geschwindigkeit der Flüssigkeit zu der Großblasengeschwindigkeit hinzu, erhält man wesentlich höhere Werte für die Großblasengeschwindigkeiten. In Abbildung 6-13 sind die so ermittelten Großblasengeschwindigkeiten über den gemessenen Großblasengeschwindigkeiten als Paritätsplot aufgetragen. Im Mittel erhält man mit der Berechnung sehr gute Werte. Bei sehr großen Geschwindigkeiten werden zu niedrige Werte berechnet. Dabei könnte es sich aber um Messfehler handeln, da sich große Geschwindigkeiten nur ungenau bestimmen lassen. In solchen Fällen sind die Blasen in weniger als einer Sekunde aus dem Messvolumen entwichen. Ein Messfehler von 0,1 s entspricht dann einem Fehler von über $10 \%$. 
Bei kleinen Blasenaufstiegsgeschwindigkeiten hingegen ergibt die Berechnung zu hohe Werte. Solche Fälle sind typisch für Betriebszustände, bei denen die Gasleerrohrgeschwindigkeit knapp über der Übergangsgeschwindigkeit $\mathrm{u}_{\mathrm{G}, \mathrm{t}}$ liegt. In solchen Fällen erreichen die Großblasen nicht ihre maximale Größe und sind langsamer, als in der Berechnung vorhergesagt.

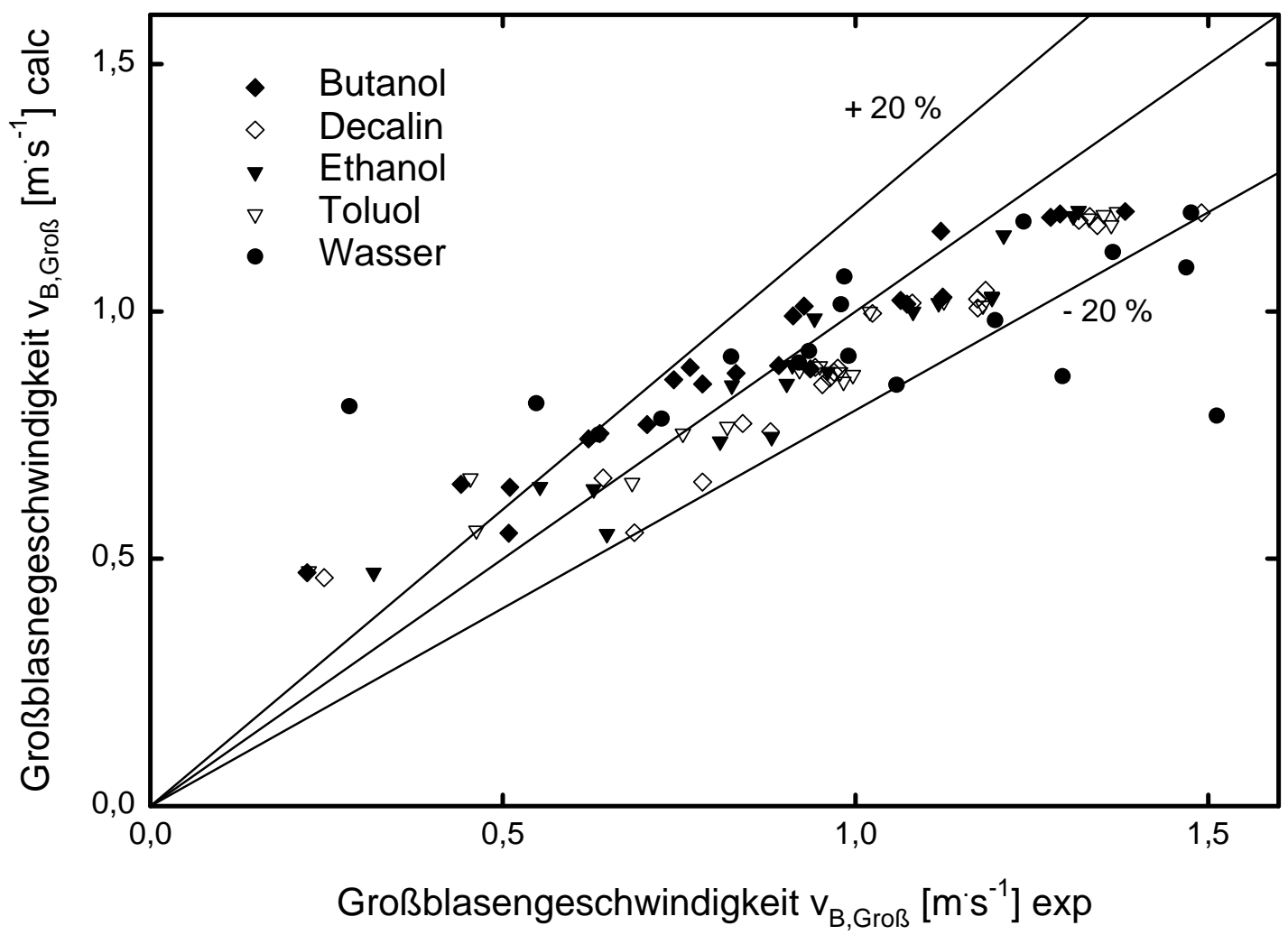

Abbildung 6-13: Vergleich der experimentell ermittelten und der berechneten Großblasengeschwindigkeiten

\subsection{Gasgehalt und Stoffübergangszahlen}

Die Korrelation des Gasgehaltes ist komplex, da einerseits Stoff- und Betriebsparameter einen Einfluss auf die Blasenbildung und -zerfall haben und andererseits sehr komplexe Vorgänge in dem Zweiphasengemisch ablaufen. Ein interessanter Ansatz zur Lösung des Problems ist die Korrelation von Öztürk (ÖzTÜRK ET. AL. 1987). Dabei werden dimensionslose Kennzahlen miteinander verknüpft. So erstellte Korrelationen sind geeignet, auf andere Systeme (andere Apparate, andere Stoffsysteme) übertragen zu werden. Die hier vorgestellten Korrelationen wurden bereits veröffentlicht (JORDAN, SCHUMPE 2001)

Die zur Anwendung kommenden dimensionslosen Kennzahlen sind im Formelverzeichnis aufgeführt.

Die in dieser Arbeit gewonnen Daten wurden für die Anpassung von Korrelationen verwendet (JORDAN, SCHUMPE 2001). Dabei sind folgende Korrelationen entstanden: 


$$
\mathrm{Sh}^{\prime}=\mathrm{a}_{1} \cdot \mathrm{Sc}^{0,5} \cdot \mathrm{Bo}^{0,34} \cdot \mathrm{Ga}^{0,27} \cdot \mathrm{Fr}^{0,72} \cdot\left(1+13,2 \cdot \mathrm{Fr}^{0,37} \cdot\left(\frac{\rho_{\mathrm{G}}}{\rho_{\mathrm{L}}}\right)^{0,49}\right)
$$

Gleichung 6-16: Korrelation der Stoffübergangszahlen $\mathrm{k}_{\mathrm{L}} \mathrm{a}^{*}$ von Jordan, Schumpe 2001

$$
\frac{\varepsilon_{\mathrm{G}}}{1-\varepsilon_{\mathrm{G}}}=\mathrm{b}_{1} \cdot \mathrm{Bo}^{0,16} \cdot \mathrm{Ga}^{0,04} \cdot \mathrm{Fr}^{0,7} \cdot\left(1+27,0 \cdot \mathrm{Fr}^{0,52} \cdot\left(\frac{\rho_{\mathrm{G}}}{\rho_{\mathrm{L}}}\right)^{0,58}\right)
$$

Gleichung 6-17: Korrelation des Gasgehaltes $\varepsilon_{\mathrm{G}}$ von Jordan, Schumpe 2001

Die Konstanten $a_{1}$ und $b_{1}$ sind begaserspezifisch

Tabelle 6-4: Begaserspezifische Faktoren der Gleichung 6-16 und Gleichung 6-17

\begin{tabular}{|c|c|c|c|c|c|}
\hline Daten & Begasertyp & $\begin{array}{c}\text { Länge } \\
\text { Kolonne [m] }\end{array}$ & $\begin{array}{c}\text { Durchmesser } \\
\text { Kolonne [m] }\end{array}$ & $\mathrm{a}_{1}$ & $\mathrm{~b}_{1}$ \\
\hline diese Arbeit & $\begin{array}{c}\text { Lochplatte } \\
19 \times 1 \mathrm{~mm}\end{array}$ & 1,3 & 0,1 & 0,522 & 0,112 \\
\hline diese Arbeit & $\begin{array}{c}\text { Lochplatte } \\
1 \times 1 \mathrm{~mm}\end{array}$ & 1,3 & 0,1 & 0,599 & 0,122 \\
\hline diese Arbeit & $\begin{array}{c}\text { Lochplatte } \\
1 \times 4,3 \mathrm{~mm}\end{array}$ & 1,3 & 0,1 & 0,488 & 0,109 \\
\hline $\begin{array}{c}\text { ÖzTÜRK } \\
\text { ET.AL. 1987 }\end{array}$ & $\begin{array}{c}\text { Begaserrohr } \\
3 \mathrm{~mm}\end{array}$ & 0,85 & 0,095 & 0,553 & 0,135 \\
\hline diese Arbeit & $\begin{array}{c}\text { Lochplatte } \\
7 \times 1 \mathrm{~mm}\end{array}$ & 1,0 & 0,115 & 0,669 & 0,153 \\
\hline
\end{tabular}

Der Gültigkeitsbereich für die Korrelationen erstreckt sich über die folgenden Bereiche der dimensionslosen Kennzahlen:

$71 \leq \mathrm{Sc} \leq 68,9 \cdot 10^{3}$

$1,21 \leq$ Bo $\leq 5,39$

$825 \leq \mathrm{Ga} \leq 1,55 \cdot 10^{6}$

$0,06 \leq$ Fr $\leq 1,22$

$93 \cdot 10^{-6} \leq\left(\rho_{\mathrm{G}} / \rho_{\mathrm{L}}\right) \leq 0,059$

In diese Korrelation sind die Messdaten von ÖZTÜRK et.al von 1987 und die Messdaten von Ethanol, 1-Butanol, Decalin und Toluol aus dieser Arbeit eingeflossen. Der mittlere Fehler für den Gasgehalt $\varepsilon_{\mathrm{G}}$ liegt bei $11,2 \%$ und für die Stoffübergangszahl $\mathrm{k}_{\mathrm{L}} \mathrm{a}^{\prime}$ bei $13,8 \%$. 
Nun soll geklärt werden, wie gut die experimentellen Ergebnisse aller Stoffe durch die verschiedenen Korrelationen widergespiegelt werden. Zunächst wird der Gasgehalt betrachtet.

Tabelle 6-5: Vergleich der mittleren Fehler für die Korrelationen des Gasgehaltes

\begin{tabular}{|l|c|}
\hline Korrelation & mittlerer Fehler [\%] \\
\hline HIKITA 1980 & $30,0 \%$ \\
\hline HAMMER 1984 & $39,1 \%$ \\
\hline IDOGAWA 1985 & $58,3 \%$ \\
\hline REILLY 1986 & $112 \%$ \\
\hline IDOGAWA 1987 & $83,6 \%$ \\
\hline WILKINSON 1992 & $75,5 \%$ \\
\hline LETZEL 1999 & $46,2 \%$ \\
\hline JORDAN SCHUMPE 2001 & $15,1 \%$ \\
\hline BEKISH 2008 & $62,5 \%$ \\
\hline
\end{tabular}

Erwartungsgemäß zeigt die Korrelation von Jordan Schumpe 2001 den kleinsten Fehler. Die hier ausgewerteten Daten lagen dieser Korrelation zugrunde. Allerdings ist der Fehler, der sich mit der Korrelation Gleichung 6-17 ergibt größer, als in der zitierten Literatur (JoRDAN; SCHUMPE 2001). In dieser Publikation wurden nur die Messwerte für Ethanol, Butanol, Toluol und Decalin ausgewertet. In der Tabelle 6-5 sind auch die Messwerte für Wasser und Ethylenglycol eingeflossen. Schlüsselt man die mittleren Fehler nach den eingesetzten Stoffen auf, dann zeigt sich, dass vor allem die Werte mit Wasser die größten Abweichungen aufweisen. Werden die Werte mit Wasser ausgeschlossen, dann ergibt sich ein mittlerer Fehler von $11,3 \%$.

Tabelle 6-6: Vergleich der mittleren Fehler für die Korrelationen des Gasgehaltes für die einzelnen Substanzen

\begin{tabular}{|l|c|c|c|c|c|c|}
\hline Korrelation & Butanol & Decalin & Ethanol & Ethylenglycol & Wasser & Toluol \\
\hline JORDAN SCHUMPE 2001 & $9,7 \%$ & $15,5 \%$ & $11,4 \%$ & $8,0 \%$ & $21,7 \%$ & $12,6 \%$ \\
\hline
\end{tabular}

In Abbildung 6-14 sind die gemessenen und berechneten Gesamtgasgehalte gegeneinander aufgetragen. Es lässt sich erkennen, dass gerade die Werte für Wasser am stärksten von der Parität abweichen. 


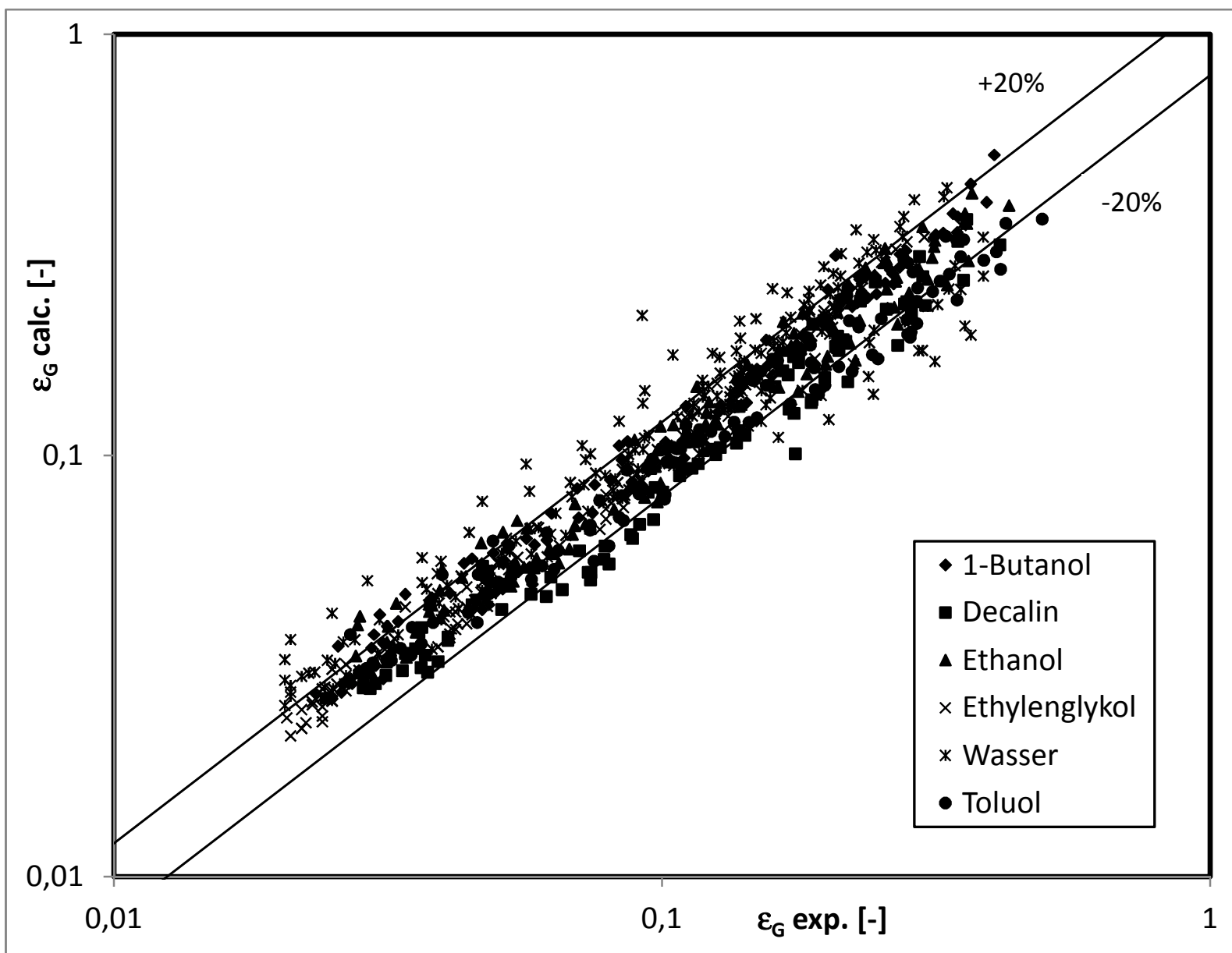

Abbildung 6-14: Paritätsplot der Gesamtgasgehalte aller Messungen dieser Arbeit für Gleichung 6-17(Jordan, Schumpe 2001)

Bei der Auswertung der Stoffübergangszahlen ergibt sich ein ganz anderes Bild

Tabelle 6-7: Vergleich der mittleren Fehler für die Korrelationen der Desorptionsraten $\mathrm{k}_{\mathrm{L}} \mathrm{a}^{*}$

\begin{tabular}{|l|c|}
\hline Korrelation & mittlerer Fehler [\%] \\
\hline ÖZTÜRK 1987 & $38,5 \%$ \\
\hline WILKINSON 1992 & $86,9 \%$ \\
\hline LETZEL 1999 & $183 \%$ \\
\hline JORDAN SCHUMPE 2001 & $44,6 \%$ \\
\hline LEMOINE 2008 & $36,9 \%$ \\
\hline
\end{tabular}

Die Korrelation von JORDAN /SCHUMPE weist mit 44,6\% einen größeren Fehler auf, als die Korrelationen von LEMOINE und ÖZTÜRK. In diesem Fall wurden die Daten für die Flüssigkeiten Wasser und Ethylenglycol mit ausgewertet. In der Korrelation von JORDAN/SCHUMPE wurden diese Werte verworfen. Beim Wasser bestand der Verdacht, dass biologischer Bewuchs die Eigenschaften des Wassers verändert hat. Das Ethylenglycol fällt aufgrund seiner hohen Viskosität aus dem Rahmen. In Abbildung 5-21 stammen alle Werte mit einer Viskosität > $3 \mathrm{mPas}$ vom Ethylenglycol. Gerade in diesem Bereich ist der Einfluss der Viskosität auf die Stoffübergangszahl besonders hoch.

Betrachtet man die Fehler der Korrelationen aufgeschlüsselt auf die einzelnen Flüssigkeiten, dann erkennt man, dass Ethylenglycol und Wasser, also die beiden Stoffe, deren Daten bei der Erstellung der Korrelation nicht eingeflossen sind, die größte Abweichung zeigen. 
Tabelle 6-8: Vergleich der mittleren Fehler für die Korrelationen der Desorptionsraten $\mathrm{k}_{\mathrm{L}} \mathrm{a}^{*}$ aufgeschlüsselt auf die Flüssigkeiten

\begin{tabular}{|l|c|c|c|c|c|c|}
\hline & 1-Butanol & Decalin & Ethanol & $\begin{array}{c}\text { Ethylen- } \\
\text { glycol }\end{array}$ & Wasser & Toluol \\
\hline ÖZTÜRK 1987 & $58,2 \%$ & $58,3 \%$ & $41,9 \%$ & $27,8 \%$ & $29,2 \%$ & $27,9 \%$ \\
\hline WILKINSON 1992 & $90,1 \%$ & $92,3 \%$ & $87,0 \%$ & $77,1 \%$ & $89,1 \%$ & $87,7 \%$ \\
\hline LETZEL 1999 & $69,7 \%$ & $48,0 \%$ & $52,9 \%$ & $735,1 \%$ & $116,4 \%$ & $25,2 \%$ \\
\hline JORDAN SCHUMPE 2001 & $12,5 \%$ & $23,5 \%$ & $31,8 \%$ & $113,4 \%$ & $51,9 \%$ & $12,3 \%$ \\
\hline LEMOINE 2008 & $42,8 \%$ & $43,6 \%$ & $36,4 \%$ & $36,6 \%$ & $38,5 \%$ & $23,3 \%$ \\
\hline
\end{tabular}

Auch hier ist es so, dass die Werte für Wasser und Ethylenglycol nicht in die Korrelation von JORDAN SCHUMPE 2001 eingeflossen ist. Bei diesen beiden Substanzen zeigen sich die größten Abweichungen zwischen berechneten und gemessenen Werte. In dem Paritätsplot in Abbildung 6-15 ist gut zu sehen, dass die berechneten Werte für den Stoffübergangszahlen in Wasser und Ethylenglycol zu hoch sind.

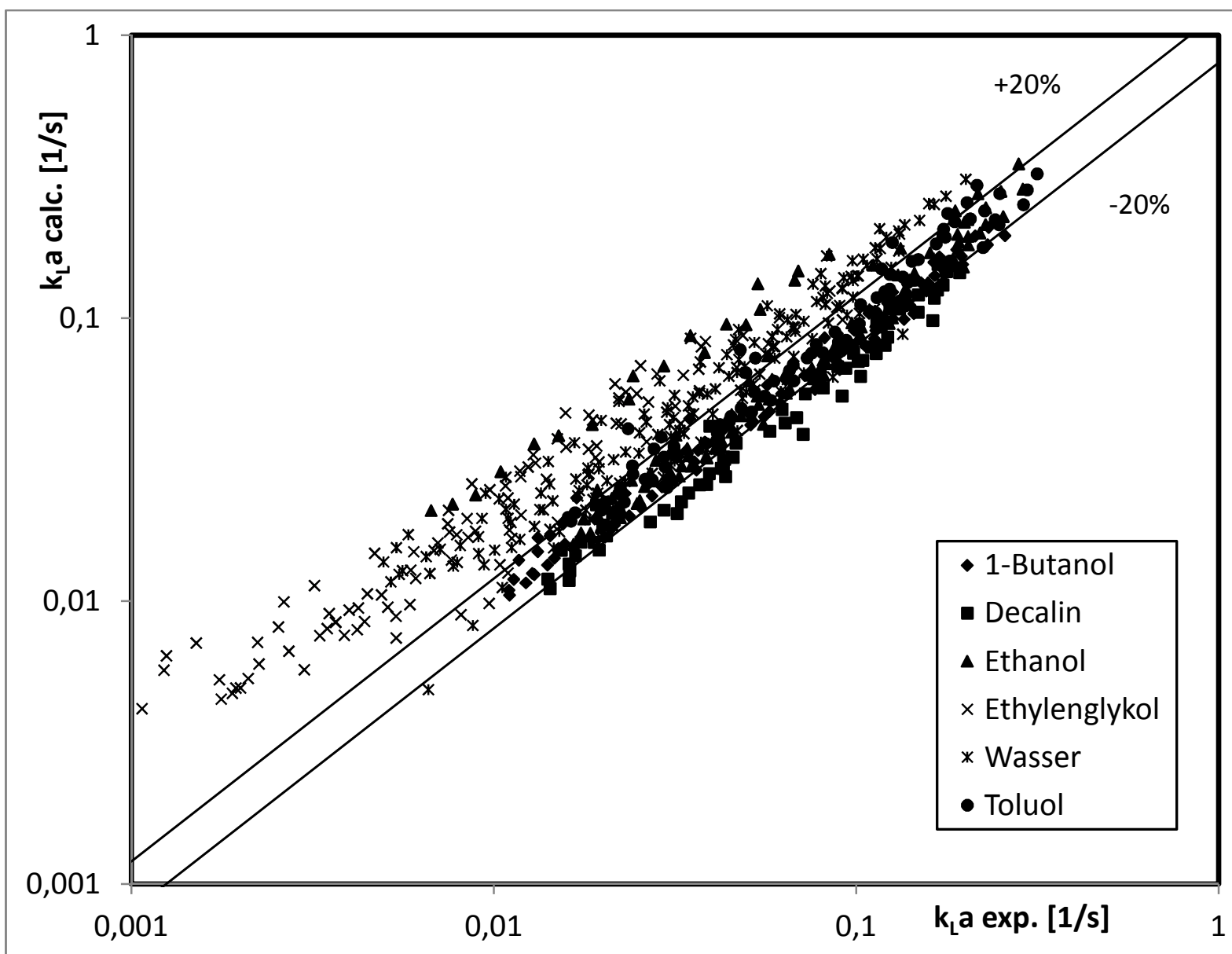

Abbildung 6-15: Paritätsplot der Stoffübergangszahlen dieser Arbeit für Gleichung 6-16 (Jordan, Schumpe 2001)

Fazit:

Für technische Anwendungen sind die hier vorgestellten Korrelationen für Stoffübergang und Gasgehalt hinreichend, wenn bei der Planung solcher Anlagen eine ausreichend große Reserve von ca. 30\% einkalkuliert wird. Für wissenschaftliche Ansprüche sind die Abweichungen immer noch viel zu groß.

Das System der Blasensäulen ist immer noch nicht ausreichend verstanden, um präzise Aussagen über das Verhalten zu treffen. Für solche Aussagen ist anscheinend ein tieferes 
Verständnis der turbulenten Vorgänge in den Fluiden notwendig. Derartige Versuche werden bereits unternommen (NedELTCHEV 2006; NedeltCHEV 2007; NeDELTCHEV 2010).

Die Forschungen $\mathrm{zu}$ Gas-Flüssig-Reaktoren im Allgemeinen und zu Blasensäulen im speziellen sind weltweit ein großes Thema. Allein für 2014 ergibt eine Suche nach Publikationen zu „Bubble Column“ und „Mass Transfer“ in Google Scholar über 1000 Treffer.

Ein aktueller Trend ist die Beschreibung von Blasensäulen mittels numerischer Simulationen (XING et. al. 2013; Xu et. al 2013; Pourtousi et. al. 2015(1) ; Pourtousi et. al. 2015(2)). Der Rechenaufwand für solche Herangehensweise ist je nach Auflösung des Modells beliebig hoch. Da die verfügbaren Rechenleistungen ständig steigen, ist dies ein gangbarer Weg für ein realistisches Modell für Blasensäulen. Allerdings müssen auch die numerischen Modelle an realen Versuchsreihen geprüft und kalibriert werden.

Dabei gilt es alle potentiellen Einflussparameter in einem weiten Bereich zu variieren. Idealerweise sollten alle betrachteten Parameter unabhängig voneinander variiert werde. In dieser Arbeit wurde vor allem Wert auf die Variation der Gasdichte und ihres Einflusses auf den Gasgehalt und den Stoffübergang gelegt.

Der Einfluss der verwendeten Flüssigkeit auf Gasgehalt und Stoffübergang wurde ebenfalls untersucht. Allerdings lassen sich die Parameter wie Oberflächenspannung, Viskosität und Dichte der Flüssigkeit nicht unabhängig voneinander variieren, sondern sind an die verwendeten Stoffsysteme gebunden. Die Anzahl der veränderbaren Parameter erhöht sich, wenn keine reinen Flüssigkeiten, sondern Gemische von Flüssigkeiten verwendet werden. Der Einsatz von oberflächenaktiven Komponenten und suspendierten Feststoffen erweitert die Variationsmöglichkeiten noch weiter.

Aber auch die Geometrie des Versuchsaufbaus hat Einfluss auf die ermittelten Parameter Gasgehalt und Stoffübergangszahl. Vor allem der Begaser hat einen signifikanten Einfluss auf die untersuchten Größen (siehe Abschnitt 5.1.5 und 5.2.4). Der Begaser ist auch Gegenstand jüngerer Untersuchungen (SINAN et. al. 2013; POURTOUSI et al 2014). Allgemein lässt sich sagen: je größer die Öffnungen im Begaser sind, desto größer ist die durchschnittliche Blasengröße und der Übergang zum heterogenen Strömungsregime erfolgt bei kleineren Gasleerrohrgeschwindigkeiten. Eine numerische Simulation der Begaserregion scheint gegenwärtig noch nicht möglich zu sein (POURTOUSI et. al. 2015(1)). Die gegenwärtigen Modelle müssen mit Messungen an realen Systemen kalibriert werden.

Die große Anzahl potentieller Einflussparameter auf die Vorgänge in Blasensäulen erschwert die Bewertung von publizierten Messreihen in Blasensäulenreaktoren: Werden nicht alle Parameter exakt dokumentiert, und fließen so in die Modelle ein, kann das zu irreführenden Ergebnissen führen. In dieser Arbeit ist im Kapitel „Stand der Forschung“ bereits auf solche irreführenden Modelle eingegangen worden (siehe KoJIMA et al 1995 in Abschnitt 2.2).

Ein weiterer Weg, die Modelle für Blasensäulen zu verbessern, ist die Integration neuer Teilmodelle in das Gesamtmodell. In dieser Arbeit wurde von einer homogen durchmischten flüssigen Phase in Blasensäulen ausgegangen (siehe Abschnitt 4.2.2 bis 4.2.4). Korrekt wäre die Annahme einer endlichen Durchmischungsgeschwindigkeit (NEDELTCHEV et al 2015).

Dies alles ist jedoch nicht Bestandteil dieser Arbeit. Eine weitere Verfeinerung der Modelle und ein tieferes Verständnis bleibt künftigen Arbeiten vorbehalten. 


\section{Symbolverzeichnis}

Lateinische Buchstaben

\begin{tabular}{|c|c|c|}
\hline Symbol & Einheit & Beschreibung \\
\hline $\mathrm{a}$ & $\mathrm{m}^{-1}$ & spezifische Phasengrenzfläche: $\mathrm{a}=\mathrm{A} \mathrm{V}^{-1}$ \\
\hline $\mathrm{A}$ & $\mathrm{m}^{2}$ & Grenzfläche; Fläche allgemein \\
\hline$\overline{A_{B S}}$ & $\mathrm{~m}^{3}$ & Querschnittsfläche der Blasensäule \\
\hline $\mathrm{c}$ & $\mathrm{kmol} \mathrm{m}^{-3}$ & Konzentration \\
\hline $\mathrm{c}_{\mathrm{O} 2}$ & $\%$ Luftsättigung & $\begin{array}{l}\text { Sauerstoffkonzentration; } 100 \% \text { Luftsättigung entsprechen } \\
0,21 \text { bar Sauerstoffpartialdruck bzw. dem dazu im } \\
\text { Gleichgewicht stehenden gelöstem Sauerstoff }\end{array}$ \\
\hline $\mathrm{c}_{\mathrm{P}}$ & $\mathrm{J} \mathrm{kmol}^{-1} \mathrm{~K}^{-1}$ & Wärmekapazität bei konstantem Druck \\
\hline $\mathrm{c}_{\mathrm{W}}$ & - & Widerstandsbeiwert umströmter Körper \\
\hline $\mathrm{D}_{\mathrm{ax}}$ & $\mathrm{m}^{2} \mathrm{~s}^{-1}$ & axialer Dispersionskoeffizient \\
\hline $\mathrm{D}_{\mathrm{L}}$ & $\mathrm{m}^{2} \mathrm{~s}^{-1}$ & Diffusionskoeffizient in der Flüssigkeit \\
\hline $\mathrm{d}_{\mathrm{S}}$ & $\mathrm{m}$ & Sauterdurchmesser \\
\hline $\mathrm{d}_{\mathrm{B}}$ & $\mathrm{m}$ & Blasendurchmesser \\
\hline $\mathrm{d}_{\mathrm{BS}}$ & $\mathrm{m}$ & Blasensäulendurchmesser \\
\hline $\mathrm{d}_{\mathrm{O}}$ & $\mathrm{m}$ & Durchmesser der Begaseröffnung \\
\hline Eo & - & EÖTVÖS-Zahl: Eo $=\mathrm{g} \cdot \Delta \rho \cdot \mathrm{d}_{\mathrm{B}}{ }^{2} \cdot \sigma^{-1}$ \\
\hline $\mathrm{g}$ & $\mathrm{m} \mathrm{s}^{-2}$ & Fallbeschleunigung $\left(\mathrm{g}=9,81 \mathrm{~m}^{-2}\right)$ \\
\hline $\mathrm{h}$ & $\mathrm{m}$ & Höhe über dem Begaser \\
\hline $\mathrm{I}$ & & Fluoreszenzintensität \\
\hline $\mathrm{I}_{0}$ & & Fluoreszenzintensität ohne Sauerstoff \\
\hline $\mathrm{K}_{\mathrm{SV}}$ & $\mathrm{m}^{3} \mathrm{kmol}^{-1}$ & STERN-VOLLMER-Konstante \\
\hline $\mathrm{k}_{\mathrm{L}} \mathrm{a}$ & $\mathrm{s}^{-1}$ & volumenbezogene Stoffübergangszahl \\
\hline $\mathrm{k}_{\mathrm{L}}$ & $\mathrm{m} \mathrm{s}^{-1}$ & flüssigkeitsseitiger Stoffübergangskoeffizient \\
\hline $\mathrm{k}_{\mathrm{L}} \mathrm{a}^{\prime}$ & $\mathrm{s}^{-1}$ & $\begin{array}{l}\text { auf das Flüssigkeitsvolumen bezogene Stoffübergangszahl: } \\
\mathrm{k}_{\mathrm{L}} \mathrm{a}^{\prime}=\mathrm{k}_{\mathrm{L}} \mathrm{a}\left(1-\varepsilon_{\mathrm{G}}\right)^{-1}\end{array}$ \\
\hline $\mathrm{k}_{\mathrm{L}} \mathrm{a}^{*}$ & $\mathrm{~s}^{-1}$ & Desorptionsrate aus den Stoffübergangsmessungen \\
\hline $\mathrm{L}_{\mathrm{O} 2}$ & - & OsTWALD-Koeffizient für Sauerstoff: $\mathrm{L}=\mathrm{c}_{\mathrm{L}} \mathrm{c}_{\mathrm{G}}{ }^{-1}$ \\
\hline $\mathrm{m}$ & $\mathrm{kg}$ & Masse \\
\hline$\dot{\mathrm{m}}$ & $\mathrm{kg} \mathrm{s}^{-1}$ & Massestrom \\
\hline Mo & - & MORTON-Zahl: $\mathrm{Mo}=\mathrm{g} \cdot \Delta \rho \mu_{\mathrm{L}}^{4} \rho_{\mathrm{L}}^{-2 \cdot} \sigma_{\mathrm{L}}^{-3}$ \\
\hline $\mathrm{p}$ & bar & Druck \\
\hline$\Delta \mathrm{p}_{0}$ & bar & Differenzdruck mit Begasung \\
\hline$\Delta \mathrm{p}_{\mathrm{E}}$ & bar & Differenzdruck ohne Begasung \\
\hline $\mathrm{P}$ & $\mathrm{kg} \mathrm{m}^{2} \mathrm{~s}^{-3}$ & Leistung \\
\hline $\mathrm{P}_{\mathrm{Q}}$ & $\mathrm{J} \mathrm{s}^{-1}$ & Heizleistung \\
\hline $\mathrm{T}$ & $\mathrm{K}$ & Temperatur \\
\hline $\mathrm{t}$ & $\mathrm{s}$ & Zeit \\
\hline $\mathrm{U}$ & $\mathrm{V}$ & Spannung des Photomultipliers \\
\hline $\mathrm{U}_{0}$ & $\mathrm{~V}$ & $\begin{array}{l}\text { Spannung des Photomultipliers bei Abwesenheit von } \\
\text { Sauerstoff }\end{array}$ \\
\hline $\mathrm{U}_{100}$ & $\mathrm{~V}$ & Spannung des Photomultipliers bei $100 \%$ Sauerstoffsättigung \\
\hline $\mathrm{u}_{\mathrm{G}}$ & $\mathrm{m} \mathrm{s}^{-1}$ & Gasleerrohrgeschwindigkeit \\
\hline $\mathrm{V}$ & $\mathrm{m}^{3}$ & Volumen \\
\hline
\end{tabular}




\begin{tabular}{|l|l|l|}
\hline$\dot{\mathrm{V}}$ & $\mathrm{m}^{3} \mathrm{~s}^{-1}$ & Volumenstrom \\
\hline $\mathrm{v}$ & $\mathrm{m} \mathrm{s}^{-1}$ & Geschwindigkeit \\
\hline $\mathrm{v}_{\mathrm{O}}$ & $\mathrm{m} \mathrm{s}^{-1}$ & Gasgeschwindigkeit in der Begaseröffnung \\
\hline
\end{tabular}

Griechische Buchstaben

\begin{tabular}{|l|l|l|}
\hline Symbol & Einheit & Beschreibung \\
\hline$\varepsilon_{\mathrm{G}}$ & - & Gasgehalt \\
\hline$\rho$ & $\mathrm{kg} \mathrm{m}^{-3}$ & Dichte \\
\hline$\eta$ & $\mathrm{Pa} \mathrm{s}$ & dynamische Viskosität \\
\hline$\sigma$ & $\mathrm{N} \mathrm{m}^{-1}$ & Oberflächenspannung \\
\hline$\nu$ & $\mathrm{m}^{2} \mathrm{~s}^{-1}$ & kinematische Viskosität \\
\hline
\end{tabular}

Indices

\begin{tabular}{|l|l|l|}
\hline Symbol & Einheit & Beschreibung \\
\hline 0 & - & zum Zeitpunkt $\mathrm{t}=0$ \\
\hline B & - & in der Einzelblase \\
\hline G & - & Gasphase \\
\hline Groß & - & Großblasen \\
\hline Klein & - & Kleinblasen \\
\hline L & - & Flüssigphase \\
\hline max & - & Maximalwert \\
\hline $\mathrm{O}_{2}$ & - & Sauerstoff \\
\hline $\mathrm{o}$ & - & oben \\
\hline $\mathrm{u}$ & - & unten \\
\hline
\end{tabular}

Dimensionslose Kennzahlen

\begin{tabular}{|l|l|l|}
\hline Symbol & Name & Gleichung \\
\hline Bo & BonD-Zahl & $\mathrm{Bo}=\frac{\mathrm{g} \cdot \rho_{\mathrm{L}} \cdot \mathrm{d}^{2}}{\sigma}$ \\
\hline Fl & Fluid-Kennzahl & $\mathrm{Fl}=\frac{\sigma_{\mathrm{L}} \cdot \rho_{\mathrm{L}}}{\eta_{\mathrm{L}} \cdot \Delta \rho \cdot \mathrm{v}}$ \\
\hline Fr & FrOUDE-Zahl & $\mathrm{Fr}=\frac{\mathrm{v}}{\sqrt{\mathrm{d} \cdot \mathrm{g}}}$ \\
\hline Ga & Frweiterte & $\mathrm{Fr}=\frac{\mathrm{v}^{2} \cdot \rho_{\mathrm{L}}}{\mathrm{d} \cdot \Delta \rho \cdot \mathrm{g}}$ \\
\hline Mo & GALILEI-Zahl & $\mathrm{Ga}=\frac{\mathrm{g} \cdot \mathrm{d}^{3}}{\mathrm{v}_{\mathrm{L}}^{2}}$ \\
\hline Re & MoRTON-Zahl & $\mathrm{Mo}=\frac{\eta_{\mathrm{L}}^{4} \cdot \mathrm{g} \cdot \Delta \rho}{\rho_{\mathrm{L}}^{2} \cdot \sigma^{3}}$ \\
\hline Sc & SCHMIDT-Zahl & $\mathrm{Sc}=\frac{\eta_{\mathrm{L}}}{\rho_{\mathrm{L}} \cdot \mathrm{D}_{\mathrm{L}}}$ \\
\hline
\end{tabular}




\begin{tabular}{|l|l|l|}
\hline $\mathrm{Sh}^{\prime}$ & SHERWOOD-Zahl & $\mathrm{Sh}^{\prime}=\frac{\mathrm{k}_{\mathrm{L}} \mathrm{a}^{*} \cdot \mathrm{d}_{\mathrm{B}}^{2}}{\mathrm{D}_{\mathrm{L}}}$ \\
\hline $\mathrm{St}$ & STANTON-Zahl & $\mathrm{St}=\frac{\mathrm{k}_{\mathrm{L}} \mathrm{a} \cdot \mathrm{V}_{\text {Disp }}}{\dot{\mathrm{V}}_{\mathrm{G}}}$ \\
\hline $\mathrm{We}$ & WEBER-Zahl & $\mathrm{We}=\frac{\mathrm{v}^{2} \cdot \mathrm{d} \cdot \rho}{\sigma}$ \\
\hline
\end{tabular}




\section{Literaturverzeichnis}

"Ullmann's encyclopaedia of industrial chemistry", Fifth edition, Volume B4

Editors: BARbara Elvers, STEPHEN HAWkins, GAIL SCHUlZ

VCH Verlagsgesellschaft mbH D-6940 Weinheim; ISBN: 3-527-20134-3.

AKITA K.; YoshidA F.

"Bubble Size, Interfacial Area, and Liquid-Phase Mass Transfer Coefficient in Bubble

Columns"

Industrial \& Engineering Chemistry Process Design and Development, 1974, 13. Jg., Nr.

1, S. 84-91.

BACH, H. F.; PILHOFER,

"Variation of Gas Hold-up in Bubble Columns with Physical Properties of Liquids and

Operating Parameters of Columns."

German Chemical Engineering, 1978, 1. Jg., S. 270-275.

BehKish, A., LEMoINE, R., OUKACI, R., \& Morsi, B. I.

"Novel Correlations for Gas Holdup in Large-Scale Slurry Bubble Column Reactors

Operating under Elevated Pressures and Temperatures"

Chemical Engineering Journal, 2006, 115. Jg., Nr. 3, S. 157-171.

Clift R., Grace J. R., Weber M. E.

"Bubbles, Drops and Particles"

1978 by Academic Press Inc. New York, San Francisco, London; ISBN0-12-176950-X.

CockX A., Roustan M., Line A., Hebrard G.

"Modelling of Mass Transfer Coefficient $\mathrm{k}_{\mathrm{L}}$ in Bubble Columns"

Transactions on IChemE, 1995, 73. Jg., S. A627-A631.

CRC Handbook of Chemistry and Physics

Published 1968 by The Chemical Rubber Co.

18901 Cranwood Parkway, Cleveland, Ohio 44128.

DEWES I.

"Untersuchung zum Einfluss der Gasdichte auf Stoffübergang und Gasgehalt in

Blasensäulen und Suspensionsblasensäulen“

Dissertation, Friedrich-Alexander-Universität Erlangen-Nürnberg, 1996.

Dudukovic A., Milosevic V., PJanovic R.

"Gas-Solid and Gas-Liquid Mass-Transfer Coefficients"

AIChE Journal, 1996, 42. Jg., Nr. 1, S. 269-270. 
ELLENBERGER J., KRISHNA R.

„A unified Approach to the Scale-Up of Gas-Solid Fluidized Bed and Gas-Liquid Bubble Column Reactors"

Chemical Engineering Science, 1994, 49. Jg., Nr. 24, S. 5391-5411.

FleisCHER C., BECKER S., EIGENBERGER G.

„Transient Hydrodynamics, Mass Transfer, and Reaction in Bubble Columns: $\mathrm{CO}_{2}$

Absorption into $\mathrm{NaOH}$ Solutions“"

Chemical Engineering Research \& Design, 1995, 73. Jg., Nr. 6, S. 649-653.

GAdDis E. S., Vogelpohl A.

"Bubble Formation in Quiescent Liquids under constant Flow Condition"

Chemical Engineering Science, 1986, 41. Jg., Nr. 1, S. 97-105.

GESTRICH, Wolfgang; RÄHSE, Wilfried.

„Der relative Gasgehalt von Blasenschichten“

Chemie Ingenieur Technik, 1975, 47. Jg., Nr. 1, S. 8-13.

Groen, J. S., Oldeman, R. G. C., Mudde, R. F., \& Van Den Akker, H. E. A.

"Coherent Structures and Axial Dispersion in Bubble Column Reactors."

Chemical Engineering Science, 1996, 51. Jg., Nr. 10, S. 2511-2520.

Hammer, H., Schrag, H., HeKtor, K., Schonau, K., Kusters, W., Soemarno, A., NapP, W.

"New Subfunctions on Hydrodynamics, Heat and Mass Transfer for Gas/Liquid and

Gas/Liquid/Solid Chemical and Biochemical Reactors"

Frontiers of Chemical Science and Engineering, 1984, 464. Jg.

Hammer, H., Küsters, W., Schrag, H. J., Soemarno, A., Sahabi, U., SchÖnaU, H., NapP, W.

“19. Recent Research Work on Bubble Column Reactors with and without Suspended

Solids."

Recent advances in the engineering analysis of chemically reacting systems, 1984, S. 379.

Hikita, H., Asai, S., Tanigawa, K., Segawa, K., \& KitaO, M.

"Gas Hold-up in Bubble Columns"

The Chemical Engineering Journal, 1980, 20. Jg., Nr. 1, S. 59-67.

HikitA, H., \& KiKUKAWA, H.

"Liquid-Phase Mixing in Bubble Columns: Effect of Liquid Properties."

The Chemical Engineering Journal, 1974, 8. Jg., Nr. 3, S. 191-197.

HOEFSLOOT H.C.J., KRISHNA R.

"Influence of Gas Density on the Stability of Homogeneous Flow in Bubble Columns"

Industrial \& Engineering Chemistry Research, 1993, 32. Jg., Nr. 4, S. 747-750. 
HUGHMARK G.A.

"Holdup and Mass Transfer in Bubble Columns"

Industrial \& Engineering Chemistry Process Design and Development, 1967, 6. Jg., Nr. 2,

S. 218-220.

IDOGAWA, K., IKEDA, K., FUKUDA, T., MOROOKA, S.

"Effects of Gas and Liquid Properties on the Behaviour of Bubbles in a Bubble Column under High-Pressure".

Kagaku Kogaku Ronbunshu, 1985, 11. Jg., Nr. 4, S. 432-437.

IDOGAWA K., IKEDA K., FuKUdA T. MOROOKA S.

"Effect of Gas and Liquid Properties on the Behaviour of Bubbles in a Column under

High Pressure"

International Journal of Chemical Engineering, 1987, 27. Jg., Nr. 3.

IDOGEWA K., IKEDA K., FuKUdA T., MOROOKA S.

"Formation and Flow of Gas Bubbles in a Pressurized Bubble Column with a single

Orifice or Nozzle Gas Distributor"

Chemical Engineering Communications, 1987, 59. Jg., Nr. 1-6, S. 201-212.

JORDAN, Uwe; SAXENA, Alok K.; SCHUMPE, Adrian

"Dynamic Gas Disengagement in a High - Pressure Bubble Column"

The Canadian Journal of Chemical Engineering, 2003, 81. Jg., Nr. 3 - 4, S. 491-498. JORDAN, Uwe; SCHUMPE, Adrian.

“The Gas Density Effect on Mass Transfer in Bubble Columns with Organic Liquids"

Chemical Engineering Science, 2001, 56. Jg., Nr. 21, S. 6267-6272.

KLING G.

"Über die Dynamik der Blasenbildung beim Begasen von Flüssigkeiten unter Druck"

International Journal of Heat and Mass Transfer, 1962, 5. Jg., Nr. 3, S. 211-223.

KoJima H., SAwai J., SUZUKi H.

"Effect of Pressure on Volumetric Mass Transfer Coefficient and Gas Holdup in Bubble

Column"

Chemical Engineering Science, 1997, 52. Jg., Nr. 21, S. 4111-4116.

KRISHNA R., ELLENBERGER J.

„Gas Holdup in Bubble Column Reactors Operating in the Churn-Turbulent Flow

Regime“

AIChE journal, 1996, 42. Jg., Nr. 9, S. 2627-2634. 
KRISHNA R., WILKINSON P. M., VAN DIERENDONCK L. L.

„A Model for Gas Holdup in Bubble Columns incorporating the Influence of Gas Density in Flow Regime Transition“

Chemical Engineering Science, 1991, 46. Jg., Nr. 10, S. 2491-2496.

Krishna, R., URSEANU, M. I., VAN BATEN, J. M., \& EllenBerger, J.

„Influence of Scale on the Hydrodynamics of Bubble Columns operating in the Churn-

Turbulent Regime: Experiments vs. Eulerian Simulations"

Chemical Engineering Science, 1999, 54. Jg., Nr. 21, S. 4903-4911.

Lemoine, R., Behkish, A., Sehabiague, L., Heintz, Y. J., Oukaci, R., \& Morsi, B. I.

"An Algorithm for predicting the Hydrodynamic and Mass Transfer Parameters in Bubble

Column and Slurry Bubble Column Reactors"

Fuel Processing Technology, 2008, 89. Jg., Nr. 4, S. 322-343.

LetZel H.M., SCHOUten J.C., KRISHNA R., VAN DEN BLEeK C.M.

"Gas Holdup and Mass Transfer in Bubble Column Reactors operated at elevated

Pressure“

Chemical Engineering Science, 1999, 54. Jg., Nr. 13, S. 2237-2246.

LETZEL H.M., SCHOUTEN J.C., KRISHNA R., VAN DEN BLEEK C.M.

„Effect of Gas Density on Large-Bubble Holdup in Bubble Column Reactors”

AIChE journal, 1998, 44. Jg., Nr. 10, S. 2333-2336.

LIN T.-J., TSUCHIYA K., FAN L.-S.

"Bubble Flow Characteristics in Bubble Columns at elevated Pressure and Temperature" AIChE Journal, 1998, 44. Jg., Nr. 3, S. 545-560.

MANERI C. C.

"New Look at Wave Analogy for Prediction of Bubble Terminal Velocity"

AIChE Journal, 1995, 41. Jg., Nr. 3, S. 481-487.

MARQueZ A. L., WiLd G., Midoux N.

"A Review of Recent Chemical Techniques for the Determination of the Volumetric

Mass-Transfer Coefficient $\mathrm{k}_{\mathrm{L}} \mathrm{a}$ in Gas-Liquid Reactors"

Chemical Engineering and Processing: Process Intensification, 1994, 33. Jg., Nr. 4, S. 247-260.

MENDELSON H. D.

"The Prediction of Bubble Terminal Velocity from Wave Theory"

AIChE Journal, 1967, 13. Jg., Nr. 2, S. 250-253. 
MERSMANN A.

"Auslegung und Maßstabsvergrößerungen von Blasen- und Tropfensäulen"

Chemie Ingenieur Technik, 1977, 49. Jg., Nr. 9, S. 679-691.

Mersmann A.

"Design and scale-up of bubble and spray columns."

German Chemical Engineering, 1978, 1. Jg., Nr. 1, S. 1-11.

MiYAhiRA T., TANAKA A.

"Size of Bubbles generated from Porous Plates"

Journal of Chemical Engineering of Japan, 1997, 30. Jg., Nr. 2, S. 353-355.

NedeltcheV, S., JoRdAn, U., LORENZ, O., \& SCHUMPE, A .

"Identification of Various Transition Velocities in a Bubble Column based on

Kolmogorov Entropy"

Chemical Engineering \& Technology, 2007, 30. Jg., Nr. 4, S. 534-539.

Nedeltchev, S., Jordan, U., \& Schumpe, A.

"A new Correction Factor for Theoretical Prediction of Mass Transfer Coefficients in

Bubble Columns"

Journal of Chemical Engineering of Japan, 2006, 39. Jg., Nr. 12, S. 1237-1242.

Nedeltchev, S., JoRdAn, U., \& SCHUMPe, A.

"Correction of the Penetration Theory applied to the Prediction of $\mathrm{k}_{\mathrm{L}} \mathrm{a}$ in a Bubble Column with Organic Liquids"

Chemical Engineering \& Technology, 2006, 29. Jg., Nr. 9, S. 1113-1117.

Nedeltchev, S., Jordan, U., \& Schumpe, A.

"Semi - Theoretical Prediction of Volumetric Mass Transfer Coefficients in Bubble

Columns with Organic Liquids at Ambient and Elevated Temperatures"

The Canadian Journal of Chemical Engineering, 2010, 88. Jg., Nr. 4, S. 523-532.

Nedeltchev, S., Jordan, U., \& Schumpe, A.

"Correction of the Penetration Theory based on Mass-Transfer Data from Bubble

Columns operated in the Homogeneous Regime under High Pressure"

Chemical Engineering Science, 2007, 62. Jg., Nr. 22, S. 6263-6273.

NedeltCheV, S, Schubert, M.

„Statistical Validation of the Mixing Length Concept in Bubble Columns Operated in the Transition Flow Regime.“

Journal of Chemical Engineering of Japan, 2015, 48. Jg., Nr. 2, S. 107-111. 
OYeVAar M. H., Bos R., WeSTERTERP K. R.

„Interfacial areas and Gas Holdups in Gas-Liquid Contactors at Elevated Pressures”

Chemical Engineering Science, 1991, 46. Jg., Nr. 5, S. 1217-1231.

ÖZTÜRK S. S., SCHUMPE A., DECKWER W.-D.

„Organic Liquids in a Bubble Column: Holdups and Mass Transfer Coefficients”

AIChE journal, 1987, 33. Jg., Nr. 9, S. 1473-1480.

PATEl S. A., DAly J. G., BuKur D. B.

"Holdup and Interfacial Area Measurements using Dynamic Gas Disengagement"

AIChE journal, 1989, 35. Jg., Nr. 6, S. 931-942.

PEEBlEs F. N., GARBER H. J.

"Studies on the Motion of Gas Bubbles in Liquids"

Chemical Engineering Progress, 1953, 49. Jg., Nr. 2, S. 88-97.

Pourtousi, M., Sahu, J. N., \& Ganesan, P.

"Effect of Interfacial Forces and Turbulence Models on Predicting Flow Pattern inside the

Bubble Column"

Chemical Engineering and Processing: Process Intensification, 2014, 75. Jg., S. 38-47.

Pourtousi, M., GANESAN, P., \& SAHU, J. N.

„Effect of Bubble Diameter Size on Prediction of Flow Pattern in Euler-Euler Simulation of Homogeneous Bubble Column Regime“"

Measurement, 2015, 76. Jg., S. 255-270.

Pourtousi, M., Sahu, J. N., Ganesan, P., Shamshirband, S., \& Redzwan, G.

„A Combination of Computational Fluid Dynamics (CFD) and adaptive Neuro-Fuzzy

System (ANFIS) for Prediction of the Bubble Column Hydrodynamics““

Powder Technology, 2015, 274. Jg., S. 466-481.

Reilly I. G., Scott D. S., De Bruijn T. J. W., Macintyre D.

"The Role of Gas Phase Momentum in Determining Gas Holdup and Hydrodynamic Flow

Regimes in Bubble Column Operations"

The Canadian Journal of Chemical Engineering, 1994, 72. Jg., Nr. 1, S. 3-12.

Reilly I.G., Scott D.S., De Bruijn T., Jain A., Piskorz J.

"A Correlation for Gas Holdup in Turbulent Coalescing Bubble Columns"

The Canadian Journal of Chemical Engineering, 1986, 64. Jg., Nr. 5, S. 705-717.

SARrafi A., Jamialahmadi M., MÜller-Steinhagen H., SMith J. M.

„Gas Holdup in Homogeneous and Heterogeneous Gas-Liquid Bubble Column Reactors”

The Canadian Journal of Chemical Engineering, 1999, 77. Jg., Nr. 1, S. 11-21. 
ŞAL, SINAN; GÜL, ÖMER F.; ÖZDEMIR, MUSTAFA.

„The Effect of Sparger Geometry on Gas Holdup and Regime Transition Points in a

Bubble Column equipped with Perforated Plate Spargers“.

Chemical Engineering and Processing: Process Intensification, 2013, 70. Jg., S. 259-266. SPRingmann H.-G., STEIFF A., WeInSPACH P.-M.

„Bestimmung des volumenspezifischen Stoffübergangskoeffizienten in

Suspensionsblasensäulen mit einer modifizierten Sulfit-Oxidations-Methode“

Chemie Ingenieur Technik, 1991, 63. Jg., Nr. 2, S. 162-163.

SRIRAM K., MANN R.

„Dynamic Gas Disengagement: a new Technique for assessing the Behaviour of Bubble

Columns“

Chemical Engineering Science, 1977, 32. Jg., Nr. 6, S. 571-580.

Stegeman D., KnOP P. A., WijnAnds A. J. G., Westerterp K. R.

"Interfacial Area and Gas Holdup in a Bubble Column Reactor at Elevated Pressures"

Industrial \& Engineering Chemistry Research, 1996, 35. Jg., Nr. 11, S. 3842-3847.

Tsuge H., Terasaka K., Koshida W., Matsue H.

„Bubble Formation at Submerged Nozzles for small Gas Flow Rate under low Gravity“

Chemical Engineering Science, 1997, 52. Jg., Nr. 20, S. 3415-3420.

TSUGE H.; TANAKA Y., HiBINO S.

„Effect of the Physical Properties of Gas on the Volume of Bubble Formed from a

Submerged Single Orifice"

The Canadian Journal of Chemical Engineering, 1981, 59. Jg., Nr. 5, S. 569-572.

VANDU, C. O.; KRISHNA, R.

"Volumetric Mass Transfer Coefficients in Slurry Bubble Columns operating in the

Churn-Turbulent Flow Regime"

Chemical Engineering and Processing: Process Intensification, 2004, 43. Jg., Nr. 8, S. 987-995.

VANDU, C. O.; KRISHNA, R.

"Influence of Scale on the Volumetric Mass Transfer Coefficients in Bubble Columns"

Chemical Engineering and Processing: Process Intensification, 2004, 43. Jg., Nr. 4, S. $575-579$ 


\section{VAUCK, MÜLLER}

"Grundoperationen chemischer Verfahrenstechnik"

10. Auflage 1994; Dt. Verlag für Grundstoffindustrie Leipzig, Stuttgart

ISBN: 3-342-00629-3

Vial, C., Laine, R., Poncin, S., Midoux, N., \& Wild, G.

"Influence of Gas Distribution and Regime Transitions on Liquid Velocity and Turbulence in a 3-D Bubble Column."

Chemical Engineering Science, 2001, 56. Jg., Nr. 3, S. 1085-1093.

WILKINSON P. M.

"Physical Aspects and Scale-up of High Pressure Bubble Columns"

Dissertation Rijksuniversiteit Groningen; 1991

WILKINSON P. M., DIERENDONCK L. V.

"Pressure and Gas Density Effects on Bubble Break-up and Gas Hold-up in Bubble

Columns"

Chemical Engineering Science, 1990, 45. Jg., Nr. 8, S. 2309-2315.

WiLKINSON P. M., SPEK A. P., VAN DiERENDONCK L. L.

„Design Parameters Estimation for Scale-Up of High-Pressure Bubble Columns“

AIChE Journal, 1992, 38. Jg., Nr. 4, S. 544-554.

XING, C., WANG, T., \& WANG, J.

„Experimental Study and Numerical Simulation with a coupled CFD-PBM Model of the Effect of Liquid Viscosity in a Bubble Column.“

Chemical Engineering Science, 2013, 95. Jg., S. 313

Xu, L., YuAn, B., Ni, H., \& Chen, C.

„Numerical Simulation of Bubble Column Flows in Churn-Turbulent Regime:

Comparison of Bubble Size Models.

Industrial \& Engineering Chemistry Research, 2013, 52. Jg., Nr. 20, S. 6794-6802.

ZEHNER, P.

"Momentum, Mass and Heat Transfer in Bubble Columns. Part 1. Flow Model of the Bubble Column and Liquid Velocities."

International Journal of Chemical Engineering, 1986, 26. Jg., S. 22. 


\section{Ergebnisübersicht}

In der Ergebnisübersicht sind die Ergebnisse aller Messreihen dieser Arbeit tabellarisch zusammengefasst. Zunächst die variierten Versuchsparameter:

- fortlaufende Nummer

- Flüssigkeit

- Gas

- Lochanzahl des Begaser

- Lochgröße des Begasers

- Durchmesser der Blasensäule

- Höhe über Begaser des unteren Drucksensors für die Differenzdruckmessung

- Höhe über Begaser des oberen Drucksensors für die Differenzdruckmessung

- Füllhöhe der Flüssigkeit ohne Begasung

- Temperatur

- Druck

- Normvolumenstrom des Gas

Und die erhaltenen Resultate aus den Stoffübergangsmessungen und den

Entgasungsmessungen. Leere Felder bedeuten, dass keine Werte ermittelt werden konnten.

- Gesamtgasgehalt

- Standardabweichung des Gasgehalts

- Desorptionsrate $\mathrm{k}_{\mathrm{L}} \mathrm{a}^{*}$

- Standardabweichung der Stoffübergangszahl

- Gasgehalt 0-65 cm über dem Begaser

- Gasgehalt 65 - $120 \mathrm{~cm}$ über dem Begaser

- Gasgehalt der Großblasen

- Aufstiegsgeschwindigkeit der Großblasen

- Gasgehalt der Kleinblasen

- Aufstiegsgeschwindigkeit der Kleinblasen 


\begin{tabular}{|c|c|c|c|c|c|c|c|c|c|c|c|c|c|c|c|c|c|c|c|c|c|}
\hline$\underline{3}$ & 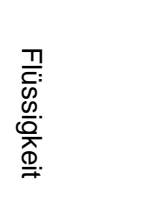 & D & 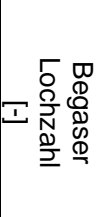 & 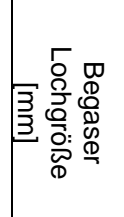 & 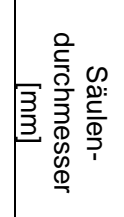 & 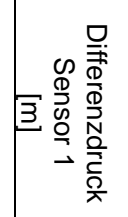 & 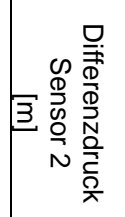 & 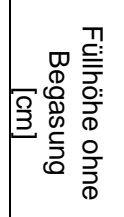 & 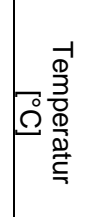 & 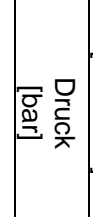 & 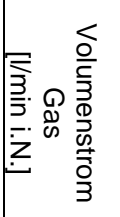 & 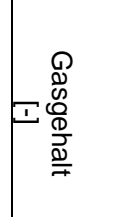 & 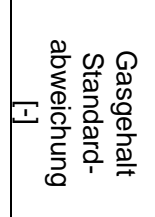 & 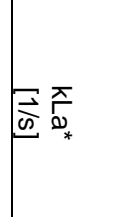 & 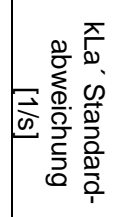 & 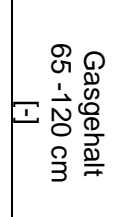 & 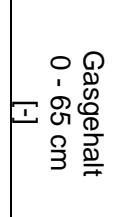 & 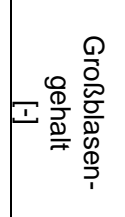 & 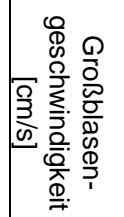 & 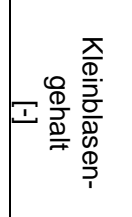 & 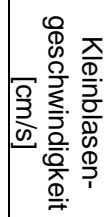 \\
\hline 1 & 1-Butanol & Stickstoff & 19 & 1 & 102 & 0 & 1,2 & 130 & 20 & 1 & 4,7 & 0,031 & 0,0000 & 0,0129 & 0,0002 & 0,038 & 0,019 & 0,010 & 60,9 & 0,018 & 23,5 \\
\hline 2 & 1-Butanol & Stickstoff & 19 & 1 & 102 & 0 & 1,2 & 130 & 20 & 1 & 9,4 & 0,058 & 0,0000 & 0,0255 & 0,0003 & 0,067 & 0,044 & 0,017 & 70,9 & 0,038 & 23,1 \\
\hline 3 & 1-Butanol & Stickstoff & 19 & 1 & 102 & 0 & 1,2 & 130 & 20 & 1 & 23,6 & 0,110 & 0,0006 & 0,0557 & 0,0021 & 0,119 & 0,090 & 0,047 & 83,1 & 0,057 & 22,3 \\
\hline 4 & 1-Butanol & Stickstoff & 19 & 1 & 102 & 0 & 1,2 & 130 & 20 & 1 & 47,1 & 0,162 & 0,0006 & 0,0916 & 0,0031 & 0,169 & 0,142 & 0,080 & 107,3 & 0,074 & 23,4 \\
\hline 5 & 1-Butanol & Stickstoff & 19 & 1 & 102 & 0 & 1,2 & 130 & 20 & 1 & 94,2 & 0,245 & 0,0026 & 0,1751 & 0,0079 & 0,247 & 0,216 & 0,147 & 127,7 & 0,084 & 22,9 \\
\hline 6 & 1-Butanol & Stickstoff & 19 & 1 & 102 & 0 & 1,2 & 130 & 20 & 2 & 9,4 & 0,030 & 0,0000 & 0,0141 & 0,0005 & 0,039 & 0,018 & 0,012 & 62,1 & 0,016 & 22,7 \\
\hline 7 & 1-Butanol & Stickstoff & 19 & 1 & 102 & 0 & 1,2 & 130 & 20 & 2 & 18,9 & 0,057 & 0,0006 & 0,0273 & 0,0006 & 0,071 & 0,043 & 0,018 & 67,1 & 0,038 & 23,1 \\
\hline 8 & 1-Butanol & Stickstoff & 19 & 1 & 102 & 0 & 1,2 & 130 & 20 & 2 & 47,1 & 0,114 & 0,0006 & 0,0617 & 0,0001 & 0,127 & 0,098 & 0,047 & 78,3 & 0,064 & 22,9 \\
\hline 9 & 1-Butanol & Stickstoff & 19 & 1 & 102 & 0 & 1,2 & 130 & 20 & 2 & 94,3 & 0,187 & 0,0000 & 0,1133 & 0,0043 & 0,196 & 0,162 & 0,092 & 91,2 & 0,086 & 22,4 \\
\hline 10 & 1-Butanol & Stickstoff & 19 & 1 & 102 & 0 & 1,2 & 130 & 20 & 2 & 188,5 & 0,278 & 0,0015 & 0,1951 & 0,0035 & 0,282 & 0,245 & 0,164 & 112,1 & 0,098 & 22,9 \\
\hline 11 & 1-Butanol & Stickstoff & 19 & 1 & 102 & 0 & 1,2 & 130 & 20 & 5 & 23,6 & 0,031 & 0,0000 & 0,0150 & 0,0000 & 0,041 & 0,021 & 0,011 & 53,8 & 0,020 & 22,2 \\
\hline 12 & 1-Butanol & Stickstoff & 19 & 1 & 102 & 0 & 1,2 & 130 & 20 & 5 & 47,1 & 0,062 & 0,0006 & 0,0300 & 0,0005 & 0,073 & 0,048 & 0,021 & 57,4 & 0,038 & 21,9 \\
\hline 13 & 1-Butanol & Stickstoff & 19 & 1 & 102 & 0 & 1,2 & 130 & 20 & 5 & 117,8 & 0,143 & 0,0046 & 0,0767 & 0,0006 & 0,152 & 0,118 & 0,056 & 62,2 & 0,078 & 22,2 \\
\hline 14 & 1-Butanol & Stickstoff & 19 & 1 & 102 & 0 & 1,2 & 130 & 20 & 5 & 235,6 & 0,227 & 0,0026 & 0,1407 & 0,0046 & 0,232 & 0,196 & 0,107 & 74,2 & 0,106 & 22,5 \\
\hline 15 & 1-Butanol & Stickstoff & 19 & 1 & 102 & 0 & 1,2 & 130 & 20 & 5 & 471,3 & 0,339 & 0,0057 & 0,2319 & 0,0136 & 0,342 & 0,294 & 0,193 & 92,7 & 0,124 & 22,6 \\
\hline 16 & 1-Butanol & Stickstoff & 19 & 1 & 102 & 0 & 1,2 & 130 & 20 & 10 & 47,1 & 0,033 & 0,0000 & 0,0143 & 0,0010 & 0,041 & 0,023 & 0,004 & 85,5 & 0,028 & 24,0 \\
\hline 17 & 1-Butanol & Stickstoff & 19 & 1 & 102 & 0 & 1,2 & 130 & 20 & 10 & 94,3 & 0,075 & 0,0012 & 0,0358 & 0,0012 & 0,085 & 0,061 & 0,028 & 41,1 & 0,044 & 21,4 \\
\hline 18 & 1-Butanol & Stickstoff & 19 & 1 & 102 & 0 & 1,2 & 130 & 20 & 10 & 235,6 & 0,165 & 0,0025 & 0,0882 & 0,0036 & 0,173 & 0,138 & 0,060 & 51,0 & 0,094 & 22,1 \\
\hline 19 & 1-Butanol & Stickstoff & 19 & 1 & 102 & 0 & 1,2 & 130 & 20 & 10 & 471,3 & 0,261 & 0,0031 & 0,1509 & 0,0021 & 0,267 & 0,228 & 0,117 & 63,7 & 0,129 & 22,2 \\
\hline 20 & 1-Butanol & Stickstoff & 19 & 1 & 102 & 0 & 1,2 & 130 & 20 & 10 & 942,5 & 0,366 & & & & 0,394 & 0,341 & 0,229 & 76,6 & 0,137 & 22,7 \\
\hline 21 & 1-Butanol & Stickstoff & 19 & 1 & 102 & 0 & 1,2 & 130 & 20 & 20 & 94,3 & 0,041 & 0,0000 & 0,0191 & 0,0004 & 0,049 & 0,030 & 0,018 & 33,6 & 0,021 & 20,6 \\
\hline 22 & 1-Butanol & Stickstoff & 19 & 1 & 102 & 0 & 1,2 & 130 & 20 & 20 & 188,5 & 0,081 & 0,0007 & 0,0382 & 0,0014 & 0,090 & 0,063 & 0,022 & 44,2 & 0,053 & 21,2 \\
\hline 23 & 1-Butanol & Stickstoff & 19 & 1 & 102 & 0 & 1,2 & 130 & 20 & 20 & 471,3 & 0,182 & 0,0021 & 0,0818 & 0,0008 & 0,189 & 0,153 & 0,056 & 50,8 & 0,114 & 21,6 \\
\hline 24 & 1-Butanol & Stickstoff & 19 & 1 & 10 & 0 & 1,2 & 13 & 20 & 20 & 942,5 & 0,316 & 0,0047 & 0,1695 & 0,0226 & 0,324 & 0,280 & 0,176 & 44,1 & 0,125 & 20,7 \\
\hline 25 & 1-Butanol & Stickstoff & 19 & 1 & 102 & 0 & 1,2 & 130 & 20 & 20 & 1885 & 0,404 & & & & 0,442 & 0,369 & 0,241 & 70,4 & 0,162 & 22,9 \\
\hline 26 & 1-Butanol & Stickstoff & 19 & 1 & 102 & 0 & 1,2 & 130 & 20 & 40 & 188,5 & 0,045 & 0,0000 & 0,0169 & 0,0017 & 0,053 & 0,032 & 0,027 & 27,9 & 0,014 & 18,6 \\
\hline 27 & 1-Butanol & Stickstoff & 19 & 1 & 102 & 0 & 1,2 & 130 & 20 & 40 & 377 & 0,087 & 0,0021 & 0,0347 & 0,0016 & 0,094 & 0,073 & 0,042 & 30,9 & 0,040 & 18,6 \\
\hline 28 & 1-Butanol & Stickstoff & 19 & 1 & 102 & 0 & 1,2 & 130 & 20 & 40 & 942,5 & 0,246 & 0,0038 & 0,1127 & 0,0072 & 0,252 & 0,209 & 0,118 & 22,2 & 0,115 & 21,6 \\
\hline
\end{tabular}




\begin{tabular}{|c|c|c|c|c|c|c|c|c|c|c|c|c|c|c|c|c|c|c|c|c|c|}
\hline$\underline{z}$ & 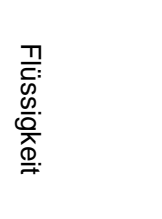 & $\stackrel{\circledR}{\circledR}$ & 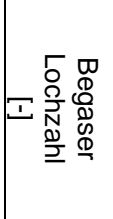 & 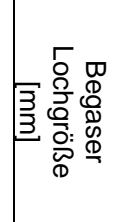 & 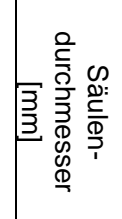 & 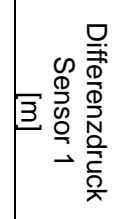 & 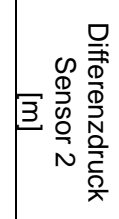 & 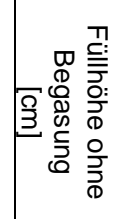 & 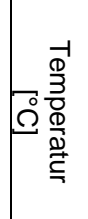 & 总 몰 & 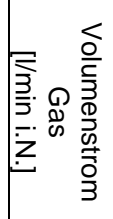 & 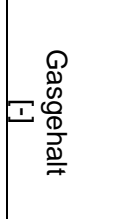 & 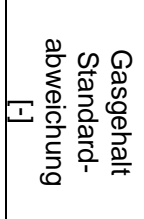 & 空 $\frac{\bar{a}}{a_{*}}$ & 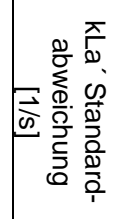 & 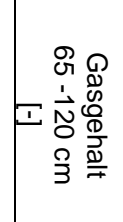 & 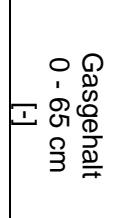 & 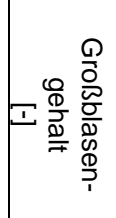 & 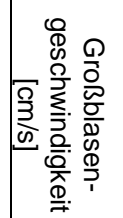 & 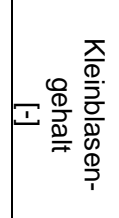 & 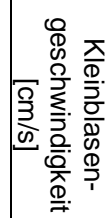 \\
\hline 29 & 1-Butanol & Helium & 19 & 1 & 102 & 0 & 1,2 & 130 & 20 & 1 & 4,7 & 0,023 & & & & 0,034 & 0,013 & 0,012 & 64,5 & 0,011 & 24,3 \\
\hline 30 & 1-Butanol & Helium & 19 & 1 & 102 & 0 & 1,2 & 130 & 20 & 1 & 9,4 & 0,048 & & & & 0,062 & 0,036 & 0,023 & 66,4 & 0,025 & 23,1 \\
\hline 31 & 1-Butanol & Helium & 19 & 1 & 102 & 0 & 1,2 & 130 & 20 & 1 & 23,6 & 0,093 & & & & 0,107 & 0,079 & 0,046 & 89,1 & 0,046 & 23,2 \\
\hline 32 & 1-Butanol & Helium & 19 & 1 & 102 & 0 & 1,2 & 130 & 20 & 1 & 47,1 & 0,135 & & & & 0,153 & 0,116 & 0,080 & 112,4 & 0,053 & 24,5 \\
\hline 33 & 1-Butanol & Helium & 19 & 1 & 102 & 0 & 1,2 & 130 & 20 & 1 & 94,2 & 0,200 & & & & 0,225 & 0,175 & 0,138 & 138,3 & 0,060 & 24,8 \\
\hline 34 & 1-Butanol & Helium & 19 & 1 & 102 & 0 & 1,2 & 130 & 20 & 3 & 14,1 & 0,026 & & & & 0,038 & 0,016 & 0,011 & 61,7 & 0,015 & 25,1 \\
\hline 35 & 1-Butanol & Helium & 19 & 1 & 102 & 0 & 1,2 & 130 & 20 & 3 & 28,2 & 0,050 & & & & 0,063 & 0,037 & 0,018 & 74,3 & 0,031 & 23,2 \\
\hline 36 & 1-Butanol & Helium & 19 & 1 & 102 & 0 & 1,2 & 130 & 20 & 3 & 70,8 & 0,094 & & & & 0,107 & 0,081 & 0,042 & 93,6 & 0,051 & 24,4 \\
\hline 37 & 1-Butanol & Helium & 19 & 1 & 102 & 0 & 1,2 & 130 & 20 & 3 & 141,3 & 0,148 & & & & 0,161 & 0,133 & 0,082 & 106,4 & 0,064 & 25,1 \\
\hline 38 & 1-Butanol & Helium & 19 & 1 & 102 & 0 & 1,2 & 130 & 20 & 3 & 282,6 & 0,215 & & & & 0,236 & 0,192 & 0,148 & 129,0 & 0,065 & 24,5 \\
\hline 39 & 1-Butanol & Stickstoff & 1 & 4,3 & 102 & 0 & 1,2 & 130 & 20 & 1 & 4,7 & 0,027 & 0,0004 & 0,0123 & 0,0006 & & & & & & \\
\hline 40 & 1-Butanol & Stickstoff & 1 & 4,3 & 102 & 0 & 1,2 & 130 & 20 & 1 & 9,4 & 0,051 & 0,0004 & 0,0236 & 0,0003 & & & & & & \\
\hline 41 & 1-Butanol & Stickstoff & 1 & 4,3 & 102 & 0 & 1,2 & 130 & 20 & 1 & 23,6 & 0,098 & 0,0038 & 0,0511 & 0,0008 & & & & & & \\
\hline 42 & 1-Butanol & Stickstoff & 1 & 4,3 & 102 & 0 & 1,2 & 130 & 20 & 1 & 47,1 & 0,149 & 0,0021 & 0,0878 & 0,0010 & & & & & & \\
\hline 43 & 1-Butanol & Stickstoff & 1 & 4,3 & 102 & 0 & 1,2 & 130 & 20 & 1 & 94,2 & 0,231 & 0,0030 & 0,1579 & 0,0021 & & & & & & \\
\hline 44 & 1-Butanol & Stickstoff & 1 & 4,3 & 102 & 0 & 1,2 & 130 & 20 & 2 & 9,4 & 0,028 & 0,0010 & 0,0128 & 0,0002 & & & & & & \\
\hline 45 & 1-Butanol & Stickstoff & 1 & 4,3 & 102 & 0 & 1,2 & 130 & 20 & 2 & 18,9 & 0,055 & 0,0006 & 0,0252 & 0,0000 & & & & & & \\
\hline 46 & 1-Butanol & Stickstoff & 1 & 4,3 & 102 & 0 & 1,2 & 130 & 20 & 2 & 47,1 & 0,109 & 0,0000 & 0,0576 & 0,0007 & & & & & & \\
\hline 47 & 1-Butanol & Stickstoff & 1 & 4,3 & 102 & 0 & 1,2 & 130 & 20 & 2 & 94,3 & 0,176 & 0,0010 & 0,1122 & 0,0014 & & & & & & \\
\hline 48 & 1-Butanol & Stickstoff & 1 & 4,3 & 102 & 0 & 1,2 & 130 & 20 & 2 & 188,5 & 0,272 & 0,0025 & 0,1965 & 0,0016 & & & & & & \\
\hline 49 & 1-Butanol & Stickstoff & 1 & 4,3 & 102 & 0 & 1,2 & 130 & 20 & 5 & 23,6 & 0,030 & 0,0007 & 0,0147 & 0,0004 & & & & & & \\
\hline 50 & 1-Butanol & Stickstoff & 1 & 4,3 & 102 & 0 & 1,2 & 130 & 20 & 5 & 47,1 & 0,059 & 0,0007 & 0,0291 & 0,0001 & & & & & & \\
\hline 51 & 1-Butanol & Stickstoff & 1 & 4,3 & 102 & 0 & 1,2 & 130 & 20 & 5 & 117,8 & 0,136 & 0,0006 & 0,0770 & 0,0022 & & & & & & \\
\hline 52 & 1-Butanol & Stickstoff & 1 & 4,3 & 102 & 0 & 1,2 & 130 & 20 & 5 & 235,6 & 0,222 & 0,0015 & 0,1442 & 0,0051 & & & & & & \\
\hline 53 & 1-Butanol & Stickstoff & 1 & 4,3 & 102 & 0 & 1,2 & 130 & 20 & 5 & 471,3 & 0,347 & 0,0007 & 0,2574 & 0,0105 & & & & & & \\
\hline 54 & 1-Butanol & Stickstoff & 1 & 4,3 & 102 & 0 & 1,2 & 130 & 20 & 10 & 47,1 & 0,032 & 0,0007 & 0,0157 & 0,0006 & & & & & & \\
\hline 55 & 1-Butanol & Stickstoff & 1 & 4,3 & 102 & 0 & 1,2 & 130 & 20 & 10 & 94,3 & 0,071 & 0,0007 & 0,0363 & 0,0001 & & & & & & \\
\hline 56 & 1-Butanol & Stickstoff & 1 & 4,3 & 102 & 0 & 1,2 & 130 & 20 & 10 & 235,6 & 0,159 & 0,0006 & 0,0900 & 0,0013 & & & & & & \\
\hline
\end{tabular}




\begin{tabular}{|c|c|c|c|c|c|c|c|c|c|c|c|c|c|c|c|c|c|c|c|c|c|}
\hline$\underline{z}$ & 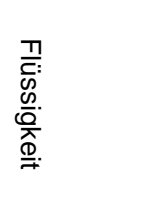 & $\stackrel{\wp}{\circledR}$ & 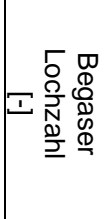 & 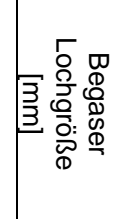 & 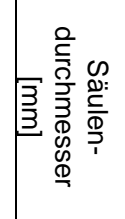 & 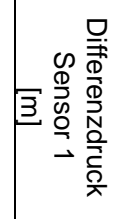 & 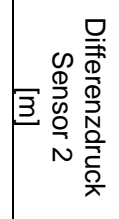 & 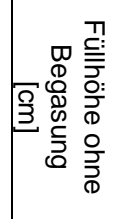 & 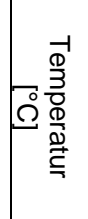 & 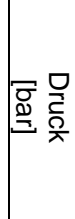 & 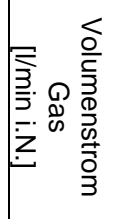 & 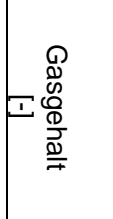 & 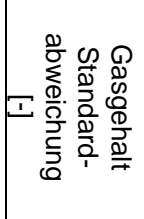 & 至 $\frac{\pi}{D_{*}}$ & 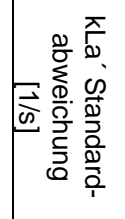 & 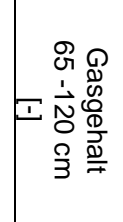 & 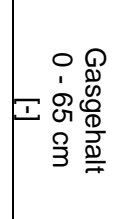 & 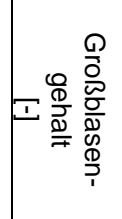 & 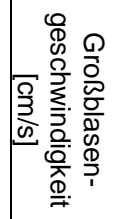 & 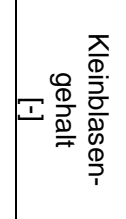 & 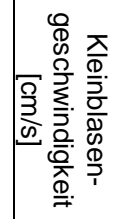 \\
\hline 57 & 1-Butanol & Stickstoff & 1 & 4,3 & 102 & 0 & 1,2 & 130 & 20 & 10 & 471,3 & 0,263 & 0,0029 & 0,1560 & 0,0041 & & & & & & \\
\hline 58 & 1-Butanol & Stickstoff & 1 & 4,3 & 102 & 0 & 1,2 & 130 & 20 & 20 & 94,3 & 0,038 & 0,0000 & 0,0204 & 0,0006 & & & & & & \\
\hline 59 & 1-Butanol & Stickstoff & 1 & 4,3 & 102 & 0 & 1,2 & 130 & 20 & 20 & 188,5 & 0,075 & 0,0015 & 0,0366 & 0,0055 & & & & & & \\
\hline 60 & 1-Butanol & Stickstoff & 1 & 4,3 & 102 & 0 & 1,2 & 130 & 20 & 20 & 471,3 & 0,182 & 0,0026 & 0,1059 & 0,0038 & & & & & & \\
\hline 61 & 1-Butanol & Stickstoff & 1 & 4,3 & 102 & 0 & 1,2 & 130 & 20 & 20 & 942,5 & 0,313 & 0,0014 & 0,1851 & 0,0024 & & & & & & \\
\hline 62 & 1-Butanol & Stickstoff & 1 & 4,3 & 102 & 0 & 1,2 & 130 & 20 & 40 & 188,5 & 0,044 & 0,0007 & 0,0223 & 0,0005 & & & & & & \\
\hline 63 & 1-Butanol & Stickstoff & 1 & 4,3 & 102 & 0 & 1,2 & 130 & 20 & 40 & 377 & 0,084 & 0,0021 & 0,0434 & 0,0002 & & & & & & \\
\hline 64 & 1-Butanol & Stickstoff & 1 & 4,3 & 102 & 0 & 1,2 & 130 & 20 & 40 & 942,5 & 0,237 & 0,0014 & 0,1355 & 0,0025 & & & & & & \\
\hline 65 & 1-Butanol & Stickstoff & 1 & 4,3 & 102 & 0 & 1,2 & 130 & 20 & 40 & 1885 & 0,391 & 0,0085 & 0,2131 & 0,0111 & & & & & & \\
\hline 66 & 1-Butanol & Helium & 1 & 4,3 & 102 & 0 & 1,2 & 130 & 20 & 1 & 4,7 & 0,025 & 0,0000 & 0,0111 & 0,0002 & & & & & & \\
\hline 67 & 1-Butanol & Helium & 1 & 4,3 & 102 & 0 & 1,2 & 130 & 20 & 1 & 9,4 & 0,047 & 0,0000 & 0,0208 & 0,0008 & & & & & & \\
\hline 68 & 1-Butanol & Helium & 1 & 4,3 & 102 & 0 & 1,2 & 130 & 20 & 1 & 23,6 & 0,087 & 0,0007 & 0,0416 & 0,0002 & & & & & & \\
\hline 69 & 1-Butanol & Helium & 1 & 4,3 & 102 & 0 & 1,2 & 130 & 20 & 1 & 47,1 & 0,128 & 0,0007 & 0,0640 & 0,0011 & & & & & & \\
\hline 70 & 1-Butanol & Helium & 1 & 4,3 & 102 & 0 & 1,2 & 130 & 20 & 1 & 94,2 & 0,196 & 0,0000 & 0,1084 & 0,0018 & & & & & & \\
\hline 71 & 1-Butanol & Helium & 1 & 4,3 & 102 & 0 & 1,2 & 130 & 20 & 2 & 9,4 & 0,026 & & 0,0110 & & & & & & & \\
\hline 72 & 1-Butanol & Helium & 1 & 4,3 & 102 & 0 & 1,2 & 130 & 20 & 2 & 18,9 & 0,047 & 0,0000 & 0,0216 & 0,0002 & & & & & & \\
\hline 73 & 1-Butanol & Helium & 1 & 4,3 & 102 & 0 & 1,2 & 130 & 20 & 2 & 47,1 & 0,088 & 0,0000 & 0,0420 & 0,0012 & & & & & & \\
\hline 74 & 1-Butanol & Helium & 1 & 4,3 & 102 & 0 & 1,2 & 130 & 20 & 2 & 94,3 & 0,137 & 0,0007 & 0,0754 & 0,0024 & & & & & & \\
\hline 75 & 1-Butanol & Helium & 1 & 4,3 & 102 & 0 & 1,2 & 130 & 20 & 2 & 188,5 & 0,210 & 0,0021 & 0,1220 & 0,0016 & & & & & & \\
\hline 76 & 1-Butanol & Helium & 1 & 4,3 & 102 & 0 & 1,2 & 130 & 20 & 7 & 33 & 0,027 & 0,0000 & 0,0114 & 0,0002 & & & & & & \\
\hline 77 & 1-Butanol & Helium & 1 & 4,3 & 102 & 0 & 1,2 & 130 & 20 & 7 & 66 & 0,050 & 0,0000 & 0,0210 & 0,0007 & & & & & & \\
\hline 78 & 1-Butanol & Helium & 1 & 4,3 & 102 & 0 & 1,2 & 130 & 20 & 7 & 164,9 & 0,103 & 0,0000 & 0,0520 & 0,0006 & & & & & & \\
\hline 79 & 1-Butanol & Helium & 1 & 4,3 & 102 & 0 & 1,2 & 130 & 20 & 7 & 329,9 & 0,156 & 0,0012 & 0,0870 & 0,0030 & & & & & & \\
\hline 80 & 1-Butanol & Helium & 1 & 4,3 & 102 & 0 & 1,2 & 130 & 20 & 7 & 659,8 & 0,228 & 0,0035 & 0,1471 & 0,0028 & & & & & & \\
\hline 81 & 1-Butanol & Stickstoff & 7 & 1 & 115 & 0,1 & 0,905 & 100 & 20 & 1 & 5 & 0,026 & 0,0004 & 0,0117 & & & & 0,013 & 46,9 & 0,012 & 21,0 \\
\hline 82 & 1-Butanol & Stickstoff & 7 & 1 & 115 & 0,1 & 0,905 & 100 & 20 & 1 & 10 & 0,049 & 0,0006 & 0,0230 & 0,0005 & & & 0,021 & 54,4 & 0,028 & 21,2 \\
\hline 83 & 1-Butanol & Stickstoff & 7 & 1 & 115 & 0,1 & 0,905 & 100 & 20 & 1 & 20 & 0,084 & 0,0018 & 0,0440 & 0,0011 & & & 0,033 & 73,4 & 0,052 & 20,9 \\
\hline 84 & 1-Butanol & Stickstoff & 7 & 1 & 115 & 0,1 & 0,905 & 100 & 20 & 1 & 30 & 0,111 & 0,0012 & 0,0568 & 0,0005 & & & 0,049 & 79,1 & 0,061 & 21,6 \\
\hline
\end{tabular}




\begin{tabular}{|c|c|c|c|c|c|c|c|c|c|c|c|c|c|c|c|c|c|c|c|c|c|}
\hline$\underline{z}$ & 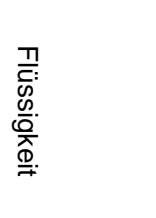 & $\underset{\aleph}{\stackrel{\wp}{~}}$ & 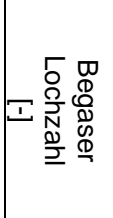 & 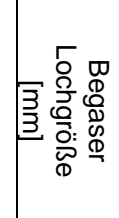 & 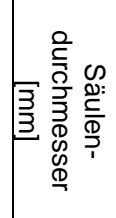 & 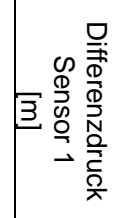 & 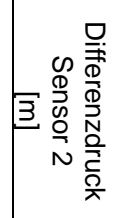 & 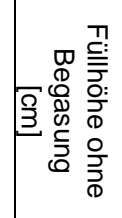 & 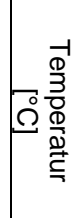 & 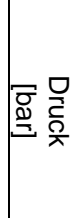 & 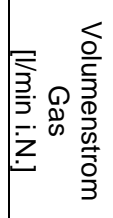 & 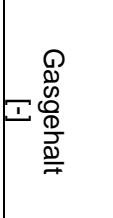 & 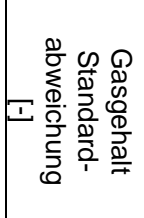 & 点西 & 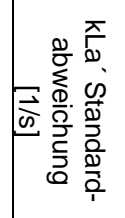 & 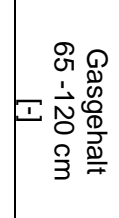 & 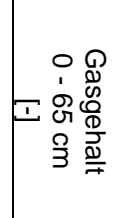 & 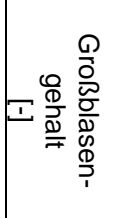 & 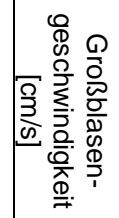 & 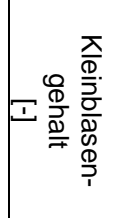 & 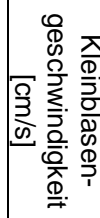 \\
\hline 85 & 1-Butanol & Stickstoff & 7 & 1 & 115 & 0,1 & 0,905 & 100 & 20 & 1 & 50 & 0,155 & 0,0019 & 0,0887 & 0,0030 & & & 0,067 & 101,5 & 0,087 & 21,0 \\
\hline 86 & 1-Butanol & Stickstoff & 7 & 1 & 115 & 0,1 & 0,905 & 100 & 20 & 1 & 75 & 0,201 & 0,0135 & 0,1260 & 0,0015 & & & 0,088 & 124,9 & 0,113 & 21,8 \\
\hline 87 & 1-Butanol & Stickstoff & 7 & 1 & 115 & 0,1 & 0,905 & 100 & 20 & 1 & 100 & 0,207 & 0,0039 & 0,1637 & 0,0071 & & & 0,081 & 180,8 & 0,127 & 22,0 \\
\hline 88 & 1-Butanol & Stickstoff & 7 & 1 & 115 & 0,1 & 0,905 & 100 & 20 & 2 & 10 & 0,030 & 0,0004 & 0,0132 & & & & 0,015 & 38,9 & 0,015 & 20,1 \\
\hline 89 & 1-Butanol & Stickstoff & 7 & 1 & 115 & 0,1 & 0,905 & 100 & 20 & 2 & 20 & 0,057 & 0,0008 & 0,0268 & 0,0004 & & & 0,027 & 42,2 & 0,029 & 19,9 \\
\hline 90 & 1-Butanol & Stickstoff & 7 & 1 & 115 & 0,1 & 0,905 & 100 & 20 & 2 & 40 & 0,102 & 0,0012 & 0,0505 & 0,0006 & & & 0,045 & 52,1 & 0,057 & 20,1 \\
\hline 91 & 1-Butanol & Stickstoff & 7 & 1 & 115 & 0,1 & 0,905 & 100 & 20 & 2 & 60 & 0,140 & 0,0027 & 0,0792 & 0,0040 & & & 0,058 & 60,6 & 0,083 & 20,6 \\
\hline 92 & 1-Butanol & Stickstoff & 7 & 1 & 115 & 0,1 & 0,905 & 100 & 20 & 2 & 100 & 0,196 & 0,0180 & 0,1261 & 0,0069 & & & 0,099 & 68,7 & 0,097 & 20,5 \\
\hline 93 & 1-Butanol & Stickstoff & 7 & 1 & 115 & 0,1 & 0,905 & 100 & 20 & 2 & 150 & 0,271 & 0,0029 & 0,1648 & 0,0083 & & & 0,166 & 65,6 & 0,105 & 20,1 \\
\hline 94 & 1-Butanol & Stickstoff & 7 & 1 & 115 & 0,1 & 0,905 & 100 & 20 & 2 & 200 & 0,326 & 0,0053 & 0,2304 & 0,0243 & & & 0,216 & 70,2 & 0,110 & 20,4 \\
\hline 95 & 1-Butanol & Stickstoff & 7 & 1 & 115 & 0,1 & 0,905 & 100 & 20 & 5 & 25 & 0,031 & 0,0004 & 0,0132 & 0,0008 & & & 0,014 & 38,3 & 0,016 & 19,5 \\
\hline 96 & 1-Butanol & Stickstoff & 7 & 1 & 115 & 0,1 & 0,905 & 100 & 20 & 5 & 50 & 0,063 & 0,0011 & 0,0298 & 0,0003 & & & 0,029 & 37,2 & 0,034 & 19,1 \\
\hline 97 & 1-Butanol & Stickstoff & 7 & 1 & 115 & 0,1 & 0,905 & 100 & 20 & 5 & 100 & 0,127 & 0,0022 & 0,0622 & 0,0020 & & & 0,065 & 35,6 & 0,062 & 18,7 \\
\hline 98 & 1-Butanol & Stickstoff & 7 & 1 & 115 & 0,1 & 0,905 & 100 & 20 & 5 & 150 & 0,179 & 0,0051 & 0,0925 & 0,0009 & & & 0,090 & 38,7 & 0,089 & 19,0 \\
\hline 99 & 1-Butanol & Stickstoff & 7 & 1 & 115 & 0,1 & 0,905 & 100 & 20 & 5 & 250 & 0,265 & 0,0021 & 0,1393 & 0,0153 & & & 0,155 & 42,1 & 0,110 & 19,7 \\
\hline 100 & 1-Butanol & Stickstoff & 7 & 1 & 115 & 0,1 & 0,905 & 100 & 20 & 5 & 375 & 0,345 & 0,0015 & 0,1891 & 0,0188 & & & 0,222 & 47,7 & 0,124 & 20,4 \\
\hline 101 & 1-Butanol & Stickstoff & 7 & 1 & 115 & 0,1 & 0,905 & 100 & 20 & 10 & 50 & 0,034 & 0,0007 & 0,0155 & 0,0003 & & & 0,019 & 30,8 & 0,015 & 18,5 \\
\hline 102 & 1-Butanol & Stickstoff & 7 & 1 & 115 & 0,1 & 0,905 & 100 & 20 & 10 & 100 & 0,070 & 0,0020 & 0,0339 & 0,0006 & & & 0,030 & 33,1 & 0,040 & 18,8 \\
\hline 103 & 1-Butanol & Stickstoff & 7 & 1 & 115 & 0,1 & 0,905 & 100 & 20 & 10 & 200 & 0,141 & 0,0037 & 0,0721 & 0,0020 & & & 0,063 & 33,7 & 0,078 & 18,3 \\
\hline 104 & 1-Butanol & Stickstoff & 7 & 1 & 115 & 0,1 & 0,905 & 100 & 20 & 10 & 300 & 0,202 & 0,0032 & 0,0987 & 0,0076 & & & 0,119 & 31,5 & 0,083 & 17,5 \\
\hline 105 & 1-Butanol & Stickstoff & 7 & 1 & 115 & 0,1 & 0,905 & 100 & 20 & 10 & 400 & 0,256 & 0,0043 & & & & & 0,162 & 34,0 & 0,093 & 15,4 \\
\hline 106 & Decalin & Stickstoff & 1 & 4,3 & 102 & 0 & 1,2 & 130 & 20 & 1 & 4,7 & 0,029 & 0,0005 & 0,0143 & 0,0007 & & & & & & \\
\hline 107 & Decalin & Stickstoff & 1 & 4,3 & 102 & 0 & 1,2 & 130 & 20 & 1 & 9,4 & 0,058 & 0,0004 & 0,0270 & 0,0006 & & & & & & \\
\hline 108 & Decalin & Stickstoff & 1 & 4,3 & 102 & 0 & 1,2 & 130 & 20 & 1 & 23,6 & 0,113 & 0,0005 & 0,0578 & 0,0040 & & & & & & \\
\hline 109 & Decalin & Stickstoff & 1 & 4,3 & 102 & 0 & 1,2 & 130 & 20 & 1 & 47,1 & 0,170 & 0,0020 & 0,1013 & 0,0082 & & & & & & \\
\hline 110 & Decalin & Stickstoff & 1 & 4,3 & 102 & 0 & 1,2 & 130 & 20 & 2 & 9,4 & 0,031 & 0,0006 & 0,0141 & 0,0005 & & & & & & \\
\hline 111 & Decalin & Stickstoff & 1 & 4,3 & 102 & 0 & 1,2 & 130 & 20 & 2 & 18,9 & 0,063 & 0,0006 & 0,0295 & 0,0012 & & & & & & \\
\hline 112 & Decalin & Stickstoff & 1 & 4,3 & 102 & 0 & 1,2 & 130 & 20 & 2 & 47,1 & 0,128 & 0,0006 & 0,0685 & 0,0050 & & & & & & \\
\hline
\end{tabular}




\begin{tabular}{|c|c|c|c|c|c|c|c|c|c|c|c|c|c|c|c|c|c|c|c|c|c|}
\hline$\underline{\underline{s}}$ & 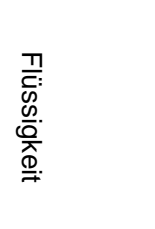 & ळ & 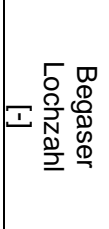 & 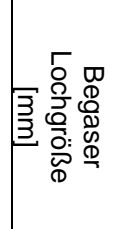 & 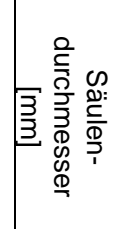 & 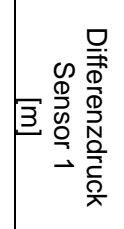 & 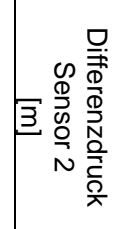 & 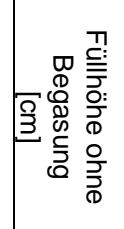 & 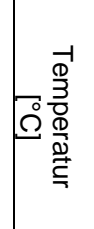 & \begin{tabular}{|l|} 
\\
$\mathscr{g}$
\end{tabular} & 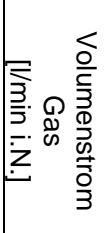 & 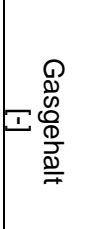 & 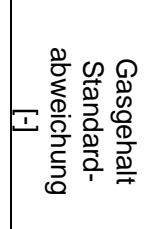 & 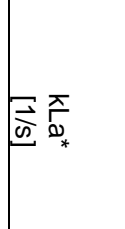 & 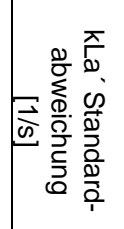 & 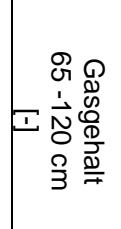 & 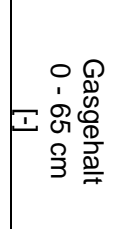 & 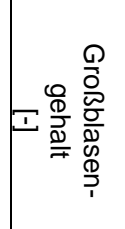 & 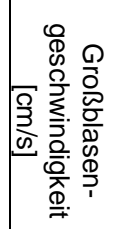 & 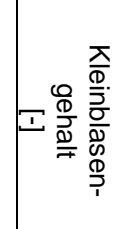 & 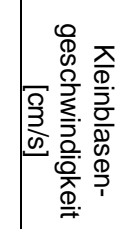 \\
\hline 113 & Decalin & Stickstoff & 1 & 4,3 & 102 & 0 & 1,2 & 130 & 20 & 2 & 94,3 & 0,210 & 0,0026 & 0,1201 & 0,0064 & & & & & & \\
\hline 114 & Decalin & Stickstoff & 1 & 4,3 & 102 & 0 & 1,2 & 130 & 20 & 5 & 23,6 & 0,034 & 0,0000 & 0,0161 & 0,0011 & & & & & & \\
\hline 115 & Decalin & Stickstoff & 1 & 4,3 & 102 & 0 & 1,2 & 130 & 20 & 5 & 47,1 & 0,071 & 0,0006 & 0,0345 & 0,0006 & & & & & & \\
\hline 116 & Decalin & Stickstoff & 1 & 4,3 & 102 & 0 & 1,2 & 130 & 20 & 5 & 117,8 & 0,174 & 0,0010 & 0,0915 & 0,0038 & & & & & & \\
\hline 117 & Decalin & Stickstoff & 1 & 4,3 & 102 & 0 & 1,2 & 130 & 20 & 5 & 235,6 & 0,285 & 0,0012 & 0,1627 & 0,0132 & & & & & & \\
\hline 118 & Decalin & Stickstoff & 1 & 4,3 & 102 & 0 & 1,2 & 130 & 20 & 10 & 47,1 & 0,036 & 0,0000 & 0,0154 & 0,0030 & & & & & & \\
\hline 119 & Decalin & Stickstoff & 1 & 4,3 & 102 & 0 & 1,2 & 130 & 20 & 10 & 94,3 & 0,091 & 0,0000 & 0,0436 & 0,0009 & & & & & & \\
\hline 120 & Decalin & Stickstoff & 1 & 4,3 & 102 & 0 & 1,2 & 130 & 20 & 10 & 235,6 & 0,218 & 0,0026 & 0,1028 & 0,0042 & & & & & & \\
\hline 121 & Decalin & Stickstoff & 1 & 4,3 & 102 & 0 & 1,2 & 130 & 20 & 10 & 471,3 & 0,355 & 0,0041 & 0,1642 & 0,0133 & & & & & & \\
\hline 122 & Decalin & Stickstoff & 1 & 4,3 & 102 & 0 & 1,2 & 130 & 20 & 20 & 94,3 & 0,045 & 0,0000 & 0,0204 & 0,0009 & & & & & & \\
\hline 123 & Decalin & Stickstoff & 1 & 4,3 & 102 & 0 & 1,2 & 130 & 20 & 20 & 188,5 & 0,100 & 0,0006 & 0,0458 & 0,0031 & & & & & & \\
\hline 124 & Decalin & Stickstoff & 1 & 4,3 & 102 & 0 & 1,2 & 130 & 20 & 20 & 471,3 & 0,269 & 0,0175 & 0,1136 & 0,0134 & & & & & & \\
\hline 125 & Decalin & Stickstoff & 1 & 4,3 & 102 & 0 & 1,2 & 130 & 20 & 20 & 942,5 & 0,413 & 0,0019 & 0,1930 & 0,0229 & & & & & & \\
\hline 126 & Decalin & Stickstoff & 1 & 4,3 & 102 & 0 & 1,2 & 130 & 20 & 40 & 188,5 & 0,048 & 0,0000 & 0,0213 & 0,0004 & & & & & & \\
\hline 127 & Decalin & Stickstoff & 1 & 4,3 & 102 & 0 & 1,2 & 130 & 20 & 40 & 377 & 0,175 & 0,0046 & 0,0714 & 0,0058 & & & & & & \\
\hline 128 & Decalin & Stickstoff & 1 & 4,3 & 102 & 0 & 1,2 & 130 & 20 & 40 & 942,5 & 0,302 & 0,0088 & \begin{tabular}{|l|l|}
0,1171 \\
\end{tabular} & 0,0091 & & & & & & \\
\hline 129 & Decalin & Stickstoff & 19 & 1 & 102 & 0 & 1,2 & 130 & 20 & 1 & 4,7 & 0,030 & 0,0005 & 0,0161 & 0,0010 & & & 0,002 & 166,9 & 0,028 & 26,1 \\
\hline 130 & Decalin & Stickstoff & 19 & 1 & 102 & 0 & 1,2 & 130 & 20 & 1 & 9,4 & 0,066 & 0,0005 & 0,0320 & 0,0020 & & & 0,008 & 112,2 & 0,058 & 22,3 \\
\hline 131 & Decalin & Stickstoff & 19 & 1 & 102 & 0 & 1,2 & 130 & 20 & 1 & 23,6 & 0,116 & 0,0011 & 0,0637 & 0,0051 & & & 0,037 & 105,8 & 0,079 & 18,9 \\
\hline 132 & Decalin & Stickstoff & 19 & 1 & 102 & 0 & 1,2 & 130 & 20 & 1 & 47,1 & 0,166 & 0,0010 & 0,0983 & 0,0098 & & & 0,069 & 130,4 & 0,097 & 17,6 \\
\hline 133 & Decalin & Stickstoff & 19 & 1 & 102 & 0 & 1,2 & 130 & 20 & 1 & 94,2 & 0,245 & & & & & & 0,129 & 146,8 & 0,115 & 16,5 \\
\hline 134 & Decalin & Stickstoff & 19 & 1 & 102 & 0 & 1,2 & 130 & 20 & 2 & 9,4 & 0,034 & 0,0000 & 0,0162 & 0,0005 & & & 0,006 & 174,0 & 0,027 & 23,8 \\
\hline 135 & Decalin & Stickstoff & 19 & 1 & 102 & 0 & 1,2 & 130 & 20 & 2 & 18,9 & 0,074 & 0,0006 & 0,0329 & 0,0017 & & & 0,011 & 77,0 & 0,064 & 21,4 \\
\hline 136 & Decalin & Stickstoff & 19 & 1 & 102 & 0 & 1,2 & 130 & 20 & 2 & 47,1 & 0,137 & 0,0004 & 0,0624 & 0,0048 & & & 0,038 & 94,0 & 0,099 & 18,3 \\
\hline 137 & Decalin & Stickstoff & 19 & 1 & 102 & 0 & 1,2 & 130 & 20 & 2 & 94,3 & 0,207 & 0,0014 & 0,1221 & 0,0276 & & & 0,082 & 105,5 & 0,125 & 16,9 \\
\hline 138 & Decalin & Stickstoff & 19 & 1 & 102 & 0 & 1,2 & 130 & 20 & 2 & 188,5 & 0,295 & & & & & & 0,138 & 137,8 & 0,157 & 15,9 \\
\hline 139 & Decalin & Stickstoff & 19 & 1 & 102 & 0 & 1,2 & 130 & 20 & 5 & 23,6 & 0,036 & 0,0000 & 0,0168 & 0,0009 & & & 0,002 & 151,2 & 0,034 & 24,7 \\
\hline 140 & Decalin & Stickstoff & 19 & 1 & 102 & 0 & 1,2 & 130 & 20 & 5 & 47,1 & 0,079 & 0,0006 & 0,0370 & 0,0016 & & & 0,006 & 108,4 & 0,072 & 20,7 \\
\hline
\end{tabular}




\begin{tabular}{|c|c|c|c|c|c|c|c|c|c|c|c|c|c|c|c|c|c|c|c|c|c|}
\hline$\underline{z}$ & 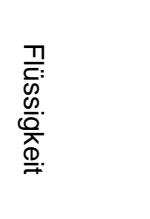 & 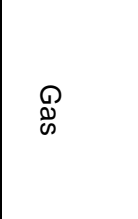 & 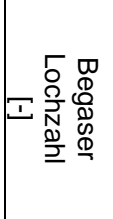 & 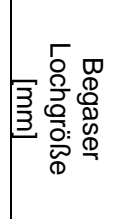 & 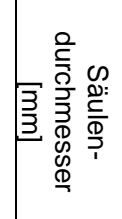 & 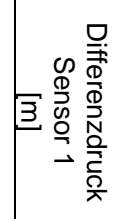 & 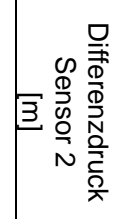 & 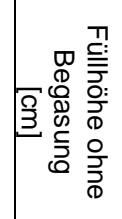 & 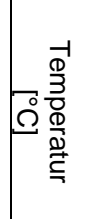 & 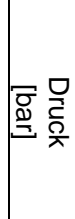 & 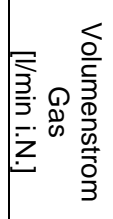 & 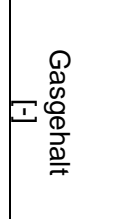 & 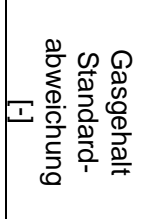 & 离 $\frac{\pi}{\infty}$ & 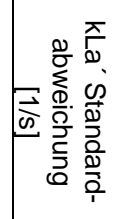 & 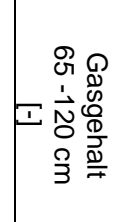 & 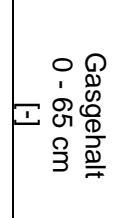 & 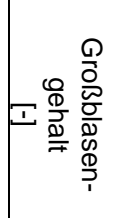 & 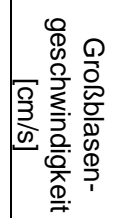 & 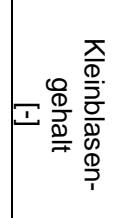 & 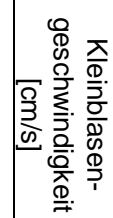 \\
\hline 141 & Decalin & Stickstoff & 19 & 1 & 102 & 0 & 1,2 & 130 & 20 & 5 & 117,8 & 0,171 & 0,0008 & 0,0812 & 0,0056 & & & 0,033 & 89,6 & 0,137 & 17,5 \\
\hline 142 & Decalin & Stickstoff & 19 & 1 & 102 & 0 & 1,2 & 130 & 20 & 5 & 235,6 & 0,256 & 0,0010 & 0,1482 & 0,0264 & & & 0,079 & 99,7 & 0,177 & 16,0 \\
\hline 143 & Decalin & Stickstoff & 19 & 1 & 102 & 0 & 1,2 & 130 & 20 & 5 & 471,3 & 0,360 & & & & & & 0,158 & 115,8 & 0,202 & 14,8 \\
\hline 144 & Decalin & Stickstoff & 19 & 1 & 102 & 0 & 1,2 & 130 & 20 & 10 & 47,1 & 0,036 & 0,0000 & 0,0174 & 0,0003 & & & 0,002 & 165,1 & 0,034 & 23,2 \\
\hline 145 & Decalin & Stickstoff & 19 & 1 & 102 & 0 & 1,2 & 130 & 20 & 10 & 94,3 & 0,096 & 0,0000 & 0,0430 & 0,0007 & & & 0,015 & 38,0 & 0,082 & 19,4 \\
\hline 146 & Decalin & Stickstoff & 19 & 1 & 102 & 0 & 1,2 & 130 & 20 & 10 & 235,6 & 0,198 & 0,0015 & 0,0936 & 0,0041 & & & 0,032 & 77,0 & 0,166 & 17,6 \\
\hline 147 & Decalin & Stickstoff & 19 & 1 & 102 & 0 & 1,2 & 130 & 20 & 10 & 471,3 & 0,299 & 0,0015 & 0,1677 & 0,0275 & & & 0,088 & 83,7 & 0,211 & 16,0 \\
\hline 148 & Decalin & Stickstoff & 19 & 1 & 102 & 0 & 1,2 & 130 & 20 & 20 & 94,3 & 0,046 & 0,0006 & 0,0201 & 0,0006 & & & 0,026 & 29,2 & 0,020 & 15,2 \\
\hline 149 & Decalin & Stickstoff & 19 & 1 & 102 & 0 & 1,2 & 130 & 20 & 20 & 188,5 & 0,097 & 0,0015 & 0,0420 & 0,0036 & & & 0,011 & 46,3 & 0,086 & 19,2 \\
\hline 150 & Decalin & Stickstoff & 19 & 1 & 102 & 0 & 1,2 & 130 & 20 & 20 & 471,3 & 0,216 & 0,0017 & 0,0978 & 0,0084 & & & 0,031 & 67,2 & 0,185 & 17,7 \\
\hline 151 & Decalin & Stickstoff & 19 & 1 & 102 & 0 & 1,2 & 130 & 20 & 20 & 942,5 & 0,346 & 0,0043 & 0,1680 & 0,0615 & & & 0,110 & 63,7 & 0,236 & 15,8 \\
\hline 152 & Decalin & Stickstoff & 19 & 1 & 102 & 0 & 1,2 & 130 & 20 & 40 & 188,5 & 0,047 & 0,0006 & 0,0192 & 0,0013 & & & 0,007 & 57,4 & 0,040 & 20,3 \\
\hline 153 & Decalin & Stickstoff & 19 & 1 & 102 & 0 & 1,2 & 130 & 20 & 40 & 377 & 0,101 & 0,0006 & 0,0395 & 0,0021 & & & 0,017 & 39,5 & 0,084 & 19,0 \\
\hline 154 & Decalin & Stickstoff & 19 & 1 & 102 & 0 & 1,2 & 130 & 20 & 40 & 942,5 & 0,289 & 0,0035 & 0,1158 & 0,0161 & & & 0,140 & 22,3 & 0,149 & 15,5 \\
\hline 155 & Decalin & Helium & 19 & 1 & 102 & 0 & 1,2 & 130 & 20 & 1 & 4,7 & 0,024 & & & & & & 0,009 & 85,6 & 0,016 & 25,5 \\
\hline 156 & Decalin & Helium & 19 & 1 & 102 & 0 & 1,2 & 130 & 20 & 1 & 9,4 & 0,051 & & & & & & 0,017 & 80,5 & 0,034 & 23,2 \\
\hline 157 & Decalin & Helium & 19 & 1 & 102 & 0 & 1,2 & 130 & 20 & 1 & 23,6 & 0,094 & & & & & & 0,042 & 102,7 & 0,053 & 21,0 \\
\hline 158 & Decalin & Helium & 19 & 1 & 102 & 0 & 1,2 & 130 & 20 & 1 & 47,1 & 0,135 & & & & & & 0,073 & 129,4 & 0,061 & 20,1 \\
\hline 159 & Decalin & Helium & 19 & 1 & 102 & 0 & 1,2 & 130 & 20 & 1 & 94,2 & 0,188 & & & & & & 0,122 & 165,3 & 0,066 & 19,1 \\
\hline 160 & Decalin & Helium & 19 & 1 & 102 & 0 & 1,2 & 130 & 20 & 3 & 14,1 & 0,028 & & & & & & 0,005 & 124,7 & 0,023 & 26,0 \\
\hline 161 & Decalin & Helium & 19 & 1 & 102 & 0 & 1,2 & 130 & 20 & 3 & 28,2 & 0,061 & & & & & & 0,016 & 71,6 & 0,045 & 22,2 \\
\hline 162 & Decalin & Helium & 19 & 1 & 102 & 0 & 1,2 & 130 & 20 & 3 & 70,8 & 0,108 & & & & & & 0,042 & 96,9 & 0,065 & 19,7 \\
\hline 163 & Decalin & Helium & 19 & 1 & 102 & 0 & 1,2 & 130 & 20 & 3 & 141,3 & 0,161 & & & & & & 0,085 & 110,0 & 0,076 & 18,9 \\
\hline 164 & Decalin & Helium & 19 & 1 & 102 & 0 & 1,2 & 130 & 20 & 3 & 282,6 & 0,230 & & & & & & 0,147 & 136,3 & 0,083 & 18,4 \\
\hline 165 & Decalin & Stickstoff & 19 & 1 & 102 & 0 & 1,2 & 130 & 50 & 1 & 4,3 & 0,037 & 0,0006 & 0,0195 & 0,0004 & & & & & & \\
\hline 166 & Decalin & Stickstoff & 19 & 1 & 102 & 0 & 1,2 & 130 & 50 & 1 & 8,5 & 0,074 & 0,0000 & 0,0387 & 0,0006 & & & & & & \\
\hline 167 & Decalin & Stickstoff & 19 & 1 & 102 & 0 & 1,2 & 130 & 50 & 1 & 21,4 & 0,125 & 0,0006 & 0,0723 & 0,0028 & & & & & & \\
\hline 168 & Decalin & Stickstoff & 19 & 1 & 102 & 0 & 1,2 & 130 & 50 & 1 & 42,7 & 0,177 & 0,0011 & 0,1140 & 0,0052 & & & & & & \\
\hline
\end{tabular}




\begin{tabular}{|c|c|c|c|c|c|c|c|c|c|c|c|c|c|c|c|c|c|c|c|c|c|}
\hline$\underline{3}$ & 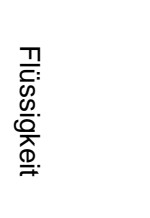 & ه్ల & 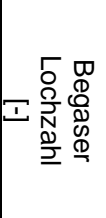 & 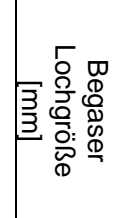 & 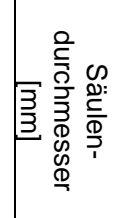 & 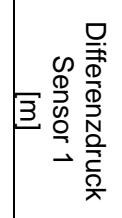 & 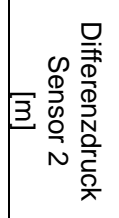 & 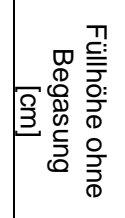 & 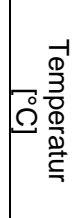 & 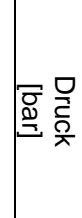 & 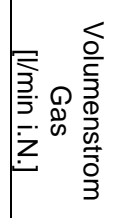 & 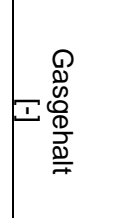 & 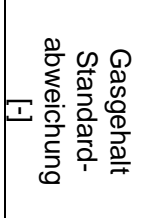 & 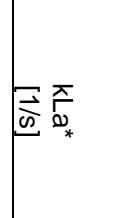 & 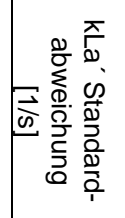 & 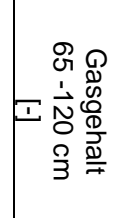 & 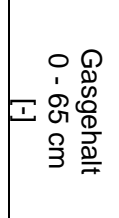 & 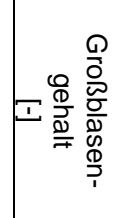 & 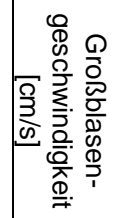 & 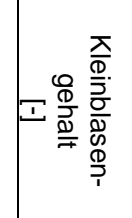 & 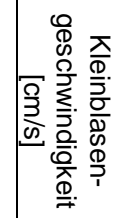 \\
\hline 169 & Decalin & Stickstoff & 19 & 1 & 102 & 0 & 1,2 & 130 & 50 & 2 & 8,5 & 0,039 & 0,0010 & 0,0189 & 0,0004 & & & & & & \\
\hline 170 & Decalin & Stickstoff & 19 & 1 & 102 & 0 & 1,2 & 130 & 50 & 2 & 17,1 & 0,080 & 0,0000 & 0,0393 & 0,0014 & & & & & & \\
\hline 171 & Decalin & Stickstoff & 19 & 1 & 102 & 0 & 1,2 & 130 & 50 & 2 & 42,7 & 0,142 & 0,0005 & 0,0794 & 0,0020 & & & & & & \\
\hline 172 & Decalin & Stickstoff & 19 & 1 & 102 & 0 & 1,2 & 130 & 50 & 2 & 85,5 & 0,214 & 0,0007 & 0,1316 & 0,0079 & & & & & & \\
\hline 173 & Decalin & Stickstoff & 19 & 1 & 102 & 0 & 1,2 & 130 & 50 & 5 & 21,4 & 0,041 & 0,0006 & 0,0200 & 0,0003 & & & & & & \\
\hline 174 & Decalin & Stickstoff & 19 & 1 & 102 & 0 & 1,2 & 130 & 50 & 5 & 42,7 & 0,088 & 0,0006 & 0,0431 & 0,0022 & & & & & & \\
\hline 175 & Decalin & Stickstoff & 19 & 1 & 102 & 0 & 1,2 & 130 & 50 & 5 & 106,9 & 0,187 & 0,0004 & 0,1043 & 0,0053 & & & & & & \\
\hline 176 & Decalin & Stickstoff & 19 & 1 & 102 & 0 & 1,2 & 130 & 50 & 5 & 213,7 & 0,277 & 0,0008 & 0,1736 & 0,0048 & & & & & & \\
\hline 177 & Decalin & Stickstoff & 19 & 1 & 102 & 0 & 1,2 & 130 & 70 & 1 & 4 & 0,036 & 0,0006 & 0,0205 & 0,0004 & & & & & & \\
\hline 178 & Decalin & Stickstoff & 19 & 1 & 102 & 0 & 1,2 & 130 & 70 & 1 & 8,1 & 0,073 & 0,0006 & 0,0425 & 0,0012 & & & & & & \\
\hline 179 & Decalin & Stickstoff & 19 & 1 & 102 & 0 & 1,2 & 130 & 70 & 1 & 20,1 & 0,123 & 0,0008 & 0,0764 & 0,0030 & & & & & & \\
\hline 180 & Decalin & Stickstoff & 19 & 1 & 102 & 0 & 1,2 & 130 & 70 & 1 & 40,3 & 0,174 & 0,0011 & 0,1239 & 0,0048 & & & & & & \\
\hline 181 & Decalin & Stickstoff & 19 & 1 & 102 & 0 & 1,2 & 130 & 70 & 2 & 8,1 & 0,037 & 0,0000 & 0,0207 & 0,0003 & & & & & & \\
\hline 182 & Decalin & Stickstoff & 19 & 1 & 102 & 0 & 1,2 & 130 & 70 & 2 & 16,1 & 0,078 & 0,0012 & 0,0433 & 0,0025 & & & & & & \\
\hline 183 & Decalin & Stickstoff & 19 & 1 & 102 & 0 & 1,2 & 130 & 70 & 2 & 40,3 & 0,138 & 0,0004 & 0,0889 & 0,0038 & & & & & & \\
\hline 184 & Decalin & Stickstoff & 19 & 1 & 102 & 0 & 1,2 & 130 & 70 & 2 & 80,5 & 0,209 & 0,0014 & 0,1485 & 0,0096 & & & & & & \\
\hline 185 & Decalin & Stickstoff & 19 & 1 & 102 & 0 & 1,2 & 130 & 70 & 5 & 20,1 & 0,041 & 0,0006 & 0,0221 & 0,0004 & & & & & & \\
\hline 186 & Decalin & Stickstoff & 19 & 1 & 102 & 0 & 1,2 & 130 & 70 & 5 & 40,3 & 0,088 & 0,0006 & 0,0466 & 0,0010 & & & & & & \\
\hline 187 & Decalin & Stickstoff & 19 & 1 & 102 & 0 & 1,2 & 130 & 70 & 5 & 100,6 & 0,188 & 0,0018 & 0,1101 & 0,0015 & & & & & & \\
\hline 188 & Decalin & Stickstoff & 19 & 1 & 102 & 0 & 1,2 & 130 & 70 & 5 & 201,3 & 0,280 & 0,0015 & 0,1777 & 0,0175 & & & & & & \\
\hline 189 & Ethanol & Stickstoff & 1 & 4,3 & 102 & 0 & 1,2 & 130 & 20 & 1 & 4,7 & 0,031 & 0,0005 & 0,0185 & 0,0005 & & & & & & \\
\hline 190 & Ethanol & Stickstoff & 1 & 4,3 & 102 & 0 & 1,2 & 130 & 20 & 1 & 9,4 & 0,058 & 0,0005 & 0,0350 & 0,0002 & & & & & & \\
\hline 191 & Ethanol & Stickstoff & 1 & 4,3 & 102 & 0 & 1,2 & 130 & 20 & 1 & 23,6 & 0,110 & 0,0012 & 0,0751 & 0,0011 & & & & & & \\
\hline 192 & Ethanol & Stickstoff & 1 & 4,3 & 102 & 0 & 1,2 & 130 & 20 & 1 & 47,1 & 0,164 & 0,0022 & 0,1293 & 0,0039 & & & & & & \\
\hline 193 & Ethanol & Stickstoff & 1 & 4,3 & 102 & 0 & 1,2 & 130 & 20 & 1 & 94,3 & 0,254 & 0,0029 & 0,2195 & 0,0040 & & & & & & \\
\hline 194 & Ethanol & Stickstoff & 1 & 4,3 & 102 & 0 & 1,2 & 130 & 20 & 2 & 9,4 & 0,034 & 0,0000 & 0,0198 & 0,0003 & & & & & & \\
\hline 195 & Ethanol & Stickstoff & 1 & 4,3 & 102 & 0 & 1,2 & 130 & 20 & 2 & 18,9 & 0,064 & 0,0007 & 0,0385 & 0,0010 & & & & & & \\
\hline 196 & Ethanol & Stickstoff & 1 & 4,3 & 102 & 0 & 1,2 & 130 & 20 & 2 & 47,1 & 0,126 & 0,0012 & 0,0828 & 0,0011 & & & & & & \\
\hline
\end{tabular}




\begin{tabular}{|c|c|c|c|c|c|c|c|c|c|c|c|c|c|c|c|c|c|c|c|c|c|}
\hline$\underline{3}$ & 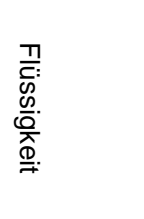 & ه్ల & 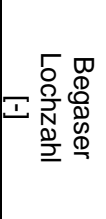 & 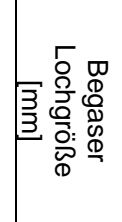 & 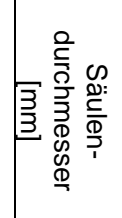 & 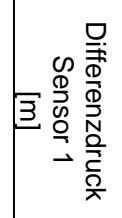 & 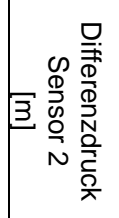 & 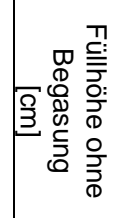 & 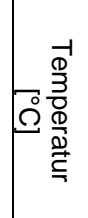 & 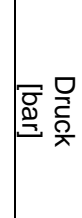 & 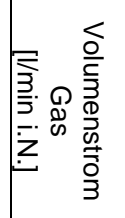 & 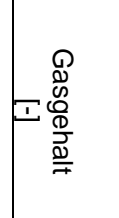 & 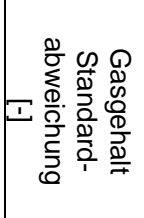 & 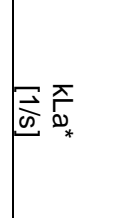 & 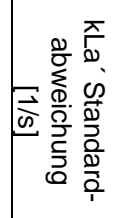 & 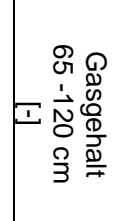 & 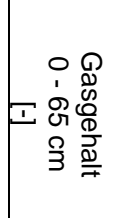 & 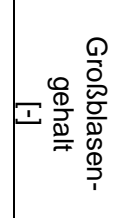 & 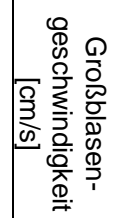 & 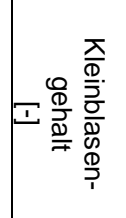 & 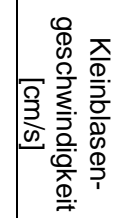 \\
\hline 197 & Ethanol & Stickstoff & 1 & 4,3 & 102 & 0 & 1,2 & 130 & 20 & 2 & 94,3 & 0,206 & 0,0031 & 0,1637 & 0,0045 & & & & & & \\
\hline 198 & Ethanol & Stickstoff & 1 & 4,3 & 102 & 0 & 1,2 & 130 & 20 & 2 & 188,5 & 0,315 & 0,0025 & 0,2545 & 0,0149 & & & & & & \\
\hline 199 & Ethanol & Stickstoff & 1 & 4,3 & 102 & 0 & 1,2 & 130 & 20 & 5 & 23,6 & 0,036 & 0,0000 & 0,0213 & 0,0007 & & & & & & \\
\hline 200 & Ethanol & Stickstoff & 1 & 4,3 & 102 & 0 & 1,2 & 130 & 20 & 5 & 47,1 & 0,074 & 0,0000 & 0,0425 & 0,0004 & & & & & & \\
\hline 201 & Ethanol & Stickstoff & 1 & 4,3 & 102 & 0 & 1,2 & 130 & 20 & 5 & 117,8 & 0,177 & 0,0010 & 0,1151 & 0,0019 & & & & & & \\
\hline 202 & Ethanol & Stickstoff & 1 & 4,3 & 102 & 0 & 1,2 & 130 & 20 & 5 & 235,6 & 0,282 & 0,0021 & 0,1976 & 0,0074 & & & & & & \\
\hline 203 & Ethanol & Stickstoff & 1 & 4,3 & 102 & 0 & 1,2 & 130 & 20 & 5 & 471,3 & 0,430 & 0,0012 & 0,2883 & 0,0052 & & & & & & \\
\hline 204 & Ethanol & Stickstoff & 1 & 4,3 & 102 & 0 & 1,2 & 130 & 20 & 10 & 47,1 & 0,040 & 0,0000 & 0,0212 & 0,0011 & & & & & & \\
\hline 205 & Ethanol & Stickstoff & 1 & 4,3 & 102 & 0 & 1,2 & 130 & 20 & 10 & 94,3 & 0,098 & 0,0000 & 0,0554 & 0,0014 & & & & & & \\
\hline 206 & Ethanol & Stickstoff & 1 & 4,3 & 102 & 0 & 1,2 & 130 & 20 & 10 & 235,6 & 0,225 & 0,0021 & 0,1233 & 0,0050 & & & & & & \\
\hline 207 & Ethanol & Stickstoff & 1 & 4,3 & 102 & 0 & 1,2 & 130 & 20 & 10 & 471,3 & 0,362 & 0,0055 & 0,2038 & 0,0010 & & & & & & \\
\hline 208 & Ethanol & Stickstoff & 1 & 4,3 & 102 & 0 & 1,2 & 130 & 20 & 20 & 94,3 & 0,049 & 0,0000 & 0,0278 & 0,0006 & & & & & & \\
\hline 209 & Ethanol & Stickstoff & 1 & 4,3 & 102 & 0 & 1,2 & 130 & 20 & 20 & 188,5 & 0,109 & 0,0014 & 0,0538 & 0,0034 & & & & & & \\
\hline 210 & Ethanol & Stickstoff & 1 & 4,3 & 102 & 0 & 1,2 & 130 & 20 & 20 & 471,3 & 0,269 & 0,0154 & 0,1266 & 0,0026 & & & & & & \\
\hline 211 & Ethanol & Stickstoff & 1 & 4,3 & 102 & 0 & 1,2 & 130 & 20 & 40 & 188,5 & 0,052 & 0,0000 & 0,0280 & 0,0037 & & & & & & \\
\hline 212 & Ethanol & Stickstoff & 1 & 4,3 & 102 & 0 & 1,2 & 130 & 20 & 40 & 377 & 0,111 & 0,0028 & 0,0506 & 0,0023 & & & & & & \\
\hline 213 & Ethanol & Stickstoff & 1 & 4,3 & 102 & 0 & 1,2 & 130 & 20 & 40 & 942,5 & 0,331 & 0,0072 & 0,1447 & 0,0038 & & & & & & \\
\hline 214 & Ethanol & Helium & 1 & 4,3 & 102 & 0 & 1,2 & 130 & 20 & 1 & 4,7 & 0,029 & 0,0007 & 0,0166 & 0,0000 & & & & & & \\
\hline 215 & Ethanol & Helium & 1 & 4,3 & 102 & 0 & 1,2 & 130 & 20 & 1 & 9,4 & 0,053 & 0,0000 & 0,0312 & 0,0006 & & & & & & \\
\hline 216 & Ethanol & Helium & 1 & 4,3 & 102 & 0 & 1,2 & 130 & 20 & 1 & 23,6 & 0,095 & 0,0015 & 0,0642 & 0,0011 & & & & & & \\
\hline 217 & Ethanol & Helium & 1 & 4,3 & 102 & 0 & 1,2 & 130 & 20 & 1 & 47,1 & 0,140 & 0,0012 & 0,1033 & 0,0025 & & & & & & \\
\hline 218 & Ethanol & Helium & 1 & 4,3 & 102 & 0 & 1,2 & 130 & 20 & 2 & 9,4 & 0,030 & 0,0007 & 0,0171 & 0,0001 & & & & & & \\
\hline 219 & Ethanol & Helium & 1 & 4,3 & 102 & 0 & 1,2 & 130 & 20 & 2 & 18,9 & 0,054 & 0,0007 & 0,0325 & 0,0010 & & & & & & \\
\hline 220 & Ethanol & Helium & 1 & 4,3 & 102 & 0 & 1,2 & 130 & 20 & 2 & 47,1 & 0,097 & 0,0006 & 0,0655 & 0,0005 & & & & & & \\
\hline 221 & Ethanol & Helium & 1 & 4,3 & 102 & 0 & 1,2 & 130 & 20 & 2 & 94,3 & 0,149 & 0,0000 & 0,1132 & 0,0045 & & & & & & \\
\hline 222 & Ethanol & Helium & 1 & 4,3 & 102 & 0 & 1,2 & 130 & 20 & 7 & 33 & 0,030 & & 0,0174 & & & & & & & \\
\hline 223 & Ethanol & Helium & 1 & 4,3 & 102 & 0 & 1,2 & 130 & 20 & 7 & 66 & 0,056 & 0,0000 & 0,0330 & 0,0000 & & & & & & \\
\hline 224 & Ethanol & Helium & 1 & 4,3 & 102 & 0 & 1,2 & 130 & 20 & 7 & 164,9 & 0,114 & 0,0010 & 0,0813 & 0,0012 & & & & & & \\
\hline
\end{tabular}




\begin{tabular}{|c|c|c|c|c|c|c|c|c|c|c|c|c|c|c|c|c|c|c|c|c|c|}
\hline$\underline{z}$ & 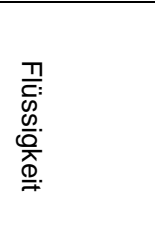 & D & 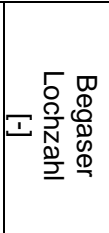 & 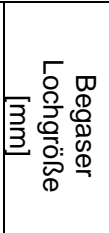 & 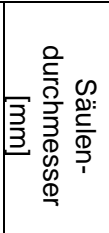 & 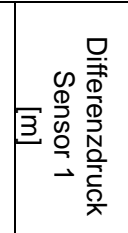 & 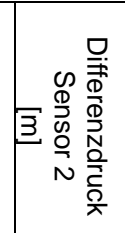 & 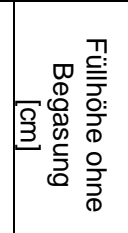 & 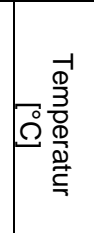 & 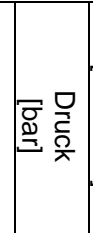 & 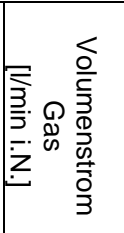 & 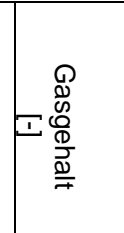 & 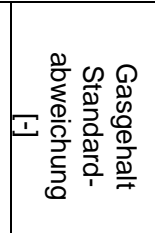 & 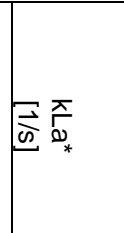 & 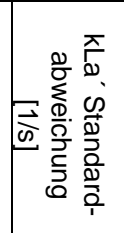 & 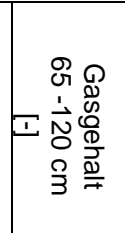 & 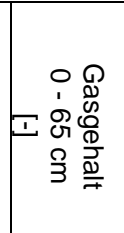 & 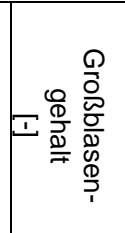 & 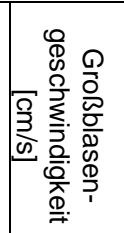 & 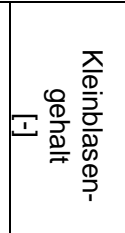 & 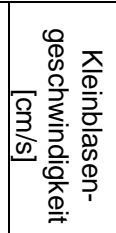 \\
\hline 2225 & Ethanol & Helium & 1 & 4,3 & 102 & 0 & 1,2 & 130 & 20 & 7 & 329,9 & 0,172 & 0,0006 & 0,1337 & 0,0067 & & & & & & \\
\hline 226 & Ethanol & Stickstoff & 19 & 1 & 102 & 0 & 1,2 & 130 & 20 & 1 & 4,7 & 0,034 & 0,0018 & 0,0212 & 0,0014 & & & 0,007 & 177,1 & 0,027 & 23,1 \\
\hline 227 & Ethanol & Stickstoff & 19 & 1 & 102 & 0 & 1,2 & 130 & 20 & 1 & 9,4 & 0,065 & 0,0027 & 0,0388 & 0,0016 & & & 0,014 & 83,0 & 0,051 & 22,6 \\
\hline 228 & Ethanol & Stickstoff & 19 & 1 & 102 & 0 & 1,2 & 130 & 20 & 1 & 23,6 & 0,117 & 0,0015 & 0,0799 & 0,0028 & & & 0,041 & 91,2 & 0,076 & 21,2 \\
\hline 229 & Ethanol & Stickstoff & 19 & 1 & 102 & 0 & 1,2 & 130 & 20 & 1 & 47,1 & 0,172 & 0,0030 & 0,1336 & 0,0034 & & & 0,081 & 110,1 & 0,091 & 20,2 \\
\hline 230 & Ethanol & Stickstoff & 19 & 1 & 102 & 0 & 1,2 & 130 & 20 & 1 & 94,3 & 0,257 & 0,0007 & 0,2275 & 0,0030 & & & 0,147 & 131,8 & 0,109 & 19,6 \\
\hline 231 & Ethanol & Stickstoff & 19 & 1 & 102 & 0 & 1,2 & 130 & 20 & 2 & 9,4 & 0,032 & 0,0035 & 0,0223 & 0,0002 & & & 0,005 & 191,0 & 0,028 & 24,8 \\
\hline 232 & Ethanol & Stickstoff & 19 & 1 & 102 & 0 & 1,2 & 130 & 20 & 2 & 18,9 & 0,068 & 0,0009 & 0,0408 & 0,0001 & & & 0,016 & 66,1 & 0,052 & 21,7 \\
\hline 233 & Ethanol & Stickstoff & 19 & 1 & 102 & 0 & 1,2 & 130 & 20 & 2 & 47,1 & 0,125 & 0,0008 & 0,0848 & 0,0004 & & & 0,039 & 93,4 & 0,086 & 20,3 \\
\hline 234 & Ethanol & Stickstoff & 19 & 1 & 102 & 0 & 1,2 & 130 & 20 & 2 & 94,3 & 0,201 & 0,0010 & 0,1578 & 0,0010 & & & 0,089 & 96,7 & 0,111 & 19,0 \\
\hline 235 & Ethanol & Stickstoff & 19 & 1 & 102 & 0 & 1,2 & 130 & 20 & 5 & 23,6 & 0,032 & 0,0013 & 0,0248 & 0,0023 & & & 0,003 & 199,5 & 0,029 & 24,1 \\
\hline 236 & Ethanol & Stickstoff & 19 & 1 & 102 & 0 & 1,2 & 130 & 20 & 5 & 47,1 & 0,072 & 0,0009 & 0,0453 & 0,0013 & & & 0,008 & 101,5 & 0,065 & 21,5 \\
\hline 237 & Ethanol & Stickstoff & 19 & 1 & 102 & 0 & 1,2 & 130 & 20 & 5 & 117,8 & 0,163 & 0,0011 & 0,1027 & 0,0003 & & & 0,039 & 79,4 & 0,124 & 18,5 \\
\hline 238 & Ethanol & Stickstoff & 19 & 1 & 102 & 0 & 1,2 & 130 & 20 & 5 & 235,6 & 0,257 & 0,0006 & 0,1851 & 0,0007 & & & 0,089 & 87,9 & 0,168 & 17,3 \\
\hline 239 & Ethanol & Stickstoff & 19 & 1 & 102 & 0 & 1,2 & 130 & 20 & 10 & 47,1 & 0,038 & 0,0008 & 0,0193 & 0,0001 & & & 0,005 & 138,5 & 0,033 & 21,7 \\
\hline 240 & Ethanol & Stickstoff & 19 & 1 & 102 & 0 & 1,2 & 130 & 20 & 10 & 94,3 & 0,093 & 0,0019 & 0,0481 & 0,0002 & & & 0,008 & 93,9 & 0,085 & 19,3 \\
\hline 241 & Ethanol & Stickstoff & 19 & 1 & 102 & 0 & 1,2 & 130 & 20 & 10 & 235,6 & 0,200 & 0,0012 & 0,1198 & 0,0064 & & & 0,018 & 127,5 & 0,182 & 17,4 \\
\hline 242 & Ethanol & Stickstoff & 19 & 1 & 102 & 0 & 1,2 & 130 & 20 & 10 & 471,3 & 0,311 & 0,0049 & 0,2035 & 0,0015 & & & 0,076 & 89,1 & 0,235 & 16,8 \\
\hline 243 & Ethanol & Stickstoff & 19 & 1 & 102 & 0 & 1,2 & 130 & 20 & 20 & 94,3 & 0,043 & 0,0012 & 0,0307 & 0,0001 & & & 0,006 & 87,3 & 0,037 & 20,4 \\
\hline 244 & Ethanol & Stickstoff & 19 & 1 & 102 & 0 & 1,2 & 130 & 20 & 20 & 188,5 & 0,093 & 0,0018 & 0,0533 & 0,0027 & & & 0,008 & 70,0 & 0,086 & 19,2 \\
\hline 245 & Ethanol & Stickstoff & 19 & 1 & 102 & 0 & 1,2 & 130 & 20 & 20 & 471,3 & 0,229 & 0,0016 & 0,1358 & 0,0006 & & & 0,043 & 52,1 & 0,187 & 17,0 \\
\hline 246 & Ethanol & Stickstoff & 19 & 1 & 102 & 0 & 1,2 & 130 & 20 & 20 & 942,5 & 0,360 & 0,0040 & 0,1876 & 0,0033 & & & 0,134 & 53,8 & 0,226 & 15,8 \\
\hline 247 & Ethanol & Stickstoff & 19 & 1 & 102 & 0 & 1,2 & 130 & 20 & 40 & 188,5 & 0,047 & 0,0020 & 0,0323 & 0,0008 & & & 0,005 & 70,3 & 0,041 & 20,3 \\
\hline 248 & Ethanol & Stickstoff & 19 & 1 & 102 & 0 & 1,2 & 130 & 20 & 40 & 377 & 0,099 & 0,0019 & 0,0640 & 0,0020 & & & 0,010 & 49,6 & 0,089 & 18,5 \\
\hline 249 & Ethanol & Stickstoff & 19 & 1 & 102 & 0 & 1,2 & 130 & 20 & 40 & 942,5 & 0,267 & 0,0104 & 0,1114 & 0,0026 & & & 0,065 & 33,0 & 0,201 & 16,2 \\
\hline 250 & Ethanol & Stickstoff & 7 & 1 & 115 & 0,1 & 0,905 & 100 & 20 & 1 & 5 & 0,028 & 0,0008 & 0,0067 & & & & 0,011 & 48,8 & 0,016 & 19,4 \\
\hline 251 & Ethanol & Stickstoff & 7 & 1 & 115 & 0,1 & 0,905 & 100 & 20 & 1 & 10 & 0,051 & 0,0011 & 0,0129 & & & & 0,015 & 66,8 & 0,036 & 20,2 \\
\hline 252 & Ethanol & Stickstoff & 7 & 1 & 115 & 0,1 & 0,905 & 100 & 20 & 1 & 20 & 0,089 & 0,0014 & 0,0242 & & & & 0,029 & 81,0 & 0,060 & 19,3 \\
\hline
\end{tabular}




\begin{tabular}{|c|c|c|c|c|c|c|c|c|c|c|c|c|c|c|c|c|c|c|c|c|c|}
\hline$\underline{z}$ & 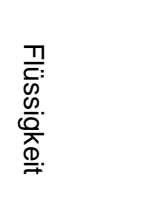 & $\underset{\aleph}{\circledR}$ & 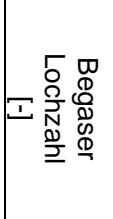 & 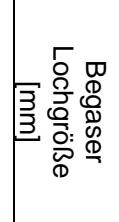 & 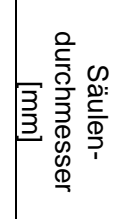 & 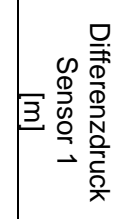 & 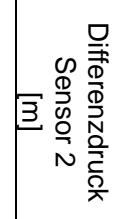 & 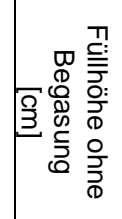 & 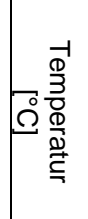 & 总 몰 & 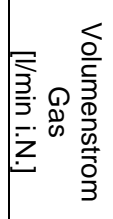 & 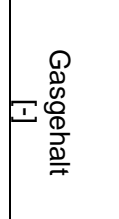 & 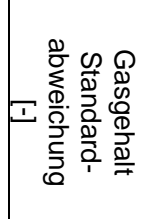 & 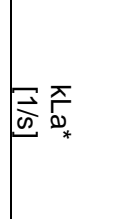 & 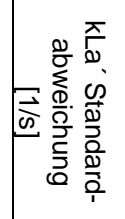 & 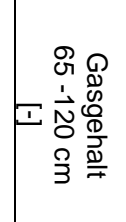 & 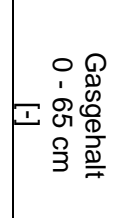 & 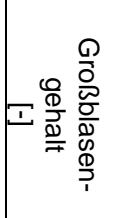 & 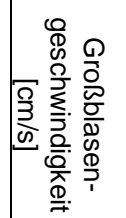 & 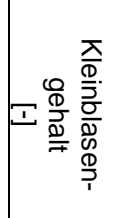 & 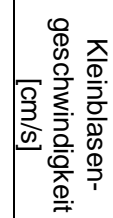 \\
\hline 253 & Ethanol & Stickstoff & 7 & 1 & 115 & 0,1 & 0,905 & 100 & 20 & 1 & 30 & 0,116 & 0,0023 & 0,0349 & 0,0008 & & & 0,044 & 89,9 & 0,072 & 18,3 \\
\hline 254 & Ethanol & Stickstoff & 7 & 1 & 115 & 0,1 & 0,905 & 100 & 20 & 1 & 50 & 0,166 & 0,0044 & 0,0535 & 0,0012 & & & 0,072 & 96,9 & 0,095 & 17,7 \\
\hline 255 & Ethanol & Stickstoff & 7 & 1 & 115 & 0,1 & 0,905 & 100 & 20 & 2 & 10 & 0,028 & 0,0011 & 0,0077 & & & & 0,012 & 47,1 & 0,016 & 18,6 \\
\hline 256 & Ethanol & Stickstoff & 7 & 1 & 115 & 0,1 & 0,905 & 100 & 20 & 2 & 20 & 0,054 & 0,0039 & 0,0151 & & & & 0,014 & 81,0 & 0,041 & 19,1 \\
\hline 257 & Ethanol & Stickstoff & 7 & 1 & 115 & 0,1 & 0,905 & 100 & 20 & 2 & 40 & 0,105 & 0,0018 & 0,0295 & 0,0004 & & & 0,027 & 76,8 & 0,078 & 17,7 \\
\hline 258 & Ethanol & Stickstoff & 7 & 1 & 115 & 0,1 & 0,905 & 100 & 20 & 2 & 60 & 0,146 & 0,0018 & 0,0439 & 0,0019 & & & 0,034 & 95,1 & 0,112 & 17,6 \\
\hline 259 & Ethanol & Stickstoff & 7 & 1 & 115 & 0,1 & 0,905 & 100 & 20 & 2 & 100 & 0,221 & 0,0042 & 0,0692 & 0,0061 & & & 0,076 & 87,0 & 0,144 & 17,1 \\
\hline 260 & Ethanol & Stickstoff & 7 & 1 & 115 & 0,1 & 0,905 & 100 & 20 & 4 & 20 & 0,033 & 0,0009 & 0,0089 & & & & 0,012 & 40,5 & 0,021 & 19,0 \\
\hline 261 & Ethanol & Stickstoff & 7 & 1 & 115 & 0,1 & 0,905 & 100 & 20 & 4 & 40 & 0,069 & 0,0006 & 0,0187 & & & & 0,018 & 44,5 & 0,051 & 18,0 \\
\hline 262 & Ethanol & Stickstoff & 7 & 1 & 115 & 0,1 & 0,905 & 100 & 20 & 4 & 80 & 0,139 & 0,0046 & 0,0382 & 0,0013 & & & 0,036 & 47,2 & 0,103 & 17,0 \\
\hline 263 & Ethanol & Stickstoff & 7 & 1 & 115 & 0,1 & 0,905 & 100 & 20 & 4 & 120 & 0,209 & 0,0010 & 0,0544 & 0,0009 & & & 0,072 & 41,4 & 0,137 & 16,3 \\
\hline 264 & Ethanol & Stickstoff & 7 & 1 & 115 & 0,1 & 0,905 & 100 & 20 & 4 & 200 & 0,306 & 0,0027 & 0,0842 & 0,0078 & & & 0,119 & 46,7 & 0,187 & 16,7 \\
\hline 265 & Ethanol & Stickstoff & 7 & 1 & 115 & 0,1 & 0,905 & 100 & 20 & 7 & 40 & 0,040 & 0,0011 & 0,0105 & 0,0002 & & & 0,010 & 47,8 & 0,030 & 18,7 \\
\hline 266 & Ethanol & Stickstoff & 7 & 1 & 115 & 0,1 & 0,905 & 100 & 20 & 7 & 80 & 0,097 & 0,0035 & 0,0236 & & & & 0,025 & 31,9 & 0,072 & 16,7 \\
\hline 267 & Ethanol & Stickstoff & 7 & 1 & 115 & 0,1 & 0,905 & 100 & 20 & 7 & 160 & 0,199 & 0,0046 & 0,0497 & 0,0018 & & & 0,067 & 28,8 & 0,132 & 15,5 \\
\hline 268 & Ethanol & Stickstoff & 7 & 1 & 115 & 0,1 & 0,905 & 100 & 20 & 7 & 240 & 0,265 & 0,0027 & 0,0677 & 0,0004 & & & 0,098 & 33,4 & 0,168 & 15,9 \\
\hline 269 & Ethanol & Stickstoff & 1 & 1 & 102 & 0 & 1,2 & 130 & 20 & 1 & 4,7 & 0,031 & 0,0013 & 0,0223 & 0,0021 & & & 0,009 & 62,3 & 0,023 & 23,0 \\
\hline 270 & Ethanol & Stickstoff & 1 & 1 & 102 & 0 & 1,2 & 130 & 20 & 1 & 9,4 & 0,062 & 0,0005 & 0,0403 & 0,0036 & & & 0,015 & 74,8 & 0,048 & 21,9 \\
\hline 271 & Ethanol & Stickstoff & 1 & 1 & 102 & 0 & 1,2 & 130 & 20 & 1 & 23,6 & 0,120 & 0,0015 & 0,0863 & 0,0052 & & & 0,037 & 101,4 & 0,083 & 19,9 \\
\hline 272 & Ethanol & Stickstoff & 1 & 1 & 102 & 0 & 1,2 & 130 & 20 & 1 & 47,1 & 0,174 & 0,0020 & 0,1403 & 0,0038 & & & 0,075 & 118,9 & 0,099 & 18,7 \\
\hline 273 & Ethanol & Stickstoff & 1 & 1 & 102 & 0 & 1,2 & 130 & 20 & 1 & 94,3 & 0,255 & 0,0030 & 0,2278 & 0,0091 & & & 0,144 & 135,4 & 0,111 & 18,2 \\
\hline 274 & Ethanol & Stickstoff & 1 & 1 & 102 & 0 & 1,2 & 130 & 20 & 2 & 9,4 & 0,036 & 0,0000 & 0,0253 & 0,0024 & & & 0,010 & 50,7 & 0,026 & 22,7 \\
\hline 275 & Ethanol & Stickstoff & 1 & 1 & 102 & 0 & 1,2 & 130 & 20 & 2 & 18,9 & 0,069 & 0,0007 & 0,0459 & 0,0013 & & & 0,013 & 73,4 & 0,055 & 21,3 \\
\hline 276 & Ethanol & Stickstoff & 1 & 1 & 102 & 0 & 1,2 & 130 & 20 & 2 & 47,1 & 0,138 & 0,0006 & 0,1011 & 0,0026 & & & 0,039 & 91,6 & 0,100 & 18,6 \\
\hline 277 & Ethanol & Stickstoff & 1 & 1 & 102 & 0 & 1,2 & 130 & 20 & 2 & 94,3 & 0,209 & 0,0012 & 0,1785 & 0,0033 & & & 0,079 & 107,6 & 0,130 & 17,5 \\
\hline 278 & Ethanol & Stickstoff & 1 & 1 & 102 & 0 & 1,2 & 130 & 20 & 2 & 188,5 & 0,299 & 0,0024 & 0,2508 & 0,0122 & & & 0,151 & 126,1 & 0,148 & 17,1 \\
\hline 279 & Ethanol & Stickstoff & 1 & 1 & 102 & 0 & 1,2 & 130 & 20 & 5 & 23,6 & 0,038 & 0,0007 & 0,0261 & 0,0008 & & & 0,006 & 73,4 & 0,031 & 21,9 \\
\hline 280 & Ethanol & Stickstoff & 1 & 1 & 102 & 0 & 1,2 & 130 & 20 & 5 & 47,1 & 0,082 & 0,0000 & 0,0513 & 0,0003 & & & 0,008 & 89,9 & 0,074 & 19,7 \\
\hline
\end{tabular}




\begin{tabular}{|c|c|c|c|c|c|c|c|c|c|c|c|c|c|c|c|c|c|c|c|c|c|}
\hline$\underline{3}$ & 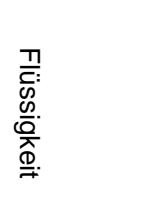 & § & 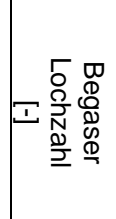 & 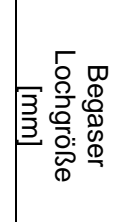 & 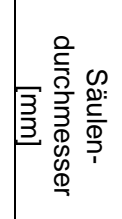 & 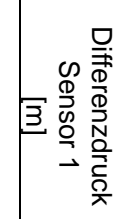 & 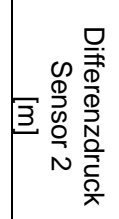 & 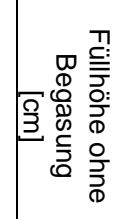 & 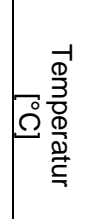 & 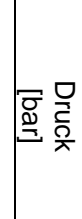 & 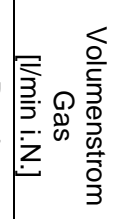 & 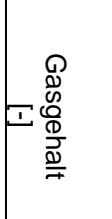 & 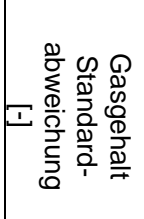 & $\vec{F}_{*}{ }_{\infty}$ & 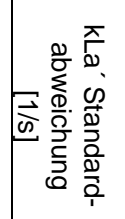 & 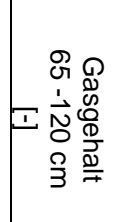 & 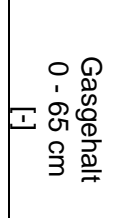 & 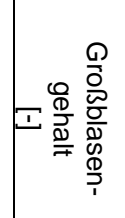 & 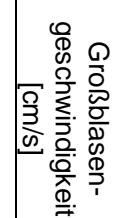 & 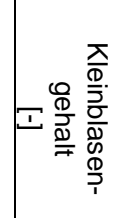 & 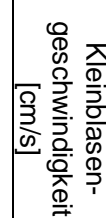 \\
\hline 281 & Ethanol & Stickstoff & 1 & 1 & 102 & 0 & 1,2 & 130 & 20 & 5 & 117,8 & 0,184 & 0,0006 & 0,1259 & 0,0019 & & & 0,036 & 78,7 & 0,148 & 17,2 \\
\hline 282 & Ethanol & Stickstoff & 1 & 1 & 102 & 0 & 1,2 & 130 & 20 & 5 & 235,6 & 0,268 & 0,0038 & 0,1920 & 0,0015 & & & 0,079 & 95,4 & 0,189 & 16,4 \\
\hline 283 & Ethanol & Stickstoff & 1 & 1 & 102 & 0 & 1,2 & 130 & 20 & 5 & 471,3 & 0,368 & 0,0010 & 0,2807 & 0,0112 & & & 0,162 & 112,1 & 0,205 & 16,4 \\
\hline 284 & Ethanol & Stickstoff & 1 & 1 & 102 & 0 & 1,2 & 130 & 20 & 10 & 47,1 & 0,039 & 0,0000 & 0,0270 & 0,0007 & & & 0,003 & 150,5 & 0,037 & 21,5 \\
\hline 285 & Ethanol & Stickstoff & 1 & 1 & 102 & 0 & 1,2 & 130 & 20 & 10 & 94,3 & 0,099 & 0,0021 & 0,0575 & 0,0051 & & & 0,007 & 64,6 & 0,092 & 18,3 \\
\hline 286 & Ethanol & Stickstoff & 1 & 1 & 102 & 0 & 1,2 & 130 & 20 & 10 & 235,6 & 0,220 & 0,0025 & 0,1388 & 0,0061 & & & 0,034 & 67,6 & 0,186 & 16,3 \\
\hline 287 & Ethanol & Stickstoff & 1 & 1 & 102 & 0 & 1,2 & 130 & 20 & 10 & 471,3 & 0,314 & 0,0026 & 0,2033 & 0,0093 & & & 0,090 & 80,7 & 0,224 & 15,7 \\
\hline 288 & Ethanol & Stickstoff & 1 & 1 & 102 & 0 & 1,2 & 130 & 20 & 20 & 94,3 & 0,054 & 0,0014 & 0,0346 & 0,0066 & & & & & & \\
\hline 289 & Ethanol & Stickstoff & 1 & 1 & 102 & 0 & 1,2 & 130 & 20 & 20 & 188,5 & 0,119 & 0,0007 & 0,0586 & 0,0013 & & & 0,010 & 35,9 & 0,109 & 17,1 \\
\hline 290 & Ethanol & Stickstoff & 1 & 1 & 102 & 0 & 1,2 & 130 & 20 & 20 & 471,3 & 0,258 & 0,0021 & 0,1304 & 0,0042 & & & 0,020 & 85,0 & 0,238 & 15,6 \\
\hline 291 & Ethanol & Stickstoff & 1 & 1 & 102 & 0 & 1,2 & 130 & 20 & 20 & 942,5 & 0,357 & 0,0050 & 0,2168 & 0,0045 & & & 0,118 & 60,6 & 0,239 & 15,1 \\
\hline 292 & Ethanol & Stickstoff & 1 & 1 & 102 & 0 & 1,2 & 130 & 20 & 40 & 188,5 & 0,057 & 0,0007 & 0,0319 & 0,0004 & & & & & & \\
\hline 293 & Ethanol & Stickstoff & 1 & 1 & 102 & 0 & 1,2 & 130 & 20 & 40 & 377 & 0,121 & 0,0029 & 0,0570 & 0,0031 & & & 0,003 & 64,5 & 0,117 & 16,4 \\
\hline 294 & Ethanol & Stickstoff & 1 & 1 & 102 & 0 & 1,2 & 130 & 20 & 40 & 942,5 & 0,295 & & 0,1326 & & & & & & & \\
\hline 295 & Ethanol & Helium & 1 & 1 & 102 & 0 & 1,2 & 130 & 20 & 1 & 4,7 & 0,028 & 0,0000 & 0,0177 & 0,0001 & & & 0,008 & 71,9 & 0,020 & 26,9 \\
\hline 296 & Ethanol & Helium & 1 & 1 & 102 & 0 & 1,2 & 130 & 20 & 1 & 9,4 & 0,053 & 0,0007 & 0,0346 & 0,0006 & & & 0,023 & 64,5 & 0,031 & 22,7 \\
\hline 297 & Ethanol & Helium & 1 & 1 & 102 & 0 & 1,2 & 130 & 20 & 1 & 23,6 & 0,098 & 0,0010 & 0,0754 & 0,0011 & & & 0,045 & 93,4 & 0,053 & 22,1 \\
\hline 298 & Ethanol & Helium & 1 & 1 & 102 & 0 & 1,2 & 130 & 20 & 1 & 47,1 & 0,143 & 0,0006 & 0,1245 & 0,0037 & & & 0,076 & 122,9 & 0,067 & 21,3 \\
\hline 299 & Ethanol & Helium & 1 & 1 & 102 & 0 & 1,2 & 130 & 20 & 1 & 94,3 & 0,219 & 0,0157 & 0,1904 & 0,0027 & & & 0,142 & 140,2 & 0,077 & 21,0 \\
\hline 300 & Ethanol & Helium & 1 & 1 & 102 & 0 & 1,2 & 130 & 20 & 3 & 14,1 & 0,032 & 0,0000 & 0,0193 & 0,0000 & & & & & & \\
\hline 301 & Ethanol & Helium & 1 & 1 & 102 & 0 & 1,2 & 130 & 20 & 3 & 28,3 & 0,059 & 0,0000 & 0,0342 & 0,0030 & & & & & & \\
\hline 302 & Ethanol & Helium & 1 & 1 & 102 & 0 & 1,2 & 130 & 20 & 3 & 70,7 & 0,113 & 0,0007 & 0,0810 & 0,0016 & & & & & & \\
\hline 303 & Ethanol & Helium & 1 & 1 & 102 & 0 & 1,2 & 130 & 20 & 3 & 141,4 & 0,167 & 0,0035 & 0,1353 & 0,0020 & & & & & & \\
\hline 304 & Ethanol & Helium & 1 & 1 & 102 & 0 & 1,2 & 130 & 20 & 3 & 282,8 & 0,235 & 0,0014 & 0,1990 & 0,0138 & & & & & & \\
\hline 305 & Ethanol & Stickstoff & 1 & 4,3 & 102 & 0 & 1,2 & 130 & 50 & 1 & 4,3 & 0,039 & 0,0006 & 0,0240 & 0,0000 & & & & & & \\
\hline 306 & Ethanol & Stickstoff & 1 & 4,3 & 102 & 0 & 1,2 & 130 & 50 & 1 & 8,5 & 0,069 & 0,0006 & 0,0453 & 0,0004 & & & & & & \\
\hline 307 & Ethanol & Stickstoff & 1 & 4,3 & 102 & 0 & 1,2 & 130 & 50 & 1 & 21,4 & 0,122 & 0,0011 & 0,0972 & 0,0017 & & & & & & \\
\hline 308 & Ethanol & Stickstoff & 1 & 4,3 & 102 & 0 & 1,2 & 130 & 50 & 1 & 42,7 & 0,180 & 0,0015 & 0,1599 & 0,0055 & & & & & & \\
\hline
\end{tabular}




\begin{tabular}{|c|c|c|c|c|c|c|c|c|c|c|c|c|c|c|c|c|c|c|c|c|c|}
\hline$\leq$ & 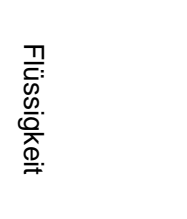 & $\stackrel{\infty}{\infty}$ & 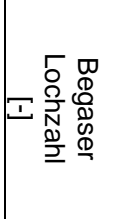 & 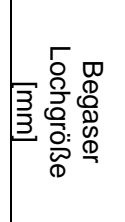 & 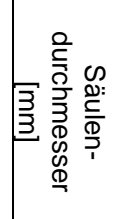 & 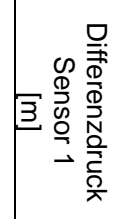 & 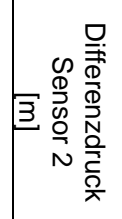 & 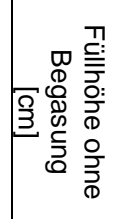 & 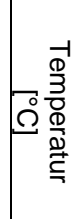 & 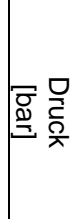 & 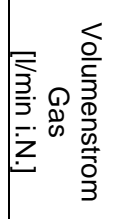 & 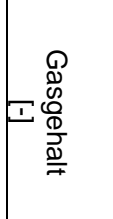 & 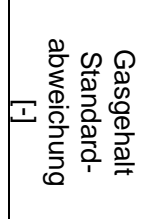 & $\underset{\omega}{\vec{\omega}} \bar{d}_{*}$ & 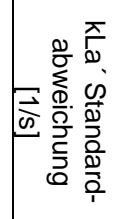 & 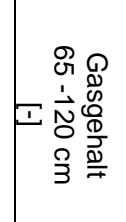 & 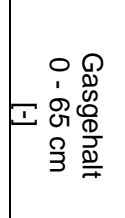 & 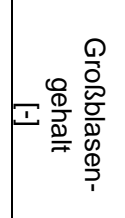 & 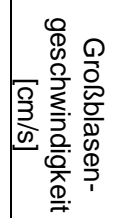 & 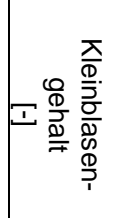 & 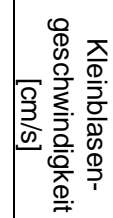 \\
\hline 309 & Ethylenglycol & Stickstoff & 1 & 4,3 & 102 & 0 & 1,2 & 130 & 10 & 1 & 4,9 & 0,022 & & 0,0011 & & & & & & & \\
\hline 310 & Ethylenglycol & Stickstoff & 1 & 4,3 & 102 & 0 & 1,2 & 130 & 10 & 1 & 9,8 & 0,041 & & 0,0022 & & & & & & & \\
\hline 311 & Ethylenglycol & Stickstoff & 1 & 4,3 & 102 & 0 & 1,2 & 130 & 10 & 1 & 24,4 & 0,079 & & 0,0047 & & & & & & & \\
\hline 312 & Ethylenglycol & Stickstoff & 1 & 4,3 & 102 & 0 & 1,2 & 130 & 10 & 1 & 48,8 & 0,125 & & 0,0087 & & & & & & & \\
\hline 313 & Ethylenglycol & Stickstoff & 1 & 4,3 & 102 & 0 & 1,2 & 130 & 10 & 1 & 97,6 & 0,199 & 0,0021 & 0,0158 & 0,0014 & & & & & & \\
\hline 314 & Ethylenglycol & Stickstoff & 19 & 1 & 102 & 0 & 1,2 & 130 & 20 & 1 & 4,7 & 0,021 & 0,0002 & 0,0017 & 0,0003 & & & & & & \\
\hline 315 & Ethylenglycol & Stickstoff & 19 & 1 & 102 & 0 & 1,2 & 130 & 20 & 1 & 9,4 & 0,042 & 0,0006 & 0,0035 & 0,0000 & & & & & & \\
\hline 316 & Ethylenglycol & Stickstoff & 19 & 1 & 102 & 0 & 1,2 & 130 & 20 & 1 & 23,6 & 0,085 & 0,0010 & 0,0075 & 0,0001 & & & & & & \\
\hline 317 & Ethylenglycol & Stickstoff & 19 & 1 & 102 & 0 & 1,2 & 130 & 20 & 1 & 47,1 & 0,133 & 0,0027 & 0,0128 & 0,0010 & & & & & & \\
\hline 318 & Ethylenglycol & Stickstoff & 19 & 1 & 102 & 0 & 1,2 & 130 & 20 & 1 & 94,2 & 0,208 & 0,0048 & 0,0216 & 0,0027 & & & & & & \\
\hline 319 & Ethylenglycol & Stickstoff & 19 & 1 & 102 & 0 & 1,2 & 130 & 20 & 2 & 9,4 & 0,023 & 0,0000 & 0,0012 & 0,0001 & & & & & & \\
\hline 320 & Ethylenglycol & Stickstoff & 19 & 1 & 102 & 0 & 1,2 & 130 & 20 & 2 & 18,9 & 0,044 & 0,0000 & 0,0026 & 0,0003 & & & & & & \\
\hline 321 & Ethylenglycol & Stickstoff & 19 & 1 & 102 & 0 & 1,2 & 130 & 20 & 2 & 47,1 & 0,088 & 0,0000 & 0,0075 & 0,0006 & & & & & & \\
\hline 322 & Ethylenglycol & Stickstoff & 19 & 1 & 102 & 0 & 1,2 & 130 & 20 & 2 & 94,3 & 0,149 & 0,0010 & 0,0150 & 0,0021 & & & & & & \\
\hline 323 & Ethylenglycol & Stickstoff & 19 & 1 & 102 & 0 & 1,2 & 130 & 20 & 2 & 188,5 & 0,225 & 0,0035 & 0,0254 & 0,0030 & & & & & & \\
\hline 324 & Ethylenglycol & Stickstoff & 19 & 1 & 102 & 0 & 1,2 & 130 & 20 & 5 & 23,6 & 0,026 & 0,0007 & 0,0013 & 0,0003 & & & & & & \\
\hline 325 & Ethylenglycol & Stickstoff & 19 & 1 & 102 & 0 & 1,2 & 130 & 20 & 5 & 47,1 & 0,047 & 0,0000 & 0,0032 & 0,0004 & & & & & & \\
\hline 326 & Ethylenglycol & Stickstoff & 19 & 1 & 102 & 0 & 1,2 & 130 & 20 & 5 & 117,8 & 0,102 & 0,0000 & 0,0098 & 0,0003 & & & & & & \\
\hline 327 & Ethylenglycol & Stickstoff & 19 & 1 & 102 & 0 & 1,2 & 130 & 20 & 5 & 235,6 & 0,165 & 0,0010 & 0,0183 & 0,0014 & & & & & & \\
\hline 328 & Ethylenglycol & Stickstoff & 19 & 1 & 102 & 0 & 1,2 & 130 & 20 & 5 & 471,3 & 0,267 & 0,0026 & 0,0349 & 0,0014 & & & & & & \\
\hline 329 & Ethylenglycol & Stickstoff & 19 & 1 & 102 & 0 & 1,2 & 130 & 20 & 10 & 47,1 & 0,027 & 0,0007 & 0,0015 & 0,0004 & & & & & & \\
\hline 330 & Ethylenglycol & Stickstoff & 19 & 1 & 102 & 0 & 1,2 & 130 & 20 & 10 & 94,3 & 0,055 & 0,0007 & 0,0059 & 0,0003 & & & & & & \\
\hline 331 & Ethylenglycol & Stickstoff & 19 & 1 & 102 & 0 & 1,2 & 130 & 20 & 10 & 235,6 & 0,112 & 0,0007 & 0,0118 & 0,0014 & & & & & & \\
\hline 332 & Ethylenglycol & Stickstoff & 19 & 1 & 102 & 0 & 1,2 & 130 & 20 & 10 & 471,3 & 0,198 & 0,0049 & 0,0248 & 0,0001 & & & & & & \\
\hline 333 & Ethylenglycol & Stickstoff & 19 & 1 & 102 & 0 & 1,2 & 130 & 20 & 20 & 94,3 & 0,033 & 0,0021 & 0,0025 & 0,0020 & & & & & & \\
\hline 334 & Ethylenglycol & Stickstoff & 19 & 1 & 102 & 0 & 1,2 & 130 & 20 & 20 & 188,5 & 0,059 & 0,0000 & 0,0060 & 0,0003 & & & & & & \\
\hline 335 & Ethylenglycol & Stickstoff & 19 & 1 & 102 & 0 & 1,2 & 130 & 20 & 20 & 471,3 & 0,157 & 0,0014 & 0,0183 & 0,0001 & & & & & & \\
\hline 336 & Ethylenglycol & Stickstoff & 19 & 1 & 102 & 0 & 1,2 & 130 & 20 & 20 & 942,5 & 0,240 & 0,0064 & 0,0368 & 0,0011 & & & & & & \\
\hline
\end{tabular}




\begin{tabular}{|c|c|c|c|c|c|c|c|c|c|c|c|c|c|c|c|c|c|c|c|c|c|}
\hline$\underline{s}$ & 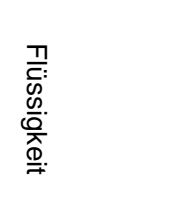 & 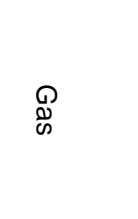 & 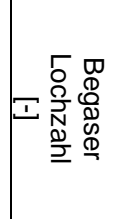 & 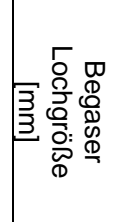 & 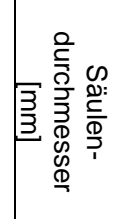 & 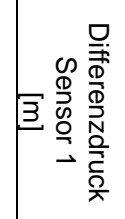 & 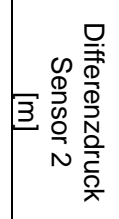 & 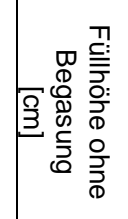 & 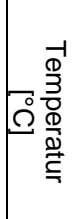 & סृ & 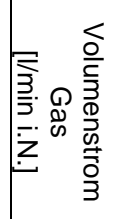 & 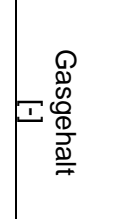 & 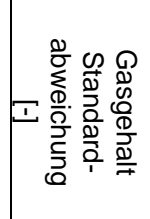 & 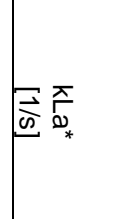 & 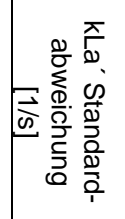 & 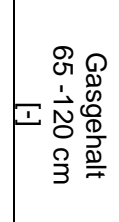 & 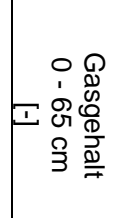 & 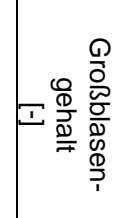 & 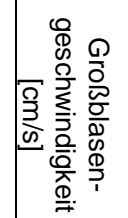 & 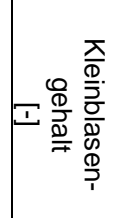 & 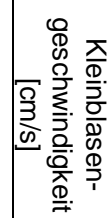 \\
\hline 337 & Ethylenglycol & Stickstoff & 19 & 1 & 102 & 0 & 1,2 & 130 & 20 & 40 & 188,5 & 0,034 & & 0,0042 & & & & & & & \\
\hline 338 & Ethylenglycol & Stickstoff & 19 & 1 & 102 & 0 & 1,2 & 130 & 20 & 40 & 377 & 0,092 & 0,0078 & 0,0110 & 0,0010 & & & & & & \\
\hline 339 & Ethylenglycol & Stickstoff & 19 & 1 & 102 & 0 & 1,2 & 130 & 20 & 40 & 942,5 & 0,182 & 0,0028 & 0,0225 & 0,0023 & & & & & & \\
\hline 340 & Ethylenglycol & Stickstoff & 19 & 1 & 102 & 0 & 1,2 & 130 & 20 & 40 & 1885 & 0,301 & & 0,0384 & & & & & & & \\
\hline 341 & Ethylenglycol & Stickstoff & 1 & 4,3 & 102 & 0 & 1,2 & 130 & 20 & 1 & 4,7 & 0,022 & 0,0005 & 0,0019 & 0,0001 & & & & & & \\
\hline 342 & Ethylenglycol & Stickstoff & 1 & 4,3 & 102 & 0 & 1,2 & 130 & 20 & 1 & 9,4 & 0,041 & 0,0005 & 0,0037 & 0,0001 & & & & & & \\
\hline 343 & Ethylenglycol & Stickstoff & 1 & 4,3 & 102 & 0 & 1,2 & 130 & 20 & 1 & 23,6 & 0,081 & 0,0005 & 0,0076 & 0,0001 & & & & & & \\
\hline 344 & Ethylenglycol & Stickstoff & 1 & 4,3 & 102 & 0 & 1,2 & 130 & 20 & 1 & 47,1 & 0,127 & 0,0005 & 0,0130 & 0,0003 & & & & & & \\
\hline 345 & Ethylenglycol & Stickstoff & 1 & 4,3 & 102 & 0 & 1,2 & 130 & 20 & 1 & 94,2 & 0,201 & 0,0020 & 0,0231 & 0,0012 & & & & & & \\
\hline 346 & Ethylenglycol & Stickstoff & 1 & 4,3 & 102 & 0 & 1,2 & 130 & 20 & 2 & 9,4 & 0,022 & & 0,0021 & & & & & & & \\
\hline 347 & Ethylenglycol & Stickstoff & 1 & 4,3 & 102 & 0 & 1,2 & 130 & 20 & 2 & 18,9 & 0,042 & & 0,0040 & & & & & & & \\
\hline 348 & Ethylenglycol & Stickstoff & 1 & 4,3 & 102 & 0 & 1,2 & 130 & 20 & 2 & 47,1 & 0,083 & & 0,0084 & & & & & & & \\
\hline 349 & Ethylenglycol & Stickstoff & 1 & 4,3 & 102 & 0 & 1,2 & 130 & 20 & 2 & 94,3 & 0,142 & & 0,0158 & & & & & & & \\
\hline 350 & Ethylenglycol & Stickstoff & 1 & 4,3 & 102 & 0 & 1,2 & 130 & 20 & 2 & 188,5 & 0,218 & & 0,0282 & & & & & & & \\
\hline 351 & Ethylenglycol & Stickstoff & 1 & 4,3 & 102 & 0 & 1,2 & 130 & 20 & 5 & 23,6 & 0,024 & & 0,0023 & & & & & & & \\
\hline 352 & Ethylenglycol & Stickstoff & 1 & 4,3 & 102 & 0 & 1,2 & 130 & 20 & 5 & 47,1 & 0,044 & & 0,0045 & & & & & & & \\
\hline 353 & Ethylenglycol & Stickstoff & 1 & 4,3 & 102 & 0 & 1,2 & 130 & 20 & 5 & 117,8 & 0,098 & & 0,0109 & & & & & & & \\
\hline 354 & Ethylenglycol & Stickstoff & 1 & 4,3 & 102 & 0 & 1,2 & 130 & 20 & 5 & 235,6 & 0,159 & & 0,0218 & & & & & & & \\
\hline 355 & Ethylenglycol & Stickstoff & 1 & 4,3 & 102 & 0 & 1,2 & 130 & 20 & 5 & 471,3 & 0,255 & & 0,0374 & & & & & & & \\
\hline 356 & Ethylenglycol & Stickstoff & 1 & 4,3 & 102 & 0 & 1,2 & 130 & 20 & 10 & 47,1 & 0,025 & & 0,0027 & & & & & & & \\
\hline 357 & Ethylenglycol & Stickstoff & 1 & 4,3 & 102 & 0 & 1,2 & 130 & 20 & 10 & 94,3 & 0,053 & & 0,0061 & & & & & & & \\
\hline 358 & Ethylenglycol & Stickstoff & 1 & 4,3 & 102 & 0 & 1,2 & 130 & 20 & 10 & 235,6 & 0,111 & & 0,0140 & & & & & & & \\
\hline 359 & Ethylenglycol & Stickstoff & 1 & 4,3 & 102 & 0 & 1,2 & 130 & 20 & 10 & 471,3 & 0,184 & & 0,0267 & & & & & & & \\
\hline 360 & Ethylenglycol & Stickstoff & 1 & 4,3 & 102 & 0 & 1,2 & 130 & 20 & 20 & 94,3 & 0,032 & & 0,0039 & & & & & & & \\
\hline 361 & Ethylenglycol & Stickstoff & 1 & 4,3 & 102 & 0 & 1,2 & 130 & 20 & 20 & 188,5 & 0,061 & & 0,0076 & & & & & & & \\
\hline 362 & Ethylenglycol & Stickstoff & 1 & 4,3 & 102 & 0 & 1,2 & 130 & 20 & 20 & 471,3 & 0,126 & & 0,0191 & & & & & & & \\
\hline 363 & Ethylenglycol & Stickstoff & 1 & 4,3 & 102 & 0 & 1,2 & 130 & 20 & 40 & 188,5 & 0,041 & & 0,0054 & & & & & & & \\
\hline 364 & Ethylenglycol & Stickstoff & 1 & 4,3 & 102 & 0 & 1,2 & 130 & 20 & 40 & 377 & 0,079 & & 0,0112 & & & & & & & \\
\hline
\end{tabular}




\begin{tabular}{|c|c|c|c|c|c|c|c|c|c|c|c|c|c|c|c|c|c|c|c|c|c|}
\hline$\leq$ & 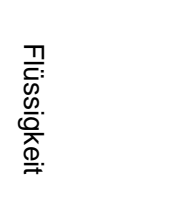 & $\stackrel{\infty}{\infty}$ & 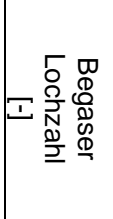 & 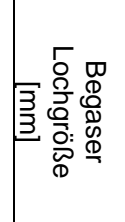 & 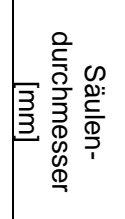 & 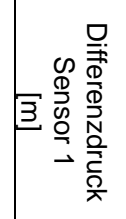 & 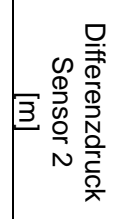 & 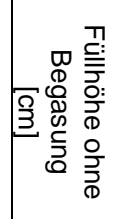 & 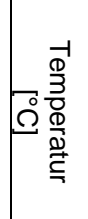 & 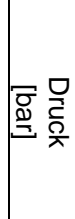 & 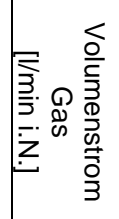 & 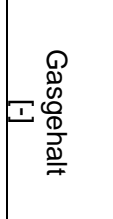 & 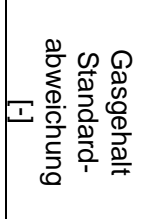 & 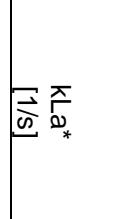 & 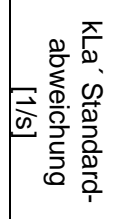 & 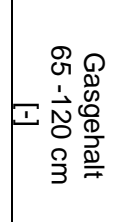 & 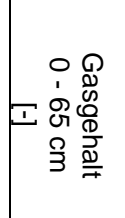 & 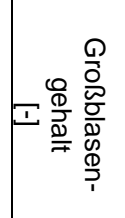 & 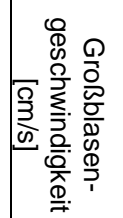 & 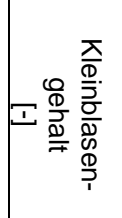 & 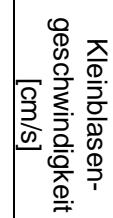 \\
\hline 365 & Ethylenglycol & Helium & 1 & 4,3 & 102 & 0 & 1,2 & 130 & 20 & 1 & 4,7 & 0,021 & & 0,0018 & & & & & & & \\
\hline 366 & Ethylenglycol & Helium & 1 & 4,3 & 102 & 0 & 1,2 & 130 & 20 & 1 & 9,4 & 0,039 & & 0,0033 & & & & & & & \\
\hline 367 & Ethylenglycol & Helium & 1 & 4,3 & 102 & 0 & 1,2 & 130 & 20 & 1 & 23,6 & 0,077 & & 0,0068 & & & & & & & \\
\hline 368 & Ethylenglycol & Helium & 1 & 4,3 & 102 & 0 & 1,2 & 130 & 20 & 1 & 47,1 & 0,119 & & 0,0108 & & & & & & & \\
\hline 369 & Ethylenglycol & Helium & 1 & 4,3 & 102 & 0 & 1,2 & 130 & 20 & 1 & 94,2 & 0,190 & & 0,0187 & & & & & & & \\
\hline 370 & Ethylenglycol & Helium & 1 & 4,3 & 102 & 0 & 1,2 & 130 & 20 & 2 & 9,4 & 0,022 & & 0,0019 & & & & & & & \\
\hline 371 & Ethylenglycol & Helium & 1 & 4,3 & 102 & 0 & 1,2 & 130 & 20 & 2 & 18,9 & 0,041 & & 0,0035 & & & & & & & \\
\hline 372 & Ethylenglycol & Helium & 1 & 4,3 & 102 & 0 & 1,2 & 130 & 20 & 2 & 47,1 & 0,079 & & 0,0070 & & & & & & & \\
\hline 373 & Ethylenglycol & Helium & 1 & 4,3 & 102 & 0 & 1,2 & 130 & 20 & 2 & 94,3 & 0,130 & & 0,0119 & & & & & & & \\
\hline 374 & Ethylenglycol & Helium & 1 & 4,3 & 102 & 0 & 1,2 & 130 & 20 & 4 & 18,8 & 0,024 & & 0,0020 & & & & & & & \\
\hline 375 & Ethylenglycol & Helium & 1 & 4,3 & 102 & 0 & 1,2 & 130 & 20 & 4 & 37,7 & 0,042 & & 0,0037 & & & & & & & \\
\hline 376 & Ethylenglycol & Helium & 1 & 4,3 & 102 & 0 & 1,2 & 130 & 20 & 4 & 94,3 & 0,085 & & 0,0079 & & & & & & & \\
\hline 377 & Ethylenglycol & Helium & 1 & 4,3 & 102 & 0 & 1,2 & 130 & 20 & 4 & 188,5 & 0,132 & & 0,0124 & & & & & & & \\
\hline 378 & Ethylenglycol & Helium & 1 & 4,3 & 102 & 0 & 1,2 & 130 & 20 & 4 & 377 & 0,192 & & 0,0221 & & & & & & & \\
\hline 379 & Ethylenglycol & Stickstoff & 1 & 4,3 & 102 & 0 & 1,2 & 130 & 30 & 1 & 4,6 & 0,024 & & 0,0030 & & & & & & & \\
\hline 380 & Ethylenglycol & Stickstoff & 1 & 4,3 & 102 & 0 & 1,2 & 130 & 30 & 1 & 9,1 & 0,044 & & 0,0059 & & & & & & & \\
\hline 381 & Ethylenglycol & Stickstoff & 1 & 4,3 & 102 & 0 & 1,2 & 130 & 30 & 1 & 22,8 & 0,085 & & 0,0119 & & & & & & & \\
\hline 382 & Ethylenglycol & Stickstoff & 1 & 4,3 & 102 & 0 & 1,2 & 130 & 30 & 1 & 45,6 & 0,131 & 0,0007 & 0,0192 & 0,0005 & & & & & & \\
\hline 383 & Ethylenglycol & Stickstoff & 1 & 4,3 & 102 & 0 & 1,2 & 130 & 30 & 1 & 91,1 & 0,203 & 0,0014 & 0,0333 & 0,0047 & & & & & & \\
\hline 384 & Ethylenglycol & Stickstoff & 19 & 1 & 102 & 0 & 1,2 & 130 & 50 & 1 & 4,3 & 0,023 & 0,0000 & 0,0042 & 0,0003 & & & & & & \\
\hline 385 & Ethylenglycol & Stickstoff & 19 & 1 & 102 & 0 & 1,2 & 130 & 50 & 1 & 8,5 & 0,046 & 0,0000 & 0,0104 & 0,0003 & & & & & & \\
\hline 386 & Ethylenglycol & Stickstoff & 19 & 1 & 102 & 0 & 1,2 & 130 & 50 & 1 & 21,4 & 0,091 & 0,0007 & 0,0182 & 0,0006 & & & & & & \\
\hline 387 & Ethylenglycol & Stickstoff & 19 & 1 & 102 & 0 & 1,2 & 130 & 50 & 1 & 42,7 & 0,138 & 0,0000 & 0,0317 & 0,0020 & & & & & & \\
\hline 388 & Ethylenglycol & Stickstoff & 19 & 1 & 102 & 0 & 1,2 & 130 & 50 & 1 & 85,5 & 0,212 & 0,0006 & 0,0487 & 0,0008 & & & & & & \\
\hline 389 & Ethylenglycol & Stickstoff & 19 & 1 & 102 & 0 & 1,2 & 130 & 50 & 2 & 8,5 & 0,027 & 0,0007 & 0,0044 & 0,0006 & & & & & & \\
\hline 390 & Ethylenglycol & Stickstoff & 19 & 1 & 102 & 0 & 1,2 & 130 & 50 & 2 & 21,4 & 0,050 & 0,0000 & 0,0089 & 0,0001 & & & & & & \\
\hline 391 & Ethylenglycol & Stickstoff & 19 & 1 & 102 & 0 & 1,2 & 130 & 50 & 2 & 42,7 & 0,097 & 0,0000 & 0,0195 & 0,0001 & & & & & & \\
\hline 392 & Ethylenglycol & Stickstoff & 19 & 1 & 102 & 0 & 1,2 & 130 & 50 & 2 & 85,5 & 0,156 & 0,0006 & 0,0369 & 0,0005 & & & & & & \\
\hline
\end{tabular}




\begin{tabular}{|c|c|c|c|c|c|c|c|c|c|c|c|c|c|c|c|c|c|c|c|c|c|}
\hline$\underline{3}$ & 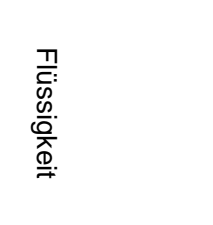 & $\stackrel{\infty}{\infty}$ & 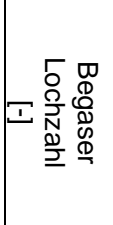 & 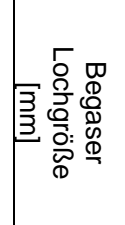 & 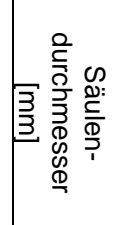 & 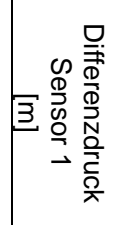 & 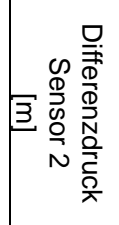 & 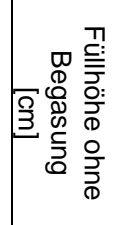 & 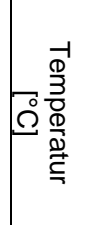 & 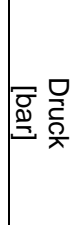 & 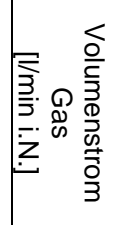 & 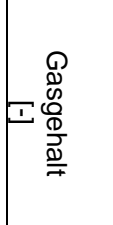 & 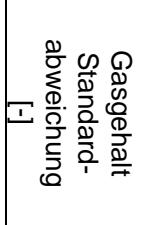 & 居离 & 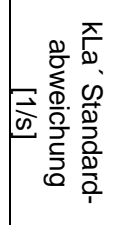 & 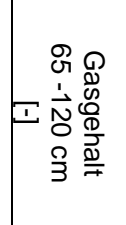 & 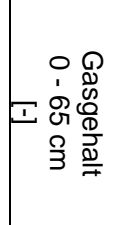 & 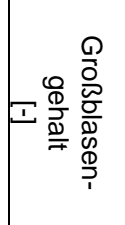 & 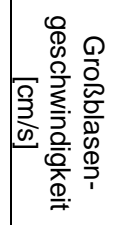 & 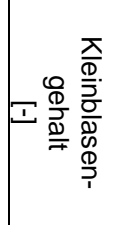 & 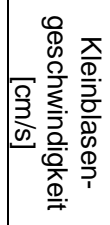 \\
\hline 393 & Ethylenglycol & Stickstoff & 19 & 1 & 102 & 0 & 1,2 & 130 & 50 & 2 & 171 & 0,232 & 0,0010 & 0,0614 & 0,0052 & & & & & & \\
\hline 394 & Ethylenglycol & Stickstoff & 19 & 1 & 102 & 0 & 1,2 & 130 & 50 & 5 & 21,4 & 0,028 & 0,0000 & 0,0051 & 0,0007 & & & & & & \\
\hline 395 & Ethylenglycol & Stickstoff & 19 & 1 & 102 & 0 & 1,2 & 130 & 50 & 5 & 42,7 & 0,053 & 0,0007 & 0,0085 & 0,0010 & & & & & & \\
\hline 396 & Ethylenglycol & Stickstoff & 19 & 1 & 102 & 0 & 1,2 & 130 & 50 & 5 & 106,9 & 0,115 & 0,0000 & 0,0264 & 0,0007 & & & & & & \\
\hline 397 & Ethylenglycol & Stickstoff & 19 & 1 & 102 & 0 & 1,2 & 130 & 50 & 5 & 213,7 & 0,179 & 0,0006 & 0,0468 & 0,0019 & & & & & & \\
\hline 398 & Ethylenglycol & Stickstoff & 19 & 1 & 102 & 0 & 1,2 & 130 & 50 & 5 & 427,5 & 0,280 & 0,0015 & 0,0845 & 0,0036 & & & & & & \\
\hline 399 & Ethylenglycol & Stickstoff & 19 & 1 & 102 & 0 & 1,2 & 130 & 50 & 10 & 42,7 & 0,032 & 0,0007 & 0,0049 & 0,0013 & & & & & & \\
\hline 400 & Ethylenglycol & Stickstoff & 19 & 1 & 102 & 0 & 1,2 & 130 & 50 & 10 & 85,5 & 0,065 & 0,0000 & 0,0150 & 0,0006 & & & & & & \\
\hline 401 & Ethylenglycol & Stickstoff & 19 & 1 & 102 & 0 & 1,2 & 130 & 50 & 10 & 213,7 & 0,133 & 0,0010 & 0,0328 & 0,0007 & & & & & & \\
\hline 402 & Ethylenglycol & Stickstoff & 19 & 1 & 102 & 0 & 1,2 & 130 & 50 & 10 & 427,5 & 0,217 & 0,0021 & 0,0595 & 0,0004 & & & & & & \\
\hline 403 & Ethylenglycol & Stickstoff & 1 & 4,3 & 102 & 0 & 1,2 & 130 & 50 & 1 & 4,3 & 0,024 & 0,0005 & 0,0054 & 0,0001 & & & & & & \\
\hline 404 & Ethylenglycol & Stickstoff & 1 & 4,3 & 102 & 0 & 1,2 & 130 & 50 & 1 & 8,5 & 0,045 & 0,0005 & 0,0109 & 0,0001 & & & & & & \\
\hline 405 & Ethylenglycol & Stickstoff & 1 & 4,3 & 102 & 0 & 1,2 & 130 & 50 & 1 & 21,4 & 0,087 & 0,0005 & 0,0223 & 0,0001 & & & & & & \\
\hline 406 & Ethylenglycol & Stickstoff & 1 & 4,3 & 102 & 0 & 1,2 & 130 & 50 & 1 & 42,7 & 0,133 & 0,0005 & 0,0349 & 0,0003 & & & & & & \\
\hline 407 & Ethylenglycol & Stickstoff & 1 & 4,3 & 102 & 0 & 1,2 & 130 & 50 & 1 & 85,5 & 0,205 & 0,0020 & 0,0572 & 0,0012 & & & & & & \\
\hline 408 & Ethylenglycol & Stickstoff & 1 & 4,3 & 102 & 0 & 1,2 & 130 & 70 & 1 & 4 & 0,025 & & 0,0081 & & & & & & & \\
\hline 409 & Ethylenglycol & Stickstoff & 1 & 4,3 & 102 & 0 & 1,2 & 130 & 70 & 1 & 8,1 & 0,048 & & 0,0159 & & & & & & & \\
\hline 410 & Ethylenglycol & Stickstoff & 1 & 4,3 & 102 & 0 & 1,2 & 130 & 70 & 1 & 20,1 & 0,091 & & 0,0340 & & & & & & & \\
\hline 411 & Ethylenglycol & Stickstoff & 1 & 4,3 & 102 & 0 & 1,2 & 130 & 70 & 1 & 40,3 & 0,136 & & 0,0525 & & & & & & & \\
\hline 412 & Ethylenglycol & Stickstoff & 1 & 4,3 & 102 & 0 & 1,2 & 130 & 70 & 1 & 80,5 & 0,209 & & 0,0917 & & & & & & & \\
\hline 413 & Ethylenglycol & Stickstoff & 1 & 4,3 & 102 & 0 & 1,2 & 130 & 80 & 1 & 3,9 & 0,024 & & 0,0097 & & & & & & & \\
\hline 414 & Ethylenglycol & Stickstoff & 1 & 4,3 & 102 & 0 & 1,2 & 130 & 80 & 1 & 7,8 & 0,047 & & 0,0197 & & & & & & & \\
\hline 415 & Ethylenglycol & Stickstoff & 1 & 4,3 & 102 & 0 & 1,2 & 130 & 80 & 1 & 19,6 & 0,091 & & 0,0420 & & & & & & & \\
\hline 416 & Ethylenglycol & Stickstoff & 1 & 4,3 & 102 & 0 & 1,2 & 130 & 80 & 1 & 39,1 & 0,139 & & 0,0646 & & & & & & & \\
\hline 417 & Ethylenglycol & Stickstoff & 1 & 4,3 & 102 & 0 & 1,2 & 130 & 80 & 1 & 78,2 & 0,211 & & 0,1121 & & & & & & & \\
\hline 418 & Leitungswasser & Stickstoff & 7 & 1 & 115 & 0,1 & 0,905 & 100 & 10 & 1 & 10 & 0,049 & 0,0011 & & & & & 0,010 & 93,8 & 0,039 & 25,3 \\
\hline 419 & Leitungswasser & Stickstoff & 7 & 1 & 115 & 0,1 & 0,905 & 100 & 10 & 1 & 20 & 0,082 & 0,0014 & & & & & 0,026 & 79,6 & 0,057 & 23,0 \\
\hline 420 & Leitungswasser & Stickstoff & 7 & 1 & 115 & 0,1 & 0,905 & 100 & 10 & 1 & 30 & 0,109 & 0,0005 & & & & & 0,040 & 87,5 & 0,069 & 21,3 \\
\hline
\end{tabular}




\begin{tabular}{|c|c|c|c|c|c|c|c|c|c|c|c|c|c|c|c|c|c|c|c|c|c|}
\hline$\leq$ & 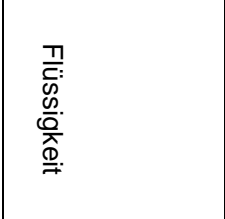 & $\stackrel{\infty}{\infty}$ & 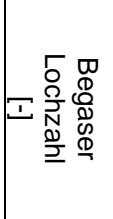 & 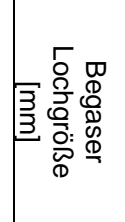 & 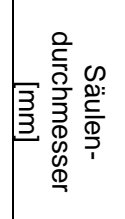 & 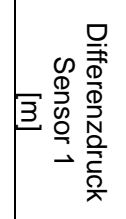 & 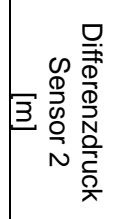 & 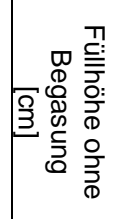 & 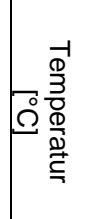 & 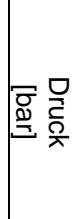 & 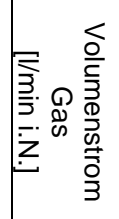 & 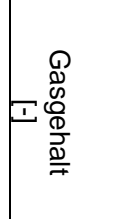 & 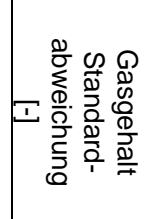 & 常否 & 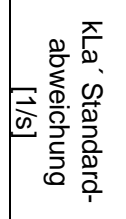 & 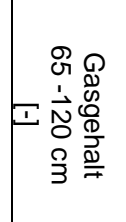 & 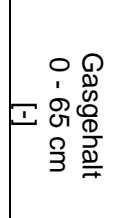 & 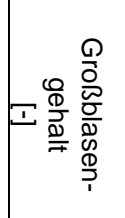 & 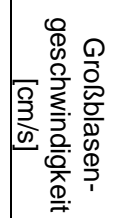 & 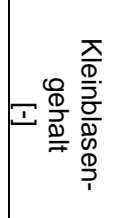 & 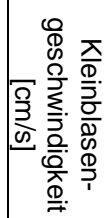 \\
\hline 421 & Leitungswasser & Stickstoff & 7 & 1 & 115 & 0,1 & 0,905 & 100 & 10 & 1 & 40 & 0,137 & 0,0028 & & & & & 0,056 & 90,5 & 0,081 & 19,8 \\
\hline 422 & Leitungswasser & Stickstoff & 7 & 1 & 115 & 0,1 & 0,905 & 100 & 10 & 1 & 50 & 0,159 & 0,0037 & & & & & 0,062 & 105,4 & 0,097 & 19,2 \\
\hline 423 & Leitungswasser & Stickstoff & 1 & 4,3 & 102 & 0 & 1,2 & 130 & 20 & 1 & 5 & & & 0,0094 & 0,0008 & & & & & & \\
\hline 424 & Leitungswasser & Stickstoff & 1 & 4,3 & 102 & 0 & 1,2 & 130 & 20 & 1 & 10 & & & 0,0191 & 0,0029 & & & & & & \\
\hline 425 & Leitungswasser & Stickstoff & 1 & 4,3 & 102 & 0 & 1,2 & 130 & 20 & 1 & 20 & & & 0,0326 & 0,0026 & & & & & & \\
\hline 426 & Leitungswasser & Stickstoff & 1 & 4,3 & 102 & 0 & 1,2 & 130 & 20 & 1 & 30 & & & 0,0480 & 0,0039 & & & & & & \\
\hline 427 & Leitungswasser & Stickstoff & 1 & 4,3 & 102 & 0 & 1,2 & 130 & 20 & 1 & 50 & & & 0,0756 & 0,0068 & & & & & & \\
\hline 428 & Leitungswasser & Stickstoff & 1 & 4,3 & 102 & 0 & 1,2 & 130 & 20 & 1 & 75 & & & 0,0979 & 0,0138 & & & & & & \\
\hline 429 & Leitungswasser & Stickstoff & 1 & 4,3 & 102 & 0 & 1,2 & 130 & 20 & 1 & 100 & & & 0,1249 & 0,0157 & & & & & & \\
\hline 430 & Leitungswasser & Stickstoff & 1 & 4,3 & 102 & 0 & 1,2 & 130 & 20 & 1 & 150 & & & 0,1361 & \#DIV/0! & & & & & & \\
\hline 431 & Leitungswasser & Stickstoff & 1 & 4,3 & 102 & 0 & 1,2 & 130 & 20 & 1 & 4,7 & 0,021 & 0,0014 & 0,0056 & 0,0008 & & & & & & \\
\hline 432 & Leitungswasser & Stickstoff & 1 & 4,3 & 102 & 0 & 1,2 & 130 & 20 & 1 & 9,4 & 0,038 & 0,0028 & 0,0114 & 0,0019 & & & & & & \\
\hline 433 & Leitungswasser & Stickstoff & 1 & 4,3 & 102 & 0 & 1,2 & 130 & 20 & 1 & 23,6 & 0,076 & 0,0054 & 0,0260 & 0,0040 & & & & & & \\
\hline 434 & Leitungswasser & Stickstoff & 1 & 4,3 & 102 & 0 & 1,2 & 130 & 20 & 1 & 47,1 & 0,119 & 0,0089 & 0,0458 & 0,0075 & & & & & & \\
\hline 435 & Leitungswasser & Stickstoff & 1 & 4,3 & 102 & 0 & 1,2 & 130 & 20 & 1 & 94,2 & 0,186 & 0,0020 & 0,0799 & 0,0056 & & & & & & \\
\hline 436 & Leitungswasser & Stickstoff & 1 & 4,3 & 102 & 0 & 1,2 & 130 & 20 & 2 & 9,4 & 0,021 & 0,0007 & 0,0050 & 0,0004 & & & & & & \\
\hline 437 & Leitungswasser & Stickstoff & 1 & 4,3 & 102 & 0 & 1,2 & 130 & 20 & 2 & 18,9 & 0,037 & 0,0007 & 0,0095 & 0,0002 & & & & & & \\
\hline 438 & Leitungswasser & Stickstoff & 1 & 4,3 & 102 & 0 & 1,2 & 130 & 20 & 2 & 47,1 & 0,074 & 0,0000 & 0,0222 & 0,0005 & & & & & & \\
\hline 439 & Leitungswasser & Stickstoff & 1 & 4,3 & 102 & 0 & 1,2 & 130 & 20 & 2 & 94,3 & 0,127 & 0,0006 & 0,0474 & 0,0014 & & & & & & \\
\hline 440 & Leitungswasser & Stickstoff & 1 & 4,3 & 102 & 0 & 1,2 & 130 & 20 & 2 & 188,5 & 0,197 & 0,0015 & 0,0829 & 0,0064 & & & & & & \\
\hline 441 & Leitungswasser & Stickstoff & 1 & 4,3 & 102 & 0 & 1,2 & 130 & 20 & 3 & 15 & & & 0,0101 & 0,0001 & & & & & & \\
\hline 442 & Leitungswasser & Stickstoff & 1 & 4,3 & 102 & 0 & 1,2 & 130 & 20 & 3 & 30 & & & 0,0205 & 0,0037 & & & & & & \\
\hline 443 & Leitungswasser & Stickstoff & 1 & 4,3 & 102 & 0 & 1,2 & 130 & 20 & 3 & 50 & & & 0,0447 & 0,0210 & & & & & & \\
\hline 444 & Leitungswasser & Stickstoff & 1 & 4,3 & 102 & 0 & 1,2 & 130 & 20 & 3 & 100 & & & 0,0672 & 0,0018 & & & & & & \\
\hline 445 & Leitungswasser & Stickstoff & 1 & 4,3 & 102 & 0 & 1,2 & 130 & 20 & 3 & 150 & & & 0,1021 & 0,0115 & & & & & & \\
\hline 446 & Leitungswasser & Stickstoff & 1 & 4,3 & 102 & 0 & 1,2 & 130 & 20 & 3 & 250 & & & 0,1636 & 0,0400 & & & & & & \\
\hline 447 & Leitungswasser & Stickstoff & 1 & 4,3 & 102 & 0 & 1,2 & 130 & 20 & 5 & 23,6 & 0,021 & 0,0007 & 0,0054 & 0,0000 & & & & & & \\
\hline 448 & Leitungswasser & Stickstoff & 1 & 4,3 & 102 & 0 & 1,2 & 130 & 20 & 5 & 47,1 & 0,037 & 0,0007 & 0,0108 & 0,0003 & & & & & & \\
\hline
\end{tabular}




\begin{tabular}{|c|c|c|c|c|c|c|c|c|c|c|c|c|c|c|c|c|c|c|c|c|c|}
\hline$\leq$ & 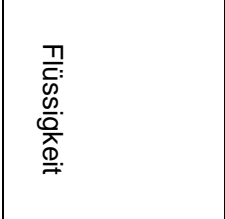 & $\stackrel{\infty}{\infty}$ & 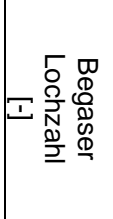 & 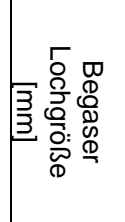 & 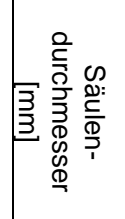 & 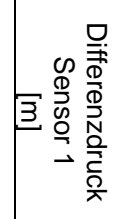 & 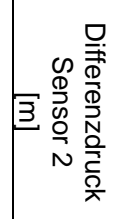 & 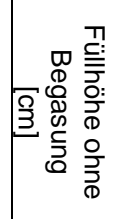 & 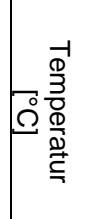 & 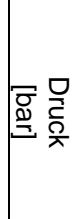 & 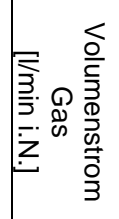 & 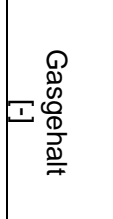 & 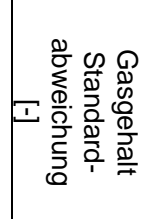 & 灾 $\mathbb{d}_{*}$ & 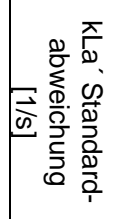 & 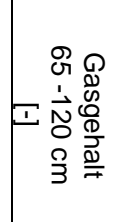 & 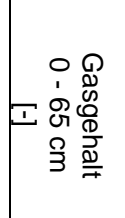 & 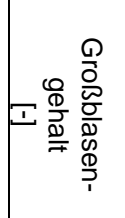 & 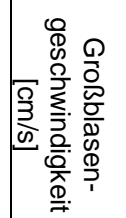 & 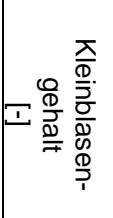 & 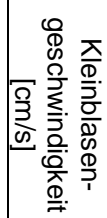 \\
\hline 449 & Leitungswasser & Stickstoff & 1 & 4,3 & 102 & 0 & 1,2 & 130 & 20 & 5 & 117,8 & 0,084 & 0,0021 & 0,0287 & 0,0001 & & & & & & \\
\hline 450 & Leitungswasser & Stickstoff & 1 & 4,3 & 102 & 0 & 1,2 & 130 & 20 & 5 & 235,6 & 0,139 & 0,0021 & 0,0569 & 0,0006 & & & & & & \\
\hline 451 & Leitungswasser & Stickstoff & 1 & 4,3 & 102 & 0 & 1,2 & 130 & 20 & 5 & 471,3 & 0,226 & 0,0028 & 0,1160 & 0,0072 & & & & & & \\
\hline 452 & Leitungswasser & Stickstoff & 1 & 4,3 & 102 & 0 & 1,2 & 130 & 20 & 10 & 47,1 & 0,021 & & 0,0058 & 0,0002 & & & & & & \\
\hline 453 & Leitungswasser & Stickstoff & 1 & 4,3 & 102 & 0 & 1,2 & 130 & 20 & 10 & 94,3 & 0,045 & 0,0007 & 0,0141 & 0,0002 & & & & & & \\
\hline 454 & Leitungswasser & Stickstoff & 1 & 4,3 & 102 & 0 & 1,2 & 130 & 20 & 10 & 235,6 & 0,093 & 0,0014 & 0,0372 & 0,0002 & & & & & & \\
\hline 455 & Leitungswasser & Stickstoff & 1 & 4,3 & 102 & 0 & 1,2 & 130 & 20 & 10 & 471,3 & 0,159 & 0,0028 & 0,0760 & 0,0013 & & & & & & \\
\hline 456 & Leitungswasser & Stickstoff & 1 & 4,3 & 102 & 0 & 1,2 & 130 & 20 & 10 & 942,5 & 0,289 & 0,0116 & 0,1642 & 0,0057 & & & & & & \\
\hline 457 & Leitungswasser & Stickstoff & 1 & 4,3 & 102 & 0 & 1,2 & 130 & 20 & 20 & 94,3 & 0,025 & & 0,0093 & 0,0011 & & & & & & \\
\hline 458 & Leitungswasser & Stickstoff & 1 & 4,3 & 102 & 0 & 1,2 & 130 & 20 & 20 & 188,5 & 0,047 & & 0,0167 & 0,0016 & & & & & & \\
\hline 459 & Leitungswasser & Stickstoff & 1 & 4,3 & 102 & 0 & 1,2 & 130 & 20 & 20 & 471,3 & 0,105 & 0,0015 & 0,0465 & 0,0013 & & & & & & \\
\hline 460 & Leitungswasser & Stickstoff & 1 & 4,3 & 102 & 0 & 1,2 & 130 & 20 & 20 & 942,5 & 0,212 & 0,0058 & 0,1046 & 0,0057 & & & & & & \\
\hline 461 & Leitungswasser & Stickstoff & 1 & 4,3 & 102 & 0 & 1,2 & 130 & 20 & 40 & 188,5 & 0,029 & 0,0014 & 0,0104 & 0,0001 & & & & & & \\
\hline 462 & Leitungswasser & Stickstoff & 1 & 4,3 & 102 & 0 & 1,2 & 130 & 20 & 40 & 377 & 0,057 & 0,0021 & 0,0198 & 0,0002 & & & & & & \\
\hline 463 & Leitungswasser & Stickstoff & 1 & 4,3 & 102 & 0 & 1,2 & 130 & 20 & 40 & 942,5 & 0,092 & 0,0042 & 0,0615 & 0,0054 & & & & & & \\
\hline 464 & Leitungswasser & Helium & 1 & 4,3 & 102 & 0 & 1,2 & 130 & 20 & 1 & 4,7 & 0,021 & 0,0007 & 0,0052 & 0,0001 & & & & & & \\
\hline 465 & Leitungswasser & Helium & 1 & 4,3 & 102 & 0 & 1,2 & 130 & 20 & 1 & 9,4 & 0,040 & & 0,0110 & 0,0001 & & & & & & \\
\hline 466 & Leitungswasser & Helium & 1 & 4,3 & 102 & 0 & 1,2 & 130 & 20 & 1 & 23,6 & 0,078 & 0,0007 & 0,0253 & 0,0001 & & & & & & \\
\hline 467 & Leitungswasser & Helium & 1 & 4,3 & 102 & 0 & 1,2 & 130 & 20 & 1 & 47,1 & 0,123 & 0,0006 & 0,0418 & 0,0005 & & & & & & \\
\hline 468 & Leitungswasser & Helium & 1 & 4,3 & 102 & 0 & 1,2 & 130 & 20 & 1 & 94,2 & 0,196 & 0,0050 & 0,0778 & 0,0033 & & & & & & \\
\hline 469 & Leitungswasser & Helium & 1 & 4,3 & 102 & 0 & 1,2 & 130 & 20 & 3 & 14,1 & 0,021 & & 0,0055 & & & & & & & \\
\hline 470 & Leitungswasser & Helium & 1 & 4,3 & 102 & 0 & 1,2 & 130 & 20 & 3 & 28,3 & 0,038 & 0,0007 & 0,0108 & 0,0001 & & & & & & \\
\hline 471 & Leitungswasser & Helium & 1 & 4,3 & 102 & 0 & 1,2 & 130 & 20 & 3 & 70,7 & 0,072 & & 0,0264 & 0,0006 & & & & & & \\
\hline 472 & Leitungswasser & Helium & 1 & 4,3 & 102 & 0 & 1,2 & 130 & 20 & 3 & 141,4 & 0,120 & 0,0012 & 0,0440 & 0,0009 & & & & & & \\
\hline 473 & Leitungswasser & Helium & 1 & 4,3 & 102 & 0 & 1,2 & 130 & 20 & 3 & 282,8 & 0,186 & 0,0010 & 0,0822 & 0,0042 & & & & & & \\
\hline 474 & Leitungswasser & Stickstoff & 1 & 4,3 & 102 & 0 & 1,2 & 133 & 20 & 1 & 5 & 0,026 & & & & & & 0,018 & 50,8 & 0,008 & 27,2 \\
\hline 475 & Leitungswasser & Stickstoff & 1 & 4,3 & 102 & 0 & 1,2 & 133 & 20 & 1 & 10 & 0,047 & & & & & & 0,019 & 78,2 & 0,028 & 29,6 \\
\hline 476 & Leitungswasser & Stickstoff & 1 & 4,3 & 102 & 0 & 1,2 & 133 & 20 & 1 & 20 & 0,083 & & & & & & 0,038 & 88,7 & 0,044 & 26,3 \\
\hline
\end{tabular}




\begin{tabular}{|c|c|c|c|c|c|c|c|c|c|c|c|c|c|c|c|c|c|c|c|c|c|}
\hline$\underline{z}$ & 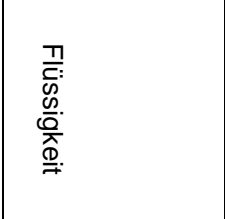 & $\underset{\aleph}{\circledR}$ & 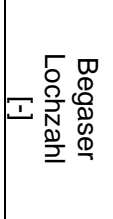 & 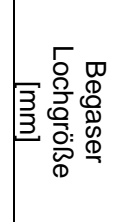 & 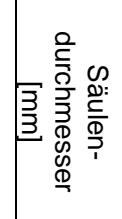 & 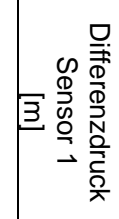 & 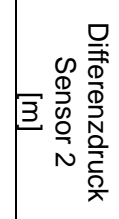 & 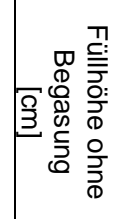 & 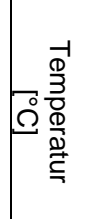 & 总 몰 & 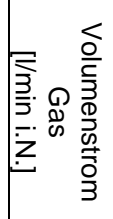 & 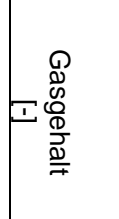 & 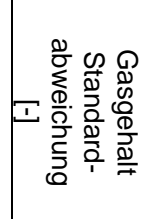 & 居 & 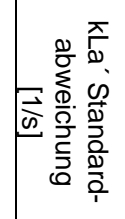 & 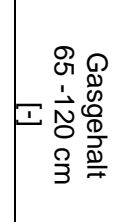 & 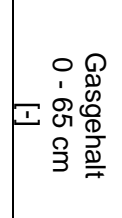 & 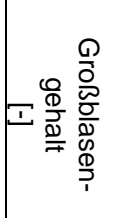 & 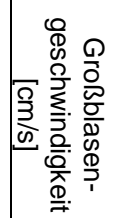 & 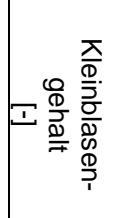 & 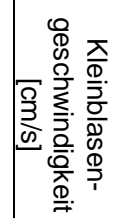 \\
\hline 477 & Leitungswasser & Stickstoff & 1 & 4,3 & 102 & 0 & 1,2 & 133 & 20 & 1 & 30 & 0,111 & & & & & & 0,055 & 98,5 & 0,056 & 25,8 \\
\hline 478 & Leitungswasser & Stickstoff & 1 & 4,3 & 102 & 0 & 1,2 & 133 & 20 & 1 & 50 & 0,148 & & & & & & 0,074 & 130,5 & 0,074 & 24,8 \\
\hline 479 & Leitungswasser & Stickstoff & 1 & 4,3 & 102 & 0 & 1,2 & 133 & 20 & 1 & 75 & 0,197 & & & & & & 0,103 & 145,0 & 0,094 & 24,6 \\
\hline 480 & Leitungswasser & Stickstoff & 1 & 4,3 & 102 & 0 & 1,2 & 133 & 20 & 1 & 100 & 0,229 & & & & & & 0,142 & 146,1 & 0,087 & 23,7 \\
\hline 481 & Leitungswasser & Stickstoff & 1 & 4,3 & 102 & 0 & 1,2 & 133 & 20 & 1 & 150 & 0,275 & & & & & & 0,174 & 181,9 & 0,100 & 25,3 \\
\hline 482 & Leitungswasser & Stickstoff & 1 & 4,3 & 102 & 0 & 1,2 & 133 & 20 & 3 & 15 & 0,025 & & & & & & 0,008 & 75,1 & 0,017 & 31,6 \\
\hline 483 & Leitungswasser & Stickstoff & 1 & 4,3 & 102 & 0 & 1,2 & 133 & 20 & 3 & 30 & 0,050 & & & & & & 0,018 & 76,3 & 0,032 & 27,7 \\
\hline 484 & Leitungswasser & Stickstoff & 1 & 4,3 & 102 & 0 & 1,2 & 133 & 20 & 3 & 50 & 0,081 & & & & & & 0,026 & 90,2 & 0,056 & 26,4 \\
\hline 485 & Leitungswasser & Stickstoff & 1 & 4,3 & 102 & 0 & 1,2 & 133 & 20 & 3 & 100 & 0,152 & & & & & & 0,054 & 100,2 & 0,098 & 22,7 \\
\hline 486 & Leitungswasser & Stickstoff & 1 & 4,3 & 102 & 0 & 1,2 & 133 & 20 & 3 & 150 & 0,202 & & & & & & 0,069 & 126,2 & 0,133 & 20,9 \\
\hline 487 & Leitungswasser & Stickstoff & 1 & 4,3 & 102 & 0 & 1,2 & 133 & 20 & 3 & 250 & 0,291 & & & & & & 0,110 & 141,3 & 0,181 & 19,5 \\
\hline 488 & Leitungswasser & Stickstoff & 1 & 4,3 & 102 & 0 & 1,2 & 133 & 20 & 3 & 350 & 0,351 & & & & & & 0,140 & 162,0 & 0,212 & 19,3 \\
\hline 489 & Leitungswasser & Stickstoff & 1 & 4,3 & 102 & 0 & 1,2 & 133 & 20 & 6 & 25 & 0,023 & & & & & & 0,005 & 192,2 & 0,018 & 30,4 \\
\hline 490 & Leitungswasser & Stickstoff & 1 & 4,3 & 102 & 0 & 1,2 & 133 & 20 & 6 & 50 & 0,048 & & & & & & 0,017 & 61,1 & 0,031 & 28,5 \\
\hline 491 & Leitungswasser & Stickstoff & 1 & 4,3 & 102 & 0 & 1,2 & 133 & 20 & 6 & 100 & 0,105 & & & & & & 0,021 & 85,5 & 0,084 & 24,3 \\
\hline 492 & Leitungswasser & Stickstoff & 1 & 4,3 & 102 & 0 & 1,2 & 133 & 20 & 6 & 150 & 0,155 & & & & & & 0,044 & 75,1 & 0,111 & 22,0 \\
\hline 493 & Leitungswasser & Stickstoff & 1 & 4,3 & 102 & 0 & 1,2 & 133 & 20 & 6 & 250 & 0,244 & & & & & & 0,053 & 110,4 & 0,190 & 19,2 \\
\hline 494 & Leitungswasser & Stickstoff & 1 & 4,3 & 102 & 0 & 1,2 & 133 & 20 & 6 & 400 & 0,342 & & & & & & 0,098 & 112,4 & 0,245 & 17,9 \\
\hline 495 & Leitungswasser & Stickstoff & 1 & 4,3 & 102 & 0 & 1,2 & 133 & 20 & 6 & 500 & 0,385 & & & & & & 0,108 & 129,7 & 0,277 & 18,1 \\
\hline 496 & Leitungswasser & Stickstoff & 1 & 4,3 & 102 & 0 & 1,2 & 133 & 20 & 11 & 50 & 0,026 & & & & & & 0,008 & 80,2 & 0,018 & 29,8 \\
\hline 497 & Leitungswasser & Stickstoff & 1 & 4,3 & 102 & 0 & 1,2 & 133 & 20 & 11 & 100 & 0,063 & & & & & & 0,018 & 54,7 & 0,044 & 26,2 \\
\hline 498 & Leitungswasser & Stickstoff & 1 & 4,3 & 102 & 0 & 1,2 & 133 & 20 & 11 & 200 & 0,144 & & & & & & 0,025 & 61,6 & 0,119 & 22,2 \\
\hline 499 & Leitungswasser & Stickstoff & 1 & 4,3 & 102 & 0 & 1,2 & 133 & 20 & 11 & 300 & 0,223 & & & & & & 0,035 & 78,7 & 0,189 & 19,7 \\
\hline 500 & Leitungswasser & Stickstoff & 1 & 4,3 & 102 & 0 & 1,2 & 133 & 20 & 11 & 500 & 0,334 & & & & & & 0,073 & 78,3 & 0,260 & 17,7 \\
\hline 501 & Leitungswasser & Stickstoff & 1 & 4,3 & 102 & 0 & 1,2 & 133 & 20 & 21 & 200 & 0,081 & & & & & & 0,010 & 60,1 & 0,072 & 23,4 \\
\hline 502 & Leitungswasser & Stickstoff & 1 & 4,3 & 102 & 0 & 1,2 & 133 & 20 & 21 & 300 & 0,135 & & & & & & 0,009 & 77,7 & 0,126 & 21,4 \\
\hline 503 & Leitungswasser & Stickstoff & 1 & 4,3 & 102 & 0 & 1,2 & 133 & 20 & 21 & 500 & 0,294 & & & & & & 0,086 & 18,7 & 0,208 & 19,8 \\
\hline 504 & Leitungswasser & Stickstoff & 1 & 4,3 & 102 & 0 & 1,2 & 133 & 20 & 31 & 150 & 0,037 & & & & & & 0,013 & 38,0 & 0,024 & 25,0 \\
\hline
\end{tabular}




\begin{tabular}{|c|c|c|c|c|c|c|c|c|c|c|c|c|c|c|c|c|c|c|c|c|c|}
\hline$\leq$ & 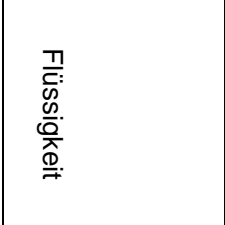 & ه్ల & 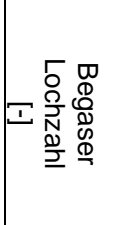 & 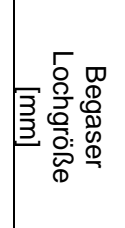 & 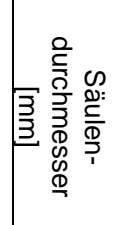 & 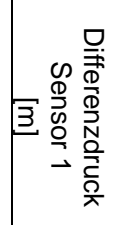 & 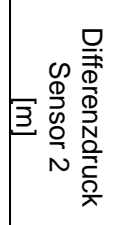 & 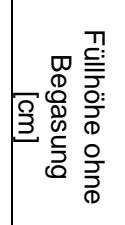 & 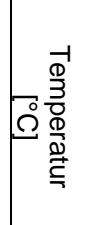 & 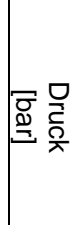 & 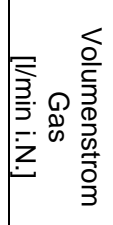 & 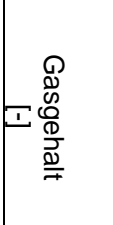 & 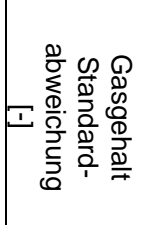 & 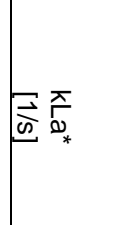 & 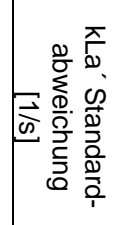 & 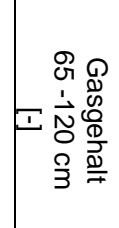 & 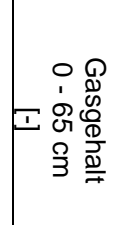 & 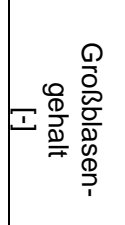 & 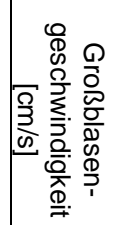 & 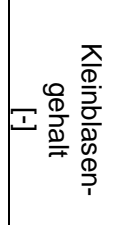 & 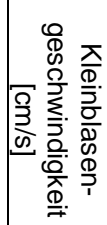 \\
\hline 505 & Leitungswasser & Stickstoff & 1 & 4,3 & 102 & 0 & 1,2 & 133 & 20 & 31 & 300 & 0,090 & & & & & & 0,009 & 87,6 & 0,082 & 21,7 \\
\hline 506 & Leitungswasser & Stickstoff & 1 & 4,3 & 102 & 0 & 1,2 & 133 & 20 & 31 & 500 & 0,180 & & & & & & 0,045 & 25,2 & 0,135 & 19,1 \\
\hline 507 & Leitungswasser & Stickstoff & 1 & 4,3 & 102 & 0 & 1,2 & 133 & 20 & 40 & 1000 & 0,306 & & & & & & 0,091 & 24,2 & 0,215 & 16,5 \\
\hline 508 & Leitungswasser & Stickstoff & 1 & 4,3 & 102 & 0 & 1,2 & 133 & 20 & 41 & 200 & 0,039 & & & & & & 0,008 & 48,6 & 0,030 & 25,6 \\
\hline 509 & Leitungswasser & Stickstoff & 1 & 4,3 & 102 & 0 & 1,2 & 133 & 20 & 41 & 500 & 0,202 & & & & & & & & & \\
\hline 510 & Leitungswasser & Stickstoff & 19 & 1 & 102 & 0 & 1,2 & 130 & 20 & 1 & 4,7 & 0,024 & 0,0010 & 0,0079 & 0,0006 & & & & & & \\
\hline 511 & Leitungswasser & Stickstoff & 19 & 1 & 102 & 0 & 1,2 & 130 & 20 & 1 & 9,4 & 0,049 & 0,0063 & 0,0171 & 0,0014 & & & & & & \\
\hline 512 & Leitungswasser & Stickstoff & 19 & 1 & 102 & 0 & 1,2 & 130 & 20 & 1 & 23,6 & 0,090 & 0,0051 & 0,0345 & 0,0023 & & & & & & \\
\hline 513 & Leitungswasser & Stickstoff & 19 & 1 & 102 & 0 & 1,2 & 130 & 20 & 1 & 47,1 & 0,138 & 0,0054 & 0,0586 & 0,0025 & & & & & & \\
\hline 514 & Leitungswasser & Stickstoff & 19 & 1 & 102 & 0 & 1,2 & 130 & 20 & 1 & 94,2 & 0,210 & 0,0012 & 0,1071 & 0,0031 & & & & & & \\
\hline 515 & Leitungswasser & Stickstoff & 19 & 1 & 102 & 0 & 1,2 & 130 & 20 & 2 & 9,4 & 0,027 & 0,0007 & 0,0091 & 0,0002 & & & & & & \\
\hline 516 & Leitungswasser & Stickstoff & 19 & 1 & 102 & 0 & 1,2 & 130 & 20 & 2 & 18,9 & 0,049 & 0,0007 & 0,0181 & 0,0014 & & & & & & \\
\hline 517 & Leitungswasser & Stickstoff & 19 & 1 & 102 & 0 & 1,2 & 130 & 20 & 2 & 47,1 & 0,092 & 0,0012 & 0,0369 & 0,0015 & & & & & & \\
\hline 518 & Leitungswasser & Stickstoff & 19 & 1 & 102 & 0 & 1,2 & 130 & 20 & 2 & 94,3 & 0,152 & 0,0021 & 0,0717 & 0,0068 & & & & & & \\
\hline 519 & Leitungswasser & Stickstoff & 19 & 1 & 102 & 0 & 1,2 & 130 & 20 & 2 & 188,5 & 0,229 & 0,0031 & 0,1129 & 0,0033 & & & & & & \\
\hline 520 & Leitungswasser & Stickstoff & 19 & 1 & 102 & 0 & 1,2 & 130 & 20 & 5 & 23,6 & 0,031 & 0,0007 & 0,0118 & 0,0001 & & & & & & \\
\hline 521 & Leitungswasser & Stickstoff & 19 & 1 & 102 & 0 & 1,2 & 130 & 20 & 5 & 47,1 & 0,051 & 0,0000 & 0,0195 & 0,0000 & & & & & & \\
\hline 522 & Leitungswasser & Stickstoff & 19 & 1 & 102 & 0 & 1,2 & 130 & 20 & 5 & 117,8 & 0,107 & 0,0006 & 0,0469 & 0,0014 & & & & & & \\
\hline 523 & Leitungswasser & Stickstoff & 19 & 1 & 102 & 0 & 1,2 & 130 & 20 & 5 & 235,6 & 0,172 & 0,0015 & 0,0810 & 0,0030 & & & & & & \\
\hline 524 & Leitungswasser & Stickstoff & 19 & 1 & 102 & 0 & 1,2 & 130 & 20 & 5 & 471,3 & 0,272 & 0,0031 & 0,1495 & 0,0077 & & & & & & \\
\hline 525 & Leitungswasser & Stickstoff & 19 & 1 & 102 & 0 & 1,2 & 130 & 20 & 10 & 47,1 & 0,033 & 0,0000 & 0,0130 & 0,0001 & & & & & & \\
\hline 526 & Leitungswasser & Stickstoff & 19 & 1 & 102 & 0 & 1,2 & 130 & 20 & 10 & 94,3 & 0,060 & 0,0007 & 0,0252 & 0,0005 & & & & & & \\
\hline 527 & Leitungswasser & Stickstoff & 19 & 1 & 102 & 0 & 1,2 & 130 & 20 & 10 & 235,6 & 0,119 & 0,0015 & 0,0562 & 0,0015 & & & & & & \\
\hline 528 & Leitungswasser & Stickstoff & 19 & 1 & 102 & 0 & 1,2 & 130 & 20 & 10 & 471,3 & 0,195 & 0,0042 & 0,1013 & 0,0059 & & & & & & \\
\hline 529 & Leitungswasser & Stickstoff & 19 & 1 & 102 & 0 & 1,2 & 130 & 20 & 10 & 942,5 & 0,327 & 0,0088 & 0,1769 & 0,0068 & & & & & & \\
\hline 530 & Leitungswasser & Stickstoff & 19 & 1 & 102 & 0 & 1,2 & 130 & 20 & 20 & 94,3 & 0,043 & 0,0007 & 0,0180 & 0,0004 & & & & & & \\
\hline 531 & Leitungswasser & Stickstoff & 19 & 1 & 102 & 0 & 1,2 & 130 & 20 & 20 & 188,5 & 0,069 & 0,0007 & 0,0284 & 0,0005 & & & & & & \\
\hline 532 & Leitungswasser & Stickstoff & 19 & 1 & 102 & 0 & 1,2 & 130 & 20 & 20 & 471,3 & 0,137 & 0,0015 & 0,0680 & 0,0021 & & & & & & \\
\hline
\end{tabular}




\begin{tabular}{|c|c|c|c|c|c|c|c|c|c|c|c|c|c|c|c|c|c|c|c|c|c|}
\hline$\underline{z}$ & 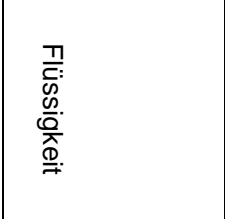 & $\underset{ఱ}{\circledast}$ & 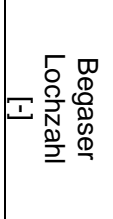 & 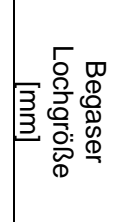 & 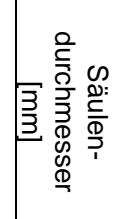 & 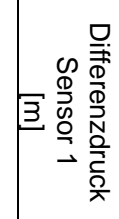 & 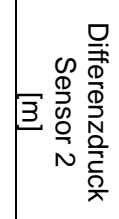 & 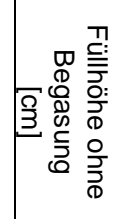 & 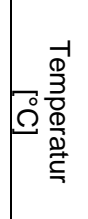 & 总 몰 & 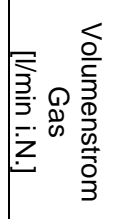 & 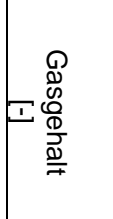 & 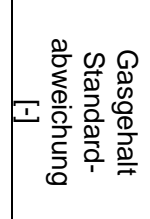 & $\vec{E}$ & 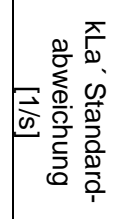 & 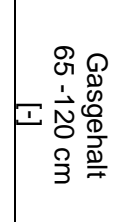 & 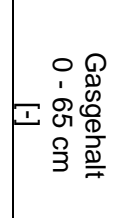 & 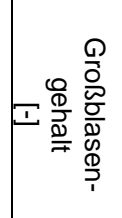 & 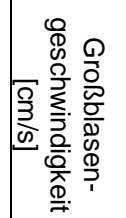 & 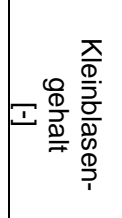 & 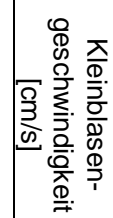 \\
\hline 533 & Leitungswasser & Stickstoff & 19 & 1 & 102 & 0 & 1,2 & 130 & 20 & 20 & 942,5 & 0,246 & 0,0040 & 0,1311 & 0,0045 & & & & & & \\
\hline 534 & Leitungswasser & Stickstoff & 19 & 1 & 102 & 0 & 1,2 & 130 & 20 & 40 & 188,5 & 0,043 & 0,0000 & 0,0172 & 0,0010 & & & & & & \\
\hline 535 & Leitungswasser & Stickstoff & 19 & 1 & 102 & 0 & 1,2 & 130 & 20 & 40 & 377 & 0,073 & 0,0007 & 0,0300 & 0,0001 & & & & & & \\
\hline 536 & Leitungswasser & Stickstoff & 19 & 1 & 102 & 0 & 1,2 & 130 & 20 & 40 & 942,5 & 0,186 & 0,0067 & 0,0900 & 0,0029 & & & & & & \\
\hline 537 & Leitungswasser & Helium & 19 & 1 & 102 & 0 & 1,2 & 130 & 20 & 1 & 4,7 & 0,025 & 0,0000 & 0,0067 & 0,0001 & & & & & & \\
\hline 538 & Leitungswasser & Helium & 19 & 1 & 102 & 0 & 1,2 & 130 & 20 & 1 & 9,4 & 0,045 & 0,0007 & 0,0137 & 0,0001 & & & & & & \\
\hline 539 & Leitungswasser & Helium & 19 & 1 & 102 & 0 & 1,2 & 130 & 20 & 1 & 23,6 & 0,086 & 0,0007 & 0,0306 & 0,0006 & & & & & & \\
\hline 540 & Leitungswasser & Helium & 19 & 1 & 102 & 0 & 1,2 & 130 & 20 & 1 & 47,1 & 0,130 & 0,0000 & 0,0474 & 0,0019 & & & & & & \\
\hline 541 & Leitungswasser & Helium & 19 & 1 & 102 & 0 & 1,2 & 130 & 20 & 1 & 94,2 & 0,205 & 0,0051 & 0,0831 & 0,0036 & & & & & & \\
\hline 542 & Leitungswasser & Helium & 19 & 1 & 102 & 0 & 1,2 & 130 & 20 & 3 & 14,1 & 0,025 & & 0,0077 & & & & & & & \\
\hline 543 & Leitungswasser & Helium & 19 & 1 & 102 & 0 & 1,2 & 130 & 20 & 3 & 28,3 & 0,046 & 0,0007 & 0,0146 & 0,0011 & & & & & & \\
\hline 544 & Leitungswasser & Helium & 19 & 1 & 102 & 0 & 1,2 & 130 & 20 & 3 & 70,7 & 0,092 & 0,0014 & 0,0356 & 0,0003 & & & & & & \\
\hline 545 & Leitungswasser & Helium & 19 & 1 & 102 & 0 & 1,2 & 130 & 20 & 3 & 141,4 & 0,140 & 0,0014 & 0,0590 & 0,0022 & & & & & & \\
\hline 546 & Leitungswasser & Helium & 19 & 1 & 102 & 0 & 1,2 & 130 & 20 & 3 & 282,8 & 0,206 & 0,0028 & 0,0924 & 0,0058 & & & & & & \\
\hline 547 & Leitungswasser & Stickstoff & 1 & 1 & 102 & 0 & 1,2 & 130 & 20 & 1 & 4,7 & 0,023 & 0,0005 & 0,0081 & 0,0005 & & & & & & \\
\hline 548 & Leitungswasser & Stickstoff & 1 & 1 & 102 & 0 & 1,2 & 130 & 20 & 1 & 9,4 & 0,043 & 0,0013 & 0,0169 & 0,0006 & & & & & & \\
\hline 549 & Leitungswasser & Stickstoff & 1 & 1 & 102 & 0 & 1,2 & 130 & 20 & 1 & 23,6 & 0,087 & 0,0018 & 0,0409 & 0,0010 & & & & & & \\
\hline 550 & Leitungswasser & Stickstoff & 1 & 1 & 102 & 0 & 1,2 & 130 & 20 & 1 & 47,1 & 0,138 & 0,0025 & 0,0646 & 0,0027 & & & & & & \\
\hline 551 & Leitungswasser & Stickstoff & 1 & 1 & 102 & 0 & 1,2 & 130 & 20 & 1 & 94,2 & 0,212 & 0,0065 & 0,1165 & 0,0083 & & & & & & \\
\hline 552 & Leitungswasser & Stickstoff & 1 & 1 & 102 & 0 & 1,2 & 130 & 20 & 2 & 9,4 & 0,025 & 0,0007 & 0,0091 & 0,0006 & & & & & & \\
\hline 553 & Leitungswasser & Stickstoff & 1 & 1 & 102 & 0 & 1,2 & 130 & 20 & 2 & 18,9 & 0,046 & 0,0014 & 0,0182 & 0,0008 & & & & & & \\
\hline 554 & Leitungswasser & Stickstoff & 1 & 1 & 102 & 0 & 1,2 & 130 & 20 & 2 & 47,1 & 0,095 & 0,0006 & 0,0445 & 0,0007 & & & & & & \\
\hline 555 & Leitungswasser & Stickstoff & 1 & 1 & 102 & 0 & 1,2 & 130 & 20 & 2 & 94,3 & 0,160 & 0,0010 & 0,0822 & 0,0033 & & & & & & \\
\hline 556 & Leitungswasser & Stickstoff & 1 & 1 & 102 & 0 & 1,2 & 130 & 20 & 2 & 188,5 & 0,237 & 0,0031 & 0,1309 & 0,0055 & & & & & & \\
\hline 557 & Leitungswasser & Stickstoff & 1 & 1 & 102 & 0 & 1,2 & 130 & 20 & 5 & 23,6 & 0,028 & 0,0007 & 0,0112 & 0,0001 & & & & & & \\
\hline 558 & Leitungswasser & Stickstoff & 1 & 1 & 102 & 0 & 1,2 & 130 & 20 & 5 & 47,1 & 0,052 & 0,0000 & 0,0231 & 0,0003 & & & & & & \\
\hline 559 & Leitungswasser & Stickstoff & 1 & 1 & 102 & 0 & 1,2 & 130 & 20 & 5 & 117,8 & 0,114 & 0,0012 & 0,0577 & 0,0021 & & & & & & \\
\hline 560 & Leitungswasser & Stickstoff & 1 & 1 & 102 & 0 & 1,2 & 130 & 20 & 5 & 235,6 & 0,182 & 0,0006 & 0,0980 & 0,0062 & & & & & & \\
\hline
\end{tabular}




\begin{tabular}{|c|c|c|c|c|c|c|c|c|c|c|c|c|c|c|c|c|c|c|c|c|c|}
\hline$\underline{3}$ & 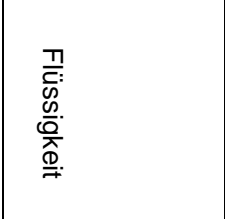 & 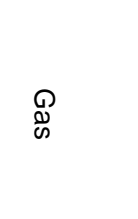 & 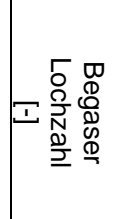 & 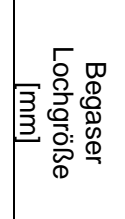 & 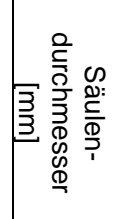 & 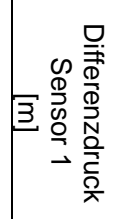 & 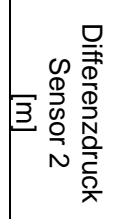 & 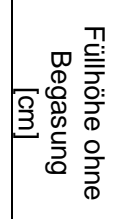 & 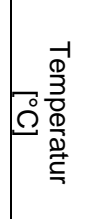 & 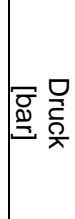 & 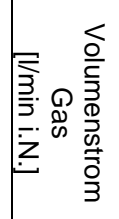 & 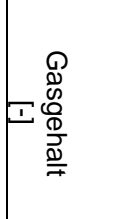 & 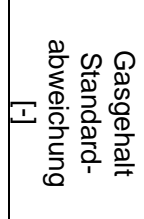 & 居离 & 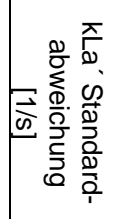 & 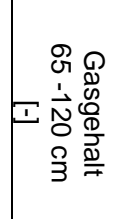 & 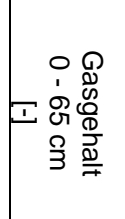 & 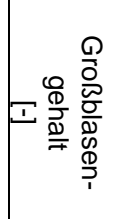 & 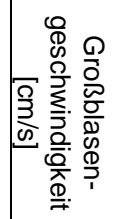 & 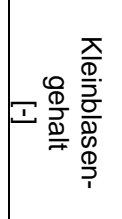 & 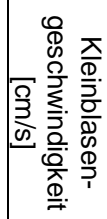 \\
\hline 561 & Leitungswasser & Stickstoff & 1 & 1 & 102 & 0 & 1,2 & 130 & 20 & 5 & 471,3 & 0,276 & 0,0035 & 0,1586 & 0,0099 & & & & & & \\
\hline 562 & Leitungswasser & Stickstoff & 1 & 1 & 102 & 0 & 1,2 & 130 & 20 & 10 & 47,1 & 0,031 & 0,0007 & 0,0134 & 0,0003 & & & & & & \\
\hline 563 & Leitungswasser & Stickstoff & 1 & 1 & 102 & 0 & 1,2 & 130 & 20 & 10 & 94,3 & 0,064 & 0,0000 & 0,0308 & 0,0004 & & & & & & \\
\hline 564 & Leitungswasser & Stickstoff & 1 & 1 & 102 & 0 & 1,2 & 130 & 20 & 10 & 235,6 & 0,128 & 0,0006 & 0,0643 & 0,0015 & & & & & & \\
\hline 565 & Leitungswasser & Stickstoff & 1 & 1 & 102 & 0 & 1,2 & 130 & 20 & 10 & 471,3 & 0,205 & 0,0035 & 0,1141 & 0,0088 & & & & & & \\
\hline 566 & Leitungswasser & Stickstoff & 1 & 1 & 102 & 0 & 1,2 & 130 & 20 & 10 & 942,5 & 0,331 & 0,0057 & 0,2007 & 0,0155 & & & & & & \\
\hline 567 & Leitungswasser & Stickstoff & 1 & 1 & 102 & 0 & 1,2 & 130 & 20 & 20 & 94,3 & 0,039 & 0,0000 & 0,0194 & 0,0004 & & & & & & \\
\hline 568 & Leitungswasser & Stickstoff & 1 & 1 & 102 & 0 & 1,2 & 130 & 20 & 20 & 188,5 & 0,068 & 0,0000 & 0,0314 & 0,0002 & & & & & & \\
\hline 569 & Leitungswasser & Stickstoff & 1 & 1 & 102 & 0 & 1,2 & 130 & 20 & 20 & 471,3 & 0,139 & 0,0017 & 0,0682 & 0,0013 & & & & & & \\
\hline 570 & Leitungswasser & Stickstoff & 1 & 1 & 102 & 0 & 1,2 & 130 & 20 & 20 & 942,5 & 0,243 & 0,0050 & 0,1316 & 0,0014 & & & & & & \\
\hline 571 & Leitungswasser & Stickstoff & 1 & 1 & 102 & 0 & 1,2 & 130 & 20 & 40 & 188,5 & 0,040 & 0,0007 & 0,0183 & 0,0000 & & & & & & \\
\hline 572 & Leitungswasser & Stickstoff & 1 & 1 & 102 & 0 & 1,2 & 130 & 20 & 40 & 377 & 0,072 & 0,0007 & 0,0315 & 0,0011 & & & & & & \\
\hline 573 & Leitungswasser & Stickstoff & 1 & 1 & 102 & 0 & 1,2 & 130 & 20 & 40 & 942,5 & 0,185 & 0,0071 & 0,0916 & 0,0033 & & & & & & \\
\hline 574 & Leitungswasser & Helium & 1 & 1 & 102 & 0 & 1,2 & 130 & 20 & 1 & 4,7 & 0,021 & 0,0000 & 0,0065 & 0,0002 & & & & & & \\
\hline 575 & Leitungswasser & Helium & 1 & 1 & 102 & 0 & 1,2 & 130 & 20 & 1 & 9,4 & 0,042 & 0,0000 & 0,0135 & 0,0001 & & & & & & \\
\hline 576 & Leitungswasser & Helium & 1 & 1 & 102 & 0 & 1,2 & 130 & 20 & 1 & 23,6 & 0,081 & 0,0012 & 0,0302 & 0,0003 & & & & & & \\
\hline 577 & Leitungswasser & Helium & 1 & 1 & 102 & 0 & 1,2 & 130 & 20 & 1 & 47,1 & 0,126 & 0,0017 & 0,0523 & 0,0020 & & & & & & \\
\hline 578 & Leitungswasser & Helium & 1 & 1 & 102 & 0 & 1,2 & 130 & 20 & 1 & 94,2 & 0,197 & 0,0025 & 0,0969 & 0,0054 & & & & & & \\
\hline 579 & Leitungswasser & Helium & 1 & 1 & 102 & 0 & 1,2 & 130 & 20 & 3 & 14,1 & 0,022 & 0,0000 & 0,0071 & 0,0001 & & & & & & \\
\hline 580 & Leitungswasser & Helium & 1 & 1 & 102 & 0 & 1,2 & 130 & 20 & 3 & 28,3 & 0,041 & 0,0007 & 0,0143 & 0,0002 & & & & & & \\
\hline 581 & Leitungswasser & Helium & 1 & 1 & 102 & 0 & 1,2 & 130 & 20 & 3 & 70,7 & 0,087 & 0,0006 & 0,0354 & 0,0003 & & & & & & \\
\hline 582 & Leitungswasser & Helium & 1 & 1 & 102 & 0 & 1,2 & 130 & 20 & 3 & 141,4 & 0,136 & 0,0021 & 0,0606 & 0,0033 & & & & & & \\
\hline 583 & Leitungswasser & Helium & 1 & 1 & 102 & 0 & 1,2 & 130 & 20 & 3 & 282,8 & 0,203 & 0,0007 & 0,0977 & 0,0011 & & & & & & \\
\hline 584 & Leitungswasser & Stickstoff & 7 & 1 & 115 & 0,1 & 0,905 & 100 & 20 & 1 & 5 & & & 0,0112 & 0,0001 & & & & & & \\
\hline 585 & Leitungswasser & Stickstoff & 7 & 1 & 115 & 0,1 & 0,905 & 100 & 20 & 1 & 10 & 0,050 & 0,0013 & 0,0230 & 0,0015 & & & 0,021 & 58,0 & 0,029 & 25,1 \\
\hline 586 & Leitungswasser & Stickstoff & 7 & 1 & 115 & 0,1 & 0,905 & 100 & 20 & 1 & 20 & 0,085 & 0,0032 & 0,0402 & 0,0008 & & & 0,029 & 78,5 & 0,056 & 23,0 \\
\hline 587 & Leitungswasser & Stickstoff & 7 & 1 & 115 & 0,1 & 0,905 & 100 & 20 & 1 & 25 & & & 0,0498 & 0,0006 & & & & & & \\
\hline 588 & Leitungswasser & Stickstoff & 7 & 1 & 115 & 0,1 & 0,905 & 100 & 20 & 1 & 30 & 0,114 & 0,0023 & 0,0542 & 0,0013 & & & 0,038 & 93,5 & 0,076 & 21,7 \\
\hline
\end{tabular}




\begin{tabular}{|c|c|c|c|c|c|c|c|c|c|c|c|c|c|c|c|c|c|c|c|c|c|}
\hline$\underline{s}$ & 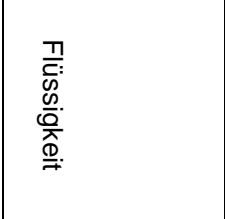 & Ф) & 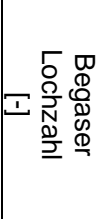 & 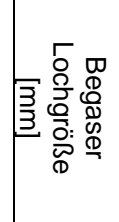 & 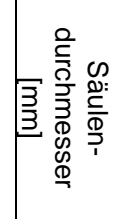 & 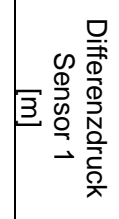 & 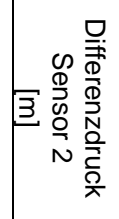 & 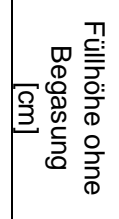 & 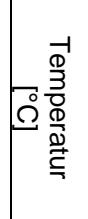 & 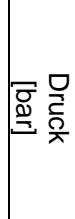 & 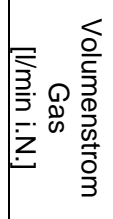 & 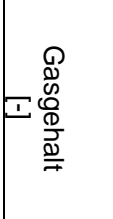 & 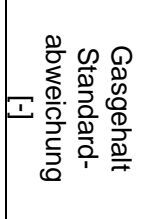 & $\overrightarrow{\omega_{0}}$ & 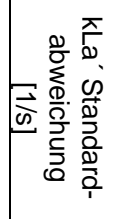 & 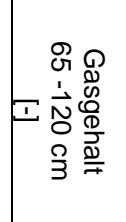 & 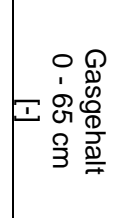 & 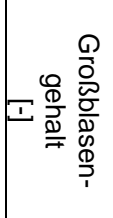 & 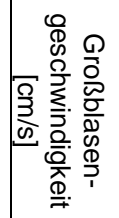 & 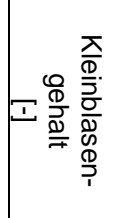 & 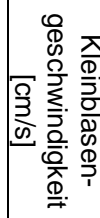 \\
\hline 589 & Leitungswasser & Stickstoff & 7 & 1 & 115 & 0,1 & 0,905 & 100 & 20 & 1 & 40 & 0,141 & 0,0018 & & & & & 0,062 & 85,6 & 0,078 & 20,2 \\
\hline 590 & Leitungswasser & Stickstoff & 7 & 1 & 115 & 0,1 & 0,905 & 100 & 20 & 1 & 50 & 0,166 & 0,0015 & 0,0838 & 0,0081 & & & 0,063 & 104,1 & 0,103 & 20,1 \\
\hline 591 & Leitungswasser & Stickstoff & Sinte & scheibe & 102 & 0 & 1,2 & 185 & 20 & 1 & 5 & 0,043 & & & & & & 0,001 & 141,9 & 0,042 & 23,4 \\
\hline 592 & Leitungswasser & Stickstoff & Sinte & scheibe & 102 & 0 & 1,2 & 185 & 20 & 1 & 10 & 0,095 & & & & & & 0,003 & 110,4 & 0,092 & 21,6 \\
\hline 593 & Leitungswasser & Stickstoff & Sinte & scheibe & 102 & 0 & 1,2 & 185 & 20 & 1 & 20 & 0,154 & & & & & & 0,005 & 332,3 & 0,149 & 20,4 \\
\hline 594 & Leitungswasser & Stickstoff & Sinte & scheibe & 102 & 0 & 1,2 & 185 & 20 & 1 & 30 & 0,186 & & & & & & 0,031 & 117,0 & 0,155 & 20,5 \\
\hline 595 & Leitungswasser & Stickstoff & Sinte & scheibe & 102 & 0 & 1,2 & 185 & 20 & 3 & 10 & 0,029 & & & & & & 0,005 & 71,7 & 0,024 & 24,1 \\
\hline 596 & Leitungswasser & Stickstoff & Sinte & scheibe & 102 & 0 & 1,2 & 185 & 20 & 3 & 20 & 0,063 & & & & & & 0,002 & 86,4 & 0,061 & 22,3 \\
\hline 597 & Leitungswasser & Stickstoff & Sinte & scheibe & 102 & 0 & 1,2 & 185 & 20 & 3 & 40 & 0,128 & & & & & & 0,004 & 200,2 & 0,124 & 18,6 \\
\hline 598 & Leitungswasser & Stickstoff & Sinte & scheibe & 102 & 0 & 1,2 & 185 & 20 & 6 & 25 & 0,038 & & & & & & 0,001 & 53,9 & 0,037 & 24,1 \\
\hline 599 & Leitungswasser & Stickstoff & Sinte & scheibe & 102 & 0 & 1,2 & 185 & 20 & 6 & 50 & 0,082 & & & & & & 0,003 & 74,5 & 0,079 & 21,4 \\
\hline 600 & Leitungswasser & Stickstoff & Sinte & scheibe & 102 & 0 & 1,2 & 185 & 20 & 6 & 100 & 0,191 & & & & & & 0,012 & 43,1 & 0,179 & 18,3 \\
\hline 601 & Leitungswasser & Stickstoff & Sinte & scheibe & 102 & 0 & 1,2 & 185 & 20 & 11 & 50 & 0,041 & & & & & & & & & \\
\hline 602 & Leitungswasser & Stickstoff & Sinte & scheibe & 102 & 0 & 1,2 & 185 & 20 & 11 & 100 & 0,112 & & & & & & & & & \\
\hline 603 & Leitungswasser & Stickstoff & Sinte & scheibe & 102 & 0 & 1,2 & 185 & 20 & 11 & 200 & 0,197 & & & & & & 0,020 & 46,5 & 0,177 & 18,1 \\
\hline 604 & Leitungswasser & Stickstoff & Sinte & scheibe & 102 & 0 & 1,2 & 185 & 20 & 21 & 100 & 0,053 & & & & & & & & & \\
\hline 605 & Leitungswasser & Stickstoff & Sinte & scheibe & 102 & 0 & 1,2 & 185 & 20 & 21 & 200 & 0,108 & & & & & & & & & \\
\hline 606 & Leitungswasser & Stickstoff & Sinte & scheibe & 102 & 0 & 1,2 & 185 & 20 & 21 & 400 & 0,201 & & & & & & 0,029 & 43,4 & 0,173 & 18,0 \\
\hline 607 & Leitungswasser & Stickstoff & Sinte & scheibe & 102 & 0 & 1,2 & 185 & 20 & 31 & 150 & 0,051 & & & & & & & & & \\
\hline
\end{tabular}




\begin{tabular}{|c|c|c|c|c|c|c|c|c|c|c|c|c|c|c|c|c|c|c|c|c|c|}
\hline$\underline{z}$ & 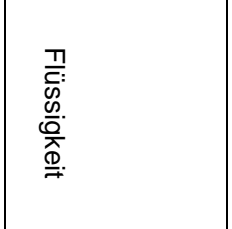 & 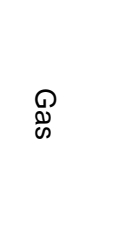 & 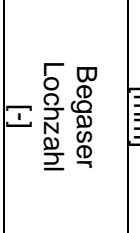 & 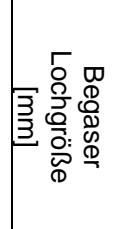 & 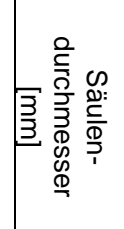 & 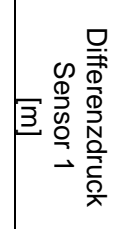 & 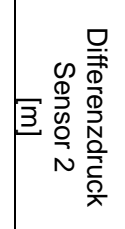 & 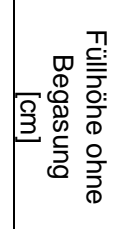 & م) & 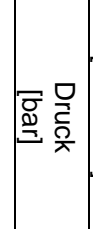 & 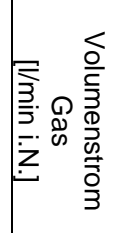 & 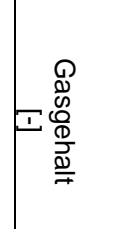 & 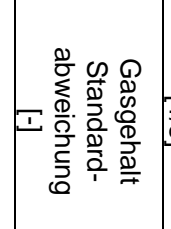 & 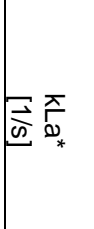 & 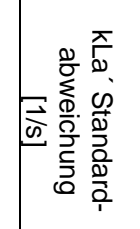 & 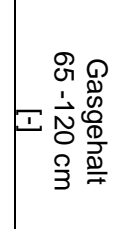 & 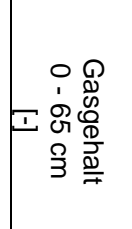 & 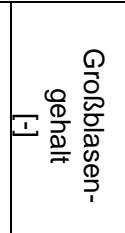 & 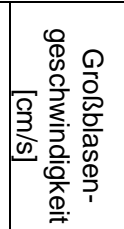 & 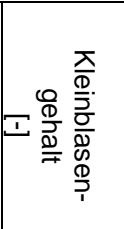 & 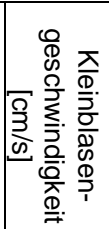 \\
\hline 608 & Leitungswasser & Stickstoff & Sintersc & scheibe & 102 & 0 & 1,2 & 185 & 20 & 31 & 300 & 0,108 & & & & & & 0,012 & 22,1 & 0,096 & 20,1 \\
\hline 609 & Leitungswasser & Stickstoff & Sintersc & scheibe & 102 & 0 & 1,2 & 185 & 20 & 31 & 500 & 0,162 & & & & & & 0,005 & 131,0 & 0,157 & 19,4 \\
\hline 610 & Leitungswasser & Stickstoff & \begin{tabular}{|c|}
$\begin{array}{c}\text { Sintersch } \\
\text { eibe }\end{array}$ \\
\end{tabular} & & 102 & 0 & 1,2 & 185 & 20 & 41 & 100 & 0,026 & & & & & & & & & \\
\hline 611 & Leitungswasser & Stickstoff & $\begin{array}{c}\text { Sintersch } \\
\text { eibe }\end{array}$ & & 102 & 0 & 1,2 & 185 & 20 & 41 & 200 & 0,051 & & & & & & & & & \\
\hline 612 & Leitungswasser & Stickstoff & \begin{tabular}{|c} 
Sintersch \\
eibe
\end{tabular} & & 102 & 0 & 1,2 & 185 & 20 & 41 & 400 & 0,116 & & & & & & 0,043 & 25,4 & 0,073 & 16,1 \\
\hline 613 & Leitungswasser & Stickstoff & $\begin{array}{c}\text { Sintersch } \\
\text { eibe }\end{array}$ & & 102 & 0 & 1,2 & 185 & 20 & 41 & 500 & 0,132 & & & & & & 0,004 & 126,8 & 0,128 & 18,0 \\
\hline 614 & Leitungswasser & Stickstoff & $\begin{array}{c}\text { Sintersch } \\
\text { eibe }\end{array}$ & & 102 & 0 & 1,2 & 185 & 20 & 51 & 250 & 0,049 & & & & & & 0,042 & 23,8 & 0,007 & 18,2 \\
\hline 615 & Leitungswasser & Stickstoff & $\begin{array}{c}\text { Sintersch } \\
\text { eibe }\end{array}$ & & 102 & 0 & 1,2 & 150 & 20 & 1 & 5 & 0,044 & & & & & & 0,006 & 82,3 & 0,038 & 23,2 \\
\hline 616 & Leitungswasser & Stickstoff & $\begin{array}{c}\text { Sintersch } \\
\text { eibe }\end{array}$ & & 102 & 0 & 1,2 & 150 & 20 & 1 & 10 & 0,097 & & & & & & 0,003 & 70,2 & 0,094 & 22,1 \\
\hline 617 & Leitungswasser & Stickstoff & $\begin{array}{c}\text { Sintersch } \\
\text { eibe }\end{array}$ & & 102 & 0 & 1,2 & 150 & 20 & 1 & 20 & 0,158 & & & & & & 0,038 & 49,9 & 0,121 & 22,2 \\
\hline 618 & Leitungswasser & Stickstoff & $\begin{array}{c}\text { Sintersch } \\
\text { eibe }\end{array}$ & & 102 & 0 & 1,2 & 150 & 20 & 1 & 30 & 0,175 & & & & & & 0,060 & 72,2 & 0,115 & 22,3 \\
\hline 619 & Leitungswasser & Stickstoff & $\begin{array}{c}\text { Sintersch } \\
\text { eibe }\end{array}$ & & 102 & 0 & 1,2 & 150 & 20 & 1 & 50 & 0,175 & & & & & & 0,074 & 130,4 & 0,101 & 23,2 \\
\hline 620 & Leitungswasser & Stickstoff & $\begin{array}{c}\text { Sintersch } \\
\text { eibe }\end{array}$ & & 102 & 0 & 1,2 & 150 & 20 & 1 & 75 & 0,205 & & & & & & 0,109 & 136,6 & 0,096 & 23,4 \\
\hline 621 & Leitungswasser & Stickstoff & $\begin{array}{c}\text { Sintersch } \\
\text { eibe }\end{array}$ & & 102 & 0 & 1,2 & 150 & 20 & 1 & 100 & 0,227 & & & & & & 0,134 & 154,5 & 0,094 & 23,1 \\
\hline 622 & Leitungswasser & Stickstoff & $\begin{array}{c}\text { Sintersch } \\
\text { eibe }\end{array}$ & & 102 & 0 & 1,2 & 150 & 20 & 3 & 20 & 0,066 & & & & & & 0,025 & 25,7 & 0,042 & 21,1 \\
\hline 623 & Leitungswasser & Stickstoff & $\begin{array}{c}\text { Sintersch } \\
\text { eibe }\end{array}$ & & 102 & 0 & 1,2 & 150 & 20 & 3 & 40 & 0,138 & & & & & & 0,012 & 44,7 & 0,126 & 20,2 \\
\hline 624 & Leitungswasser & Stickstoff & $\begin{array}{c}\text { Sintersch } \\
\text { eibe }\end{array}$ & & 102 & 0 & 1,2 & 150 & 20 & 3 & 60 & 0,201 & & & & & & 0,070 & 29,3 & 0,131 & 19,3 \\
\hline 625 & Leitungswasser & Stickstoff & $\begin{array}{c}\text { Sintersch } \\
\text { eibe }\end{array}$ & & 102 & 0 & 1,2 & 150 & 20 & 3 & 100 & 0,202 & & & & & & 0,073 & 70,9 & 0,129 & 21,2 \\
\hline 626 & Leitungswasser & Stickstoff & \begin{tabular}{|c|} 
Sintersch \\
eibe
\end{tabular} & & 102 & 0 & 1,2 & 150 & 20 & 3 & 200 & 0,223 & & & & & & 0,127 & 102,1 & 0,096 & 23,5 \\
\hline 627 & Leitungswasser & Stickstoff & \begin{tabular}{|c|} 
Sintersch \\
eibe
\end{tabular} & & 102 & 0 & 1,2 & 150 & 20 & 3 & 300 & 0,265 & & & & & & 0,161 & 126,2 & 0,104 & 24,4 \\
\hline
\end{tabular}




\begin{tabular}{|c|c|c|c|c|c|c|c|c|c|c|c|c|c|c|c|c|c|c|c|c|c|}
\hline$\underline{3}$ & 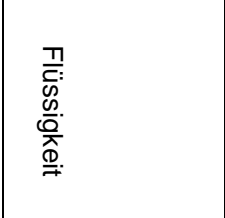 & $\underset{\aleph}{\stackrel{\wp}{~}}$ & 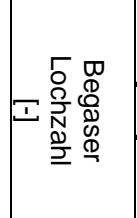 & 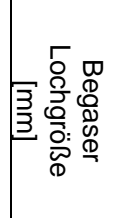 & 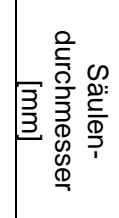 & 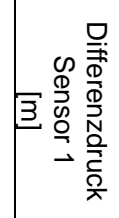 & 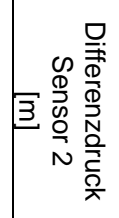 & 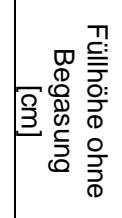 & 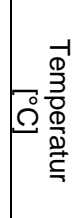 & 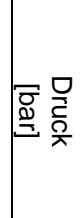 & 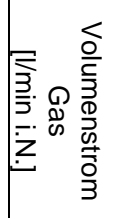 & 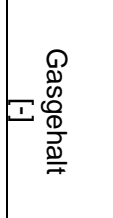 & 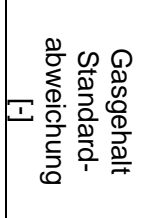 & 空西 & 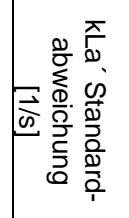 & 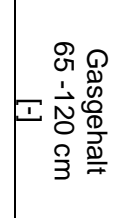 & 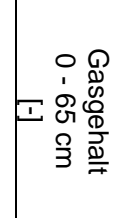 & 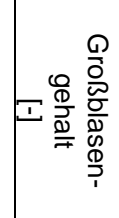 & 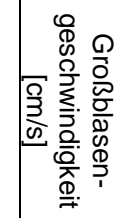 & 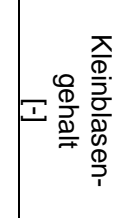 & 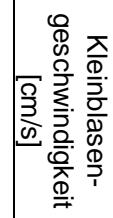 \\
\hline 628 & Leitungswasser & Stickstoff & $\begin{array}{c}\text { Sintersch } \\
\text { eibe }\end{array}$ & & 102 & 0 & 1,2 & 150 & 20 & 6 & 25 & 0,039 & & & & & & 0,004 & 36,9 & 0,035 & 22,9 \\
\hline 629 & Leitungswasser & Stickstoff & \begin{tabular}{|c|}
$\begin{array}{c}\text { Sintersch } \\
\text { eibe }\end{array}$ \\
\end{tabular} & & 102 & 0 & 1,2 & 150 & 20 & 6 & 50 & 0,080 & & & & & & 0,004 & 64,3 & 0,076 & 22,0 \\
\hline 630 & Leitungswasser & Stickstoff & $\begin{array}{c}\text { Sintersch } \\
\text { eibe }\end{array}$ & & 102 & 0 & 1,2 & 150 & 20 & 6 & 100 & 0,174 & & & & & & 0,085 & 25,2 & 0,089 & 18,8 \\
\hline 631 & Leitungswasser & Stickstoff & $\begin{array}{c}\text { Sintersch } \\
\text { eibe }\end{array}$ & & 102 & 0 & 1,2 & 150 & 20 & 6 & 150 & 0,220 & & & & & & 0,062 & 42,9 & 0,158 & 19,5 \\
\hline 632 & Leitungswasser & Stickstoff & $\begin{array}{c}\text { Sintersch } \\
\text { eibe }\end{array}$ & & 102 & 0 & 1,2 & 150 & 20 & 6 & 250 & 0,228 & & & & & & 0,082 & 80,2 & 0,146 & 21,0 \\
\hline 633 & Leitungswasser & Stickstoff & $\begin{array}{c}\text { Sintersch } \\
\text { eibe }\end{array}$ & & 102 & 0 & 1,2 & 150 & 20 & 6 & 375 & 0,246 & & & & & & 0,118 & 96,7 & 0,129 & 22,5 \\
\hline 634 & Leitungswasser & Stickstoff & $\begin{array}{c}\text { Sintersch } \\
\text { eibe }\end{array}$ & & 102 & 0 & 1,2 & 150 & 20 & 6 & 500 & 0,292 & & & & & & 0,107 & 143,5 & 0,185 & 19,5 \\
\hline 635 & Leitungswasser & Stickstoff & $\begin{array}{c}\text { Sintersch } \\
\text { eibe }\end{array}$ & & 102 & 0 & 1,2 & 150 & 20 & 11 & 50 & 0,041 & & & & & & 0,009 & 48,3 & 0,032 & 22,9 \\
\hline 636 & Leitungswasser & Stickstoff & $\begin{array}{c}\text { Sintersch } \\
\text { eibe }\end{array}$ & & 102 & 0 & 1,2 & 150 & 20 & 11 & 100 & 0,110 & & & & & & & & & \\
\hline 637 & Leitungswasser & Stickstoff & $\begin{array}{c}\text { Sintersch } \\
\text { eibe }\end{array}$ & & 102 & 0 & 1,2 & 150 & 20 & 11 & 200 & 0,231 & & & & & & 0,053 & 21,0 & 0,178 & 17,1 \\
\hline 638 & Leitungswasser & Stickstoff & $\begin{array}{c}\text { Sintersch } \\
\text { eibe }\end{array}$ & & 102 & 0 & 1,2 & 150 & 20 & 11 & 300 & 0,253 & & & & & & 0,036 & 64,3 & 0,218 & 18,2 \\
\hline 639 & Leitungswasser & Stickstoff & $\begin{array}{c}\text { Sintersch } \\
\text { eibe }\end{array}$ & & 102 & 0 & 1,2 & 150 & 20 & 11 & 500 & 0,272 & & & & & & 0,059 & 110,9 & 0,213 & 18,8 \\
\hline 640 & Leitungswasser & Stickstoff & 7 & 1 & 115 & 0,1 & 0,905 & 100 & 25 & 1 & 1 & & & 0,0066 & 0,0001 & & & & & & \\
\hline 641 & Leitungswasser & Stickstoff & 7 & 1 & 115 & 0,1 & 0,905 & 100 & 25 & 1 & 2 & & & 0,0088 & 0,0001 & & & & & & \\
\hline 642 & Leitungswasser & Stickstoff & 7 & 1 & 115 & 0,1 & 0,905 & 100 & 25 & 1 & 3 & & & 0,0106 & 0,0001 & & & & & & \\
\hline 643 & Leitungswasser & Stickstoff & 7 & 1 & 115 & 0,1 & 0,905 & 100 & 25 & 1 & 5 & 0,038 & 0,0008 & 0,0183 & 0,0010 & & & & & & \\
\hline 644 & Leitungswasser & Stickstoff & 7 & 1 & 115 & 0,1 & 0,905 & 100 & 25 & 1 & 10 & 0,062 & 0,0008 & 0,0274 & 0,0048 & & & 0,027 & 36,5 & 0,035 & 22,2 \\
\hline 645 & Leitungswasser & Stickstoff & 7 & 1 & 115 & 0,1 & 0,905 & 100 & 25 & 1 & 15 & 0,078 & 0,0012 & & & & & 0,036 & 48,3 & 0,042 & 21,0 \\
\hline 646 & Leitungswasser & Stickstoff & 7 & 1 & 115 & 0,1 & 0,905 & 100 & 25 & 1 & 20 & 0,096 & 0,0024 & & & & & 0,041 & 57,3 & 0,055 & 21,5 \\
\hline 647 & Leitungswasser & Stickstoff & 7 & 1 & 115 & 0,1 & 0,905 & 100 & 25 & 1 & 25 & 0,109 & 0,0035 & 0,0520 & 0,0049 & & & 0,044 & 72,2 & 0,065 & 20,0 \\
\hline 648 & Leitungswasser & Stickstoff & 7 & 1 & 115 & 0,1 & 0,905 & 100 & 25 & 1 & 30 & 0,117 & 0,0066 & & & & & 0,049 & 78,8 & 0,068 & 21,2 \\
\hline 649 & Leitungswasser & Stickstoff & 7 & 1 & 115 & 0,1 & 0,905 & 100 & 25 & 1 & 35 & 0,133 & 0,0042 & & & & & 0,052 & 88,6 & 0,081 & 19,6 \\
\hline 650 & Leitungswasser & Stickstoff & 7 & 1 & 115 & 0,1 & 0,905 & 100 & 25 & 1 & 40 & 0,140 & 0,0122 & & & & & 0,059 & 94,4 & 0,081 & 19,4 \\
\hline
\end{tabular}




\begin{tabular}{|c|c|c|c|c|c|c|c|c|c|c|c|c|c|c|c|c|c|c|c|c|c|}
\hline$\underline{n}$ & 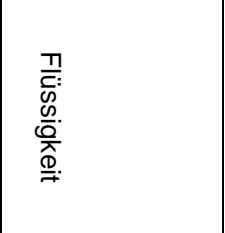 & $\stackrel{\mathscr{D}}{\mathscr{\infty}}$ & 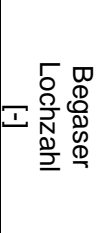 & 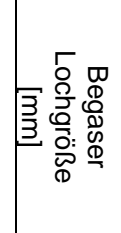 & 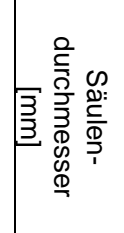 & 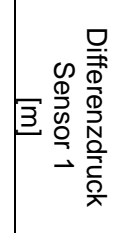 & 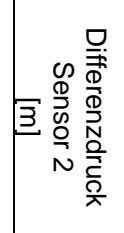 & 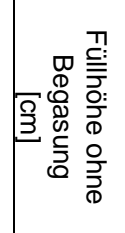 & 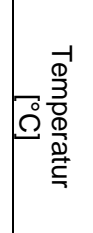 & 몰 올 & 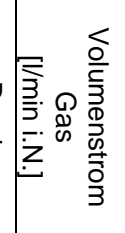 & 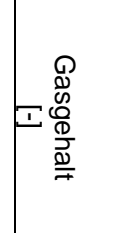 & 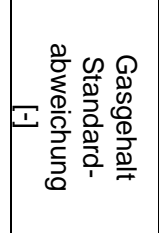 & 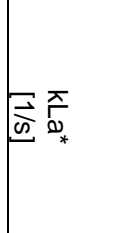 & 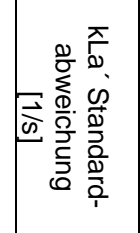 & 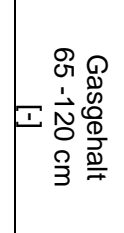 & 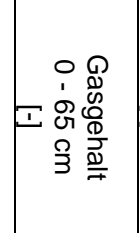 & 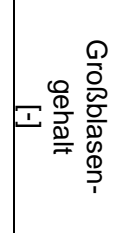 & 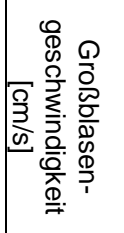 & 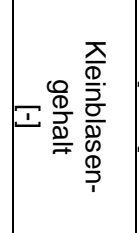 & 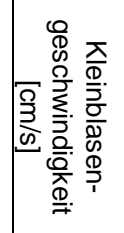 \\
\hline 651 & Leitungswasser & Stickstoff & 7 & 1 & 115 & 0,1 & 0,905 & 100 & 25 & 1 & 45 & 0,162 & 0,0032 & & & & & 0,069 & 88,7 & 0,093 & 19,1 \\
\hline 652 & Leitungswasser & Stickstoff & 7 & 1 & 115 & 0,1 & 0,905 & 100 & 25 & 1 & 50 & 0,160 & 0,0153 & 0,0944 & 0,0109 & & & 0,073 & 99,4 & 0,087 & 18,5 \\
\hline 653 & Leitungswasser & Stickstoff & 7 & 1 & 115 & 0,1 & 0,905 & 100 & 25 & 1 & 60 & 0,186 & 0,0037 & & & & & 0,087 & 100,3 & 0,099 & 18,5 \\
\hline 654 & Leitungswasser & Stickstoff & 7 & 1 & 115 & 0,1 & 0,905 & 100 & 25 & 1 & 80 & 0,231 & 0,0044 & & & & & 0,100 & 116,9 & 0,131 & 18,5 \\
\hline 655 & Leitungswasser & Stickstoff & 7 & 1 & 115 & 0,1 & 0,905 & 100 & 25 & 1 & 100 & 0,252 & 0,0054 & & & & & 0,123 & 126,3 & 0,129 & 17,8 \\
\hline 656 & Leitungswasser & Stickstoff & 7 & 1 & 115 & 0,1 & 0,905 & 100 & 25 & 3 & 10 & 0,025 & 0,0006 & & & & & & & & \\
\hline 657 & Leitungswasser & Stickstoff & 7 & 1 & 115 & 0,1 & 0,905 & 100 & 25 & 3 & 20 & 0,047 & 0,0005 & & & & & 0,020 & 29,5 & 0,027 & 21,4 \\
\hline 658 & Leitungswasser & Stickstoff & 7 & 1 & 115 & 0,1 & 0,905 & 100 & 25 & 3 & 30 & 0,067 & 0,0008 & & & & & 0,029 & 34,1 & 0,037 & 20,4 \\
\hline 659 & Leitungswasser & Stickstoff & 7 & 1 & 115 & 0,1 & 0,905 & 100 & 25 & 3 & 40 & 0,084 & 0,0027 & & & & & 0,025 & 47,2 & 0,060 & 21,1 \\
\hline 660 & Leitungswasser & Stickstoff & 7 & 1 & 115 & 0,1 & 0,905 & 100 & 25 & 3 & 50 & 0,103 & 0,0024 & & & & & 0,030 & 50,6 & 0,073 & 20,4 \\
\hline 661 & Leitungswasser & Stickstoff & 7 & 1 & 115 & 0,1 & 0,905 & 100 & 25 & 3 & 60 & 0,119 & 0,0019 & & & & & 0,026 & 63,4 & 0,093 & 20,5 \\
\hline 662 & Leitungswasser & Stickstoff & 7 & 1 & 115 & 0,1 & 0,905 & 100 & 25 & 3 & 80 & 0,158 & 0,0029 & & & & & 0,047 & 54,7 & 0,111 & 19,2 \\
\hline 663 & Leitungswasser & Stickstoff & 7 & 1 & 115 & 0,1 & 0,905 & 100 & 25 & 3 & 100 & 0,196 & 0,0014 & & & & & 0,061 & 56,8 & 0,136 & 18,0 \\
\hline 664 & Leitungswasser & Stickstoff & 7 & 1 & 115 & 0,1 & 0,905 & 100 & 25 & 3 & 120 & 0,239 & 0,0027 & & & & & 0,075 & 55,5 & 0,164 & 17,8 \\
\hline 665 & Leitungswasser & Stickstoff & 7 & 1 & 115 & 0,1 & 0,905 & 100 & 25 & 3 & 140 & 0,277 & 0,0026 & & & & & 0,079 & 60,1 & 0,198 & 17,4 \\
\hline 666 & Leitungswasser & Stickstoff & 7 & 1 & 115 & 0,1 & 0,905 & 100 & 25 & 3 & 160 & 0,318 & 0,0055 & & & & & 0,092 & 61,8 & 0,227 & 17,4 \\
\hline 667 & Leitungswasser & Stickstoff & 7 & 1 & 115 & 0,1 & 0,905 & 100 & 25 & 3 & 180 & 0,351 & 0,0033 & & & & & 0,127 & 54,3 & 0,223 & 16,6 \\
\hline 668 & Leitungswasser & Stickstoff & 7 & 1 & 115 & 0,1 & 0,905 & 100 & 25 & 3 & 200 & 0,386 & 0,0026 & & & & & 0,139 & 54,2 & 0,247 & 17,3 \\
\hline 669 & Leitungswasser & Stickstoff & 7 & 1 & 115 & 0,1 & 0,905 & 100 & 25 & 2 & 25 & & & 0,0373 & 0,0059 & & & & & & \\
\hline 670 & Leitungswasser & Stickstoff & 7 & 1 & 115 & 0,1 & 0,905 & 100 & 25 & 2 & 50 & & & 0,0717 & 0,0116 & & & & & & \\
\hline 671 & Leitungswasser & Stickstoff & 7 & 1 & 115 & 0,1 & 0,905 & 100 & 25 & 2,5 & 12,5 & & & 0,0143 & 0,0009 & & & & & & \\
\hline 672 & Leitungswasser & Stickstoff & 7 & 1 & 115 & 0,1 & 0,905 & 100 & 25 & 2,5 & 25 & & & 0,0315 & 0,0005 & & & & & & \\
\hline 673 & Leitungswasser & Stickstoff & 7 & 1 & 115 & 0,1 & 0,905 & 100 & 25 & 2,5 & 50 & & & 0,0619 & 0,0007 & & & & & & \\
\hline 674 & Leitungswasser & Stickstoff & 7 & 1 & 115 & 0,1 & 0,905 & 100 & 25 & 4 & 25 & & & 0,0207 & 0,0003 & & & & & & \\
\hline 675 & Leitungswasser & Stickstoff & 7 & 1 & 115 & 0,1 & 0,905 & 100 & 25 & 4 & 50 & & & 0,0470 & 0,0010 & & & & & & \\
\hline 676 & Leitungswasser & Stickstoff & 7 & 1 & 115 & 0,1 & 0,905 & 100 & 25 & 5 & 20 & 0,030 & 0,0009 & & & & & & & & \\
\hline 677 & Leitungswasser & Stickstoff & 7 & 1 & 115 & 0,1 & 0,905 & 100 & 25 & 5 & 25 & & & 0,0184 & 0,0004 & & & & & & \\
\hline- & ggswasser & toff & 7 & 1 & 115 & 0,1 & 05 & 100 & 25 & 5 & 40 & 0,058 & 0,0011 & & & & & & & & \\
\hline
\end{tabular}




\begin{tabular}{|c|c|c|c|c|c|c|c|c|c|c|c|c|c|c|c|c|c|c|c|c|c|}
\hline$\leq$ & 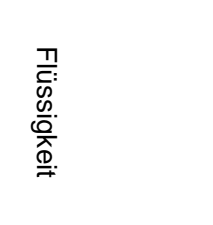 & 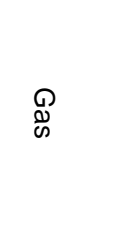 & 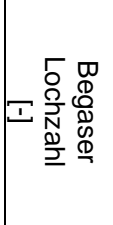 & 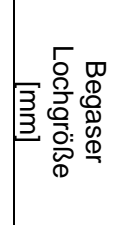 & 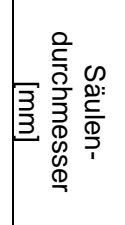 & 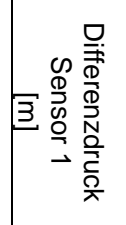 & 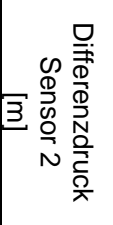 & 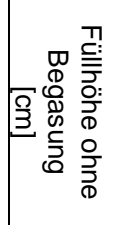 & 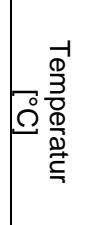 & 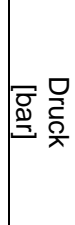 & 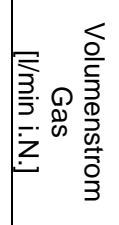 & 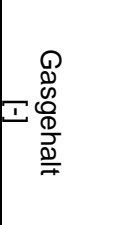 & 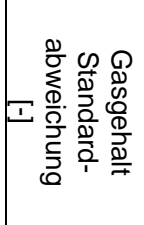 & 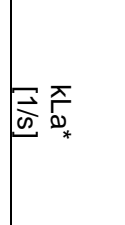 & 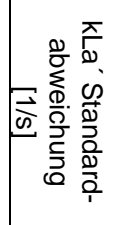 & 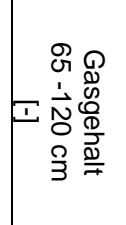 & 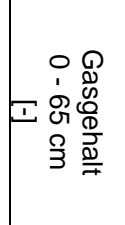 & 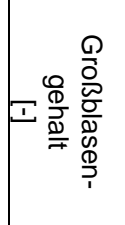 & 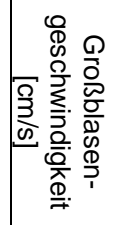 & 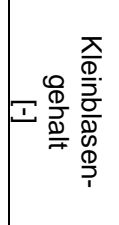 & 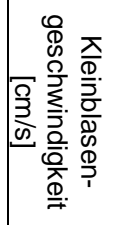 \\
\hline 679 & Leitungswasser & Stickstoff & 7 & 1 & 115 & 0,1 & 0,905 & 100 & 25 & 5 & 50 & & & 0,0387 & 0,0002 & & & & & & \\
\hline 680 & Leitungswasser & Stickstoff & 7 & 1 & 115 & 0,1 & 0,905 & 100 & 25 & 5 & 60 & 0,085 & 0,0022 & & & & & 0,034 & 32,9 & 0,051 & 20,7 \\
\hline 681 & Leitungswasser & Stickstoff & 7 & 1 & 115 & 0,1 & 0,905 & 100 & 25 & 5 & 80 & 0,117 & 0,0027 & & & & & 0,029 & 39,0 & 0,087 & 19,9 \\
\hline 682 & Leitungswasser & Stickstoff & 7 & 1 & 115 & 0,1 & 0,905 & 100 & 25 & 5 & 100 & 0,152 & 0,0027 & 0,0862 & 0,0019 & & & 0,030 & 38,8 & 0,121 & 19,4 \\
\hline 683 & Leitungswasser & Stickstoff & 7 & 1 & 115 & 0,1 & 0,905 & 100 & 25 & 5 & 120 & 0,195 & 0,0028 & & & & & 0,062 & 29,3 & 0,133 & 18,2 \\
\hline 684 & Leitungswasser & Stickstoff & 7 & 1 & 115 & 0,1 & 0,905 & 100 & 25 & 5 & 150 & & & 0,1344 & 0,0054 & & & & & & \\
\hline 685 & Leitungswasser & Stickstoff & 7 & 1 & 115 & 0,1 & 0,905 & 100 & 25 & 7 & 70 & 0,073 & 0,0011 & & & & & & & & \\
\hline 686 & Leitungswasser & Stickstoff & 7 & 1 & 115 & 0,1 & 0,905 & 100 & 25 & 7 & 105 & 0,121 & 0,0019 & & & & & 0,037 & 30,8 & 0,084 & 18,8 \\
\hline 687 & Leitungswasser & Stickstoff & 7 & 1 & 115 & 0,1 & 0,905 & 100 & 25 & 7 & 140 & 0,172 & 0,0051 & & & & & 0,054 & 25,4 & 0,119 & 17,8 \\
\hline 688 & Leitungswasser & Stickstoff & 7 & 1 & 115 & 0,1 & 0,905 & 100 & 25 & 7 & 175 & 0,238 & 0,0056 & & & & & 0,067 & 26,0 & 0,171 & 16,2 \\
\hline 689 & Leitungswasser & Stickstoff & 7 & 1 & 115 & 0,1 & 0,905 & 100 & 25 & 7 & 210 & 0,298 & 0,0062 & & & & & 0,124 & 20,9 & 0,174 & 15,1 \\
\hline 690 & Leitungswasser & Stickstoff & 7 & 1 & 115 & 0,1 & 0,905 & 100 & 25 & 7 & 250 & 0,357 & 0,0079 & & & & & 0,153 & 21,6 & 0,204 & 14,3 \\
\hline 691 & Leitungswasser & Stickstoff & 7 & 1 & 115 & 0,1 & 0,905 & 100 & 25 & 7 & 25 & & & 0,0146 & 0,0008 & & & & & & \\
\hline 692 & Leitungswasser & Stickstoff & 7 & 1 & 115 & 0,1 & 0,905 & 100 & 25 & 7 & 50 & & & 0,0294 & 0,0002 & & & & & & \\
\hline 693 & Leitungswasser & Stickstoff & 7 & 1 & 115 & 0,1 & 0,905 & 100 & 25 & 10 & 50 & 0,039 & 0,0009 & & & & & & & & \\
\hline 694 & Leitungswasser & Stickstoff & 7 & 1 & 115 & 0,1 & 0,905 & 100 & 25 & 10 & 100 & 0,085 & 0,0009 & & & & & & & & \\
\hline 695 & Leitungswasser & Stickstoff & 7 & 1 & 115 & 0,1 & 0,905 & 100 & 25 & 10 & 150 & 0,163 & 0,0063 & & & & & & & & \\
\hline 696 & Leitungswasser & Stickstoff & 7 & 1 & 115 & 0,1 & 0,905 & 100 & 25 & 10 & 200 & 0,243 & 0,0022 & & & & & & & & \\
\hline 697 & Leitungswasser & Stickstoff & 7 & 1 & 115 & 0,1 & 0,905 & 100 & 25 & 10 & 250 & 0,315 & 0,0097 & & & & & & & & \\
\hline 698 & Leitungswasser & Stickstoff & 7 & 1 & 115 & 0,1 & 0,905 & 100 & 25 & 10 & 300 & 0,366 & 0,0059 & & & & & & & & \\
\hline 699 & Leitungswasser & Stickstoff & 7 & 1 & 115 & 0,1 & 0,905 & 100 & 30 & 1 & 5 & & & 0,0152 & 0,0006 & & & & & & \\
\hline 700 & Leitungswasser & Stickstoff & 7 & 1 & 115 & 0,1 & 0,905 & 100 & 30 & 1 & 10 & 0,053 & 0,0011 & 0,0308 & 0,0004 & & & 0,030 & 43,3 & 0,023 & 22,1 \\
\hline 701 & Leitungswasser & Stickstoff & 7 & 1 & 115 & 0,1 & 0,905 & 100 & 30 & 1 & 20 & 0,087 & 0,0023 & 0,0485 & 0,0019 & & & 0,036 & 68,8 & 0,051 & 22,6 \\
\hline 702 & Leitungswasser & Stickstoff & 7 & 1 & 115 & 0,1 & 0,905 & 100 & 30 & 1 & 30 & 0,114 & 0,0026 & 0,0595 & 0,0014 & & & 0,047 & 83,2 & 0,067 & 21,7 \\
\hline 703 & Leitungswasser & Stickstoff & 7 & 1 & 115 & 0,1 & 0,905 & 100 & 30 & 1 & 40 & 0,139 & 0,0030 & & & & & 0,061 & 90,3 & 0,079 & 21,0 \\
\hline 704 & Leitungswasser & Stickstoff & 7 & 1 & 115 & 0,1 & 0,905 & 100 & 30 & 1 & 50 & 0,166 & 0,0033 & 0,0885 & 0,0030 & & & 0,062 & 112,1 & 0,104 & 20,8 \\
\hline 705 & Leitungswasser & Stickstoff & 7 & 1 & 115 & 0,1 & 0,905 & 100 & 40 & 1 & 10 & 0,054 & 0,0009 & & & & & 0,018 & 55,3 & 0,036 & 23,7 \\
\hline 706 & Leitungswasser & Stickstoff & 7 & 1 & 115 & 0,1 & 0,905 & 100 & 40 & 1 & 20 & 0,089 & 0,0028 & & & & & 0,035 & 69,5 & 0,054 & 22,7 \\
\hline
\end{tabular}




\begin{tabular}{|c|c|c|c|c|c|c|c|c|c|c|c|c|c|c|c|c|c|c|c|c|c|}
\hline$\underline{3}$ & 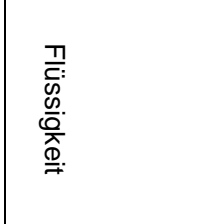 & ळ & 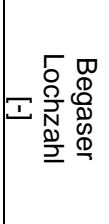 & 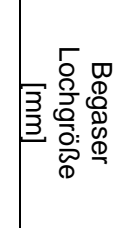 & 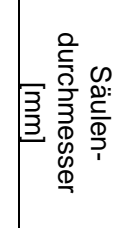 & 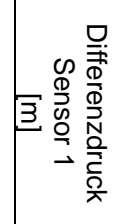 & 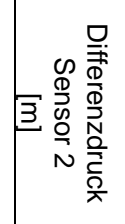 & 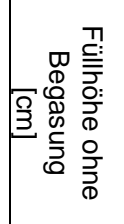 & 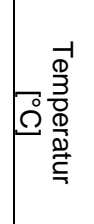 & 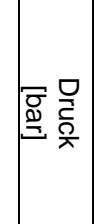 & 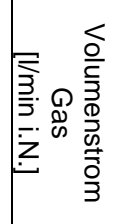 & 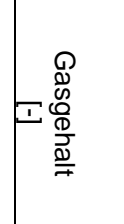 & 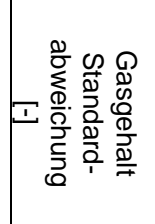 & 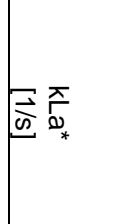 & 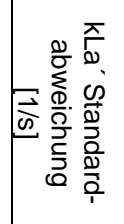 & 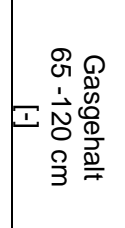 & 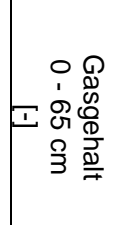 & 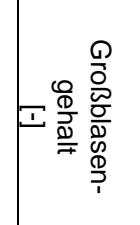 & 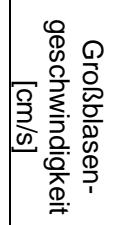 & 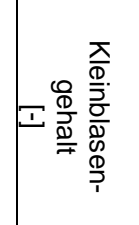 & 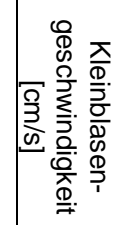 \\
\hline 707 & Leitungswasser & Stickstoff & 7 & 1 & 115 & 0,1 & 0,905 & 100 & 40 & 1 & 30 & 0,119 & 0,0056 & & & & & 0,053 & 78,0 & 0,066 & 22,0 \\
\hline 708 & Leitungswasser & Stickstoff & 7 & 1 & 115 & 0,1 & 0,905 & 100 & 40 & 1 & 40 & 0,141 & 0,0011 & & & & & 0,063 & 91,5 & 0,078 & 21,4 \\
\hline 709 & Leitungswasser & Stickstoff & 7 & 1 & 115 & 0,1 & 0,905 & 100 & 40 & 1 & 50 & 0,169 & 0,0022 & & & & & 0,080 & 92,1 & 0,088 & 20,7 \\
\hline 710 & Leitungswasser & Stickstoff & 7 & 1 & 115 & 0,1 & 0,905 & 100 & 50 & 1 & 3 & 0,029 & 0,0005 & & & & & & & & \\
\hline 711 & Leitungswasser & Stickstoff & 7 & 1 & 115 & 0,1 & 0,905 & 100 & 50 & 1 & 5 & 0,040 & 0,0011 & 0,0188 & & & & & & & \\
\hline 712 & Leitungswasser & Stickstoff & 7 & 1 & 115 & 0,1 & 0,905 & 100 & 50 & 1 & 10 & 0,057 & 0,0043 & 0,0335 & 0,0030 & & & 0,027 & 46,3 & 0,031 & 23,1 \\
\hline 713 & Leitungswasser & Stickstoff & 7 & 1 & 115 & 0,1 & 0,905 & 100 & 50 & 1 & 15 & 0,079 & 0,0019 & & & & & 0,043 & 48,6 & 0,035 & 21,4 \\
\hline 714 & Leitungswasser & Stickstoff & 7 & 1 & 115 & 0,1 & 0,905 & 100 & 50 & 1 & 20 & 0,093 & 0,0041 & 0,0492 & 0,0059 & & & 0,048 & 59,6 & 0,045 & 21,7 \\
\hline 715 & Leitungswasser & Stickstoff & 7 & 1 & 115 & 0,1 & 0,905 & 100 & 50 & 1 & 30 & 0,122 & 0,0041 & 0,0670 & 0,0057 & & & 0,060 & 74,0 & 0,062 & 21,4 \\
\hline 716 & Leitungswasser & Stickstoff & 7 & 1 & 115 & 0,1 & 0,905 & 100 & 50 & 1 & 40 & 0,148 & 0,0065 & & & & & 0,080 & 77,7 & 0,067 & 21,5 \\
\hline 717 & Leitungswasser & Stickstoff & 7 & 1 & 115 & 0,1 & 0,905 & 100 & 50 & 1 & 50 & 0,168 & 0,0057 & 0,1007 & 0,0075 & & & 0,090 & 87,7 & 0,078 & 20,8 \\
\hline 718 & Leitungswasser & Stickstoff & 7 & 1 & 115 & 0,1 & 0,905 & 100 & 70 & 1 & 5 & & & 0,0214 & 0,0038 & & & & & & \\
\hline 719 & Leitungswasser & Stickstoff & 7 & 1 & 115 & 0,1 & 0,905 & 100 & 70 & 1 & 10 & 0,057 & 0,0004 & 0,0388 & 0,0032 & & & 0,043 & 40,9 & 0,015 & 18,5 \\
\hline 720 & Leitungswasser & Stickstoff & 7 & 1 & 115 & 0,1 & 0,905 & 100 & 70 & 1 & 20 & 0,092 & 0,0020 & 0,0675 & 0,0051 & & & 0,063 & 54,1 & 0,029 & 22,1 \\
\hline 721 & Leitungswasser & Stickstoff & 7 & 1 & 115 & 0,1 & 0,905 & 100 & 70 & 1 & 30 & 0,124 & 0,0063 & 0,0914 & 0,0088 & & & 0,082 & 62,6 & 0,041 & 21,9 \\
\hline 722 & Leitungswasser & Stickstoff & 7 & 1 & 115 & 0,1 & 0,905 & 100 & 70 & 1 & 40 & 0,148 & 0,0071 & & & & & 0,105 & 68,3 & 0,043 & 21,2 \\
\hline 723 & Leitungswasser & Stickstoff & 7 & 1 & 115 & 0,1 & 0,905 & 100 & 70 & 1 & 50 & 0,169 & 0,0082 & 0,1212 & 0,0099 & & & 0,122 & 75,0 & 0,047 & 21,8 \\
\hline 724 & Toluol & Stickstoff & 19 & 1 & 102 & 0 & 1,2 & 130 & 20 & 1 & 4,7 & 0,035 & 0,0005 & 0,0229 & 0,0004 & & & 0,008 & 73,0 & 0,027 & 24,1 \\
\hline 725 & Toluol & Stickstoff & 19 & 1 & 102 & 0 & 1,2 & 130 & 20 & 1 & 9,4 & 0,075 & 0,0005 & 0,0417 & 0,0052 & & & 0,014 & 58,5 & 0,061 & 21,7 \\
\hline 726 & Toluol & Stickstoff & 19 & 1 & 102 & 0 & 1,2 & 130 & 20 & 1 & 23,6 & 0,130 & 0,0008 & 0,0776 & 0,0029 & & & 0,037 & 98,1 & 0,093 & 19,2 \\
\hline 727 & Toluol & Stickstoff & 19 & 1 & 102 & 0 & 1,2 & 130 & 20 & 1 & 47,1 & 0,186 & 0,0008 & 0,1239 & 0,0106 & & & 0,075 & 116,7 & 0,111 & 17,9 \\
\hline 728 & Toluol & Stickstoff & 19 & 1 & 102 & 0 & 1,2 & 130 & 20 & 1 & 94,2 & 0,276 & 0,0013 & 0,2022 & 0,0210 & & & 0,135 & 134,9 & 0,141 & 16,9 \\
\hline 729 & Toluol & Stickstoff & 19 & 1 & 102 & 0 & 1,2 & 130 & 20 & 2 & 9,4 & 0,036 & 0,0014 & 0,0221 & 0,0024 & & & 0,005 & 92,7 & 0,032 & 23,9 \\
\hline 730 & Toluol & Stickstoff & 19 & 1 & 102 & 0 & 1,2 & 130 & 20 & 2 & 18,9 & 0,080 & 0,0000 & 0,0421 & 0,0004 & & & 0,013 & 61,9 & 0,067 & 21,1 \\
\hline 731 & Toluol & Stickstoff & 19 & 1 & 102 & 0 & 1,2 & 130 & 20 & 2 & 47,1 & 0,149 & 0,0006 & 0,0874 & 0,0018 & & & 0,035 & 95,0 & 0,114 & 18,4 \\
\hline 732 & Toluol & Stickstoff & 19 & 1 & 102 & 0 & 1,2 & 130 & 20 & 2 & 94,3 & 0,227 & 0,0014 & 0,1483 & 0,0152 & & & 0,082 & 101,6 & 0,145 & 16,7 \\
\hline 733 & Toluol & Stickstoff & 19 & 1 & 102 & 0 & 1,2 & 130 & 20 & 2 & 188,5 & 0,329 & 0,0016 & 0,2153 & 0,0327 & & & 0,139 & 133,1 & 0,190 & 15,8 \\
\hline 734 & Toluol & Stickstoff & 19 & 1 & 102 & 0 & 1,2 & 130 & 20 & 5 & 23,6 & 0,038 & 0,0000 & 0,0261 & 0,0009 & & & 0,010 & 58,6 & 0,029 & 22,7 \\
\hline
\end{tabular}




\begin{tabular}{|c|c|c|c|c|c|c|c|c|c|c|c|c|c|c|c|c|c|c|c|c|c|}
\hline$\underline{z}$ & 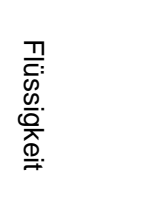 & 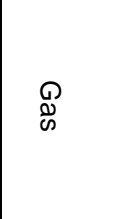 & 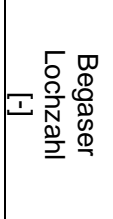 & 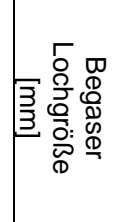 & 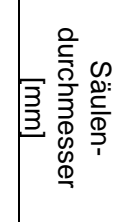 & 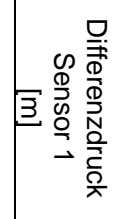 & 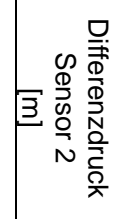 & 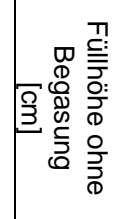 & 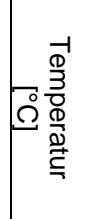 & 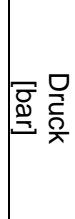 & 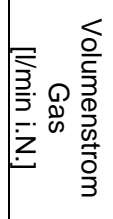 & 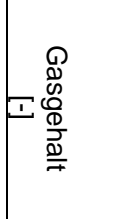 & 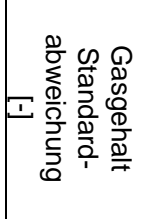 & 至 $\frac{\pi}{\omega_{*}}$ & 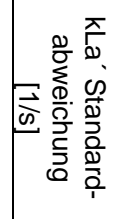 & 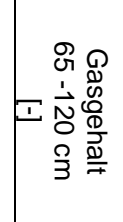 & 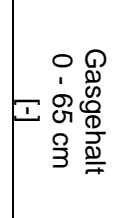 & 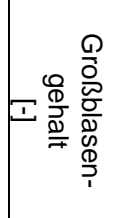 & 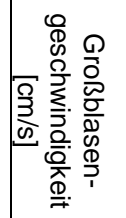 & 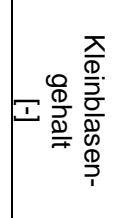 & 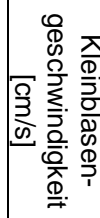 \\
\hline 735 & Toluol & Stickstoff & 19 & 1 & 102 & 0 & 1,2 & 130 & 20 & 5 & 47,1 & 0,085 & 0,0006 & 0,0481 & 0,0007 & & & 0,009 & 73,9 & 0,076 & 20,4 \\
\hline 736 & Toluol & Stickstoff & 19 & 1 & 102 & 0 & 1,2 & 130 & 20 & 5 & 117,8 & 0,198 & 0,0010 & 0,1087 & 0,0022 & & & 0,037 & 71,7 & 0,162 & 17,0 \\
\hline 737 & Toluol & Stickstoff & 19 & 1 & 102 & 0 & 1,2 & 130 & 20 & 5 & 235,6 & 0,293 & 0,0030 & 0,1733 & 0,0112 & & & 0,079 & 92,8 & 0,213 & 15,9 \\
\hline 738 & Toluol & Stickstoff & 19 & 1 & 102 & 0 & 1,2 & 130 & 20 & 10 & 47,1 & 0,038 & 0,0000 & 0,0241 & 0,0008 & & & 0,002 & 151,6 & 0,036 & 23,2 \\
\hline 739 & Toluol & Stickstoff & 19 & 1 & 102 & 0 & 1,2 & 130 & 20 & 10 & 94,3 & 0,101 & 0,0006 & 0,0525 & 0,0024 & & & 0,010 & 41,5 & 0,091 & 19,0 \\
\hline 740 & Toluol & Stickstoff & 19 & 1 & 102 & 0 & 1,2 & 130 & 20 & 10 & 235,6 & 0,242 & 0,0005 & 0,1199 & 0,0065 & & & 0,031 & 65,0 & 0,211 & 16,2 \\
\hline 741 & Toluol & Stickstoff & 19 & 1 & 102 & 0 & 1,2 & 130 & 20 & 10 & 471,3 & 0,351 & 0,0053 & 0,1788 & 0,0024 & & & 0,086 & 79,0 & 0,264 & 15,0 \\
\hline 742 & Toluol & Stickstoff & 19 & 1 & 102 & 0 & 1,2 & 130 & 20 & 20 & 94,3 & 0,046 & 0,0010 & 0,0277 & 0,0010 & & & 0,013 & 58,0 & 0,033 & 20,2 \\
\hline 743 & Toluol & Stickstoff & 19 & 1 & 102 & 0 & 1,2 & 130 & 20 & 20 & 188,5 & 0,106 & 0,0010 & 0,0496 & 0,0026 & & & 0,009 & 41,6 & 0,097 & 18,2 \\
\hline 744 & Toluol & Stickstoff & 19 & 1 & 102 & 0 & 1,2 & 130 & 20 & 20 & 471,3 & 0,280 & 0,0050 & 0,1174 & 0,0074 & & & 0,037 & 44,4 & 0,242 & 15,3 \\
\hline 745 & Toluol & Stickstoff & 19 & 1 & 102 & 0 & 1,2 & 130 & 20 & 20 & 942,5 & 0,424 & & & & & & 0,142 & 47,6 & 0,282 & 14,0 \\
\hline 746 & Toluol & Stickstoff & 19 & 1 & 102 & 0 & 1,2 & 130 & 20 & 40 & 188,5 & 0,049 & 0,0010 & 0,0235 & 0,0020 & & & 0,010 & 53,5 & 0,039 & 19,5 \\
\hline 747 & Toluol & Stickstoff & 19 & 1 & 102 & 0 & 1,2 & 130 & 20 & 40 & 377 & 0,111 & 0,0000 & 0,0479 & 0,0020 & & & 0,007 & 48,7 & 0,103 & 17,4 \\
\hline 748 & Toluol & Stickstoff & 19 & 1 & 102 & 0 & 1,2 & 130 & 20 & 40 & 942,5 & 0,321 & 0,0051 & 0,1258 & 0,0142 & & & 0,093 & 23,9 & 0,228 & 14,2 \\
\hline 749 & Toluol & Helium & 19 & 1 & 102 & 0 & 1,2 & 130 & 20 & 1 & 4,7 & 0,029 & & & & & & 0,003 & 210,3 & 0,026 & 26,5 \\
\hline 750 & Toluol & Helium & 19 & 1 & 102 & 0 & 1,2 & 130 & 20 & 1 & 9,4 & 0,058 & & & & & & 0,017 & 71,7 & 0,041 & 22,8 \\
\hline 751 & Toluol & Helium & 19 & 1 & 102 & 0 & 1,2 & 130 & 20 & 1 & 23,6 & 0,102 & & & & & & 0,044 & 93,3 & 0,058 & 21,5 \\
\hline 752 & Toluol & Helium & 19 & 1 & 102 & 0 & 1,2 & 130 & 20 & 1 & 47,1 & 0,145 & & & & & & 0,075 & 123,3 & 0,071 & 21,5 \\
\hline 753 & Toluol & Helium & 19 & 1 & 102 & 0 & 1,2 & 130 & 20 & 1 & 94,2 & 0,217 & & & & & & 0,141 & 141,1 & 0,076 & 20,8 \\
\hline 754 & Toluol & Helium & 19 & 1 & 102 & 0 & 1,2 & 130 & 20 & 3 & 14,1 & 0,032 & & & & & & 0,004 & 83,3 & 0,028 & 25,5 \\
\hline 755 & Toluol & Helium & 19 & 1 & 102 & 0 & 1,2 & 130 & 20 & 3 & 28,2 & 0,064 & & & & & & 0,018 & 61,5 & 0,045 & 22,8 \\
\hline 756 & Toluol & Helium & 19 & 1 & 102 & 0 & 1,2 & 130 & 20 & 3 & 70,8 & 0,108 & & & & & & 0,043 & 92,4 & 0,065 & 21,1 \\
\hline 757 & Toluol & Helium & 19 & 1 & 102 & 0 & 1,2 & 130 & 20 & 3 & 141,3 & 0,163 & & & & & & 0,080 & 113,3 & 0,082 & 20,2 \\
\hline 758 & Toluol & Helium & 19 & 1 & 102 & 0 & 1,2 & 130 & 20 & 3 & 282,6 & 0,233 & & & & & & 0,145 & 136,4 & 0,088 & 20,2 \\
\hline 759 & Toluol & Stickstoff & 1 & 4,3 & 102 & 0 & 1,2 & 130 & 20 & 1 & 4,7 & 0,031 & 0,0005 & 0,0198 & 0,0005 & & & & & & \\
\hline 760 & Toluol & Stickstoff & 1 & 4,3 & 102 & 0 & 1,2 & 130 & 20 & 1 & 9,4 & 0,060 & 0,0005 & 0,0385 & 0,0011 & & & & & & \\
\hline 761 & Toluol & Stickstoff & 1 & 4,3 & 102 & 0 & 1,2 & 130 & 20 & 1 & 23,6 & 0,118 & 0,0005 & 0,0808 & 0,0027 & & & & & & \\
\hline 762 & Toluol & Stickstoff & 1 & 4,3 & 102 & 0 & 1,2 & 130 & 20 & 1 & 47,1 & 0,179 & 0,0008 & 0,1385 & 0,0064 & & & & & & \\
\hline
\end{tabular}




\begin{tabular}{|c|c|c|c|c|c|c|c|c|c|c|c|c|c|c|c|c|c|c|c|c|c|}
\hline$\underline{z}$ & 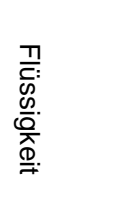 & 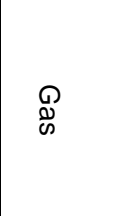 & 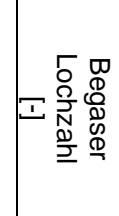 & 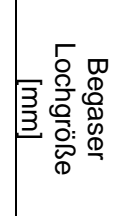 & 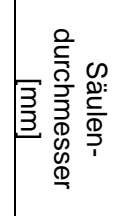 & 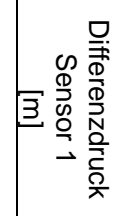 & 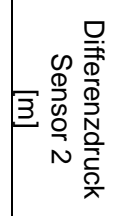 & 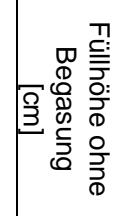 & 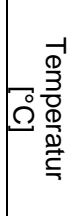 & סृ & 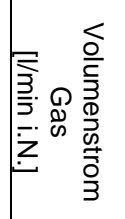 & 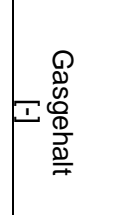 & 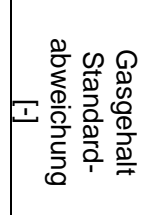 & $\begin{array}{l}\vec{\sigma} \\
\vec{\omega} \\
D_{*}\end{array}$ & 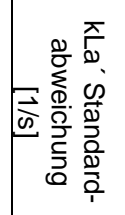 & 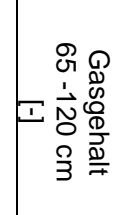 & 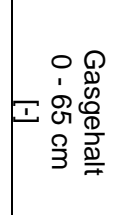 & 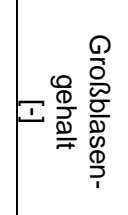 & 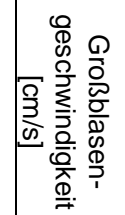 & 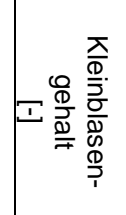 & 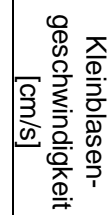 \\
\hline 763 & Toluol & Stickstoff & 1 & 4,3 & 102 & 0 & 1,2 & 130 & 20 & 1 & 94,2 & 0,281 & 0,0037 & 0,2261 & 0,0171 & & & & & & \\
\hline 764 & Toluol & Stickstoff & 1 & 4,3 & 102 & 0 & 1,2 & 130 & 20 & 2 & 9,4 & 0,033 & 0,0006 & 0,0204 & 0,0008 & & & & & & \\
\hline 765 & Toluol & Stickstoff & 1 & 4,3 & 102 & 0 & 1,2 & 130 & 20 & 2 & 18,9 & 0,065 & 0,0006 & 0,0413 & 0,0008 & & & & & & \\
\hline 766 & Toluol & Stickstoff & 1 & 4,3 & 102 & 0 & 1,2 & 130 & 20 & 2 & 47,1 & 0,135 & 0,0006 & 0,0938 & 0,0010 & & & & & & \\
\hline 767 & Toluol & Stickstoff & 1 & 4,3 & 102 & 0 & 1,2 & 130 & 20 & 2 & 94,3 & 0,228 & 0,0030 & 0,1743 & 0,0051 & & & & & & \\
\hline 768 & Toluol & Stickstoff & 1 & 4,3 & 102 & 0 & 1,2 & 130 & 20 & 2 & 188,5 & 0,355 & 0,0034 & 0,2489 & 0,0195 & & & & & & \\
\hline 769 & Toluol & Stickstoff & 1 & 4,3 & 102 & 0 & 1,2 & 130 & 20 & 5 & 23,6 & 0,035 & 0,0000 & 0,0223 & 0,0009 & & & & & & \\
\hline 770 & Toluol & Stickstoff & 1 & 4,3 & 102 & 0 & 1,2 & 130 & 20 & 5 & 47,1 & 0,074 & 0,0000 & 0,0451 & 0,0008 & & & & & & \\
\hline 771 & Toluol & Stickstoff & 1 & 4,3 & 102 & 0 & 1,2 & 130 & 20 & 5 & 117,8 & 0,190 & 0,0006 & 0,1140 & 0,0037 & & & & & & \\
\hline 772 & Toluol & Stickstoff & 1 & 4,3 & 102 & 0 & 1,2 & 130 & 20 & 5 & 235,6 & 0,312 & 0,0023 & 0,1662 & 0,0034 & & & & & & \\
\hline 773 & Toluol & Stickstoff & 1 & 4,3 & 102 & 0 & 1,2 & 130 & 20 & 5 & 471,3 & & & & & & & & & & \\
\hline 774 & Toluol & Stickstoff & 1 & 4,3 & 102 & 0 & 1,2 & 130 & 20 & 10 & 47,1 & 0,038 & 0,0006 & 0,0242 & 0,0014 & & & & & & \\
\hline 775 & Toluol & Stickstoff & 1 & 4,3 & 102 & 0 & 1,2 & 130 & 20 & 10 & 94,3 & 0,101 & 0,0000 & 0,0580 & 0,0025 & & & & & & \\
\hline 776 & Toluol & Stickstoff & 1 & 4,3 & 102 & 0 & 1,2 & 130 & 20 & 10 & 235,6 & 0,248 & 0,0025 & 0,1225 & 0,0049 & & & & & & \\
\hline 777 & Toluol & Stickstoff & 1 & 4,3 & 102 & 0 & 1,2 & 130 & 20 & 10 & 471,3 & 0,386 & 0,0035 & 0,1868 & 0,0056 & & & & & & \\
\hline 778 & Toluol & Stickstoff & 1 & 4,3 & 102 & 0 & 1,2 & 130 & 20 & 20 & 94,3 & 0,048 & 0,0000 & 0,0295 & 0,0015 & & & & & & \\
\hline 779 & Toluol & Stickstoff & 1 & 4,3 & 102 & 0 & 1,2 & 130 & 20 & 20 & 188,5 & 0,109 & 0,0014 & 0,0593 & 0,0030 & & & & & & \\
\hline 780 & Toluol & Stickstoff & 1 & 4,3 & 102 & 0 & 1,2 & 130 & 20 & 20 & 471,3 & 0,292 & 0,0031 & 0,1345 & 0,0072 & & & & & & \\
\hline 781 & Toluol & Stickstoff & 1 & 4,3 & 102 & 0 & 1,2 & 130 & 20 & 20 & 942,5 & & & & & & & & & & \\
\hline 782 & Toluol & Stickstoff & 1 & 4,3 & 102 & 0 & 1,2 & 130 & 20 & 40 & 188,5 & 0,053 & 0,0007 & 0,0290 & 0,0004 & & & & & & \\
\hline 783 & Toluol & Stickstoff & 1 & 4,3 & 102 & 0 & 1,2 & 130 & 20 & 40 & 377 & 0,117 & 0,0000 & 0,0527 & 0,0014 & & & & & & \\
\hline 784 & Toluol & Stickstoff & 1 & 4,3 & 102 & 0 & 1,2 & 130 & 20 & 40 & 942,5 & & & & & & & & & & \\
\hline 785 & Toluol & Stickstoff & 1 & 4,3 & 102 & 0 & 1,2 & 130 & 20 & 40 & 1885 & & & & & & & & & & \\
\hline 786 & Toluol & Helium & 1 & 4,3 & 102 & 0 & 1,2 & 130 & 20 & 1 & 4,7 & 0,028 & 0,0000 & 0,0163 & 0,0002 & & & & & & \\
\hline 787 & Toluol & Helium & 1 & 4,3 & 102 & 0 & 1,2 & 130 & 20 & 1 & 9,4 & 0,052 & 0,0006 & 0,0319 & 0,0013 & & & & & & \\
\hline 788 & Toluol & Helium & 1 & 4,3 & 102 & 0 & 1,2 & 130 & 20 & 1 & 23,6 & 0,094 & 0,0006 & 0,0654 & 0,0017 & & & & & & \\
\hline 789 & Toluol & Helium & 1 & 4,3 & 102 & 0 & 1,2 & 130 & 20 & 1 & 47,1 & 0,137 & 0,0021 & 0,1028 & 0,0023 & & & & & & \\
\hline 790 & Toluol & Helium & 1 & 4,3 & 102 & 0 & 1,2 & 130 & 20 & 1 & 94,2 & 0,211 & 0,0019 & 0,1760 & 0,0220 & & & & & & \\
\hline
\end{tabular}




\begin{tabular}{|c|c|c|c|c|c|c|c|c|c|c|c|c|c|c|c|c|c|c|c|c|c|}
\hline$\underline{3}$ & 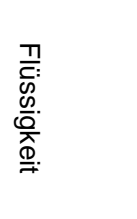 & ه్ల & 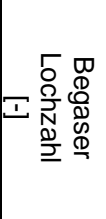 & 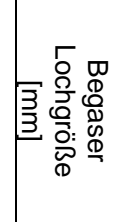 & 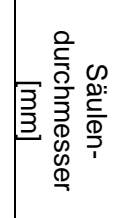 & 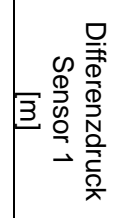 & • & 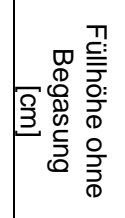 & 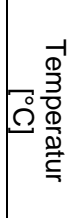 & 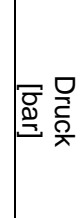 & 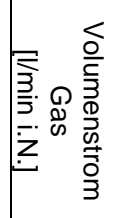 & 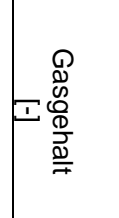 & 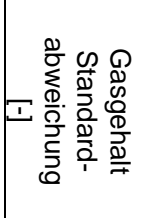 & 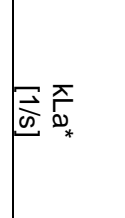 & 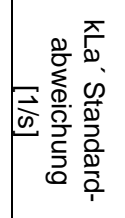 & 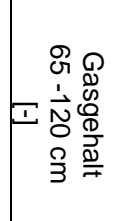 & 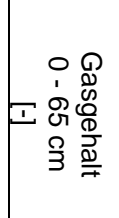 & 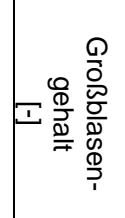 & 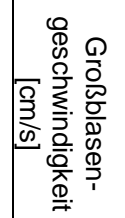 & 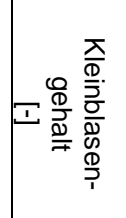 & 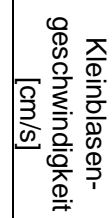 \\
\hline 791 & Toluol & Helium & 1 & 4,3 & 102 & 0 & 1,2 & 130 & 20 & 2 & 9,4 & 0,029 & 0,0000 & 0,0160 & 0,0003 & & & & & & \\
\hline 792 & Toluol & Helium & 1 & 4,3 & 102 & 0 & 1,2 & 130 & 20 & 2 & 18,9 & 0,053 & 0,0000 & 0,0314 & 0,0004 & & & & & & \\
\hline 793 & Toluol & Helium & 1 & 4,3 & 102 & 0 & 1,2 & 130 & 20 & 2 & 47,1 & 0,096 & 0,0010 & 0,0670 & 0,0015 & & & & & & \\
\hline 794 & Toluol & Helium & 1 & 4,3 & 102 & 0 & 1,2 & 130 & 20 & 2 & 94,3 & 0,151 & 0,0011 & 0,1141 & 0,0075 & & & & & & \\
\hline 795 & Toluol & Helium & 1 & 4,3 & 102 & 0 & 1,2 & 130 & 20 & 2 & 188,5 & 0,218 & 0,0023 & 0,1742 & 0,0242 & & & & & & \\
\hline 796 & Toluol & Helium & 1 & 4,3 & 102 & 0 & 1,2 & 130 & 20 & 4 & 18,8 & 0,030 & 0,0006 & 0,0168 & 0,0005 & & & & & & \\
\hline 797 & Toluol & Helium & 1 & 4,3 & 102 & 0 & 1,2 & 130 & 20 & 4 & 37,7 & 0,053 & 0,0006 & 0,0314 & 0,0003 & & & & & & \\
\hline 798 & Toluol & Helium & 1 & 4,3 & 102 & 0 & 1,2 & 130 & 20 & 4 & 94,2 & 0,106 & 0,0006 & 0,0732 & 0,0016 & & & & & & \\
\hline 799 & Toluol & Helium & 1 & 4,3 & 102 & 0 & 1,2 & 130 & 20 & 4 & 188,5 & 0,159 & 0,0010 & 0,1236 & 0,0010 & & & & & & \\
\hline 800 & Toluol & Helium & 1 & 4,3 & 102 & 0 & 1,2 & 130 & 20 & 4 & 377 & 0,235 & 0,0042 & 0,2065 & 0,0194 & & & & & & \\
\hline 801 & Toluol & Stickstoff & 7 & 1 & 115 & 0,1 & 0,905 & 100 & 20 & 1 & 6,2 & 0,048 & 0,0011 & & & & & 0,024 & 26,3 & 0,025 & 18,5 \\
\hline 802 & Toluol & Stickstoff & 7 & 1 & 115 & 0,1 & 0,905 & 100 & 20 & 1 & 12,5 & 0,077 & 0,0007 & & & & & 0,022 & 49,6 & 0,055 & 19,1 \\
\hline 803 & Toluol & Stickstoff & 7 & 1 & 115 & 0,1 & 0,905 & 100 & 20 & 1 & 18,7 & 0,104 & 0,0021 & & & & & 0,030 & 61,0 & 0,074 & 18,7 \\
\hline 804 & Toluol & Stickstoff & 7 & 1 & 115 & 0,1 & 0,905 & 100 & 20 & 1 & 31,2 & 0,146 & 0,0009 & & & & & 0,050 & 73,9 & 0,096 & 17,4 \\
\hline 805 & Toluol & Stickstoff & 7 & 1 & 115 & 0,1 & 0,905 & 100 & 20 & 1 & 43,6 & 0,184 & 0,0024 & & & & & 0,058 & 91,8 & 0,126 & 17,4 \\
\hline 806 & Toluol & Stickstoff & 7 & 1 & 115 & 0,1 & 0,905 & 100 & 20 & 1 & 62,3 & 0,231 & 0,0082 & & & & & 0,074 & 109,9 & 0,157 & 17,2 \\
\hline 807 & Toluol & Stickstoff & 7 & 1 & 115 & 0,1 & 0,905 & 100 & 20 & 1 & 5 & 0,046 & & 0,0301 & 0,0004 & & & & & & \\
\hline 808 & Toluol & Stickstoff & 7 & 1 & 115 & 0,1 & 0,905 & 100 & 20 & 1 & 10 & 0,074 & & 0,0447 & 0,0005 & & & & & & \\
\hline 809 & Toluol & Stickstoff & 7 & 1 & 115 & 0,1 & 0,905 & 100 & 20 & 1 & 20 & 0,118 & & 0,0753 & 0,0027 & & & & & & \\
\hline 810 & Toluol & Stickstoff & 7 & 1 & 115 & 0,1 & 0,905 & 100 & 20 & 1 & 30 & 0,155 & & 0,1133 & 0,0036 & & & & & & \\
\hline 811 & Toluol & Stickstoff & 7 & 1 & 115 & 0,1 & 0,905 & 100 & 20 & 1 & 50 & 0,220 & & 0,1426 & 0,0063 & & & & & & \\
\hline 812 & Toluol & Stickstoff & 7 & 1 & 115 & 0,1 & 0,905 & 100 & 20 & 1 & 75 & 0,287 & & 0,2419 & 0,0232 & & & & & & \\
\hline 813 & Toluol & Stickstoff & 7 & 1 & 115 & 0,1 & 0,905 & 100 & 20 & 1 & 100 & 0,344 & & 0,2964 & 0,0314 & & & & & & \\
\hline 814 & Toluol & Stickstoff & 7 & 1 & 115 & 0,1 & 0,905 & 100 & 20 & 2 & 10 & 0,044 & & 0,0274 & 0,0015 & & & & & & \\
\hline 815 & Toluol & Stickstoff & 7 & 1 & 115 & 0,1 & 0,905 & 100 & 20 & 2 & 20 & 0,084 & & 0,0515 & 0,0045 & & & & & & \\
\hline 816 & Toluol & Stickstoff & 7 & 1 & 115 & 0,1 & 0,905 & 100 & 20 & 2 & 40 & 0,144 & & 0,0892 & 0,0050 & & & & & & \\
\hline 817 & Toluol & Stickstoff & 7 & 1 & 115 & 0,1 & 0,905 & 100 & 20 & 2 & 60 & 0,189 & & 0,1322 & 0,0113 & & & & & & \\
\hline 818 & Toluol & Stickstoff & 7 & 1 & 115 & 0,1 & 0,905 & 100 & 20 & 2 & 100 & 0,291 & & 0,2238 & 0,0148 & & & & & & \\
\hline
\end{tabular}




\begin{tabular}{|c|c|c|c|c|c|c|c|c|c|c|c|c|c|c|c|c|c|c|c|c|c|}
\hline$\underline{3}$ & 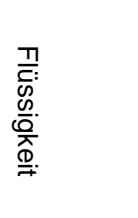 & 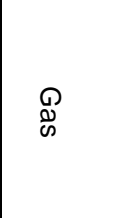 & 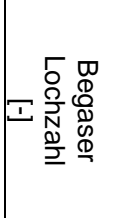 & 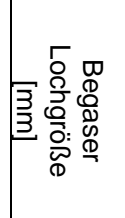 & 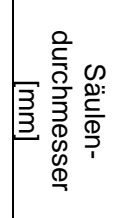 & 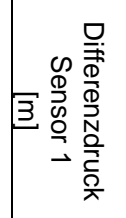 & 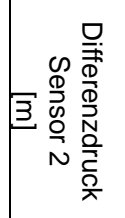 & 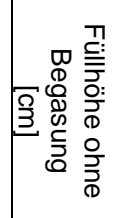 & 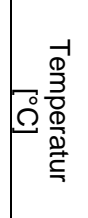 & 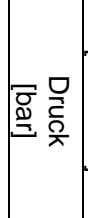 & 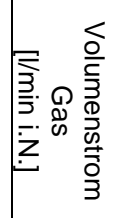 & 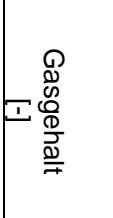 & 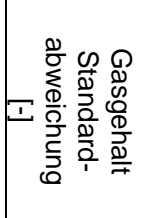 & 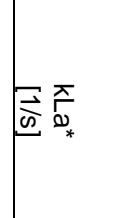 & 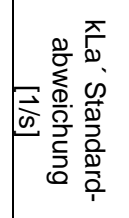 & 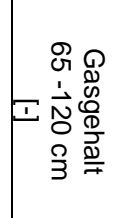 & 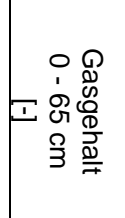 & 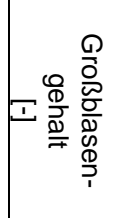 & 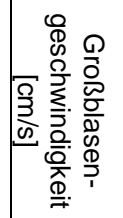 & 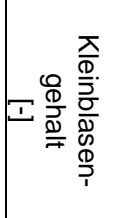 & 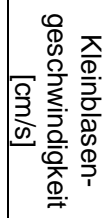 \\
\hline 819 & Toluol & Stickstoff & 7 & 1 & 115 & 0,1 & 0,905 & 100 & 20 & 2 & 150 & 0,407 & & 0,2894 & 0,0382 & & & & & & \\
\hline 820 & Toluol & Stickstoff & 7 & 1 & 115 & 0,1 & 0,905 & 100 & 20 & 2 & 200 & 0,494 & & 0,3157 & 0,0404 & & & & & & \\
\hline 821 & Toluol & Stickstoff & 7 & 1 & 115 & 0,1 & 0,905 & 100 & 20 & 2 & 12,5 & 0,050 & 0,0009 & & & & & & & & \\
\hline 822 & Toluol & Stickstoff & 7 & 1 & 115 & 0,1 & 0,905 & 100 & 20 & 2 & 24,9 & 0,088 & 0,0010 & & & & & 0,021 & 43,4 & 0,067 & 18,4 \\
\hline 823 & Toluol & Stickstoff & 7 & 1 & 115 & 0,1 & 0,905 & 100 & 20 & 2 & 37,4 & 0,123 & 0,0008 & & & & & 0,026 & 55,9 & 0,097 & 18,3 \\
\hline 824 & Toluol & Stickstoff & 7 & 1 & 115 & 0,1 & 0,905 & 100 & 20 & 2 & 62,3 & 0,187 & 0,0039 & & & & & 0,051 & 61,0 & 0,136 & 17,4 \\
\hline 825 & Toluol & Stickstoff & 7 & 1 & 115 & 0,1 & 0,905 & 100 & 20 & 2 & 87,3 & 0,251 & 0,0030 & & & & & 0,073 & 63,1 & 0,179 & 17,1 \\
\hline 826 & Toluol & Stickstoff & 7 & 1 & 115 & 0,1 & 0,905 & 100 & 20 & 2 & 124,6 & 0,334 & 0,0014 & & & & & 0,091 & 73,5 & 0,243 & 16,7 \\
\hline 827 & Toluol & Stickstoff & 7 & 1 & 115 & 0,1 & 0,905 & 100 & 20 & 5 & 25 & 0,048 & & 0,0297 & 0,0016 & & & & & & \\
\hline 828 & Toluol & Stickstoff & 7 & 1 & 115 & 0,1 & 0,905 & 100 & 20 & 5 & 50 & 0,091 & & 0,0559 & 0,0026 & & & & & & \\
\hline 829 & Toluol & Stickstoff & 7 & 1 & 115 & 0,1 & 0,905 & 100 & 20 & 5 & 100 & 0,193 & & 0,1019 & 0,0029 & & & & & & \\
\hline 830 & Toluol & Stickstoff & 7 & 1 & 115 & 0,1 & 0,905 & 100 & 20 & 5 & 150 & 0,284 & & 0,1449 & 0,0145 & & & & & & \\
\hline 831 & Toluol & Stickstoff & 7 & 1 & 115 & 0,1 & 0,905 & 100 & 20 & 5 & 250 & 0,415 & & 0,2477 & 0,0419 & & & & & & \\
\hline 832 & Toluol & Stickstoff & 7 & 1 & 115 & 0,1 & 0,905 & 100 & 20 & 5 & 31,2 & 0,051 & 0,0000 & & & & & & & & \\
\hline 833 & Toluol & Stickstoff & 7 & 1 & 115 & 0,1 & 0,905 & 100 & 20 & 5 & 62,3 & 0,104 & 0,0011 & & & & & 0,014 & 39,2 & 0,090 & 17,7 \\
\hline 834 & Toluol & Stickstoff & 7 & 1 & 115 & 0,1 & 0,905 & 100 & 20 & 5 & 93,5 & 0,171 & 0,0035 & & & & & 0,047 & 26,1 & 0,125 & 16,0 \\
\hline 835 & Toluol & Stickstoff & 7 & 1 & 115 & 0,1 & 0,905 & 100 & 20 & 5 & 155,8 & 0,285 & 0,0015 & & & & & 0,069 & 30,9 & 0,215 & 15,2 \\
\hline 836 & Toluol & Stickstoff & 7 & 1 & 115 & 0,1 & 0,905 & 100 & 20 & 7 & 25 & 0,027 & & & & & & & & & \\
\hline 837 & Toluol & Stickstoff & 7 & 1 & 115 & 0,1 & 0,905 & 100 & 20 & 7 & 50 & 0,062 & & & & & & & & & \\
\hline 838 & Toluol & Stickstoff & 7 & 1 & 115 & 0,1 & 0,905 & 100 & 20 & 7 & 100 & 0,138 & & & & & & & & & \\
\hline 839 & Toluol & Stickstoff & 7 & 1 & 115 & 0,1 & 0,905 & 100 & 20 & 7 & 150 & 0,222 & & & & & & & & & \\
\hline 840 & Toluol & Stickstoff & 7 & 1 & 115 & 0,1 & 0,905 & 100 & 20 & 7 & 250 & 0,345 & & & & & & & & & \\
\hline 841 & Toluol & Stickstoff & 7 & 1 & 115 & 0,1 & 0,905 & 100 & 20 & 10 & 50 & 0,040 & & 0,0311 & 0,0025 & & & & & & \\
\hline 842 & Toluol & Stickstoff & 7 & 1 & 115 & 0,1 & 0,905 & 100 & 20 & 10 & 100 & 0,086 & & 0,0672 & 0,0008 & & & & & & \\
\hline 843 & Toluol & Stickstoff & 7 & 1 & 115 & 0,1 & 0,905 & 100 & 20 & 10 & 200 & 0,210 & & 0,1365 & 0,0110 & & & & & & \\
\hline 844 & Toluol & Stickstoff & 7 & 1 & 115 & 0,1 & 0,905 & 100 & 20 & 10 & 250 & 0,281 & & & & & & & & & \\
\hline 845 & Toluol & Stickstoff & 7 & 1 & 115 & 0,1 & 0,905 & 100 & 20 & 10 & 300 & 0,000 & & 0,1815 & 0,0097 & & & & & & \\
\hline
\end{tabular}





\section{Lebenslauf \\ Dipl.-Chem. Uwe Jordan}

Wohnort: Worpswede, Niedersachsen, Deutschland

Geburtsdatum, -ort: 10.08.1971 Potsdam

Beruflicher Werdegang

seit Mai $2015 \quad$ Friedrich-Vorwerk KG, Tostedt

Projektingenieur

$2008-2015 \quad$ MT-Biomethan $\mathrm{GmbH}$, Zeven

Verfahrenstechnische Entwicklung

$2002-2008 \quad$ Dr. Wolfgang Koczott chem.-techn. Betriebs-GmbH, Ritterhude

Produktionsleitung

Studium

1997 - $2002 \quad$ Institut für technische Chemie der TU Braunschweig

Doktorand

1991 - $1997 \quad$ TU Braunschweig,

Abschluss: Diplom-Chemiker

\section{Schule / Zivildienst}

$1990-1991 \quad$ Zivildienst

1978 - $1990 \quad$ Schule

Abschluss: Abitur 